\section{Poster Session I}

Palm Springs, California, December 3-7, 2017

Sponsorship Statement: Publication of this supplement is sponsored by the ACNP.

Individual contributor disclosures may be found within the abstracts. Part 1: All Financial Involvement with a pharmaceutical or biotechnology company, a company providing clinical assessment, scientific, or medical products or companies doing business with or proposing to do business with ACNP over past 2 years (Calendar Years 2014-Present); Part 2: Income Sources \& Equity of $\$ 10,000$ per year or greater (Calendar Years 2014 - Present): List those financial relationships which are listed in part one and have a value greater than $\$ 10,000$ per year, OR financial holdings that are listed in part one and have a value of $\$ 10,000$ or greater as of the date of disclosure; Part 3: Financial Involvement with a pharmaceutical or biotechnology company, a company providing clinical assessment, scientific, or medical products or companies doing business with or proposing to do business with ACNP which constitutes more than 5\% of personal income (Calendar Years 2014 - Present); Part 4: Grants from pharmaceutical or biotechnology company, a company providing clinical assessment, scientific, or medical products directly, or indirectly through a foundation, university, or any other organization (Calendar Years 2014 - Present); Part 5: My primary employer is a pharmaceutical/biotech/medical device company.

Asterisks in the author lists indicate presenter of the abstract at the annual meeting.

M1. Alterations in Glucocorticoid, Immune, and Metabolic Regulation are Associated With Post-Traumatic Stress Disorder in African-American Women With Type II Diabetes Mellitus

Charles Gillespie*, Drew Dixon, Rachel Gluck, Adam Munoz, Hadrian Mendoza, Cleo Rochat, Sierra Carter, Tanja Jovanovic, Negar Fani, Abigail Lott, Bekh Bradley, Kerry Ressler, Guillermo Umpierrez, Ann Schwartz, Thaddeus Pace, Vasiliki Michopoulos

Emory University School of Medicine, Atlanta, Georgia, United States

Background: Post-traumatic stress disorder (PTSD) is a stress-related psychiatric disorder that is associated with increased risk for type 2 diabetes mellitus (T2DM). However, the biological mechanisms by which PTSD increases risks for T2DM remain unclear. A growing body of data suggests that dysregulation of the stress axis, including altered glucocorticoid sensitivity and innate immune activity, may act to disrupt glycemic control in trauma-exposed individuals with PTSD. To evaluate the association between PTSD, glycemic regulation, neuroendocrine function and immune activity, we examined associations between PTSD diagnosis and PTSD symptom severity and metabolic, glucocorticoid, and immune biomarkers in trauma-exposed women with T2DM.
Methods: We recruited a sample of $(N=63)$ AfricanAmerican women with T2DM and high rates of lifetime trauma exposure from the primary care and diabetes specialty clinic waiting rooms of Grady Memorial Hospital, a large county hospital and level 1 trauma center in Atlanta, Georgia. PTSD diagnosis and PTSD symptom severity were determined using the Clinician-Administered PTSD Scale (CAPS). A fasting blood sample was collected and a study physician performed a general medical assessment for each participant. Blood was assayed for hemoglobin A1c (HbA1c) and calculation of average estimated glucose (AEG) concentrations. Peripheral blood mononuclear cells (PBMCs) were extracted and assayed for nuclear factor $-\kappa B(N F-\kappa B)$ pathway activity and monocyte glucocorticoid sensitivity (expressed as dexamethasone [DexIC50]) using previously validated methods.

Results: Women with current PTSD had significantly higher levels of HbAlc $(p=0.006)$ and AEG $(p=0.008)$ compared to women without PTSD. Furthermore, DexIC50 $(p=0.003)$ and NF- $\kappa \mathrm{B}$ pathway activity $(p=0.013)$ were increased significantly in women with PTSD as compared to women without PTSD. PTSD symptom severity was predictive of higher DexIC50 $(\mathrm{r}=0.27 ; p=0.05)$, denoting decreased glucocorticoid sensitivity), higher $\mathrm{HbA1c}(\mathrm{r}=0.28 ; p=0.024)$, higher AEG $(\mathrm{r}=0.27 ; p=0.033)$, and higher $\mathrm{NF}-\kappa \mathrm{B}$ pathway activity $(\mathrm{r}=0.38 ; p=0.023)$. Increased NF- $\kappa \mathrm{B}$ pathway activity was associated with higher HbAlc $(r=0.34 ; p=0.05)$, but not with DexIC50 ( $r=0.023 ; p=0.91)$.

Conclusions: PTSD diagnosis and PTSD symptom severity are positively associated with worsened glycemic regulation in African-American women with T2DM. In addition, PTSD diagnosis and PTSD symptom severity are also positively associated with increased monocyte glucocorticoid resistance and increased innate immune activity. These data suggest mechanisms of shared pathophysiology and risk connecting PTSD and T2DM which are both significant public health problems. Increased understanding of shared pathophysiology between stress-related psychiatric and medical disorders such as PTSD and T2DM may lead to novel approaches to prevention and progression of disease and argues for improved integration of care for psychiatric and medical disorders.

Keywords: Post-Traumatic Stress Disorder, Type-2 Diabetes, Women, Innate Immunity, Neuroendocrine

Disclosure: Nothing to Disclose.

M2. The Endogenous Hallucinogen N,N-Dimethyltryptamine Displays Protective Effects Against Hypoxia in Cultured Human Cortical Neurons and Glial-Like Cells and Protects Against Renal Ischemia-Reperfusion Injury

Ede Frecska*, Attila Szabo

University of Debrecen, Debrecen, Hungary

Background: N,N-dimethyltryptamine (DMT) is a potent endogenous hallucinogen present in the mammalian 
brain. Recently, DMT has been found to activate the sigma-1 receptor (Sig-1 R), an intracellular chaperone fulfilling an interface role between the endoplasmic reticulum and mitochondria. It ensures signaling of endoplasmic reticulum overload into the nucleus resulting in the enhanced production of antistress and antioxidant proteins. Based on its central cellular role the activation of Sig-1 R can mitigate the outcome of hypoxia or oxidative stress and helps cell survival by attenuating endoplasmic reticulum stress.

Methods: The first aim was to test the hypothesis that DMT plays a protective role in the brain by activating the Sig-1 R. We tested 'in vitro' whether DMT can alleviate hypoxic stress (under $0.5 \% \mathrm{O} 2$ ) in cultured human cortical neurons derived from induced pluripotent stem cells, monocyte-derived macrophages, and dendritic cells. The second aim was to investigate 'in vivo' the protective role of DMT in renal ischemia-reperfusion injury of rats after clamping of the left renal artery and vein for up to $120 \mathrm{~min}$.

Results: Findings showed that DMT robustly increases the survival of the cultured cell types in severe hypoxia through the Sig-1 R. Furthermore, this phenomenon is associated with the decreased expression of the hypoxia-inducible factor 1-alpha (HIF-1 A) suggesting that DMT may alleviate hypoxic stress and increase cell survival in an HIF-1 Aindependent manner. Pretreatment of rats with DMT improved red blood cell deformability, renal function, and tissue morphology after 30-60-120 minutes of renal ischemia-reperfusion injury.

Conclusions: Our results reveal a novel and important role of DMT in human cellular physiology. We postulate that this compound may ameliorate the adverse effects of hypoxic/ ischemic insult to the brain and other organs.

Keywords: Dimethyltryptamine, Hypoxia, IschemiaReperfusion Injury, Sigma-1 Receptor

Disclosure: Nothing to Disclose.

\section{M3. Association Between Inflammation and Increased} Alcohol Dependence in Post-Traumatic Stress Disorder

Samantha Friend*, Daniel Glenn, Caroline Nievergelt, Adam Maihofer, Dewleen Baker, Victoria Risbrough

University of California, San Diego School of Medicine, San Diego, California, United States

Background: Post-traumatic stress disorder (PTSD) and Alcohol Use Disorder (AUD) are both highly prevalent, and comorbidity between PTSD and AUD is high both in the general population and among combat Veterans. Prevalence of AUD among those with PTSD is $28 \%$ for women and $52 \%$ for men. Rates of PTSD among patients with AUD are 30$59 \%$. Currently we do not understand the biological mechanisms promoting development of AUD in some PTSD patients, which will require high-resolution prospective studies in understanding development vs. maintenance of symptoms of each disorder. Evidence supports a role for inflammation as a potential pathophysiological mechanism underlying both PTSD and AUD. C-reactive protein (CRP) specifically has been identified as a risk factor for PTSD, while chronically elevated blood alcohol levels can increase CRP. Alcohol craving and consumption are associated with elevated plasma levels of inflammatory cytokines in alcoholics. Here we tested the hypothesis that inflammation, as measured by CRP, may interact with PTSD symptoms to predict alcohol dependence behaviors.

Methods: In a study of combat trauma in active duty service members (Marine Resiliency Study), Marines and Navy Corpsmen $(N=1890)$ were given the AUD Identification Test (AUDIT) as well as the Clinician Administered PTSD Scale (CAPS) 3 months after a combat deployment to Afghanistan or Iraq. AUDIT scores were totaled separately for alcohol consumption (i.e. frequency and quantity of drinking) and dependence behaviors (i.e. difficulty controlling drinking and functional impairment). Plasma was collected for measurement of CRP by enzyme-linked immunosorbent assay. Associations between PTSD, AUD, and CRP at 3-month post-deployment were analyzed using linear regression or zero-inflated negative binomial regression (ZINBR), to account for skewing from an excess in zero responses in the AUDIT score.

Results: While a PTSD diagnosis predicted alcohol consumption $(N=1446 ; \beta=0.07, p<0.01)$, there were no main effects of CRP or interactions. PTSD diagnosis predicted a roughly $10 \%$ lower chance of not demonstrating any dependence behavior $(N=1446, \beta=0.98, p<0.001$, ZINBR zero model). In those endorsing dependence symptoms, PTSD diagnosis alone did not predict dependence severity but did interact with CRP to predict dependence severity ( $N=353, \beta=0.24, p<0.02$, count model regression), with increasing CRP associated with increased dependence symptoms only in the PTSD group. Similar findings were found using total CAPS severity scores instead of PTSD diagnosis. It should be noted that of those reporting at least some dependence behaviors $(>0)$, symptom severity in subjects with PTSD was 2.51 (range $=1-11$ ) and those without PTSD averaged 2.37 (range $=1-10)(p>0.5)$ suggesting that the association of CRP with dependence severity in the PTSD group is not simply due to greater dependence in that group. Post-hoc analysis on separate CAPS subscales (re-experiencing, avoidance, numbing, and arousal subscales) indicated that the findings were primarily related to increased CRP and numbing symptoms associated with increased dependence symptoms.

Conclusions: These data support the hypothesis that there is a synergistic relationship between PTSD and CRP associated with greater alcohol dependence but not consumption. These effects may be particularly driven by the relationship between CRP and numbing PTSD symptomatology. Although directionality cannot be inferred from these preliminary data, one possibility is that PTSD in combination with inflammation may increase vulnerability for alcohol use. Interestingly, similar amounts of dependence symptoms occurred between individuals with and without PTSD, indicating that higher inflammation is not simply an artifact of higher dependence in the PTSD group. Future longitudinal analyses as well as studies with animal models will provide a greater mechanistic understanding of potential causal relationships between inflammation, trauma, and alcohol dependence.

Keywords: PTSD, Alcohol, CRP, Inflammation

Disclosure: Nothing to Disclose. 
M4. Stress Correlates in Bipolar I Disorder: An Ecological Momentary Assessment Study

Jessica Carrasco*, Sheena Dev, Tanya Nguyen, Ashley Sutherland, Nana Kori, Rudolph Benitez, Lisa Eyler

University of California, San Diego, La Jolla, California, United States

Background: Bipolar disorder (BD) is characterized by mood shifts potentially triggered by stressful daily life events. Research has been hampered by use of retrospective selfreport over weeks, which is susceptible to bias and does not measure daily stress variability. To address this, we measured daily stress over a two-week period using ecological momentary assessment in those with and without $\mathrm{BD}$, compared demographic and inflammatory correlates of stress between the two groups, and related stress to clinical variables in the $\mathrm{BD}$ group.

Methods: Thirty-four BD and sixty-five healthy comparison (HC) participants completed three daily surveys over two weeks via study-provided smartphone. Participants rated their momentary stress level from 1-7, with 7 being the highest level of stress. Individual mean and standard deviation of thrice-daily ratings across two weeks were calculated and compared between groups. Relationship to baseline demographic and blood-based inflammation levels and how these might differ between groups was assessed with regression models that included the interaction between group and each potential predictor. Finally, correlations between clinical variables and daily stress were examined within the $\mathrm{BD}$ group.

Results: Group differences in mean and individual standard deviation of daily stress were found, such that BD showed higher mean levels of daily stress and higher variability over the two weeks compared to HC. After controlling for individual mean stress levels, group differences in individual standard deviation were no longer significant. Thus, all further analyses were conducted with individual mean stress levels. A gender by diagnosis interaction $(\mathrm{p}=0.009)$ was observed such that women with $\mathrm{BD}$ were significantly more stressed than men with $\mathrm{BD}$ and $\mathrm{HC}$, whereas $\mathrm{HC}$ men were slightly more stressed than $\mathrm{HC}$ women. We also found a group by age interaction $(p=0.009)$, wherein older adults with $\mathrm{BD}$ reported higher levels of stress than younger BD and there was no age relationship in the HC group. In addition, an interaction between group and C-reactive protein (CRP) levels was found $(p=0.22)$, such that $\mathrm{BD}$ with higher levels of CRP reported more daily stress, and no such association was seen in the $\mathrm{HC}$ group. Within $\mathrm{BD}$, more severe psychotic and depression symptoms and later age of onset were all associated with higher levels of stress (all p's $<0.05$ ).

Conclusions: Momentary assessment of stress suggests that women with BD suffer from more daily stress than men and those without the disorder. In addition, aging is associated with greater stress in the BD group but not the HC group, perhaps reflecting the greater age-related burden of medical and mental health issues in those with the disorder. Consistent with this, higher C-reactive protein levels, an indicator of poorer cardiovascular health were found in those with greater daily stress, and $\mathrm{BD}$ patients with more recent onset and higher symptom burden were also more stressed. In addition, more severe psychiatric symptoms typically contribute to financial burden, unemployment, and other environmental factors which could lead to more stressful lives. These baseline observations help to suggest which $\mathrm{BD}$ patients might be at greatest risk for accelerated declines over time; we plan to pursue these questions in future analyses of data from this ongoing longitudinal study.

Keywords: Aging, Bipolar Disorder, Ecological Momentary Assessment

Disclosure: Nothing to Disclose.

\section{M5. Anterior Cingulate Cortical Metabolism at Rest is a Mediating Factor in the Relationship Between Age and Executive Function in Healthy Elderly Independent of Amyloid Deposition}

\author{
Jose Pardo*, Joel Lee, Shantal Nyabwari
}

University of Minnesota, Minneapolis, Minnesota, United States

Background: Given the increasing number of aging baby boomers and the inevitability of Age-Associated Cognitive Decline (AACD) sure to plague this population, it is important to identify and understand the role of potential biomarkers for cognitive decline in the elderly. We showed previously the anterior cingulate cortex (ACC) and adjacent regions are a major focus of aging-related metabolic dysfunction correlating with AACD (Pardo et al., 2007). Here, we examine using the ADNI dataset the hypothesis that ACC metabolism functions as a mediator in the relationship between age and executive function driven by amyloid deposition. Such relationships would suggest the decline in ACC metabolism with age is an important explanatory factor in the observed relationship between age and cognition related to the neural dysfunction induced by fibrillar amyloid during otherwise healthy aging.

Methods: [18]F-fluorodeoxyglucose (FDG) and [18]F-AV-45 (florbetapir) PET scans were obtained from the Alzheimer's Disease Neuroimaging Initiative (ADNI @ LONI). These were stereotactically normalized to standard Talairach space via the Neurostat program (S. Minoshima, U. Washington). The FDG data were normalized through proportional scaling to whole brain glucose uptake (1000 counts). The standard uptake value ratio (SUVr) data of the florbetapir scans were normalized through proportional scaling to the mean SUVr in the cerebellum. We performed a region-of-interest (ROI) analysis $(11 \mathrm{~mm}$ diameter sphere centered on $\mathrm{x}=+3 \mathrm{~mm}$ (right), $y=+17 \mathrm{~mm}, \mathrm{z}=+36 \mathrm{~mm}$ from the maximum from Pardo et al., 2007) for each scan. The PET imaging protocols are documented at the ADNI website (http://adni.loni.usc. edu/data-samples/pet/). We obtained data from 231 participants (age 59-93 years, mean 74, SD 6) assessed as cognitively normal (see ADNI for diagnostic criteria). Participants' ages and animal fluency scores were also obtained from ADNI and were appropriately matched to their respective ACC amyloid SUVr and FDG uptake ROI means. The animal fluency scores served as a measure of executive performance.

Results: In agreement with our previous study, there were significant correlations between age and metabolism 
$[\mathrm{r}=-0.44, p=2(10)-12]$; metabolism and fluency $[\mathrm{r}=-0.23$, $p=4(10)-4]$; and age and fluency $[\mathrm{r}=-0.26, p=2(10)-4]$. These findings motivated a mediation model in which ACC metabolism functions as a mediator in the relationship between age and fluency scores. The major causal assumptions of mediation were met. The Sobel test for mediation showed significance in the indirect effect of ACC metabolism on the relationship between age and fluency at the 0.05 level of significance. This suggests ACC metabolism functions as a mediator in the relationship between age and fluency. Of note, the direct effect or the effect of age on fluency when controlling for ACC metabolism (path $c^{\prime}$ in the mediation model) was not zero. In other words, the coefficient of the regression analysis of age predicting fluency when controlling for ACC metabolism was not zero $\left(c^{\prime}=-0.21\right)$ but was reduced in absolute size when compared to the coefficient indicating the total effect of age on fluency $(c=-0.26)$. This indicates that ACC metabolism is not a complete mediator between age and fluency, but is instead a partial mediator. There was no significant correlation between age and amyloid $(r=0.123, p=0.06)$ or amyloid and fluency $(\mathrm{r}=0.091, p=0.17)$. Surprisingly, resting metabolism was positively correlated with amyloid in these healthy elders $(r=+0.153, p=0.02)$.

Conclusions: The results show that ACC metabolism mediates in part the relationship between age and cognitive function. However, amyloid does not appear responsible for this relationship in this age range. These findings provide insight into mechanisms of cognitive aging. Furthermore, the results support the creation and use of novel interventions targeting the ACC to prevent or treat the decline in cognitive function associated with normal aging.

Keywords: PET Resting Metabolism, Cognitive Aging, Amyloid, Executive Function, Healthy Subjects

Disclosure: Nothing to Disclose.

\section{M6. fMRI- and Computationally-Guided rTMS Enhances Performance in Working Memory Manipulation}

Lysianne Beynel, Simon W. Davis, Courtney Crowell, Susan A. Hilbig, Wesley Lim, Duy Nguyen,

Angel V. Peterchev, Sarah H. Lisanby, Roberto Cabeza, Greg Appelbaum, Bruce Luber*

National Institute of Mental Health, Bethesda, Maryland, United States

Background: A core element of human working memory is the ability to perform mental operations on information that is stored in a flexible, limited capacity, on-line mental buffer. In our previous work, we developed an fMRI-guided repetitive transcranial magnetic stimulation (rTMS) paradigm that led to long-lasting performance enhancements in tasks involving the maintenance of items in working memory. However, given the profound importance of working memory manipulation (WM-M) abilities and their noted decline through the aging process, there is a concerted effort aimed at developing approaches to improve these abilities. Past research has identified neural substrates of WM-M centered in the dorsolateral prefrontal cortex (DLPFC), thereby providing a plausible and accessible target for noninvasive neuromodulatory stimulation that can be used to alter cortical excitability and potentially lead to facilitation of WM-M. In this study, we tested the capacity to enhance WM-M through functional-guided rTMS to the left DLPFC in a group of 31 healthy younger adults. Using the information from functional imaging to localize individual WM targets, we investigated whether the pattern of fMRI brain activations could predict individual differences in the magnitude of the rTMS WM benefit.

Methods: This study involved 6 days of participant interactions. On the first visit participants were trained on an adapted Sternberg delayed-match-to-sample memory task in which an array containing 3 to 9 letters was presented, followed by a delay period during which subjects were asked to keep the stimulus in mind and reorganize the letters into alphabetical order. At the end of the delay, a letter with a number above it appeared on the screen and participants indicated whether the number matched the letter position in its reorganized alphabetical order, using a button response. During the second visit, the same task was performed in the fMRI scanner, and we identified an individualized statistical map predicting the parametric increase in BOLD activity associated with increasing set size (or difficulty). Individual modeling of induced electric field distribution was combined with this fMRI activation to provide DLPFC targets to guide placement of the TMS coil for each participant.

On days 3 through 6, rTMS was delivered to the identified PFC target using stereotactic neuro-navigation and real-time robotic guidance to maintain accurate and reproducible targeting. Twenty-five pulses of $5 \mathrm{~Hz}$-rTMS were delivered at $100 \%$ of resting motor threshold on each trial, either with active magnetic stimulation or with an electrical sham stimulation that produced similar clicking sounds and somatosensory sensations as the active coil, but without a magnetic flux. Analysis involved repeated measures ANOVA to assess the rTMS effect on behavioral performance and correlations between rTMS effects and fMRI parametric activations to investigate the link between brain activation and rTMS induced behavioral plasticity.

Results: Results showed a significant interaction between Difficulty and Stimulation Type when the cued number indicating item position was false $(\mathrm{F}(2,56)=5.15, \mathrm{p}<.01)$, but not when it was true $(\mathrm{F}(2,56)<1)$. Post-hoc analysis revealed that subjects tended to be more accurate with active rTMS than with sham rTMS in the hardest difficulty level ( $F$ $(1,28)=11.21, p=.12)$, while their performance did not differ for the easy or medium difficulty level. To confirm this trend in the condition when the cued number indicating item position was false, active minus sham differences for each individual were tested using binomial statistics. For the hardest difficulty level, 21 subjects out of 29 showed accuracy improvement with active rTMS relative to sham $(p=.024)$, while this probability does not reach significance in the easy and the medium difficulty level, where both conditions had 13 out of 29 subjects showing better accuracy with active rTMS $(p=.71)$.

To explore whether the rTMS-induced changes were predicted by brain activations, we correlated the $\mathrm{z}$-statistic from the parametric set size effect in the targeted brain region with the percentage of change in accuracy in active rTMS, as compared to sham. We found a negative correlation between the parametric statistic and task accuracy emerge in the hardest difficulty level, suggesting 
that individuals who showed a more shallow increase in activity associated with WM difficulty show a greater benefit from TMS. This effect became significant $(r=-0.62 ; p=.02)$ when removing the single outlier subject who showed a decrease in performance associated with active rTMS. Performance was not correlated with resting motor threshold or E-field magnitude.

Conclusions: These results indicate for the first time that online $5 \mathrm{~Hz}$-rTMS to DLPFC can enhance working memory abilities. Interestingly, we found that this performance enhancement for difficult WM trials was negatively correlated with parametric fMRI activation in the targeted brain regions. The present findings therefore provide promising evidence that rTMS may enhance WM-M abilities and that these enhancements may be tied to state-dependency of activity within the DLPFC during this task. These findings therefore provide important implications towards the use of non-invasive neuromodulation to enhance cognitive function and provide specific prescriptive recommendations towards the conditions and individual differences that may lead to optimal efficacy.

Keywords: TMS, TMS Targeting, Working Memory, Functional MRI (fMRI), Cognitive Enhancement

Disclosure: Nothing to Disclose.

\section{M7. Measuring the Distribution of the Alpha7 Nicotinic Acetylcholine Receptor in Healthy Aging: An in Vivo Positron Emission Tomography Study With [18F]ASEM}

Jennifer Coughlin*, Yong Du, Hailey Rosenthal, Stephanie Slania, Yuchuan Wang, Wojciech Lesniak, Il Minn, Gwenn Smith, Hiroto Kuwabara, Andrew Horti, Dean Wong, Martin Pomper

Johns Hopkins University School of Medicine, Baltimore, Maryland, United States

Background: The alpha7 nicotinic acetylcholine receptor $(\alpha 7-n A C h R)$ is expressed on neurons and has functional roles in synaptic transmission, neurotransmitter release, intracellular signaling, and synaptic plasticity, which collectively mediate cognitive function. Altered function of the $\alpha 7-$ $\mathrm{nAChR}$ is implicated in several neuropsychiatric diseases. Nevertheless, studies of the human cerebral $\alpha 7-n A C h R$ even in healthy aging are limited in number and to postmortem tissue. The use of the radioligand, [18 F]ASEM, with positron emission tomography (PET) to image the $\alpha 7-n A C h R$ in vivo promises to help elucidate changes in the distribution of the cerebral $\alpha 7-n A C h R$ in healthy aging and disease.

Methods: The distribution of the cerebral $\alpha 7-n A C h R$ was estimated in nine cortical and subcortical brain regions in 25 healthy volunteers (ages 21-86 years; median 57 years, interquartile range 52 years) using [18 F]ASEM with PET imaging. Regional total distribution volume (VT) measurements were calculated using the Logan method from each subject's 90- min dynamic PET data and their metabolitecorrected plasma input function. Spearman's rank or Pearson's correlation analysis was used depending on the normality of the data. First, correlation between age and regional [18 F]ASEM VT was tested. Second, correlation between regional volume relative to intracranial volume (volume ratio) and 1) age or 2) [18 F]ASEM VT was tested.
Finally, the relationship between [18 F]ASEM VT and neuropsychological measures in six cognitive domains was investigated in a subpopulation of 15 elderly healthy participants (those over 50 years of age). Bonferroni correction for multiple comparisons was applied to statistical analyses.

Results: A positive correlation between [18 F]ASEM VT and age was observed in all nine brain regions of interest (ROIs). A negative correlation between tissue volume ratio and age was observed in six of the nine brain regions including striatum and five cortical (temporal, occipital, cingulate, frontal, or parietal) regions. There was no correlation between [18 F]ASEM VT and volume ratio in any ROI after controlling for age. Regional [18 F]ASEM VT and neuropsychological performance on each of the eight representative subtests were not correlated among the well-performing subpopulation of elderly healthy participants.

Conclusions: Our results suggest an increase in cerebral $\alpha 7$ $\mathrm{nAChR}$ distribution over the course of healthy aging that should be tested in future longitudinal studies. The preservation of the $\alpha 7-\mathrm{nAChR}$ in the aging human brain supports the development of therapeutic agents that target this receptor for use in the elderly. Further study of the relationship between $\alpha 7-n A C h R$ availability and cognitive impairment over aging is needed.

Keywords: Aging, PET Imaging, Alpha7 Nicotinic Acetylcholine Receptor, [18 F]ASEM

Disclosure: Nothing to Disclose.

M8. Colitis Promotes Anxiety Through a CRF-R1Mediated Suppression of Central Anandamide Signaling

Haley Vecchiarelli, Kaitlyn Tan, Maria Morena, Alessia Santori, Catherine Keenan, Martin Sticht, Kira Leitl, Winnie Ho, Keith Sharkey, Matthew Hill

University of Calgary, Calgary, Canada

Background: It is well established that peripheral inflammatory diseases (e.g. inflammatory bowel disease, arthritis) are commonly associated with stress-associated neuropsychiatric disorders (e.g. anxiety, depression). To date, however, the mechanisms underlying these comorbidities have not been fully elucidated. The endocannabinoid system regulates both anxiety and inflammation, making it a potential candidate mediating these comorbidities. To examine the hypothesis that endocannabinoids are mediators of the emotional comorbidities of peripheral inflammatory conditions, we employed an animal model of colitis to explore the potential role of endocannabinoids in these processes.

Methods: Colitis was induced by intracolonic administration of trinitrobenzene sulfonic acid (TNBS, $0.45 \mathrm{~mL}, 50 \mathrm{mg} / \mathrm{mL}$, $50 \%[\mathrm{vol} / \mathrm{vol}]$ in ethanol/water) to adult male rats, while control rats received the same volume of saline. Central levels of anandamide were measured using liquid chromatography/ tandem mass spectrometry, and the activity of its metabolic enzyme, fatty acid amide hydrolase (FAAH), was measured using a radioligand enzymatic activity assay. Anxiety-like behaviour was assayed using the elevated plus maze during the lights-on phase of the light cycle. To investigate central mechanisms, unilateral cannulation surgeries targeting the lateral ventricle were performed one week prior to colitis 
SII6

onset, with acute administration of a FAAH inhibitor (PF-04457845) occurring two hours prior to behaviour testing, or sustained administration of a corticotrophin releasing factor receptor 1 antagonist (antalarmin) through an Alzet mini-pump.

Results: There were no differences in anandamide (AEA) levels in any region measured (amygdala, hippocampus, hypothalamus and medial prefrontal cortex), three days after the onset of colitis, when the disease is peaking; however, AEA levels were decreased in the amygdala, hippocampus and medial prefrontal cortex seven days after the induction of colitis. Concomitantly, seven days after the onset of colitis, there was an increase in fatty acid amide hydrolase (FAAH) activity, indicating that peripheral inflammation can increase central AEA hydrolysis. Additionally, we observed an increase in anxiety like-behaviour in the elevated plus maze at this time point. The increase in anxiety was reversed with an acute intracerebroventricular administration of a FAAH inhibitor (PF-04457845), which increases levels of AEA. These data suggest that AEA levels are dynamically regulated in response to colitis, with the anxiety-related reduction in AEA levels occurring following the peak of disease activity, but after a sustained period of peripheral inflammation. Additionally, central administration of an antagonist of the corticotrophin releasing factor receptor 1 (CRF-R1; antalarmin) throughout the duration of colitis reversed the AEA reductions in the amygdala and hippocampus.

Conclusions: Together these findings add to the understanding of central mechanisms underlying anxiety-like behaviours associated with peripheral inflammation. They suggest that similar to stress-induced anxiety, inflammationinduced decreases in AEA signaling (due to CRF driving FAAH activity), are relevant for the change in anxiety-like behaviours associated with inflammation.

Keywords: Endocannabinoid, Anxiety, Systemic Inflammation, CRF-R1

Disclosure: Nothing to Disclose.

M9. Use of an Individual-Level Approach to Identify Cortical Connectivity Biomarkers of Global and Dimensional Symptom Severity and Improvement in Obsessive-Compulsive Disorder

Brian Brennan*, Danhong Wang, Meiling Li, Christine Perriello, Jason Elias, Nathaniel Van Kirk, Jason Krompinger, Harrison Pope, Suzanne Haber, Scott Rauch, Justin T. Baker, Hesheng Liu

Harvard Medical School, McLean Hospital, Belmont, Massachusetts, United States

Background: Studies using resting state functional connectivity magnetic resonance imaging (rs-fcMRI) have demonstrated evidence of global dysconnectivity in OCD involving both a core cortico-striato-thalamo-cortical circuit as well as broader cortical networks. However, one critical limitation of these studies is that they define important functional regions based on either a nominal "average" brain or on subjects' anatomy - approaches that ignore the substantial interindividual variability in functional and structural brain organization, thus potentially obscuring biologically important signals. In an effort to address the above limitation, we conducted a study to 1) identify cortical connectivity biomarkers of both global (using the Yale-Brown Obsessive-Compulsive Scale; YBOCS) and dimension-specific (using the Dimensional Obsessive Compulsive Scale; DOCS) symptom severity in OCD patients using a novel methodology to localize functional networks at the individual level; and 2) detect functional connections that track changes in global and dimension-specific symptom severity longitudinally, using rs-fcMRI data acquired from OCD patients preand post-treatment in an intensive residential treatment program.

Methods: We recruited 41 participants with a DSM-IV primary diagnosis of OCD, and scoring $\geq 16$ on the YBOCS, from the Obsessive-Compulsive Disorder Institute at McLean Hospital. All participants received MRI scans within one week of admission and during the last week prior to discharge. All imaging data were collected on a $3 \mathrm{~T}$ Tim Trio scanner (Siemens) with a 12-channel phased-array head coil using the following parameters: repetition time, $3000 \mathrm{~ms}$; echo time, $30 \mathrm{~ms}$; flip angle, $85^{\circ} ; 3 \times 3 \times 3 \mathrm{~mm}$ voxels; field of view, 216; and 47 axial sections collected with interleaved acquisition and no gap. Each functional run lasted $6.2 \mathrm{~min}$ (124 time points) and 2 runs were acquired per participant. For each participant, 116 cortical regions of interest (ROIs) were localized and an "individualized connectome" was constructed. Then, a support vector machine for regression (SVR) model was trained to predict total scores on the YBOCS based on ROI-to-ROI connectivity, using the data from 40 participants. The resulting model was then applied to predict the YBOCS score of the remaining participant. This procedure was repeated 41 times to predict the YBOCS scores of all participants. In a subsequent analysis, to pinpoint changes in brain connections that tracked changes in symptom severity, we used a longitudinal, within-subjects design to identify connections (from within the previously identified set of pre-treatment connections related to global symptom severity) that changed significantly from pre- to post-treatment. The above procedures were then repeated using each of the four categories of the DOCS (1-concerns about germs and contamination; 2-concerns about being responsible for harm, injury, or bad luck; 3-unacceptable thoughts; 4-concerns about symmetry, completeness, and the need for things to be "just right"; DOCS1-4) to identify dimensional connectivity biomarkers of symptom severity and treatment response.

Results: The YBOCS scores predicted by pre-treatment connectivity among the individually-specified ROIs were significantly correlated with the YBOCS scores observed in participants $(r=0.52, p=4.96 \times 10-4)$. Connections contributing to YBOCS scores were mostly between-network connections that involved the limbic, dorsal attention, and fronto-parietal control networks. Repeat analysis, using ROIs determined in the conventional manner from the brain atlas, found that connectivity was less predictive of YBOCS scores $(\mathrm{r}=0.393, p=0.011)$. Focusing on the 64 pre-treatment connections that were related to YBOCS, we found that changes in connectivity from pre- to post-treatment could predict YBOCS improvement $(\mathrm{r}=0.334, p=0.024)$. In contrast, using template-based ROIs, functional connectivity changes did not predict YBOCS improvement $(r=0.176$, $p=0.270$ ). 
Pre-treatment connectivity among individually-specified functional regions predicted DOCS1 $(r=0.377 ; p=0.015)$ and DOCS2 $(r=0.414 ; p=0.007)$ scores, but not DOCS3 or DOCS4. Furthermore, functional connectivity changes of the 19 connections that were predictive of DOCS1 scores could predict DOCS1 improvement $(r=0.512, p=6.17 \times 10-4)$. Connectivity changes in DOCS2-4 were not predictive of improvement within those symptom dimensions. Repeat analyses examining connectivity based on template-based ROIs failed to predict symptom severity or improvement on any DOCS category.

Conclusions: Using an individual-specific approach, we have 1) identified cortical connectivity biomarkers of global and dimensional OCD symptom severity and 2) identified functional connections in specific brain regions that track changes in global and dimensional OCD symptoms over time. The above findings provide evidence that identifying functional regions in individuals is a critical step towards the discovery of connectivity abnormalities in OCD. Future studies using an individual-specific approach to examine cortical and subcortical functional networks in larger, phenotypically diverse, OCD samples are warranted in order to parse out the neurobiological distinctions between different OCD symptom dimensions - a necessary step toward personalized therapeutics for OCD.

Keywords: Obsessive-Compulsive Disorder, OCD, Connectivity, Biomarker

Disclosure: Part 1: Rugen Therapeutics, Consultant.

M10. Characterizing Neurotrophic Systems in the Primate Amygdala That are Relevant to Mediating and Treating Anxiety Disorders

Rothem Kovner*, Tade Souaiaia, Yi Dong, Ali Fathi, Yezheng Tao, Delores A. French, Patrick H. Roseboom, Andrew Fox, Alexander Shackman, Jonathan A. Oler, Su-Chun Zhang, Anita Bhattacharyya, Julie Fudge, James A. Knowles, Ned Kalin

University of Wisconsin, Madison, Madison, Wisconsin, United States

Background: Anxiety and depression are common and debilitating illnesses that frequently begin early in life. Evidence from our laboratory and others support the hypothesis that altered neuroplasticity is important in the development of neuropsychiatric disorders, including anxiety and depression. While extensive work has examined the hippocampal BDNF-TrkB system, our work focuses on the relatively understudied neurotrophic factor system (NT3TrkC) in the central nucleus of the amygdala (Ce) of nonhuman primates. In previous studies, we found that decreased levels of the TrkC transcript in the Ce of young rhesus monkeys predicted high levels of anxiety and that increasing the activity of this system had anxiolytic effects. These findings support in-depth studies aimed at understanding the molecular substrates of neuroplasticity in the primate Ce as they relate to psychopathology.

Methods: We used laser capture microdissection (LCM) combined with RNAseq, to characterize the expression of TrkC mRNA levels in neurons from the Ce, basolateral amygdala, temporal cortex, caudate, and putamen of 2 rhesus monkeys. We also performed in situ hybridization in 10 monkeys to characterize levels of TrkC mRNA expression within the different amygdala nuclei. To further explore TrkC mechanisms relevant to anxiety, we developed an in vitro model of Ce neurons by capitalizing on the ability to derive mature neurons from rhesus inducible pluripotent stem cells (iPSCs). Based on the embryological origins of Ce neurons, the Ce iPSCs were differentiated into GABAergic, medium-spiny like neurons. Electrophysiology was performed to investigate the effects of TrkC activation on neuronal firing.

Results: RNAseq data demonstrated that TrkC mRNA was expressed in neurons throughout the brain regions investigated. Within the amygdala, higher levels of the transcript were found in the basolateral amygdala compared to the Ce. Consistent with this, in situ hybridization of TrkC mRNA expression revealed higher levels of the transcript in the basolateral, basomedial, and paralaminar nuclei compared to the Ce. The iPSC derived neurons shared neuropeptide (e.g. somatostatin, corticotrophin-releasing hormone, and neurotensin), transcription factor, and TrkC expression patterns with the mature Ce neurons collected from the same animals from which the iPSCs were derived. Electrophysiology studies demonstrated that application of NT3 $(0.05 \mu \mathrm{g} / \mathrm{ml})$, the ligand for TrkC, increased spontaneous action potentials by $228.2 \%$. Two groups of Ce-like neurons, derived from different embryological origins, were tested revealing selectivity in responding. Specifically, those derived from the lateral ganglionic eminence were affected by NT3 whereas medial ganglionic eminence derived neurons did not respond to NT3. Conclusions: We previously demonstrated an inverse relationship between Ce TrkC mRNA and extreme anxiety. We also found that overexpressing NT3 in the Ce resulted in anxiolytic effects. Here, we have fully characterized the distribution of TrkC mRNA in the primate amygdala focusing on the Ce. These findings set the stage for future mechanistic studies in non-human primates. Additionally, we developed an in vitro model of Ce-like neurons that has provided initial insights into TrkC function in relation to modulating amygdala neuroplasticity. Taken together, the data reported here provide a framework for future studies aimed at understanding mechanisms of, and new treatments for, human anxiety disorders.

Keywords: Amygdala, NTRK3, Neuroplasticity, Anxiety, Non-Human Primate

Disclosure: Nothing to Disclose.

\section{M11. Performance Monitoring in Obsessive-Compulsive Disorder: A Meta-Analysis of fMRI Studies Using Whole-Brain T-Maps}

Luke Norman*, Kate Fitzgerald, Yanni Liu, Joaquim Radua, Yann Chye, Stella De Wit, Chaim Huyser, Isik Karahanoglu, Tracy Luks, Dara Manoach, Carol Mathews, Katya Rubia, Chao Suo, Odile van den Heuvel, Murat Yucel, Stephan Taylor

University of Michigan, Ann Arbor, Michigan, United States

Background: Performance monitoring involves error detection and a subsequent recalibration of behavior in order to 
meet environmental demands. A number of functional magnetic resonance imaging (fMRI) studies have reported differences between patients with obsessive-compulsive disorder (OCD) and healthy controls during performance monitoring in inhibitory control tasks. These studies have tended to report altered activation in patients with OCD within key cingulo-opercular performance monitoring regions. However, the literature is not without inconsistencies and current conclusions are limited by the small sample sizes and region of interest analysis methods employed in previous studies. Here, a voxel-wise meta-analysis of fMRI brain activation during error-processing and inhibitory control was performed based primarily on whole-brain unthresholded statistical brain maps from individual studies (this substantially increasing the statistical power as compared to the use of only peak coordinates).

Methods: A preliminary analysis of the 9 studies for which data has been acquired thus far was performed using Seedbased d Mapping (SDM) software (www.sdmproject.com). All studies examined group differences in error-processing between patients with OCD $(N=224$; mean age range $=14$ 39; mean CY-BOCS range $=11-27$ ) and healthy controls $(N=216$; mean age range $=14-40)$ during tasks of motorresponse (Stop, go/no-go) or interference (flanker, multisource interference, Stroop, antisaccade) inhibition. For the errors analysis, error versus correct or error versus baseline contrasts were included. For the inhibitory control analysis, the included contrasts compared stop/no-go/incongruent/ antisaccade trials against go/congruent/prosaccade trials or baseline.

Results: In the comparison between patients with OCD and controls during error-processing, patients with OCD showed hyperactivation in right insula, bilateral inferior frontal gyrus (IFG), dorsolateral prefrontal cortex (DLPFC), dorsal/rostral anterior cingulate cortex (ACC) and presupplementary motor area, as well as reduced activation in bilateral middle temporal lobe. In the analysis of group differences during inhibitory control, patients with OCD showed hypoactivation in dorsal/rostral ACC, right insula/putamen and orbitofrontal cortex as well as in bilateral caudate and thalamus relative to controls, but hyperactivation in supplementary motor area.

Conclusions: Findings are in line with most previous reports in smaller cohorts, showing evidence for hyperactivation in medial frontal and insular regions during error-processing, and extend these reports by showing novel group differences outside of canonical cingulo-opercular performance monitoring regions in bilateral lateral prefrontal cortex and middle temporal lobe. Interestingly, similar medial frontal and right insular regions were also hypoactive during inhibitory control in OCD relative to healthy controls. Findings suggest an inefficiency in performance monitoring brain networks in OCD, whereby even increased responsiveness during error processing is insufficient to initiate appropriate recruitment of performance monitoring circuitry during behavioral adjustment and control, and this may underlie poor monitoring and control over interfering, OCD-related cognitions and behaviors.

Keywords: Obsessive-Compulsive Disorder (OCD), Functional MRI (fMRI), Meta-Analysis, Error Processing, Inhibitory Control

Disclosure: Nothing to Disclose.
M12. Medial Prefrontal Cortex Gamma-Aminobutyric Acid in Association With PTSD and Fear Extinction Recall

Isabelle Rosso, J. Eric Jensen, Elizabeth Olson, Antonia Seligowski, Scott Rauch*

McLean Hospital, Belmont, Massachusetts, United States

Background: Posttraumatic stress disorder (PTSD) has been associated with hypometabolism of the medial prefrontal cortex (mPFC; including rostral anterior cingulate cortex), a region that is critical for fear extinction. mPFC hypometabolism is thought to impair fear extinction, contributing to hyperarousal symptoms and other clinical manifestations of conditioned fear in PTSD. Although the neurochemical underpinnings of mPFC hypofunction in PTSD are unknown, there is compelling preclinical evidence that modulation of GABA signaling within the rodent $\mathrm{mPFC}$ (infralimbic cortex) can influence fear extinction. Moreover, lower mPFC GABA has been identified in major depressive disorder (MDD), which is frequently comorbid with PTSD. This study utilized magnetic resonance spectroscopy (MRS) to examine the associations of mPFC GABA with PTSD diagnosis and hyperarousal symptoms. We also tested the hypothesis that mPFC GABA correlates with individual differences in fear extinction recall.

Methods: 34 DSM-IV PTSD, 34 trauma-exposed non-PTSD (TENP), and 40 healthy control (HC) participants completed a Structured Clinical Interview for DSM-IV, MRS at 3 Tesla, and a two-day fear conditioning and extinction paradigm. PTSD and TENP participants also were interviewed using the Clinician Administered PTSD Scale. MEGAPRESS MRS spectra were collected from a 2.5 X 2.5 X 3 mL mPFC voxel, and GABA was normalized to creatine $(\mathrm{Cr})$. Skin conductance responses (SCR) were recorded during fear conditioning and extinction learning, and during extinction recall a day later. An extinction recall index was calculated for each participant as the mean SCR to the first four extinguished CS + stimuli presented during extinction recall, divided by maximum SCR to the CS+ during the conditioning phase.

Results: ANCOVA identified a significant group difference in mPFC GABA/Cr $(\mathrm{F}(2,108)=5.03, p=.008)$, controlling for MDD comorbidity $(\mathrm{F}(1,108)=1.92, p=.17)$. Follow-up least square mean contrasts showed that both PTSD patients and TENP participants had significantly lower GABA/Cr than HC (p's<.05). In the PTSD group, GABA/Cr was significantly negatively correlated with hyperarousal symptoms $(r=-0.40, p=.02)$. Extinction recall index scores were not significantly different between groups, but were significantly positively correlated with GABA/Cr levels across all participants $(\mathrm{r}=0.24, n=103, p=.02)$. When examining the latter correlation separately for each group, significant positive correlations between extinction recall and GABA/Cr were seen for both $\mathrm{HC}(\mathrm{r}=0.36, n=39, p=.02)$ and TENP $(\mathrm{r}=0.39, n=30, p=.03)$ participants, but not for PTSD patients $(\mathrm{r}=0.01, n=0.01, p=.95)$.

Conclusions: Using in vivo magnetic resonance spectroscopy, mPFC GABA was lower in trauma-exposed participants with and without PTSD compared with healthy nontraumatized controls, and was associated with more severe hyperarousal symptoms among PTSD patients. In addition, 
the observed pattern of brain-behavior correlations is consistent with GABAergic modulation of extinction recall capacity in healthy and trauma-exposed non-PTSD subjects, and with a possible loss of this modulation in people with PTSD.

Keywords: Posttraumatic Stress Disorder, Magnetic Resonance Spectroscopy, GABA, Fear Extinction, Medial Prefrontal Cortex

Disclosure: Part 1: SOBP, Board Member, Project 375, Board Member, NNDC, Board Member, ADAA, Board Member, APA, Advisory Board, Oxford Press, Honoraria, McLean Hospital, Employee, Football Players Health Study, Honoraria, SOBP Thompson Award, Honoraria, McKinsey, Consultant, VA Research Advisory Committee, Honoraria, Part 2: McLean Hospital, Employee, Part 3: McLean Hospital, Employee.

M13. Examination of an Acute Role for Thyroid Hormone Regulation of Trauma-Related Plasticity and Memory Formation in the Amygdala

Stephanie A. Maddox*, Michelle Chen, Anya Levendusky, Brianpaul Robert, Alicia K. Smith, Kerry Ressler

McLean Hospital, Harvard Medical School, Belmont, Massachusetts, United States

Background: The thyroid hormone (TH) system has been associated with anxiety, depression and well implicated in early nervous system development. Despite this early progress, almost nothing is known about the potential role that the TH system may play in mediating synaptic plasticity within the amygdala that accompanies trauma exposure and underlies long-term traumatic memory formation.

Methods: Non-biased RNA-sequencing and targeted qPCR was used to examine the regulation of genes in the amygdala in fear conditioned mice. Local amygdala infusions of the $\mathrm{TH}$ triiodothyronine (T3) or the dual $\mathrm{TH}$ receptor (TR) TRalpha (TRa) and TR-beta (TRb) antagonist 1-850 were used to examine the role of TRs in fear memory consolidation and associated gene transcription. Additional experiments examined the effect of these pharmacological agents on anxiety behaviors using the open field test.

Results: Using nonbiased RNA-sequencing from amygdala punches taken $2 \mathrm{hr}$ after fear conditioning, we revealed dynamic regulation of seven $\mathrm{TH}$ related genes in the amygdala in response to trauma, $\operatorname{Ttr}(\lg 2 \mathrm{fc}=-2.52$, $\mathrm{q}<0.05)$, Trhr $(\lg 2 \mathrm{fc}=-0.44, \mathrm{q}, 0.05)$, Trh $(\lg 2 \mathrm{fc}=-1.21$, $\mathrm{q}<0.05)$, Trip11 $(\lg 2 \mathrm{fc}=1.05, \mathrm{q}<0.05), \operatorname{Rxrg}(\lg 2 \mathrm{fc}=0.31$, $\mathrm{q}<0.05)$, Dio2 $(\lg 2 \mathrm{fc}=0.41, \mathrm{q}<0.05)$, and Med12 $(\lg 2 \mathrm{fc}=-$ $0.28, \mathrm{q}<0.05$. Additionally, using targeted $\mathrm{qPCR}$, we demonstrated a significant fear conditioning-related increase in $\mathrm{TRa}(\mathrm{T}(17)=-2.48, p<0.05)$ and $\mathrm{TRb}(\mathrm{T}(17)=-1.47$, $p=0.05)$ mRNA in the amygdala compared to control animals and $\mathrm{TRa}$ and $\mathrm{TRb}$ protein expression levels in the amygdala using immunohistochemistry. Next, using directed intra-amygdala infusions of either T3 or 1-850, we examined the effect of TR activation and inhibition at the time of fear conditioning on fear memory consolidation $24 \mathrm{~h}$ later. We found that mice receiving infusions of T3 $30 \mathrm{~min}$ prior to conditioning had enhanced levels of freezing during the conditioning session $(\mathrm{T}(21)=4.101, p<0.05)$ and enhanced levels of freezing $24 \mathrm{~h}$ later during the long-term memory test $(\mathrm{T}(21)=1.65, p=0.05)$ compared to vehicle controls. Conversely, we found that infusions of $1-850$ resulted in no difference in freezing level during fear conditioning $(\mathrm{T}(22)=$ $-0.28, p>0.05)$ but dramatically reduced long-term memory $24 \mathrm{hr}$ later $(\mathrm{T}(22)=2.96, p<0.05)$ suggesting impaired memory consolidation. We also examined the effect of intra-amygdala T3 on anxiety behavior using the open field test and T3 on fear memory with infusions occurring immediately after conditioning. Infusions of T3 resulted in increased time spent in the periphery of the open field arena $(\mathrm{T}(13)=-2.11, p=0.027)$. Infusions immediately after fear conditioning failed to alter fear memory expression $24 \mathrm{hr}$ later $(\mathrm{T}(13)=0.41, p>0.05)$.

Conclusions: These data suggest that $\mathrm{T} 3$ is anxiogenic and also suggest that activation of TRs at the time of trauma exposure or fear conditioning is rapid and must occur at the initiation of trauma to impact subsequent fear expression. Additional experiments have revealed that infusions of 1-850 at the time of fear conditioning alter the expression of many fear and memory related genes suggesting that TRs may mediate trauma-related gene expression in the amygdala. In sum, these data strongly implicate for the first time a dynamic role for the thyroid hormone axis in the amygdala in modulating fear memory related neuroplasticity.

Keywords: Thyroid Hormone, Amygdala, Fear Memory, Anxiety, Gene Expression

Disclosure: Nothing to Disclose.

M14. The Effect of Prazosin on Individual PTSD Symptoms and on the Covariance of Symptoms Over Time: Evidence for Pathophysiologically-Related Clustering

Rebecca Hendrickson*, Steve Millard, Shofer Jane, Elaine Peskind, Murray Raskind

University of Washington, Seattle, Washington, United States

Background: The $\alpha 1$ adrenoreceptor antagonist prazosin has been found to be effective for PTSD associated nightmares, hyperarousal symptoms, and total symptom severity. The particular efficacy of prazosin for nightmares (categorized as a reexperiencing symptom) and hyperarousal symptoms has led to the hypothesis that these symptoms may represent a subset of PTSD symptoms that are more tightly associated with an $\alpha 1$ adrenoreceptor mediated noradrenergic mechanism. This would suggest the existence of a pathophysiologically-related clustering of symptoms that crosses traditional symptom groupings.

The grouping of psychiatric symptoms into clusters is most often accomplished using factor analysis of single timepoint symptom profiles. An approach that is less frequently used is to use a longitudinal data set to observe whether the fluctuations of particular subsets of symptoms tend to move together in time, consistent with the presence of a shared pathophysiologic mechanism. A particularly powerful version of this approach is one where this is combined with using a pharmacologic probe, and observing whether certain subsets of symptoms are more likely to change together in 
the presence and absence of the probe. However, this has rarely been done in PTSD, and never with a pharmacologic agent that is as specific as prazosin for a single aspect of PTSD pathophysiology.

Here, we provide the first report of the efficacy of prazosin for individual symptoms other than nightmares and sleep disruption. We then examine whether the distribution of prazosin-responsiveness in PTSD symptoms can be explained by the distribution of these symptoms in the subject population, and whether there is evidence in the individual subject-level fluctuations in symptom endorsement in the presence and absence of prazosin for a pathophysiologicallyrelated cluster of prazosin-responsive symptoms.

Methods: In a post hoc reanalysis of a previously published, randomized controlled trial of twice daily prazosin for PTSD, we examined the relative effect of prazosin on individual items of the CAPS for DSM-IV. We examined the relationship of prazosin-responsiveness to the baseline distribution of symptoms in the population, and tested whether prazosin responsiveness predicted the partial correlation of the changes in symptom intensity at the level of individual subjects.

Results: Prazosin showed the largest effect for distressing dreams, anhedonia, difficulty falling or staying asleep, difficulty concentrating, and hypervigilance. These items were both higher in the subject population at baseline, and more related in how they fluctuated at the level of individual subjects, a finding most pronounced in the group receiving active study drug. In addition, subjects with more of their initial symptoms in the group of prazosin-responsive symptoms were most likely to respond to prazosin.

Conclusions: Twice daily prazosin significantly reduces not only nightmares and sleep disruption, but the majority of hyperarousal symptoms, with some evidence of efficacy for avoidance symptoms. The pattern of symptom endorsement at the level of individual subjects was consistent with prazosin-responsive items sharing a common pathophysiologic mechanism.

Keywords: PTSD, Noradrenergic System, Prazosin

Disclosure: Nothing to Disclose.

\section{M15. TMS-EEG Biomarkers for Combat-Related PTSD}

Wei $\mathrm{Wu}^{*}$, Corey Keller, Amit Etkin

Stanford University, Mountain View, California, United States

Background: Post-traumatic stress disorder (PTSD) is a severe psychiatric disorder that occurs after a psychological traumatic life event and increases individual vulnerability to adverse health outcomes. Combat-related PTSD is experienced by men and women who have been in combat. Despite the large proportion of veterans returning from war with PTSD, little is known regarding the underlying circuit abnormalities that are responsible for residual posttraumatic symptoms. Lack of this knowledge currently impedes the ability to develop circuit-informed novel therapeutic interventions.

Methods: We performed concurrent single-pulse TMS-EEG (spTMS-EEG) to a large number of healthy controls $(N=$ $80)$ and PTSD combat veterans $(N=80)$ who were deployed to either Iraq, Afghanistan, or both. Sites in key cognitive and emotional networks, including posterior dorsal lateral prefrontal cortex (pDLPFC) in the central executive network (CEN) and anterior dorsal lateral prefrontal cortex (aDLPFC) in the salience network (SN), as well as the primary motor cortex as the control sites were stimulated. The EEG data were cleaned using our in-house fully automated artifact rejection algorithm [1]. The spatiotemporal dynamics following the spTMS were then examined. The EEG responses were quantitated using TMSevoked potentials (TEPs) and event-related spectral perturbations (ERSPs), in both the sensor and source spaces.

Results: Following pDLPFC and aDLPFC stimulation, significant differences were observed for the early $(<100$ $\mathrm{ms}$ ) and late ( $>100 \mathrm{~ms}$ ) TEPs between the PTSD and healthy control groups $(p<0.05$, unpaired t-tests on the sensor and source level, FDR corrected for multiple comparisons). These changes were identified across a number of key regions within the CEN, SN, and default mode network (DMN), including the DLPFC, inferior parietal lobule, anterior cingular cortex, insula, medial PFC, and angular gyrus.

Conclusions: These results reveal for the first time, abnormalities of casual information processing in PTSD patients across key cognitive and emotional brain networks. In particular, there is more inhibition of brain activity at $\sim 100 \mathrm{~ms}$ in these networks following the stimulation for PTSD patients. These biomarkers are critical in understanding the mechanisms underlying PTSD. Potential future avenues of this work include development and personalization of rTMS to normalize these circuit abnormalities.

Keywords: TMS-EEG, Postramatic Stress Disorder, Biomarker

Disclosure: Nothing to Disclose.

M16. Meta-Analysis of DNA Methylation and PTSD in the Psychiatric Genomics Consortium PTSD Epigenetics Workgroup

Alicia Smith*, Andrew Ratanatharathorn, Marco Boks, Mark Logue, Adam Maihofer, Varun Kilaru, Murray Stein, Eric Vermetten, Karestan Koenen, Allison Aiello, Dewleen Baker, Michael Hauser, Nathan Kimbrel, Allison Ashley-Koch, Ben Luft, Evelyn Bromet, Mark Miller, Kerry Ressler, Monica Uddin, Caroline Nievergelt

Emory University School of Medicine, Atlanta, Georgia, United States

Background: Post-traumatic stress disorder (PTSD) results from trauma, but not all individuals develop PTSD after trauma. Differences in susceptibility to PTSD may be related to epigenetic differences between cases and trauma-exposed controls that can provide insight into the biological processes underlying the disorder. The Psychiatric Genomics Consortium (PGC) PTSD Epigenetics Workgroup is working to identify DNA methylation-based epigenetic biomarkers of PTSD that can serve as important indicators of the disorder, and to characterize which of these biomarkers identify individuals who may be most at risk following trauma. 
Methods: Military and civilian studies contributed bloodderived DNA methylation microarray data (HumanMethylation450 BeadChip) from PTSD cases and trauma-exposed controls from three civilian and seven military cohorts. To facilitate a cross-sectional meta-analysis, a common pipeline was applied to all 1,896 samples. Each CpG site was tested for association with current PTSD using linear models that control for age, genomic-derived ancestry estimates, leukocyte cell proportions as well as study-specific covariates if applicable (e.g. sex) followed by meta-analysis using inverse normal p-value combination and false discovery rate (FDR) estimation.

Results: The primary analyses of all samples revealed that the top four $\mathrm{CpG}$ sites fell within the aryl-hydrocarbon receptor repressor (AHRR) locus $(\mathrm{FDR}<0.0003)$ and were all associated with lower DNA methylation in PTSD cases. Sensitivity analyses that controlled for smoking substantially attenuated associations at all four sites, with the fifth ranked $\mathrm{CpG}$ site in ring finger protein $6(\mathrm{RNF} 6$; FDR $=0.007)$ to the top rank for association with PTSD. However, results did not maintain statistical significance $(F D R=0.09)$ after including smoking as a covariate. The meta-analysis of civilian cohorts $(N=545)$ yielded two PTSD-associated CpG sites in neuregulin 1 (NRG1; FDR $=0.023)$ and hepatocyte growth factor-regulated tyrosine kinase substrate (HGS; FDR = 0.033). Both loci remained significant after controlling for smoking.

Conclusions: This meta-analysis has identified associations between CpG sites in multiple biologically relevant genes and PTSD. Future studies will explicate the effect of trauma types across civilian and military cohorts, evaluate the association of identified $\mathrm{CpG}$ sites in longitudinal samples, and integrate GWAS data into ongoing EWAS analyses.

Keywords: DNA Methylation, Epigenetic, Posttraumatic Stress Disorder, PTSD

Disclosure: Nothing to Disclose.

\section{M17. Hippocampal-Prefrontal Theta Transmission Regulates Anxiety-Like Behavior}

Nancy Padilla-Coreano*, Sarah Canetta, Alvaro Garcia, Christoph Kellendonk, Joshua Gordon

Massachusetts Institute of Technology, Cambridge, Massachusetts, United States

Background: Despite enormous advances in our understanding of neural transmission and neural circuitry, the nuances of the brain's neural code remain unknown. Neural oscillations have been observed at different frequencies in the human and animal brain and have been associated to distinct behavioral states. Frequency-specific synchronization of these oscillations across regions has been associated with a variety of important behaviors and has been hypothesized to reflect increased functional connectivity and neural transmission. However, the evidence to suggest a causal role for frequency-specific oscillations in determining behavior remains largely associational and therefore speculative. To address this issue, we turned to a rodent paradigm-the elevated plus maze-where synchrony between the ventral hippocampus (vHPC) and medial prefrontal cortex (mPFC) in the theta-frequency $(4-12 \mathrm{~Hz})$ range has been tightly linked to anxiety-like behavior. Exposure to anxiogenic environments enhances theta synchrony between the vHPC and $\mathrm{mPFC} 4$, facilitating the construction of neural representations of aversion within the $\mathrm{mPFC}$ and optogenetic inhibition of the direct projections from the vHPC to the mPFC ablates these representations and reduces both thetafrequency synchrony and avoidance behavior.

Methods: To test the causal relevance of theta-frequency communication between the vHPC and $\mathrm{mPFC}$ in the production of anxiety-like behavior, we dynamically modulated vHPC-mPFC terminal activity at different frequencies using optogenetic stimulation in mice.

Results: Terminal stimulation with an $8 \mathrm{~Hz}$, but not $20 \mathrm{~Hz}$, was sufficient to increase avoidance behavior, but only when the stimulus was delivered in an oscillatory, rather than pulsatile, stimulus manner. Furthermore, the $8 \mathrm{~Hz}$ oscillatory stimulus enhanced vHPC-to-mPFC neurotransmission and entrained theta-frequency neural activity in the entire vHPCmPFC network, particularly during exposure to an anxiogenic environment.

Conclusions: These data demonstrate that theta-frequency communication between the vHPC and $\mathrm{mPFC}$ plays a privileged role in the generation of anxiety-like avoidance behavior, and provide direct evidence that synchronized oscillations between brain regions play a role in neural communication.

Keywords: Anxiety Circuitry, Medial Prefrontal Cortex, Theta

Disclosure: Nothing to Disclose.

\section{M18. Epigenetic Relationships With Response to Mind- fulness Based Stress Reduction for PTSD}

Jeffrey Bishop ${ }^{\star}$, Adam Lee, Lauren Mills, Paul Thuras, Christopher Erbes, Melissa Polusny, Gregory Lamberty, Kelvin Lim

University of Minnesota, Minneapolis, Minnesota, United States

Background: Mindfulness Based Stress Reduction (MBSR) is an effective non-pharmacologic treatment for Veterans with PTSD. Extensive work has identified epigenetic factors related to PTSD disease risk and pathophysiology, but how these factors influence treatment response is unclear. Serotonin signaling and hypothalamic-pituitary-adrenal (HPA) axis functioning may be perturbed in PTSD and are molecular pathways targeted by treatments for PTSD. We compared methylation levels in functional regions of the serotonin transporter (SLC6A4/5HTT) and FK506 binding protein 5 (FKBP5) genes between responders and nonresponders to MBSR.

Methods: Genomic DNA samples from veterans with PTSD who received MBSR as part of a previously conducted clinical trial were examined. Responders $(n=11)$ and nonresponders $(n=11)$ (defined by $\geq 10 \mathrm{pt}$ improvement on PTSD Checklist total scores after 9 weeks of treatment were selected and matched for age, sex, race, childhood trauma, current smoking status, and medication exposure. A targeted next generation sequencing approach of bisulfite-converted DNA generating 300-500 reads per region examined methylation (CpG) sites (chr17:28562752-28563675) in 
SLC6A4 previously associated with expression and depression outcomes as well as the FKBP5 Intron 7 region (chr6:35558312-35558806) identified as a functional regulator of glucocorticoid signaling. Percent methylation was compared between responders and non-responders at baseline (pre-MBSR treatment). Additionally, percent change in methylation from before to after treatment was compared between responders and non-responders.

Results: Lower baseline methylation (previously associated with increased 5HTT expression) of CpG.28563225 was observed in those who eventually responded to MBSR $(1.4 \pm 0.8 \%)$ compared to non-responders $(2.6 \pm 1.2 \%)$ ( $\mathrm{T}$ $=-2.78, p=0.012$ ). Mean methylation across FKBP5 Intron 7 , changed differentially after treatment by responder status. Decreased methylation (indicative of normalizing stress signaling) was observed in responders while increased methylation was observed in non-responders (time $\mathrm{x}$ responder group interaction $p=0.024$ ).

Conclusions: These finding highlight the potential importance of epigenetic factors in determining response to meditation treatment in PTSD. In this carefully matched sample of responders and non-responders to MBSR, baseline methylation as well as changes in methylation after treatment were observed. Baseline differences in 5HTT methylation indicate that efficient serotonin signaling may be important for meditation to effectively reduce PTSD symptoms. Additionally, decreases in FKBP5 methylation indicative of normalizing stress were observed after treatment in responders as compared to increases in non-responders. This suggests that effective meditation may positively modify stress-related pathways at the molecular level.

Keywords: PTSD, Epigenetics, Mindfulness

Disclosure: Nothing to Disclose.

\section{M19. Reorganization of Resting Connectivity Patterns Following Psychotherapy for Posttraumatic Stress Disorder}

Gregory Fonzo*, Madeleine Goodkind, Desmond Oathes, Yevgeniya Zaiko, Meredith Harvey, Kathy Peng, Elizabeth Weiss, Allison Thompson, Sanno Zack, Colleen Mills-Finnerty, Benjamin Rosenberg, Raleigh Edelstein, Rachael Wright, Carena Kole, Barbara Rothbaum, Amit Etkin

\section{Stanford University, Stanford, California, United States}

Background: Posttraumatic stress disorder (PTSD) is a highly impairing, increasingly prevalent mental disorder with a chronic course and low rates of spontaneous remission. Effective treatments have been developed, the most widely utilized of which are trauma-focused psychotherapies such as prolonged exposure. Although psychotherapy is widely utilized and the only empirically efficacious PTSD treatment, it requires a considerable investment of time and effort from both patient and practitioner, with roughly one-quarter of patients not completing treatment and one-third to one-half of those who complete treatment remaining symptomatic and impaired. Neuroscience offers promising tools to inform both clinical practice and theoretical understanding of the mechanism of exposure-based psychotherapy, which remains poorly understood. Non-invasive imaging tools such as functional magnetic resonance imaging (fMRI) provide an opportunity to understand how brain function is changed by the process of undergoing treatment, which can provide testable hypotheses for mechanisms that may underlie the cessation of PTSD symptoms and therapeutic recovery from the disorder. We recently published taskbased fMRI findings on the largest sample to date of individuals with PTSD receiving treatment in a randomized clinical trial of prolonged exposure therapy vs. patient waitlist. These findings provided much-needed insight regarding the pretreatment neural phenotype during emotional reactivity and regulation that best responds to prolonged exposure treatment, as well as how brain function in these emotional contexts is remediated by psychotherapy. Of particular generalizability and easily-disseminated to the clinic setting is the metric of fMRI resting state connectivity, which requires less infrastructure to acquire and generates voluminous amounts of potentially useful signal for predicting treatment outcome and understanding mechanisms. Scientific understanding of how resting state connectivity in PTSD predicts treatment response and changes following effective treatment is incredibly sparse. This metric's relatively quick and easy acquisition, increasing popularity, and flexible applications qualify resting state connectivity as a prime candidate for biomarker development and informer of mechanisms. This presentation will report on how seeded resting state connectivity patterns of brain structures crucial to PTSD pathophysiology and potential psychotherapy mechanisms are altered by prolonged exposure treatment. Methods: Sixty-six individuals with PTSD were individuallyrandomized to undergo immediate treatment with prolonged exposure $(N=36)$ or to a 10 -week patient waitlist $(N=30)$. Each patient underwent an 8- minute eyes-open resting state fMRI scan prior to randomization, and individuals randomized to immediate treatment underwent a post-treatment resting state fMRI scan roughly 4 weeks following their last therapy session (with a comparable delay for those in patient waitlist). Resting state scans were also acquired in traumaexposed (TEHC; $N=37$ ) and non trauma-exposed (NTHC; $N=31$ ) healthy comparison subjects to determine whether treatment-related changes reflect normalization of abnormalities or compensatory adaptations. Subregional patterns of resting connectivity were computed using the CONN toolbox on a voxel-wise level in the amygdala, hippocampus, insula, and frontopolar cortex to assess treatment-related general regional changes as well as those specific to functional subdivisions of relevant brain structures.

Results: Across all seed regions, we observed prominent whole brain significant shifts in patterns of resting state connectivity that were specific to the treatment group. These shifts were characterized predominantly by region-general changes, i.e. the same across subregions, such that the insula, amygdala, hippocampus, and frontopolar cortex become more tightly coupled with one another across time, while also showing increased connectivity with ventral visual and sensory-motor cortices and decreased connectivity with dorsolateral and dorsomedial prefrontal regions implicated in salience and executive control.

Conclusions: These findings demonstrate a prominent effect of exposure-based psychotherapy on resting brain dynamics in PTSD. This effect is characterized by a general pattern of 
enhanced connectivity amongst core limbic brain structures implicated in the pathophysiology of PTSD and the most anterior, higher-order region of the prefrontal cortex that we previously demonstrated to show treatment-dependent recruitment during emotion regulation in this same sample. Analyses to determine abnormality correction vs. compensatory adaptation via comparison to healthy controls will follow. Given observations for increased connectivity of these seeds with visual and sensory-motor cortices and decreased connectivity with dorsal prefrontal control regions, these findings suggest one downstream therapeutic effect of psychotherapy may be to reorganize communication patterns of emotional/salience processing streams away from deliberate top-down control and towards greater integration with the internal and external environmental percepts. This neuro-mechanistic account is consistent with the putative psychological mechanism of prolonged exposure, i.e. adaptive learning via emotional engagement with internal and external trauma cues while abstaining from deliberate efforts to regulate one's emotional state.

Keywords: PTSD, Connectivity, Psychotherapy, Resting State, Trauma

Disclosure: Nothing to Disclose.

M20. Therapeutic Transcranial Magnetic Stimulation Improves Cortical Information Flow in Posttraumatic Stress Disorder

Noah Philip* ${ }^{*}$ Jennifer Barredo, Emily Aiken, Mascha van 't Wout, Benjamin Greenberg, Linda Carpenter

\section{Brown University, Providence, Rhode Island, United States}

Background: Posttraumatic stress disorder (PTSD) is consistently associated with ventromedial prefrontal cortex (VMPFC) dysregulation and other neural network disruptions. However, prior research has not evaluated systemslevel network organization in PTSD, nor how functional architecture might change due to clinical interventions impacting functional networks. Convergence is a voxellevel density metric describing the architecture of functional networks (Bell et al., 2015). Networks exhibiting higher convergence are biased toward integrative processing, whereas low convergence networks are more functionally segregated. This approach has been used in healthy controls to describe systems-level integration, but has yet to be utilized in clinical populations, and is a promising method to describe network-based etiologies underlying functional connectivity changes observed in psychiatric illnesses. To this end, we 1) evaluated how convergence differed between posttraumatic stress disorder (PTSD) patients and controls, and then tested 2) how convergence changed after therapeutic transcranial magnetic stimulation (TMS).

Methods: First, we computed individual-level cortical parcellations (Wang et al., 2015) to define functional networks in patients with PTSD $(N=32)$ and healthy controls $(N=32)$. We then computed network convergence as the sum of networks (Yeo et al., 2011) exhibiting suprathreshold representation (bootstrapped 90th percentile) at a given vertex on the cortical surface. We defined a region of interest (ROI) matrix incorporating areas dysregulated in
PTSD: caudate, thalamus, rostal anterior cingulate cortex (ACC), dorsolateral prefrontal cortex (DLPFC), VMPFC, pars orbitalis, opercularis, triangularis, and precuneus). Subjects' network convergence statistics were entered into general linear models to evaluate the impact of convergence on ROI-to-ROI connectivity, covarying for age, gender, and signal-to-noise. These analyses were repeated in PTSD patients $(n=24)$ after a course of $5 \mathrm{~Hz}$ TMS to the DLPFC (Philip et al., 2017).

Results: Consistent with prior imaging research, we observed broad-based reductions in connectivity in PTSD compared to controls, including VMPFC-to-left DLPFC $(\mathrm{t}(60)=-3.49$, $\mathrm{p}-\mathrm{FDR}=.02)$, VMPFC-to-right pars orbitalis $(\mathrm{t}(60)=-3.29$, $\mathrm{p}-\mathrm{FDR}=.02)$, and ACC-to-right orbitalis $(\mathrm{t}(60)=-3.07$, $\mathrm{p}-\mathrm{FDR}=.04)$ connectivity. Of these relationships, increased salience network convergence was associated with reduced VMPFC-to-DLPFC connectivity $(\mathrm{t}(59)=1.96, p=0.05)$. Increased salience network $(\mathrm{t}(59)=2.10, p=0.04)$ and default mode network $(\mathrm{DMN})(\mathrm{t}(59)=3.27, p=<0.01)$ convergence were also associated with reduced VMPFC-to-orbitalis connectivity in PTSD.

TMS did not change within-network convergence (all $p>.1$ ). However, after stimulation, increased VMPFC-to-DLPFC connectivity was observed and this increase was associated with greater precuneus/DMN convergence $(\mathrm{t}(19)=2.47$, $p=0.02)$. There were also statistical trends between increased DMN convergence and increased VMPFC-to-orbitalis connectivity $(\mathrm{t}(19)=1.84, p=0.08)$, and reduced attention network convergence and increased ACC-to-orbitalis connectivity $(\mathrm{t}(19)=-1.96, p=0.06)$ after TMS.

Conclusions: Consistent with prior research, we found PTSD was associated with diminished connectivity in threatdetection and inhibitory control regions, and that hypoconnectivity was related to differences in the salience network, rather than changes to top-down executive control. While TMS did not change the capacity for information flow within networks themselves, stimulation resulted in normalization of prefrontal connectivity and a shift in VMPFC information flow across networks from salience to default network. These results demonstrate that network convergence can be used to characterize multi-network interactions and potentially uncover new potential mechanisms of action of brain stimulation.

Keywords: Repetitive Transcranial Magnetic Stimulation, MDD, PTSD, Resting State Functional Connectivity

Disclosure: Part 1: Neuronetics, Grant, Part 4: Neuronetics, Grant.

\section{M21. Robust Prediction of PTSD Likelihood From Early Symptoms Results From the International Consortium to Predict PTSD (ICPP) Pooled Data Analysis}

Arieh Shalev*, Andrew Ratanatharathorn, Wei Qi, Martin Gevonden, Ron Kessler, Karestan Koenen, Eugene Laska

New York University Langone Medical Center, New York, New York, United States

Background: Reliable determination of the risk of PTSD among recent trauma survivors is a pre-requisite for efficient prevention, service planning and exploring the disorder's 
pathogenesis. Studies to date have identified numerous, scattered risk indicators that did not converge into a reliable risk-assessment tool. The International Consortium to Predict PTSD (ICPP) overarching goal was to develop a function that reliably produces the probability of PTSD given a set of risk indicators.

Methods: To generate such a tool, ICPP members shared item-level data from 10 longitudinal studies of PTSD early development. PTSD and PTSD symptoms were longitudinally evaluated in 2,473 adult civilians admitted to acute care center following traumatic events, using the Clinician Administered PTSD Scale (CAPS). The probability of chronic PTSD was estimated using logistic regression and early PTSD symptom severity (CAPS total scores) as the predictor variable. Smoothed probabilities for PTSD, based on the fraction of chronic PTSD cases at each baseline severity score, were calculated and compared with predicted probabilities from the logistic regression to measure model fit. The contributions of participants' age, gender, trauma type, lifetime trauma exposure, relationship status, and education to prediction accuracy were evaluated. Prediction using a Random Forest model was compared to the logistic regression model using 10-fold cross-validation.

Results: Traumatic events leading to acute care admission included motor vehicle accidents (69.4\%) other accidents (25.1\%) and interpersonal violence (5.5\%). 36.8\% of the participants were females. The sample's average age was $39 \pm 14$. Baseline assessments took place an average of $10.3 \pm 10.6$ days after the traumatic events (range 1-60), Endpoint interviews averaged $300.7 \pm 98.1$ days after trauma exposure (range 122-456). The overall prevalence of PTSD at endpoint was $11.8 \%(n=292 ; 9.1 \%$ for male and $16.4 \%$ for female participants).

Intriguingly, the distribution of baseline PTSD symptoms for participants who developed PTSD spread across the full range of early CAPS severity scores (e.g., 25\% of all PTSD cases originating in each of the following intervals: 0-39, 4060, 61-80, and 81-126).

In the logistic regression model, the CAPS total score at baseline predicted the probability of PTSD remarkably well (smoothed probability correlation $=0.978$; Efron' $\mathrm{R} 2=0.23$; Brier score $=0.080)$. The predicted probability of PTSD increased with baseline CAPS severity scores (e.g., respectively, $9 \%, 36 \%, 75 \%$ and $94 \%$ for CAPS score of $34,68,102$, and 136, representing the four quartiles of the instrument's severity range).

Despite the higher prevalence of PTSD among female participants, at any given baseline CAPS score, the probability of PTSD for males and females were the same. Similarly, despite their well-recognized role as risk factors, the probability of PTSD conditional on the baseline CAPS score was independent of age, trauma type, and relationship status. Lower education (less than high school) and lifetime exposure to interpersonal trauma increased the risk for chronic PTSD by approximately $8 \%$ across baseline severity score, but did not affect the model's fit. Random Forest analysis' area under the curve of the receiver operator characteristic (0.850) did not differ from that obtained by the logistic regression model (0.846). Prediction from CAPS scores obtained within 30 days of the traumatic events, and prediction of 9-15 months' PTSD status did not affect the predictive accuracy of the model.
Conclusions: Early PTSD symptoms accurately predicted the probability of chronic PTSD. Furthermore, the contribution of well documented risk factors for PTSD was robustly mediated by these symptoms. Indeed, these risk factors were associated with the severity of the initial response. The uniform distribution of future PTSD cases among CAPS initial scores has not been previously described. It illustrates the previously encountered difficulty of predicting PTSD status (rather than probability) from early risk indicators. The remarkable fit of the model in data generated in separate projects, centers and countries, suggests that the logistic predictor has the potential for describing the likelihood of PTSD among survivors admitted to acute care centers globally. It, thus, provides the first predictive model that can be productively used by clinicians and health care planners. Early PTSD symptoms express many of the putative effects of known and yet unidentified predictors, and as such constitute a valid object for PTSD etiologic research.

Keywords: Post Traumatic Stress Disorder, PTSD, Prediction, Causal Modeling, Trajectory Modeling, Cortisol, Predictive Models

Disclosure: Nothing to Disclose.

\section{M22. Pre-Deployment Anhedonia is a Risk Factor for} Posttraumatic Psychopatholgy After Combat Trauma

Victoria Risbrough*, Tyler Moore, Dean Acheson, Caroline Nievergelt, Tallie Z. Baram, Hal Stern, Dewleen Baker

University of California, San Diego, La Jolla, California, United States

Background: Anhedonia, the diminished ability to experience pleasure, is an important dimensional entity linked to depression, schizophrenia and other emotional disorders. Alterations in circuits and cells underlying hedonia/reward sensitivity may be associated with risk for development of mood and anxiety disorders such as posttraumatic stress disorder (PTSD). Studies indicate that (1) low reward sensitivity in adolescence predicts later depression in adulthood and (2) remitted PTSD subjects continue to show deficient reward learning. Here we tested the hypothesis that anhedonia in early adulthood may be a risk factor for development of PTSD after trauma exposure.

Methods: The Marine Resiliency Studies (MRS) is a prospective longitudinal study of risk factors for combatrelated stress disorders in Marines deployed to Afghanistan and Iraq. Participants underwent psychiatric symptom assessments (clinician-administered PTSD scale, CAPS) and completed self-report scales for anxiety, mood and substance use symptoms prior to deployment and again at 3 and 6 months after returning from deployment. The MRS battery assessed anhedonia via the Beck Depression Inventory (A-BDI). An exploratory factor analysis (EFA) with iterated-target rotation was performed on all 21 Beck Depression Inventory (BDI) items rated at pre-deployment $(n=2600)$. This EFA revealed a 3 -factor structure, including an "anhedonia factor" A-BDI). The A-BDI combines the scores of item 4 (loss of pleasure), item 12 (loss of interest), and item 21 (loss of interest in sex). The 3 items composing the anhedonia factor were used to calculate the A-BDI We 
then used logistic regression to examine if anhedonia at predeployment in non-clinical subjects (i.e. no moderate-severe depression or PTSD symptoms) predicted subsequent PTSD diagnosis (DSM-IV) and a zero-inflated negative binomial model to examine if anhedonia predicted change in PTSD symptoms after deployment. Participants with a PTSD or depression diagnosis at pre-deployment were removed before analysis, and analysis was restricted to subjects with follow up assessments at 6 months to quantify a change vector for PTS symptoms $(N=1972)$. Deployment trauma was included in the model by using a composite of the postbattle and combat experiences subscales of the deployment risk and resiliency index.

Results: Pre-deployment anhedonia significantly increased the likelihood of post deployment PTSD diagnosis as assessed by DSM-IV symptom via CAPS [Main effect of Pre-deployment Anhedonia beta $=0.21, \mathrm{z}$ value $=4.61$, $p<1 \mathrm{e}-05$; Main effect of Deployment trauma beta $=0.37, \mathrm{z}$ value $=12.784, p<1 \mathrm{e}-05$; trauma $\mathrm{X}$ anhedonia interaction was not significant]. In addition, anhedonia significantly predicted increases in CAPS scores from pre- to postdeployment.

Conclusions: These data support the hypothesis that anhedonia in early adulthood is associated with increased vulnerability for development of future pathology after trauma exposure. Future studies are needed to determine if anhedonia in early adulthood and PTSD share common underlying etiologies and mechanisms. Supported by 1I01BX002558, MR141217, P50 MH096889 and Veterans Affairs Center of Excellence for Stress and Mental Health. Note: This poster is linked to Bolton et al. describing brain circuit changes underlying anhedonia-like phenotypes in adolescence. Please view poster W114, poster session III.

Keywords: PTSD, Anhedonia, Brain Development, Depression, Longitudinal Study

Disclosure: Nothing to Disclose.

M23. Distinct Subpopulations of Somatostatin Interneurons in Dorsolateral Septum Relay Fear and Anxiety Signals to Govern Activation of Subcortical Circuits

\section{Amar Sahay*}

Massachusetts General Hospital, Harvard Medical School, Boston, Massachusetts, United States

Background: Adaptive fear responses to ambiguous environmental threats rely upon hippocampal contextual encoding and subsequent relay of these computations to limbic circuits mediating behavioral fear responses. Contextual information processed in the hippocampus maybe relayed via the thalamus, prefrontal cortex and/or dorsolateral septum to subcortical anxiety and fear circuits. We sought to identify the neural pathways that are recruited under conditions of high and low contextual fear discrimination.

Methods: We carried out brain-wide analysis of co-activated ensembles by c-Fos immunohistochemistry under conditions of high and low contextual fear discrimination and identified neural pathways (dentate gyrus (DG)-CA3-Dorsolateral septal (DLS) circuit) and cellular relays (somatostatin interneurons in the DLS) that link hippocampal computations underlying resolution of contextual threats with subcortical fear and anxiety circuits. We examined physiological patterns of activation of specific cellular relays using in vivo longitudinal optical imaging in awake behaving mice in different fear and anxiety tasks and over time. We optogenetically manipulated these cellular relays and assessed the impact on behavior. We used whole-cell electrophysiology, pseudotyped rabies tracing and optogenetics to map monosynaptic inputs onto these cellular relays. We asked if mice engineered to harbor high levels of neurogenesis and that exhibit better contextual fear discrimination also show increased activation of these cellular relays.

Results: Brain-wide analysis of co-activated ensembles under conditions of high and low contextual fear discrimination identified a non-canonical dentate gyrus (DG)-CA3-Dorsolateral septal (DLS) circuit, whose activity was most highly correlated to discrimination performance. Within DLS, Somatostatin expressing interneurons (SST-INs) were highly activated during contextual fear discrimination. Longitudinal in vivo calcium imaging of SST-INs in DLS of awake, behaving mice identified three distinct, non-stochastically allocated subpopulations. One subpopulation of cells was preferentially activated by unconditioned stimuli (US, mild footshock) during conditioning and its activity was predictive of non-freezing epochs during recall. A second nonoverlapping subpopulation of SST-INs in DLS was recruited only during exploration of open arms in elevated plus maze and its activity did not change in response to footshocks and was not predictive of non-freezing epochs during recall. A third subpopulation of SST-INs in DLS responded to both footshocks and open arms and activity of these cells was elevated in the open arms and predicted non-freezing epochs. Retrograde mono-synaptic tracing, electrophysiological whole-cell recordings and optogenetic interrogation of DLS SST-INs demonstrated that these cells receive direct CA3 inputs and when artificially activated, recruit diverse proximal and distant subcortical targets to decrease anxiety and expression of fear. Furthermore, DLS SST-INs activity was increased in mice in which adult hippocampal neurogenesis was genetically enhanced to improve contextual fear discrimination.

Conclusions: Together, these observations, uncover previously unrecognized functional heterogeneity in subcortical SST-INs and suggest a role for distinct DLS SST-IN subpopulations in sensing and relaying computations from higher cortical areas to govern context specific activation of fear and anxiety circuits.

Keywords: Lateral Septum, Hippocampus, Fear Generalization, Somatostatin, Anxiety Circuitry

Disclosure: Nothing to Disclose.

\section{M24. Behavioral Effects of Optogenetic Induction of LTP in the Rat Medial Prefrontal Cortex After Chronic Unpredictable Stress}

Sarah Bulin*, Kelly Hohl, David Morilak

University of Texas Health Science Center, San Antonio, Texas, United States

Background: Stress-related mood and anxiety disorders, like depression and posttraumatic stress disorder (PTSD), are highly prevalent yet poorly treated. Relapse and residual 
$\overline{S 126}$

symptoms remain problematic, and a poor understanding of the neurobiology underlying these illnesses has limited the development of new treatments. Imaging studies have shown reduced activity in the $\mathrm{MPFC}$ in both depression and PTSD, associated with deficits in executive functions, including impaired cognitive flexibility, that not only represent symptoms of these illnesses, but also contribute causally to their development and maintenance. Such changes presumably involve aberrant forms of neural plasticity in the mPFC. Similarly, chronic stress compromises cognitive flexibility, and is likely to disrupt the plasticity that normally underlies this executive process. Cognitive flexibility mediated in the $\mathrm{mPFC}$ can be measured using the attentional set-shifting test, and chronic unpredictable stress (CUS) induces a deficit of cognitive flexibility on this test. Further, we have previously shown that CUS attenuates the response of the mPFC to stimulation of the excitatory afferent from the mediodorsal thalamus (MDT). Thus, we are using optogenetics to investigate if directly inducing plastic changes in the mPFC is sufficient to restore cognitive flexibility after CUS.

Methods: Glutamatergic neurons in the MDT were selectively infected either with the ChETA variant of channelrhodopsin by injecting an AAV5-CAMKII-ChETA viral vector, or with control AAV5-CAMKII-GFP, and allowed 6 weeks for expression and trafficking of the channel to terminals innervating the mPFC. Rats were anesthetized with chloral hydrate and baseline field potentials evoked by MDT stimulation were recorded in the $\mathrm{mPFC}$ for $15 \mathrm{~min}$. Optical LTP was induced by stimulating mPFC terminals and field potentials were collected for an additional hour to confirm synaptic potentiation. Then using this procedure, we are currently inducing LTP in awake CUS rats to attempt to rescue stress-induced deficits on set-shifting.

Results: The mean amplitude of evoked responses, recorded for $1 \mathrm{hr}$ after stimulation, was increased by $25 \%$ of mean baseline, indicating a potentiated response $(n=4 ; \mathrm{p}<0.002)$. This response was not observed in control GFP animals.

Conclusions: We are able to optogenetically induce LTP within the rat medial prefrontal cortex. Ongoing experiments include inducing LTP in awake CUS rats to attempt to rescue stress-induced deficits on set-shifting.

Keywords: Optogenetics, Medial Prefrontal Cortex, Chronic Stress, PTSD

Disclosure: Nothing to Disclose.

\section{M25. Association of Dysphoria With Brain Morphome-} try Among Patients With PTSD

\section{Alvaro Camacho*, Ruth Klaming, Andrea Spadoni, Alan Simmons \\ University of California, San Diego, Imperial, California, United States}

Background: The multiple comorbidities found among patients with PTSD points towards a multiple psychopathological dimensional structure of this disorder. Thus, it has been suggested that identification of the different structural components of PTSD symptomatology might help to elucidate the neurobiological basis of specific disorders. Dysphoria has been considered an important structural component of PTSD. Dysphoria is a factor that has been categorize in major depression, generalized anxiety and PTSD and corresponds to high levels of distress. Based on this rationale, we aim to study the association of the dysphoric construct with amygdala and hippocampal morphometry. These anatomic landmarks have been frequently cited as biological markers for patients with PTSD. Methods: The data includes 113 subjects combat exposed veterans with Post-Traumatic Syndrome (PTS) that received brain MRI scanning. Symptoms were measured using the PTSD Symptoms Check List (PCL) scale. Amygdala and hippocampus morphometry was measured using the fsl function FIRST, which uses meshes to calculate differences is shape and volume of brain structures. A confirmatory factor analysis (CFA) was used to obtain the best fitting models based on the published PTSD domains of introversion, arousal, avoidance and dysphoria extracted from the PCL items (based on factor analysis from Rademaker et al, 2012). We hypothesized a significant correlation between dysphoria and volume of the amygdala and hippocampus.

Results: The CFA showed an adequate fit for the model $(\mathrm{RMSEA}=0.3 ; \mathrm{CTI}=0.9)$. There was an inverse correlation $(\mathrm{r}=-0.24 ; \mathrm{df}=113 ; p=0.01)$ between dysphoria and the right amygdala. All the other correlations between dysphoria and the hippocampus and left amygdala were non-significant.

Conclusions: In this cross-sectional morphometric study of individuals with PTSD high levels of dysphoria were correlated with small right amygdala volume. Longitudinal imaging studies are needed to examine the causal association of dysphoria with morphometry and brain function.

Keywords: PTSD, MR Imaging, Brain Volume, Dysphoria Disclosure: Nothing to Disclose.

M26. Translating Arrows to Anxiety: An Ern-Based Biomarker of Risk for Pediatric Anxiety Predicts Altered Brain Function During Ecologically Valid Social Interactions in $\mathrm{fMRI}$

Johanna Jarcho*, Tessa Clarkson, Hung-Wei Chen, Megan Quarmley, Brady Nelson, Greg Hajcak

Stony Brook University, Stony Brook, New York, United States

Background: The error-related negativity (ERN) is an electrophysiological response to errors generated in the anterior cingulate (ACC). Heightened ERN is a promising biomarker of risk for, and expression of, anxiety in children and adults. The ERN is typically measured during a 'flanker' task when participants make a mistake in identifying the direction of a central arrowhead that is quickly flashed on a computer screen (i.e., " $<<><<$ "). Yet, real world contexts that elicit symptoms of anxiety often involve a greater degree of uncertainty, both in terms of interpreting stimuli, the selection of potential behavioral responses, and in the determination of whether an error has been made. It is unclear whether heightened ERN predicts altered brain function during the type of interactions that typically elicit symptoms of anxiety in day-to-day life. Given the increased risk of developing persistent anxiety symptoms in adolescence, we sought to test these relations in youths 11-14 years of age. We collected fMRI data as adolescents, with a wellcharacterized ERN, completed the Virtual School Paradigm 
(2013, 2016). This ecologically valid social interaction task models the threat of peer evaluation, a common elicitor of anxiety in adolescents.

Methods: In an ongoing study, adolescents $(N=17$; $12.68 \pm 1.00$ years) first had ERN measured. Less than 2weeks later, participants returned for an fMRI scan. They were told they would visit a Virtual School and interact with purported "Other Students". Before completing the fMRI paradigm, participants learned each student had a reputation for being nice, mean, or unpredictable. While scanning, participants entered classrooms with the Other Students, and were cued to anticipate social evaluation from each peer, prior to receiving $50 \%$ positive and negative evaluation from unpredictable peers, and $100 \%$ positive or negative evaluation from nice and mean peers, respectively. Participants then made a behavioral response to this evaluation. Analyses were performed using a mixed-effects model with ERN as a continuous between-subject variable, and reputation or feedback type as repeated, within-subject variables.

Results: Replicating prior studies using the Virtual School paradigm, participants learned Other Student reputations and utilized distinct behavioral responses depending on the predictability and valence of the social evaluation they received. Region of interest analyses tested whether ERN differentially predicted patterns of ACC engagement as adolescents anticipated, and then received unpredictable and predictable positive and negative peer evaluation. Larger ERN amplitudes were associated with greater brain activity in the dorsal ACC $(6,4,37 ; p<0.005 ; \mathrm{ke}=27$ voxels), specifically while anticipating unpredictable, but not predictably positive or negative evaluation. No relations with dACC were observed during the receipt of social evaluation. Conclusions: Adolescents with a heightened ERN had altered neural engagement during an ecologically valid social interaction task. This alteration was observed in dorsal ACC, a region implicated in generating the ERN, and only during the anticipation of social evaluation from unpredictable peers. Elevated ERN has been associated with symptoms of anxiety, biases toward more rapid processing of negative information, and increased intolerance of uncertainty. This study demonstrates that elevated ERN, measured in a highly controlled non-social context, may be related to increased neural reactivity in social contexts when forthcoming peerbased evaluation is unpredictable. Implementing therapeutic techniques aimed at reducing ERN in unpredictable social contexts may be beneficial, particularly among youth at risk for anxiety disorders.

Keywords: Adolescent Anxiety, Social Interactions, dACC, EEG Biomarkers, Functional MRI (fMRI)

Disclosure: Nothing to Disclose.

\section{M27. Characteristic Changes of Hippocampal Subfield} and Shape in Drug Naive Obsessive-Compulsive Disorder

Lianqing Zhang, Kaili Liang, Lu Lu, Xuan Bu, Hailong Li, Yanchun Yang, Qiyong Gong, Xiaoqi Huang*

West China Hospital of Sichuan University, Chengdu, China

Background: Obsessive-Compulsive disorder (OCD) is a common psychiatric disorder with obsessions and compulsions as the main symptom profile. It has been suggested to associate with dysfunction in cortico-striatothalamo-cortical (CSTC) circuit. However, apart from this traditional neural circuitry deficit, hippocampal volume reductions have been confirmed by recent multicenter metaand mega-analysis. Since the hippocampus is a heterogeneous structure, with functionally distinct subfields, it is worthwhile to determine whether certain subfield is selectively implicated in OCD. In addition, whether this change is directly attributed to OCD pathology or associated with comorbid depressive symptoms remains controversial. Thus, in current study, we aim to explore the volume (with subfield) and shape of hippocampus in a relatively large sample of drug-naive OCD patients. We hypothesis that specific subfield would been affected differently in OCD and the contribution of core symptoms and affective symptoms would be distinguishable in hippocampal structural alterations in OCD.

Methods: 81 OCD patients (26 with HAMD score less than 7 were defined as none depressive OCD (ND-OCD), 55 with HAMD score greater than 7 and less than 17 defined as mild depressive OCD (D-OCD)) diagnosed according DSM-IV criteria and 95 healthy control subjects were recruited. High resolution $\mathrm{T} 1$ weighted images were obtained sing a volumetric 3-dimensional Spoiled Gradient Recall (SPGR) sequence $(\mathrm{TR} / \mathrm{TE}=8.5 / 3.4 \mathrm{~ms}$; flip angle $=12 \mathrm{o} ; 156$ axial slices with thickness $=1 \mathrm{~mm}$, field of view $=24 \times 24 \mathrm{~cm} 2$ and data matrix $=512 \times 512$ ) via a GE $3.0 \mathrm{~T}$ scanner.

Shape analysis of subcortical nucleus was conducted with FIRST vertex analysis tool to yield a localized measure of shape differences between groups. Statistical of shape differences between two groups were also performed using a general linear model (GLM).

The structural data was automatically segmented using the latest FreeSurfer software (V. 6.0)[2]. Volume of whole hippocampus and 7 subfields were extracted as well as intracranial volume (ICV). Volume differences between two groups were compared using a general linear model (GLM) with age and intracranial volume as covariates. Diagnose, hemisphere were entered as fixed factors. Correlations between volumetric measurements with illness duration, scores of YBOCS (Yale-Brown Obsessive Compulsive Scale), HAMA (Hamilton Anxiety Scale), and HAMD (Hamilton Depression Scale) were examined by using Pearson's correlation under SPSS 17.

Results: Comparing to healthy controls, Vertex based shape analysis demonstrated that OCD patients shown expansion on the lateral and compression in the medial region of bilateral hippocampus. Right whole hippocampus and subfields of subiculum and presubiculum was found significantly decreased in both ND-OCD and D-OCD groups comparing with healthy control subjects, while the right fimbria enlarged. Right CA2/3 and hippocampal tail were smaller in both OCD groups, but only reached significance in D-OCD group when comparing with HC. No significant volume difference was found between ND-OCD and D-OCD. Negative association between compulsion and volumes of right $\mathrm{CA} 2 / 3$, subiculum and presubiculum was found in ND-OCD patients.

Conclusions: As far as we are known, this is the largest sample size study on drug naive OCD patients concerning hippocampal subfields and shape. We demonstrated that 
hippocampus structural alterations in OCD was subfields specific and associated with compulsion symptoms. It was more prominent in the right hemisphere, especially as volume reduction in certain regions. Our finding also suggest that hippocampal abnormality is associated with OCD's core symptom, especially compulsion, although depressive symptom may add the effect of alterations in certain subfields. We assumed that hippocampus may play a more prominent role in OCD which had been overlooked in the traditional CSTC circuit hypothesis.

Keywords: Obsessive Compulsive Disorder, Magnetic Resonance Imaging, Hippocampal Subfields

Disclosure: Nothing to Disclose.

\section{M28. Challenges Testing Intranasal Ketamine in Obsessive-Compulsive Disorder (OCD)}

Carolyn Rodriguez ${ }^{\star}$, Kyle Lapidus, Jordana Zwerling, Amanda Levinson, Amanda Mahnke, Shari Steinman, Eyal Kalanthroff, Helen Simpson

Stanford University School of Medicine, Stanford, California, United States

Background: Given prior reports that a single intravenous (IV) dose of ketamine, a glutamate receptor modulator, rapidly decreases symptoms in unmedicated patients with Obsessive-Compulsive Disorder (OCD), we explored whether an intranasal delivery system is a practical alternative (e.g., lower cost and easier administration) to IV infusion. Lapidus et al. reported that intranasal ketamine was tolerable and effective in major depression. Thus, we hypothesized that intranasal ketamine administration in OCD patients would be similarly tolerated and yield a greater proportion of treatment responders than midazolam at 1 week post-administration.

Methods: With IRB approval, OCD outpatients (aged 18-55) were recruited (September 2014 to May 2015). Eligible patients met criteria for OCD (both DSM-IV and DSM-5), were at least moderately symptomatic (Yale-Brown Obsessive-Compulsive Scale [Y-BOCS $(6,7)$ ] score $\geq 16$ ), and on stable psychotropic medication doses for at least 6 weeks prior to enrollment. Exclusion criteria included severe depression (Hamilton Depression Rating Scale [17 item; $\operatorname{HDRS}(8)>25$ ) or comorbid psychiatric or medical conditions that made participation unsafe.

Patients were randomized 1:1 to receive intranasal ketamine $50 \mathrm{mg}$ or intranasal midazolam $4 \mathrm{mg}$. Ketamine was delivered using the administration protocol of a prior intranasal ketamine study in depression. An independent evaluator, blind to treatment, evaluated OCD and depression symptoms at baseline and one week after drug administration. Treatment response was defined a priori as $\geq 35 \%$ Y-BOCS score reduction. Patients randomized to midazolam were offered open-label intranasal ketamine $50 \mathrm{mg}$ after study completion.

Results: Of the 23 adults with OCD who contacted the clinic to participate in pharmacological studies, 20 (87\%) were screened and determined eligible for study participation. Of those 20 adults, 15 (75\%) refused study participation due to not wanting intranasal medication, $6(40 \%)$ of whom endorsed fear of contamination from the nasal applicator.
After two participants completed the study, we discontinued it due to poor tolerability and low enrollment rate as detailed below.

Subject \#1 was a 36-year-old African-American male with moderate OCD without depression $($ YBOCS $=21$; HDRS = 1), and taking no medications at baseline. He was randomized to $50 \mathrm{mg}$ intranasal ketamine. Upon administration, he showed visible signs of discomfort (e.g., wrinkled nose, upper lip retraction, recoiling), stating "this is very unpleasant in my nose - isn't there another way for me to get this?" but wanted to continue the study. Side effects included dissociation (e.g., body feeling unusually large, colors seemed brighter than expected, and time slowed) that lasted for 45 min after administration. One week after administration, he did not meet treatment response criteria (YBOCS $=19$; HDRS $=0$ ).

Subject \#2 was a 20-year-old Caucasian female (on stable dose of sertraline $125 \mathrm{mg}$ daily for OCD and divalproate $250 \mathrm{mg}$ daily for migraines) with severe OCD and moderate depression (YBOCS $=33$ and HDRS $=18$ ). She was randomized to midazolam $4 \mathrm{mg}$. She wrinkled her nose and complained of an unpleasant "bitter taste" and "stinging" in the nose; but wanted to continue the study. She reported lightheadedness and perceptual changes (i.e., sensation of having shrunk) 30 min after administration, which resolved by $60 \mathrm{~min}$ after administration; she also reported headache that resolved by $180 \mathrm{~min}$ after administration. One week post midazolam administration, she did not meet OCD response criteria, but her depressive symptoms improved from moderate to mild $(\mathrm{YBOCS}=35 ; \mathrm{HDRS}=10)$. She was offered and accepted open treatment with intranasal ketamine. Upon administration she frowned, wrinkled her nose, opened her mouth with tongue extension and complained of unpleasant taste, stating, "I would much prefer this in my vein than in my nose." She also reported nausea and headache that resolved 110 minutes postadministration. One week after ketamine administration, she did not meet OCD response criteria, though her depressive symptom severity met criteria for remission (YBOCS $=32$, HDRS $=2$ ).

Conclusions: Contrary to our hypotheses, 1) nasal delivery was poorly tolerated in the first two study participants, and 2) neither patient met OCD response criteria one week after ketamine administration. As a result of observed verbal and physical cues of patient's discomfort during nasal delivery and difficulty enrolling study participants, the study was discontinued. The discomfort reactions to intranasal ketamine were specific to the insertion of the nasal applicator and the spray method of delivery, rather than to medication side effects. In contrast, in prior studies of ketamine conducted by C.I.R., placement of the IV and ketamine administration were well tolerated. Enrollment rates for this study were very low (of the 20 screened eligible participants, only 2 [10\%] agreed to participate over 9 months), compared to significantly higher rates in C.I.R.'s studies in the same clinic with IV ketamine. Regarding our finding that neither patient met OCD treatment response criteria, our results may simply reflect subgroups of OCD patients that do and do not respond to ketamine (as indicated by contrasting prior publications on the efficacy of IV ketamine in OCD). By chance, the study participants $(n=2)$ may be in the subgroup that does not respond to ketamine. Approaches to 
properly assess efficacy of intranasal ketamine in OCD in future studies may require utilizing a more comfortable selfadministration device (Janssen, NCT01998958).

Keywords: OCD, Obsessive-Compulsive Disorder (OCD), Ketamine, Intransal, NMDA

Disclosure: Part 4: Naurex, Grant, Allergan, Consultant, Rugen Therapeutics, Consultant, BlackThorn Therapeutics, Consultant.

\section{M29. Monoaminoxidase A (MAOA) Gene Hypermethyla- tion in Posttraumatic Stress Disorder - Novel Insights From a European PTSD Cohort}

Christiane Ziegler, Christiane Wolf, Sabina Kucukalic, Emina Sabic Dzananovic, Elma Feric Bojic,

Nermina Kravic, Mirnesa Muminovic, Romana Babic, Ana Cima Franc, Nenad Jaksic, Blerina Hoxha, Valdete Haxhibeqiri, Dragan Babic, Esmina

Avdibegovic, Aferdita Goci Uka, Miro Jakovljevic, Abdulah Kucukalic, Alma Dzubur-Kulenovic, Jürgen Deckert, Katharina Domschke*

\section{University of Freiburg, Freiburg, Germany}

Background: Posttraumatic Stress Disorder (PTSD) is a severe and disabling trauma- and stress-related disorder which can occur after experiencing or witnessing severe traumatic events. The lifetime prevalence is estimated at $6.8 \%$ in the US-American population; in populations from traumatized, war-affected regions such as BosnianHerzegovina or Kosovo, point prevalence rates of PTSD rise up to $35 \%$ or $18 \%$, respectively. One major symptom cluster of PTSD comprises extreme hyperarousal and sleep problems and is suggested to be mediated by noradrenergic dysfunction. Epigenetic mechanisms such as DNA methylation are known to mediate the interaction between environmental influences such as traumatic experiences and a genetic predisposition. Thus, in the present study monoamine oxidase A (MAOA) gene methylation governing noradrenergic turn-over was investigated for its function in PTSD.

Methods: MAOA methylation at $13 \mathrm{CpGs}$ in the exon I / intron I gene region was analyzed in 216 current PTSD patients $[\mathrm{m}=157$, age (mean \pm s.d.): $50.08 \pm 6.74$ years $], 151$ remitted PTSD patients $[\mathrm{m}=98$, age (mean \pm s.d.): $49.48 \pm 8.20$ years] and 349 healthy controls $[\mathrm{m}=232$, age (mean \pm s.d.): $48.81 \pm 8.50$ years] recruited in five psychiatric centres in Bosnia-Herzegovina, Croatia and Kosovo via direct sequencing of sodium bisulfite-treated DNA extracted from peripheral blood samples. Furthermore, severity of PTSD symptoms was assessed by the Clinician-Administered PTSD Scale (CAPS). Number and intensity of stressful life events were assessed by a 22-item life stressor list.

Results: Patients with current PTSD displayed hypermethylation of three CpGs (CpG3=43,656,362; CpG12= 43,656,514; CpG13=43,656,553, GRCh38.p2 Assembly) as compared to remitted PTSD patients and healthy controls in the male, but not in the female subsample. In addition, symptom severity as represented by CAPS scores significantly correlated with MAOA methylation, particularly at CpG sites 12 and 13 in male PTSD patients. Furthermore, the total intensity of experienced stressful life events was positively correlated with average MAOA methylation in male PTSD patients.

Conclusions: The present multicentre-study for the first time reports evidence for a possible role of MAOA hypermethylation in male PTSD patients. Hypermethylation of the investigated exon I/intron I gene region - leading to a decreased MAOA expression - might contribute to the increased noradrenergic tone known to cause the disabling and often treatment resistant symptoms of PTSD contained in the hyperarousal cluster. MAOA hypermethylation might also serve as a biological marker of PTSD risk/severity in male patients, and this association might be further moderated by the intensity of experienced life events. Provided robust replication, MAOA hypermethylation might serve as a biomarker allowing for personalized treatment options buffering increased noradrenergic signalling conferred by MAOA hypermethylation in PTSD.

Keywords: Post Traumatic Stress Disorder, DNA Methylation, Monoamine Oxidase A

Disclosure: Nothing to Disclose.

M30. A Novel Orally Active Triple Reuptake Inhibitor for the Treatment of Post-Traumatic Stress Disorder (PTSD): D-578 Attenuates Abnormal Fear Behavior in a Rodent Model of Traumatic Stress

Frank Bymaster*, Timothy Hsu, Michael Lisieski, Arman Harutyunyan, Banibrata Das, Israel Liberzon, Maarten Reith, Shane Perrine, Aloke Dutta

TRImaran Pharma, Inc., Brownsburg, Indiana, United States

Background: Post-traumatic stress disorder (PTSD) is a common disorder which often results in marked functional impairment. Currently, the only approved drugs for the pharmacotherapy of PTSD are two serotonin selective reuptake inhibitors (SSRIs), paroxetine and sertraline. These drugs are only moderately effective and have significant adverse events. Clinical research and animal models of traumatic stress exposure have shown that, in addition to serotonin (5-HT), norepinephrine (NE) and dopamine (DA) systems are also dysfunctional in PTSD, suggesting that multi-modal modulation of these monoamines could result in improved pharmacotherapy of PTSD. Therefore, we have investigated the effects of a novel triple reuptake inhibitor (TRI) D-578 in a rodent model of traumatic stress. D-578 inhibits NE, 5-HT, and DA transporters with Ki values of 6, 21 , and $30 \mathrm{nM}$, respectively, and exhibits little to no affinity for other CNS receptors.

Methods: The effects of D-578 and paroxetine were evaluated in a rat model for traumatic stress exposure - the single prolonged stress (SPS) model - which has been shown to have construct, predictive, and behavioral validity for PTSD. Adult male Sprague-Dawley rats were exposed to SPS, which involves the serial application of three stressors (restraint, forced swim, ether). On the 8th day after sham treatment or SPS exposure, rats began a cued fear conditioning procedure, which consisted of acquisition (day 8), extinction (day 9), and extinction retention (day 10) sessions. Rats were treated intraperitoneally with vehicle, paroxetine $(5 \mathrm{mg} / \mathrm{kg})$, or D-578 $(10 \mathrm{mg} / \mathrm{kg}) 90 \mathrm{~min}$ prior to 
all sessions. For acquisition of fear conditioning, there were 5 tone/foot shock presentations. Freezing behavior upon presentation of the tone/shock or tone alone was measured using a computerized system.

Results: SPS had no significant effect on the acquisition of conditioned fear (as indicated by freezing behavior) or extinction behavior compared to sham treatment, but SPS significantly impaired the retention of extinction learning. Neither drug in SPS-treated rats significantly altered acquisition or extinction learning. However, D-578 treatment of SPS exposed rats, but not paroxetine treatment of SPS exposed rats, significantly attenuated the extinction-retention deficit induced by SPS. The SPS+D-578 group was not significantly different from the sham treatment group on retention of extinction learning.

Conclusions: These findings suggest that D-578 in a model for PTSD has greater efficacy in normalizing traumatic-stress induced extinction-retention learning deficits compared to paroxetine. Further supporting the efficacy of D-578 in pharmacotherapy of PTSD, D-578, like classical antidepressants used in treatment of PTSD, was highly efficacious in the rat forced swim test and did not have motor stimulant properties. Overall these results suggest that D-578 may attenuate maladaptive retention of fearful memories and support further testing of this agent for the pharmacotherapy of PTSD. Supported by NIH/ NIMH MH084888 (AKD).

Keywords: PTSD, Serotonin, Dopamine, Norepinephrine, Reuptake Inhibitor

Disclosure: Part 2: Neurovance, Inc., Employee, Part 5: TRImaran Pharma, Inc., Employee.

\section{M31. Gene Expression Analysis in Rett Patients Treated} With Insulin-Like Growth Factor 1

Daniela Tropea*, Stephen Shovlin, Albert Sanfeliu, Giorgio Pini

\section{Trinity College Dublin, Dublin, Ireland}

Background: Rett syndrome (RTT) has often been considered by investigators to be a prototypical model for researching the treatment of neuropsychiatric disorders. Its limited aetiology and yet complex presentation and development provide a unique challenge for geneticists and for identifying candidate treatments with potential applications to other brain disorders.

RTT is characterised typically by a regression of acquired skills including language and motor skills, accompanied by hand stereotypies, growth abnormalities and autonomic dysfunctions. Although the presentation of the disease is highly variable (Pini et al., 2016b), over 90\% of typical RTT patients have de novo mutations to the global transcriptional regulator Methyl CpG binding protein 2 (MECP2). There is no current approved medication for Rett, but preclinical studies (Tropea et al., 2009; Castro et al., 2014) showed that Insulin-like growth factor 1 (IGF1) and derivatives are potential treatments. Such treatments demonstrated to be safe in RTT patients (Pini et al., 2012; Khwaja et al., 2014).

Methods: In this study, we used RNA-sequencing to provide transcriptomic analysis of whole blood of RTT patients $(n=4)$, to determine what are the effects of IGF-1 on RTT patients. Whole blood samples were taken from patients before and after treatment with IGF-1 in an Open Label Trial (Pini et al., 2016), RNA was extracted and prepared for mRNA Sequencing (Illumina).

The Sequence data was transformed from FastQ to raw counts and FPKM using "New Tuxedo" protocol as described by Pertea et al. (2016) Differential Gene Expression (DGE) was then analysed across the samples using DESeq2 and EdgeR Bioconductor packages in RStudio and was examined in respect to time interval between before and after treatment.

Results: Based on the DGE produced from the sequencing data, samples were analysed comparing the blood samples before and after the IGF-1 treatment, of these comparisons 1 gene was found only in DESeq2, 19 genes were only found in EdgeR and 6 were overlapping with both. The Top DGE genes whose expression changed in response to IGF-1 effects were: EEF1A1P7, PLLP, CCDC85C, NDUFA1 (upregulated after IGF1 treatment), and ERCC5, HLA-G, MT-ATP6, MT-CO2 (downregulated after IGF1 treatment).

Conclusions: Single gene analysis of the top significant genes shows an effect of the treatment on mitochondrial metabolism and intracellular trafficking. Network analysis (Genemania, Gene Ontology Biological Function) identified a strong contribution in the $\mathrm{T}$ cell apoptotic process $(\mathrm{P}$-value $=0.001)$. These results have implications in the interpretation of the clinical trials involving IGF1 and derivatives.

Keywords: Rett Syndrome, Insulin-Like Growth Factor 1, Transcriptomics

Disclosure: Nothing to Disclose.

\section{M32. Oxytocin Receptor DNA Methylation and Gray Matter Volume in Maltreated Children}

Shota Nishitani*, Takashi X. Fujisawa, Shinichiro Takiguchi, Koji Shimada, Alicia K. Smith, Akemi Tomoda

Emory University School of Medicine, Atlanta, Georgia, United States

Background: Child maltreatment increases risk for psychiatric disorders throughout childhood and into adulthood. Previous neuroimaging studies using structural magnetic resonance imaging (MRI) have revealed that exposure to early adversity is strongly associated with alterations in brain structure especially in children with reactive attachment disorder (Shimada $\mathrm{K}$ et al., 2015). However, it remains unknown how such changes to brain structure occur. Epigenetic regulation of genes can differ in response to child maltreatment (Mehta D et al., 2013), and such changes may influence brain development during childhood. There is a growing body of evidence that oxytocin (OT) plays an important role in secure attachment formation with a primary care-giver, and formation of such bonding is insufficient in many maltreated children. Indeed, urine OT in neglected children was lower than in control children (Wismer Fries AB et al., 2005). Recently, hypermethylation of the oxytocin receptor (OXTR) gene has been associated 
with emotion processing and temporal-limbic and prefrontal brain volumes in psychotic disorders (Rubin LH et al., 2016). However, little is known the relationship between OXTR methylation, child maltreatment and its impact on the brain structures of children.

Methods: Forty-four medication-naive children who experienced maltreatment (CM) (27 boys and 17 girls; mean age $=11.4,2.9 \mathrm{SD}$ ) and 41 typically developing (TD) children ( 28 boys and 13 girls; mean age $=14.5,2.2 \mathrm{SD}$ ) with no history of maltreatment were recruited. All of the CM had experienced physical, emotional abuse, and/or neglect early in life prior to coming into care and were living within a stable placement in a child welfare facility. All participants provided saliva samples, and DNA methylation of $20 \mathrm{CpG}$ fragments in the MT2 region of the OXTR promoter (chr3: 8,811,074 - 8,810,603: GRCh37/hg19 build) were measured by using EpiTYPER (MassARRAY system). High-resolution MRI datasets for grey matter volume (GMV) were obtained from a subset of participants (CM $N=24$; TD $N=31)$ who fulfilled inclusion criteria (full scale IQ $>70$, right-handedness). We conducted multiple linear regression analyses in all participants adjusted for age and gender as covariates to identify associations between CpG sites and child maltreatment. To determine the number of tests performed, we examined pairwise correlation between each $\mathrm{CpG}$ site. Based on the direction and significance of the correlation, we estimate 12 methylation blocks. This estimate was used to calculate the appropriate number of independent tests considered for our Bonferroni correction for associations between methylation and child maltreatment (12 tests, $\alpha=0.0042)$. We conducted ROI analyses for orbitofrontal cortex (OFC) and dorsal striatum controlling for age, full scale IQ and total brain volume to find the brain structural differences associated with OXTR methylation.

Results: We found that DNA methylation of OXTR CpG sites (chr3 (8810993-8810995; -1121-1119 from the transcription start site) associated with child maltreatment after controlling for multiple comparisons using a Bonferroni correction $(p<.01$ [corrected]), with higher methylation in the CM compared to TD groups. Methylation of these $\mathrm{CpG}$ sites was negatively correlated with GMV in the OFC (peak level: $p<.001$ [uncorrected], cluster level: $p<.05$ [corrected]). There was no association between OXTR methylation and the dorsal striatum.

Conclusions: In this study, we identified DNA methylation differences in the MT2 region of OXTR that associate with child maltreatment and with GMV for the OFC in CM and TD children. The methylation differences did not affect GMV of dorsal striatum, a region we previously observed to respond to intranasal OT administration in maltreated children. Our results suggest that hypermethylation of OXTR, and presumably lower OXTR expression, may reinforce the negative feedback of OT secretion in maltreated children, thus influencing OFC development and attachment formation.

Keywords: Child Maltreatment, DNA Methylation, Epigenetics, Structural MRI, Reactive Attachment Disorder Disclosure: Nothing to Disclose.
M33. Network Circuitry Changes as a Function of Adolescent Nicotine Exposure in an Animal Model

Robin Keeley*, Pei-Jung Tsai, Tom Mayer, Hanbing Lu, Yihong Yang, Elliot Stein

National Institute on Drug Abuse, Baltimore, Maryland, United States

Background: Smoking remains a worldwide health concern and is mediated by its main addictive component, nicotine. Despite active anti-smoking health initiatives, smoking prevalence rates remains at roughly $20 \%$ in the US. Approximately $88 \%$ of smokers report their first cigarette before the age of 15, namely during the adolescent period. Adolescence, an evolutionarily conserved transition between the juvenile and adult state, is characterized by marked changes in brain circuitry and hence, behavior. Importantly, smoking regularly during the adolescent period is associated with increased difficulty in quitting smoking as an adult. Using resting state functional connectivity ( $\mathrm{rsFC}$ ), circuits have been identified that functionally change with smoking in direct relation to nicotine dependence. Similarly, nicotine exposure in adolescent animal models results in long-term changes in brain and behavior. In humans, however, it is unclear whether these observed differences are due to predispositions towards smoking or were a direct result of smoking during a critical period of brain development. Furthermore, longitudinal assessment of network connectivity dynamics due to nicotine exposure in adolescence has never been explored. Critically, a causal relationship between these two events, smoking and functional connectivity changes, can only be explored in preclinical models using longitudinal experimental designs. Given the preponderance of smoking, as well as the natural changes in the brain that occur during the adolescent period, it is important to understand how smoking, and specifically nicotine, directly affect brain circuits and behavior.

Methods: Using a rat model, we exposed rats to 6 weeks of continuous nicotine exposure, either beginning in adolescence and extending to the adult period or exposed for the same length of time but only as adults. Nicotine, at a dose of 1.2 or $4.8 \mathrm{mg} / \mathrm{kg} / \mathrm{d}$ or saline, was administered passively using osmotic minipumps which were replaced every 2 weeks to accommodate for weight gain. Beginning at the onset of administration and continuing every 2 weeks, rats were assessed in behavioral tests of withdrawal symptoms followed by magnetic resonance imaging. Withdrawal was precipitated using the nicotinic receptor antagonist mecamylamine $(1.5 \mathrm{mg} / \mathrm{kg})$. Characteristic withdrawal signs, including shakes, ptosis and tremors, were quantified in a distinct contextual chamber and compared to withdrawal signs after a vehicle injection in a separate yet distinct contextual chamber, for next day assessment of conditioned place avoidance (CPA). Immediately after CPA testing, rats were lightly anesthetized (isoflurane and Domitor) and imaged at 9.4 T. Imaging consisted of anatomical images, MR spectroscopy (for quantification of glutamate and GABA) and functional MRI (fMRI) for rsFC. Connectivity analyses explored changes that occurred as a function of age, nicotine exposure dose and duration and the interaction between these factors. 
Results: The amount of time spent showing behavioral withdrawal increased as a function of length of exposure and was most prominent in high dose nicotine groups and in adults. Nicotine exposure changed the normal developmental trajectory of network connectivity from adolescence to adulthood. Connectivity between the prelimbic cortex, an area that undergoes significant development during the adolescent period, varied significantly as a function of nicotine dose and the interaction between nicotine dose, length of exposure and age. Prelimbic and dorsal striatal functional connectivity increased with increasing doses of nicotine. Functional connectivity between the prelimbic cortex and both the dorsal hippocampus and the anterior cingulate cortex showed differing effects of nicotine depending on the age of exposure; functional connectivity between the prelimbic cortex and these areas decreased in adolescents and increased in adults exposed to $4.8 \mathrm{mg} / \mathrm{kg} / \mathrm{d}$ of nicotine. Conclusions: This study is the first of its kind to longitudinally observe changes in functional connectivity and behavior as a function of age of nicotine exposure. Results from this study will provide insight into the consequences of nicotine exposure in adolescence on brain and behavior, ultimately to help guide clinical treatment options.

Supported by the NIDA-IRP and a grant from the FDA Center for Tobacco Products (grant number NDA 13001001-00000 to EAS)

Keywords: Adolescence, Nicotine Dependence, Resting State Functional Connectivity, Rat Models

Disclosure: Nothing to Disclose.

\section{M34. Stress During Adolescence Programs Sex-Specific Disruption of the HPA Axis Into Adulthood}

Kathleen Morrison*, Liisa Hantsoo, C. Neill Epperson, Tracy Bale

University of Pennsylvania, Philadelphia, Pennsylvania, United States

Background: Adverse childhood experiences are one of the greatest predictors for affective disorder presentation in women. As the pubertal transition is marked by dynamic hormonal changes and ensuing reorganization of the brain, it represents a window of vulnerability for adversity to result in long-term programming. Further, periods of hormonal flux across the female lifespan, including pregnancy and postpartum, exacerbate the risk for affective disturbances and promote hypothalamic-pituitary-adrenal (HPA) axis dysregulation, a key feature of affective disorders. However, little is understood as to how stress experienced during the pubertal transition alters ongoing brain maturation and its interaction with later-life events, such as pregnancy and postpartum. We have established a translationally relevant mouse model in which peripubertal stress results in a blunted stress response to an acute stressor during pregnancy and postpartum in females, but produces no long-term effect in males. This suggests that brain re-programming by peripubertal stress intersects with the hormonal state of pregnancy and postpartum to expose dysregulation. Importantly, our collaborators have demonstrated that postpartum women with high adverse childhood experiences prior to adolescence show a similar blunting of the cortisol response to an acute stressor, suggesting that the mouse model recapitulates clinical phenotypes. In mice, RNA-Seq analysis of the paraventricular nucleus of the hypothalamus (PVN) during pregnancy revealed changes in the GABA system. As the GABA system is critical in regulating PVN stress responsiveness and is modulated by allopregnanolone, we hypothesized that the rise in this hormone during pregnancy may be mechanistically important.

Methods: Female mice were exposed to chronic variable stress (CVS) from postnatal days 21-34. At 8-10 weeks old, females were bred with naïve males. On 17.5 days post conception (dpc), pregnant females were administered indomethacin $(5 \mathrm{mg} / \mathrm{kg})$ or vehicle (25\% beta-cyclodextrin) via intraperitoneal injection 2 hours prior to testing for corticosterone response to stress. HPA axis responsiveness to a 15- min restraint stress was tested. A cohort of adult male subjects that underwent the same chronic variable stress were treated with allopregnanolone $(3 \mathrm{mg} / \mathrm{kg}, 20 \mathrm{~h}$ before testing; $1 \mathrm{mg} / \mathrm{kg}, 2 \mathrm{~h}$ before testing) or vehicle (20\% betacyclodextrin) via two subcutaneous injections prior to testing for corticosterone response to stress.

Results: Pharmacological reduction of allopregnanolone by systemic injection of indomethacin resulted in the recovery of a normal corticosterone response at $17.5 \mathrm{dpc}$ in females stressed during peripuberty. Further, the blunted corticosterone response normally observed only in pregnant females was recapitulated in CVS adult males treated with allopregnanolone, further supporting a role for this neurosteroid. Preliminary data suggests that disrupting allopregnanolone binding at the GABA-A receptor in the PVN leads to similar recovery of a normal HPA axis response in females stressed during peripuberty.

Conclusions: These pharmacological studies support a role for pregnancy-induced increases in allopregnanolone as necessary to produce the blunted corticosterone response in females stressed during peripuberty. Together, these studies with a translationally-relevant mouse model provide novel insight into the mechanisms underlying risk factors for stress dysregulation, a central endophenotype of affective disorders.

Keywords: Adolescence, Allopregnanolone, Sex Differences, Neuroendocrinology, Mouse Models

Disclosure: Nothing to Disclose.

\section{M35. Social Anxiety Severity and Age Influence Neural Responses to Social Feedback}

Ashley Smith*, Eric Nelson, Katharina Kircanski, Brent Rappaport, Quyen Do, Ellen Leibenluft, Daniel Pine, Johanna Jarcho

National Institute of Mental Health, Bethesda, Maryland, United States

Background: Developmental changes in brain function and peer dynamics during the transition from childhood to adolescence often coincide with increases in social anxiety symptoms. However, most imaging research compares clinically anxious to non-anxious youth collapsed across age. Thus, little is known about potential interactive effects of the full spectrum of social anxiety symptoms and age on brain function during peer interactions. The present study 
aims to characterize these relations using an ecologically valid paradigm that models ongoing social interactions with peers among youths 8 to 18 years of age with a range of social anxiety symptoms.

Methods: In this study, 112 youth (8-18 years old, $M=12.8$ 2.7 years) completed a computerized social interaction task during fMRI. Of the recruited participants, 57 (17 males) met DSM-5 criteria for at least one anxiety disorder and 55 (20 males) participants were free of psychiatric illness. To quantify social anxiety dimensionally, we employed a latent factor analysis using multiple self-report measures that assess distinct symptoms of social anxiety. Self-report scores on the Screen for Child Anxiety Related Disorders (SCARED) Social Anxiety subscale, the Fear of Negative Evaluation Scale (FNE), and the Social Anxiety Scale (SAS) were tested as three observed variables reflecting one common latent variable, social anxiety. Indeed, all three measures had high factor loadings $(\mathrm{SCARED}=.80, \mathrm{FNE}=.80, \mathrm{SAS}=.84)$ on the latent social anxiety factor. The standardized factor scores for each participant, which we refer to as the Social Anxiety Composite (SAC), was extracted and used as a continuous variable in all analyses. SAC scores were not correlated with age $(\mathrm{r}=.10, p=\mathrm{ns})$, puberty $(\mathrm{r}=.08, p=\mathrm{ns})$, or IQ $(\mathrm{r}=.00$, $p=n s)$ and were well-distributed across both anxious and control participants.

Participants completed a dynamic social interaction task, Virtual School (Jarcho et al., 2013), during fMRI. In this task, participants believe they are receiving evaluative feedback from other adolescents in real time. In line with previous research demonstrating that evaluative feedback from peers is particularly salient to socially anxious youth, the task was designed such that specific peers provided $100 \%$ positive feedback, $100 \%$ negative feedback ("predictable"), or $50 \%$ negative and $50 \%$ positive feedback ("unpredictable"). We ran a mixed-effects model with SAC and age as continuous, between-subject variables and feedback type as a repeated, within-subject variable.

Results: Whole brain analyses $(p<.001, \mathrm{k}>41)$ revealed a significant SAC X Age X Feedback interaction in the superior temporal gyrus, hippocampus, and caudate, regions broadly involved in social and emotional learning. Youth with low, but not high, SAC showed developmentally-dependent responses to negative feedback based on its predictability. Specifically, among those with low SAC, younger participants showed greater activation during predictable, relative to unpredictable, negative feedback, while older participants showed the opposite pattern [STG: $\mathrm{F}(1,54)=4.77, p=.03$; $\mathrm{p} 2=.08$; hippocampus: $\mathrm{F}(1,54)=11.41, p=.001 ; \mathrm{p} 2=.17$; caudate: $\mathrm{F}(1,54)=7.13, p=.01 ; \mathrm{p} 2=.12]$.

Conclusions: The present study demonstrated that there are unique and interactive relations between age and severity of social anxiety that impact brain responses in youth during distinct forms of peer-based social interactions. These findings highlight the need to consider the impact of development on social information processing across both clinical and sub-clinical levels of social anxiety. Future research aimed at understanding how age, symptom severity, and social processing interact may help inform prevention and treatment programs for socially anxious youth.

Keywords: Human Neuroimaging, Adolescence, Social Interactions, Social Anxiety

Disclosure: Nothing to Disclose.
M36. Meta-Analysis: Risk of Behavioral Activation Symptoms and Mania in Pediatric SSRI Trials

Jennifer Dwyer*, Shipa Telang, Margot Reed, Michael Bloch

\author{
Yale Child Study Center, New Haven, Connecticut, \\ United States
}

Background: Selective serotonin reuptake inhibitors (SSRIs) are the first line pharmacologic for anxiety, OCD and depressive disorders in the pediatric population. Many clinicians report that many children prescribed SSRI medication experience a Pediatric Behavioral Activation Syndrome, which exists along a spectrum from mild activation, increased energy, insomnia or irritability up through agitation, hyperactivity, and possibly even manic behavior. However, evidence of Pediatric Behavioral Activation Syndrome from randomized, placebo-controlled trials of SSRI medications remains sparse. Efforts to quantify the degree of risk have been hampered by inconsistent reporting across clinical trials, and the range of behaviors which might constitute an activation syndrome. Here we have undertaken a meta-analysis of pediatric SSRI clinical trials in an effort to better define the risk of symptoms of the behavioral activation syndromes in pediatric populations.

Methods: PubMed was searched for randomized, placebocontrolled trials of SSRIs in pediatric populations (less than 18 years of age). The reference list from previous metaanalyses examining the efficacy of antidepressant medications in pediatric populations was searched for further eligible trials. We extracted the rates of several adverse event outcomes associated with the behavioral activation syndrome in children (insomnia, irritability, agitation, hyperactivity, anxiety/nervousness and akathisia/restlessness) as well as mania (manic behavior). Comprehensive Meta-Analysis version 3.0 was used to examine the risk ratio of adverse events on SSRI medications compared to placebo.

Results: A minority of studies reported adverse event rates for the outcome of interest, especially manic behavior. SSRI use was associated with a significantly increased risk of insomnia, irritability, agitation and hyperactivity in children compared to placebo. There was no evidence of publication bias or significant heterogeneity among included trials. We plan to additionally examine the FDA database of medication trials as well as industry trial databases to identify additional data on side-effects from trials that may not have reported these outcomes in peer-reviewed publications.

Conclusions: Peer-reviewed published reports of pediatric SSRI trials appear to support that symptoms of Pediatric Behavioral Activation Syndrome are more common with these medications as compared to placebo. Caution is warranted as these spontaneously reported adverse events may have issues with reporting and measurement bias that may have affected the suicidality literature with these same medications. Additionally, although we did not detect publication or reporting bias in the included trials, there exists a strong possibility that the risk of adverse events reported in peer-reviewed publication may systematically differ from those not published.

Keywords: SSRI, Adolescent Depression, Adolescent Anxiety Disclosure: Nothing to Disclose. 
M37. Polygenic Risk, Functional Glucocorticoid Response and Depressive Symptoms in Adolescents: The Moderating Role of Childhood Trauma

Thorhildur Halldorsdottir*, Ana Paula Matos, Yvonne Awaloff, Eiríkur Örn Arnarson, W. Edward Craighead, Elisabeth Binder

\section{Max Planck Institute of Psychiatry, Munchen, Germany}

Background: With the rising prevalence of depression, identifying risk factors associated with this disorder in premorbid populations is important. Genetic and epidemiological studies suggest depression comes about through the complex interplay between multiple genes and environmental factors. Accordingly, gene-by-environment interaction studies using polygenic risk scores (PRS) provide a promising framework to understand the development of depression. Previous studies examining the interaction between PRS derived from Psychiatric Genomics Consortium (PGC) genome-wide association studies (GWAS) and stressful life events on depression risk have, however, yielded inconsistent findings (Mullins et al., 2016; Musliner et al., 2015; Peyrot et al., 2014). In addition to PRS derived from case/control association studies, investigating polygenic scores that lead to functional differences in the response to environmental risk factors of depression may provide further insight into the disorder. In a recent study (Arloth et al., 2015), a functional genetic risk score, composed of approximately 300 common genetic variants associated with differences in the transcriptional response to glucocorticoid receptor activation, was also associated with depression and dysfunctional amygdala activity (a brain region implicated in the etiology of depression). Building on these findings, we examine the three-way interaction between the case/control major depression disorder (MDD) PRS, the functional glucocorticoid response score (fGRS), and exposure to childhood trauma in shaping the risk for depressive symptoms in a community sample of adolescents.

Methods: Participants included 1,345 genotyped adolescents (Mage $=13.95$, 64.2\% female; 100\% Europeans of Portuguese descent) who completed self-report measures on exposure to childhood trauma (Childhood Trauma Questionnaire; Bernstein \& Fink, 1998) and depressive symptoms (Children's Depression Inventory; Kovacs, 1992). The MDD PRS was derived from the PGC GWAS of MDD (Hyde et al., 2016; Ripke et al., 2013). The fGRS was based on genetic variants previously found to moderate the immediate transcriptional response to GR activation (Arloth et al., 2015). Biological sex, SES, residency and population stratification were controlled for in the analyses.

Results: The main effect of the fGRS $(p=.049)$ was nominally significant but not the main effect of the MDD PRS $(p=.540)$ and exposure to childhood trauma $(p=.190)$ on depressive symptoms. The triple interaction between MDD PRS, fGRS and exposure to childhood trauma, however, significantly predicted depressive symptoms $(p=.005)$. Posthoc analyses revealed that in children with lower to average levels of exposure to childhood trauma, higher depression symptoms were seen in children with both, a high MDD PRS and a high fGRS, while low scores of these genetic scores were protective. This relationship was lost at high levels of childhood trauma exposure.
Conclusions: The present study suggests that following low to moderate exposure to childhood trauma, both a genetic risk for MDD and higher glucocorticoid sensitivity best predict depressive symptoms in adolescents. At high levels of childhood trauma, the impact of these two PRS is absent. If replicated, the findings could have important implications in terms of identifying at-risk youth.

Keywords: Adolescent Depression, Polygenic Risk Score, Glucocorticoid Receptor Gene, Early Identification of Risk, Childhood Trauma

Disclosure: Nothing to Disclose.

\section{M38. Association Between Cortisol Reactivity and Conditioned Pain Modulation in Adolescents With Tension-Type Headache}

Matthew Morris*, Brooklynn Bailey, Lynn Walker, Stephen Bruehl, Uma Rao

Meharry Medical College, Nashville, Tennessee, United States

Background: Tension-type headache (TTH) is highly prevalent in adolescents and associated with significant impairment across multiple spheres of functioning. Although stress levels and depressive symptoms are associated with TTH in adults, the extent to which these factors influence pain experiences in adolescents with TTH remains unclear. The present study sought to examine whether cortisol responses during evoked pain stimuli are associated with extent of conditioned pain modulation (CPM; an index of endogenous pain inhibitory function) in adolescents with TTH over and above the influence of depressive symptoms.

Methods: Participants were recruited as part of a randomized controlled trial examining the impact of a yoga intervention on headache intensity and disability compared to a waitlist control group. The current findings are focused on the baseline thermal pain protocol, which occurred prior to randomization. Participants were 27 youth (mean age $=17.2, \mathrm{SD}=1.3$ ) with TTH, including 23 females and 4 males. Anxiety prior to pain testing was rated by participants on a 0 to 100 scale $(0=$ "none" and $100=$ "worst possible"). Perceived pain intensity to thermal stimuli was rated by participants on a 0 to 100 scale $(0=$ "no pain" and $100=$ "worst imaginable pain"). CPM, also called diffuse noxious inhibitory controls, was determined as the percentage reduction in pain ratings from a preconditioning phase (test stimulus $=30 \times 30 \mathrm{~mm}$ thermode applied to ventral forearm) to a conditioning phase (conditioning stimulus = hot water bath maintained at a steady temperature of $46.5^{\circ} \mathrm{C}$ ). Weaker CPM (i.e., less decrease in pain ratings) is believed to reflect dysfunction of descending endogenous pain modulatory systems. Five saliva samples were obtained throughout the pain testing protocol to determine cortisol reactivity (20 minutes prior to testing, immediately prior to testing, and at 20,40, and 60 minutes after pain protocol initiation). Prior to pain testing, depressive symptoms were assessed by self-report and headache frequency and intensity (rated on a Likert Scale from 0 to 10) were assessed by selfreport for the preceding two-week period.

Results: Preliminary analyses revealed that TTH participants reported a mean of 3.3 tension-type headaches $(\mathrm{SD}=2.4), 1.2$ migraines $(\mathrm{SD}=2.2)$, and a mean headache intensity of 4.3 $(\mathrm{SD}=1.9)$ over the prior 2 weeks. Participants reported a 
mean CPM reduction of $14.0 \%(\mathrm{SD}=24.6)$ and mean depressive symptom score of $11.1(\mathrm{SD}=7.0)$. None of the headache variables were correlated with CPM or depressive symptoms (p's >.05), and CPM was not correlated with depressive symptoms. Higher pre-testing anxiety levels predicted more rapid increases in cortisol levels during the pain protocol. Multilevel models revealed that both CPM and depressive symptoms predicted quadratic change in cortisol levels during the protocol. Simple slope analyses showed rapidly increasing cortisol levels during the pain protocol for youth who exhibited weaker CPM $(b=.0001, t=3.2, p$ $=.002$ ); in contrast, cortisol levels did not change significantly during the protocol for youth who exhibited stronger CPM (i.e., greater reductions in pain ratings). For youth with lower depressive symptoms, cortisol levels decreased at the start of the protocol but then rapidly increased $(b=.0001$, $\mathrm{t}=2.5, \mathrm{p}=.014$ ); in contrast, youth with higher depressive symptoms showed no significant change in cortisol levels during the protocol. Removing the 4 males from analyses resulted in the same interaction patterns, though the interaction terms were reduced to non-significant trends for CPM $(\mathrm{p}=.055)$ and depressive symptoms $(\mathrm{p}=.060)$.

Conclusions: The present study found that adolescents with TTH who exhibited weaker CPM had higher pre-testing cortisol levels compared to those with stronger CPM and showed rapidly increasing cortisol levels throughout the pain testing session. The relation between cortisol response and CPM was significant over and above the influence of depressive symptoms, which characterize youth with TTH and are independently associated with cortisol reactivity. Greater cortisol reactivity to pain testing was also associated with higher pre-testing anxiety levels. These findings add to a growing literature demonstrating hypothalamic-pituitaryadrenal (HPA) alterations across a variety of chronic pain conditions and suggest a potential role for cortisol reactivity in pain modulation among adolescents with TTH.

Keywords: Adolescence, Cortisol, Pain

Disclosure: Nothing to Disclose.

\section{M39. Characterizing Intrinsic Functional Connectivity in Relations to Impaired Self-Regulation in High- Functioning Male Youth With Autism Spectrum Disorder}

Susan Shur-Fen Gau*, Hsiang-Yuan Lin, Hsing-Chang $\mathrm{Ni}$, Kathleen R. Merikangas

National Taiwan University Hospital and College of Medicine, Taipei, Taiwan (Republic of China)

Background: While a substantial amount of youth with autism spectrum disorder (ASD) present impaired selfregulation (dysregulation), e.g., emotional lability, tantrums, low frustration tolerance, aggression, and impulsivity, besides the core symptoms, little is known about the neural correlates of dysregulation and its impacts on brain connectivity in youth with ASD [Mazefsky et al., 2013]. The present study aimed to investigate the categorical and dimensional neural correlates of dysregulation in ASD, based on resting-state functional MRI (rs-fMRI) data, and to explore the impacts of dysregulation on resting-state functional connectivity in youth with ASD.
Methods: The sample consisted of 48 boys with highfunctioning ASD and 49 typically developing (TD) boys. Their age ranges are 7-17 years old. The ASD group was further divided into ASD boys with dysregulation (ASD+ Dysregulation, $\mathrm{n}=32$ ) and those without dysregulation (ASD-Dysregulation, $n=16$ ). Their ASD diagnosis was based on clinical evaluation, Autism Diagnostic Interview-Revised interview with their parents, and Autism Diagnostic Observation Scale assessment with the participants. Their parents also completed the Child Behavior Checklist (CBCL) about the youth participants. All the participants received intelligence and rsfMRI assessments. Dysregulation was defined by the sum of T-scores of Attention, Aggression, and Anxiety/Depression subscales on the CBCL greater than 180 . We conducted a multivariate connectome-wide association study [Shehzad et al., 2014] to examine the intrinsic functional connectivity with rsfMRI. We estimated the impact of dysregulation on restingstate functional connectivity in ASD (i.e., categorical diagnostic comparisons covarying dysregulation level), and also explored the dimensional neural correlates of dysregulation. Furthermore, we also investigated the categorical subgroup differences between ASD boys with and without dysregulation (defined as categorical neural correlates of dysregulation in ASD).

Results: We found that both categorical and dimensional neural correlates of dysregulation in ASD youth involve atypical cross-talk between multiple large functional brain networks, especially between those subserving sensorimotor processing and salience encoding, beyond higher-level cognitive control circuitries. Moreover, interaction within the attention network serves as ASD-specific dimensional neural correlates underpinning impaired-self regulation. When levels of individual dysregulation are taken into consideration, overall dysconnectivity patterns associated with ASD alter substantially, benchmarked against the pure categorical diagnostic group differences.

Conclusions: Our results highlight inter-individual variability in dysregulation may contribute to the inconsistency in the neuroimaging literature of ASD. Collectively, the present findings provide evidence to suggest that dysregulation, beyond core autistic symptoms, may be considered as both categorical and dimensional moderators to parse heterogeneity of high-functioning male youths with ASD. The finding also echoes the approach in the second iteration of the Autism Brain Imaging Data Exchange (ABIDE II), which emphasizes the pressing needs to consider co-occurring psychopathology when characterizing ASD pathophysiology. Keywords: Autism Spectrum Disorder, Resting-State Functional MRI, Self-Regulation, A Multivariate ConnectomeWide Association Study

Disclosure: Nothing to Disclose.

\section{M40. In-Vivo Measurement of a Neurogenic Signal and Pattern Separation in Adolescent Depression}

Chadi Calarge*, Kellen Gandy, Griselda Barba Villalobos, Jacqueline Nguyen, Allison Gregg, Seon Young Kim, Mirjana Maletic-Savatic

\section{Baylor College of Medicine, Houston, Texas, United States}

Background: Neurogenesis has been implicated in major depressive disorder (MDD) although research has been 
$\overline{S 136}$

hampered by inability to measure it in-vivo. Our group has previously identified a 18:1 mono-unsaturated fatty acid that resonates at $1.28 \mathrm{ppm}$ and is highly enriched in neuroprogenitor cells (referred to as neurogenic signal). We examined whether this signal is associated with MDD in adolescents and its correlation with performance on pattern separation, a cognitive process that appears to depend on neurogenesis.

Methods: Medically-healthy 12 to 17-year-old un-medicated patients with MDD and healthy controls were enrolled. They underwent a diagnostic evaluation following the DSM-5 and the Child Depression Rating Scale-Revised (CDRS-R) was completed. The Mnemonic Similarity Task, which taps into pattern separation, was administered. It consists of displaying images that were either 1) "New", displayed only once, 2) "Old", displayed twice, whereby the participant must recognize they are new the first time they are displayed and that they are "old" the second time around, or 3) "Similar", whereby the participant must recognize the images are new the first time they are displayed and that they are similar to the first ones, but not identical, when they are displayed again. In addition, the participants underwent a magnetic resonance spectroscopy (MRS) scan to measure the neurogenic signal in the left hippocampus and in a control region of the parietal white matter.

Results: Twenty-four participants (16 with MDD, 20 앙 age: $14.7 \pm 1.6$ yrs) completed the Mnemonic Similarity Task, with MDD patients exhibiting a significantly lower lure hit rate compared to controls (56\% vs $68 \%, p=0.0001)$. Lure discrimination index (LDI), which corrects for response bias, was inversely associated with CDRS-R T score (Pearson's $r=-0.42, p=0.0385)$. Sixteen participants underwent MRS ( 8 with MDD, 14 o, age: $14.1 \pm 1.7 \mathrm{yrs}$ ). While the neurogenic signal in the left hippocampus was about half the magnitude of that in the parietal white matter in MDD, it was nearly twice as high in healthy controls $(p=0.01)$. In fact, the CDRS$\mathrm{R}$ T score was inversely correlated with the relative magnitude of the neurogenic signal in the left hippocampus to that in the parietal white matter (Spearman's $r=-0.65, p=0.012$ ). LDI was positively associated with the relative magnitude of the neurogenic signal in the left hippocampus to that in the parietal white matter (Spearman's $r=0.46, p=0.112$ ).

Conclusions: Using state-of-the-art methodology, adolescent MDD was found to be associated with reduced neurogenic signal in the left hippocampus and impaired performance on pattern separation, suggesting that these might serve as valid biomarkers of depression.

Keywords: MR Spectroscopy, Adolescent Depression, Adult Hippocampal Neurogenesis, Cognitive Neuroscience

Disclosure: Nothing to Disclose.

M41. Toward a Neurobiological Understanding of Williams Syndrome Using a Graph Theoretical Approach and Resting-State fMRI

Michael Gregory*, Philip Kohn, J. Shane Kippenhan, Tiffany Nash, Daniel Eisenberg, Jasmin Czarapata, Dwight Dickinson, Carolyn Mervis, Karen Berman

National Institute of Mental Health, Bethesda, Maryland, United States

Background: Williams syndrome (WS) is caused by hemizygous deletion of approximately 1.5 megabases on chromosome $7 \mathrm{q} 11.23$ and is characterized by significant visuospatial construction deficits, hypersociability, and mild to moderate intellectual disability with relative sparing of concrete language. Because the genes within the hemideleted region of chromosome 7 are known, WS has been used as a model for understanding how genetic changes translate into alterations in complex behaviors through brain function. Though structural and functional neuroimaging studies have identified the insula and intraparietal sulcus (IPS), among other regions, as hubs of neural dysfunction in WS, little work has been done to investigate alterations in the underlying network architecture. Recent advances in mathematics, neuroimaging, and computing have provided analytic methods for understanding these brain dynamics using a graph theoretical framework, where real-world networks are represented by a graph of the pair-wise relationships between objects. When this model is applied to the brain, nodes of the graph correspond to individual brain regions and the edges between nodes correspond to "connectivity" between regions. Here, we utilized graph theory analytics to describe neural circuit-based functional characteristics of children with WS, and we tested for relations between these brain measures and cognition.

Methods: Twenty-one children with WS (age 10.9+/-3.9, 15 girls) and 22 typically developing (TD) children (age 12.1 $+/-3.2,11$ girls) underwent resting state functional MRI and neuropsychological testing with KBIT-2 and DAS-II. All participants had a KBIT-2 composite IQ in the normal to low-normal range. Resting state EPI sequences were collected on each participant (two runs of 6.13 minutes each, TR/ $\mathrm{TE}=2000 / 24 \mathrm{~ms}, 1.875 \times 1.875 \times 3 \mathrm{~mm}$ voxels, 184 volumes $)$, as was a T1-weighted structural scan to be used for alignment. After pre-processing of EPI images and alignment to a studyspecific, combined WS and TD template, mean time-series from 200 regions-of-interest (nodes) based on a previously reported whole-brain parcellation (Craddock 2012) were extracted. Then, after wavelet decomposition, graph theory metrics of nodal degree (number of edges emanating from a node), average shortest path length (average of the number of edges necessary to get from a node to all other nodes), and clustering coefficient (a measure of whether neighbors of a node are connected with each other) were computed using the $\mathrm{R}$ package iGraph. Nodal degree was compared across groups, and between-group differences were thresholded at $p<0.005$, uncorrected. Finally, in each group, we tested the relation between network architecture and cognition by correlating individual neuropsychological standard scores (KBIT-2 nonverbal reasoning and DAS-II spatial cluster) with graph theory measures derived from regions where group differences in nodal degree were identified. Correlations with a statistical value of $p<0.05$ are reported.

Results: Six brain regions showed altered nodal degree in children with WS as compared to TD children. These included bilateral posterior insula, right hippocampus, left IPS, right temporal pole and right dorsolateral prefrontal cortex (DLPFC). All regions except DLPFC exhibited a decrease in nodal degree in the WS group. There were no correlations between neuropsychological standard scores and graph theory measures in DLPFC or temporal pole in either group. However, in children with WS, the IPS, hippocampus, and insula showed associations with neuropsychological 
measures: there were negative relations between IPS clustering coefficient and hippocampal nodal degree and both neuropsychological measures $\left(r^{\prime} s<-0.47\right)$, and there was a positive relation between insula clustering coefficient and KBIT-2 nonverbal score $(r=0.54)$. In TD children, the only correlation was a negative relation between hippocampus nodal degree and KBIT-2 nonverbal reasoning score $(\mathrm{r}=$ -0.67).

Conclusions: This work provides the first description of alterations in functional network architecture of the WS brain using a graph theoretical approach. With this data-driven analytic approach, we found that regions previously implicated in both visuospatial abilities (IPS and hippocampus) and social functioning (bilateral insula) had lower nodal degree (i.e. decreased functional connectivity with all other regions). Thus, the regional pattern of the results is consistent with the neurobehavioral phenotype that typifies individuals with WS: hypersociability and impaired visuospatial constructional abilities. Additionally, the results are consistent with prior neuroimaging studies using structural MRI as well as taskbased and resting fMRI. Hippocampal network properties were significantly related to nonverbal reasoning in both groups, whereas associations of the IPS with nonverbal reasoning and spatial abilities were found in children with WS but not TD children. These findings suggest that in the context of WS, an individual's levels of nonverbal reasoning and spatial abilities may reflect the extent to which the IPS is "connected".

Keywords: Williams Syndrome, Resting State Functional Connectivity, Visuospatial Ability, Nonverbal Reasoning, Neural Network Connectivity

Disclosure: Nothing to Disclose.

\section{M42. Assessing Relationships Between Childhood Trau- ma and Brain Morphology in Large Community-Based Adult Sample}

Ashley Clausen*, Robin Aupperle, Henry Yeh, Darcy

Waller, Janelle Payne, Elisabeth Akeman, Martin

Paulus, Tulsa 1000 Investigators

Laureate Institute for Brain Research, Tulsa, Oklahoma, United States

Background: Approximately $77 \%$ of adults in the United States endorse experiencing at least one form of childhood trauma, which can include emotional abuse and neglect, physical abuse and neglect, and sexual abuse. Greater severity of childhood trauma has been associated with increased rates of chronic mental health diagnoses such as depression, anxiety, PTSD and substance use disorder. Childhood trauma and adult psychopathology have been linked to changes in morphology within overlapping brain regions, including the anterior cingulate, dorsal lateral prefrontal and orbitofrontal cortices, corpus callosum, and hippocampus. Limited sample sizes of previous studies have made it difficult to identify what effects may be uniquely related to childhood trauma, rather than being primarily driven by current mental health diagnoses. This study used magnetic resonance imaging (MRI) data from a large community-based adult sample to delineate relationships between childhood trauma and regional grey matter (GM) volume that may be distinct from the impact of psychopathology.

Methods: Data were combined across studies completed at the same research institute between November 2010 September 2015. Subjects included both males $(n=207)$ and females $(n=370)$ between the ages of 18-59 (mean age $=32, \mathrm{SD}=11$ years) and consisted of both healthy controls who did not meet diagnostic criteria for a psychiatric disorder $(n=276)$ and individuals who met criteria for at least one psychological disorder $(n=301)$. The Childhood Trauma Questionnaire (CTQ) was used to assess traumatic experiences during childhood. Adult psychopathology was assessed using either the Mini International Neuropsychiatric Inventory or the Structured Clinical Interview for DSM-IV Disorders. High resolution T1-weighted anatomical scans optimized for tissue contrast were acquired on a $3 \mathrm{~T}$ GE MR750 scanner with an 8- or a 32-channel head coil. Autosegmentation of regional GM volume was completed using FreeSurfer (version 5.3.0), controlling for total GM volume. Elastic net machine learning (with cross-validation) was conducted to identify regions for which GM volume related to CTQ severity. A follow-up regression analysis identified which regions contributed unique variance in CTQ severity. Separate hierarchical regression analyses were used to assess relationships between CTQ and GM volume for each region identified via elastic net, controlling for age, education, gender, and MRI coil type (step 1), and presence of adult psychopathology (step 2).

Results: Elastic net analysis revealed 38 brain regions that related to CTQ score, spanning primarily frontal and parietal regions. Of those, the right inferior portion of the circular sulcus of the insula (a portion of the mid-posterior insula; = 0.007, $p=0.004$ ), and the right marginal branch of the cingulate sulcus (medial frontal lobe; $=-0.005, p=0.021$ ) accounted for unique variance in CTQ severity. When controlling for age, education, gender, MRI coil type and adult psychopathology, increased CTQ severity was significantly associated with larger GM volume of the right inferior portion of the circular sulcus of the insula $(=0.12$, $p=0.013$ ), while the relationship with the cingulate sulcus was no longer significant $(=-0.08, p=0.085)$.

Conclusions: Prior research on childhood trauma has focused primarily on amygdala and prefrontal regions, given that these regions are implicated in emotional processing. However, theoretically-driven approaches may limit understanding of how childhood trauma impacts regions outside of these networks. To overcome this limitation, the present study utilized a data-drive, machine learning approach in a large community-based adult sample to explore relationships between regional GM volume and CTQ severity. Results suggest that CTQ severity is uniquely associated with higher GM volume within the right inferior portion of the circular sulcus of the insula, even when controlling for demographic factors and adult psychopathology. This region of the insula is thought to be heavily involved in viscerosensory processing. Thus, it is plausible that extended periods of physiological arousal secondary to childhood trauma may potentiate neural development within the inferior portion of the circular sulcus of the insula. Increased GM volume within this region 
could potentially lead to increased processing and awareness of visceral (such as increased heart rate or startle response) and/or nociceptive information. This in turn could contribute to hyperarousal and altered pain processing often observed for trauma populations. Research using longitudinal designs and the use of interoception-related tasks could further clarify the functional and clinical significance of these findings.

Keywords: Gray Matter Volumes, Childhood Trauma, Machine Learning, MRI

Disclosure: Nothing to Disclose.

\section{M43. Abnormal Prefrontal Structure and Development in Pediatric Posttraumatic Stress Disorder}

Sara Heyn, Taylor Keding, Jeanette Mumford, Ryan Herringa*

University of Wisconsin School of Medicine and Public Health, Madison, Wisconsin, United States

Background: PTSD is common in youth, affecting an estimated $5 \%$ of children by age 18 . While the neurobiology of pediatric PTSD remains poorly understood, accumulating evidence suggests abnormal structure and function in frontolimbic circuitry underlying emotion processing and regulation. Furthermore, our recent cross-sectional age analyses suggest abnormal frontolimbic development which could contribute to persistence of illness as youth age (Keding \& Herringa, 2015; Wolf \& Herringa, 2016; Keding \& Herringa, 2016). To date, however, there are no reported whole brain studies examining longitudinal neurodevelopment in pediatric PTSD. Such information is critical for identifying both compensatory and maladaptive neurodevelopmental trajectories which could serve as treatment targets in affected youth.

Methods: To address these knowledge gaps, we conducted a naturalistic longitudinal neuroimaging study of youth with PTSD compared to non-traumatized healthy youth matched for age and sex. At baseline, youth were aged 8-18 years and free of psychiatric medications. Longitudinal assessment and imaging were repeated one year later. The study sample includes 25 youth with PTSD (16 F, avg. 13.9 years at baseline) and 21 nontraumatized healthy youth (16 F, average 13.7 years at baseline). At each time point, youth were assessed with structured psychiatric interviews, and completed T1weighted MRI scans. Parent/child reports of PTSD, anxiety, and depressive symptoms were obtained at both time points using standardized questionnaires and reduced to primary symptom dimensions using principal component analysis (Patriat et al, 2016). Longitudinal voxel-based morphometry (VBM) was conducted using CAT12/SPM12. Longitudinal gray matter surface processing was conducted in Freesurfer. Here, primary measures included cortical thickness and surface area using automated cortical parcellation (Destrieux atlas). All data were processed using linear mixed effects (LME) models including a group ${ }^{\star}$ age interaction, and covaried for sex. VBM group level analyses were conducted in AFNI (whole-brain pFWE <.05). Cortical surface measures were processed in $\mathrm{R}$ with FDR correction $(\mathrm{q}<.05)$. Finally, we conducted exploratory resting state functional connectivity analyses examining prefrontalamygdala connectivity using amygdala subnuclei (SFA, CM, BL; SPM Anatomy Toolbox) as seeds and prefrontal VBM clusters as targets. Longitudinal resting state data were processed in AFNI with motion scrubbing. Extracted beta weights were analyzed in $\mathrm{R}$ for group level differences using LME.

Results: In the VBM analysis, both healthy and PTSD youth showed decreasing grey matter volume (GMV) with age in widespread cortical areas, consistent with normative cortical thinning. In group main effects, PTSD youth showed reduced GMV in multiple cortical regions including the vmPFC (BA 10/11), bilateral ventrolateral (vl)PFC (BA 45/47), and posterior cingulate cortex (BA 31). Of these regions, only the vlPFC showed a relationship to symptom severity, and was inversely associated with social aversion scores. Next, a group ${ }^{\star}$ age interaction was present in left dorsolateral (dl)PFC (BA 9). Here, dlPFC GMV decreased over time in healthy youth, a pattern that was absent in youth with PTSD. In the cortical surface analysis, group ${ }^{\star}$ age interactions were detected in left dlPFC surface area, overlapping with the VBM-dlPFC cluster. Mirroring the VBM results, dlPFC surface area decreased over time in healthy youth, but not in youth with PTSD. However, neither dlPFC GMV or surface area related to symptom severity over time, limiting a clear assessment of whether abnormal neurodevelopment may represent compensatory versus maladaptive change.

To explore the functional correlates of these prefrontal abnormalities, we conducted targeted resting state connectivity analyses, using anatomically defined amygdala subnuclei as seeds and VBM prefrontal clusters (vmPFC, R/L vlPFC, L dlPFC) as targets. Here, only the dlPFC showed abnormal coupling with the amygdala in PTSD youth. Specifically, group ${ }^{\star}$ age interactions were present between the dlPFC and both left and right basolateral (BL) amygdala. In each case, healthy youth showed decreasing (more negative) coupling over time, while PTSD youth showed increasing (more positive) coupling over time. Furthermore, dlPFCright BL coupling was positively associated with reexperiencing symptoms of PTSD. Finally, in post hoc analyses, the above findings were not accounted for by age at index trauma, pubertal stage, IQ, medication or psychotherapy history, or trauma load.

Conclusions: Together, these novel findings point to sustained structural abnormalities, as well as abnormal neurodevelopment, in frontolimbic circuitry in pediatric PTSD. Notably, abnormal dlPFC development appears to be maladaptive for youth with PTSD, given reduced dlPFCamygdala negative coupling over time and, in turn, increasing reexperiencing symptoms. Given the known role of the dlPFC in cognitive-emotional control, this provides a potential neurobiological substrate of impaired fear and emotion regulation in pediatric PTSD. Sustained abnormalities in the vmPFC and vlPFC may underlie further deficits in fear extinction and emotion regulation which have been implicated in pediatric PTSD.

Keywords: Pediatric PTSD, Structural Neuroimaging, Prefrontal-Amygdala-Connectivity, Longitudinal Imaging

Disclosure: Nothing to Disclose. 
M44. Increased Insula Activity During Inhibitory Control After Slow-Wave Sleep Disruption in Adolescents

Lora Cope ${ }^{\star}$, Meghan Martz, Deirdre Conroy, Philip Cheng, Robert Hoffman, Todd Arnedt, Mary Heitzeg

University of Michigan, Ann Arbor, Michigan, United States

Background: An increase in impulsive, risk-taking, and sensation-seeking behavior during adolescence relative to childhood and adulthood is thought to be related in part to an imbalance between bottom-up reward and top-down cognitive control system developmental trajectories. During this same period, significant changes in sleep architecture are taking place that may also contribute to these adolescenttypical behaviors. Specifically, slow-wave sleep, which is thought to restore inhibition to the cortex, is reduced by approximately $66 \%$ between the ages of 11 and 17 . However, it remains unknown how brain maturation and sleep alteration processes interact in adolescents to produce the risky behaviors commonly seen during this developmental period. To this end, the purpose of this analysis was to examine the relation between experimental slow-wave sleep disruption and brain activity/performance associated with inhibitory control during adolescence.

Methods: Participants were 30 healthy adolescents (ages $15-17 ; 53 \%$ female) with no or low levels of lifetime drug and alcohol use (i.e., $\leq 15$ lifetime alcoholic drinks and no binge drinking; $\leq 9$ occasions of marijuana use and none in the past month; no nicotine use in the past month; no other substance use). Over the course of the two-week study, participants kept a person-specific and consistent sleep schedule, verified by actigraphy and a self-report sleep diary. Participants slept three nights in the sleep lab: an adaptation night (Night 6), a slow-wave disruption night (Night 7 or 13), and a control night (Night 7 or 13). Disruption and control nights were counter-balanced across participants. During the disruption night, a sleep technician monitored electroencephalographic activity for two delta waves occurring within $15 \mathrm{sec}$ and delivered a series of $1000 \mathrm{~Hz}$ tones at an intensity of 20-100 dB emitted through a speaker attached to the headboard of the bed in order to suppress slow-wave sleep without waking the participant. Procedures were the same during the adaptation and control nights except that tones were not delivered. On the mornings following the disruption and control nights, participants were scanned with functional magnetic resonance imaging while performing a go/no-go task. Two contrasts of interest were examined to probe failed and successful inhibition, respectively: false alarms vs. implicit baseline (FA vs. BL) and correct rejections vs. implicit baseline (CR vs. BL). The following bilateral anatomical regions of interest (ROI) from the go/no-go literature were created in WFU PickAtlas: anterior cingulate cortex, insula, dorsolateral prefrontal cortex, and inferior frontal gyrus. Mean signal change in each ROI from each contrast was extracted and imported into SPSS for further analysis.

Results: There were no significant differences in go/no-go task performance between disruption and control nights ( $p$-values ranged from.093 for hit reaction time variability to.993 for hit reaction time). As designed, total sleep time was not significantly different between control and disruption nights $(\mathrm{t}[29]=0.09, \mathrm{p}=.931)$, but time spent in slowwave sleep was significantly shorter during the disruption night than during the control night $(\mathrm{t}[29]=5.60, \mathrm{p}<.001)$. Failed inhibition: The left insula showed significantly greater activation after the disruption night than after the control night during FA vs. BL $(\mathrm{p}=.019)$. The right insula also trended toward significance $(\mathrm{p}=.081)$ in the same direction. Successful inhibition: The left insula showed significantly greater activation after the disruption night than after the control night during CR vs. BL ( $\mathrm{p}=.014)$, as did the right insula $(\mathrm{p}=.027)$. Furthermore, greater insula activation after the disruption night was associated with more false alarms (left: $\mathrm{r}=.50, \mathrm{p}=.005$; right: $\mathrm{r}=.42, \mathrm{p}=.022$ ) and greater variability in false alarm reaction time (left: $\mathrm{r}=.37, \mathrm{p}=.044$; right: n.s.) during the same session. Greater insula activation after the disruption night was also associated with fewer hits (left: $\mathrm{r}=-.43, \mathrm{p}=.019$; right: $\mathrm{r}=-.38, \mathrm{p}=.040$ ) and greater variability in hit reaction time (left: $\mathrm{r}=.57, \mathrm{p}=.001$; right: $\mathrm{r}$ $=.45, \mathrm{p}=.013)$ during the same session. No other ROIs showed significantly different activation between disruption and control nights.

Conclusions: In summary, insula activity during both failed and successful inhibition was greater following slow-wave sleep disruption than following a non-disruption night in a sample of healthy adolescents. The insula is frequently identified by studies of inhibitory control, including those utilizing the go/no-go task, but its specific role is still unknown. Some studies have linked insula activity during inhibitory control to working memory. Here, performing the go/no-go task after a night of slow-wave sleep disruption may have elicited compensatory activation patterns that resulted in similar task performance between disruption and control nights. Future mediational analyses may shed light on this mechanism, as greater insula activity was also associated with more false alarms, fewer hits, and greater reaction time variability. These results point to an interesting target for future analyses that examine the association between adolescent sleep changes and impulsive, risktaking behavior.

Keywords: Brain Imaging, fMRI, Impulsivity, SlowWave Sleep

Disclosure: Nothing to Disclose.

M45. Accelerated Development of Anatomical Innervation and Tract Integrity Between Amygdala and Prefrontal Cortex Following Maternal Separation is Sex-Specific and Relates to Anxiety Susceptibility and Resilience in Rats

Heather Brenhouse*, Jennifer Honeycutt, Praveen Kulkarni, Craig Ferris

Northeastern University, Boston, Massachusetts, United States

Background: Recent human imaging and animal studies highlight the importance of corticolimbic circuitry in the sexspecific regulation of emotional behavior and its disruption in mental disorders. Growing evidence suggests that childhood adversity in humans leads to life-long changes in 
$\overline{\mathrm{S} I 40}$

connectivity between limbic and cortical regions, with consequential emotional and cognitive deficits. Surprisingly little, however, is known about the interaction of sex and early life stress (ELS) on corticolimbic development, despite a striking gender difference in the clinical time-course and symptomology of ELS effects. Diffusion tensor imaging has shown that fractional anisotropy (FA) - a parameter linked to axon packing and myelination-increases between childhood and early adulthood, with frontal-temporal connections demonstrating the most prolonged development. These age-related differences in DTI measures have been linked to a range of behavioral measures, documenting their functional consequences. Here, a rodent model of ELS allowed us to investigate longitudinal sex differences in corticolimbic tract integrity after ELS, which is currently largely unknown. We also examined the anatomical effects of ELS on innervation of the basolateral amygdala (BLA) to the PFC over development. We hypothesized that alterations in corticolimbic connectivity begin early in development, with fiber development following a precocial trajectory after ELS exposure.

Methods: Male and female rats were either subjected to maternal separation between postnatal days (P)2-20 ( $n=5-7 /$ group in tracer study; $n=3$ /group in DTI study) or were undisturbed and weaned at P21. DTI: Male and female rats were imaged on a Bruker 7 T MRI scanner at P25 and again at P45. Subjects were anesthetized with $3 \%$ isofluorane, and a high-resolution anatomical data set was collected using the RARE pulse sequence. DTI was acquired two times for signal averaging. Tracer Study: Subjects underwent stereotaxic microinjection of the anterograde tracer biotin dextran amine (BDA) into the BLA at P21, P31, or P41. Five days post-injection, rats were intracardially perfused and brains were processed for labeling of BDA in the PFC. Total length of labeled fibers in the prelimbic and infralimbic PFC were evaluated using stereological procedures. Twenty-four hours before imaging (DTI) or sacrifice (tracer), all subjects were tested for anxiety-like behavior in an elevated plus maze.

Results: ELS-exposed females (multiplicity-corrected $p=0.0498)$, but not males $(p=0.66)$ displayed more BLA $\rightarrow$ PFC terminals in the infralimbic PFC by P21. By P31, a main effect of group $(\mathrm{F} 1,21=5.9, p=0.02)$ showed that both males and females exposed to ELS had increased BLA $\rightarrow$ PFC innervation. Preliminary analyses suggest that while at P31, higher BLA $\rightarrow$ PFC innervation was related to less time in the open arms $(p=0.015)$, at P41, higher BLA $\rightarrow$ PFC innervation was related to more time spent in the open arms $(p=0.04)$. Preliminary DTI analyses suggest that tract integrity between the amygdala and PFC increases over adolescence, and is higher in ELS-exposed females compared to controls, while no effect was observed in males.

Conclusions: These data suggest that maternal separation accelerates the innervation of the PFC from the BLA, with an earlier innervation in females. In both males and females, earlier innervation (P31) correlated with higher anxiety-like behavior, however later in adolescence (P41), higher innervation was related to lower anxiety-like behavior following early life stress. One hypothesis that arises from these relationships is that accelerated innervation confers higher anxiety during early adolescence, yet may yield resilience, or at least lower anxiety, later on. Further analyses of longitudinal DTI will allow us to investigate whether those animals with earlier changes to corticolimbic tracts behave differently later in adolescence, compared to those with laterdeveloping connectivity. Taken together, these findings point to circuitry changes that develop through adolescence and correlate with clinical reports of aberrant corticolimbic maturation and anxiety-like behaviors after ELS. Further investigation into the relationship between innervation, tract integrity, and functional connectivity over development are necessary and currently underway.

Keywords: Maternal Separation, Sex-Differences, Corticolimbic, Diffusion Tensor Imaging, Anterograde Tracer

Disclosure: Nothing to Disclose.

M46. Large-Scale Brain Organization During Facial Emotion Processing as a Function of Early Life Trauma Among Adolescent Girls

Joshua Cisler*, Anthony Privratsky, Sonet Smitherman, Ryan Herringa, Clinton Kilts

University of Wisconsin, Madison, Madison, Wisconsin, United States

Background: A wealth of research has investigated the impact of early life trauma exposure on functional brain activation during facial emotion processing and has often demonstrated amygdala hyperactivity and weakened connectivity between amygdala and medial PFC (mPFC). There have been notably limited investigations linking these previous node-specific findings into larger-scale network models of brain organization.

Methods: To address these gaps, we applied graph theoretical analyses to fMRI data collected during a facial emotion processing task among 88 adolescent girls ( $n=59$ exposed to direct physical or sexual assault; $n=29$ healthy controls) during fMRI. Large-scale organization indices of modularity, assortativity, and global efficiency were calculated for stimulus-specific functional connectivity using an 883 region-of-interest parcellation.

Results: Among the entire sample, more severe early life trauma was associated with more modular and assortative, but less globally efficient, network organization across all stimulus categories. Among the assaulted girls, severity of early life trauma and PTSD diagnoses were both simultaneously related to increased modular brain organization. We also found that more modularized network organization was related both to amygdala hyperactivation and weakened connectivity between amygdala and medial PFC.

Conclusions: These results demonstrate that early life trauma is associated with enhanced brain organization during facial emotion processing and that this pattern of brain organization might explain the commonly observed association between childhood trauma and amygdala hyperactivity and weakened connectivity with mPFC. Implications of these results for neurocircuitry models are discussed

Keywords: Childhood Trauma, Functional Neuroimaging, Large Scale Networks

Disclosure: Nothing to Disclose. 
M47. Peak Alpha Frequency and Phase Coherence as Electrophysiological Biomarkers of Autism Spectrum Disorder (ASD)

Abigail Dickinson, Damla Senturk, Shafali Jeste*

David Geffen School of Medicine, Los Angeles, California, United States

Background: The dynamics of neural oscillations in the alpha range $(6-12 \mathrm{~Hz})$ are exquisitely sensitive to developmental changes in brain networks. Measures such as peak alpha frequency, alpha power and alpha band connectivity shed light on the integrity of neural networks and, moreover, are easily measured through quantitative electroencephalography (EEG). In neurodevelopmental disorders, such as autism spectrum disorder (ASD), where behavioral manifestations and outcomes are heterogeneous, there has been an increasing interest in the identification of quantitative, mechanistic biomarkers that can serve several purposes: (1) diagnostic classification, (2) clinical stratification, (3) objective measurement of change with intervention and outcome with treatment. Over the last three years, as part of the UCLA Autism Center of Excellence biomarkers core, we have examined characteristics of alpha band oscillations in a heterogeneous, clinically representative sample of children with ASD. We asked two questions. First, do peak alpha frequency and alpha band coherence in both long and shortrange networks distinguish children with ASD from typically developing (TD) children? Secondly, do these alpha band oscillations relate to developmental level and cognitive function within the autism spectrum?

Methods: We studied 61 children with ASD (mean age 69 months; range: 25-126) with nonverbal IQ ranging from 10-145 (mean NVIQ: 75) and 40 age matched TD children (mean age: 72 months; range: 29-146; mean NVIQ: 113). Cognitive and language assessments with convergent validity were administered based on the ability and age of the child. EEG was recorded using a high density 128 channel hydrocel geodesic sensor net (EGI inc.) while children passively viewed bouncing soap bubbles on a computer screen. Spontaneous EEG data were then extensively cleaned through both manual inspection and independent component analysis (ICA). Peak alpha frequency was computed by performing spectral power analyses on EEG data, before removing the $1 / \mathrm{f}$ trend from the calculated power spectra, and then fitting a Gaussian curve to accurately capture power modulations in the alpha range. This process was conducted separately for frontal, central, and occipital regions. To calculate alpha band coherence, EEG data were transformed into current source density (CSD) using a spherical spline Laplacian transform to mitigate the effects of volume conduction on coherence estimates. CSD estimates were then decomposed into the time-frequency domain using fast Fourier transform. Phase coherence within the alpha range was calculated between every possible electrode pair. A permutation test with a false discovery rate (FDR) adjustment for multiple testing was used to identify electrode pairs which demonstrated significantly different phase coherence between ASD and TD participants.

Results: Peak alpha frequency was significantly lower in ASD compared to TD children in frontal $(\mathrm{t}(89)=2.71, p=0.008)$ and central regions $(\mathrm{t}(93)=2.50, p=0.014)$, and this difference approached significance for the occipital region $(\mathrm{t}(92)=1.92), p=0.058)$. Instead of showing the typical increase with age, peak alpha frequency correlated strongly with non-verbal cognition in children with ASD (frontal: $\mathrm{R}=.373, p=.008$; central: $\mathrm{R}=.337, p=.013$ ). Using a conservative FDR correction of 0.05 , one long range central interhemispheric electrode pair showed significantly decreased phase coherence in ASD compared to TD children ( $\mathrm{t}$ $(96)=4.20, p=.000)$. Alpha phase coherence for this electrode pair was not associated with either age or IQ in the ASD group (age: $p=.193$; non-verbal IQ: $p=.267$, verbal IQ: $p=.215)$.

Conclusions: Both peak alpha frequency and long-range alpha band coherence distinguish ASD from TD children. Children with ASD show significantly decreased peak alpha frequency and significantly reduced long range interhemispheric connections. Each of these metrics suggest that there is disrupted network-level brain activity in ASD. The phase coherence result replicates previous studies of long-range alpha hypoconnectivity in ASD, but in a larger and more cognitively heterogeneous sample. The relationship between peak alpha frequency and non-verbal cognition in the ASD group suggests that deviations in network development may underlie cognitive impairment in individuals with ASD. These results support the value of peak alpha frequency and phase coherence as biomarkers of ASD, with peak alpha frequency also serving as a stratification marker. Continued and future studies will examine these measures earlier in development and will determine their stability and change over time and with intervention.

Keywords: Autism Spectrum Disorder, EEG/ERP Electrophysiology, Alpha Oscillation, EEG Biomarkers Disclosure: Nothing to Disclose.

\section{M48. Prefrontal Parvalbumin Interneurons in Control of Social Behavior}

Lucy Bicks, Beth Lucas, Michelle Peng, Roger Clem, Schahram Akbarian, Hirofumi Morishita*

Icahn School of Medicine at Mount Sinai, New York, New York, United States

Background: Social processing is a domain that is commonly dysregulated in psychiatric disorders, and is poorly treated by available psychiatric medications. In humans and rodents, portions of the evolutionarily conserved medial prefrontal cortex (mPFC) are part of a network that regulates social behavior. Many disorders with shared social processing deficits show impairments in inhibitory neurotransmission within the brain, particularly in the MPFC, suggesting a role for PFC inhibitory action in regulating social behavior. Parvalbumin expressing interneurons (PVIs) are one of the major subclasses of inhibitory neurons, implicated in psychiatric disorders. Here we aim to examine a causal contribution of PVIs in mPFC for social behavior in mice.

Methods: To test whether PVIs are activated by social exploration in the $\mathrm{MPFC}$, we exposed adult male mice in a 3chamber apparatus to either a novel mouse (social) or a novel object (object) under a wire corral. We perfused mice $90 \mathrm{~min}$ after the end of the exposure, and stained for egr 1 , a 
$\overline{\mathrm{S} I 42}$

marker of neuronal activity, as well as PV to identify PVIs. To investigate the causal contribution of mPFC-PVIs in social behavior of adult mice, we leveraged chemogenetic technologies. We selectively expressed hM4Di, an inhibitory DREADD (Designer Receptor Exclusively Activated by Designer Drugs), or hM3Dq an excitatory DREADD in the adult mPFC-PVIs.

Results: While both object and social groups showed overall increased activity reflected by egr1 induction across regions of the of $\mathrm{mPFC}$ regions including the dorsal anterior cingulated (dACC), the prelimbic region (PL) and the vACC, only the social group showed significant activation of PVIs in dACC, suggesting that social exploration engages dACC PVI activity specifically. Acute selective suppression of dmPFC PV interneurons in adult mice decreased sociability in a 3-chamber test and disrupted social recognition in a habituation-dishabituation paradigm. Suppression of PVIs did not affect olfactory discrimination, or anxietyrelated behaviors, suggesting a specific effect of PVI suppression on social behavior. We next tested whether increasing PVI activity could rescue a social deficit caused by social isolation during a 2 -week adolescent window, by expressing the excitatory DREADD in mPFC-PVIs. We found activating PVIs in adult $\mathrm{MPFC}$ rescued a persistent social deficit induced by transient adolescent social isolation. Conclusions: These results demonstrate that PVIs activity in the $\mathrm{mPFC}$ is necessary and sufficient for appropriate social behavior in mice. Our study implicates mPFC PVIs as promising cellular targets for future therapeutic development on social impairments in neurodevelopmental and psychiatric disorders.

Keywords: Social Behavior, Parvalbumin Neurons, Medial Prefrontal Cortex, Chemogenetics, Adolescence

Disclosure: Nothing to Disclose.

\section{M49. GABAB Correlates of Resting-State Functional Connectivity in Depressed Adolescents}

Paul Croarkin*, Irena Balzekas, Charles Lewis, John Port, Hang Joon Jo

Mayo Clinic, Rochester, Minnesota, United States

Background: Recently, functional magnetic resonance (fMRI)-based resting state (RS) functional connectivity features have identified distinct biotypes in Major Depressive Disorder (MDD) that may predict treatment efficacy. However, there are practical barriers for the clinical implementation of fMRI biomarkers. The neurochemical underpinnings of RS connectivity features are also incompletely understood. Transcranial magnetic stimulation (TMS) is an inexpensive, noninvasive technique to assess cortical physiology at the bedside. TMS indirectly measures GABAB receptor mediated tone via long interval cortical inhibition (LICI) and cortical silent period (CSP). In adolescents with MDD, deficits of LICI may be markers of treatment resistance and suicide risk. The purpose of this study was to pilot methodology focused on examining associations with LICI and CSP and functional connectivity measures in adolescents with MDD. This work could inform common substrates for specific TMS and RS AMRI based biomarkers and provide opportunities for pragmatic biomarker development.

Methods: Six participants with MDD (mean age $16.7+1.7$ years, 4 females) underwent MRI sessions and TMS testing (LICI and CSP). For MRI sessions, participants were instructed to keep their eyes open and focus on a spot, and their respiration and cardiac pulse were also recorded. T1 anatomy (MPRAGE, TR/TE $=7.37 / 3.04 \mathrm{~ms}$, inversion time $=$ $900 \mathrm{~ms}$, flip angle $=8^{\circ}$, voxel size $=1 \times 1 \times 1.2 \mathrm{~mm} 3$ ) and RS fMRI (GE EPI, 3.3mm isocubic voxel size, 48 slices, total acquisition time $=448 \mathrm{~s}, \mathrm{TR} / \mathrm{TE}=2900 / 30 \mathrm{~ms}$, flip angle $=$ $90^{\circ}$ ) data were collected using a GE 3 Tesla scanner equipped with 8-ch head-coil array. All imaging data were aligned with the N27 brain template and RS fMRI data were preprocessed with ANATICOR and PESTICA. For each participant, a connectivity matrix was obtained from correlations between timeseries separately averaged within FreeSurfer's regions of interest (ROIs), and standardized via Fisher transformation. During concurrent CSP and LICI testing sessions, participants wore earplugs and were seated comfortably. Motor evoked potential (MEP) data were collected with surface electromyography. Participants were instructed to maintain muscle relaxation, which was monitored with audio feedback. The TMS coil was placed tangentially the participant's head at a 45-degree angle over the motor hotspot. Cortical silent period measures were collected during muscle contraction at $20 \%$ of the participant's maximum with a TMS pulse intensity $140 \%$ motor threshold. LICI measures were collected with interstimulus intervals of $100 \mathrm{~ms}, 150 \mathrm{~ms}$, and $200 \mathrm{~ms}$. Correlation coefficients between connectivity matrices and LICI/CSP scores were generated for each participant and their statistical significance was corrected by false discovery rate control (FDR $\mathrm{q}<0.05$ ).

Results: Means (SD) of cortical inhibition measures in this sample reflected prior reports of cortical inhibition in adolescents with MDD: LICI $100=0.142(0.086)$; LICI $150=0.238$ (0.141), LICI $200=0.406 \quad(0.42), \quad C S p=0.158$ (0.022). Heat maps demonstrated correlations between connectivity patterns and LICI 100, LICI 150, LIC 200, and CSP. Generally, impaired cortical inhibition as determined by higher LICI values was associated with an increased number of strong correlations with connectivity patterns. Notably, LICI 200 demonstrated the largest number of correlations and the strongest correlations as compared to the other measures tested. For LICI 200, the left lingual gyrus had the highest number of correlated connectivity patterns. The number of correlated connectivity patterns was greatest for LICI 100, LICI 150, and CSP in the right precentral gyrus, left declive, and left declive, respectively.

Conclusions: These correlations between deficits in LICI and discrete RS connectivity features demonstrate that concurrent measurement of GABAergic neurotransmission and RS functional connectivity may have utility in biomarker studies of adolescent MDD. Although the small sample size is a limitation in the interpretation of these preliminary results, these data suggest the feasibility and utility of this approach. RS studies may further validate TMS measures of cortical inhibition such as LICI and CSP, enhancing our understanding of the neurochemical substrate for functional connectivity. An efficient and accessible proxy for RS studies would have great utility in future clinical research and practice. 
Keywords: Resting-State fMRI, GABA Transmission, Transcranial Magnetic Stimulation, fMRI Functional Connectivity, Adolescents

Disclosure: Part 4: Neuronetics, Grant, Assurex, Grant, Pfizer, Inc., Grant.

M50. Breadth and Depth of Working Memory and Executive Function Deficits in Mild Cognitive Impairment and Relationships to Brain Structure and Functional Competence

Terry Goldberg*, Jesus Gomar

New York State Psychiatric Institute/Columbia University, New York, New York, United States

Background: Although working memory (WM) and executive function (EF) impairment may be evident in MCI, its characterization has remained incomplete. This study aimed to investigate the breadth and depth of WM/EF in individuals with Mild Cognitive Impairment (MCI) as compared to normal elderly control (NEC) participants, and Alzheimer's disease (AD) patients and the relationship of WM/EF to brain morphometrics and everyday functional competence.

Methods: We assessed $48 \mathrm{MCI}$ individuals, 58 probable AD individuals, and a sample of 124 NECs, with a wide range of $\mathrm{WM} / \mathrm{EF}$ tests that covered functions such as attention, information manipulation, set-shifting, psychomotor speed, problem solving, and cognitive control (e.g., N Back, Tower of London, Stroop test, trail making, Letter-Number span, digit span) as well as tests of episodic memory (Logical Memory, Selective Reminding). We assessed regional brain morphometrics using Freesurfer image analyses after structural MRI and functional performance using the UPSA to analyze their relationship with WM/EF.

Results: MCI subjects demonstrated significantly worse performance on nearly all tests of WM/EF. Effect sizes (Cohen's d) were in the.70 to 1.05 range. The modal MCI participant demonstrated impairment on five of 12 measures. As expected $\mathrm{AD}$ subjects performed worse than the MCI group and the modal $\mathrm{AD}$ participant demonstrated nine impaired test performances. Factor analysis revealed an interpretable four-factor solution that included, cognitive speed, working memory involving short term information maintenance, and executive function involving simultaneous maintenance and manipulation of information, and episodic memory as a "control." These factors, in turn, were related in stepwise regression models to both specific and global cortical thickness reductions. WM/EF factors were also correlated with performance based measures of functional competence.

Conclusions: To the best of our knowledge this is the most comprehensive study of WM/EF to date in MCI. It is also the largest non-ADNI study on WM/EF in MCI and it offers complementary findings that usefully extend and refine the heavily mined ADNI database. Tasks included 1. Assays of simple WM storage that engages the phonological loop; 2. more complex measures involving simultaneous storage and manipulation of information involving such processes as multiple re-sequencing of items, planning, and updating of information; and 3. set switching in the context of speed demands and organized visual search. Impairment severity in the latter two types of task were in the medium to large ES range in MCI and differences were highly significant. Moreover, the modal MCI subject demonstrated impairment on four to six measures. For comparison, the modal EHC demonstrated no impairments and the modal $\mathrm{AD}$ subject demonstrated seven or nine impairments. Impairments were significantly associated reductions in dorso- and ventrolateral cortical thickness for EF. Speed determined in part by set switching and eye movement control was associated with superior gyrus thickness reductions. Global cortical thickness reductions were also associated with all WM/EF subdomains. Memory performance was related to hippocampal volume. These findings suggest that several critical neural circuits that underlie "frontal" tasks are already compromised before $\mathrm{AD}$ is diagnosed. Last, several $\mathrm{WM} / \mathrm{EF}$ subdomains were associated with functional competence. In summary, the results that we report suggest that WM/EF impairments in amnestic MCI are present, substantial, may be related to both dedicated circuitry and more global morphometric measures, and even at this potentially prodromal stage of $\mathrm{AD}$, have clinically deleterious consequences.

Keywords: Mild Cognitive Impairment Due to AD, Executive Function, Aging and Dementia

Disclosure: Nothing to Disclose.

M51. The Alzheimer's Prevention Initiative (API) Generation Program: Evaluating the Efficacy of the BACE-1 Inhibitor CNP520 in Preclinical Alzheimer's Disease

Pierre Tariot*, Cristina Lopez-Lopez, Angelika Caputo, Ronald G. Thomas, Jessica Langbaum, Robert Lenz, Gabriel Vargas, Vissia Viglietta, Eric M. Reiman, Ana Graf

Banner Alzheimer's Institute, Phoenix, Arizona, United States

Background: CNP520, a Beta-site-APP cleaving enzyme-1 (BACE-1) inhibitor, is in clinical development for the treatment of preclinical Alzheimer's disease (AD) in the "Generation Program".

Methods: The Generation Program, with funding sources that include NIA, philanthropy and Novartis/Amgen, comprises two international pivotal studies with similar designs and the aim of assessing the potential therapeutic benefit of CNP520. Under the auspices of the Alzheimer's Prevention Registry, we developed a novel, trial-independent APOE4 recruitment registry known as GeneMatch to support enrollment into these and other studies.

Generation Study 1 is a randomized, double-blind, placebocontrolled, two-cohort parallel group study to evaluate the efficacy of CAD106 (an active immunotherapy against beta-amyloid) and CNP520 (at a single dose), each vs its placebo, in cognitively unimpaired participants at particularly elevated risk for the imminent onset of clinical symptoms of $\mathrm{AD}$ by virtue of their age (60-75 years) and carriage of 2 copies of APOE4 (representing about $2 \%$ of the population). By age 85 , these homozygotes have a risk of up to $55 \%$ of developing symptomatic AD (Blacker et al 2017). 
About $70-80 \%$ of APOE4 homozygotes age $60-75$ will have extensive fibrillar A $\beta$ deposition (Jansen et al., 2015).

Generation Study 2 is a randomized, double-blind, placebocontrolled, parallel group study to evaluate the efficacy of two doses of CNP520 in cognitively unimpaired 60-75 yo participants also at increased risk for the onset of clinical symptoms due to $\mathrm{AD}$ : APOE4 homozygotes as well as APOE4 heterozygotes (about 25\% of the population) with elevated brain amyloid as measured by amyloid PET imaging or A $\beta$ levels in CSF. These 2 groups have about the same risk of developing symptoms of $\mathrm{AD}$ and represent a form of "preclinical" AD.

Efficacy of CNP520 will be evaluated by dual primary endpoint variables: time to diagnosis of mild cognitive impairment (MCI) due to $\mathrm{AD}$ or dementia due to $\mathrm{AD}$, and/ or decline in score in the API preclinical composite cognitive (APCC) test, a sensitive cognitive battery used as continuous measure of cognition from unimpaired through mild impairment stages expected to occur in the study. The diagnostic criteria proposed by the NIA-Alzheimer's Association working group will be used to establish the events of progression to symptomatic $\mathrm{AD}$, in conjunction with a centralized adjudication process. The Clinical Dementia Rating (CDR), Repeatable Battery for the Assessment of Neuropsychological Status (RBANS), and Measurement of Everyday Cognition (ECog) scale will be included as secondary endpoints in order to more fully characterize potential drug effects and to contribute to the assessment of their clinical relevance. The program will also investigate the effects of CNP520 on underlying AD pathology as assessed by additional biomarker studies including amyloid and (pending) tau PET scans. APOE4 has been linked to development of cerebral amyloid angiopathy (CAA). BACE inhibitors such as CNP520 might have potential to reduce vascular amyloid load and therefore have beneficial effects on CAA and ultimately reduce the risk for microbleeds. Appropriate MRI monitoring will be implemented to monitor for these events.

Results: Generation Study 1 began multicenter enrollment in 2016; Generation Study 2 has just launched. Trial progress to date will be summarized. GeneMatch serves as the primary recruitment mechanism in the US and is proving to be effective: to date, over 42,000 volunteers have been enrolled; of those genotyped, nearly $4 \%$ are APOE 4 homozygotes and $30 \%$ are heterozygotes, and $>800$ have been referred to the program.

Conclusions: The Generation Program is designed to provide efficacy, safety and tolerability data for CNP520 compared to placebo in people at risk for the onset of clinical symptoms of $\mathrm{AD}$.

Keywords: Alzheimer's Disease, Prevention of Alzheimer's Disease, CNS Clinical Trials

Disclosure: Part 1: Genentech, Grant, Roche, Grant, Novartis, Grant, Amgen, Grant, Lilly, Grant, Avid, Grant, Merck, Advisory Board, Self, Merck, Grant, Self, Lundbeck, Advisory Board, Self, Lundbeck, Grant, Self, Pfizer, Grant, Self, Pfizer, Consultant, Self, Takeda, Grant, Acadia, Consultant, AstraZeneca, Grant, AbbVie, Grant, AbbVie, Consultant, ACImmune, Advisory Board, Boehringer-Ingelheim, Advisory Board, Adamas, Stock / Equity, Part 2: Lilly, Advisory Board, Part 4: Genentech, Grant, Roche, Grant,
Novartis, Grant, Amgen, Grant, Lundbeck, Grant, Takeda, Grant, AstraZeneca, Grant, Avid, Grant.

\section{M52. Lithium as a Treatment To prevent Impairment of Cognition in Elders (LATTICE)}

Ariel Gildengers*, Howard Aizenstein, Stewart Anderson, Meryl Butters, James Emanuel, Tamer Ibrahim, Francis Lotrich, Chester Mathis, Dana Tudorascu

University of Pittsburgh School of Medicine, Pittsburgh, Pennsylvania, United States

Background: Lithium as a Treatment To prevent Impairment of Cognition in Elders (LATTICE) is a National Institute on Aging funded study to examine the effects of lithium on delaying conversion to dementia in older adults with mild cognitive impairment (MCI).

Over the past several years, there has been increasing interest in repurposing the use of lithium for diseases involving neurodegeneration (Lazzara and Kim, 2015). Lithium treatment has been associated with neurogenesis in the hippocampus (van Erp et al., 2012), up-regulation of important neurotrophic factors such as B-cell lymphoma 2 (Bcl-2) and brain-derived neurotrophic factor (BDNF) (Schloesser et al., 2012), and, relevant to this project, inhibition of glycogen synthase kinase 3 (GSK-3) isoforms $\alpha$ and $\beta$. In particular, GSK-3 $\alpha$ interacts with gammasecretase playing a critical role in the conversion of amyloid precursor protein (APP) to amyloid-beta $(A \beta)$; lithium has been shown to reduce $A \beta$ production and memory deficits in AD transgenic mouse models (Matsunaga et al., 2015). GSK- $3 \beta$ phosphorylates tau, a critical step in the formation of neurofibrillary tangles, and lithium has been shown to reduce tau phosphorylation in vivo and in vitro (Phiel et al., 2003). That lithium may alter the AD trajectory is supported by numerous observational reports showing delay of dementia onset in those treated with it (Gerhard et al., 2015, Nunes et al., 2007, Kessing et al., 2010, Kessing et al., 2008, Terao et al., 2006). However, the results of the few human lithium trials conducted have been mixed (Hampel et al., 2009, Macdonald et al., 2008, Forlenza et al., 2011, Nunes et al., 2013). At this time, it is difficult to draw definitive conclusions and make recommendations for widespread use of lithium based on the current evidence as well as the unclear risk/benefit profile of lithium treatment in older adults at risk for developing AD. Research is needed to determine whether lithium has a role as an anti-dementia agent. In contrast to previous studies, we will implement an RCT with a more integrative, comprehensive approach than done before involving state-of-the-art ultra-high field (7 T) human MRI, neurocognitive assessment, and blood-/CSFbased biomarker measurement to investigate the role of lithium as an anti-dementia agent.

Methods: Enroll and randomly assign 80 individuals 60 years and older with MCI (amnestic type; single or multiple domain) to take lithium, titrated as tolerated to a blood level of 0.6 to $0.8 \mathrm{mEq} / \mathrm{L}$, or placebo for two years to assess lithium's effects on preserving cognition and delaying conversion to dementia. Participants will receive annual neurocognitive assessment, ultra-high field (7 T) brain MRI 
(e.g., high-resolution imaging of hippocampal and total cortical gray volumes, white matter integrity, dynamic imaging), blood- (e.g., GSK-3 $\beta$, BDNF, cytokines) and CSFbased (e.g., A $\beta$, total tau, and phospho tau) biomarker measurement. All subjects will undergo baseline PET imaging of $\mathrm{A} \beta$ on which to stratify randomization. This study is powered to detect medium/large effect sizes for $\mathrm{H} 1$ 2 , and will serve as the "go/no-go" for a subsequent, largerscale, confirmatory study.

Results: Once data collection is completed, we will address the following hypotheses -

H1: a) Participants randomized to take lithium for two years, compared to placebo, will better maintain cognitive function, primarily in memory, which b) will be associated with changes in biomarkers (e.g., GSK-3 $\beta$ activity, BDNF).

H2: a) Participants randomized to take lithium for two years, compared to placebo, will have larger hippocampal volumes and lower total gray matter thinning, which $b$ ) will be associated with changes in biomarkers (e.g., GSK-3 $\beta$ activity, $\mathrm{BDNF}$ ) and c) better cognitive function, primarily in memory.

Exploratory H3: To examine whether lithium is related to additional markers of enhanced brain integrity (e.g., lower level of microbleeds, higher white matter integrity, better network connectivity, or decreased CSF phospho tau levels). Conclusions: Enrollment is expected to continue until 2020 with final study assessment in early 2022. Data analysis will be completed in mid-2022 with study findings reported later in the year.

Keywords: Prevention of Alzheimer's Disease, Lithium, Mild Cognitive Impairment Due to AD

Disclosure: Nothing to Disclose.

M53. Sex Differences in the Relationship Between Abdominal Fat Distribution and Structural Brain Networks

Matthias Heinrich, Frauke Beyer, Markus Loeffler, Michael Stumvoll, Shahrzad Kharabian, Matthias Raschpichler, Karsten Mueller, Juergen Kratzsch, Matthias L. Schroeter, George Slavich, Arno Villringer, Veronica Witte, Julia Sacher*

Max Planck Institute for Cognitive and Brain Sciences, Clinic for Cognitive Neurology, University of Leipzig, Leipzig, Germany

Background: Accumulating evidence from neuroimaging studies in obesity suggests an inverse relationship between gray matter volume and abdominal fat accumulation. Abdominal visceral adiposity correlates negatively with verbal memory and attention, and represents a risk factor for dementia. Women seem to be more vulnerable to risk of developing cognitive impairment and dementia in association with abdominal visceral adiposity than with overall adiposity, as measured by BMI. Early patterns of grey matter loss can already be detected during mid-life, a time of substantial changes in body fat distribution, increased visceral fat accumulation, and ovarian-hormone fluctuations in women. So far, we do not know whether sex hormones affect the relationship between visceral abdominal fat accumulation and grey matter structure, in part because large cohort studies simultaneously including brain, abdominal and hormonal data are lacking. To address this issue, therefore, we examined whether sex hormones modulate the relationship between visceral adipose tissue volume (VAT) and structural grey matter (GM) networks.

Methods: All analyses were performed in the comprehensively phenotyped adult cohort of the population-based Leipzig Research Center for Civilization Diseases (LIFE) study ( $N=975$, 474 females, 19-79 years of age). Participants with previous stroke, cancer, major neuropsychiatric pathologies and any current medication affecting the central nervous system were excluded. High-resolution T1-weighted images were assessed at a Siemens Verio 3 Tesla Scanner with a 32-channel head coil with an MPRAGE (ADNI) sequence with $1 \mathrm{~mm}$ isotropic voxels, 176 slices, $\mathrm{TR}=2300$ $\mathrm{ms}, \mathrm{TE}=2.98 \mathrm{~ms}$, and inversion time $(\mathrm{TI})=900 \mathrm{~ms}$. Gray matter (GM) was preprocessed using FreeSurfer (www. freesurfer.net) and FSL-VBM (fsl.fmrib.ox.ac.uk). For the neuroimaging data-analysis, we applied a linked independent component analysis (FLICA: FMRIB's Linked Independent Component Analysis) of (a) grey matter volume, (b) cortical thickness and (3) pial surface to identify structural neural networks. For the abdominal MR imaging data analysis, we segmented visceral and subcutaneous adipose tissue using a semi automatic macro in ImageJ. Serum levels for estrogen, progesterone and testosterone were analyzed by liquid chromatography tandem-mass spectrometry (LC-MS/MS) techniques.

Results: Inter-rater variability for abdominal adipose tissue segmentation was excellent for both visceral (VAT, ICC = 0.98 ) and subcutaneous adipose tissue (SCAT, ICC $=0.97$ ). VAT was significantly higher in men than women $(t$ $(918.71)=12.22, p<0.001$, alpha $=0.05)$. The best model predicting VAT from age was a quadratic fit for men (adjusted R2 $=0.931, p<0.001$ ) a polynomial fit of third degree (adjusted R2 $=0.851, p<0.001$ ), with an inflectionpoint at 47 years of age, for women. VAT was significantly negatively associated with a large-scale, age-sensitive grey matter network, previously associated with heightened risk for dementia. Men showed a significantly steeper negative association between VAT and structural grey matter network than women $(p<0.0024)$. Furthermore, estradiol (adjusted $\mathrm{R} 2=0.05, p<0.002$ ) and progesterone (adjusted $\mathrm{R} 2=0.12$, $p<0.001)$ levels show a positive association with this structural network in women, even when correcting for age. Conclusions: The present data indicate a sex-specific interaction between visceral adipose fat and a structural grey matter network previously linked to cognitive impairment and dementia: While in men this inverse relationship can be consistently displayed throughout all age groups, premenopausal women do not show a significant negative association between visceral abdominal fat and structural grey matter load. Furthermore, our findings provide first evidence for a protective role of ovarian hormones estrogen and progesterone in maintaining the structural grey matter network organization, that, when compromised, has been associated with increased dementia-risk. This evidence supports a perimenopausal vulnerability model for the female brain during midlife, when women, possibly due to the loss of ovarian hormone production, start to experience increased visceral fat accumulation, which represents a risk factor for structural grey matter loss. 
$\overline{S \mid 46}$

Keywords: MR Imaging, Sex Steroids, Visceral Obesity, Aging and Dementia, Depression

Disclosure: Nothing to Disclose.

M54. Very Large Effects of a Comprehensive, Individualized, Person-Centered Management (CI-PCM) Program on Persons With Moderately Severe Alzheimer's Disease (AD) are not due to Differential Antipsychotic Medication Usage

Barry Reisberg*, Munther Alshalabi, Jaspreet Sangha, Mudasar Hassan, James Golomb, Isabel Monteiro, Carol Torossian, Istvan Boksay, Melanie Shulman, Sloane Heller, Yongzhao Shao, Sunnie Kenowsky

New York University Alzheimer's Disease Center, New York, New York, United States

Background: On the basis of observations that cognitively, functionally, and in other ways, the Alzheimer's disease (AD) process reverses normal development (a process we termed retrogenesis), we proposed a science of $\mathrm{AD}$ management (Reisberg et al., Am J Alzheimer's Dis Other Demen, 2002). We investigated the utility of this management science in a 28-week randomized, controlled trial (Reisberg et al., Dement Geriatr Cogn Disord, 2017). The duration, overall design, and measures in the management study were similar to those employed in our memantine pivotal trial (Reisberg, et al., N Eng J Med, 2003). An important addition to the 2017 study was a Comprehensive, Individualized, PersonCentered Management (CI-PCM) program developed by Sunnie Kenowsky. This study was a 28 week, clinician blinded, randomized, controlled, parallel group trial. Eligible subjects had a caregiver participant and probable AD. All subjects had a functional assessment staging (FAST) stage $\geq$ $6 \mathrm{a}$ and an MMSE score of 3-14. Subjects $(n=54)$ were randomized and assessed for eligibility. Twenty were enrolled. Ten subject-caregiver dyads had been randomized to the CI-PCM program and 10 to the Usual Community Care and Financial Compensation (UCC+FC) study group. All subjects received memantine as tolerated, titrated to a maximum of $10 \mathrm{mg}$ twice daily.

Dyads in the CI-PCM intervention group received caregiver training, management assessment, therapeutic home visits, carer support groups and memory coaching based on retrogenesis observations that $\mathrm{AD}$ persons can still learn, just as children can learn. Therefore, CI-PCM subjects were memory coached in diminished skills, such as dressing, continence, etc.

At the trial's conclusion, both primary outcome measures, the NYU Clinician's Interview Based Impression of Change Plus Caregiver Input (NYU-CIBIC-Plus) and the abbreviated Alzheimer's Disease Cooperative Study Activities of Daily Living Inventory modified for more severe dementia, (ADCS-ADLsev-abv), showed better scores in the CI-PCM subjects than in the UCC+FC subject group $(p<0.01$ and $p<0.001$, respectively). Secondary outcomes showed a significant benefit of the CI-PCM program on the FAST $(p<0.01)$ and on 3 assessments of behavioral disturbance ( $p$ values $<0.05$ ) and no effect on cognition. Globally, the magnitude of benefit of the CI-PCM program subjects versus the UCC+FC subjects on the NYU-CIBIC Plus was 2.9 points. This was compared with the benefit in the 2003 NEJM trial of memantine versus placebo in very similar subjects (e.g., baseline MMSE $=7.9$ in both studies), of 0.3 points. Therefore, globally, the CI-PCM program showed an improvement of $967 \%$ over memantine treatment alone. However, our 2017 published study did not examine possible differential effects of antipsychotic medication usage which could influence behavioral disturbances, and conceivably also functioning and cognitive scores.

Methods: Study books from the 2017 published trial were reviewed by investigators blinded to group assignment. All antipsychotic medication usage at each evaluation period, i.e., baseline, and weeks 4, 12 and 28 had previously been recorded. When medication had been started or discontinued between visits or it's dosing schedule changed affecting daily use and the starting or discontinuation date was unknown, the midpoint time interval was used in calculation of the estimated treatment duration. The starting and ending study visit days were each counted as one day.

Results: Both groups had 2 subjects (20\% of each group) who received antipsychotic medication. Group 1 had one subject who received risperidone, $0.5 \mathrm{mg}$ daily, for 82 days, and one subject who received risperidone, $0.25 \mathrm{mg}$ to $0.5 \mathrm{mg}$ daily, on an as necessary basis (p.r.n.), for an estimated 58.75 days. Group 2 had 1 subject who received quetiapine, $50 \mathrm{mg}$ daily p.r.n., alternated every other month with trazodone p.r.n., for an estimated 27.5 days, plus $50 \mathrm{mg}$ daily for an estimated 56.25 days, for an estimated total of 83.75 days. A second subject took aripiprazole for 197 days ( $2.5 \mathrm{mg}$ daily for 78 days, then $3.5 \mathrm{mg}$ daily). Hence the mean number of estimated days on antipsychotic medication for group 1 was 70.4 and for group 2 was 140.375. After completing the analysis, the blind was broken. Group 1 subjects had been randomized to the CI-PCM program and group 2 subjects to the UCC+FC condition. Subjects from group 1 were on antipsychotic medications for $\sim$ half (50.15\%) of the time that subjects from group 2 received these medications. Additionally, both subjects in the CIPCM group were on antipsychotics at baseline but were discontinued before the week 12 study visit; whereas subjects in the UCC+FC group were started on antipsychotic medication on or shortly after baseline and had their dosages increased, reaching maximal dosage levels at week 28.

Conclusions: The CI-PCM program produced dramatically large functional and global benefits, as well as significant improvement in behavioral disturbances in our recently published study. The present results do not support the hypothesis that the improvements were due to differential antipsychotic medication usage. In fact, the results seem to point to the possibility of discontinuing antipsychotic medication usage and the elimination of associated risks of these medications, with the CI-PCM program. The CI-PCM program can likely improve health outcomes for many of the most disturbed $\mathrm{AD}$ persons with the most burdened caregivers. (Supported primarily by non-profit foundations). Keywords: Antipsychotic Medication, Alzheimer's Disease, Functional Impairment, Behavioral Disturbances, Global Changes

Disclosure: Part 1: Zachary and Elizabeth M. Fischer Center for Alzheimer's Research Foundation, Grant, Forest Research Institute of Forest Laboraties, Grant, United States Department of Health and Human Services (DHHS) grant P30 
AG08051 from the US National Institute on Aging, Grant, Louis J. Kay and June E. Kay Foundation, Grant, The Hagedorn Fund, Grant, Mrs. Miriam Glaubach and Dr. Felix Glaubach, Grant, Part 2: Zachary and Elizabeth M. Fischer Center for Alzheimer's Research Foundation, Grant, Forest Research Institute of Forest Laboraties, Grant, United States Department of Health and Human Services (DHHS) grant P30 AG08051 from the US National Institute on Aging, Grant, Louis J. Kay and June E. Kay Foundation, Grant, The Hagedorn Fund, Grant, Mrs. Miriam Glaubach and Dr. Felix Glaubach, Grant.

M55. Decoding Ventral Striatal Oscillations Related to Feeding Behavior: Toward Real-Time Models That Generalize Across Individual Animals and Brain States

Lucas Dwiel, Megan Cheng, Michael Connerney, Alan Green, Jibran Khokhar, Wilder Doucette*

Dartmouth Hitchcock Medical Center, Lebanon, New Hampshire, United States

Background: Binge eating is a disruptive and treatmentresistant behavior that complicates the management of obesity and many comorbid psychiatric disorders. Binge eating is characterized by the overconsumption of highly palatable foods. It has also been modeled in animals using intermittent limited access to palatable food that over time produces "binge like" feeding behavior. The distributed circuit that governs feeding behavior, particularly of palatable food, has a nexus point within the ventral striatum (VS) - integrating information from energy homeostasis circuits of the hypothalamus and brain stem, cognitive control regions of the prefrontal cortex and central nodes of learning and memory. Emerging evidence suggests that dynamic changes in local field potential (LFP) oscillations recorded in the VS reflect underlying neuronal processes supported by previous work that has shown the entrainment of single-unit firing to oscillations recorded in VS. The VS has also been evaluated as a target for circuit based interventions (deep brain stimulation) in appetitive disorders and if information related to feeding behavior could be extracted from stimulating electrodes, then it could be used for real-time feedback in closed-loop and adaptive systems. Here, we tested the hypothesis that information contained within VS oscillations could be used to predict real-time feeding behavior, food palatability and the quantity of food consumed.

Methods: Sprague-Dawley rats were implanted with electrodes and local field potentials were recorded with paired video during limited access sessions with offline video analysis to identify relevant behavioral intervals (Plexon system). Custom code written in Matlab was used to extract LFP features of power, phase coherence and power coupling from 4 VS nodes (nucleus accumbens core and shell bilaterally). These were assessed across 6 established frequency bands (delta, theta, alpha, beta, low gamma and high gamma). The LFP features were used as predictors in statistical modeling (logistic regression) and machine learning (lasso and support vector machine) approaches to build and test models to classify the type and predict the quantity of food consumed, classify rat behavior (feeding or not feeding) in real time
(5 second windows), and predict the impending onset of future feeding bouts. The stability and performance of the real-time models were examined across individuals, hunger states and food palatability. To determine if models were outperforming chance, all analysis was also performed on permuted data using Monte Carlo sampling and then the two distributions of model performance (area under the receiver operator characteristic curve [AUC] or cross validation error) were compared with the Mann-Whitney U test and converting the U-statistic into an effect size (ES). Real-time models were validated with naïve data while the models predicting quantity and food type used a 5-fold crossvalidation within lasso.

Results: VS oscillations recorded during the limited access session were able to outperform permuted data to predict how much food was eaten $(E S=0.5)$, the increase in food consumption from baseline to sessions following 24 and 48 hours of food deprivation $(\mathrm{ES}=1.15)$ and the type of food consumed (high sugar/high fat or house chow - ES = 0.89). When predicting concurrent behavior (feeding or not feeding) a model built from one rat performed well on left out data from itself (average $\mathrm{AUC}=0.91$ ) but did not perform as well when applied to other individuals. Models built from a subset of the population data (equal contribution from individuals) performed well across all individuals (average $\mathrm{AUC}=0.83$ and $\mathrm{ES}=3.64$ ). The best univariate (single LFP feature) model built using the population data was able to perform with an average AUC $=0.75$. Finally, VS oscillations recorded in the 5 seconds preceding the initiation of feeding was able to be classified as distinct from other notfeeding time windows (average $\mathrm{AUC}=0.75$ ). The capacity to predict future feeding was possible up to one minute before the initiation of feeding behavior.

Conclusions: These findings suggest that current or future feeding behavior can be decoded from ventral striatal oscillations and that estimates of food palatability and the quantity consumed is feasible. Together these findings suggest that VS oscillations are a rich source of information related to feeding behavior that could be further evaluated as targets for treatment development or for real-time feedback for circuit-based interventions to treat eating disorders or obesity.

Keywords: Local Field Potentials, Feeding Behavior, Machine Learning

Disclosure: Nothing to Disclose.

M56. Reduced Default Mode Network and Reward Circuitry Connectivity 12-Months Post-Operative in Bariatric Surgery Patients

Hilal Cerit*, Paul Davidson, Taryn Hye, Priyanka Moondra, Florina Haimovici, Stephanie Sogg, Scott Shikora, Jill Goldstein, A. Eden Evins, Luke Stoeckel, Laura Holsen

Harvard Medical School/Brigham \& Women's Hospital, Boston, Massachusetts, United States

Background: Bariatric surgery is an effective intervention to achieve weight loss in severely obese individuals. Profound short-term effects of bariatric surgery on the brain have been noted in recent years. Among longitudinal studies, findings 
$\overline{\mathrm{S} I 48}$

suggest significant reductions post-surgery in response to high-calorie food images in food motivation and reward circuitry using task-based fMRI paradigms, although similar studies examining connectivity between regions are sparse. A handful of studies have examined differences between bariatric surgery patients and comparison groups in functional connectivity cross-sectionally post-surgery or in in the short term following surgery, reporting reduced default mode network connectivity in patients vs. BMI-matched controls, and attenuation from pre- to post-gastric bypass in fractional amplitude of low-frequency fluctuation in limbic and reward regions. Recently, findings emerged suggesting reduced connectivity between the precuneus and the STG and SMG, and between the posterior cingulate cortex and STG, MTG, and fusiform gyrus in gastric bypass patients at 1-year post-surgery, providing preliminary data of lasting effects of bariatric surgery on coordinated activity between key neural circuits regions guiding food motivation, bodily sensations, and self-regulation. It is unclear whether similar alterations might generalize to other bariatric procedures, such as vertical sleeve gastrectomy (SG), currently the most prominent bariatric procedure which has been demonstrated to induce different effects on task-based BOLD activity compared to gastric bypass. The current study aimed to investigate the effects of SG on resting state brain connectivity from pre- $(<1$-month prior $)$ to 12 -months post-SG.

Methods: We recruited 15 patients (13 females; 39.3 years at baseline; 41.7 BMI at baseline) scheduled to undergo vertical SG. Patients completed a baseline study visit within one month prior to their procedure and completed another study visit 12-months after their surgery. At both visits, patients underwent resting state fMRI scanning on a Siemens $3 \mathrm{~T}$ Skyra scanner. Functional connectivity pre- and post-SG was assessed in using CONN v17.b. First, an ROI-to-ROI approach was used in a priori ROIs (nucleus accumbens, putamen, and amygdala) based on our findings from taskrelated BOLD data acquired in the same patients ( $p$ FDR $<0.05)$. In addition, changes in functional connectivity were assessed default mode and salience network regions. Following ROI-to-ROI analysis, seed-to voxel analysis was conducted (cluster size p-FDR corrected, cluster threshold

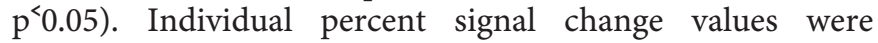
extracted from selected ROIs using REX.

Results: In the ROI-to ROI analysis, there was overall network-level reduction in connectivity post-SG in the left NAcc $[F(3,12)=3.51, p-F D R=0.0493]$. Seed-to-voxel analyses with left NAcc as the seed showed attenuated connectivity with clusters in the right postcentral gyrus ( $p$ $\mathrm{FDR}<0.001)$ and right cerebellum (p-FDR $=0.002)$. Network-level connectivity within the default mode network was also significantly reduced post-SG $[F(3,12)=10.69$, $\mathrm{p}-\mathrm{FDR}=0.001]$. At the ROI-to-ROI individual ROI connection level, the left putamen showed reduced connectivity post-SG with the brainstem $[\mathrm{t}(1,14)=4.41, \mathrm{p}-\mathrm{FDR}=0.0496]$, which was confirmed in the seed-to-voxel analysis (pFDR $<0.001)$. Following SG, ROI-to-ROI connectivity between the right amygdala and regions in the temporal cortex [right parahippocampal gyrus (PHG), fusiform cortex, middle temporal gyrus (MTG)] was reduced $[\mathrm{F}$ $(3,12)=3.18, \mathrm{p}-\mathrm{FDR}=0.003]$. Seed-to-voxel analysis in the right amygdala confirmed these findings, with reduced connectivity with the left temporal pole ( $\mathrm{p}-\mathrm{FDR}<0.001$ ), right hippocampus/PHG (p-FDR $=0.001)$, and MTG (p$\mathrm{FDR}=0.006$ ). Finally, the lateral parietal cortex within the default mode network showed significant widespread reduced connectivity with several regions post-SG [ROI-toROI seed level: $(\mathrm{F}(3,12)=4.21, \mathrm{p}-\mathrm{FDR}=0.03)$. Seed-to-voxel analyses revealed reductions in connectivity between the left lateral parietal cortex and a cluster spanning the left superior parietal lobule, left supramarginal gyrys, left lateral occipital cortex, and left angular gyrus (p-FDR $<0.001$ ), the left middle temporal gyrus $(\mathrm{p}-\mathrm{FDR}=0.002)$, and the left occipital pole $(\mathrm{p}-\mathrm{FDR}=0.003)$, and between the right lateral parietal cortex and clusters within the right supramarginal gyrus (p$\mathrm{FDR}=<0.001$, right cerebellum $(\mathrm{p}-\mathrm{FDR}=0.002)$, and right middle temporal gyrus $(\mathrm{p}-\mathrm{FDR}=0.004)$. There were no significant Pre- to Post-surgery changes in salience network connectivity.

Conclusions: Our preliminary analyses demonstrate changes in connectivity between regions within the default mode network (MPFC, lateral parietal cortex) and reward/motivation regions (NAcc, putamen, amygdala) in sleeve gastrectomy patients, with significant reductions from baseline to 12months post-surgery. These changes mimic literature on taskbased BOLD activity, and suggest robust effects of bariatric surgery on multi-modal aspects of brain functioning. Further, comparison of these findings to previous reports on gastric bypass patients indicate potentially distinct mechanisms through which these procedures affect brain connectivity, improving the understanding of gut-brain interaction.

Keywords: Bariatric Surgery, Resting State Functional Connectivity, Default Mode Network (DMN), Reward Circuitry

Disclosure: Nothing to Disclose.

\section{M57. Reward Learning Capacity in Binge Eating Disorder}

Iris Balodis*, Fiza Arshad, Kiran Punia, Michele Laliberte McMaster University, Hamilton, Canada

Background: Reward processing alterations may represent critical factors in the onset, maintenance and relapse of disordered eating. Many therapies emphasize developing new associations to stimuli, however, to date, few studies examine associative learning and preference formation in clinical populations. The current study examined how individuals with binge eating disorder (BED) develop preferences to rewarding non-food stimuli. Additionally, this study assesses the degree to which non-food reward conditioning relates to treatment outcome measures over time.

Methods: The Probabilistic Reward Task (PRT) is a computerized reward-learning task that measures an individual's ability to modulate their behaviour as a function of rewards. This task uses non-food reward to objectively assess hedonic capacity and differentiate between learning and motivational effects. The PRT was administered in a treatment-seeking BED group and to a comparison control group recruited from the community. BED and control groups will be compared on hedonic capacity - the propensity to modulate their behaviour as a function of reward.

Results: Results are expected to demonstrate alterations in hedonic, or reward-learning capacity in the BED group. 
Specifically, trial-by-trial probability analyses will test how BED individuals integrate reinforcement history over the course of the task and whether this results in a response bias toward a reward-paired cue. Sub-group analyses will also be conducted to examine the role of overvaluation of shape in BED as well as other clinical severity indicators such as chronicity of binge eating.

Conclusions: Results will be discussed in the context of reward learning capacity and relationship to treatment outcome, including changes in binge eating behaviour. Specifically, this study will test whether reward-learning alterations predict persistent bingeing following treatment. These findings will evaluate whether the PRT confers predictive validity of reward learning in individuals with BED. Any alterations in the ability to develop a behavioural bias to reward-paired stimuli may contribute to persistent bingeing or treatment resistance in BED.

Keywords: Binge Eating Disorder, Reward-Based DecisionMaking, Treatment-Response, Attentional Bias Modification, Reinforcement Learning

Disclosure: Nothing to Disclose.

\section{M58. Neural Correlates of Impaired Reward-Effort Integration in Remitted Bulimia Nervosa}

Stefanie Müller, Yosuke Morishima, Simon Schwab, Roland Wiest, Andrea Federspiel, Gregor Hasler*

\section{University of Bern, Bern, Switzerland}

Background: The integration of reward magnitudes and effort costs is required for an effective behavioral guidance. This reward-effort integration was reported to be dependent on dopaminergic neurotransmission. As bulimia nervosa has been associated with a dysregulated dopamine system and catecholamine depletion led to reward processing deficits in remitted bulimia nervosa, the purpose of this study was to identify the role of dopamine dysfunction and its relation to behavioral and neural reward-effort integration in bulimia nervosa.

Methods: To investigate the interaction between catecholamine functioning and behavioral, and neural responses directly, 17 remitted bulimic ( $\mathrm{rBN})$ and 21 healthy individuals (HC) received alpha-methyl-paratyrosine (AMPT) over 24 hours to achieve catecholamine depletion in a randomized, crossover study design. We used functional magnetic resonance imaging (fMRI) with the Monetary Incentive Delay (MID) task to assess reward-effort integration in relation to dopaminergic neurotransmission at the behavioral and neural-computational level.

Results: AMPT reduced the ability to integrate rewards and efforts effectively in $\mathrm{HC}$ participants. In contrast, in $\mathrm{rBN}$ participants, the reduced reward-effort integration was associated to illness duration in the sham condition and unrelated to catecholamine depletion. Regarding neural activation, AMPT decreased the reward prediction error signal in the anteroventral striatum. This decrease was associated with the AMPT-induced reduction of monetary earning in $\mathrm{HC}$ in contrast to $\mathrm{rBN}$ participants.

Conclusions: Our findings contributed to the theory of a desensitized dopaminergic system in bulimia nervosa. A disrupted processing of reward magnitudes and effort costs might increase the probability of maintenance of bulimic symptoms.

Keywords: Eating Disorders, Catecholamine Depletion, Functional MRI (fMRI), Reward Prediction Error

Disclosure: Nothing to Disclose.

\section{M59. Does Stimulating Brain Regions Associated With Inhibitory Control Have Paradoxical Effects on Eating Behavior?}

Rebecca Ashare*, Mary Falcone, James Loughead, Chan To, Erin Logue-Chamberlain, Roy Hamilton, Joseph Kable, Caryn Lerman

University of Pennsylvania Perelman School of Medicine, Philadelphia, Pennsylvania, United States

Background: Behaviors such as tobacco use, unhealthy diet, and sedentary behavior have far-reaching health implications. These modifiable behaviors account for a substantial proportion of deaths from cancer, cardiovascular disease and diabetes, and take a significant economic toll. Yet, many unhealthy behaviors are very resistant to change, despite widespread knowledge of the risks. To explain the persistence of these behaviors, we need to address a fundamental aspect of behavior change - an individual's ability to exert sufficient self-control to overcome temptations for immediate gratification and/or to maintain attention to long-term goals. Neuroimaging studies examining where and how selfcontrol is executed in the brain point to the central role of neural activity in the right inferior frontal gyrus (IFG). Importantly, emerging evidence shows that activity in the IFG and cognitive control circuits can be modulated using a noninvasive and safe intervention: direct current transcranial stimulation (tDCS). In this proof of concept study, we investigated whether one session of $2.0 \mathrm{~mA}$ anodal stimulation over the right IFG with cathodal stimulation over the left IFG (versus sham stimulation) reduced chocolate craving and chocolate consumption.

Methods: Twenty-three healthy females (ages 18-35) completed two tDCS sessions $(2.0 \mathrm{~mA}$ and sham stimulation; order counterbalanced) in a within-subject double-blind, randomized design with a 4-week washout period between sessions. Eligible participants were self-reported "chocolate cravers" and restrained eaters according to a Cognitive Restraint scale. Sessions were completed during the late luteal phase of the menstrual cycle to control for potential variation in craving levels. During each visit, baseline measures of delay discounting and inhibitory control (e.g., stop signal task) were completed prior to administration of 20 minutes of active or sham stimulation while participants completed a computerized inhibitory control training task (chocolate go/no-go task). In this task, half the participants were randomized to a condition in which chocolate images were paired with a no-go cue on all trials (chocolate no-go condition) whereas in the other condition, chocolate images were only paired with the no-go cue on $50 \%$ of trials (control condition). After the tDCS and chocolate go/no-go task, participants completed a 15- minute ad-libitum "taste test" in which they were told to eat "as much or as little chocolate as you want" and rated the chocolate on a variety of characteristics (e.g., sweetness, smell, intensity, appearance, 
taste, and persistence). The primary outcome was the total amount of chocolate consumed (g).

Results: Contrary to our hypotheses, active tDCS significantly increased the amount of chocolate consumed compared to the sham condition (mean $=42.1 \mathrm{~g}$ vs. $33.2 \mathrm{~g}$, $p=0.01$ ), but had no effect on subjective ratings of chocolate (all ps $>0.2$ ). Overall, higher levels of delay discounting and self-reported disinhibited eating and lower levels of nervousness during stimulation predicted greater chocolate consumption $(\mathrm{ps}<0.01)$. However, these relationships did not vary as a function of stimulation condition $(p>0.15)$. Significant session order effects were also found indicating that the tDCS effect on chocolate consumption was evident only for those who received sham first $(p=0.01)$; there was no session effect for those who received active first $(p=0.5)$. Conclusions: The results of this study suggest that stimulating the right IFG does not increase the ability to exert selfcontrol over eating behavior. In fact, our results indicate that stimulating this brain region associated with inhibitory control may actually reduce the ability to resist chocolate. Importantly, our finding that these effects were dependent on the order in which stimulation was administered has methodological implications for conducting within-subject tDCS studies.

Keywords: Brain Stimulation, Inhibitory Control, Eating, TDCS, Inferior Frontal Gyrus

Disclosure: Nothing to Disclose.

M60. Hypothalamic and Nucleus Accumbens Cerebral Blood Flow Vary as a Function of Long-Term Carbohydrate-To-Fat Ratio Diets

Laura Holsen*, Hilal Cerit, Belinda Lennerz, W. Scott Hoge, Taryn Hye, Priyanka Moondra, Jill Goldstein, Cara Ebbeling, David Ludwig

Harvard Medical School, Boston, Massachusetts, United States

Background: In the US, about $50 \%$ of overweight or obese adults are trying to lose weight, often by following energyrestricted diets. While many of these individuals experience some initial success, most have difficulty maintaining clinically significant weight loss over the long term. An explanation for this difficulty relates to biology, in that weight loss elicits adaptations that promote weight regain, including a decline in energy expenditure, an increase in hunger, and in some individuals, alterations in brain reward and homeostatic activity. Whether macronutrient composition influences these adaptations remains one of the most contentious debates in the field of nutrition. We conducted an ancillary study in the context of a randomized controlled trial (RCT), designed to evaluate outcomes of diets varying in macronutrient composition, specifically carbohydrate-to-fat ratio, during weight-loss maintenance. We measured resting state blood flow in appetite- and reward-related regions of the brain, examining the full dynamic spectrum of blood flow at pre- and post-prandial time points. We hypothesized that 1) regional cerebral blood flow ( $\mathrm{rCBF}$ ) to the nucleus accumbens (NAcc) at 4 hours post-prandial would differ according to diet group; and 2) rCBF to the hypothalamus at fasting (pre-prandial) would differ according to diet group.
Methods: Subjects completed a Run-In phase during which they lost $10 \%$ of their baseline body weight on a standard diet, and then were randomized to one of 3 test diets for 20 weeks. The macronutrient composition of the test diets was as follows: HIGH Carbohydrate: $60 \%$ carbohydrate $/ 20 \%$ fat/20\%protein, MODerate carbohydrate: $40 \% / 40 \% / 20 \%$, LOW carbohydrate: $20 \% / 60 \% / 20 \%$. Energy content for the 3 test diets was distributed throughout the day $(22.5 \%$ for breakfast, $32.5 \%$ for lunch, $32.5 \%$ for dinner, $12.5 \%$ for an evening snack), and the macronutrient composition of each meal and snack reflected the composition of respective test diets. Prior to randomization in the parent RCT, a subsample of subjects enrolled in the brain imaging study. Following 1420 weeks on the test diets, this subset of subjects $(n=21$ HIGH, $n=23$ MOD, $n=28$ LOW) completed a morning study visit after an overnight fast (12 hours). During this visit, they underwent scanning with an MRI imaging protocol on a $3 \mathrm{~T}$ Siemens Skyra scanner that included a fasting (pre-prandial) perfusion [pulsed arterial spin labeling (pASL) - PICORE Q2TIPS: TR $=4 \mathrm{~s}$, TE $=13 \mathrm{~ms}$, $3.4 \times 3.4 \times 4.0 \mathrm{~mm}, 24$ slices) scan; consumed their $\mathrm{HIGH}$, MOD, or LOW breakfast meal; and then rested quietly for 3.5 hours. At 4 hours following the meal, they underwent a late post-prandial brain imaging session (perfusion ASL, T1). Every 30 minutes for 4.5 hours, they also rated their hunger level, using a $10-\mathrm{cm}$ visual analogue scale, and completed the Food Craving Inventory. ASL data were analyzed using ANTs, FSL, and Freesurfer, including co-registration of segmented/labeled $\mathrm{T} 1$ image to the scaled perfusion CBF image and mean CBF values extracted for a priori ROIs (nucleus accumbens, hypothalamus). Behavioral data were analyzed using ANOVAs in SPSS; with statistical threshold set at $p<0.05$.

Results: Age, $\mathrm{BMI}$ at scan visit, $\mathrm{M}: \mathrm{F}$ ratio, and menstrual cycle day (for females) did not differ by diet group (all $p>0.4$ ). Hunger ratings at baseline and throughout the study visit (AUC) did not differ by diet group (all $p>0.1$ ). Total scores on the Food Craving Inventory did not differ by diet group $(p>0.1)$. Resting perfusion in the NAcc at 4 hours post-prandial differed significantly by group: $\mathrm{F}(2,67)=3.89$, $p=0.026$. Post-hoc comparisons demonstrated significantly higher NAcc rCBF in the MOD vs. LOW group $[\mathrm{t}$ $(1,45)=2.31, p=0.026]$ and in the HIGH vs. LOW group $[\mathrm{t}(1,44)=2.65, p=0.011]$, but no difference between the HIGH vs. MOD groups $(p>0.6)$. Pre-prandial (fasting) resting perfusion in the hypothalamus differed significantly by group: $F(2,67)=3.20, p=0.048$. Post-hoc comparisons demonstrated significantly higher hypothalamus $\mathrm{rCBF}$ in the HIGH vs. LOW group $[\mathrm{t}(1,44)=2.32, p=0.025]$, but no difference between the HIGH vs. MOD groups $(p>0.09)$ or the LOW vs. MOD groups $(p>0.5)$.

Conclusions: These data confirm the primary and secondary hypotheses regarding rCBF to homeostatic (hypothalamus) and reward (NAcc) regions of the brain. Specifically, subjects who were randomly assigned to the high carbohydrate diet exhibited significantly elevated $\mathrm{rCBF}$ in the hypothalamus at the pre-prandial timepoint, and in the NAcc at 4 hours postprandial, compared to individuals on a low carbohydrate-tofat composition diet, even in the absence of any group differences in subjective hunger or food craving ratings. Findings indicate potentially cumulative effects of long-term dietary macronutrient composition on brain reward and 
homeostatic activity, which could provide insight into the mechanism through which weight-loss maintenance on these diets produces variable success rates. For instance, elevated signaling in these systems in individuals on high carbohydrate diets could make them more vulnerable to increased food intake and suboptimal weight-loss maintenance. Although preliminary, these insights contribute to the mechanistic understanding for the challenges underlying maintenance of diet-induced weight loss, potentially informing the design of more effective therapies.

Keywords: Reward Circuitry, Dieting, Weight Loss Treatment, Perfusion

Disclosure: Nothing to Disclose.

\section{M61. Conditioned Food Avoidance in Adolescence: A Contributor to Eating Disorders?}

Cynthia Kuhn*, Elizabeth Burnette, Rylee Wander, Gabriela Ocampo, David Walker, Nancy Zucker

Duke University Medical Center, Durham, North Carolina, United States

Background: Anorexia Nervosa (AN) is the leading cause of mortality due to psychiatric causes. Research into its mechanism has been stymied because animal models have focused on anorexia associated with food restriction, extreme stress and or excess physical activity. No current animal models capture the key characteristics of visceral hypersensitivity leading to learned food avoidance, adolescent onset and female dominance. The present study developed a food aversion task that involved no food deprivation to investigate the developmental and sex specificity of conditioned food avoidance.

Methods: Male and female rats ( $\mathrm{PN} 28-30$ and PN 60-62) were placed in a feeding cage and allowed to eat a novel cereal (Cheerios, Ch) for one hour. Twenty-four hours later, they were allowed to eat a novel, palatable food (Froot Loops, $\mathrm{FL})$. and then were treated with saline or $\mathrm{LiCl}(19 \mathrm{mg} / \mathrm{kg})$. Pica was quantitated in a subset of animals for $1 \mathrm{hr}$. as a measure of nausea. Twenty-four hours later, rats were given the opportunity to eat FL or another novel cereal, Apple Jacks (AJ) and cereal intakes (g/kg bw) were quantitated. Statistics on all results were analyzed by 3-way ANOVA (age $\mathrm{x}$ sex $\mathrm{x}$ treatment) using NCSS. All experiments were approved by the Duke University IACUC and conducted in accordance with the NIH Guide for the Care and Use of Laboratory Animals.

Results: $\mathrm{LiCl}$ caused pica that was greater in adults than adolescents ( $p<.002$ effect of drug, $p<.002$ age $x$ dose). Adolescents ate more of a novel food (Ch) than adults $(\mathrm{p}<.001$ for effect of age, $\mathrm{p}<.0002$ age $\mathrm{x}$ sex $)$. All animals showed marked CFA to FL ( $p<.001$ effect of drug), and adults showed more CFA than adolescents $(p<.003$ effect of age, $p<.007$ age $x$ sex $x$ treatment). Furthermore, females generalized the CFA to include a novel food not previously paired with $\mathrm{LiCl}$ more than males $(\mathrm{AJ}) \mathrm{p}<.001$ effect of drug, ( $p<.0002$ effect of age, $p<.002$ effect of sex).

Conclusions: The results show that adults exhibit more conditioned food aversion than adolescents in this model. However, even adolescents exhibited some CFA to this low dose of $\mathrm{LiCl}$, showing more CFA than is detected using saccharin-containing water in published reports. Adult females exhibited the most evidence of nausea (pica), supporting our hypothesis that behavioral responses to visceral stimuli would increase from early adolescence (prepuberty into maturity. The most robust developmental difference observed was that females generalized more than males to avoid foods not previously associated with $\mathrm{LiCl}$ that were presented at the same time and in the same context as a food associated with $\mathrm{LiCl}$. These studies support the hypothesis that sex differences in conditioned food avoidance arise as animals mature from adolescence into adulthood, and support the possibility that maturation of neural circuits involved in organizing responses to aversive stimuli could play a role in the appearance of food -avoiding eating disorders during adolescence in humans. Supported by Duke Institute for Brain Sciences and Department of Pharmacology and Cancer Biology, Duke University Medical Center

Keywords: Eating Disorders, Adolescence, Sex Differences Disclosure: Nothing to Disclose.

\section{M62. GABA Neurotransmission in an Animal Model of Binge Eating Disorder: Effects of Lisdexamfetamine}

\author{
Yong Kee Choi, David Heal, Peter Hutson, Galen \\ Carey, Frank Tarazi*
}

Harvard Medical School, McLean Hospital, Mailman Research Center, Belmont, Massachusetts, United States

Background: Binge eating disorder (BED) is a psychiatric impulse control disorder that is characterized by compulsive overeating episodes. The main symptoms of the disorder include frequent binge eating episodes that are accompanied by feelings of extreme anxiety and helplessness during or after bingeing. We developed an animal model of binge eating in which adult, female rats were given unpredictable, limited access to chocolate (Vickers et al, 2015). These bingeeating rats showed compulsive consumption when given access to palatable food (Heal et al, 2016) and increased cognitive impulsivity in a chocolate rewarded delay discounting task (Vickers et al, 2017), suggesting that the model also mimics some of the underlying psychopathology of BED. Furthermore, all of these dysregulated behaviors were prevented by administration of lisdexamfetamine (LDX). We evaluated expression of two GABA biomarkers (GAD65 mRNA and GAD67 mRNA) using in situ hybridization in binge-eating animals, and then examined the effects of LDX, which was recently approved for treatment of BED, on same GABA biomarkers.

Methods: We collected forebrain tissue from 4 groups of rats ( $N=8$ /group):

Cohort 1, Group A: rats were given access to chow (24 hr) +empty pot (2 hr on an irregular access schedule as described by Vickers et al (2015). Rats in Cohort 1 represent the control groups.

Cohort 1, Group B: non-binge control rats were treated with LDX $(0.8 \mathrm{mg} / \mathrm{kg})$

Cohort 2, Group A: rats were given access to chow (24 hr) +junk food (powdered chocolate) for binging $2 \mathrm{hr}$ on an irregular access schedule. Animals in Cohort 2 represent a model of BED.

Cohort 2, Group B: Binge eating rats were treated with LDX $(0.8 \mathrm{mg} / \mathrm{kg})$. 
On Day 30, rats allocated to drug treatment were given LDX $(0.8 \mathrm{mg} / \mathrm{kg}$ po $) 1 \mathrm{hr}$ prior to a final $2 \mathrm{hr}$ bingeing session on chocolate or presentation of empty pots to the control groups. Rats were killed and brains were taken $1 \mathrm{hr}$ after the final test session. In situ hybridization was performed using two 49-base-long sequences from the rat GAD65 or GAD67 mRNA sequence. Sense an antisense sequence of GAD65 or GAD67 for each probe were selected from base 260-309 and 700-749 or base 275-324, 1377 1426, respectively.

Results: GAD65 mRNA expression was significantly reduced in medial prefrontal cortex (MPC; 19\%), dorsolateral frontal cortex (DFC; 19\%), core and shell of nucleus accumbens (by $30 \%$ and $29 \%$, respectively) of binge-eating rats compared to control vehicle-treated rats $[p<0.01]$. There were no changes in GAD65 and GAD67 mRNAs in caudate-putamen. Administration of LDX $(0.8 \mathrm{mg} / \mathrm{kg})$ to the control group of rats did not affect expression of GAD65 mRNA in all forebrain regions examined. In contrast, administration of LDX to binge eating rats reversed the observed reduction in GAD65 mRNA expression in MPC (by 10\%), DFC (by 10\%), NAc-C (by 17\%) and NAc-S (by 16\%) [ $p<0.05]$. GAD67 mRNA followed a similar trend as GAD65 mRNA. GAD67 mRNA expression was significantly reduced in MPC (by $20 \%$ ), DFC (by $21 \%$ ), NAc-C (by $32 \%$ ) and NAC-S (by $30 \%$ ) of binge eating rats compared to control vehicle treated rats $(p<0.01)$. Administration of $\operatorname{LDX}(0.8 \mathrm{mg} / \mathrm{kg})$ to binge eating rats reversed the observed reduction in GAD67 mRNA expression in MPC (by 10\%), DFC (by 11\%), NAc C (by $18 \%$ ) and NAc-S (by 19\%) $[p<0.05]$.

Conclusions: Conclusions. These results provide the first evidence that the expression of GABA biomarkers (GAD65 and GAD67 mRNAs) is reduced in an animal model of BED compared to control animals. These findings suggest that alterations in GABA neurotransmission in the frontal cortex and nucleus accumbens may contribute to the impulsive behaviors observed in BED patients. LDX-induced normalization of GAD65 and GAD67 mRNAs in same forebrain region may contribute to the beneficial therapeutic effects of LDX in BED patients. (Supported by Shire Pharmaceuticals).

\section{References.}

Heal DJ et al (2016). J Psychopharmacol, 30: 662-75.

Vickers SP et al (2015). J Psychopharmacol, 29: 1290-307.

Vickers SP et al (2017). J Psychopharmacol, 31: 784-797.

Keywords: Binge Eating Disorder, GABA, GAD65 mRNA, GAD67 mRNA

Disclosure: Part 4: Shire, Grant, Sunovion Pharmaceuticals, Grant.

\section{M63. Sex Differences in the Brain's Gray Matter in Cocaine Addiction}

Sivan Kinreich*, Scott Mackey, Rebecca Preston-Campbell, Keren Bachi, Thomas Maloney, Muhammad Parvaz, Hugh Garavan, Nelly Alia-Klein, Rita Goldstein, ENIGMA Addiction Working Group

Icahn School of Medicine at Mount Sinai, New York, New York, United States

Background: Over the last decade, neuroimaging studies investigated the neuroanatomical changes associated with cocaine addiction. However, although national studies show increased drug use rates in women such that $\sim 35 \%$ of cocaine users are women, the majority of this neuroimaging research has been accomplished in males. Studies in women, and those focusing on sex differences, are clearly needed especially given the following statistics indicative of enhanced severity of addiction in women: women display a faster rate of progression to addiction from first cocaine use, earlier treatment seeking, more protracted recovery from abstinence, and higher levels of cue-induced craving. These trends suggest sex differences in the involvement, and alteration of, different brain regions during the addiction/ abstinence processes. A systematic investigation of the neuroanatomical integrity in individuals with cocaine use disorders (iCUD) that takes into account the differences between the genders is lacking.

Methods: Here we used voxel-based morphometry (VBM) to study the differences in gray matter (GM) volumes between female and male iCUD. Structural MRI scans (T1-weighted) were pooled from 8 laboratories including 458 individuals (152 male \& 76 female controls, 152 male \& 78 female iCUD). All participants received a clinical interview for DSM-IV Axis I Disorders to assess for substance dependence and accordingly were divided to four groups matched on age (male control: $36.7 \pm 8.4$, female control: $36.9 \pm 10.2$, male iCUD: $37.9 \pm 6.1$, female iCUD: $39.7 \pm 7.4)$. The groups differed significantly in education (male control: $14.68 \pm 2.6$, female control: $14.2 \pm 1.89$, male iCUD: $12.51 \pm 1.45$, female iCUD: $12.21 \pm 1.4$ ). This data is part of the multi-site ENIGMA Addiction working group, an international effort by PIs worldwide, which gathered researchers in imaging genomics, neurology and psychiatry, to understand brain structure and function in relation to substance use disorders. While scanners and scanning protocols were different at each of the sites, the data was preprocessed similarly using FreeSurfer, a fully automated MRI processing pipeline, and validated by a standardized quality control protocol. Next, the data was analyzed using the SPM-VBM toolbox (CAT12; Gaser, C, University of Jena, Germany) including realignment for the origin (to be at the anterior commissure), segmentation into different tissue classes, resampling (1.5 $\mathrm{mm})$, MNI normalization and smoothing with an $8 \mathrm{~mm}$ kernel. A whole brain ANCOVA analysis (2 groups [control, iCUD] $\times 2$ genders $[\mathrm{M}, \mathrm{F}])$ was used with total intracranial volume, age, education and laboratories $(N=8)$ included in the model as covariates.

Results: Replicating previous reports, current results revealed significant $\mathrm{GM}$ reductions in the anterior prefrontal cortex (BA10) $(t=5.87$, pFWE $<0.000,9104$ voxels $)$, orbitofrontal cortex (BA 47) $(\mathrm{t}=5.08, \mathrm{pFWE}<0.000,2954$ voxels), and supplementary motor area $(\mathrm{t}=4.65, \mathrm{pFWE}<0.000,1009$ voxels) in the iCUD as compared to controls. Importantly, whereas male iCUD compared to male controls showed lower GM in the right amygdala $(t=4.97, \mathrm{pFWE}<0.000,777$ voxels) and dorsal anterior cingulate cortex $(t=4.3$, pFWE $<0.03,135$ voxels), female iCUD compared to female controls showed lower GM in the insula $(t=3.98$, $\mathrm{pFWE}<0.01,550$ voxels $)$ and fusiform cortex $(\mathrm{t}=4.57$, pFWE $<0.000,656$ voxels).

Conclusions: These findings suggest sexually dimorphic changes in the GM reductions reported in the literature in iCUD such that male iCUD showed lower GM compared to male controls in areas related to cognitive and emotional 
control while female iCUD showed lower GM compared to female controls in areas related to interoceptive awareness and face recognition. These results may ultimately help guide the use of different treatment approaches. For example, treatment for male iCUD may emphasize emotion regulation and cognitive processes such as decision-making while treatment for female iCUD may emphasize enhancements in self-awareness.

Keywords: Sexual Dimorphism, Cocaine Addiction, Voxel-Based Morphometry (VBM)

Disclosure: Nothing to Disclose.

\section{M64. Role of Subthalamic Nucleus in Interrupting Behavior}

Katie Fife, Jon Heston, Navarre Gutierrez-Reed, Vivien Zell, Julie Bailly, Christina Lewis, Adam Aron, Thomas Hnasko*

University of California, San Diego, La Jolla, California, United States

Background: Stopping is the neurocognitive process of inhibiting prepotent response tendencies. We stop our behaviors in time of motivational conflict, or in response to new or surprising information, so that we may 'buy time' to decide on the best course of action. Much correlative work has shown that the subthalamic nucleus (STN) is activated in response to stop signals or surprise, but evidence that STN activity is necessary or sufficient for stopping is scant.

Methods: We expressed ChR2 or Halorhodopsin in STN projection neurons. We developed a licking assay to test how activating the STN might influence a recently initiated bout of behavior. We also developed an assay using surprise to interrupt licking, and ask how STN inhibition might interact. We are now developing mouse stop-signal and cognitive assays to extend these results.

Results: Brief activation of the STN is sufficient to interrupt behavior, while STN inhibition is sufficient to blunt interruption caused by surprise.

Conclusions: These data support a circuit model whereby activation of the STN in response to stop signals or unexpected events interrupts behavior - to 'buy time' to consider subsequent action at times of motivational conflict. Future work is aimed at testing this hypothesis using a formal stop-signal assay where we can assess both Going and Stopping in the same assay - and testing how an STN-based stopping system also interrupt cognitive processes.

Keywords: Subthalamic Nucleus, Optogenetics, Stopping Disclosure: Nothing to Disclose.

M65. Distinct Contributions by Different Regions of the Medial Prefrontal Cortex, Nucleus Accumbens and Basolateral Amygdala in Mediating Risk/Reward Decision Making Guided by External Cues

\section{Mieke van Holstein, Maric Tse, Stan Floresco*}

University of British Columbia, Vancouver, Canada

Background: We often face decisions requiring a choice between options that vary in terms of rewards magnitude and uncertainty. In some instances, information about the likelihood of obtaining different rewards are inferred using internally-generated representations, whereas in other instances, external stimuli provide information about the relative amount of reward uncertainty. For example, an experienced Blackjack player knows the odds of winning a hand are larger when the dealer is showing a " 6 " card compared to an "ace". Similarly, studies with human participants often present choices between taking a gamble and choosing a safe alternative, with explicit cues informing participants about reward probabilities prior to the decision. Functional neuroimaging studies using these tasks have revealed a role for the nucleus accumbens (NAc) and distinct parts of the prefrontal cortex (PFC), in particular the ventromedial PFC (vmPFC) and the anterior cingulate cortex (ACC), in risky decision making. Preclinical studies with rodents have provided additional insight in the neural basis of risk/reward decision making, however, many of these studies have used tasks where choice is guided by internally-generated information. An exhaustive series of experiments using probabilistic discounting tasks has revealed critical and dissociable roles for the medial PFC, NAc shell and the basolateral amygdala (BLA) in this form of risk/reward decision making. Yet, how these regions may contribute to decision making guided by external discriminative stimuli has not been explored. To bridge this gap between assays in humans and rats we developed a novel assay we have colloquially termed the "Blackjack" task. Using this task, we assessed the roles of the medial PFC, NAc shell, and BLA in cue-guided risky decision making. Due to their potential homology to the human vmPFC and ACC, we were particularly interested in the roles of the infralimbic (IL) and prelimbic (PL) regions of the rodent medial PFC, respectively.

Methods: Male Long Evans rats were trained on the Blackjack task, consisting of 40 discrete-choice trials. On each trial, they were presented with a choice between a small/ certain ( 1 pellet) and a large/uncertain ( 4 pellet) option. The odds of obtaining the larger reward varied unpredictably from trial to trial between either $50 \%$ or $12.5 \%$. Prior to a choice, one of two tones signaled that the odds of obtaining the larger reward were either good $(50 \%)$ or poor $(12.5 \%)$. After training, rats were implanted with bilateral cannulae either in the NAc shell, BLA, IL, or the PL. After re-training, each animal received two test days: one after the infusion of saline, and one after the infusion of the GABA - A/B agonists muscimol and baclofen.

Results: Under control conditions, well-trained rats selected the large reward option more often when the odds were good vs. poor $(\sim 70 \%$ vs. $25 \%)$. Inactivation of the NAc shell increased risky choice, particularly on trials where cues signaled that the odds were poor. Subsequent studies assessed the involvement of key cortical and subcortical inputs the NAc shell in mediating this form of decision making. Inactivation of the IL increased risky choice in a manner similar to inactivation the NAc shell. Likewise, inactivation of the BLA tended to increase risky choice when the odds were poor. However, this profile was distinct in that it was accompanied by pronounced impairments in the ability to suppress the tendency to be influenced by the rewarded or non-rewarded outcome on the preceding trial. In stark contrast to the above-mentioned findings, 
inactivation of the PL reduced risky choice selectively when the odds were good, suggesting that this region may contribute to the appropriate pursuit of larger/risky rewards. Conclusions: These data reveal that the IL and the NAc shell both suppress inappropriate actions toward larger rewards when cues provide information that these rewards are unlikely. In addition, the IL and PL regions of the medial PFC play opposing roles when using cues to guide decisions associated with uncertainty, similar to the roles the vmPFC and ACC might play in humans. Finally, the BLA might act to suppress inappropriate actions toward larger rewards when cues provide information that these actions are unlikely to yield a reward. Moreover, this nucleus appears to tailor the sensitivity to wins and losses to the odds at hand. Collectively, these data demonstrate how distinct prefrontalsubcortical networks mediate dissociable aspects of risk/ reward decision making, and how dysfunction in BLA-ILNAc shell circuits may lead to impulsive, riskier behaviors. This task may thus play a valuable role in further elucidating the neural bases of normal and abnormal decision making associated with numerous psychiatric disorders.

Keywords: Decision Making, Prefrontal Cortex, Amygdala, Nucleus Accumbens Shell

Disclosure: Part 1: Pfizer, Inc., Grant.

\section{M66. Striatal Adenosine A2A Receptor Regulates Impulsivity, Goal-Directed Alcohol Seeking Behaviors}

Sa-Ik Hong, Phillip Starski, Sun Choi, Alfredo Oliveros, Doo-Sup Choi*

Mayo Clinic College of Medicine, Rochester, Minnesota, United States

Background: Two main types of GABAergic neurons denoted striatonigral and striatopallidal neurons regulate simplified "GO" or "NO-GO" circuits in the dorsal and ventral striatal regions, which may regulate goal-directed behaviors, impulsivity and alcohol-seeking behaviors. However, the precise role of striatal neurons depending on behavioral contexts and influence of other brain regions remains to be examined. The adenosine $\mathrm{A} 2 \mathrm{~A}$ receptor (A2AR) is abundantly expressed in the striatopallidal neurons in the striatum and has been implicated in increased alcohol drinking through enhancement of goal-directed behaviors and impulsivity.

Methods: We investigated whether striatal adenosine A2A receptors are implicated in alcohol drinking, goal-directed behaviors and impulsivity using mice that were exposed to differential reward of low rate (DRL) schedules during Pavlovian conditioning, second-order discrimination, and the 5-choice serial reaction time task (5-CSRTT).

Results: We demonstrate that deficits of A2AR function promote impulsive responses. In a binge-drinking paradigm, mice that were exposed to vaporized ethanol for $4 \mathrm{~h}$ in every 4th day displayed increased premature responses during the challenge tests, suggesting that binge alcohol consumption increase impulsivity through dampening A2AR. Next, we investigated whether the A2AR in the dorsomedial striatum (DMS) contributes to goal-directed behavior in mice exposed to vaporized ethanol for $16 \mathrm{~h}$ per day during 4 days. Our results show that ethanol-exposed mice elicited anxiety-like behavior in elevated plus maze test without affecting working memory in y-maze test. After acquisition of instrumental behavior training in C57BL/6 J mice, both CGS21680 $(0.1 \mathrm{mg} / \mathrm{kg}$, i.p.), A2AR agonist, and optogenetic stimulation of A2AR-expressing neurons in the DMS abolished goaldirected behavior in response to sweetened $10 \%$ ethanol, whereas ZM241385 (20 mg/kg, i.p.), A2AR antagonist, did not impede goal-directed behavior under valued condition. Interestingly, A2AR activation prevented voluntary ethanol consumption in operant chamber even after $24 \mathrm{~h}$ following CGS21680 (0.1 mg/kg, i.p.) injection.

Conclusions: Taken together, our results suggest that the DMS A2AR regulates impulsivity, goal-directed and alcohol seeking behaviors.

Keywords: Impulsivity, Goal-Directed Behaviors, AlcoholSeeking Behavior, Adenosine A2A Receptor, Dorsal Striatum Disclosure: Nothing to Disclose.

\section{M67. An Electronic Observer-Reported Outcomes Instrument (IA Diary) to Measure Impulsive Aggression in Children With Attention-Deficit/Hyperactivity Disorder}

Adelaide Robb, Robert Findling*, Daniel Connor, Steve Hwang, Christopher Evans, Stefan Schwabe, Gianpiera Ceresoli-Borroni

Johns Hopkins University \& Kennedy Krieger Institute, Baltimore, Maryland, United States

Background: For approximately $26 \%$ of children receiving optimized treatment for attention-deficit/hyperactivity disorder (ADHD), accompanying untreated symptoms of impulsive aggression (IA) persist and may complicate treatment and impact functioning.[1,2] In the absence of an existing measurement tool that the FDA found suitable to serve as a primary endpoint in clinical trials for IA, Supernus worked with clinicians and clinical outcomes assessment experts to generate a scale that complies with FDA guidance. [3] A novel measurement tool, the IA diary, was developed to assess behaviors of IA in children optimally treated for ADHD.

1. Jensen PS et al. J Am Acad Child Adolesc Psychiatry. 2007;46:309-22.

2. MTA Cooperative Group. Arch Gen Psychiatry. 1999;56:1073-86.

3. Guidance for Industry. Patient-Reported Outcome Measures: Use in Medical Product Development to Support Labeling Claims. Silver Spring, MD: US Department of Health and Human Services Food and Drug Administration, Center for Drug Evaluation and Research, Center for Biologics Evaluation and Research, Center for Devices and Radiological Health; 2009. Available at: www.fda.gov/downloads/Drugs/GuidanceComplianceRegulatoryInformation/ Guidances/UCM193282.pdf.

Methods: An initial qualitative study of a provisional IA diary was conducted, consisting of semi-structured conceptelicitation interviews followed by a round of debriefing. Interviews with pediatric patients and their caregivers were conducted to qualitatively validate a provisional 30 -item IA diary, which contained behavioral items generated by clinical experts. The sample consisted of children aged 6-12 years, with data obtained from caregivers $(n=23)$ and children 
$(n=23)$. A subsequent psychometric validation was conducted in a multicenter, non-interventional study in which the IA diary was administered over a period of 14 days to parents of children with IA and ADHD. A total of 103 parents of children (ages 6-12 years, inclusive) participated in the psychometric validation study. Among the key inclusion criteria were: a diagnosis of ADHD, as confirmed by the Diagnostic and Statistical Manual of Mental Disorders (DSM-IV-TR or DSM-5); significant signs of IA in the child, as measured by a Retrospective-Modified Overt Aggression Scale (R-MOAS) score of $\geq 20$ and a Vitiello Aggression Scale score of -2 to -5 ; and willingness to complete the electronic diary for 14 days, the paper R-MOAS twice, and the paper Nisonger Child Behavior Rating Form (NCBRFTIQ) and Caregiver Global Impression of Change once. To establish the validity/reliability of the IA diary, analyses included inter-item correlations, exploratory factor analysis (EFA), item-response theory (IRT) modeling, internal consistency, test-retest reliability, concurrent validity (estimated by correlation between the IA diary and the R-MOAS/ NCBRF-TIQ), known-groups methods, and minimal clinically important difference (using Caregiver Global Impression of Change scores).

Results: Qualitative analyses demonstrated that the concepts contained in the provisional 30-item IA diary were supported by the concept-elicitation results obtained from interviews with children and their caregivers. The IA diary then underwent further psychometric testing. Within the psychometric validation study, the prevalence of IA behavior reporting in 15 (50\%) of the items was found to be too low $(<1 \%)$ for analysis. The remaining 15 behavior items had prevalence rates of $2.9 \%-58.3 \%$; items with prevalence rates below $10 \%$ were retained if content validity was deemed high by clinical experts. EFA and IRT models confirmed the existence of two subdomains in the IA diary; these subdomains were subsumed by a general domain of IA behavior frequency, yielding a single total behavioral frequency score (TBFS). Internal consistency was high for this TBFS (marginal reliability $=0.86$ and $\alpha=0.73$ ). Testretest reliability for the TBFS, based on the intra-class correlation coefficient, was 0.8 . Convergent validity of TBFS with $\mathrm{R}-\mathrm{MOAS}$ ranged from $\mathrm{r}=0.49$ to $\mathrm{r}=0.63$. The TBFS precisely characterized highly symptomatic IA patients, those most in need of intervention.

Conclusions: The development of a reliable and valid IA measurement tool that is easily used and captures the intensity of behavior frequency allows clinicians and researchers to accurately monitor changes in IA behavioral event rates. This study is supported by a subsequent qualitative research study in adolescents, and the IA diary is currently being used in pivotal Phase 3 trials, as the FDA found this instrument appropriate for this purpose.

Keywords: ADHD, Impulsiveness, Aggression, Novel Tool, Children and Adolescents

Disclosure: Part 1: Actavis, Alcobra, Cog Cubed, Forest, GlaxoSmithKline, Lundbeck, Medgenics, U.S. National Institutes of Health, Neurim, Novartis, Otsuka, PCORI, Pfizer, Purdue, Rhodes, Roche, Shire, Sunovion, Supernus Pharmaceuticals, Syneurx, Takeda, Validus, Grant, Actavis, Akili, Alcobra, Bracket, Cognition Group, Coronado Biosciences, Elsevier, Epharma Solutions, Genentech, GlaxoSmithKline, Guilford Press, Ironshore, KemPharm, Lundbeck, Merck, U.
S. National Institutes of Health, Otsuka, Pfizer, Physicians Postgraduate Press, Shire, Sunovion, Supernus Pharmaceuticals, Teva, Tris, Validus, WebMD, Consultant, Touchpoint, Advisory Board, American Academy of Child \& Adolescent Psychiatry, Shire, Honoraria, American Psychiatric Press, Johns Hopkins University Press, Sage, Royalties.

\section{M68. An fMRI Study of Cognitive Flexibility in Trichotillomania}

Jon Grant*, Samuel Chamberlain

University of Chicago, Chicago, Illinois, United States

Background: Compulsive hair pulling, referred to as Trichotillomania, is a relatively common psychiatric condition whose neurobiological basis is unknown. Individuals with trichotillomania report being unable to stop their pulling once they have started engaging in the behavior. Abnormalities of flexible responding have been implicated in the pathophysiology of compulsivity, such as in obsessivecompulsive disorder, and thus may be relevant for Trichotillomania. The goal of this study was to probe reversal learning and attentional set-shifting in trichotillomania using a functional neuroimaging task.

Methods: Twelve adults with trichotillomania and 13 matched healthy controls undertook a previously validated functional MRI (fMRI) task of cognitive flexibility (Intradimensional Extradimensional task [IDE] task). The nature of the task's progression allows performance on specific cognitive components to be examined independently. Reinforcement learning involves a subject learning by trial and error feedback, and reversal learning looks at the subject's ability to adapt responses flexibly to a change in learning contingencies. The extra-dimensional shift (EDS) involves a switching of attention between two perceptual dimensions. The processes involved in the IDE task are associated with neural activity of the lateral PFC and the OFC. The EDS stage of the task reflects mainly frontostriatal processing and lateral $\mathrm{PFC}$ activation, whereas reversal learning involves activation of the OFC.

T2*-weighted volumes depicting blood oxygen leveldependent (BOLD) signal were acquired with the first 10 being discarded to avoid T1-equilibrium effects using the following parameters: $\mathrm{TR}=2 \mathrm{~s} ; \mathrm{TE}=30 \mathrm{~ms} ; 32$ axial $3 \mathrm{~mm} 3$ slices; FoV = 192 x 192 mm, 64 x 46 matrix. fMRI recordings were pre-processed and analyzed using SPM12. In brief, whole brain volumes were motion-corrected, slice-time acquisition corrected, co-registered to structural scans and normalized the standard Montreal Neurological Institute (MNI) echo-planar imaging template. Normalised images were smoothed with an $8-\mathrm{mm}$ full-width at half-maximum Gaussian kernel. Subject level contrast estimates were calculated using a General Linear Model (GLM) that included the task timing onsets and durations and motion estimates calculated using the higher-order regression 24parameter model. Neural responses were modelled to multiple conditions of the IDED task. Psychological events were modelled at the 1 st level using GLM. Contrast estimates for each event were elevated to the 2 nd level and entered into a 1-sample t-test to establish group-wise activation maps. A watershed algorithm was applied to each False Discovery 
Rate (FDR) cluster corrected map to derive independent Regions of Interest (ROI) in a data-driven manner. Beta estimates were extracted for each ROI across subjects and compared between groups using 2-sample t-tests, with significance defined as $p<0.05$ uncorrected.

Results: Groups did not differ significantly from each other for reaction times on the different stages of the task. Reversal events evoked the expected patterns insula and parietal regions and activity in the frontal dorsal cortex extending anterior to the frontal poles (FDR cluster corrected, $\mathrm{k}=441$, $p<0.05)$; whereas extra-dimensional shifts evoked the expected frontal dorso-lateral \& parietal pattern of activity (FDR cluster corrected, $\mathrm{k}=2109, p<0.05$ ). For reversal of responses, trichotillomania was associated with significantly increased activity in the right frontal middle gyrus and decreased activity in the left lingual gyrus and right inferior occipital gyrus, compared to controls. For ED shifting, trichotillomania was associated with significantly increased activity in the right frontal middle gyrus and decreased activity in the right occipital middle gyrus during ED switches. Effect sizes for these significant group differences were large by Cohen's D (range $\mathrm{d}=1.2$ to 2.0 ).

Conclusions: This study identified differences in brain activation during flexible responding in patients with trichotillomania, with excess frontal activation coupled with hypoactivation in more posterior brain regions in patients versus controls. Disruption in the balance of frontal versus secondary more posterior circuitry may thus contribute to the pathophysiology of this disorder. Results differ markedly from those observed previously in patients with obsessivecompulsive disorder on the same task. These patterns in a small sample reflect pilot data suggestive of potentially important neural dysfunction associated with trichotillomania.

Keywords: Human Neuroimaging, Obsessive-Compulsive and Related Disorders, Cognitive Neuroscience

Disclosure: Part 1: Takeda, Grant, Brainsway, Grant, Part 4: Takeda, Grant, Brainsway, Grant.

\section{M69. Epigenetic and Transcriptional Changes in the} CYP2D6 Gene are Related to Severity of Suicide Attempt

Jussi Jokinen*, Adrian Boström, Marie Asberg, Helgi Schiöth

\section{Umeå University, Stockholm, Sweden}

Background: Studies of epigenetics and transcriptional activity in suicide attempters may provide knowledge about possible suicide prevention strategies. To our knowledge, this is the first study investigating both epigenomics and transcriptomics in suicide severity in a cohort of suicide attempters.

Methods: The genome-wide methylation pattern was measured in whole blood using the Illumina Infinium Methylation EPIC BeadChip, measuring the methylation state of over $850 \mathrm{~K}$ CpG sites. Prior to analysis, the global DNA methylation pattern was pre-processed according to standard protocols and adjusted for white blood cell type heterogeneity. Subjects were thereafter stratified into one of two groups (high risk or low risk) based on the severity of the suicidal behaviour. Suicide attempts with a violent method, scores greater than 6 points on the Freeman suicide intent scale, and later completed suicide were considered as high risk. On the association analysis between DNA methylation and severity of suicide attempt, we included CpG sites whose methylation levels have been shown by Hannon et al. to have a high correlation between blood and brain. We further limited the analysis to CpG sites located within $2000 \mathrm{bp}$ of the transcriptional start site of the nearest gene, as these have been shown to especially influence transcription of the adjacent gene. A total of 13,191 such CpG-sites were included in the subsequent analysis. We performed multiple linear regression models of methylation $\mathrm{M}$-values to a categorical variable of severity of attempted suicide (highrisk or low-risk), adjusting for optimally determined covariates. As a second step, we made use of open-access data (http://www.ebi.ac.uk/arrayexpress/experiments/EGEOD-24095/) to investigate the expressional profile of candidate genes in matched postmortem brain samples of MDD suicide cases and controls who died of natural causes. Log fold change values were available from 11 matched samples (log2(expression MDD/expression control), sampled from both the dentate gyrus and the CA1 subregions of the hippocampus. Binomial tests were used to investigate whether there was a significant hypo- or hyperexpression of candidate genes in MDD in the respective brain regions. Results: There were significantly more males in the high-risk group $(p<0.01)$. There were no between-group differences in BMI, occurrence of borderline or any other personality disorder, alcohol dependence or substance abuse. Both groups scored equally on the Karolinska Interpersonal Violence Subscales measuring exposure to violent behavior during childhood and adulthood. Cg07016288 - located $163 \mathrm{bp}$ upstream of the transcription start site of the cytochrome P450 2D6 (CYP2D6) gene - was significantly hypomethylated in the high-risk group after corrections were made for multiple testing using the bonferroni method. CYP2D6 was further demonstrated to be significantly hypoexpressed in post-mortem brain samples from the dentate gyrus $(p<10 \mathrm{e}-4)$ of MDD suicide cases compared to controls. In linear regression models performed in healthy controls, methylation levels at cg07016288 in blood were positively correlated to cg07016288 methylation levels in all four different brain regions (prefrontal cortex; $r=0.394$, $p=0.000515 ;$ entorhinal cortex; $r=0.492, p=1.33 \mathrm{e}-05$; superior temporal gyrus; $r=0.474, p=1.76 \mathrm{e}-05$; cerebellum; $r=0.324, p=0.00591$.

Conclusions: CYP2D6 is an enzyme known to metabolize as many as $25 \%$ of commonly prescribed drugs, including antidepressants and antipsychotics. While it is primarily expressed in the liver, CYP2D6 is also highly expressed in areas of the central nervous system. It also metabolizes a plethora of endogenous substances including neurosteroids, and both m-tyramine and p-tyramine which CYP2D6 metabolizes into dopamine in the brain.

Keywords: Epigenetics, Suicide Behavior Severity, Cytochrome P450s

Disclosure: Nothing to Disclose. 
M70. Gene Expression Imputation Across Multiple Brain Regions Reveals Novel Bipolar Genes and Distinct Patterns of Risk Gene Expression Throughout Development

Laura Huckins ${ }^{\star}$, Amanda Dobbyn, Whitney McFadden, Douglas Ruderfer, Gabriel Hoffman, Weiqing Wang, Veera Rajagopal, Thomas Als, Tan-Hoang Nguyen, Panos Roussos, Menachem Fromer, Robin Kramer, Enrico Domencini, Eric Gamazon, Ditte Demontis, Anders Borglum, Bernie Devlin, Solveig Sieberts, Nancy Cox, Hae Kyung Im, Eli Stahl, Pamela Sklar

Icahn School of Medicine at Mount Sinai, New York, New York, United States

Background: Transcriptomic imputation approaches offer an opportunity to test associations between disease and gene expression in otherwise inaccessible tissues, such as brain, by combining eQTL reference panels with large-scale genotype data. These genic associations could elucidate signals in complex GWAS loci and may disentangle the role of different tissues in disease development.

Methods: Here, we use the largest eQTL reference panel for the dorso-lateral pre-frontal cortex (DLPFC), collected by the CommonMind Consortium, to create a set of gene expression predictors and demonstrate their utility. We applied these predictors, and 12 GTEx-derived brain region gene expression predictors, to 21,488 bipolar cases and 54,303 matched controls, constituting the largest transcriptomic imputation study of bipolar disorder (BPD) to date. Additionally, we analysed three specific BPD subtypes, including 14,938 individuals with subtype 1 (BD-I), 3,543 individuals with subtype 2 (BD-II), and 1,500 individuals with schizoaffective subtype (SAB).

Results: We identified 125 gene-tissue associations with BPD, of which 53 are independently associated after FINEMAP analysis. 29/53 associations were novel; i.e., did not lie within $1 \mathrm{Mb}$ of a locus identified in the recent PGC-BD GWAS. We identified 37 independent BD-I gene-tissue associations (10 novel), 2 BD-II associations, and $2 \mathrm{SAB}$ associations. Our $\mathrm{BPD}, \mathrm{BD}-\mathrm{I}$ and BD-II associations were significantly more likely to be differentially expressed in post-mortem brain tissue of BPD, BD-I and BD-II cases than we might expect by chance (no SAB samples were available for analysis).

We identified gene-tissue associations with specific BPDrelated endophenotypes using a PHEWAS-based approach. Finally, we investigated developmental expression patterns for all BPD and subtype associated genes using BRAINSPAN, and identified distinct groups of genes expressed specifically pre-natally or post-natally.

Conclusions: We have used transcriptomic imputation to harness large GWAS sample sizes and yield biologically relevant data about disease architecture. Using 13 gene expression predictors, we identify novel gene-tissue associations for bipolar disorder, and three bipolar disorder subtypes. Identifying associated genes, rather than SNPs or loci, allows us to carry out sophisticated downstream analyses such as tissue-specific PHEWAS and developmental transcriptome analysis.

Keywords: Bipolar Disorder, Transcriptome, Mood Disorder Subtypes

Disclosure: Nothing to Disclose.
M71. 6-Diazo-5-Oxo-I-Norleucine (DON)-Prodrug Normalizes Depression-Like Phenotypes Induced by Social Defeat Stress via Anti-Inflammatory Cascades

Xiaolei Zhu, Michael Nedelcovych, Ajit Thomas, Aisa Moreno-Megui, Wade Coomer, Varun Vohra, Camilo Rojas, Barbara Slusher, Atsushi Kamiya*

Johns Hopkins University School of Medicine, Baltimore, Maryland, United States

Background: There are a number of clinically effective treatments for stress-associated mental disorders, including depression. Nonetheless, a large portion of those afflicted with depression exhibit resistance to first-line treatments (e.g., SSRIs), which calls for novel interventions based on its pathological mechanisms. Alterations in immune and inflammation processes are observed in patients with depression. Consistently, preclinical studies indicate that chronic stress induces peripheral and intracerebral activation of immune and inflammation systems, leading to depression-like behavioral phenotypes. Given that excessive glutamate concentrations play a role in the mechanisms involved in neuroinflammation, we consider glutaminase the enzyme that hydrolyzes glutamine into glutamate - as a potential target protein of drug treatments for depression. Inhibition of glutaminase leads to lower levels of glutamate, thus potentially reducing neuroinflammation. One drug, 6diazo-5-oxo-L-norleucine (DON), is a glutamine analog that acts as a glutaminase inhibitor and has been shown to block glutamate release from microglia. Based on these findings, DON was proposed as a potential drug that could be used to treat diseases that are exacerbated by neuroinflammation, such as multiple sclerosis and cancer; but during early clinical studies, patients reported symptoms of systemic toxicities such as nausea, diarrhea, and neurological toxicities. In response, we have developed DON-prodrug to combat the toxicity of the activated form of DON. In this study, we investigate whether the DON-prodrug can relieve neuroinflammation and ameliorate the depression-like symptoms in a mouse model subjected to social defeat stress. Methods: We examine whether chronic oral-administration of DON-prodrug prevents observed inflammatory changes and depressive behaviors by using the chronic social defeat mouse model. The social interaction test and sucrose preference test will be used to assess the effect of DONprodrug on sociability and anhedonia-like phenotypes, respectively. The pharmacokinetics of DON-prodrug is also assessed in our mouse models along with the drug's effect on inflammatory changes in serum and brain microglia.

Results: We found that chronic treatment of DON-prodrug reversed social avoidance phenotypes induced by social defeat stress. DON-prodrug treated mice subjected to social defeat stress and control mice treated with DON-prodrug have approximately the same and a greater preference for the sucrose solution in comparison with the stressed mice given the vehicle. These finding suggest that $\mathrm{DON}$-prodrug may have antidepressant effects.

Conclusions: We are currently examining whether chronic oral-administration of DON-prodrug may have antidepressant effects by suppression of inflammatory responses caused by social defeat stress via inhibition of glutaminase activity.

S157 
The underlying mechanism of action of DON-prodrug may provide a unique opportunity to uncover glutaminase as a new target for pharmacotherapy of stress-associated psychiatric conditions, including depression and may also accelerate the understanding of pathophysiology of these conditions.

Keywords: Depression, Social Defeat Stress, Inflammation, Glutamine, Antidepressant

Disclosure: Part 1: Taisho Pharma. Co. Ltd., Consultant, Part 2: Taisho Pharma. Co. Ltd., Consultant, Part 4: Astellas Pharma, Inc., Grant, Hitachi, Ltd, Grant.

\section{M72. Aberrant Inhibition in the Dorsolateral Prefrontal Cortex Distinguishes Major Depressive Disorder From the Healthy Brain}

Daphne Voineskos*, Daniel Blumberger, Reza Zomorrodi, Nigel Rogasch, Faranak Farzan, Zafiris J. Daskalakis

\section{Centre for Addiction and Mental Health, Toronto, Canada}

Background: The inhibitory gamma-aminobutyric acid (GABA) system has become a particular focus of investigation in MDD, and GABAergic abnormalities have been found in several investigational modalities, including imaging, pathological studies and neurophysiological studies. Until recently, neurophysiological studies have been limited to the motor cortex, but TMS-EEG affords a window to directly measure evoked activity in the Dorsolateral Prefrontal Cortex (DLPFC), an area of specific interest in MDD. The aim of this study was to examine differences in TEP waveform peaks between healthy individuals and individuals with MDD when TMS-EEG is applied to the DLPFC. We hypothesize that individuals with TRD will have altered TMS-evoked potentials, specifically the N100 and N45, those previously related to GABAergic inhibition. We hypothesize that similar aberrancies may be seen in measures of excitability (ie. the P60 component and the AUC) and therefore may inform a neurophysiological measure of excitation/inhibition.

Methods: 30 Individuals with MDD and 30 age and sexmatched healthy individuals underwent single pulse TMSEEG paradigms to assess cortical inhibition. The TMSevoked potential waveform was examined through Global Mean Field Amplitude (GMFA) analysis of waveform peaks in the two groups.

Results: Individuals in the MDD group demonstrated altered cortical inhibition in the DLPFC. Specifically, larger amplitudes of the N45 $(\mathrm{t}=-4.894, p<0.001)$ and N100 peaks $(t=-3.496, p=0.001)$. As well, the P60 peak was significantly larger in the MDD group than in the group of healthy individuals $(t=-3.260, p=0.002)$. The AUC was significantly larger in the TRD group than in the group of healthy subjects $(t=-3.114, p=0.003)$. There was a positive correlation between N100 amplitude and severity of depressive symptoms in the subjects with MDD (Spearman's rho $=0.420$, $p=0.021)$. In an ROC analysis, N45 amplitude was able to predict illness group with $80 \%$ sensitivity, $73.3 \%$ specificity and $76.6 \%$ accuracy (AUC $=0.829, p<0.001$ ).

Conclusions: Our study provides the first directly measured neurophysiological evidence of altered cortical inhibition and a novel measure of cortical excitation in the DLPFC of individuals with MDD. Specifically, our study demonstrated larger evoked peaks correlating to GABA-A and GABA-B inhibitory activity in the MDD group than in the healthy individuals. It may be that these evoked peaks represent different indices of GABA-A and GABA-B activity in the DLPFC than previously found in the motor cortex.

Keywords: EEG Biomarkers, Major Depressive Disorder (MDD), Cortical Inhibition, Cortical Excitation-Inhibition Balance

Disclosure: Nothing to Disclose.

\section{M73. Oxidative Stress: The Mechanistic Basis of Resilience to Trauma}

Samina Salim, Hesong Liu

University of Houston, Houston, Texas, United States

Background: Negative consequences of early life trauma are well recognized, yet, many aspects remain unclear. For example, not all children who experience traumatic events are at equal risk of developing later life psychiatric disorders. Some are resilient despite being exposed to the same risk factors, while others remain susceptible. The mechanistic basis for resilience is not known. We postulate that oxidative stress is the biochemical sensor which determines presence of resilience or susceptibility. Here, we have utilized the rat single prolonged stress (SPS) model to investigate the role of oxidative stress as the neurobiological basis of resilience.

Methods: Male Sprague-Dawley rats were exposed to SPS on post-natal day (PND) 25. Tests to assess anxiety- and depression-like behavior, as well as learning and memory function were performed at PND32. After conclusion of behavior tests, rats were sacrificed, blood was collected and brains harvested for isolation of pre-frontal cortex (PFC), hippocampus and the amygdala regions of the brain.

Results: We observed that induction of early life stress at PND25 using SPS procedure led to the occurrence of two types of behavioral phenotypes at PND32. One group of rats exhibited anxiety-like behavior (examined using light-dark and elevated plus maze tests), depression-like behavior (assessed using forced swim test) as well as short-term but not long-term learning-memory impairment (examined using radial arm water maze test). The other group did not exhibit any behavioral or cognitive deficits. We refer to the two groups as the susceptible (with deficits) and the resistant (without deficits). Interestingly, the susceptible group exhibited increased neuronal oxidative stress. This correlated with reduced cellular pool of redox sensitive nuclear factor (erythroid-derived 2)-like 2 (Nrf2) transcription factor in the $\mathrm{PFC}$ region but not in the hippocampus or the amygdala. Reduced Nrf2 corresponded with increased levels of Keap-1 and histone deacetylase (HDAC)-2. Low levels of phase II antioxidant enzymes including Manganese $(\mathrm{Mn})$ superoxide dismutase (SOD) and Hemeoxygenase (HO)-1 were noted in this group when compared to control or resistant groups. On the other hand, the resistant group did not exhibit any change in Nrf2, keap1, HDAC2, MnSOD or HO-1 levels.

Conclusions: We conclude that the ability to acquire susceptibility or resistance to early life SPS-induced behavioral and cognitive deficits resides in oxidative-antioxidative 
balance. This balance is maintained by Nrf2. Epigenetic suppression of Nrf2 activation via methylation and histone modification disrupts oxidant/antioxidant balance damaging biochemical integrity of critical brain areas including the PFC and disrupting protective and adaptive mechanisms, causing behavioral and cognitive impairments.

Keywords: Stress, Trauma, Oxidative Stress, Anxiety, Depression

Disclosure: Nothing to Disclose.

\section{M74. The Neuronal Stem Cell Transcriptome of Pre-} menstrual Dysphoric Disorder

Allison Goff*, Allen Meyers, Jessica Hoffman, Cheryl Marietta, Pedro Martinez, Peter Schmidt, David Goldman

National Institutes of Health/National Institute on Alcohol Abuse and Alcoholism, Rockville, Maryland, United States

Background: Premenstrual dysphoric disorder (PMDD) is characterized by recurrent affective and behavioral symptoms during the luteal phase of the normal menstrual cycle. Clinical studies show that in women with PMDD, symptoms recur after re-exposure to physiologic levels of estradiol (E2) or progesterone ( $\mathrm{P} 4)$ during $\mathrm{GnRH}$-agonist-induced ovarian suppression. Intrinsic differences in mRNA and protein expression of the $\mathrm{ESC} / \mathrm{E}(\mathrm{Z})$ complex were recently found by transcriptome analysis of lymphoblastoid cells lines (LCLs) from women with PMDD. Furthermore, women with PMDD show symptom reduction after allopregnanolone (ALLO) stabilization across the menstrual cycle by blocking the conversion of P4 to ALLO with dutasteride. The effects of E2/P4 exposure on LCLs established changes in gene expression that may contribute to PMDD vulnerability, but the LCL model may not be fully informative for neuronal function. Therefore, we sought to determine genes regulated by E2, P4, or ALLO in neuronal stem cells from women with PMDD and asymptomatic controls.

Methods: Neural stem cells (NSCs) were created from induced pluripotent cell (iPSC) lines from women with PMDD and asymptomatic controls $(n=2,2$, respectively; 2 technical replicates per individual). iPSCs were differentiated across 3 passages, exposed to vehicle (DMSO), E2, P4, or ALLO for $24 \mathrm{hrs}$ at $100 \mathrm{nM}$, and examined for differences in gene expression via AmpliSeqRNA transcriptome. Furthermore, qRT-PCR using TaqMan probes was used to confirm that NSCs expressed hormone receptors. Primary data analysis was performed with the AmpliSeqRNA transcriptome plug-in Ion Torrent Suite Software and Partek Flow. We then generated a deep transcriptome representation with a mean read count of 14 million AmpliSeq reads per sample, 195x mean coverage depth, and a mean of $99.5 \%$ aligned reads.

Results: qRT-PCR on NSCs confirmed expression ESR1, ESR2, PGRMC1, PGRMC2, and GPER. Preliminary results based on deep transcriptome representation demonstrate unsupervised hierarchical clustering in PMDD cases vs controls, as well as hormone treatment-specific clustering. Gene expression heat maps show differential expression in PMDD NSCs vs controls, as well as in the presence and absence of each hormone. Pairwise comparisons of each sample show a mean Pearson correlation coefficient of 0.98 . Conclusions: NSCs from women with PMDD vs asymptomatic controls showed differential gene expression both after vehicle treatment and in response to hormone. These data suggest that E2, P4, and ALLO might contribute to differential neuronal development involved in PMDD. Additionally, the Pearson correlation matrix shows a high degree of similarity across all samples (i.e., cases and controls and hormone treatment-specific) NSCs. This suggests cellular homogeneity, signifying that NSCs are a useful model for studying the effects of hormone on neurons.

Keywords: Neural Stem Cell, Premenstrual Dysphoric Disorder, Next Generation Sequencing

Disclosure: Nothing to Disclose.

\section{M75. rTMS Effects in Patients With Co-Morbid Somatic Pain and Depressive Mood Disorders}

\section{Angela Phillips, Robert Burr, David Dunner*}

Center for Anxiety and Depression, Mercer Island, Washington, United States

Background: Pain is a common co-morbidity among clinically depressed individuals and greater pain severity within the depressed population often results in greater treatment resistance. This study investigated a group of patients who were treated with repetitive transcranial magnetic stimulation (rTMS) for treatment resistant depression (TRD) and who were assessed for severity of both depression and somatic pain at baseline and throughout treatment.

Methods: Records of 71 patients treated for TRD with rTMS at the Center for Anxiety and Depression from January 2008 to June 2017 were reviewed. All patients received the standard, FDA approved, high frequency rTMS protocol over the left dorsolateral prefrontal cortex. IRB approval for chart review analysis was obtained from the University of Washington and all patients had signed a written consent form for rTMS which included review of records for research purposes. Data were extracted from patient charts, deidentified using alphanumeric coding and stored in RedCap, an online data collection and storage tool. Primary outcome measures, which included depression severity using the Quick Inventory of Depressive Symptomatology (QIDS) and a 0-10 pain rating, were assessed after every 5 treatments throughout the usual course of 30 treatments. Data were available for 68 patients. All patients had failed at least 2 adequate trials of antidepressant pharmacotherapy prior to being treated with rTMS. Longitudinal QIDS and pain ratings were assessed by rTMS treatment number for each patient, for the entire sample, and for subgroups based on the median split of pain, and for subgroups defined by the QIDS for depression severity. Across-patient correlations of pain and depression were computed at each of the assessment points (every 5 treatments) for the whole sample, and also within the high and low baseline pain and depression severity subgroups. Within and across patient relationships of pain with respect to treatment session, depression with respect to treatment session, and pain with respect to depression were assessed using linear mixed models 
$\mathrm{SI} 60$

including high/low baseline pain and/or depression severity as a group factor. SPSS software package was used to compute univariate (means, SDs, counts, frequencies) and bivariate (correlations, regression coefficients, and categorical model associations) descriptive statistics.

Results: The mean QIDS at baseline was 19.5 (SD =4.9). The median pain score at baseline was 2 with a range of $0-8$. No patient reported pain above 8 at any point during the study. Patient baseline pain scores were dichotomized into two groups at the empirical sample medians (median split): low (pain scores of 0-2) and high (pain scores of 3-8). Pain scores showed a significant, modest correlation with depression ratings at baseline $(r=0.243, p=0.046)$. When dichotomizing patients into the median split pain groups, the low baseline pain group had a nonsignificant correlation with depression scores $(r=0.153, p=.372)$, while the high baseline pain group had a significant correlation with depression scores $(r=0.501, p=.003) .58 .8 \%$ of the sample showed an antidepressant response to rTMS, and $66.7 \%$ of patients with low baseline pain responded whereas $50 \%$ of patients with high pain scores responded. Depression severity by pain group cross tabulation was non-significant (X-square $=$ 2.349; $p=0.503$ ). Two patients in the high pain group showed improvement in depression but not pain. Both patients were early rTMS responders ( $>50 \%$ reduction in QIDS) at treatment 5. One patient in the high pain group showed no improvement of pain or depression. None of the patients demonstrated substantial worsening of pain or depression during treatment. There were no significant differences in depression or pain severity at baseline comparing unipolar with bipolar groups, men vs women, or older vs younger patients.

Conclusions: Half of this sample of patients with TRD had low pain levels at baseline. Worsening of somatic pain during rTMS treatment was unusual and of no clinical significance during rTMS treatment. Our data suggest that improvement in depression and somatic pain in a group of patients with TRD and comorbid somatic pain can be effected with rTMS treatment. It remains unclear if pain response is dependent upon depression response in the high pain group.

Keywords: Repetitive Transcranial Magnetic Stimulation (rTMS), Pain, Depression

Disclosure: Part 1: Neuronetics-I own a NeuroStar rTMS device and the type of disclosure is not pertinent so I chose one, Royalties, Part 2: Neuronetics same as above, Royalties.

M76. Lumateperone Uniquely Enhances Glutamatergic Neurotransmission Through Activation of Both NMDA and AMPA Channels via a Dopamine D1 ReceptorDependent Mechanism: Implications for Treatment of Mood Disorders

Torgny Svensson*, Sophie Dutheil, Joseph Hendrick, Lei Zhang, Lawrence Wennogle, Cedric O'Gorman, Gretchen Snyder, Monica Marcus, Mates Sharon, Kimberly Vanover, Robert Davis

Karolinska Institutet, Stockholm, Sweden

Background: Lumateperone (ITI-007) is an investigational new drug that provides selective and simultaneous modulation of serotonin, dopamine and glutamate neurotransmission. Lumateperone is a potent serotonin 5HT2A receptor antagonist, a dopamine receptor phosphoprotein modulator (DPPM) acting as a presynaptic partial agonist and postsynaptic antagonist at dopamine D2 receptors, a dopamine D1 receptor-dependent indirect modulator of glutamatergic transmission, and a serotonin reuptake inhibitor (Li et al., 2014; Snyder et al., 2015). ITI-007 is in Phase III clinical development as a treatment for schizophrenia, bipolar depression and agitation associated with dementia, including Alzheimer's Disease (Lieberman et al., 2016).

Solid evidence supports the utility of adjunctive treatment with modest doses of antipsychotic drugs (APD) to enhance the effectiveness of antidepressants in patients suffering from treatment-resistant depression (TRD) (Nelson and Papakostas 2009, Tohen et al., 2010). At the molecular level, combined application of antipsychotic drugs with selective serotonin uptake inhibitors (SSRIs) robustly and synergistically increases NMDA mediated currents on pyramidal neurons in the rat mPFC. Further, combined application of an APD plus an SSRI enhances AMPA receptor currents in this brain region - an effect not seen with either treatment alone (Björkholm, et al., 2015, 2017). Interestingly, this enhancement of glutamatergic neurotransmission in $\mathrm{mPFC}$ via both NMDA and AMPA receptors was found to be mimicked by non-anesthetic doses of ketamine, which provides rapid antidepressant efficacy in patients with TRD (Berman et al., 2000). Together, these data may help explain that a combination of an APD with a SSRI may be effective for alleviating TRD. Unique among APDs, lumateperone exhibits both dopamine receptor modulation and SSRI activity in a single compound. Thus, based on the data reported below, lumateperone may represent a new rapid acting treatment for mood disorders.

Methods: We used both intracellular recordings of NMDA and AMPA receptor-activated currents in prefrontal cortical rat slice preparations and biochemical measurements of protein phosphorylation in the mTOR signaling pathway in rat $\mathrm{MPFC}$ after exposure to lumateperone.

Results: Experimental results support antidepressant activity for lumateperone. Unique among APDs, lumateperone is a potent SSRI. In receptor binding studies using $3 \mathrm{H}-$ Imipramine binding to recombinant human serotonin reuptake transporter expressed in $\mathrm{CHO}$ cells, lumateperone exhibited a $\mathrm{Ki}$ of $26 \mathrm{nM}$ (imipramine standard $\mathrm{Ki}$ was $10 \mathrm{nM}$ ). This activity is more potent than any currently approved antipsychotic agent, most of which lack activity as SSRIs. Moreover, lumateperone was active in the mouse social defeat model of antidepressant activity and significantly reduced symptoms of depression in schizophrenic patients with comorbid depression.

Using intracellular recordings of NMDA and AMPA receptor-activated currents in prefrontal cortical rat slice preparations, lumateperone was shown to amplify both NMDA and AMPA induced currents. NMDA-current amplification can thus be induced by co-administration of an antipsychotic agent (D2R antagonist) plus a potent antidepressant (SERT inhibitor)1, or by lumateperone alone. Potentiation of AMPA currents has not been shown by any other APD making this a unique finding for lumateperone. These effects of lumateperone were blocked by the D1 receptor antagonist, SCH233390. Additionally, like ketamine, 
lumateperone $(3.0 \mathrm{mg} / \mathrm{kg})$ rapidly activated the mTOR signaling pathway in rat $\mathrm{mPFC}$.

Conclusions: Like other rapid acting antidepressants such as ketamine, lumateperone enhances both NMDA and AMPAinduced currents in rat mPFC pyramidal neurons via activation of D1 receptors, and increases phosphorylation of key proteins of the mTOR signaling pathway. We thus hypothesize that lumateperone achieves enhanced glutamatergic neurotransmission in the $\mathrm{mPFC}$, owing to multiple pharmacological properties, including its uniquely potent activity (among antipsychotic medications) as a SERT inhibitor and its ability to enhance dopamine neurotransmission via D1 receptors.

Lumateperone has been shown to improve symptoms of depression in patients with acute schizophrenia (Lieberman et al., 2016) and also in stable schizophrenic patients switched to lumateperone from other antipsychotics (Vanover et al. ACNP 2017). Lumateperone is currently in Phase III clinical trials in patients with bipolar depression as well as in Phase III development for the treatment of schizophrenia and for agitation associated with dementia, including Alzheimer's Disease. Based on these data, lumateperone may be useful as a single, stand-alone, orally-available, rapidacting treatment for depression, lacking the adverse side effects of ketamine and other current pharmacological approaches.

Keywords: Lumateperone, NMDA, AMPA, Glutamate, Depression

Disclosure: Part 1: Swedish Brain Foundation and Research Council, Grant, AstraZeneca, Grant, Otsuka, Grant, Astellas, Grant, Lundbeck A/S, Grant, Intra-Cellular Therapies, Inc., Grant, CDRD Berolina AB, Grant, Lundbeck A/S, Honoraria, AstraZeneca, Honoraria, Otsuka, Honoraria, CDRD Berolina AB, Honoraria, The Carnegie Health Care Funds, Honoraria.

\section{M77. Antidepressant Effects of Ketamine and Buprenorphine in a Rodent Model of LPS-Induced Depression}

Caroline Browne*, Bruno Carabelli, Aylar Berenji Kalkhoran, Kaitlyn Gouty, Sahana Aiyer, Irwin Lucki

Uniformed Services University of the Health Sciences, Bethesda, Maryland, United States

Background: Activation of the immune system is implicated in the development of Major Depressive Disorder (MDD). $30 \%$ of patients treated with pro-inflammatory cytokines for cancer, multiple sclerosis and other illnesses develop clinical depression. Moreover, serum levels of inflammatory cytokines such as TNFa and IL-6 are higher in subjects with MDD, and anti-inflammatory compounds have been shown to alleviate depressive symptoms. The purpose of these studies was to determine whether novel fast-acting antidepressants reverse depressive-like behavior produced by an immune challenge. Systemic administration of lipopolysaccharide (LPS, the endotoxic component of the outer cell wall of gram negative bacteria), induces time-dependent behavioral alterations in sickness behavior peaking 6-8 h post administration and depressive-like behavior which peaks approximately $24 \mathrm{~h}$ following exposure. In this study, we investigated the effects of ketamine and buprenorphine. These are two putative non-monoaminergic antidepressants currently being studied in clinical trials for treatmentresistant MDD.

Methods: All studies were approved by the Institutional Animal Care and Use Committee for the Uniformed Services University of the Health Sciences. Male C57BL/6 J mice ( 8 weeks, Jackson Laboratories, Bar Harbor, ME), were singly housed 24 hours prior to the onset of study. Buprenorphine hydrochloride $(0.25 \mathrm{mg} / \mathrm{kg}$; Tocris $)$ was prepared in vehicle (sterile molecular grade deionized water) and ketamine hydrochloride (1, 3 and $10 \mathrm{mg} / \mathrm{kg}$; Ketalar, Mylan Industries,) was dissolved in sterile saline (Hospira Inc.). All compounds were given by intraperitoneal (IP) injection $1 \mathrm{~h}$ prior to immune challenge. LPS (Escherichia coli, O111:B4, Sigma Aldrich, Catalogue \# L2630) was dissolved in saline and administered a dose of $0.83 \mathrm{mg} / \mathrm{Kg}$. For all injections, the injection volume was $10 \mathrm{ml} / \mathrm{kg}$. Sickness behavior, temperature, nesting and kaolin intake was evaluated throughout the first 8 hours following immune challenge. Motor activity was assessed using an open field $24 \mathrm{~h}$ post LPS. Two hours later mice were exposed to the forced swim test (FST) to evaluate depressive-like behavior. Mice were euthanized $2.5 \mathrm{~h}$ post FST, when blood and tissue were collected for investigation. Results: 1) In both experiments. LPS induced a peak of sickness behavior $8 \mathrm{H}$ post injection, which was accompanied by hypothermia, poor nesting and increased intake of kaolin versus chow (pica). 2) Ketamine produced significant interactions for kaolin intake $(\mathrm{F}(1,36)=23.4, p<0.001)$ and sickness behavior $(\mathrm{F}(3,88)=3.84, p=0.012)$, where ketamine $(1 \mathrm{mg} / \mathrm{kg} p<0.05$, and $10 \mathrm{mg} / \mathrm{kg} p<0.01)$ attenuated the effects of LPS. Ketamine was ineffective at modifying the hypothermia and nesting behavior responses. 3) Significant buprenorphine ${ }^{\star}$ LPS interactions were measured for sickness behavior $(\mathrm{F}(1,36)=5.384, p=0.026)$ and temperature, $(\mathrm{F}(6,81)=4.074, p=0.001)$ only, where buprenorphine diminished both responses. 4) In the open field, LPS treated mice spent more time exploring the periphery than the center region of the arena. This behavior was not modified by either compound. 5) In the FST, a significant LPS ${ }^{\star}$ ketamine interaction was measured on immobility $\mathrm{F}(3,79)=4.416, p=0.006$, where ketamine $(10 \mathrm{mg} / \mathrm{kg}, p<0.05)$ diminished the time spent immobile in LPS-treated mice. 6) Similarly, a significant LPS*buprenorphine interaction was observed on immobility $\mathrm{F}(1,37)$ $=4.693, p=0.038$, where buprenorphine significantly reduced immobility in LPS-treated mice $(p<0.05) .7)$ Levels of pERK in the hippocampus remained elevated at $28 \mathrm{~h}$ post LPS treatment. Both ketamine $(10 \mathrm{mg} / \mathrm{kg})$ and buprenorphine normalized these levels.

Conclusions: Ketamine and buprenorphine are two compounds that have already been shown to benefit patients with severe and refractory depression. Here, our data suggest that these compounds also have activity in a model of immune mediated depression. Moreover, these data suggest that despite the different mode of action of buprenorphine and ketamine, both compounds are capable of regulating the aberrant molecular alterations induced by stress and immune dysfunction.

Keywords: Buprenorphine, Ketamine, LPS

Disclosure: Nothing to Disclose. 
M78. Preliminary Analysis of Resting State Functional Connectivity in Young Adults With Bipolar Disorder: Comparison of Subtypes and Relation With Symptom Measures

Sarah Thomas*, Rachel Christensen, Amanda Ruggieri, Lauren Kasoff, Kerri Kim, Steven Giavasis, Michael Milham, Daniel Dickstein

Alpert Medical School, Brown University, East Providence, Rhode Island, United States

Background: Greater knowledge of neural mechanisms underlying bipolar disorder $(\mathrm{BD})$ is crucial to improve diagnosis and treatment of $\mathrm{BD}$ - the most expensive mental health disorder including direct healthcare costs and lost productivity. BD is also linked to high rates of substance abuse and suicidal behavior despite our best current treatments. Towards this goal, resting state functional connectivity (RSFC) provides an opportunity to see how different expressions of $\mathrm{BD}$, including those with distinct episodes of mania or hypomania (BD type I and II, respectively) differ from those with a more sub-syndromal course (BD "not otherwise specified" [BD-NOS]) and from healthy controls (HC) without psychopathology. Whole-brain RSFC methods include Regional Homogeneity (ReHo; Zang et al., 2004) that identifies clusters of voxels that are similarly active to or synchronized with one another, based on the time series of those voxels, and also amplitude of low-frequency fluctuations (ALFF), measuring slower activity oscillations between the frequency of 0.01 and $0.1 \mathrm{~Hz}$, typically within grey matter (Yu-Feng et al., 2007). While ReHo and ALFF have been used separately in studies of BD adults (Liu et al., 2012, 2013), no studies have investigated these metrics within different clinical presentations of $\mathrm{BD}$, using both methods in combination. Therefore, we sought to use both ReHo and ALFF as complementary techniques to compare RSFC alterations in BD-I/II vs. BD-NOS participants vs. HCs. We hypothesized that BD-I/II and BD-NOS differ from $\mathrm{HC}$ and each other, and that RSFC indices would be differentially related to psychiatric symptom measures.

Methods: After informed consent, we enrolled two groups of young adults (ages 17-30): 1) those with either BD-I/II or BDNOS followed since childhood by Brown's site of the COBY study; 2) HCs without lifetime psychopathology history. All participants completed the Structured Clinical Interview for DSM-IV. BD participants completed the Beck Depression Inventory, Hamilton Depression and Anxiety Rating Scales (HAM-D \& HAM-A, respectively), Young Mania Rating Scale, and received a Global Assessment of Functioning (GAF) score. Participants underwent structural (MPRAGE, $1 \mathrm{~mm}, \mathrm{TR}=$ 2300) and RSFC scans ( $3 \mathrm{~mm}, 256$ volumes) on a Siemens $3 \mathrm{~T}$ Tim Trio. Image analysis used the Configurable Pipeline for the Analysis of Connectomes (CPAC; Craddock et al., 2013). CPAC RSFC processing includes slice timing correction, motion correction (excluding $>2$ standard deviations of Power framewise displacement), skull stripping, and normalization. The sample consisted of 109 young adults; however, during preprocessing, 5 participants were excluded for excessive motion and 11 participants did not have a smoothed ReHo or ALFF image. Thus, the final sample was 93 participants, divided into 3 groups for the purpose of analyses (BD-I/II, BD-NOS, and HC). ReHo uses Kendall's coefficient of concordance for each voxel to determine time series similarity within clusters of 27 neighboring voxels, which are then standardized with $\mathrm{z}$-scores during group analysis. ALFF, calculated in native space, is derived by converting voxels' time series frequency to the power domain, summing amplitudes between frequencies of 0.01 and $0.1 \mathrm{~Hz}$, converting amplitudes to $\mathrm{z}$-scores for individual-level spatial maps, and then transforming spatial maps to MNI standard space (Craddock et al., 2013). For both ReHo and ALFF, significant clusters were those that survived thresholding of $\mathrm{z}=3.1$, $p=0.05$ using spatially smoothed images (4-mm FWHM Gaussian kernel) in a GLM. Covariates were age, sex, full scale IQ, and head motion parameters.

Results: The BD-I/II group had significantly greater ReHo values than the BD-NOS group in the precuneus and L \& R cerebral cortex. BD-NOS participants had significantly decreased ReHo compared to HCs in the R cerebral cortex, superior frontal gyrus, and paracingulate gyrus, plus a cluster with the $\mathrm{L} \& \mathrm{R}$ cerebral cortex and intracalcarine cortex. Among BD-I/II participants, greater ReHo values were inversely associated with mania $(r=-0.48, p=0.01)$ and depression $(r=-0.50, p=0.007)$. For BD-NOS participants, ReHo values extracted from the cluster including the superior frontal gyrus, $\mathrm{R}$ cerebral cortex, and paracingulate gyrus were associated with the GAF score $(r=0.59, p=0.03)$. The BD-I/II group had significantly greater ALFF values relative to the $\mathrm{BD}$ NOS group in the occipital pole and L \& R cerebral cortex, plus a cluster in the frontal operculum cortex, L cerebral cortex, insular cortex, and inferior frontal gyrus (pars triangularis). BD-NOS participants had significantly decreased ALFF compared to HCs in the frontal pole and L cerebral cortex. The BD-I/II group had significantly decreased ALFF relative to $\mathrm{HCs}$ in $\mathrm{R}$ cerebral white matter. Among BD-NOS participants, decreased ALFF values compared to HCs were significantly associated with HAM-A $(r=-0.57, p=0.05)$ and HAM-D symptoms $(r=-0.61, p=0.03)$.

Conclusions: Findings from RSFC analyses across groups of BD-I/II, BD-NOS, and HCs indicate group-related ReHo and ALFF differences, which in turn are also related to BDrelated symptoms. Significant clusters were in brain regions also identified in other studies of ReHo and ALFF in BD, including the insula and regions central to the default mode network (precuneus). Importantly, these findings advance the scientific field by demonstrating how BD without distinct episodes has pathophysiology distinct from HCs and BD-I/II. Keywords: Bipolar Disorder, Human Neuroimaging, Resting State Functional Connectivity, Young Adults Disclosure: Nothing to Disclose.

M79. A Phase 2, Double-Blind, Placebo-Controlled Study of NSI-189 Phosphate, a Neurogenic Compound Among Out-Patients With Major Depressive Disorder

George Papakostas*, Karl Johe, Bettina Hoeppner, Marlene Freeman, Martina Flynn, Holly Hand, Rutendo Kashambwa, Maurizio Fava

Massachusetts General Hospital, Boston, Massachusetts, United States

Background: NSI-189, a benzylpiperizine-aminopyridine, is a novel compound being developed for the treatment of 
major depressive disorder (MDD), based on preclinical evidence of neurogenesis in human hippocampus-derived neural stem cells in vitro and in mouse hippocampus in vivo, as well as promising results from a double-blind, placebocontrolled phase Ib study in MDD in-patients. The goal of the present study was to evaluate the efficacy, safety and tolerability of two different doses of oral NSI-189 as monotherapy in out-patients with MDD. For this purpose, the sequential-parallel comparison design (SPCD) was chosen, which was masked for both subjects and raters.

Methods: 220 out-patients with recurrent MDD were randomized in a double-blind fashion to one of three treatment groups (1:1:3 probability): NSI-189 $40 \mathrm{mg}$ daily $(n=43)$, NSI- $80 \mathrm{mg}$ daily $(n=43)$, and placebo $(n=132)$ for 6 weeks (stage 1). At the end of 6 weeks, placebo-treated subjects who were non-responders (defined as subjects with less than a 50\% reduction from baseline in the clinician-rated Montgomery-Asberg Depression Rating Scale (MADRS) score and a score of 16 or greater on the MADRS) were re-randomized (1:1:1 probability), in a double-blind fashion to 6 weeks of additional treatment with NSI-189 $40 \mathrm{mg}$ daily $(n=22)$, NSI-80 mg daily $(n=22)$, or placebo $(n=22)$ (stage 2). Patients who completed 6 weeks of treatment on any dose of NSI-189 continued the same dose for an additional 6 weeks (stage 2). The primary outcome measure for the study was the MADRS. Other outcome measures included the clinician-rated 17-item Hamilton Depression Rating Scale (HAMD-17), the patient-rated Symptoms of Depression Questionnaire (SDQ), the patient-rated Cognitive and Physical Functioning Scale (CPFQ), and the patient-rated version of the Quick Inventory of Depressive Symptomatology Scale (QIDS-SR).

Results: 220 out-patients with recurrent MDD were randomized in a double-blind fashion to one of three treatment groups (1:1:3 probability): NSI-189 $40 \mathrm{mg}$ daily $(n=44)$, NSI-80 mg daily $(n=44)$, and placebo $(n=132)$ for 6 weeks (stage 1). At the end of 6 weeks, placebo-treated subjects who were non-responders (defined as subjects with less than a $50 \%$ reduction from baseline in the clinician-rated Montgomery-Asberg Depression Rating Scale (MADRS) score and a score of 16 or greater on the MADRS) were re-randomized (1:1:1 probability), in a double-blind fashion to 6 weeks of additional treatment with NSI-189 $40 \mathrm{mg}$ daily $(n=22)$, NSI-80 mg daily $(n=22)$, or placebo $(n=22)$ (stage 2). Patients who completed 6 weeks of treatment on any dose of NSI-189 continued the same dose for an additional 6 weeks (stage 2). The primary outcome measure for the study was the MADRS. Other outcome measures included the clinician-rated 17-item Hamilton Depression Rating Scale (HAMD-17), the patient-rated Symptoms of Depression Questionnaire (SDQ), the patient-rated Cognitive and Physical Functioning Scale (CPFQ), and the patient-rated version of the Quick Inventory of Depressive Symptomatology Scale (QIDS-SR).

Conclusions: Although this placebo-controlled, SPCD, phase II study of NSI-189 failed to show a statistically significant advantage over placebo on the primary outcome measure and on the other clinician-rated secondary measure of depression, all three self-rated measures of depression and cognition showed significant advantages of NSI-189 over placebo. These results warrant further evaluation of the antidepressant and pro-cognitive effects of this compound.
Keywords: Cognition, Major Depressive Disorder (MDD), Novel Therapeutics, Randomized, Clinical Trial

Disclosure: Part 1: Neuralstem, Inc., Grant.

\section{M80. Novel Transcriptome-Based Polygenic Risk Score for Depression is Associated With Reduced DLPFC Function and Stress-Related Depressive Symptoms}

Yuliya Nikolova*, Arin Bakht, Leon French, Ahmad Hariri, Etienne Sibille

\section{Centre for Addiction and Mental Health, Toronto,} Canada

Background: Neuroimaging studies have associated major depressive disorder (MDD) with dysfunction of the dorsolateral prefrontal cortex (dlPFC), which may reflect reduced top-down regulation of emotion and behavior. Parallel work in human postmortem brain has identified depressionrelated transcriptomic changes in the dlPFC, but the functional impact of these changes is unknown. To bridge these lines of research, we converted transcriptomic changes into a novel polygenic risk score (PRS), using common gene expression-altering SNPs, and mapped it onto MRI-assessed dlPFC function and self-reported depressive symptoms.

Methods: Case-control comparisons in postmortem dlPFC (Brodmann area 9; BA9) expression levels of 10,680 gene transcripts were conducted between patients with depression and age- and gender-matched controls $(n=14$ matched pairs). The PrediXcan tool with cis-eQTL SNPs from the Genotype-Tissue Expression (GTEx) project BA9 tissue was then used to "impute" dlPFC expression of 1,805 of these transcripts in participants of the Duke Neurogenetics Study (DNS; $n=276,156$ women, mean age 19.86 \pm 1.25 ). Using weights reflecting the direction and magnitude of depression-related change in gene transcript levels from the postmortem case-control comparisons, we summed imputed gene expression values into a weighted PRS capturing subtle shifts toward, or away from, a depression-like dlPFC transcriptome. As male- and female-specific weights were markedly different, separate male- and female-pattern PRS values (PRS-M and PRS-F, respectively) were generated. These scores were then mapped onto dlPFC activity in a working memory fMRI task, as well as self-reported depressive symptoms.

Results: Higher PRS-M was associated with lower left dlPFC activity in the combined male and female cohort, but only in Non-Hispanic Caucasians $(b=-0.194, p=0.022)$. The effect was strongly driven by males $(b=-0.307, p=0.028)$, rather than females $(b=-0.127, p=0.233)$. In the combined cohort, lower dlPFC function was in turn associated with increased depressive symptoms across ethnicities, but only in those reporting higher levels of recent life stress (dlPFC $\mathrm{x}$ stress interaction: $\mathrm{b}=-0.147, p=0.020$; dlPFC effect at stress $1 \mathrm{SD}$ above mean: $\mathrm{b}=-0.240, p=0.006)$. Furthermore, dlPFC activity mediated an indirect positive association between PRS-M and stress-related depressive symptoms $(b=0.046$, 95\% CI: 0.007-0.133). PRS-F did not correlate with dlPFC function or depressive symptoms (all $p>0.20$ ).

Conclusions: Our results suggest that, in Caucasian males, common genetic variants capturing subtle transcriptomic shifts toward a depression-like molecular phenotype in the 
dlPFC may reduce working memory-related dlPFC activity, which may in turn predispose to heightened depressive symptoms particularly in the context of stress.

Keywords: Depression, DLPFC, Human Genetics, Stress Reactivity

Disclosure: Nothing to Disclose.

M81. Convergence of Active and Passive Assessments of Affective Instability in Predicting the Prospective Course of Bipolar Disorder: The BiAffect Study

Jonathan Stange, John Zulueta, Scott Langenecker, Kelly Ryan, Andrea Piscitello, Olusola Ajilore, Jennifer Duffecy, Melvin McInnis, Peter Nelson, Alex Leow*

University of Illinois at Chicago, Chicago, Illinois, United States

Background: The recent development of technological innovations has opened new frontiers in the active and passive assessment of risk factors in mood disorders. One technique that can be administered via smartphone is ecological momentary assessment (EMA), which has important potential for the identification of periods of risk and for translation to real-time interventions. EMA can be used to assess variability in actively reported indices such as negative affect, as well as fluctuations in passively recorded metrics such as keyboard typing speed. However, little work, if at all, has studied changes in general typing kinematics, a novel category of "digital phenotyping" data streams, in the context of bipolar disorder. We examined whether day-today instability (large, frequent fluctuations) in self-reported indices of negative affect, as well as instability in typing speed, would be associated with a poorer prospective symptom course among individuals with bipolar disorder.

Methods: As part of the BiAffect-PRIORI consortium, 18 members of the Prechter Longitudinal Study of Bipolar Disorder participated in a naturalistic, longitudinal study. Subjects used BiAffect-enabled Android smartphones (Samsung Note 3) and completed a 7-10 day baseline EMA period during which they were prompted, once daily, to rate their mood, energy, rapidity of thinking, and impulsive feelings and actions. Simultaneously, BiAffect unobtrusively collected typing kinematics metadata (how a person typed instead of what was typed) as subjects interacted with their phones as usual, allowing us to extract average typing speed (time since last key) within each day in their natural environments (i.e., in the real world) across the EMA period. Instability metrics were created for each rated affective word and for daily typing speed, using the root mean square successive difference (rMSSD). Subjects also completed the Hamilton Depression Rating Scale and Young Mania Rating Scale at baseline (before EMA) and weekly for 6-8 weeks after the EMA period, yielding 120 separate mood-rating observations across the prospective period. Multilevel modeling examined whether individuals with greater baseline instability (day-today variability) in affect and typing speed over the EMA period would exhibit elevated trajectories of mood symptoms over time. To conduct a conservative test of hypotheses, we examined the influence of instability metrics on prospective mood symptoms after covarying the influence of baseline mood symptoms and each person's average levels of affect and typing speed.

Results: Greater instability of mood during the baseline EMA period significantly predicted elevated prospective symptoms of depression $(\mathrm{t}=2.91, \mathrm{p}<.005)$ and mania $(t=2.72, p<.01)$ across the prospective period, beyond the influence of baseline symptoms and average mood during EMA. Instability of energy predicted elevated prospective symptoms of mania $(\mathrm{t}=2.20, \mathrm{p}=.03)$, but not depression $(t=1.78, p=.08)$. Furthermore, instability of typing speed predicted elevated symptoms of depression $(t=2.18, p$ $=.03)$, but not mania $(\mathrm{t}=0.73, \mathrm{p}=.46)$. Instability of typing speed was positively but not significantly correlated with indices of affective instability ( $\mathrm{rs}=.12-.37$ ), suggesting unique contributions of actively- and passively-assessed metrics of instability to the prediction of illness course.

Conclusions: These results support the utility of using active and passive indices of affective and motor instability in the prediction of future mood symptoms among adults with bipolar disorder. These findings suggest that the use of EMA and related smartphone technologies may be useful for identifying patients who may be in need of more intensive treatments, or even as early markers of phase shift in bipolar disorder. Future work might examine how such metrics of instability can be used as part of a person-centered approach to identifying periods of elevated risk for recurrence.

Keywords: Ecological Momentary Assessment, SmartphoneBased App, Bipolar Disorder, Neuropsychology, Emotion Disclosure: Nothing to Disclose.

\section{M82. Plasma Serotonin and Kynurenine Pathway Metabolite Levels in Depression Patients Treated With Escitalopram and Desvenlafaxine}

Yu Sun*, Wayne Drevets, Gustavo Turecki, Qingqin Li

Janssen Pharmaceutical, Pennington, New Jersey, United States

Background: Previous studies have shown that levels of serotonin and metabolites in kynurenine pathways are affected by antidepressant treatments. Among the metabolites in kynurenine pathways, kynurenic acid (KynA) is hypothesized to be neuroprotective, while 3hydroxykynurenine $(3 \mathrm{HK})$ and quinolinic acid (QuinA) are hypothesized to be neurotoxic. In addition, the KynA/QuinA and $\mathrm{KynA} / 3 \mathrm{HK}$ ratios were proposed to be a putative neuroprotective index. Kynurenine (Kyn)/tryptophan (Trp) ratio is thought to be a proxy measure of indoleamine 2,3dioxygenase (IDO) activity, a heme enzyme that catalyzes the first and rate-limiting step in tryptophan catabolism to $\mathrm{N}$-formyl-kynurenine. The increases in the Kyn/Trp ratio arise from pro-inflammatory cytokine driven IDO and stress/cortisol driven tryptophan-2,3-dioxygenase (TDO), which drive Trp down the Kyn pathway and away from serotonin (5HT) and melatonin synthesis. Therefore, reduced Trp, increased Kyn and Kyn/Trp ratio is suggestive of higher inflammation state. Here we investigate the relationship between baseline levels of plasma serotonin and kynurenine metabolites and treatment outcome, as well as treatment-elicit changes in serotonin and kynurenine pathway. 
Methods: The study dataset contains 28 healthy controls and 161 MDD patients in two treatment arms (78 treated with escitalopram; 83 treated with desvenlafaxine). HAMD-21 and plasma samples were collected at baseline before the treatment and 8 weeks after treatment. Plasma levels of serotonin (5HT), Trp, Kyn, KynA, 3HK and QuinA were measured using liquid chromatography-mass spectrometry. Responders to antidepressant treatment are defined as MDD patients with $\geq 50 \%$ reduction from baseline in HAMD-21 score at week 8 . Remitters are defined as MDD patients whose week 8 HAMD-21 score are $\leq 7$. We used general linear model to compare baseline biomarker levels between patients with different treatment responses correcting for age, gender and measurement batch. Changes in serotonin and kynurenine pathway after antidepressant treatments were compared using mixed models with repeated measurements (MMRM).

Results: Baseline plasma levels of serotonin were significantly lower in the MDD patients than in healthy controls $(p=0.0043)$. No significant differences in levels of kynurenine pathway metabolites were observed between healthy controls and MDD patients at baseline. Baseline disease severity as measured by HAMD-21 score is significantly lower in remitters than in non-remitters in all MDD patients ( $\mathrm{p}<0.0001)$, and in both treatment arms $(\mathrm{p}=0.0014$ in escitalopram arm and $p=0.0061$ in desvenlafaxine arm). No other baseline markers showed significant difference between remitters and non-remitters. Baseline HAMD-21 score is also significantly lower in responders in all MDD patients $(p=0.0215)$, but not significant in either treatment arm. Escitalopram responders had higher baseline serotonin levels $(p=0.0226)$ and lower Kyn/Trp ratios $(p=0.0132)$ than non-responders. No baseline markers showed significant difference between desvenlafaxine responders and non-responders. After treatments, serotonin levels were further reduced in both treatment arms $(p<0.0001)$ but escitalopram arm showed more reduction $(\mathrm{p}=0.0013)$. Both treatments reduced the plasma serotonin levels irresponsive of the response status. No ratios of kynurenine metabolites showed significant change after antidepressant treatment in either arm. In escitalopram treated patients, $\mathrm{KynA} / 3 \mathrm{HK}$ ratios are significantly increased after treatment $(p=0.0118)$ only in the remitters. No ratios of kynurenine metabolites were significantly different between responders and nonresponders in either arm.

Conclusions: Current data support previous observations that lower baseline severity predicts better antidepressant treatment outcomes. Lower baseline Kyn/Trp ratio, which reflects a lower inflammation state, and higher baseline serotonin levels may be also serve as predictors for response to escitalopram. Furthermore, remitters to escitalopram treatment showed putatively beneficiary changes in kynurenine metabolite ratios than non-remitters, suggesting that in these patients, escitalopram treatment may help turn the kynurenine pathway in a neuroprotective direction. Additional studies are needed to validate these findings.

Keywords: Major Depressive Disorder (MDD), Kynurenine Pathway, Serotonin, Escitalopram, Desvenlafaxine

Disclosure: Part 1: Janssen Research \& Development, LLC, Employee, Stock / Equity, Part 2: Janssen Research \& Development, LLC, Employee, Stock / Equity, Part 3: Janssen Research \& Development, LLC, Employee, Part 4: Janssen
Research \& Development, LLC, Employee, Stock / Equity, Part 5: Janssen Research \& Development, LLC, Employee.

\section{M83. A Behavioral Measure of Probabilistic Reward Learning Predicts Clinical Response to Pramipexole in Individuals With Major Depressive Disorder}

Alexis Whitton*, Jenna Reinen, Mark Slifstein, Patrick McGrath, Dan Iosifescu, Anissa Abi-Dargham, Diego Pizzagalli, Franklin Schneier

\section{Harvard Medical School, Belmont, Massachusetts,} United States

Background: Studies linking major depressive disorder (MDD) to dysfunction in the mesolimbic dopamine (DA) reward system have suggested that agents that enhance DA neurotransmission may be useful for treating depression. In particular, DA agonists such as pramipexole (PPX) - a D3preferring DA agonist - may be especially useful for treating depressed individuals who present with reward-related deficits, such as anhedonia. Preliminary support for this notion comes from preclinical studies showing that chronic treatment with PPX causes an increased expression of D3 receptors and tonic $\mathrm{DA}$ in the striatum, which in turn increases incentive salience for rewards. In humans, PPX has also shown efficacy in treatment of MDD, however no studies to date have linked these effects to the neurobiology of MDD. Importantly, it remains unknown whether measures of reward circuit functioning may be used to identify depressed individuals who may respond most favorably to a DA agonist. The current study aimed to evaluate whether reward circuit function, measured using an objective behavioral measure of reward learning, would predict clinical response to PPX. We hypothesized that blunted reward learning would predict greater clinical response to PPX, as PPX may normalize DA function in these individuals.

Methods: Twenty-six adults with MDD aged 18-50, with no history of medication treatment or substance use disorder, and 26 age- and sex-matched healthy comparison subjects were recruited. At baseline, all completed a behavioral probabilistic reward task (PRT) that used an asymmetrical reinforcement schedule to produce a behavioral response bias. This bias has been shown to be attenuated in individuals with MDD and enhanced under conditions that increase phasic DA signaling in the striatum. Individuals with MDD completed a counterbalanced version of the PRT again following six weeks of open label treatment with PPX 0.5-2.5 mg/day. Assessments of DA receptor density using positron emission tomography (PET) imaging with [11C]-(+)-PHNO before and after oral administration of dextroamphetamine $0.5 \mathrm{mg} / \mathrm{kg}$, as well as fMRI BOLD signal change in the striatum during a reinforcement learning task, were conducted in the same sample and are being reported separately.

Results: Five subjects from each group were excluded from PRT analyses for failing to pass quality control criteria. A Group (controls, MDD) x Block $(1,2,3)$ ANOVA revealed a main effect of Group for PRT response bias, $\mathrm{F}(1,40)=8.71$, $p=.005, \eta p 2=0.18$, where across blocks of the task, the MDD group exhibited an overall blunting in response bias relative to controls. Within the MDD group, there were significant improvements in depression severity on the 
SI66

Hamilton Depression Scale (HAM-D; week 0 mean $20.23 \pm 2.47$ vs. week 6 mean 8.36 $\pm 5.40, p<.001)$ and anhedonia on the Snaith-Hamilton Pleasure Scale (SHAPS; week 0 mean $31.74 \pm 6.65$ vs. week 6 mean 25.29 \pm 6.89 , $p=.002$ ) from pre- to post-treatment with PPX. Although there were no significant changes in response bias from preto post-treatment $(p=.49)$, linear mixed models revealed that baseline response bias predicted changes in depression on the HAM-D $(\beta=-2.61, p=0.002, z=-3.13, S E=0.84)$, and anhedonia on the SHAPS $(\beta=-1.96, p=0.007, z=-2.68, \mathrm{SE}=0.73)$. Contrary to hypotheses, improvement in depression and anhedonia was significantly greater in individuals with more intact baseline reward learning. These behavioral findings converged with fMRI findings in the same sample, which showed that greater reward prediction error (RPE) in the left ventral striatum was associated with a significantly greater reduction in depression severity on the HAM-D over the course of treatment $(\beta=-0.54, p=0.03, \mathrm{z}=-2.25, \mathrm{SE}=0.24)$.

Conclusions: The current findings suggest that the D3preferring DA agonist PPX improves depression and anhedonia to a greater extent in MDD individuals who show evidence of more intact response bias and ventral striatal RPE - two measures that have been linked to phasic DA signaling. Chronic treatment with PPX on the other hand, has been found to increase motivation for rewards via an increase in tonic DA. It is therefore possible that individuals with MDD who display more intact reward learning and RPEs may preferentially benefit from PPX as it induces a complementary increase in motivation to obtain rewards, rather than simply remediating an overall DA deficit. The implication of these findings for the use of dopaminergic medications in individuals with MDD will be discussed.

Keywords: Depression, Dopamine Agonists, Reward Circuitry, Functional MRI (fMRI), Pramipexole

Disclosure: Nothing to Disclose.

M84. The Role of Dentate Gyrus Activity in the Behavioral Response to Antidepressant Treatment

Christine Yohn, Benjamin Samuels*

Rutgers University, Piscataway, New Jersey, United States

Background: Approximately 32-35 million adults in the US population (16\%) experience an episode of major depression in their lifetime, and commonly used treatments, such as selective serotonin reuptake inhibitors (SSRIs), are not ideal since only a subset of patients $(\sim 33 \%)$ achieves remission with initial treatment. The reasons why some individuals remit to antidepressant treatments while others do not are unknown. Our overall research program addresses this question by assessing antidepressant treatment resistance in mice. Proper assessment of the antidepressant response in mice first requires manipulations that will yield negative behaviors that can then be reversed by antidepressant treatment. Chronic treatment of mice with corticosterone (CORT) or exposure to social defeat effectively induces multiple changes in behavior associated with enhanced responses to potential harm and sustained threats. Subsequent chronic treatment with antidepressants such as fluoxetine (FLX) reverses these behavioral changes in some, but not all, of the mice, permitting stratification into responders and non-responders to FLX. We found that dentate gyrus granule cell activity was significantly increased in non-responders to FLX relative to responders.

Methods: Our studies were aimed at assessing the significance of alterations in dentate gyrus activity for the behavioral response to antidepressants and determining whether manipulation of dentate gyrus activity can alter the behavioral response of mice to FLX. We used a combination of molecular markers and in vivo physiology to assess the activity of the dentate gyrus and both chemoand optogenetic approaches to manipulate the dentate gyrus. Results: Mice were grouped into responders and nonresponders to CORT+FLX or social defeat+FLX by assessing behavior in both the novelty suppressed feeding and forced swim tests. Dentate gyrus activity was assessed by counting cFos positive neurons following exposure to the novelty suppressed feeding task. We found significantly increased dentate gyrus activity in mice that were non-responders to FLX relative to responders. We then found that subsequent inhibition of the dentate gyrus using either chemo- or optogenetic tools could convert behavioral non-responders into responders.

Conclusions: Our data suggest that circuit based manipulations of the dentate gyrus may provide an effective augmentation strategy for non-responders to SSRI treatment. These data indicate that the dentate gyrus is a critical component of the neural circuitry underlying the antidepressant response.

Keywords: Treatment Resistance, Dentate Gyrus, Neural Circuitry

Disclosure: Nothing to Disclose.

M85. A Novel Approach to Link Genetics and Human Brain Imaging Identifies Parahippocampal Gyrus to Habenula Resting State Functional Connectivity as Altered in Suicidality

Ramiro Salas*, Savannah Gosnell, Guillermo Poblete, Tien Nguyen, Alok Madan, Christopher Fowler

Baylor College of Medicine, Houston, Texas, United States

Background: Suicide is a current major problem with increasing numbers of suicides and failing therapeutic interventions. There are two biological forces behind suicidality: genetics and brain function. Although both fields are collecting data at unprecedented speed, integration between these two fields is very slow. In genetics, genome wide association studies (GWAS) commonly compare up to millions of genetic variants between two samples (e.g., suicidal vs. non-suicidal in this case). Given the number of variants studied, multiple comparisons must be taken into account and $\mathrm{p}$ values of 10-7 or less are commonly necessary. This creates a very large number of false negatives and lost information in studies that cost millions of dollars. In brain imaging we typically study a relatively small number of patients with and without a certain condition (in this case, past suicide attempt). Since there are thousands of voxels in the brain, there is also a large multiple comparison problem. Thus, brain imaging has been "accused" of being a field filled with false positives (multiple comparison approaches less stringent than those used in genetics are typical). 
Methods: We have developed a computational approach (ProcessGeneLists, PGL) to partially solve both problems. Given an archived GWAS we use all gene variants with $p$ values $10-3$ or less, which are mainly discarded by traditional genetic analysis, and use the Allan Atlas (a free www resource) to average the mRNA expression levels of the genes associated with those genetic variants in each brain region studied in the Atlas. Next, for each region we compare the expression of those genes from GWAS to the expression of all other genes in the Atlas, and choose the regions in which those genes are most significantly co-expressed (as average). Those brain regions become regions of interest (ROI) in our imaging studies. The main idea is that by averaging the expression of many genes, those truly involved in suicidality (mostly false negatives in traditional genetic analysis because the $\mathrm{p}$ value was, for example, 10-6) will be expressed in brain regions relevant to suicidality, while the other genes (true negatives in traditional genetics analysis) will be expressed in a random pattern and will have little weight when the average is compared to the average of all other genes.

Results: We performed PGL on an archived GWAS on past suicide attempt in bipolar patients and found a list of 130 genes (SNPs in those genes had p values of 10-3 or less, this threshold is arbitrary and should be optimized in the future) that highlighted 7 brain regions: Globus pallidus, putamen, corpus callosum, temporal cortex, parahippocampus, cerebellum (crus 2 and $7 \mathrm{~b}$ ), and medial geniculate nucleus. Importantly, since we arrived to these ROIs through the Allen Atlas, we have a specific coordinate (or coordinates) for each ROI. Next, we studied those regions using resting state functional connectivity (RSFC) in a sample of psychiatric patients with $(n=133)$ and without $(n=284)$ past suicide attempt. We studied the RSFC between the PGL highlighted regions (anatomically defined whole regions) and all other brain regions (we used the AAL atlas to segment the brain and added a few small regions not included in the atlas but interesting to as: ventral tegmental area, substantia nigra, habenula, medial and dorsal raphe nuclei, and locus coeruleus). When all those comparisons were made between past suicide attempt and not, the parahippocampus to habenula RSFC resulted larger in suicidal than in non-suicidal patients $(p<10-5$, surviving Bonferroni comparison for all pairs of areas studied). In addition, we used the coordinates of the PGL highlighted regions and performed a whole brain study. In this case, we found that the parahippocampus coordinate (within the subiculum) showed increased RSFC with medial frontal gyrus in past suicidal attempt patients. Finally, we analyzed which of the 130 genes identified had more weight in pointing to the parahippocampus and found a short list of 3 genes (NFIA, GRIK1, and GRM8). We are currently in the process of genotyping these genes in our imaging sample. Our new hypothesis is that variants in these genes affect suicidality by increasing the RSFC of the parahippocampus with, at least, the habenula and the medial frontal gyrus.

Conclusions: If successful, our approach will create a link between genetics and brain imaging of suicide that will further our understanding of suicide and could be exploited for example, in the future development of personalized therapies and in clinically predicting which patients are at higher risk. Instead of asking a genetics question ("which genes are important for a phenotype?") and an imaging question ("which brain areas are important for a certain phenotype?") in isolation, we may now be able to ask the question "which genes, via altering brain function where in the brain, are associated with a certain phenotype?". We showed here that the PGL approach worked for suicidality, and we believe it may work for any other neuropsychiatric phenotype in which a GWAS (other methods to obtain a list of possibly associated genes can also be used) has already been performed.

Keywords: Suicide, fMRI/Imaging Genetics, Resting-State Functional MRI, GWAS, Habenula

Disclosure: Nothing to Disclose.

\section{M86. Functional Activation During Emotion Processing in Late-Life Depression: Early Markers of Treatment Response}

\author{
Helmet Karim*, Carmen Andreescu, Jordan Karp, \\ Charles Reynolds, Howard Aizenstein
}

University of Pittsburgh Western Psychiatric Institute and Clinic, Pittsburgh, Pennsylvania, United States

Background: Treatment of major depression (MDD) often requires multiple trials of medications before an effective therapy can be identified, this poses a serious issue as it is associated with an increased risk of suicide and can contribute to worsening co-morbidities. In late-life depression (LLD), as the time required to respond to a single medication is on average longer (6 weeks compared to 4) these risks may be worsened. Several studies have shown changes in functional activation/connectivity following acute doses as measured by functional magnetic resonance imaging (fMRI). In this study, we aimed to investigate early changes in functional brain activation (during emotion reactivity) that occur during a treatment trial.

Methods: LLD patients $(N=33,23 \mathrm{~F}$, greater than 60 years old) were enrolled into a 12-week Venlafaxine treatment trial where an fMRI scan was collected at baseline, 12 hours following a placebo, 12 hours following their first dose, a week after beginning treatment, and at the end of the trial. Participants performed an emotion reactivity task (faces/ shapes) and matched two faces to a cue (either angry/fearful) and a control (matching shapes). Montgomery-Asberg Depression Rating Scale (MADRS) measured depression severity, and remitters had a score less than 10 for at least two weeks. After spatial preprocessing and modeling of activation - we investigated whether there were significant group by time effects. Multiple comparisons correction was conducted using statistical non-parametric mapping (cluster forming threshold of 0.001), which controlled for the familywise error $(p<0.05)$.

Results: We found a significant group by time interaction in the left insula, superior temporal gyrus, thalamus, supramarginal gyrus, inferior parietal gyrus, and the right insula that did not occur following placebo. Remitters had relatively lower activation that increased following treatment (reversed in non-remitters). We found that the initial dose (placebo vs. acute period) showed a significant interaction in the left parahippocampus. Similarly, remitters showed a significant increase in activation while non-remitters showed a significant decrease. 
Conclusions: The changes identified across the 12-week treatment interval may reflect the gradual increased activity in implicit emotion regulation in the remitters and continued and progressive disruption in the non-remitters.

Keywords: Late-life Depression, Emotion Processing, Depression Treatment Response

Disclosure: Nothing to Disclose.

\section{M87. Cardiovascular Effects of Intravenous Ketamine for Depression: Analysis of 684 Consecutive Infusions}

Patricio Riva Posse*, Collin Reiff, Gregory Job, Gail Galendez, Johnathan Edwards, William McDonald

Emory University School of Medicine, Atlanta, Georgia, United States

Background: Ketamine is being increasingly used in psychiatry, having shown rapid antidepressant effects and antisuicidal properties. While its use is considered off-label, ketamine infusions are becoming more widespread, and there are many concerns about the potential for abuse, as well as questions about appropriate settings and monitoring. Administration of an off-label medication that is outside of the regular skills of psychiatrists needs to, first and foremost, avoid putting patients at unnecessary risk. But so far there has been limited data about the cardiovascular effects of the drug when administered by infusion at subanesthetic doses. Methods: All patients were recruited at Emory University and received their first ketamine infusion between June 2014 and June 2016. The protocol for administration of IV ketamine for the treatment of depression at Emory University was approved by the Medical Practice Committee of Emory University Hospital and the data collection was authorized by the local IRB. Participants with unipolar or bipolar depression were adults, able to provide informed consent regarding the off-label use of ketamine for the treatment of depression. Exclusion criteria included presence of psychotic symptoms, active substance use disorder, severe PTSD, clinically significant symptoms of borderline personality disorder, or medical instability.

Ketamine was administered by continuous IV infusion for 40 minutes, and dosing was determined on a weight basis $(0.5 \mathrm{mg} / \mathrm{kg})$. Blood pressure was measured at baseline, every 10 minutes from the start until the end of the infusion, and every 15 minutes thereafter. Prior to each infusion patients completed mood rating questionnaire (QIDS-SR), and were assessed clinically to evaluate response to ketamine (CGI-S and CGI-I). Participants were also monitored clinically for the appearance of cognitive or urinary side effects. The usual protocol involved administration of ketamine in a biweekly fashion for the first three weeks. Infusions could be continued if deemed clinically efficacious. Patients were continued on their antidepressant medications and were instructed to take their home medications in the morning of the infusion.

Results: 66 consecutive patients received a total of 684 infusions between June 2014 and June 2016. Forty patients $(60.6 \%)$ were female. Mean age was 56.7 years $(37.9 \%$ were over 60 years old). All infusions were done as an off-label indication to treat severe depression. QIDS-SR score was $17.38,47 \%$ of patients had received ECT in the past. $87.9 \%$ $(n=58)$ of patients had unipolar depression, 8 subjects had bipolar depression. 24 subjects (36.1\%) had essential hypertension and were taking antihypertensive medication prior to the initiation of the ketamine infusions. All infusions were tolerated and there was no need for interruption of the infusion due to either psychotomimetic side effects or unstable vital signs.

At the start of the infusion, systolic pressure was 105.27 (34.47), diastolic was $71.81(11.81)$ and mean arterial pressure (MAP) was 82.96 (19.02) $\mathrm{mmHg}$. Peaks of blood pressure were observed at 30 minutes and 40 minutes into the infusion. At 30 minutes, the blood pressure readings were: systolic 108.55 (34.97), diastolic 74.98 (11.79) and MAP 86.17 (19.51) mmHg. The biggest difference in blood pressure from the start of the infusions was measured at 30 minutes, and it was $3.28 \mathrm{mmHg}, 3.17 \mathrm{mmHg}$ and $3.21 \mathrm{mmHg}$ for systolic, diastolic and MAP, respectively.

After the infusion was finished at 40 minutes, vital signs returned to baseline values gradually, and 30 minutes after the end of the infusion (70 minutes from start) the difference in systolic pressure was $0.6 \mathrm{mmHg}$ and $1.56 \mathrm{mmHg}$ in MAP (no statistical difference with baseline readings), while remained mildly elevated in diastolic pressure by $3.03 \mathrm{mmHg}$.

Analysis on different subgroups were conducted (normotensives vs. hypertensives; patients under 60 years old or above 60; patients who received or not lorazepam prior to infusion), with small differences that were statistically significant but not clinically relevant. There was no development of tolerance between the first and the sixth infusions in terms of blood pressure.

9.21\% of infusions (in 33 patients) had elevations of at least $30 \mathrm{mmHg}$ systolic or $15 \mathrm{mmHg}$ diastolic, most of those during the infusions at the 30 - and 40 - minute time points. Conclusions: This is the most extensive report on the use of subanesthetic ketamine infusions for the treatment of depression. Infusions are overall well tolerated from the cardiovascular standpoint. Most of the concerns regarding its hypertensive effects are extrapolated from the general anesthesia literature that uses higher doses in IV push, and not extended infusions of low doses. Many patients will continue to have discrete elevations of systolic or diastolic blood pressure, but the majority of patients do not have substantial cardiovascular changes during the infusions requiring more invasive monitoring. More research needs to be conducted concerning different dosing and infusion times.

Keywords: Ketamine, Cardiovascular, Treatment Resistant Depression

Disclosure: Part 1: Johnson \& Johnson, Consultant.

M88. Association of Novel ALX4 Gene Polymorphism With Escitalopram-SSRI Treatment Response in Caucasians: Findings From the Co-Med Trial

Bharathi Gadad*, Prithvi Raj, Manish Jha, Thomas

Carmody, Igor Dozmorov, Edward Wakeland, Madhukar Trivedi

University of Texas Southwestern Medical Center at Dallas, Dallas, Texas, United States

Background: CO-MED (Combination of Medications to Enhance Depression Outcomes) is a randomized, 3-arm 
clinical trial comparing efficacy of monotherapy vs two antidepressant combinations. In the present study, we test potential common genetic polymorphisms that significantly differentiate between responder and non-responder groups using genome wide association studies (GWAS).

Methods: A total of $n=459$ patients who met the DSM-IVTR criteria were randomly assigned to receive acute 12 weeks and long-term continuation (total seven months) of double blinded treatment with i) Escitalopram+Placebo (Monotherapy); ii) Bupropion+Escitalopram and iii) Venlafaxine + Mirtazapine groups respectively (ii and iii are combinations of medications). The outcomes were measured using Clinician-rated QIDS-SR (Quick Inventory of Depressive Symptomatology, Self-Reported 16-item version). We performed genome wide variant analysis using the illumina 2.5 chip, which provided genotypes for 2.5 million single nucleotide polymorphisms (SNPs). Genotypes were analyzed for participants at week 6 (response; QIDS-SR $>50 \%$ reduction) and week 12 (remission; QIDS-SR $<5$ ) for all the three treatment groups. We also generated a multi-locus genetic variable describing the individual number of alleles of the selected SNPs associated with beneficial treatment outcome by haplotype mapping (response at week 6 \& 12). Results: Combinations of medications did not outperform monotherapy based on the treatment outcome (previous finding by Trivedi and group). GWAS identified a potentially regulatory SNP rs10769025 in ALX4 gene on chromosome 11 with strong significance ( $p$ value $=9.85925 \mathrm{E}-08)$ for responders to Escitalopram+placebo (monotherapy). Further, haplotype analysis on seven ALX4 variants showed that a regulatory haplotype CAAACTG is significantly [OR $(\mathrm{LCI}-\mathrm{UCI})=3.4(1.7-7.0), \quad p=2.00 \mathrm{E}-04]$ associated with Response to Escitalopram+ Placebo monotherapy.

Conclusions: Our genetic analysis suggests that potentially regulatory polymorphisms in ALX4 gene may be associated with Escitalopram treatment outcome in Caucasians. So far, ALX4 is known to play a critical role in craniofacial development and mental retardation. Its mechanism of action and its signaling pathway based on the antidepressant treatment need to be elucidated.

Keywords: Escitalopram, Pharmacogenetics, GWAS

Disclosure: Nothing to Disclose.

\section{M89. Social-Economic Tasks Reveal Opposite Decision- Making Biases in Schizophrenia and Depression}

Aylin Daldal, Sheila Shankar, Theodore Satterthwaite, Lillie Vandekar, Natalie Katchmar, David Roalf, Joseph Kable, Daniel Wolf*

University of Pennsylvania, Wynnewood, Pennsylvania, United States

Background: Both schizophrenia and depression are associated with abnormalities in social function, including social decisions that require integrating rational self-interest with interpretations of social norms. However, divergent symptoms and traits associated with the two disorders are likely to yield distinct patterns of abnormalities in social interactions. Here we applied the Ultimatum Game (UG) and the Dictator Game (DG) across the two disorders, in order to characterize self- vs. other-regarding biases in sensitivity to unfairness.
We hypothesized that for decisions balancing rational selfinterest and fairness, schizophrenia would be associated with a self-regarding bias while depression would be associated with an other-regarding bias.

Methods: Three groups of subjects completed UG and DG tasks: control (CT, $n=20)$, schizophrenia (SZ, $n=22)$, and major depression $(\mathrm{MD}, n=20)$. In the UG task, the participant had to either accept or reject a proposed (hypothetical) offer of some part of $\$ 10$, with the proposed offer varying across trials. Accepting the offer results in the proposed split of the money while rejecting the offer results in no money for either individual. In the DG task, participants completed a single trial, deciding how they would split $\$ 10$ between themselves and another individual, with the other individual required to accept the outcome. The amount given away in DG and the minimum amount accepted in UG were analyzed, focusing on the group $\mathrm{x}$ task interaction that compares self-regarding vs other-regarding biases between the groups. In the UG task, pure rational selfinterest would lead to accepting any offer greater than $\$ 0$, while in the DG task, it would lead to giving away $\$ 0$. In contrast, pure and consistent inequity aversion (insistence on fairness) would lead to even splits $(\$ 5 / \$ 5)$ in both tasks. Extreme self-regarding bias would lead to giving $\$ 0$ in DG but accepting only fair (\$5) offers in UG; extreme otherregarding bias would lead to giving fair offers of $\$ 5$ in DG, but accepting any offer more than $\$ 0$ in UG.

Results: All three groups (CT, SZ, MD) showed broadly normative responses in both tasks, in that average performance within each group reflected intermediate choices balancing rational self-interest against fairness considerations. However, the specific balance varied across groups, with opposite diagnostic patterns evident for DG vs. UG. In $\mathrm{DG}, \mathrm{MD}$ gave away the most money $(\$ 4.30)$, followed by CT (\$3.67) and then SZ (SZ \$2.86). In contrast in UG, MD accepted the lowest offers (\$2.65), followed by CT $(\$ 2.95)$ and then SZ (\$3.68). These divergent patterns led to a significant group $\mathrm{x}$ task interaction $(p=.019)$. Performance on the two tasks was uncorrelated across all participants $(\mathrm{r}=-0.01)$.

Conclusions: Comparing UG and DG tasks allowed us to differentiate inequity aversion in general from sensitivity to unfairness specifically to others versus oneself. While on average participants balance self-interest and fairness, analysis of individual and group differences indicate that asymmetric inequity aversion is prominent. The pattern observed in schizophrenia is consistent with elevated social distrust leading to greater insistence on being treated fairly without an equivalent focus on fairness to others. In depression, the opposite pattern could reflect elevated guilt and/or reduced self-esteem, leading to a willingness to accept unfair treatment toward oneself while maintaining an insistence on fairness to others. The results may also reflect disorder-related differences in the understanding of social norms or in their application to oneself. Future work should examine the specific state and trait dimensions of illness, cognition and personality associated with these divergent asymmetries, as well as their underlying neural circuitry, to pave the way for individualized assessment and intervention. Keywords: Social Behavior, Depression, Schizophrenia, Decision-Making, Behavioral Economics

Disclosure: Nothing to Disclose. 
M90. A Probe Into the Connection Between Inflammation, Cognition and Suicide

Ricardo Caceda*, Sue Griffin, Pedro Delgado

Stony Brook University, Stony Brook, New York, United States

Background: Elevated inflammation has recently been linked to the pathophysiology of suicide. An array of factors has been shown to influence inflammation including metabolic factors, acute and chronic medical conditions, recent trauma, psychological stress and mood disorders.

In this context, the source of the elevated inflammation associated with suicidal behavior or ideation is not entirely clear. For instance, limited data exists on the relative contribution of chronic suicidal diathesis, comorbid medical conditions or acute psychological stress to the increased inflammation levels in suicidal patients. Additionally, little is known regarding potential downstream events, if any, associated with increased inflammation. Hence, we examined: a) factors associated with inflammation, and b) factors associated with suicidal ideation in depressed patients hospitalized for acute suicidal ideation.

Methods: We recruited 70 consecutive adult depressed patients of both genders hospitalized in a psychiatric inpatient unit for severe suicidal ideation. We performed clinical characterization including suicidality characterization with the Columbia Suicide Rating Scale, measures of depression, anxiety, medical comorbidity, psychological and physical pain, impulsivity, verbal fluency, $\mathrm{C}$ reactive protein (CRP), and interleukin (IL) 6. Variable normality distribution was examined with the Shapiro-Wilk test. Two generalized linear models were performed with either CRP or suicidal ideation severity as dependent variables.

Results: A generalized linear model was significant $(r=.776$; $\mathrm{p}=.008)$ for association of CRP with IL6 $(\mathrm{r}=.984 ; \mathrm{p}<.001)$ and number of lifetime suicide attempts $(\mathrm{r}=.754 ; \mathrm{p}=.01)$. Regarding suicidal ideation severity, a generalized linear model $(\mathrm{r}=.9 ; \mathrm{p}=.02)$ showed association with current physical pain $(\mathrm{r}=.918 ; \mathrm{p}=.002)$, cognitive impulsivity $(\mathrm{r}=.699 ; \mathrm{p}=.016)$ and verbal fluency $(\mathrm{r}=.609 ; \mathrm{p}=.028)$.

Conclusions: Increased inflammation does not seem to be directly related to acute suicidal ideation or to putative risk factors for suicidal ideation (physical pain and cognitive function), but seems a trait associated with lifetime suicidal behavior.

Keywords: Suicide, Inflammation, Inpatient

Disclosure: Part 1: Neuronetics, Grant.

M91. Ketamine and (2R,6R)-Hydroxynorketamine Promote Dendrite Outgrowth and GluR1 Expression in Human iPSC-Derived Dopaminergic Neurons

Emilio Merlo Pich*, Ginetta Collo, Laura Cavalleri, Mark J. Millan, PierFranco Spano

\section{Takeda Development Center Europe, Basel, Switzerland}

Background: Dysfunctional relationships between glutamate neurotransmission and neural plasticity have been recognised as pathologic feature in depression and drug addiction. Ketamine, a dissociative anaesthetic that blocks the
N-methyl-D-aspartate (NMDA) receptor, produces rapidonset antidepressant effects when infused i.v. at subanesthesic doses for about 1 hour. The antidepressant effects persist for a week or more, phenomena whose neurobiological underpinning are unclear. One hypothesis postulates that ketamine would trigger adaptive response in neurons that extend beyond the pharmacologic exposure driving longer term structural changes in neural system connectivity. Converging findings indicate that the antidepressant effects produced by ketamine may be related to neuroplasticity changes in dendritic arborisation and synaptic terminals in the frontocortical/hippocampal networks via engagement of $\alpha$-amino-3-hydroxy-5-methyl-4-isoxazolepropionic acid receptor (AMPAR)-dependent glutamate transmission, as well as activation of BDNF/TrkB and mTOR intracellular signallings, all critical pathways involved in cell growth and plasticity $[1,2]$. However, these long-term effects could be also mediated by one of ketamine metabolites, ( $2 \mathrm{R}, 6 \mathrm{R})$ hydroxynorketamine (HNK) [3]. The levels of this metabolite increase within hours after infusion of ketamine, reaching its higher values in the submicromolar range at 612 hours [4]. Using primary cultures of mouse mesencephalic dopaminergic (DA) neurons we recently showed that in vitro exposures to ketamine can engage $\mathrm{BDNF} / \mathrm{TrkB}$ and mTOR signalling pathways, effects that are mediated by AMPAR activation. Dysfunctional DA neurons are known to be critical in determining anhedonia, a core symptom of depression and chronic stress conditions. These observations were confirmed also in human DA neurons differentiated from inducible pluripotent stem cells (iPSC) [5], suggesting a possible translational relevance. In the present work, we used both mouse primary cultures of DA neurons and human iPSC-derived DA neuron to test the pharmacologic effects of ketamine and HNK on dendritic growth and soma size, as well as their effects on AMPAR neurotransmission, including the expression levels and cellular distribution of AMPAR subunits.

Methods: Human iPSC were generated from healthy donors following the approval of the local ethics committee and differentiated into DA neurons [5]. DA neurons, cultured for 60-80 days, expressed differentiation markers, including tyrosine hydroxylase (TH), dopamine transporter (DAT), NMDA subunit NR2B, AMPA subunits GluR1 and GluR2. Mouse DA neurons were obtained from E13.5 embryo mesencephalon. Maximal dendrite length, number of primary dendrites and soma size of $\mathrm{TH}$-positive neurons were measured using computer-assisted morphometry 3 days after exposure to either ketamine or HNK, or vehicle. Semiquantitative immunofluorescence and PCR were used to assess the expression and distribution of GluR1 and GluR2 at baseline and following treatment. Ketamine was used at 0.01$1.0 \mu \mathrm{M}$ for 1-hour exposure, HNK at $0.1-0.5 \mu \mathrm{M}$ for 1 and 6- hour exposure.

Results: Ketamine dose-dependently increased dendritic arborisation and soma size in both mouse and human DA neurons. These effects were inhibited by pre-treatment with the AMPA antagonists NBQX $(10 \mu \mathrm{M})$ and GYKI52466 $(10 \mu \mathrm{M})$. Significant increases of maximal dendrite length $(p<0.01)$, number of primary dendrites $(p<0.01)$ and soma size $(p<0.01)$ were observed after HKN exposures at $0.5 \mu \mathrm{M}$, while $0.1 \mu \mathrm{M}$ delivered only a trend for increase at both 1 and 6- hour exposure. These effects were similar in mouse DA 
neurons and in human DA neurons. Semi-quantitative immunofluorescence image analysis indicated that GluR1 was preferentially expressed in the soma, while GluR2 was more expressed in dendrites of DA neurons. This distribution was not changed by ketamine exposure, that instead significant increases the levels of the GluR1.

Conclusions: Ketamine and HNK similarly induced structural plasticity in mouse and/or human DA neurons by engaging the AMPAR, as previously demonstrated in vivo, at concentrations and exposure time that are compatible with the subanaesthetic doses use in patients suffering from Mood Disorders [5]. These data suggest a possible role of DA neurons in mediating the in vivo effects of Ketamine and its metabolite.

\section{References:}

[1] Li N et al. Science 329: 959-964, 2010.

[2] Autry et al. Nature 475: 91-95, 2011.

[3] Zanos P. et al. Nature 533: 481-486 m 2016.

[4] Zhao X et al. Br J Clin Pharmacol; 74: 304-314, 2012.

[5] Cavalleri L et al. Molecular Psychiatry, under revision, 2017.

Keywords: Neural Plasticity, Translational Pharmacology, Dopamine System

Disclosure: Part 1: Takeda, Employee, Part 2: Takeda, Stock / Equity, Part 3: Takeda, Employee, Part 4: Servier, Grant, Spouse, Part 5: Takeda, Employee.

\section{M92. The Ankryin3 Psychiatric Risk Gene Regulates Neuronal Microtubule Dynamics}

Jacob Garza, Xiaoli Qi, Klaudio Gjeluci, Melanie Leussis, Gerard Martens, Geert Poelmans, Tracey Petryshen*

Harvard Medical School, Massachusetts General Hospital, Boston, Massachusetts, United States

Background: Ankyrin3 (ANK3) has been identified as a risk gene for bipolar disorder (BD) and autism spectrum disorders (ASD) through multiple genome-wide and targeted association studies as well as sequencing studies. Carriers of the $\mathrm{BD}$ risk alleles have reduced ankyrin3 expression in the brain, suggesting that ankyrin3 suppression contributes to disease. However, the molecular mechanism through which ankyrin 3 confers disease risk is unknown. Ankyrin3 encodes the ankyring protein that tethers integral membrane proteins to the cytoskeleton. We previously reported that Ank $3+/$ - heterozygous mice, which have $\sim 50 \%$ reduced ankyrinG in the brain, exhibit behavioral changes reminiscent of bipolar mania (impulsivity, increased motivation for reward), and altered expression of proteins involved in neuron axonal transport. Both the behavioral and protein expression changes in Ank3+/- mice are normalized by lithium treatment, supporting the disease relevance of these findings. This study sought to further examine the molecular mechanism underlying the behavioral and neuronal changes induced by ankyrin3 suppression in brain.

Methods: We performed RNA sequencing of hippocampus from Ank3+/- heterozygous and Ank3+/+ wildtype mice, followed by identification of differentially expressed genes using the Tuxedo package. Biological pathways implicated by RNAseq analysis were verified by Western blot analysis of hippocampal proteins and live-cell imaging of forebrain primary neurons. CRISPR/dCas9 technology was used to repress ankyrin 3 transcription in mouse Neuro2A cells in order to conduct in-depth biochemical analysis. Neurons were treated with lithium and CHIR-99021, a selective inhibitor of the primary lithium target GSK3B, as well as an inhibitor of CRMP2, an axon-specific substrate of GSK3B.

Results: RNA sequencing identified 283 genes that were differentially expressed between Ank3+/- and Ank3+/+ mouse hippocampus (fold change $>1.2$, uncorrected $p<10$ $3)$. There was enrichment of genes involved in microtubulemediated processes (e.g., cytoskeleton, cargo trafficking), implicating altered microtubule functions in Ank3+/- mice. Western blot analysis confirmed increased expression of the microtubule interacting protein EB3 in the hippocampus of Ank3+/- compared to Ank3+/+ mice (40\% increase, $p<0.01$ ), supporting instability of microtubules in Ank3+/mouse brain. Live-cell imaging of EB3 movement in primary neurons verified microtubule instability in Ank $3+/$ - mice $(p=0.002)$. This was further confirmed in ankyrin3repressed Neuro2a cells (i.e., $>20 \%$ increased EB3, $p<0.01 ; 25 \%$ lower polymerized tubulin, $p<0.01$ ), and was reversed by treatment with lithium and CHIR-99021. Increased GSK3B-specific phosphorylation of CRMP2Thr514 (>30\%, $p<0.003)$ indicated impaired CRMP2 activity following ankyrin3 repression. Treatment with a CRMP2 inhibitor blocked lithium and CHIR-99021 reversal of elevated EB3, indicating that CRMP2 dependent signaling is involved in the microtubule defects of Ank3+/- mice.

Conclusions: Ankyrin3 disruption in mice is associated with altered neuronal microtubule dynamics. Lithium reversal of both the microtubule and behavioral changes of Ank3+/mice suggests that microtubule defects may underlie maniclike behaviors observed in these mice. Our results highlight the importance of risk gene studies using mouse and neuronal models to elucidate the neural mechanisms underlying psychiatric illness.

Keywords: Bipolar Disorder, GWAS, CRISPR/Cas9, Autism Spectrum Disorder

Disclosure: Nothing to Disclose.

M93. Comparing the Associations of Circulating and Lipopolysaccharide-Induced Inflammatory Markers With Depressive Symptoms in Older Adults

\author{
Hyong Jin Cho*, Silena Te, Michael Irwin \\ University of California, Los Angeles, California, \\ United States
}

Background: Inflammation is hypothesized to play a significant role in the pathophysiology of depression, especially in older adults as aging processes involve increased inflammation. While lipopolysaccharide (LPS) induced production of proinflammatory cytokines is generally a more sensitive measure of systemic inflammation compared to circulating levels of proinflammatory cytokines, the associations of the two measures with depressive symptoms have not been directly compared. This study examined whether LPS-induced production of cytokines or circulating plasma cytokines is a more sensitive biomarker in 
association with depressive symptoms in insomniac older adults who were otherwise healthy.

Methods: A cross-sectional analysis of the baseline data from a randomized trial of insomnia treatments was conducted. The analysis included 117 community-dwelling adults aged 55 years or older with insomnia who were otherwise healthy physically and mentally. Plasma levels of tumor necrosis factor- $\alpha$ (TNF- $\alpha)$, interleukin-6 (IL-6), and C-reactive protein (CRP) were measured. Peripheral monocytes were stimulated with LPS in vitro and LPS-induced monocyte production of IL- 6 and TNF- $\alpha$ was assayed. Depressive symptoms were assessed using the clinician-rated Inventory of Depressive Symptomatology (IDS-C). Multivariate linear regression was conducted to test the associations between these inflammatory markers and depressive symptoms, first in the entire sample and then in subgroups stratified into high and low levels of each inflammatory marker based on median split.

Results: Despite their age and insomnia, the participants presented with relatively low CRP levels (median $1.1 \mathrm{mg} / \mathrm{L}$ ) compared to the US adult population (median $2.1 \mathrm{mg} / \mathrm{L}$ ), possibly because they were generally healthy and active. In the entire sample, none of the inflammatory markers, neither circulating nor LPS-induced, were significantly associated with depressive symptoms. In the high inflammation subgroups, LPS-induced cytokines were significantly positively correlated with depressive symptoms even after full adjustment for covariates: TNF- $\alpha$ (adjusted $\beta=0.28$, $p=0.04$ ), IL-6 (adjusted $\beta=0.29, p=0.04$ ), and IL-6+TNF$\alpha$ (adjusted $\beta=0.43, p=0.001$ ). However, neither plasma IL-6 nor plasma TNF- $\alpha$ was associated with depressive symptoms; and plasma CRP was unexpectedly negatively correlated with depressive symptoms (adjusted $\beta=-0.36$, $p=0.02$ ). In the low inflammation groups, none of the inflammatory markers were significantly associated with depressive symptoms except for LPS-induced TNF- $\alpha$, which was unexpectedly negatively correlated with depressive symptoms (adjusted $\beta=-0.30, p=0.02$ ).

Conclusions: LPS-induced cytokines, compared to circulating cytokines, appear to be more sensitive correlates of depressive symptoms in older adults with elevated systemic inflammation.

Keywords: Inflammation, Depressive Symptoms, Lipopolysaccharide, Cytokines, C-reactive Protein

Disclosure: Nothing to Disclose.

M94. Increased Intra-Limbic Functional Connectivity to Insula, Thalamus, Caudate, Putamen, and Cingulate Cortex in Patients With Major Depression

Keith Sudheimer*, Jennifer Keller, Lakshika

Tennakoon, Rowena Gomez, Amy Garrett,

Alan Schatzberg

\section{Stanford University, Stanford, California, United States}

Background: Proper functioning of limbic brain structures is critical for maintaining healthy emotional behaviors. Patients with major depression have abnormal patterns of activity and connectivity in several limbic brain structures which are associated with the emotional symptoms of depression. Limbic brain structures have widely distributed connections with other limbic structures and throughout the cortex. This study examines the large-scale patterns of limbic functional connectivity of 33 different limbic structures in healthy participants, patients with major depression, and patients with major depression and patients with major depression and psychotic symptoms. The hypothesis was that the subgenual cingulate cortex would show systemic disruptions in connectivity with other limbic brain regions.

Methods: A total of 113 participants completed a 5-minute resting state brain scan. This sample included 41 healthy participants, 45 patients with major depression, and 27 patients with psychotic major depression. Resting state functional connectivity scans were analyzed standard preprocessing steps (filtering, de-trending, motion correction, etc.). Next, the images were analyzed using custom Matlab scripts to perform hierarchical (agglomerative) clustering to identify the largest and most homogeneous time courses from each of 33 different limbic brain structures (Amygdala, BA25*, Brainstem, Caudate, Ant. Cingulate, Middle Cingulate, Post. Cingulate, Claustrum, Hippocampus, Hypothalamus*, Insula, Midbrain*, Pallidum, Parahippocampal cortex, Pons*, Putamen, Subcallosal Gyrus*, Thalamus, and the Uncus. ${ }^{\star}=$ not bilateral). These time courses were then tested against the whole brain for correlations using a series of 33 simple regressions for each participant in SPM12. These simple regressions yielded functional connectivity maps of each limbic brain region for each participant. These functional connectivity maps (33/participant x 113 participants) were then used as input into a 2nd-level (group $\mathrm{x}$ brain region) factorial model. 2nd-level T-tests were constructed to test for systemic effects of depression across all functional connectivity maps. These T-tests were used to characterize the functional connectivity patterns that are persistently highly correlated with the 33 limbic brain regions. They also test for persistent differences in healthy participants and patients across all limbic brain regions.

Results: Healthy participants, patients with major depression, and patients with psychotic major depression all demonstrate strong limbic functional connectivity with other limbic areas. They also showed similar patterns of consistently inverse connectivity with occipital, parietal, temporal, and (to a lesser extent) medial frontal cortices. The pooled group of patients with major depression (nonpsychotic +psychotic) showed dramatically increased limbic connectivity to the insula $(\mathrm{T}=6.69)$, thalamus $(\mathrm{T}=3.32)$, caudate $(\mathrm{T}=4.8)$, putamen $(\mathrm{T}=5.45)$ and cingulate cortex $(\mathrm{T}=5.00)$, superior/middle frontal gyrus $(\mathrm{T}=3.34)$. These tests were corrected for multiple comparisons (False Discovery Rate $<0.05)$. We did not observe group differences in the large-scale patterns limbic connectivity to the subgenual cingulate at the FDR $<0.05$ threshold. However, we did find reduced limbic connectivity to the subgenual in patients with depression at the apriori $p<0.05$ uncorrected $(\mathrm{T}=2.78)$ threshold.

Conclusions: These data suggest that large scale patterns of intra-limbic functional may provide insights into the pathophysiology of depressive symptoms that standard methods of seed-based or ICA based functional connectivity analysis may overlook. Specifically, we have identified depression-related increases in intra-limbic connectivity to the insula, thalamus, caudate, putamen, and cingulate cortex. 
Keywords: Limbic Cortex, Depression, Insula, Connectivity, Resting State Functional Connectivity

Disclosure: Nothing to Disclose.

\section{M95. Long-Term Treatment of Depression With Repeated Intramuscular Ketamine}

David Feifel* ${ }^{\star}$ Patricia Peppa, Danielle Boggie, Kelly Lee

University of California, San Diego, La Jolla, California, United States

Background: Intravenous (IV) and Intramuscular (IM) administered Ketamine produces a rapid antidepressant effect in patients with treatment-resistant depression (TRD). However, to maintain the benefit ketamine must typically be re-administered every 2-6 weeks. Very little is known about the long-term safety and efficacy of such repeated ketamine treatments and there are concerns that it may induce addiction, tolerance, or bladder damage. We report on a cohort of outpatient TRD patients who have been administered IM ketamine $(0.25-1.0 \mathrm{mg} / \mathrm{kg})$ on average every 25 days from for over a year.

Methods: A retrospective medical record review was conducted on 14 TRD outpatients who received repeated IM ketamine for at least 12 months and up to 5 years. Extracted data, including adverse effects and depression rating scales (BDI-II and PHQ-9) completed by patients just prior to and 24- hours after ketamine treatments at four time points: initiation of treatment, at 3, 6 and 12 months afterwards.

Results: Mean age of this cohort was $48.3( \pm 12)$ years and $64 \%$ were female. Dose of ketamine administered ranged between $0.25-1.0 \mathrm{mg} / \mathrm{kg}$ and average interval between injections was $25.5( \pm 20)$ days. In this cohort 7 patients were monitored with the PHQ-9 scale and the other 7 were monitored with the BDI-II scale. Average baseline BDI and PHQ-9 score was $41( \pm 13.3) 22( \pm 3.2)$, respectively. After initial treatment, BDI-II and PHQ-9 score were reduced 52\% $( \pm 11.1)$ and $33 \%( \pm 13.9)$, respectively. At 3, 6, and 12 months, there was a $76 \%( \pm 6.1), 78 \%( \pm 6.6)$ and $60 \%$ $( \pm 11)$ reduction in the BDI-II score and a $49 \%( \pm 8.2) 49 \%$ $( \pm 8.7)$, and $37 \%( \pm 9.5)$ reduction in the PHQ-9. Significant adverse events attributable to ketamine included, three episodes of significant panic and agitation due disturbing dissociative experiences in two patients and two episodes of significant nausea and vomiting in two patients. There was no evidence of addiction to ketamine or other substances nor was there evidence for cognitive impairment, bladder injury or any other serious untoward effects.

Conclusions: This retrospective chart review, describes the largest cohort to date of TRD patients whose received repeated parenteral ketamine for at least 12 months. The findings suggest that the acute antidepressant benefits of ketamine can be successfully maintained for at least one year by re-administering ketamine at individualized doses and intervals and that this approach does not induce addiction, cognitive deficits, or bladder damage.

Keywords: Ketamine, Treatment Resistant Depression, Maintenance Treatment, Long-Term Treatment

Disclosure: Nothing to Disclose.
M96. Ketamine, but not the NMDA Receptor Antagonist Lanicemine, Increases Prefrontal Connectivity in Depressed Patients

Chadi Abdallah*, Arpan Dutta, Christopher Averill, Shane McKie, Lynnette Averill, Bill Deakin

Yale University School of Medicine, West Haven, Connecticut, United States

Background: The discovery of the rapid acting antidepressant effects of ketamine have generated considerable interest in academia and industry about the prospect of targeting NMDA receptors (NMDAR) in the treatment of refractory depression. However, the results from clinical trials failing to reach primary outcomes, combined with recent preclinical evidence proposing NMDAR-independent mechanisms of ketamine, have cast doubts about the potential utility of this line of research. In this study, we investigated the impact of ketamine, and the NMDAR antagonist lanicemine, on prefrontal cortex (PFC) Global Brain Connectivity (GBC), a previously validated biomarker of depression and the rapid acting antidepressant ketamine. Prior studies have repeatedly shown reduced PFC GBC in depression, and open-label trials showed significant increases of PFC GBC $24 \mathrm{~h}$ post ketamine administration. However, the effects of ketamine on PFC GBC during infusion and placebo-controlled PFC GBC ketamine studies in depressed patients were not previously conducted.

Methods: Fifty-six unmedicated participants with major depressive disorder were randomized to intravenous placebo (normal saline; $n=18)$, ketamine $(0.5 \mathrm{mg} / \mathrm{kg} ; n=19)$, and lanicemine (100 mg; $n=19$ ). Functional connectivity magnetic resonance imaging (fc-MRI) scans were completed at baseline, during infusion, and $24 \mathrm{~h}$ post treatment. The Human Connectome Pipeline was adapted to conduct surface based preprocessing and optimize registration. PFC vertex-wise analyses were corrected for multiple comparisons using permutation testing and threshold-free cluster enhancement.

Results: Compared to placebo, ketamine, but not lanicemine, significantly increased GBC in multiple clusters within the PFC during infusion and at $24 \mathrm{~h}$ post treatment. No reduction in PFC GBC were observed. The GBC increases were found in the dorsolateral, dorsomedial, and frontomedial PFC during infusion, and in the dorsolateral and dorsomedial PFC $24 \mathrm{~h}$ following treatment. Region-ofinterest analysis showed a significant increase in average PFC GBC, compared to placebo, during (mean difference $\pm \mathrm{SEM}=0.08 \pm 0.02 ; p=0.01)$ and $24 \mathrm{~h}$ following ketamine (mean difference $\pm \mathrm{SEM}=0.13 \pm 0.04 ; p=0.02$ ). Compared to placebo, no significant changes were found during (mean difference \pm SEM $=0.03 \pm 0.03 ; p=0.45)$ or $24 \mathrm{~h}$ following lanicemine (mean difference $\pm \operatorname{SEM}=0.07 \pm 0.04 ; p=0.23$ ). In the placebo group, we found no correlation between delta PFC GBC (during minus baseline) and depression improvement at $24 \mathrm{~h}(\mathrm{r}=-0.1 ; p=0.69)$. However, delta PFC GBC positively predicted depression improvement regardless whether participants received ketamine or lanicemine $(\mathrm{r}=0.57 ; p=0.003)$.

Conclusions: The study provides the first evidence of ketamine-induced PFC GBC during infusion in depressed 
patients. It also replicates, in a placebo-controlled design, previous open label evidence of ketamine-induced PFC GBC $24 \mathrm{~h}$ post treatment. Lanicemine $(100 \mathrm{mg})$ failed to induce significant PFC GBC increases during and $24 \mathrm{~h}$ post treatment. However, it is evident that numerically the lanicemine-induced PFC GBC changes appear to be in the same direction as ketamine, which raises the question for future studies whether optimization of the lanicemine administration regimen could lead to significant prefrontal connectivity normalization - especially considering the positive relationship between PFC GBC increases and treatment response.

Keywords: Ketamine, NMDA Antagonists, fMRI Functional Connectivity, Rapid Antidepressant, Mechanism of Action

Disclosure: Part 1: Janssen, Advisory Board.

M97. Rapid Efficacy and Antisuicidal Actions of Intravenous Ketamine for Ultraresistant Depression in a Clinical Setting: A Retrospective, Database Study

Rejish Thomas*, Glen Baker, Serdar Dursun

Alberta Health Services, Edmonton, Canada

Background: An underserved and suboptimally treated population exists in depressed patients who have failed a comprehensive treatment regimen of antidepressants, augmentation strategies, psychotherapy and electroconvulsive therapy (ECT) that can be described as ultraresistant depression (URD). These patients carry a large burden of illness and have unsatisfactory and potentially tragic outcomes with conventional treatments. Therefore, this is the population in which clinicians may consider the use of experimental treatments, like intravenous ketamine. Intravenous ketamine at subanaesthetic doses has demonstrated effect sizes similar to ECT in treatment resistant depression with a novel mechanism. It also has yielded exciting antisuicidal effects independent of anxiolytic and antidepressive effects. However, the effectiveness of intravenous ketamine in non-research settings and with URD patients remains understudied.

This study aims to measure the response and remission rates in URD patients in a clinical setting by means of a retrospective, database study. Secondarily, the study will attempt to support previous findings of predictors of response to IV ketamine treatment and investigate if adverse childhood experience score and level of treatment resistance impact response.

Methods: Fifty URD patients were treated with intravenous ketamine between May 2015 and December 2016, inclusive, in two community hospitals in Edmonton using a standardized protocol. Data were collected from inpatient and outpatient charts and included demographics, past and present diagnoses, laboratory results, past and present psychopharmacology, information to estimate an adverse childhood experiences (ACE) score, body mass index (BMI), family history of addictions and mental illness, substance use, medical diagnoses, rating scales and side effects from tracking sheets. Statistical analysis to investigate predictors of response included logistic regression analysis using a dependent variable of response $(50 \%$ reduction in Beck Depression Inventory (BDI) score) and independent variables of age, gender, anxious features, suicidality, anhedonia, Maudsley Staging Method (MSM) score, first degree family history of alcoholism, BMI, ACE score, and benzodiazepine/lamotrigine/antipsychotic use during treatment.

Results: At baseline, the average treatment resistance was severe, with a MSM score of 12.1 out of $15,90.2 \%$ were resistant to ECT, and the average BDI score was 34.2. Further, $46.8 \%$ and $48.9 \%$ of the population were at least once described as having Cluster B and/or Cluster C traits, respectively. The response rate was $43.1 \%$ and remission rate was $15.7 \%$. Of those patients who reported at least mild depression or suicidality, $68.9 \%$ and $54.0 \%$, respectively, later reported complete remission after treatment with ketamine. There were no statistically significant findings correlating any of the investigated factors to response.

Conclusions: In a clinical setting, intravenous ketamine showed effectiveness in a complex, severely treatment resistant, depressed population on multiple medication profiles concurrently. This study was unable to give support to previous findings of predictors of response to ketamine treatment.

Keywords: Ketamine, Treatment Resistant Depression, Suicide, Predictors of Response, Intravenous Administration Disclosure: Nothing to Disclose.

\section{M98. Narp Mediates Antidepressant-Like Effects of Electroconvulsive Seizures}

Andrew Chang, Punit Vaidya, Edward Retzbach, Jerome Chung, Urian Kim, Katherine Baselice, Kristen Maynard, Alec Stepanian, Melissa Staley, Lan Xiao, Ashley Blouin, Sungho Han, Joanne Lee, Paul Worley, Kellie Tamashiro, Barbara Hempstead, Keri

Martinowich, Mary Ann Wilson, Jay Baraban, Irving Reti*

Johns Hopkins University, Baltimore, Maryland, United States

Background: Growing recognition of persistent cognitive defects associated with electroconvulsive therapy (ECT), a highly effective and commonly used antidepressant treatment, has spurred interest in identifying its mechanism of action to guide development of safer treatment options. However, as repeated seizure activity elicits a wide range of electrophysiological and biochemical effects, this goal has remained elusive. We have examined whether deletion of Narp, an immediate early gene induced by electroconvulsive seizures (ECS), blocks its antidepressant efficacy.

Methods: Since repeated ECS treatments have a high rate of mortality in many mouse strains commonly used for behavioral studies, we generated Narp KO mice on a CF-1 background, as this strain withstands repeated ECS well. All courses of ECS consisted of 5 daily treatments. To monitor antidepressant efficacy, mice were subjected to the forced swim test (FST) and tail suspension test (TST) 24 hours after the last ECS. We also assessed multiple behavioral and immunohistochemical measures of seizure quality. Finally, we also explored whether proliferation and differentiation of neural progenitors in the hippocampal dentate gyrus were 
affected by Narp deletion, staining for DCX and monitoring $\mathrm{DCX}+$ dendritic outgrowth in the dentate molecular layer.

Results: Narp KO mice fail to show the expected immobility reduction in the FST (two-way ANOVA shows a significant interaction between genotype and treatment: $\mathrm{F}(1,35)=5.08$, $p=0.031$; post hoc analysis of WT sham versus ECS groups, $p=0.002$; Narp KO sham versus ECS groups, $p=0.63$ ) and TST (two-way ANOVA shows a significant interaction between genotype and treatment: $\mathrm{F}(1,37)=7.83$, $p=0.00813$; post hoc analysis of WT sham versus ECS groups, $p<0.0001$; Narp KO sham versus ECS groups, $p=0.67$ ) after ECS. In theory, these deficits could be due to the inability of Narp KO mice to mount a normal seizure in response to electroconvulsive stimulation. However, we found that multiple responses elicited by seizure activity, including hippocampal neurogenesis and locomotor activity, as well as induction of c-Fos and BDNF are normal in Narp KO mice. Although Narp deletion did not suppress ECSinduced proliferation in the dentate gyrus, it did block DCX+ dendritic outgrowth of immature granule cell neurons in the dentate molecular layer induced by ECS (two-way ANOVA shows a significant interaction between genotype and treatment: $\mathrm{F}(1,20)=4.65, p=0.0435$; post hoc analysis of WT sham versus ECS groups, $p=0.000468$; Narp KO sham versus ECS groups, $p=0.273322$ ).

Conclusions: As repeated seizure activity produces a bewildering array of electrophysiological and biochemical effects in brain, it has been unclear whether it would be feasible to identify which of these play a critical role in its highly effective antidepressant effects, information that would be valuable in developing improved treatment regimens. We submit that these findings provide an important starting point for deciphering the molecular basis of ECT by focusing attention on Narp, and its role in regulating dendritic outgrowth, as a potential mediator of its antidepressant action.

Keywords: Electroconvulsive Therapy, Mechanism of Action, Hippocampus, Antidepressant

Disclosure: Nothing to Disclose.

\section{M99. Genome-Wide Analyses of Venlafaxine Response in Late-Life Depression}

Daniel Mueller*, Victoria Marshe, Malgorzata Maciukiewicz, Arun Tiwari, Daniel M. Blumberger, Jordan Karp, Charles Reynolds III, Benoit Mulsant, Etienne Sibille, James Kennedy, Eric Lenze

University of Toronto, Toronto, Canada

Background: Treatment of late-life depression (LLD) with antidepressants is challenging and often a time-consuming task. More than $50 \%$ of patients with LLD do not achieve remission, which can lead to prolonged depressive symptoms associated with greater medical complications, progressive cognitive decline and an increased risk for dementia. Therefore, developing genomic biomarkers for antidepressant treatment response will be extremely important to optimize treatment and alleviate the burden of LLD.

The objective of the current study was to conduct a genomewide association study (GWAS) to find novel variants associated with remission on venlafaxine treatment in LLD.
In addition, we used post-GWAS approaches to characterize our findings in a biological context as well as to explore the overlapping genetic architecture between depression and Alzheimer's disease.

Methods: Three-hundred and fifty-four participants $(>60$ years) of mixed ancestry, diagnosed with major depression (MADRS > 15), were treated with open-label venlafaxine (up to $300 \mathrm{mg} /$ day) for approximately 12 weeks. We genotyped 588,628 variants across the genome using the Illumina PsychArray BeadChip which was then imputed to the 1000 Genomes reference panel (Phase 3) using IMPUTE v.2.2. After imputation and restricting to a MAF $\geq 1 \%$, we included $N=7,389,525$ variants.

Associations with remission status (MADRS $\leq 10)$ and change in MADRS score were conducted using logistic/ linear regressions, adjusted for ancestry, sex, recruitment site, length in treatment, depressive episode duration, and baseline depressive severity. For associated regions, we conducted statistical and functional Bayesian fine-mapping to prioritize variants based on putative functionality, with particular enrichment across four depression-relevant brain tissues available through the Roadmap Epigenomics Project: cingulate gyrus, hippocampus, frontal lobe and substantia nigra. For a larger biological context, we conducted gene set enrichment analysis using Interval-based Enrichment Analysis (INRICH). Lastly, we compared the genomic architecture (i.e., shared genetic contributions between traits) of venlafaxine remission using GWAS summary statistics from the Psychiatric Genomics Consortium (PGC) MDD GWAS and Alzheimer's disease from the International Genomics of Alzheimer's Project (IGAP) GWAS using a polygenic risk score (PRS) approach.

Results: Although there were no variants survived corrections for multiple testing $(p<5 \times 10-8)$ within our European$(N=310)$ or African- $(N=34)$ ancestry subsamples, we observed several suggestive associations within genes relevant for neuropsychiatric disorders.

Our top hits with MADRS score change were located in variants near MIR1246 $(\beta=16.98, p=9.22 \times 10-7)$ and in ERBB4 $(\beta=6.46, p=5.35 \times 10-7)$. ERBB4 which has been implicated in risk for schizophrenia, was also associated with post-ketamine treatment down regulation of GABA and glutamate levels in the rat prefrontal cortex and hippocampus, resulting in an antidepressant effect. We also observed a suggestive association between remission status and a variant in phosphodiesterase gene PDE9A $(\mathrm{OR}=6.03, p=3.30 \times 10$ 6 ), suggesting a role in synaptic neurotransmitter signaling. PDE9A has been previously associated with risk for depression but not with response to fluoxetine or desipramine, two antidepressants of different classes. Our top empirically significant pathways included processes in telomere maintenance via recombination (GO:0000722), DNA-dependent DNA replication (GO:0006261) and positive regulation of cAMP biosynthetic process (GO:0030819). Overall, our PRS analyses revealed that there was no significant evidence for a shared genetic architecture between risk for MDD or Alzheimer's in both MADRS score change or remission status. However, there was a trend contribution to remission status from variants associated with MDD risk at a $\mathrm{p}$-value threshold of $p=0.02(\mathrm{NSNPs}=4925, \mathrm{R} 2=0.008$, $p=0.09$ ). 
Conclusions: Our study in a unique well-characterized sample of patients with LLD revealed interesting suggestive genetic associations with measures of venlafaxine remission. Notably, ERBB4 was also implicated in ketamine response and suggests an involvement of GABA and glutamate signaling. As for PDE9A, our findings suggest a different underlying genetic contribution to response with venlafaxine compared to other antidepressants of different classes. Our pathway analyses indicated that processes involved in ageing including telomere and DNA integrity maintenance might be important for the etiology of depression in older adults.

Our future steps include building integrated machine learning model to ultimately predict remission at baseline to optimize and personalized LLD treatment. In addition, we are working towards validating our findings in larger and pooled samples of depression in older adults.

Keywords: Late-Life Depression, GWAS, Antidepressant, Venlafaxine, Bioinformatics

Disclosure: Nothing to Disclose.

M100. Endogenous Kynurenic Acid Mediates Ketamineand (2R,6R)-Hydroxynorketamine-Induced Increases in Extracellular Glutamate and Antidepressant Actions

Robert Schwarcz*, Hui-Qiu Wu, Panos Zanos, Todd Gould

Maryland Psychiatric Research Center, Baltimore, Maryland, United States

Background: (R,S)-ketamine (ketamine), a non-competitive NMDA receptor (NMDAR) antagonist, causes rapid and sustained antidepressant effects in treatment-resistant patients. Production of ketamine's metabolite (2 R,6 R)-hydroxynorketamine (HNK) is essential, and (2 R,6 R)-HNK itself is sufficient to reproduce the antidepressant effects of ketamine in rodent models. The antidepressant actions of (2 R,6 R)-HNK are independent of NMDAR inhibition but appear to be critically dependent on an increase in extracellular glutamate. The mechanism by which (2 R,6 R)-HNK, as well as ketamine itself, raise glutamate levels are unknown, however. We now tested the hypothesis that the astrocyte-derived metabolite kynurenic acid (KYNA), which is known to control extracellular glutamate levels in the brain, plays a central role in the biochemical and behavioral effects of both ketamine and (2 R,6 R)-HNK.

Methods: Experiments were performed using 1) in vivo microdialysis in the medial prefrontal cortex (mPFC) of freely moving rats and 2) two depression models in mice (forced swim test and learned helplessness).

Results: Assessed by microdialysis, acute, systemic administration of either ketamine or $(2 \mathrm{R}, 6 \mathrm{R})$ - HNK (both at $20 \mathrm{mg} / \mathrm{kg}$ ) significantly reduced the extracellular levels of KYNA (maximal effect of $-30 \%$ after 2 hours), concomitant with an increase in extracellular glutamate levels (maximal effect of $+179 \%$ after 3 hours), in the mPFC. Pre-treatment with KYNA's bioprecursor L-kynurenine ( $5 \mathrm{mg} / \mathrm{kg}$, ip), or the kynurenine 3-monooxygenasease inhibitor Ro 61-8048 $(20 \mathrm{mg} / \mathrm{kg}$, ip) prevented both of these acute effects of ketamine and (2 R,6 R)-HNK. Behaviorally, pre-treatment with L-kynurenine prevented ketamine- and (2 R,6 R)-HNKinduced decreases in forced swim test immobility time, and
(2 R,6 R)-HNK-induced reversal of learned helplessness, in mice. Reverse dialysis of the sodium channel blocker tetrodotoxin $(5 \mu \mathrm{M})$, used to prevent neuron excitability, did not modify ketamine- or (2 R,6 R)-HNK-induced effects on either KYNA or glutamate in the rat $\mathrm{mPFC}$, suggesting a central role of astrocytes in the phenomena described here. Conclusions: Our data indicate that the antidepressant actions of ketamine, via the production of ( $2 \mathrm{R}, 6 \mathrm{R})-\mathrm{HNK}$, are mediated by KYNA-related modulation of glutamate neurotransmission.

Keywords: Ketamine, Kynurenic Acid, Astrocyte, Antidepressant, Glutamate

Disclosure: Nothing to Disclose.

M101. Celecoxib Augmentation of Escitalopram in Treatment-Resistant Bipolar Depression: Effects on TRYCATS

Monica Feliz Castillo*, Stephen Murata, Markus Schwarz, Brendan Martin, Angelos Halaris

Loyola University Chicago Stritch School of Medicine, Maywood, Illinois, United States

Background: In bipolar disorder, the depressive phase most adversely impairs the individual's quality of life. Failure to respond during anti-depressant therapy has been reported to be 3.4 times more common in bipolar depressed patients. It is thought that the chronic pro-inflammatory state associated with stress and depression may be associated with a pathological shunt of tryptophan metabolism toward the kynurenine pathway (TRYCATS) and thereby interfere with the ability of an antidepressant agent to exert its full therapeutic action. The purpose of this study was to determine if modulation of the inflammatory response via inhibition of cyclooxygensa-2 (COX-2) would exert a normalizing effect on specific TRYCATS, especially the neurotoxic Quinolinic Acid (QA). It was hypothesized that co-administration of the anti-inflammatory agent, celecoxib (CBX), with the SSRI antidepressant, escitalopram (ESC), would reverse treatment resistance in bipolar depression and demonstrate an augmented response while decreasing QA blood levels over time. We further hypothesized that by restoring the pathway towards the physiological pattern, anti-inflammatory agents may convert a treatment-resistant bipolar depressed patient into a partial responder or a remitter. We had previously demonstrated that administration of ESC can reduce neurotoxic metabolites of the kynurenine pathway in major depression by showing reductions of plasma levels of 3-hydroxykynurenine and QA, indicating that ESC may exert its antidepressant effect in part through inhibition of synthesis of certain neurotoxic TRYCATS and through reduction of the inflammatory response (Halaris et al, 2015). QA is synthesized by activated microglia and acts as an endogenous NMDA-receptor agonist with neurotoxic properties. Therefore, QA levels at baseline may prove to be a useful biomarker in predicting patient treatment response.

Methods: This was a randomized, double-blind, two-arm, placebo-controlled study consisting of a screening visit, a 2week washout, and a 1-week placebo run-in phase. Subjects who met study criteria and were not placebo-responders 
underwent a physical exam, medical history, routine laboratory tests, and completed a number of rating instruments. At their baseline visit, they were rated in a blinded manner, as if they had been actively treated. If they continued to score $\geq 18$ on the 17-item Hamilton Depression scale (HAM-D), they were randomized to receive ESC+CBX, or ESC+placebo. The overall study was powered for 70 patients (35 in each arm of the study) to complete 8 weeks of active medication to be considered study completers. A database of normative (untreated) data had already been collected in a previous study.

Blood levels of inflammation biomarkers and TRYCATS were determined at baseline and weeks 4 and 8. Plasma was immediately separated and frozen at $-70^{\circ} \mathrm{C}$. Blood levels of CBX and ESC were determined. A Wilcoxon Rank Sum test was used to assess for differences in QA levels between CBX and placebo receiving patients at baseline. Univariable exact logistic regression analyses were then used to compare rates of treatment response or remission by drug therapy. A binomial distribution was specified for the response variable, while a logit link was used to estimate the odds ratio for each of the explanatory variables against a referent. A linear mixed effects model was also used to estimate patients' QA values over time by drug therapy after adjusting for sex, age, and body mass index (BMI). Random intercepts were allowed for each patient to account for their multiple observations over the 8-week study period.

Results: The combination of ESC with CBX over an 8-week treatment period led to decreased levels of patient-reported depressive symptoms vs. ESC with placebo. This is based on the greater number of patients with symptom response and remission in comparison to the ESC+placebo group at 8 weeks. Patients receiving CBX were 4.13 (95 CI: 1.03-18.48) times more likely to respond to treatment compared to those receiving placebo ( $\mathrm{p}=0.04)$, and 14.34 (95 CI: 2.59-153.17) times more likely to experience remission compared to those receiving placebo $(\mathrm{p}<0.001)$. Overall, patients receiving $\mathrm{CBX}($ mean $=55.69$, standard error $[\mathrm{SE}]=6.27$ ) had comparable QA values to patients receiving placebo (mean $=64.90$, $\mathrm{SE}=7.20, p=0.34)$. Although co-administration of ESC with CBX reversed treatment resistance and showed an augmented response, we did not detect a normalization of serum kynurenine metabolites in these patients. Lastly, QA values did not change significantly over time independent of which therapy the patients received $(p=0.28)$.

Conclusions: The goals of this study were to determine if QA levels were different in bipolar depressed subjects compared to controls, and if modulation of the inflammatory response would normalize TRYCATS, especially neurotoxic QA levels, thereby reversing treatment resistance. In a prior study of MDD we had demonstrated reduction of neurotoxic TRYCATS, indicating that ESC may exert its antidepressant effect in part through inhibition of synthesis of certain neurotoxic metabolites and through reduction of the inflammatory response. Normalization of TRYCATS did not occur in the present study, since patients receiving CBX had comparable QA values as those receiving placebo, and QA levels did not change significantly over time based on drug therapy.

Keywords: Bipolar Depression, Inflammation, Celecoxib, Kynurenines

Disclosure: Nothing to Disclose.
M102. Medically Unexplained Neurologic Symptoms in Bipolar I Disorder: A Latent Class Mixture Modeling Approach

Juliet Edgcomb*, Berit Kerner

University of California, Los Angeles Semel Institute for Neuroscience and Human Behavior, Los Angeles, California, United States

Background: In the United States, medically unexplained somatic symptoms are estimated to contribute to $\$ 256$ billion in health care costs every year. Recent meta-analytic studies have suggested individuals with bipolar spectrum disorders experience physical symptoms at a rate similar to those with unipolar depression. Nearly one-third of referrals to general neurology clinics are for patients who report symptoms poorly explained by identifiable organic disease. Medically unexplained neurologic symptoms are prevalent amongst those with psychiatric disorders, contribute to lower quality of life and increased global burden of disease, and are not well understood as they relate to populations with affective disorders, especially Bipolar I Disorder (BID). This study is designed to 1) investigate markers of disease severity in those with BID and medically unexplained neurologic symptoms, and 2) assess heterogeneity amongst medically unexplained neurologic symptoms in BID to identify clinical subtypes.

Methods: Data was provided by the National Institute of Mental Health (NIMH) Repository and Genomic Resources Center for Collaborative Genomics Research on Mental Disorders. Our sample consists of 3012 unrelated adult patients with bipolar I disorder (BID) (mean age $=42.8,62 \%$ female), of whom, 667 experienced physical symptoms and 186 experienced medically unexplained neurologic symptoms (MUNS). Multivariate logistic regression and analyses of covariance were utilized to identify predictors of disease severity in the sample. Latent Class Mixture Modeling was conducted to estimate the Latent Class (LC) membership for each individual using estimation maximization algorithm based on the probability of endorsing a profile of 12 variables corresponding to varied neurologic symptoms. Comorbid anxiety disorders were added to the model as covariates. Using draws from posterior probabilities, auxiliary variables were included in the model to evaluate additional potential class predictors. A step-wise approach was adopted to incorporate predictors and investigate distal outcomes.

Results: Individuals with BID and MUNS are more likely to attempt suicide (64\%) compared with non-neurologic somatizers (58\%) and nonsomatizers (46\%) (Chi-square = $45.1, p<0.0001)$. When accounting for covariates, MUNS were $1.3 \mathrm{x}$ more likely to have attempted suicide $(\operatorname{ExpB} 1.527$, CI 1.27-1.84) compared with nonsomatizers, and reported a higher mean number of attempts (Means: MUNS 4.69, somatizers 3.81, and nonsomatizers 3.23; $\mathrm{F}=4.447$, $p=0.012$ ). Number of hospitalizations differed such that MUNS tended to have more hospitalizations (Mean 8.06) compared with nonsomatizers (6.59) and non-neurological somatizers (6.35). Age at first hospitalization, duration of most severe mania, duration of most severe depression, age of onset of most severe mania, and age of onset of most severe depression did not differ significantly amongst somatization groups. Latent mixture model fit indices 
suggested a division of the MUNS sample into three subclasses (LC1, LC2, LC3), based on the type of neurologic symptoms and their correlations. LC1 was characterized by a high probability of all neurologic somatic symptoms $(N=22)$, LC2 was characterized by musculoskeletal symptoms (weakness, difficulty walking, seizures; $N=14$ ), and LC3 was characterized by sensory somatic symptoms (blindness, hearing loss, paralysis, amnesia; $N=142$ ). After comorbid anxiety disorders were added to the model as covariates, auxiliary variables were investigated. Latent class was independently associated with disease severity, including days of duration of most severe mania, rates of comorbid substance use disorder, and rates of comorbid personality disorder. Data on prescribed medications for the individuals in the sample are available and can be used, with further analysis, as a guidance to pharmacologic treatment.

Conclusions: To our knowledge, this is the first investigation of medically unexplained neurologic symptoms in bipolar I disorder. Our results suggest that MUNS in bipolar I disorder are an independent risk factor for suicidality and hospitalization. The heterogeneity amongst MUNS may be explained by three latent classes; each of which has a unique pattern of comorbid disorders and markers of disease severity. Limitations include use of self-reported data to assess neurologic symptoms and retrospective correlational analyses. Future research should be conducted to replicate these findings using prospective methods and objective measures, such as analysis of medical records. Overall, our results emphasize the importance of jointly addressing physical symptoms and psychiatric comorbidity to mitigate burden of disease.

Keywords: Bipolar Disorder, Psychosomatic Medicine, Neurological Disorders, Affective Disorders, Mood Disorders Disclosure: Nothing to Disclose.

M103. Predicting Individual Responses to the Electroconvulsive Therapy With Hippocampal Subfield Volumes in Major Depression Disorder

Bo Cao*, Qinghua Luo, Yixiao Fu, Lian Du, Tian Qiu, Xiangying Yang, Xiaolu Chen, Qibin Chen,

Jair C. Soares, Raymond Y. Cho, Xiang Yang Zhang, Haitang Qiu

University of Texas Health Science Center at Houston, Houston, Texas, United States

Background: Electroconvulsive therapy (ECT) is the oldest yet the most effective treatment for major depression disorder (MDD). ECT can induce neurogenesis and synaptogenesis in hippocampus, which may lead to the increase of whole hippocampal volume. However, the hippocampus contains distinct subfields, e.g., the cornu ammonis (CA) subfields, a granule cell layer (GCL), a molecular layer (ML), and the subiculum. In this study, we aimed to 1) investigate which hippocampal subfields were affected by ECT in MDD and 2) predict the treatment response to ECT using subfield volumes at baseline.

Methods: Twenty-four patients with severe MDD received the modified ECT and their structural brain images were acquired with magnetic resonance imaging before and after ECT. A state-of-the-art algorithm was used for hippocampal segmentation. The ECT effect on hippocampal subfield volumes was investigated using repeated-measurement analysis of variance. A machine learning algorithm was applied on the hippocampal subfield volumes to predict the individual response to ECT.

Results: We found that ECT induced volume increases in CA subfields, GCL, ML and subiculum. We were able to predict the change in depressive symptoms $(r=0.71)$. Within the remitters, the degree of alleviation of depressive symptoms by ECT could be predicted with high accuracy $(r=0.95)$. Receiver operating characteristic (ROC) analysis also showed the robust prediction of remission with an area under the curve of 0.84 .

Conclusions: Our findings provide evidence for particular hippocampal subfields having specific roles in the response to ECT treatment in MDD. We also provide an analytic approach for generating predictions about clinical outcomes for ECT in MDD using hippocampal subfield volumes and machine learning methods.

Keywords: Major Depressive Disorder (MDD), ECT, Hippocampal Subfields

Disclosure: Nothing to Disclose.

M104. Machine Learning can Predict Clinical Response to Transcranial Magnetic Stimulation: A Resting State EEG Study of Posttraumatic Stress Disorder and Major Depression

Amin Zand Vakili*, Noah Philip, Stephanie Jones, Audrey Tyrka, Benjamin Greenberg, Linda Carpenter

Alpert Medical School, Brown University, Providence, Rhode Island, United States

Background: Repetitive transcranial magnetic stimulation (rTMS) is an FDA cleared treatment for Major Depressive Disorder (MDD), and its effectiveness is being studied in other neuropsychiatric conditions such as Posttraumatic stress disorder (PTSD). rTMS is thought to produce its effect by modulating cortical circuitry. However, the exact mechanisms of these changes are not entirely understood. Use of Machine Learning and quantitative analytics can provide a powerful tool to study changes in cortical circuitry associated with rTMS and can elucidate its mechanism of action, and in doing so, may provide a tool to predict and objectively track clinical improvement. Here we coupled Machine Learning with an inexpensive, sparse EEG array to test whether this combination could detect treatment changes and potentially predict outcomes.

Methods: We collected resting-state 8-channel EEG data in 35 patients with MDD and PTSD before and after an unblinded course of rTMS $(5 \mathrm{~Hz}$ to the left dorsolateral prefrontal cortex, 3000 pulses per session up to 40 sessions). Clinical ratings in both domains (using the PTSD checklist (PCL-5) and Inventory of Depressive Symptomatology (IDSSR) for PTSD and MDD symptoms, respectively) were acquired prior to and following rTMS. We derived connectivity of all possible recording site pairs (28) before and after rTMS and applied a Machine Learning algorithm (Support Vector Machine) to determine how connectivity changed after treatment. We also used a sparse regression model (least absolute shrinkage and selection operator) and 
tested the hypothesis that baseline connectivity could be used to predict clinical outcome.

Results: Interpretable baseline data were analyzed from 21 patients who had noise free EEG recorded pre- and posttreatment. The SVM algorithm was trained to identify preand post-rTMS EEG recordings based on coherence in 4 frequency bands. The algorithm was able to assign EEG recordings to pre- and post-treatment with notable accuracy on a cross validated sample (performance for Alpha, Beta, Theta and Delta at $75.40 \pm 1.47 \%, 77.44 \pm 1.44 \%$, $73.81 \pm 1.47 \%$ and $78.57 \pm 1.42 \%$ respectively). All performances were above chance $(50 \%$, all $p<.001)$. Post-hoc analysis revealed that for most frequency bands (Alpha, Beta, Theta) classification was achieved mostly using local modulation of connectivity in the left prefrontal cortex (the site of stimulation) as well as longer connections between left prefrontal and midline occipital-central electrodes.

We also found that clinical improvement (quantified as percent reduction in PCL-5 and IDS-SR) was predictable based on pretreatment connectivity, particularly using Alpha band coherences. Our model predicted clinical improvement based on Alpha coherence with r square of 0.33 (PCL-5) and 0.41 (IDS-SR). Both were significantly better than chance (cross validated prediction, $p=0.006$ for PCL-5 and $p=0.002$ for IDS-SR). When we define a $50 \%$ reduction of these scales as "clinical improvement," the model was able to screen for patients who would improve with a sensitivity of $55.56 \%$ and a specificity of $91.67 \%$ for PCL-5 and a sensitivity of $55.56 \%$ and a specificity of $75.00 \%$ for IDS-SR on a cross-validated sample.

Conclusions: This initial study using a sparse EEG array and Machine Learning algorithm suggests that EEG connectivity metrics have a promise both as a biomarker of mechanism and in response prediction. Machine Learning approaches were able to detect global trends in data that were not otherwise detectable. We also found that EEG based biomarkers may be useful for screening and assessment of treatment response for rTMS. The model was able to detect a significant number of future non-responders to rTMS. The results presented here indicate that EEG in an office setting can provide insight into suitability and response to treatment.

Keywords: Neuromodulation, Repetitive Transcranial Magnetic Stimulation (rTMS), Machine Learning, MDD, PTSD, EEG Biomarkers

Disclosure: Nothing to Disclose.

M105. Stress Controllability Elicits Sex-Specific Structural Plasticity in Prefrontal-Brainstem Circuits

Tina Gruene, Michael Barratta, Steven Maier, Rebecca Shansky*

Northeastern University, Boston, Massachusetts, United States

Background: In male rats, control over a stressor (escapable stress, or ES) reliably protects the animals from the negative effects of stress on measures of fear, anxiety, and social behavior. This is not the case in female rats, suggesting that the key circuits that mediate ES effects are sexually dimorphic. Our goal here was to identify sex-specific patterns of stress-related plasticity in prefrontal projections to the dorsal raphe nucleus (DRN), which is central to the protective effects of ES.

Methods: Male and female animals received intra-DRN retrobead injections and then underwent escapable stress, inescapable stress, or remained in their home cage. Retrolabeled layer $\mathrm{V}$ pyramidal neurons and unlabeled neighboring cells in the prelimbic cortex were then filled with Lucifer Yellow and imaged in 3D using confocal microscopy. Dendritic segments were analyzed for spine head diameter to assess putative alterations in synaptic strengthening.

Results: In males, we observed circuit-specific enlargement of mushroom spines after ES, while IS induced non-specific enlargement of both mushroom and thin spines. In contrast, females exhibited non-specific effects regardless of stress controllability.

Conclusions: These findings demonstrate that stress-induced structural plasticity in the prefrontal cortex is both sex- and circuit-specific. Importantly, they point to a potential mechanism by which escapable stress fails to protect females from long-term effects of stress.

Keywords: Acute Stress, Sex Differences, Prefrontal Cortex, Plasticity

Disclosure: Nothing to Disclose.

\section{M106. Neural Correlates of Treatment Response to Intravenous Ketamine in Adolescents With Treatment- Resistant Depression}

Kathryn Cullen*, Bonnie Klimes-Dougan, Palistha Amatya, Michelle Thai, Melinda Westlund Schreiner, Mark Roback, Sophia Albott, Yanan Ren, Lynn Eberly, Kelvin Lim, Bryon Mueller

University of Minnesota Medical School, Minneapolis, Minnesota, United States

Background: Depression is a severe disorder that often begins during adolescence. Resistance to standard treatments is a harbinger for severe outcomes such as chronicity and suicide. Advancement in the care of treatment-resistant depression (TRD) in adolescents is urgently needed. Adolescence is a time period notable for significant neurodevelopmental change, highlighting the importance of testing novel treatments that might take advantage of the advanced neuroplasticity that is inherent to developmental periods. Ketamine is a glutamatergic agent that has shown promise in adults with treatment-resistant depression (TRD); neuroscience research investigating potential mechanisms of this effect have suggested that ketamine may serve to restore impaired neuroplasticity to promote positive neural changes. No studies have yet been published testing the efficacy or biological effects of medication in adolescents. Application of high-spatial and temporal resolution functional magnetic resonance imaging (fMRI) to measure resting-state functional connectivity (RSFC) is one approach to assess how disease and treatment can alter neural networks. Identification of treatment-induced RSFC changes can shed light on the neural mechanisms underlying treatment response.

Methods: This is an open-label pilot study consisting of a series of 6 intravenous ketamine infusions $(0.5 \mathrm{mg} / \mathrm{kg})$ over the course of two weeks. We measured depression severity 
$\mathrm{SI} 80$

using the Child Depression Rating Scale-Revised (CDRS-R), and collected resting-state fMRI at baseline and 24 hours after the last infusion. A significant clinical response was defined as a $50 \%$ improvement on the CDRS-R at the posttreatment evaluation. Brain imaging was performed on a Siemens Prisma scanner. Resting-state imaging parameters were as follows: $2 \mathrm{~mm}$ isotropic voxel, $\mathrm{TR}=0.71 \mathrm{~s}$, field of view $=104 \times 104 \times 72,680$ volumes, multiband factor $=8$. A high-resolution $\mathrm{T} 1$ image was also collected (1mm isotropic voxel, 5 minutes). FreeSurfer was used to parcellate the anatomical data. Pre-processing steps included skull-stripping, distortion correction, high-pass temporal filtering, and spatial smoothing ( $3 \mathrm{~mm}$ kernel). Noise due to physiological signal and to motion was removed using FSL FIX. RSFC analyses focused on the amygdala, a brain region implicated in negative affect and in depression. We conducted wholebrain regression analyses using the mean time series from the FreeSurfer-based anatomically-defined left and right amygdala as "seeds", and including the time series from cerebrospinal fluid and white matter regions of interest as covariates of no interest. These first-level anlayses yielded statistical maps for left amygdala RSFC and right amygdala RSFC for each person. Pre-infusion left and right amygdala connectivity maps were subtracted from post-treatment left and right amygdala connectivity maps to yield whole-brain statistical maps representing change in amygdala connectivity at each voxel. Clinical change (post-minus-pre CDRS-R scores) were regressed against the amygdala connectivity change maps to identify neural change correlates of clinical response. Statistical images were voxel-wise thresholded at $p<0.005$; significant (alpha $<0.05)$ cluster sizes were determined using $3 \mathrm{dCl}$ ustSim (AFNI).

Results: Thirteen adolescents with TRD completed the protocol; at the time of this submission, data were available for 12 patients for analysis. After the ketamine infusions, there was a significant reduction in CDRS-R scores $(p=0.001)$. However, there was substantial variability in response, and only 5 participants met criteria for significant clinical response. Greater clinical improvement was significantly correlated with greater decrease in amygdala RSFC with anterior cingulate cortex and hippocampus/parahippocampus, but with greater increase in amygdala RSFC with superior frontal and medial frontal cortex (this pattern was noted bilaterally).

Conclusions: This study suggests that ketamine may be a promising treatment for adolescent TRD, and that the underlying mechanism of this effect may involve enhancing neuroplastic processes to re-shape fronto-limbic neural circuits -dampening connections that may be contributing to deleterious emotion processing, and promoting more healthy connections- to alleviate symptoms. Limitations of this work include the small sample and the lack of a placebo arm; future randomized controlled studies are needed to confirm the findings. Advancement in neuroscience-based treatment for depression would have a profound impact on public health by preventing negative outcomes and restoring healthy developmental trajectories for adolescents with depression.

Keywords: Ketamine, Adolescent Depression, Resting State Functional Connectivity

Disclosure: Nothing to Disclose.
M107. Ventral CA3 DeltaFosB Mediates Prophylactic Ketamine Efficacy Against Stress-Induced Depression

Alessia Mastrodonato*, Randy Martinez, Ina Pavlova, Christina LaGamma, Josephine McGowan, Rebecca Brachman, Alfred Robison, Christine Ann Denny

Columbia University, Research Foundation for Mental Hygiene, Inc., New York, New York, United States

Background: Stress exposure is a major risk factor for mood disorders, such as major depressive disorder (MDD) and post-traumatic stress disorder (PTSD). However, some individuals can successfully adapt to stress, which is known as stress resilience. We previously reported that a single injection ketamine prior to stress protects against the development of depressive-like behavior and buffers against fear in mice. However, the cellular and molecular pathways underlying ketamine-induced stress resilience are still largely unknown.

Methods: $129 \mathrm{~S} 6 / \mathrm{SvEv}$ mice were injected with saline or ketamine $(30 \mathrm{mg} / \mathrm{kg}) 1$ week before a 10 - day social defeat (SD) paradigm. Following SD, mice were sacrificed and the brains were processed to visualize deltaFosB expression. In a second set of experiments, mice were stereotaxically injected with viral vectors into ventral CA3 (vCA3) in order to upregulate or downregulate deltaFosB expression. The following viral vectors were used: adeno-associated virus (AAV2) expressing GFP alone (AAV-GFP), AAV expressing GFP and deltaFosB (AAV- $\Delta$ FosB), or AAV expressing GFP and deltaJunD (AAV- $\Delta$ JunD). Two weeks after surgeries, mice were administered a single injection of saline or ketamine $(30 \mathrm{mg} / \mathrm{kg})$. One week later, mice were subjected to a contextual fear conditioning (CFC) paradigm, forced swim test (FST), elevated plus maze (EPM) and open field test (OF). In a third set of experiments we utilized ArcCreERT2 mice, a line that allows for the indelible labeling of neural ensembles representing a single behavioral experience, in order to visualize memory traces following prophylactic ketamine administration.

Results: We found that prophylactic ketamine administration, but not SD, increases deltaFosB expression in the prefrontal cortex (PFC), specifically the infralimbic cortex (IL), prelimbic cortex (PL), and anterior cingulate $(\mathrm{CgL})$. However, in the hippocampus (HPC), prophylactic ketamine administration in the SD group increased deltaFosB expression in the ventral DG (vDG) and vCA3. In the viral injected cohort of mice, we found that blockade of deltaFosB activity in vCA3 inhibited prophylactic ketamine efficacy while overexpression of deltaFosB mimicked and occluded ketamine's effects. Finally, in the ArcCreERT2 mice, we found that ketamine administration altered memory traces relating to the stressful experience specifically in vCA3, but not in vDG.

Conclusions: Our data indicate that prophylactic ketamine may be protective by altering deltaFosB expression in the ventral HPC. By understanding how deltaFosB expression changes with depressive-like behavior, and how prophylactic ketamine influences its expression, our work elucidates both the pathophysiology of depression and provides insights into novel treatment targets.

Keywords: deltaFosB, Ketamine, Depression

Disclosure: Nothing to Disclose. 
M108. SAGE-217: A GABAA Positive Allosteric Modulator in Subjects With Major Depressive Disorder - Results From an Open-Label, Phase 2 Trial

Stephen Kanes*, Georgios Nomikos, Handan Gunduz-Bruce, Inder Kaul, Robert Riesenberg, Abdul Sankoh, Haihong Li, Steven Paul, David Rubinow, Charles Zorumski, James Doherty

Sage Therapeutics, Cambridge, Massachusetts, United States

Background: Major depressive disorder (MDD) remains a major cause of disability worldwide with significant human suffering, loss of lives and high economic burden. Current treatments offer variable improvement, require several weeks of dosing and present safety and tolerability challenges, indicating an important need to develop more effective, safe and tolerable novel antidepressants. Neuroactive steroids (NAS), such as $3 \alpha-$ hydroxy-5 $\alpha$-pregnane-20-one (allopregnanolone) are potent positive allosteric modulators (PAMs) of synaptic and extrasynaptic GABAA receptors (GABA-R). Cerebrospinal fluid allopregnanolone levels are reduced in depressed subjects and increase following treatment with selective serotonin uptake inhibitors, with a significant correlation between increasing levels and improvement. These findings suggest a critical role for GABA-R PAM NASs and GABAergic neurotransmission in the pathogenesis of depression. SAGE-217 is a novel NAS with oral availability and a pharmacokinetic profile amenable to once daily dosing. In Phase 1 studies, SAGE 217 was generally well tolerated in healthy volunteers. The objectives of this open label Phase 2 study were to assess the safety and tolerability of SAGE-217 in subjects with MDD, and to evaluate if a two-week treatment with SAGE-217 would lead to a reduction in depressive symptoms. An additional focus was to assess the speed and durability of response.

Methods: Male and female subjects between ages 18-65 years of age, experiencing a major depressive episode (diagnosed according to DSM-V, with a severity by Hamilton Depression Rating Scale [HAM-D] total score $\geq 22$ ) were enrolled in the study. Subjects who were taking antidepressants at time of enrollment remained on their stable dose throughout the study; no new psychotropics were allowed. Subjects received a $30 \mathrm{mg}$ dose of SAGE-217 Oral Solution at night on Days 114. The frequency and severity of adverse events (AEs) were summarized descriptively, and changes from baseline depression scores using HAM-D and the MontgomeryÅsberg Depression Rating Scale (MADRS), and anxiety scores using the Hamilton Anxiety Rating Scale (HAM-A), were assessed and analyzed using descriptive statistics and mixed effects models for repeated measures. Additional endpoints included Clinical Global Impression-Severity and Improvement (CGI-S and CGI-I) parameters.

Results: Thirteen subjects with a mean (SD) baseline HAMD score of 27.2 (3.06) were enrolled in the study. Five patients were receiving stable doses of antidepressants in the study; the remaining 8 patients took no concomitant medications and responses were similar in both groups.

Mean HAM-D total score was reduced from baseline by 19.9 points at the end of treatment period (Day 15) $(p<0.0001)$. Similarly, on Day 15, HAM-D response, defined as having $\geq 50 \%$ reduction in HAM-D, was $85 \%$ (11/13 subjects) and $62 \%$ (8/13 subjects) achieved remission, defined as HAM-D $\leq 7$. Statistically significant reductions from baseline HAM-D total scores were observed as early as Day $2(p=0.0009)$. By Day 7, HAM-D response was $62 \%$ (8/13 subjects) and remission was 39\% (5/13 subjects). Reductions in depressive symptoms were maintained through the end of treatment period (Day 15, $p<0.0001$, primary endpoint) and persisted during the follow-up period (Day 28, $p<0.0001$ ).

Similar improvements were observed with MADRS and HAM-A. The mean MADRS total score at baseline was 36.9 and there was a mean reduction of 26.4 points at Day 15 $(p<0.0001)$. MADRS ratings similarly showed significant reductions from baseline scores beginning with Day 2 $(p=0.02)$, throughout the treatment period at Day 15 and at follow up on Day 28 (both $p<0.0001$ ).

Baseline HAM-A scores (mean 23.2) decreased by 15.5 points at Day $15(p<0.0001)$. HAM-A scores followed a similar pattern where statistically significant reductions occurred as early as Day $3(p=0.0006)$ and maintained throughout the treatment period at Day 15 and also follow up at Day 28 (both $p<0.0001$ ).

Baseline CGI severity was $4.8(0.38)$ indicating a mean in the "moderately ill (4)" to "markedly ill (5)" range. Consistent with the large reductions observed in symptom severity, by the end of treatment at Day 15, 92\% (12/13) of the subjects were rated as "very much improved" or "much improved" on CGI-I. SAGE-217 was generally well tolerated. There were no serious or severe AEs, discontinuations or dose reductions. The most common AEs were sedation (6 subjects), headache (4 subjects), somnolence (3 subjects), dizziness (3 subjects), and myalgia (3 subjects).

Conclusions: This is the first proof-of-concept trial of SAGE-217, a GABAA PAM, in MDD. In the study, SAGE-217 was generally well tolerated. Moreover, a relatively short treatment period of 14 days with SAGE-217 was associated with a fast onset of action (within days) and clinically meaningful and durable (up to 2 weeks after the end of treatment) treatment effect. Based on these positive findings, a multicenter, double-blind, randomized clinical trial testing the efficacy and safety of SAGE-217 in MDD is currently ongoing. Overall, these results suggest a potential role for SAGE-217 in a novel class of compounds for the treatment of depression. Further studies are required.

Keywords: Major Depressive Disorder, GABA-A Receptors, Positive Allosteric Modulators

Disclosure: Part 1: Sage Therapeutics, Employee, Part 2: Sage Therapeutics, Employee, Part 3: Sage Therapeutics, Employee, Part 5: Sage Therapeutics, Employee.

\section{M109. Neuroimaging Predictors of Functional Improve- ment With Antidepressant Treatments: Findings From the EMBARC Study}

Manish Jha*, Crystal Cooper, Abu Minhajuddin, Cherise Chin-Fatt, Hanzhang Lu, Mary Phillips, Patrick McGrath, Maurizio Fava, Myrna Weissman, Madhukar Trivedi

University of Texas Southwestern Medical Center, Dallas, Texas, United States

Background: Disability due to depression extends beyond core depressive symptoms and encompasses broad-ranging 
functional impairments. Patients with major depressive disorder (MDD) continue to report significant impairment in psychosocial function even after attaining symptomatic remission. Persistent impairment in psychosocial function, in turn, is associated with lower-likelihood of sustained remission and higher-likelihood of relapse. Arterial spin labeling (ASL) is a commonly used neuroimaging tool to measure cerebral blood flow (CBF; perfusion) and has previously identified and replicated differences between depressed and healthy controls. Baseline neuroimaging biomarkers which predict persistent impairment in psychosocial function despite antidepressant medication treatment can help personalize treatment by incorporating psychosocial interventions early in course of treatment.

Methods: We used data from participants in the Establishing moderators and biosignatures of antidepressant response in clinical care (EMBARC) who completed 8 weeks of treatment with sertraline or placebo and measures of depression severity, psychosocial function, and CBF $(n=204)$. Symptomatic remission was defined as Hamilton Rating Scale for Depression 17 -item (HRSD17) score $\leq 7$. Clinically significant improvement in psychosocial function was defined as increase of $\geq 1$ standard deviation in the overall score of social adjustment scale short-form (SAS-SF). A 2x2 analysis was used to detect difference between clinically significant improvement in SASSF vs. symptomatic remission. Participants were scanned at baseline on a $3 \mathrm{~T}$ MRI, which included a pseudo-continuous ASL sequence, i.e. PCASL, that lasted approximately 6 minutes. To evaluate the neuroimaging predictors of functional improvement, whole-brain voxel-by-voxel analysis was conducted on relative $\mathrm{CBF}$ comparing remitters with clinically significant improvement in SAS-SF vs. remitters without clinically significant improvement in SAS-SF.

Results: In the EMBARC study, 56.9\% (41/72) remitters and $27.9 \%(50 / 132)$ non-remitters experienced clinically significant improvement in SAS-SF after 8 weeks of treatment. This difference was statistically significant (chi-square $=6.85, \mathrm{df}=1$, $p=0.009$ ). Remitters who did not have clinically significant improvement in SAS-SF had greater baseline relative CBF (i.e., perfusion) than remitters who experienced clinically significant improvement in SAS-SF in the cerebellum, inferior temporal gyrus, subgenual cingulate, and the inferior and orbitofrontal cortices. Clusters in these regions were significant at $\mathrm{t}=2.97$; $\mathrm{p}<.002$; with at least 75 continuous voxels.

Conclusions: Based on functional improvement after antidepressant treatment, we found cerebral perfusion differences in novel (interior temporal gyrus and cerebellum) as well as previously implicated (subgenual cingulate and orbitofrontal cortex) brain regions.

Keywords: Depression, Neuroimaging, Subgenual, Functional Impairment, Orbitofrontal Cortex

Disclosure: Nothing to Disclose.

M110. Deep Brain Stimulation of the Medial Forebrain Bundle: Continued Responses in Treatment Resistant Depression

Jair Soares*, Albert Fenoy, Paul Schulz, Christina Burrows, Giovana Zunta-Soares, Joao de Quevedo

University of Texas Houston Medical School, Houston, Texas, United States
Background: Treatment resistant depression (TRD) is a serious, debilitating disorder. Deep brain stimulation (DBS) to the superolateral branch of the medial forebrain bundle (MFB) has been reported by Schlaepfer et al. (2013) to lead to rapid anti-depressant effects. This report is an update to our recently published results (Fenoy et al., 2016).

Methods: In this pilot study, we assessed the efficacy of MFB-DBS in a cohort of six TRD patients over a 52-week period using improvement on the Montgomery-Åsberg Depression Rating Scale (MADRS) as the primary outcome measure. Implanted patients entered a 4-week single-blinded sham stimulation period prior to stimulation initiation. Deterministic fiber tracking analysis was performed to compare modulated fiber tracts between patients.

Results: Upon stimulation at target intraoperatively, responders reported immediate increases in energy and motivation. There was a significant mean change in mood during the 4week sham stimulation phase, where the mean difference in MADRS score between baseline and the end of sham stimulation was 10 pts $(28 \%$ reduction, $p=0.02)$. After initiating stimulation, 3 of 6 patients had a $>50 \%$ decrease in MADRS scores relative to baseline at 7 days. The difference in MADRS score between baseline and week 1 of active stimulation was significant (mean change $=15$ pts, $43 \%$ reduction, $p=0.005)$ as was the difference between baseline and week 2 (mean change $=17$ pts, $49 \%$ reduction, $p=0.001$ ). One patient withdrew from study participation. At 52 weeks, 4 of remaining 5 patients have $>70 \%$ decrease in MADRS scores relative to baseline; one patient failed to respond. Evaluation of modulated fiber tracts reveals significant common frontal connectivity to the target region in all responders.

Conclusions: This study of MFB-DBS confirms rapid antidepressant effects are observed with stimulation, as reported by Schlaepfer et al. (2013). Although the insertional response itself is significant, anti-depressant responses continue to be significant over time which argue against placebo, with sustained effects in responder patients.

Keywords: Deep Brain Stimulation, Treatment Resistant Depression, Medial Forebrain Bundle, MADRS

Disclosure: Part 1: BMS, Grant, Forest, Grant, Merck, Grant, Elan, Grant, Johnson \& Johnson, Grant, Astellas, Consultant, Pfizer, Honoraria, Abbott, Honoraria.

M111. Evidence for Gene Expression Biomarkers of Suicide in Patients With Mood Disorders

Derrek Hibar*, Andrew Jaffe, Joo Heon Shin, Thomas Hyde, Joel Kleinman, Daniel Weinberger, Wayne Drevets, Ziad Saad, Maura Furey, Hartmuth Kolb

Janssen Research \& Development, LLC, San Diego, California, United States

Background: Mood disorders like major depressive disorder (MDD) and bipolar disorder (BD) are among the most common psychiatric illnesses in the world. (1) One of the leading causes of death in mood disorder patients is suicide: patients show a 10 to 20 -fold increase in suicide rates compared to the general population $(2,3)$. Biomarkers of suicide which aid in the pre-emptive identification of 
patients at highest risk for suicide can be a valuable resource for reducing mortality in patients suffering from a mood disorder. Gene expression studies of postmortem brain tissue can provide biological insights into the underlying neurobiology of suicide and potentially lead to the development of novel biomarkers. In this study, we used whole genome RNA sequencing to examine gene expression differences in postmortem human dorsolateral prefrontal cortex (DLPFC) of $\mathrm{MDD} / \mathrm{BD}$ suicide completers compared to both $\mathrm{MDD} / \mathrm{BD}$ patients and medical control subjects who died from accidental or natural causes.

Methods: We conducted next-generation RNA sequencing in Phase I of the BrainSeq Consortium (4) to quantify gene expression in postmortem human DLPFC samples from 433 subjects (age range: 17-85 years; mean age: $44.3+/-14.6$ std. dev.) including $145 \mathrm{MDD}$ patients (91 suicide completers), $66 \mathrm{BD}$ patients (45 suicide completers), and 222 controls. A patient was considered a suicide completer (Sui) if manner of death was recorded as suicide after medical examination. A patient was considered a non-suicide completer (NonSui) if manner of death was recorded as accidental or natural causes after medical examination. All subjects with an indeterminant manner of death were excluded. All controls were screened for and free of significant neurological or psychiatric disorders, including any history of suicide or suicide attempts. Gene expression levels were quantified on an Illumina HiSeq 2000 (Illumina; San Diego, CA USA) following a Poly-A library protocol with 100 base pair paired-end reads and a target depth of 80-100 million reads. Reads were aligned to the hg19 transcriptome using TopHat2 (5) and counted with htseq (6). Within BD and MDD we performed three contrasts: Sui vs. NonSui, Sui vs Control, and NonSui vs. Control to test for gene expression differences using DESeq2(7). All contrast models included covariates for age of death, sex, sample RNA integrity, post mortem interval, four multidimensional components of ancestry, and ten surrogate variables for RNA degredation and quality (8). Genes were considered differentially expressed using a False Discovery Rate threshold of $\mathrm{q}<0.05(9)$.

Results: In the contrast of MDD-Sui vs Control, we found significant evidence for 976 differentially expressed genes (at FDR $\mathrm{q}<0.05 ; 504$ up-regulated and 472 down-regulated). The most significant finding was a protein coding gene TBCA $(p=6.03 \times 10-13$; log2 fold change $($ LFC $)=0.189)$. In the contrast of MDD-NonSui vs Control we found evidence for 1870 differentially expressed genes. Of the differentially expressed genes in both contrasts, 465 genes were uniquely differentially expressed in the MDD-Sui vs Control comparison (and not in MDD-NonSui vs Control) the top five by significance were: FBXW8, DYNLL1, MYCT1, CETP, and ZFYVE19. When comparing MDD-NonSui with MDD-Sui we found evidence for one differentially expressed gene, a long non-coding RNA (LINC00460; $p=2.01 \times 10-7$; $\mathrm{LFC}=0.308$ ).

In the contrast of BD-Sui vs Control, we found significant evidence for 1348 differentially expressed genes (at FDR $\mathrm{q}<0.05 ; 817$ up-regulated and 531 down-regulated). The most significant finding was a protein coding gene TUFT1 $(p=2.86 \times 10-13 ; \quad L F C=0.327)$. In the contrast of BD-NonSui vs Control we found evidence for 495 differentially expressed genes. Of the differentially expressed genes in both contrasts, 1109 genes were uniquely differentially expressed in the BD-Sui vs Control comparison (and not in BD-NonSui vs Control) the top five by significance were: MT1X, ARID5A, ANGPTL4, PIP5K1A, and MT1M. When comparing BD-NoSui with BD-Sui only one gene was differentially expressed: CTAG2 $(p=3.01 \times 10-16$; $\mathrm{LFC}=30.0$ ).

Conclusions: This study provides insight into the underlying etiology of suicide in mood disorders by applying RNA sequencing to postmortem DLPFC samples from patients with major depression and bipolar disorder. We found evidence for differential expression in 465 genes specific to MDD patients (and 1109 specific to BD patients) who were suicide completers. Direct comparisons between suicide completers vs non-suicide completers yielded few results, likely due to reduced power in the smaller sample sizes. However, TBCA in MDD and CTAG2 in BD may represent targets of interest for future studies. Interestingly, 89 genes are differentially expressed in both the MDD-Sui and BD-Sui groups (all genes are concordant in direction of effect for both disorders), which suggests that these may be general mood disorder markers of suicidality. The top gene CETP encodes a protein involved in removal of cholesterol and conversion of triglycerides into glycerol for accessing stored energy. When comparing differentially expressed genes from the MDD-Sui vs Control comparison with those found from a recent study (10) one gene was differentially expressed in both studies: TNFRSF12A, a receptor for tumor necrosis factor. These findings suggest several candidates for further study to understand the genetic mechanisms underlying suicidality in mood disorders.

Keywords: Suicide, Biomarker, Gene Expression, Mood Disorders

Disclosure: Part 1: Janssen R\&D, Employee, Part 2: Janssen R\&D, Employee, Part 3: Janssen R\&D, Employee, Part 4: Janssen R\&D, Employee, Part 5: Janssen R\&D, Employee.

\section{M112. Association Between C Reactive Protein and Nucleus Accumbens Functional Connectivity in Patients With Major Depression}

Sara Costi ${ }^{\star}$, Laurel Morris, Abigail Collins, Nicholas Van Dam, Prantik Kundu, Emily Stern, Scott Russo, James Murrough

Icahn School of Medicine at Mount Sinai, New York, New York, United States

Background: Depression is among the most disabling human illnesses worldwide, and current treatments fall short of what is required to meet this large public health need. Numerous studies have suggested that Major Depressive Disorder (MDD) is accompanied by immune dysregulation and elevation of pro-inflammatory mediators (cytokines, chemokines and acute phase proteins) in circulating serum or plasma. There is now substantial evidence that peripheral pro-inflammatory mediators impact mood state and reward responsiveness via changes within a discrete reward circuit, which includes projections from the ventral tegmental area (VTA) to the nucleus accumbens (NAc) and medial 
$\overline{S 184}$

prefrontal cortex (mPFC). In the current study, we measured peripheral C-reactive protein (CRP; an acute-phase proinflammatory protein) and resting state functional connectivity (RSFC) using advanced multi-echo multiband (MEMB) functional magnetic resonance imaging (fMRI) at 3 Tesla in subjects with MDD and healthy volunteers. The current report focused on the relationship between CRP and RSFC within the reward circuit, and represents a preliminary analysis of an ongoing project investigating inflammatory and neurocircuit mechanisms of anhedonia in depression (Grant number: R21MH109771).

Methods: The project enrolled subjects suffering from current MDD and a group of healthy control volunteers (HC). Severity of depression and anhedonia were measured by the Montgomery-Åsberg Depression Rating Scale (MADRS) and blood was collected to measure high sensitivity CRP (hs-CRP) using a latex-enhanced immunoturbidimetry prior to brain imaging procedures. Resting state functional MRI data was collected using a MEMB acquisition sequence for improved temporal resolution and signal power, particularly for subcortical structures. Four echos were acquired with a multi band acceleration factor of 5 . Anatomical data were collected with an MP2RAGE sequence. Functional data were denoised with multi-echo independent component analysis (me-ica, https://bitbucket. org/prantikk/me-ica) for robust BOLD separation based on component echo time dependence. Functional and anatomical data were coregistered and normalized to MNI space. Functional connectivity between bilateral NAc and the whole brain was computed and compared between groups. Additionally, these functional connectivity maps were correlated with hs-CRP.

Results: Twelve subjects with MDD ( $41 \pm 12$ years old; $58.3 \%$ male) and thirteen healthy controls ( $\mathrm{HC}, 33 \pm 7.7$ years old; $69.2 \%$ male) had data available for the current interim analysis. Within the MDD, group mean MADRS score was $27.61 \pm 4.42$; within the HC group, mean MADRS score was $0.84 \pm 1.21$. Mean hs-CRP in MDD was $2.2 \pm 2.85$ and in HC was $1.33 \pm .95$. Consistent with our hypotheses, we detected a positive association between hs-CRP and MADRS score, although at a trend level $(r=.364, p=.068)$. There were no group differences in functional connectivity of the NAc in this preliminary sample. Within the MDD group, there was a positive correlation between hs-CRP and connectivity of the NAc with 3 clusters, including a ventral midbrain region encompassing VTA, lateral prefrontal cortex and inferior parietal cortex [voxelwise $p<0.01$ and afni's 3dClustSim cluster correction with a non-gaussian spatial autocorrelation function (acf), cluster $>104$, alpha $<0.05$ ].

Conclusions: Consistent with basic and clinical evidence, these findings suggest a link between peripheral inflammatory markers and connectivity within the reward circuit. This circuitry may therefore serve as a target for antiinflammatory treatment strategies that, through the reduction of peripheral levels of hs-CRP and pro-inflammatory mediators, might lead to clinical improvement in patients with major depression.

Keywords: Major Depressive Disorder (MDD), Inflammation, Brain Connectivity

Disclosure: Nothing to Disclose.
M113. Clinical Qualification of [18F] JNJ-64413739, a Novel Candidate PET Ligand for the P2X7 Ion Channel

Mark Schmidt*, Michel Koole, Jan de Hoon, Anja Hijzen, Paulien Ravenstijn, Marc Ceusters, Anindya Bhattacharya, Michael Letavic, Wei Zhang, Hartmuth Kolb, Sofie Celen, Guy Bormans, Koen Van Laere

Janssen Research \& Development, LLC, Beerse, Belgium

Background: P2X7 is an ATP-gated cation channel embedded in the cell membrane. In the CNS, P2X7 receptors have been identified on several cell types with the most abundant expression on microglia. Activation of the P2X7 promotes the NLRP3 inflammasome assembly and release of neuroactive cytokines such as IL1 $\beta$ and IL-18. Overactivation of this pathway is hypothesized to play a role in the pathophysiology of mood disorders. Selective, brain penetrant inhibitors of P2X7 have been developed as candidate therapeutics. A site-specific PET ligand for the P2X7 receptor could provide evidence of target engagement and assist in clinical dose selection. [18 F] JNJ-64413739, a selective P2X7 antagonist, has been extensively evaluated in preclinical models as a candidate PET ligand. We now report the clinical qualification of the ligand in healthy adult males including dosimetry, brain distribution, test-retest (TRT) variability, and blocking by pharmacological doses of the P2X7 inhibitor JNJ-54175446.

Methods: For the radiation dosimetry, 3 healthy male subjects were given a dose of $\sim 185 \mathrm{MBq}[18 \mathrm{~F}] \mathrm{JNJ}-64413739$ and underwent 10 consecutive whole body PET/CT scans (Siemens Biograph) to assess the tracer biodistribution for approximately 5 hours post injection. Delineation of source organs allowed estimation of the normalized cumulated activity and calculation of the corresponding organ doses and effective dose (ED) with OLINDA. To evaluate the tracer distribution in the brain, 5 healthy male subjects were given 115-196 MBq [18 F] JNJ-64413739 and completed a 120- minute dynamic PET scan (PETMR GE Signa) combined with arterial blood sampling and tracer radiometabolite analysis. 1 and 2 tissue compartment models (1-2TCM) and Logan graphical analysis (LGA) were evaluated to quantify tracer kinetics and to calculate regional volumes of distribution (VT) (PMOD v.3.7). Retest scans were acquired for 3 of the 5 subjects (interscan interval 26-44 days). To determine the proportion of the PET signal associated with specific binding to P2X7 in human in vivo, one subject underwent a baseline and post dose [18 F] JNJ-64413739 PETMR scan. The latter was performed after an oral dose of JNJ-54175446 predicted to saturate binding based on PET studies in rhesus macaque. Occupancy was estimated by analyzing baseline and post dose VT values using a Lassen plot.

Results: Average ED was $22 \pm 1 \mu \mathrm{Sv} / \mathrm{MBq}$ with the highest organ absorbed doses for gall bladder, urinary bladder, small and large intestine. 2TCM proved to be the most appropriate kinetic model to estimate regional VT with very similar estimates for LGA. Regional VT values were similar across all brain volumes with slightly higher values in thalamus, striatum, and brain stem. Inter subject variability of VT was relatively high with a composite cortical region of interest VT showing an approximate 3 -fold range across subjects. Average regional TRT was $12.7 \pm 8.4 \%$ by $2 \mathrm{TCM}$ and 
$13.5 \pm 8.0 \%$ by LGA while the acquisition time could be reduced to 90 minutes with only limited increase in bias and TRT variability. A single oral dose of JNJ-54175446 resulted in $78 \%$ occupancy across all brain regions and no reference region could be identified. Tracer metabolism was not altered by pretreatment with JNJ-54175446.

Conclusions: [18 F] JNJ-64413739 appears to be a suitable PET ligand for quantification of P2X7 ion channels in human brain. As such it can be used for testing target engagement by brain permeable P2X7 antagonists, assist in guiding dose selection of JNJ-54175446 for Phase 2 clinical trials, and provide insight into the expression of P2X7 receptors in health and disease.

Keywords: Positron Emission Tomography Imaging, Precision Medicine for Mood Disorders, P2X7, Human Clinical Trial

Disclosure: Part 1: Janssen Pharmaceutical, Employee, Novartis, Stock / Equity, Johnson \& Johnson, Stock / Equity, Part 2: Janssen Pharmaceutical, Employee, Novartis, Stock / Equity, Self, Johnson \& Johnson, Stock / Equity, Part 5: Janssen Pharmaceutical, Employee.

M114. Interferon-Alpha and Anti-TNF Have Differential Effects on Amygdala Emotional Reactivity With Actions of Interferon-Alpha Predicting the Later Development of Depressive Symptoms

Charlotte Clarke, Jerry Tibble, Valerie Voon, Kevin Davies, Neil Harrison*

Brighton \& Sussex Medical School, University of Sussex, Brighton, United Kingdom

Background: $U p$ to one in three patients receiving Interferon- $\alpha$ (IFN- $\alpha)$ based therapies for Hepatitis-C develop depression. Conversely, anti-TNF therapies have been shown to improve mood and depression providing key empirical support for the 'inflammatory theory' of depression. Heightened amygdala reactivity (particularly to negatively valenced emotional stimuli) is a consistent finding within major depressive disorder (MDD); it can predict treatment efficacy and reverses following successful treatment. However, whether IFN- $\alpha$ and anti-TNF therapies act to enhance and attenuate depressive symptoms through modulation of amygdala emotional reactivity is currently unknown.

Methods: Utilising a prospective study design, we recruited 30 patients (mean $48.0 \pm 10.5$ years, 21 male) initiating IFN- $\alpha$ based therapy of Hepatitis-C and 30 patients (mean $50.4 \pm 15.7$ years, 10 male) initiating anti-TNF therapy for inflammatory arthritis. All patients completed an emotional face-processing task (sad, neutral and happy expressions) during fMRI and blood sampling before and after their first IFN- $\alpha$ (4 hours) or anti-TNF (24 hours) injection. All were then followed up with psychiatric assessments for 3-months of treatment. fMRI data were analysed in SPM12 and results reported for bilateral amygdala regions of interest at a family wise error (FWE) small-volume corrected threshold of $\alpha<0.05$ after thresholding at an uncorrected statistical threshold of $p<0.001$.

Results: IFN- $\alpha$ was associated with an acute increase in circulating cytokines: IFN- $\alpha$ : mean ( \pm s.e.) $3.12 \pm 0.95 \mathrm{pmol} / \mathrm{L}$ baseline to $43.26 \pm 7.66 \mathrm{pmol} / \mathrm{L}$ at 4 hours, $p<0.001$, IL- 6 from $1.87 \pm 0.39 \mathrm{pmol} / \mathrm{L}$ to $5.13 \pm 0.78 \mathrm{pmol} / \mathrm{L}, p<0.001$, IL-1Ra from $169.36 \pm 25.26 \mathrm{pmol} / \mathrm{L}$ to $616.53 \pm 127.17 \mathrm{pmol} / \mathrm{L}$ $p=0.001)$, IL-10 from $0.83 \pm 0.18 \mathrm{pmol} / \mathrm{L}$ to $1.44 \pm 0.28 \mathrm{pmol} /$ $\mathrm{L}, p=0.011)$. Anti-TNF was associated with a reduction in circulating cytokines that broadly mirrored those to IFN- $\alpha$ : IL-6 from $6.41 \pm 1.72 \mathrm{pmol} / \mathrm{L}$ to $3.05 \pm 0.97 \mathrm{pmol} / \mathrm{L}$ at 24 hours, $p=0.017$, IL1ra from $766.33 \pm 249.22 \mathrm{pmol} / \mathrm{L}$ to $490.74 \pm 195.21 \mathrm{pmol} / \mathrm{L}, \quad p=0.049$, IL-10 from $0.30 \pm 0.13$ $\mathrm{pmol} / \mathrm{L}$ to $0.22 \pm 0.12 \mathrm{pmol} / \mathrm{L}, p=0.017$. TNF showed a non-significant increase likely reflecting increased circulating bound TNF $(p=0.15)$.

IFN- $\alpha$ significantly increased depression symptoms (HAMD) at 4 weeks $(p<0.001)$ but not 4 - hours after first dose $(p>0.1)$. Conversely, anti-TNF significantly improved mood (HADS) at both 24 hours $(p=0.003)$ and 12 weeks $(p<0.001)$.

As anticipated, emotional face stimuli robustly activated the ventral visual stream, including bilateral fusiform face areas and amygdala regions of interest (ROI). Neither IFN- $\alpha$ nor anti-TNF had any significant effect on the visual processing pathway. However, both significantly modulated amygdala reactivity. IFN- $\alpha$ selectively enhanced right amygdala responses to sad (compared to neutral) face stimuli $(p=0.037)$. These acute changes in reactivity additionally predicted increases in HAMD depressive symptoms 4 weeks later $(p=0.038)$. Conversely, anti-TNF was associated with a significant decrease in right amygdala reactivity to all emotionally valenced faces $(p=0.033)$, though this was not significantly greater for sad compared to neutral faces $(p>0.05)$. This reduction in amygdala reactivity correlated with acute changes in depressive symptoms ( 24 hours) but did not significantly predict future depressive symptoms change.

Conclusions: Pro- and anti-inflammatory cytokines differentially modulate amygdala reactivity to emotionally valenced face stimuli. IFN- $\alpha$ rapidly reorients amygdala reactivity to negatively valenced emotional information, an action that predicts the subsequent development of depressive symptoms supporting a mechanistic role in IFN- $\alpha$ induced depression. Conversely, anti-TNF was associated with a general reduction in amygdala reactivity that was observed across emotional expression. This correlated with coincident improvement in depressive symptoms but did not significantly predict subsequent improvement in mood. These data highlight sensitivity of amygdala to circulating cytokines and support a potential mechanistic role in the development of interferon-induced depression.

Keywords: Amygdala, Depression Inflammation Cytokine, fMRI Faces Paradigm

Disclosure: Nothing to Disclose.

M115. Implication of the Mitochondrial and Immune Dysfunctions in Bipolar Disorder: New Insights Into Pathogenesis

Giselli Scaini, Gabriel Fries, Tatiana Barichello, Samira da Silva Valvassori, Sudhakar Selvaraj, Giovana Zunta-Soares, Jair Soares, Joao de Quevedo

University of Texas Health Science Center at Houston, Houston, Texas, United States

Background: Bipolar disorder (BD) is characterized by marked changes in energy which accompany mood episodes 
$\mathrm{SI} 86$

and which appear to be fundamental to understand the pathophysiology of the disorder. Moreover, several studies have suggested that impaired mitochondrial function might lead to a disruption of normal neural plasticity, contribute to the atrophic changes and promote the neurocognitive deficits and progression of bipolar disorder (BD). Among myriad downstream effects of changes in energy metabolism is immune function. Because the mechanisms underlying the inflammation as well as its relationship with mitochondrial function remain elusive, we studied immune function, NLRP3 activation and a number of markers of inflammation activate by the inflammasone.

Methods: Twenty-five patients with BD-I and 25 age- and sex-matched healthy controls were recruited from outpatient clinics at the University of Texas Health Science Center at Houston. Human blood samples were collected in heparin collection tubes. Then, PBMCs were separated using LeucoPREP brand cell separation tubes, and mixed with RPMI-1640 medium containing 10\% DMSO.

Results: Our results showed an upregulation in the NLRP3, ASC and pro-caspase 1 in BD patients, as well as an increase in the caspase- 1 activity and IL-18 levels. Since TSPO and cardiolipin levels can be involved in the NLRP3 inflammasome activation we evaluated the genes involved in the TSPO pathway (TSPO, VDAC and ANT) and the cardiolipin levels, we found an up-regulation in these genes and a significantly increase in the cardiolipin levels in PBMCs from BD patients compared to controls. Considering that TSPO is involved in intrinsic pathway of apoptosis we also evaluated the cytochrome c levels and the activity of the caspase- 3 and 9. Our results showed that $\mathrm{BD}$ patients had higher levels of cytochrome $c$ in the cytosol as well as an increase in the activity of caspase-3 and 9. Finally, we found a positive correlation between NRLP3, TSPO, and cardiolipin levels, as well as a positive correlation between TSPO, cytochrome c, caspase-3 and 9. In an attempt to explore the potential clinical correlates of our findings, we tested possible correlations between psychiatric and demographic characteristics and biochemical data. Our results showed no significant differences biochemical date in patients under treatment with lithium and antipsychotics. Moreover, no associations were found between cellular parameters and age, gender, ethnicity, education, body mass index, smoking, number of depressive episodes or number of hospitalizations.

Conclusions: Overall, data reported here are consistent with the working hypothesis that mitochondrial and immune dysfunctions coexist in bipolar disorder, and may contribute to cellular dysfunction, brain volume loss and progressive cognitive in BD. Moreover, we show an important relationship between cardiolipin, a unique phospholipid which is almost exclusively located in the inner mitochondrial membrane, and the immune and cell death pathway activation in BD patients, supporting the link between mitochondrial dysfunction and the pathophysiology of BD. Keywords: Bipolar Disorder, Depression, Inflammasome, Cardiolipin, TSPO

Disclosure: Nothing to Disclose.
M116. Prophylactic Ketamine Rescues Abnormal Fear Response Following Ablation of Adult Hippocampal Neurogenesis

Ina Pavlova*, Dong-oh Seo, Josephine McGowan, René Hen, Michael Drew, Christine Ann Denny

Research Foundation for Mental Hygiene, Inc. (RFMH), New York, New York, United States

Background: We have previously identified ketamine as a stress resilience-enhancing drug, and have reported that ketamine administration prior to stress protects against depressive-like behavior and abnormal fear responses in mice. Moreover, preliminary results show that ketamine reduces abnormal fear response in mice following ablation of adult hippocampal neurogenesis (NG), suggesting that some of ketamine's protective effect to be NG-independent.

Methods: Here, we seek to understand the pathways underlying ketamine's effect on fear responses to a stressor, and to identify if these pathways are NG-independent. To label neural ensembles representing a fearful experience following ablation of neurogenesis, we created a new triple genetic mouse line, by crossing ArcCreERT2 x EYFP mice with GFAP-TK transgenic (Tg) mice. We used a combination of behavior paradigms, the triple genetic line, and a pipeline for imaging behaviorally evoked neural ensembles, to identify the brain-wide circuits that mediate ketamine's effect on fear response in mice with ablated neurogenesis.

Results: Prophylactic administration of ketamine in our new triple genetic line decreases the immobility time in the forced swim test, and freezing response after a 2-shock contextual fear conditioning (CFC) paradigm in both NG-intact and NG-ablated mice, indicating that ketamine attenuates fear expression and prevent depressive-like behavior regardless of genotype. We are currently determining the brain-wide neural patterns associated these altered behavioral profiles.

Conclusions: Our results suggest that prophylactic ketamine treatment can protect against stress-induced depressive behavior and attenuate fear response even when neurogenesis is arrested. If results from this study translate to humans, it will be possible to develop prophylactics against stressinduced psychiatric disorders in patients with already compromised adult hippocampal neurogenesis.

Keywords: Ketamine, Adult Hippocampal Neurogenesis, Stress Induced Depressive Behavior, Stress Resilience Disclosure: Nothing to Disclose.

\section{M117. Development of a Touchscreen-Based Rodent Flanker Task: Empirical Evaluation of Visual Stimuli}

Mykel Robble*, Brian Kangas, Stefanie Nickels, Lisa Wooldridge, Emilia Cárdenas, Sarah Perlo, Jack Bergman, William Carlezon, Diego Pizzagalli

Harvard Medical School, McLean Hospital, Belmont, Massachusetts, United States

Background: Deficits in cognitive function, such as reward sensitivity and cognitive control, are a common feature of virtually all neuropsychiatric disorders. While perturbations 
in cognitive control have been studied extensively in humans, it has been challenging to examine these complex processes in laboratory animals. In turn, stagnation in the development of animal-based tasks to assess these processes has impeded the identification and development of innovative treatments for neuropsychiatric disorders. As part of a larger effort to create reliable and valid cross-species assays of cognitive function, we have begun developing a rodent version of the Eriksen Flanker Task to assess cognitive control.

Methods: Using fading and correction procedures combined with touch-sensitive response technology, we trained Sprague Dawley rats to discriminate between several distinct pairs of visual stimuli. These stimuli included arrows $(</>)$ and letters $(\mathrm{H} / \mathrm{S})$ used in traditional (human) versions of the Flanker Task, as well as colored stripes (red/green) and more detailed photographic stimuli (cherries/leaf). During training, correct responses resulted in the delivery of a $30 \%$ sweetened condensed milk solution $(0.1 \mathrm{~mL} /$ reward); incorrect responses led to a repeat of the trial. Discrimination was deemed successful when the criterion of fewer than 10 repeated trials per stimulus-type during the session was observed on two consecutive days ( $\sim 80 \%$ response accuracy). Following training, rats were first tested in sessions with flanker probe trials and, subsequently, in sessions with only flanker trials. During the latter sessions, $2 / 3$ of the trials were of the congruent type and $1 / 3$ were incongruent. Response accuracies were compared by trial type to evaluate the Flanker interference effect.

Following these initial studies, Long Evans rats (reported to have better vision than Sprague-Dawley rats) were used to evaluate additional target stimulus pairs. These stimulus pairs included green and violet geometric shapes and photographs. In addition, different geometric shapes of the same color were used to evaluate the potential to successfully discriminate contours and edges without color as a stimulus dimension. Brightness was adjusted from the initial studies in an attempt to balance stimulus intensity.

Results: Sprague Dawley rats successfully discriminate using the red/green stripes and cherry/leaf photographs but did not meet criteria for the arrow or letter stimuli. When flanker trials were introduced, performance remained high and the Flanker interference effect of reduced accuracy for incongruent stimuli was confirmed. Notably, however, a response bias on incongruent trial types was observed, i.e., performance on trials with a red target was reduced, regardless of stimulus type. To determine whether the observed response bias was due to a difference in stimulus intensity, we reduced the brightness of the green stimulus. This manipulation caused Flanker performance on all trial types to approach chance accuracy, suggesting a loss of stimulus control with the dimmer green stimulus. Two rats were retrained with the dimmer green stimulus and then transitioned back into the Flanker Task. The response bias was reduced in these subjects, further suggesting that an imbalance in stimulus intensity led to response bias in the previous Flanker tests. These initial findings emphasize the need for appropriately balanced stimuli in the Flanker Task. Ongoing studies are designed to identify the most suitable type of stimuli for the Flanker Task in rats, and further establish other experimental parameters for optimal task performance. Once appropriate stimuli are validated in the rodent task, electroencephalography (EEG) data will be collected and relevant event-related potentials (ERPs) will be evaluated and compared to those recorded in human subjects.

To parallel aspects of studies in rats, EEG data were collected from human subjects using the cherry/leaf stimuli and the more traditional arrow stimuli. Relevant outcome measures including N200, theta power, and error-related negativity (ERN) were comparable across these two types of stimuli, raising confidence that the stimuli that will ultimately be suitable for rodents also will be appropriate for studies in humans. Studies with the stimuli optimized for balanced visibility and intensity are ongoing to determine whether these stimuli are also appropriate for the human task.

Conclusions: Several types of visual stimuli have been evaluated in two rat strains to determine which would be best suited for a rodent version of the Flanker Task. Rats were able to discriminate stripes or photographic images differing in color but were unable to discriminate the stimuli commonly used in the human version of the task, i.e., arrows and letters. During subsequent Flanker tests, a response bias was apparent on incongruent trial types with both the red/ green stripes and cherry/leaf images, likely due to a perceived difference in stimulus intensity. Efforts are ongoing to balance stimulus intensity and improve performance; adjustments have included altering aspects of the stimuli (brightness, color scheme, stimulus type), as well as switching to a rodent strain with greater visual capacity. Postadjustment studies have yielded encouraging findings and diminished biases. Ultimately, cross-species behavioral and neurophysiological correspondence will serve to validate the rodent task and provide the predictive power to screen potential therapeutics for numerous neuropsychiatric conditions.

Keywords: Cognitive Control, EEG/ERP Electrophysiology, Translational Neuroscience, Cognitive/Emotional Task Performance

Disclosure: Nothing to Disclose.

\section{M118. Pharmacy Data to Identify Prescription Strategies Among Veterans With Depressive Disorders}

\section{Paulo Shiroma*, Anders Westanmo, Paul Thuras,} David Atkinson, Eric Baltutis

University of Minnesota, Minneapolis, Minnesota, United States

Background: Antidepressants are the most commonly prescribed treatment modality for depression and are often the first-line treatment in primary care settings. While several practice guidelines including the VA/DoD Clinical Practice Guideline for Management of Major Depressive Disorder (May 2009) offer consensus-based expert treatment recommendations when the initial antidepressant medication is suboptimal, little is known about real world pharmacology practice by VA providers treating depression. Methods: Study sample: Administrative data included demographics, medical and psychiatric (i.e., substance use disorders, PTSD) comorbidities. The sample included all veterans treated at the MVAHCS between 12/1/2015-11/30/ 2016 who had at least one diagnosis of depression (F32.X, F33.X, F34.X, F39. F43.21, F43.23). Codes indicating 
diagnoses of bipolar, psychosis-spectrum or dementia were excluded.

Pharmacological regimen and strategies: The identification of a regimen was done at the level of individual medications and not drug classes. Up to a total of three prescription orders issued by VA Pharmacy after index diagnosis of depression (IDD) were used to determine the FST and second step treatment (SST). For a SST to be considered valid, each prescription order had a grace period of 90 days at the end of its validity period. Any medication prescribed beyond the grace period was considered a FST. The SST consists of 4 different strategies: 1) optimization (increasing dose of same FST); 2) switching to a different antidepressant; 3) augmenting the treatment regimen with a nonantidepressant agent, and 4) combining the initial antidepressant with a second antidepressant.

Algorithm development: Overall, optimization was defined if SST of same antidepressant was both issued on a date after and on a higher dose that those of the FST. To classify other strategies, a second drug must be both different from and started after FST. We considered a 28-day overlap as parameter to differentiate cross-tapering switching from either combination or augmentation. Thus, switching was defined if FST and STT were concurrently ordered for equal or less than 4 weeks. Augmentation or combination strategy was considered if augmenting agent or second antidepressant, respectively, were concurrently prescribed for $\geq 60$ consecutive days.

Results: The study sample consists of 863 patients; $14.3 \%$ were female; mean age of 51 (S.D. +/-17) years. The median number of days from IDD and prescription of first antidepressant was 21.5 days. The average number of comorbid medical and psychiatric conditions were 9 and 4, respectively with metabolic and cardiovascular, and anxiety as the most frequent conditions, respectively.

Overall among first antidepressant trial, SSRI and sertraline were the most common class $(N=543 ; 62.9 \%)$ and single prescribed antidepressant $(N=239,27.7 \%)$, respectively. The antidepressant medications were initiated mostly by providers from the Mental Health $(\mathrm{MH})(N=459 ; 53.2 \%)$ and Primary Care $(\mathrm{PC})(N=364 ; 42.2 \%)$ services. The duration of the FST were significantly different by services $(\mathrm{MH}$ : mean $=149.1+/-68.3$ days vs PC: $207.5+/-63.9$ days; $t$ test: $7.86 ; \mathrm{df}=331 ; p<0.0001)$. Out of 863 patients, 350 remained on the same antidepressant dose during the study period. Overall, 210 patients $(60 \%)$ on a single antidepressant were considered to have an adequate trial. Sertraline had the lowest frequency of adequate dose trials $(N=28 ; 32.6 \%)$ but was also the most common prescribed antidepressant $(N=86 ; 41 \%)$.

Strategies: Five-hundred and thirteen patients had a second step strategy with optimization $(N=343 ; 66.8 \%)$ being the most common, followed by switching ( $N=128 ; 24.9 \%)$, combination $(N=30 ; 5.8 \%)$ and augmentation $(N=12$; $2.3 \%)$. There was no significant difference between frequency of strategies by services ( $\mathrm{PC}$ vs. $\mathrm{MH})(\chi 2 \mathrm{df}=3=5.32$; $p=0.15)$. Patients that underwent optimization strategy had increased doses of the same antidepressant medication during the study. There was a significant difference between frequency of adequate dose by antidepressants $(\chi 2 \mathrm{df}=8$ $=53.5 ; p<0.0001)$. Mirtazapine $(N=30)$ and fluoxetine $(N=31)$ had the lowest $(N=13,43 \%)$ and highest $(N=30$;
$97 \%$ ) frequency of adequate trials, respectively. Out of 74 patients on an initial SSRI, $46(62.2 \%)$ switched to a nonSSRI antidepressant being bupropion the preferred switching agent $(N=18)$. Among 43 patients initially on a non-SSRI, 26 $(60.5 \%)$ were switched to an SSRI with sertraline $(N=12)$ as the preferred medication. Out of 30 cases using combination strategy, 20 (66.7\%) included an SSRI with escitalopram and bupropion as the most common single combination with 5 cases (16.7\%). Finally, among 12 cases identified as augmentation strategy, quetiapine became the most common augmenting agent $(N=7)$. Other atypical antipsychotics and liothyronine accounted for other augmentation purposes. Cases with lithium or stimulants were absent in this sample. Conclusions: Based on VA Pharmacy data, optimization is the most common strategy after a sub-optimal respond to the first antidepressant treatment. Use of non-antidepressant agents is rarely use as a SST even among $\mathrm{MH}$ providers. Validation of VA Pharmacy algorithm should follow to facilitate identification of sequential strategies of depression treatment.

Keywords: Antidepressant, Depression, Veterans Disclosure: Nothing to Disclose.

\section{M119. Positive Traits in the Bipolar Spectrum: The Space Between Madness and Genius}

\section{Tiffany Greenwood*, Hagop Akiskal, John Kelsoe \\ University of California, San Diego, La Jolla, California, United States}

Background: Creativity has a historical connection with the bipolar spectrum and may result from a shared genetic vulnerability with the disorder. Previous studies have shown that creativity is particularly enhanced among unaffected first-degree relatives and those with bipolar spectrum traits, such as hypomanic personality, cyclothymic temperament, and positive schizotypy. This suggests that some aspects of the bipolar spectrum may confer advantages, while more severe expressions of symptoms negatively influence creative accomplishment. Here we have evaluated bipolar disorder as an extreme of normal and beneficial population variation in creativity, temperament, personality, and cognitive flexibility. Methods: We have completed the recruitment of 141 bipolar patients, 97 creative controls, and 114 non-creative controls for a total of 358 participants. The sample is $63 \%$ female and $76 \%$ white, and $20 \%$ Hispanic with roughly equal gender and race distributions across groups. All subjects were evaluated using a comprehensive assessment battery designed to identify potentially positive traits within the bipolar spectrum. This battery included an extensive clinical and demographic assessment, a creative achievement questionnaire, 7 self-report temperament and personality questionnaires, and 18 behavioral assessments designed to capture variation in creativity performance, cognitive flexibility, risk/reward processing, impulsivity, and general cognitive functioning.

Results: Initial analyses indicate significant group differences for several behavioral tests related to cognitive flexibility and creativity, as well as hypomania, cyclothymic temperament, impulsivity, positive schizotypy, and creative achievement. We also observe the expected correlations between measures. 
Conclusions: Creativity is a complex, multidimensional construct with both cognitive and affective components, many of which appear to reflect a shared genetic vulnerability with bipolar disorder. By investigating all traits of potential interest in the same cohort, our results confirm and extend earlier observations with regard to this shared vulnerability in a larger sample. The dimensional phenotypes investigated here are expected to represent positive human characteristics that more fully capture the dimensional aspects of bipolar disorder and may shed light on its evolutionary context. As such, these dimensional phenotypes may improve our power to identify risk genes and ultimately provide novel therapeutic targets.

Keywords: Bipolar Disorder, Temperament, Personality, Creativity

Disclosure: Nothing to Disclose.

\section{M120. Ventral Dentate Gyrus Responses to Stress are Regulated by Neurogenesis}

Christoph Anacker*, Victor Luna, Gregory Stevens, Amira Millette, Ryan Shores, Briana Chen, Rene Hen

\section{Columbia University, New York, New York, United States}

Background: Adult neurogenesis in the dentate gyrus of the hippocampus is a process that is highly regulated by environmental influences, and functionally implicated in behavioral responses to stress and antidepressants in rodents and in humans. However, how adult-born neurons regulate information processing in the dentate gyrus to protect from stress-induced anxiety-like behavior is unknown.

Methods: Here we used in vivo Ca2+-imaging with headmounted miniature microscopes (Inscopix, CA) in the dentate gyrus of freely moving mice, to investigate how young adult-born neurons regulate single cell responses of large ensembles of mature granule cells during chronic psychosocial stress. The intracellular $\mathrm{Ca} 2+$ indicator, GCamp6f, was expressed in mature granule cells of the ventral dentate gyrus (vDG) using AVV8-CamkII-GCamp6f virus (UPenn vector core). $\mathrm{Ca} 2+$ transient rates of granule cells were then imaged in mice with adult-inducible deletion of the pro-apoptotic gene, Bax, from neural stem cells and their progeny (iBax mice), which have $\sim 2$ fold more young adult-born neurons than control mice. iBax mice were imaged during 10 days of social defeat stress while being attacked by a CD1 aggressor mouse through a wire-mesh enclosure that protects from physical contact. After chronic defeat, the same mice were also imaged during behavioral testing in the social interaction test and in the open field.

Results: On the first day of imaging during social defeat, vDG granule cells did not show changes in their $\mathrm{Ca} 2+$ transient rates in response to the stress of an attack. iBax mice with increased neurogenesis showed similar $\mathrm{Ca} 2+$ transient rates as mice with normal levels of neurogenesis ( $p=0.59, N=246-460$ cells). At the end of chronic defeat stress (on day 10), granule cells showed increased $\mathrm{Ca} 2+$ transient rates in response to an attack ${ }^{* * *} p=0.0002$, $N=592-620$ cells). This neuronal response to the stress of an attack was significantly lower in iBax mice with increased neurogenesis $\left({ }^{*} p=0.017\right)$. To assess whether $\mathrm{vDG}$ granule cells display heterogeneous responses to stress, we used cell selectivity analysis and identified a subset of $\sim 34 \%$ of granule cells that responded specifically to attacks after chronic social defeat. The $\mathrm{Ca} 2+$ transient rates of these 'attack'-selective cells were significantly lower in iBax mice with increased neurogenesis $\left({ }^{* *} p<0.0001, N=169-211\right.$ cells).

At the behavioral level, socially defeated mice spend $\sim 40 \%$ less time interacting with a novel mouse $\left({ }^{*} p<0.01, N=16\right.$ mice), while iBax mice with increased neurogenesis are resilient to social defeat and exhibit higher levels of social interaction time $\left({ }^{*} p<0.01, N=14\right.$ mice). At the cellular level, iBax mice exhibit reduced $\mathrm{Ca} 2+$ transient rates in the anxiogenic interaction zone $\left({ }^{*} p=0.018, N=308-483\right.$ cells) and higher rates in the safe corners of the arena (*** $p<0.0001, N=206-301$ cells). In the open field test, defeated mice spend $\sim 42 \%$ less time in the anxiogenic center $\left({ }^{* *} p<0.01, N=16\right.$ mice), while iBax mice with increased neurogenesis spend more time in the center $\left({ }^{*} p<0.05\right.$, $N=16$ mice). iBax mice showed reduced $\mathrm{Ca} 2+$ transient rates during open field exploration $\left({ }^{* *} p<0.0001 ; N=494\right.$ 859 cells). While defeated mice showed increased neuronal activity in the brightly lit anxiogenic center of the arena $\left({ }^{* *} p=0.002\right)$, this activity increase was not observed in iBax mice with increased neurogenesis $(p=0.97)$.

To determine whether this neurogenesis-mediated inhibition of the vDG is sufficient to confer stress resilience, we injected the Gi-protein coupled inhibitory DREADD receptor, $\mathrm{hM} 4 \mathrm{Di}$, into the ventral dentate gyrus and chronically silenced mature granule cells during social defeat by daily injections of the DREADD-receptor ligand, clozapine-Noxide $(\mathrm{CNO})$. Indeed, silencing mature granule cells during chronic defeat increases social interaction time $(p<0.01$, $N=11$ mice) and time in the center of the open field $(p<0.05, N=11$ mice $)$.

Conclusions: Our data show for the first time that neurogenesis inhibits the activity of mature granule cells in the vDG during stressful experiences and during anxiety-like behavior. Moreover, we show functional heterogeneity within the population of vDG granule cells by identifying a specialized sub-population of cells that specifically responds to stressful episodes (in our model: 'attacks'), and is inhibited by adult-born neurons. Inhibiting dentate gyrus function through neurogenic or pharmacological strategies could be a promising therapeutic strategy to target stress-induced psychopathology.

Keywords: Adult Hippocampal Neurogenesis, Chronic Stress, Depression, Anxiety, in vivo Calcium Imaging

Disclosure: Nothing to Disclose.

\section{M121. Acute and Prolonged Antidepressant Effects of Ketamine in Treatment-Resistant Depression}

Jennifer Phillips, Meagan Birmingham, Taylor Hatchard, Sandhaya Norris, Jeanne Talbot, Abigail Ortiz, Pierre Blier ${ }^{*}$

University of Ottawa Institute of Mental Health Research, Ottawa, Canada

Background: Subanaesthetic doses of intravenous ketamine have elicited rapid, albeit transient reduction of depressive symptoms and suicidal ideation in patients with treatmentresistant depression (TRD) (Newport et al., 2015). Repeated 
ketamine administration may prolong these effects (Coyle and Laws, 2015). Few studies examine ketamine beyond an acute-treatment phase. As such, given the short-term efficacy of ketamine and the lack of adequate medical options among individuals suffering with TRD, further investigation into the clinical utility of ketamine is warranted. The purpose of this study was to evaluate the effects of repeated infusions on response and remission rates, and to investigate prolongation of antidepressant response with maintenance infusions. In particular, it was hypothesized that repeated ketamine infusions would enhance and maintain the antidepressant effect among participants who respond.

Methods: Thirty-two participants with TRD completed a randomized trial of ketamine. Subjects received a single ketamine infusion during a double-blind crossover with midazolam (an active placebo). Following relapse of depressive symptoms, participants received 6 open-label ketamine infusions administered thrice-weekly over two-weeks (acutetreatment phase). Responders, participants obtaining $\geq 50 \%$ decrease in Montgomery-Åsberg Depression Rating Scale [MADRS] scores, received four further infusions administered once-weekly (maintenance phase). Response and remission rates were examined throughout the study.

Results: Eighteen participants responded (56\%), and 7 remitted (22\%) following the acute-treatment phase. A repeated measures ANOVA examining the cumulative effects of each infusion revealed a significant main effect of time $(p<0.001)$, with further reductions in MADRS scores with each successive infusion. The number of remitters after the first, third, and sixth acute-treatment phase infusions, were 1,3 , and 7 , respectively. In responders, there was no further change in MADRS scores during maintenance phase infusions $(p=0.70)$.

Conclusions: Results indicated that repeated ketamine infusions had cumulative antidepressant effects among individuals with TRD. Moreover, the significant reduction in depressive symptoms was further maintained among responders through once-weekly infusions. As a result, the current findings provide preliminary insight into the efficacy of this novel treatment approach, which has important implications for improving quality of life among individuals suffering from this chronic, severe form of depression.

Keywords: Ketamine, Treatment Resistant Depression, Rapid Antidepressant Effects

Disclosure: Part 1: Bristol Myers Sqibb, Consultant, Janssen, Consultant, Sunovion, Consultant, Lundbeck, Consultant, Allergan, Consultant, Pfizer, Consultant, Otsuka, Consultant, Part 2: Forest, Consultant, Pfizer, Consultant, Part 4: Allergan, Grant, Janssen, Grant, Otsuka, Grant, Lundbeck, Grant.

M122. Early Life Exposure to SSRI Antidepressants Induces Depression-Like Behavior in Adult Offspring Through Changes in Perinatal Hippocampal DNA Methylation

Sarah Clinton*, Chelsea McCoy, Matthew Glover

Virginia Tech University, Blacksburg, Virginia, United States

Background: Selective serotonin reuptake inhibitor (SSRI) antidepressants are the mainstay treatment for the $10-20 \%$ of pregnant and postpartum women who suffer major depression. With an estimated 4 million children born in the United States each year and $2-13 \%$ of pregnant women taking an SSRI, approximately 80,000-520,000 newborns are exposed annually to an SSRI in utero. SSRIs are generally considered safe, with minimal risk for pregnancy complications or teratogenic risk, although accumulating evidence suggests that in utero SSRI exposure perturbs neurodevelopment and may elicit detrimental effects on offspring's' neuropsychological health, including increased risk for internalizing behavior, abnormal social behavior, and depression during adolescence. These findings in humans are consistent with rodent data showing that perinatal SSRI exposure disturbs multiple behavioral domains associated with mood disorders, including increased helplessness, anxiety, anhedonia, perturbed sleep, and diminished sexual performance. Neurobiological mechanisms underlying the deleterious behavioral effects of perinatal SSRI exposure are poorly understood; however, our preliminary findings suggest that an epigenetic mechanism (DNA methylation) and downstream changes in the transcription factor Foxn3 in the neonatal hippocampus contribute to adverse effects of early-life SSRI exposure. Our current experiments are testing the working hypothesis that the adverse behavioral consequences of perinatal SSRI exposure are mediated by perturbed DNA methylation and Foxn3 function in the early postnatal hippocampus.

Methods: We hypothesize that perinatal SSRI exposure downregulates DNA methylation and disrupts Foxn3 signaling in the early postnatal hippocampus and that this may provide a molecular switch that triggers adverse downstream effects on neurodevelopment and emotional behavior. To test this, we first examined global DNA methylation (5methylcytosine) levels in brains of neonatal rats that were perinatally exposed to the SSRI paroxetine. We later used next-generation sequencing to interrogate gene-specific methylation changes in the hippocampus of perinatal SSRIexposed offspring. Because our data pointed to SSRI-induced suppression of DNA methylation (with a specific decrease in the DNA methyltransferase DNMT3a), our next experiment tested the hypothesis that siRNA-mediated suppression of Dnmt3a expression in the hippocampus of early postnatal rats would elicit adverse behavioral consequences (e.g., enhanced FST immobility) akin to what occurs following perinatal SSRI exposure. Finally, because the gene Foxn3 was one of the genes most affected by SSRI exposure (showing altered methylation as well as suppressed expression relative to control subjects), we conducted another siRNA study to transiently suppress Foxn3 expression in the early postnatal hippocampus to test whether this was sufficient to induce greater depression-like behavior, similar to what occurs following early life SSRI treatment.

Results: Perinatal SSRI exposure led to decreased hippocampal DNA methylation levels relative to vehicle-exposed controls; notably no changes were observed in other limbic brain regions. Next generation sequencing analyses identified numerous gene-specific methylation differences in the hippocampus of perinatal SSRI-exposed rats, with a preponderance of hypomethylated genomic regions in the Cornu Ammonis (CA) and dentate gyrus subregions. Results of our siRNA study showed that transient knock-down of Dnmt3a in the early postnatal hippocampus lead to greater 
depression-like behavior (FST immobility) in adult offspring with no effect on anxiety-like behavior. These results were highly reminiscent of the behavioral consequences of perinatal SSRI exposure. The siRNA study examining transient Foxn 3 expression is ongoing.

Conclusions: Data from our laboratory and others highlight myriad behavioral disturbances that emerge in adult offspring exposed to SSRIs in early life. The hippocampus regulates behavioral domains that are altered by perinatal SSRI exposure, including depression-like behavior in the FST, anxiety and social behavior. Moreover, our preliminary findings of perinatal SSRI-induced decreases in early postnatal hippocampal DNA methylation, including specific changes in methylation and expression of the Foxn3 gene, suggest that perinatal SSRI exposure induces molecular changes that functionally impact hippocampal-mediated behavioral domains. The SSRI-induced methylation changes were associated with reduced neonatal hippocampal expression of the DNA methylating enzyme, Dnmt3a, and we found that siRNA-mediated Dnmt3a knockdown in the neonatal hippocampus recapitulated the behavioral effects of early-life SSRI exposure in both male and female rats. Our results may have important implications on the use of SSRIs during pregnancy and on the etiology of depression in general, as early-life changes in epigenetic mechanisms disrupt normal hippocampal development, leading to an increased risk for depression in adulthood.

Keywords: Prenatal SSRI Exposure, DNA Methylation, Hippocampus, Depression

Disclosure: Nothing to Disclose.

\section{M123. In Bipolar Patients That Respond to Lithium, Targeted Sequencing of Candidate Genes Reveals Asso- ciated Variants in the Promoter of TrkB}

Nathaniel Miller*, Seth Ament, Tatyana Shekhtman, Jared Roach, John Kelsoe

University of California, San Diego School of Medicine, San Diego, California, United States

Background: We previously identified a candidate group of genes by sequencing large families with bipolar disorder. This included calcium channels, CAM kinases, and GABA receptors. We also studied the neurotrophin signaling pathways for BDNF and NGF, which have repeatedly been associated with bipolar disorder and lithium response. In regard to BDNF, lithium induces its release which is essential for its function. We have also previously shown that genetic variants in the $\operatorname{TrkB}$ gene are associated with lithium response and bipolar disorder. The goal of this study was to identify novel and common sequence variants in these genes that affect the action of lithium in bipolar disorder.

Methods: Retrospective samples were collected in regard to lithium responsiveness. 73 good responders, (LiR), 49 moderate responders (Mod), and 47 poor responders (NR) individuals were used for a sequencing study of these two genes. We targeted exons, non-coding regions with putative regulatory functions, 5' and 3' untranslated regions, and numerous DNase hypersensitive regions. Samples were combined into pools of 23 to 37 individuals. Paired-end sequencing was done using Custom Amplicons (Illumina). Variants were called with
Genome Analysis Toolkit, or alternatively LoFreq or CRISP. Then, PLINK was used to calculate a chi-square statistic between individual groups. Annotation of identified variants was done using SIFT and PolyPhen-2.

Results: For BDNF, 35 variants were called, and 159 variants were called for TrkB. Using a conservative Bonferroni correction for 174 comparisons, a statistically significant $\mathrm{p}$-value would be $<2.8 \times 10-4$. Comparing good responders to poor, two suggestive variants were found for BDNF. In the promoter of TrkB, a minor allele variant was found more frequently than expected in the LiR samples compared to the $\mathrm{NR}$, having a p-value of $2.93 \times 10-6$. RegulomeDB predicts this variant is important for transcription factor binding.

Conclusions: Targeted sequencing revealed the overabundance of a minor allele of a common SNP in the promoter of TrkB for LiR but not in NR. Lithium efficacy for the treatment of bipolar disorder is likely to involve the BDNF/ TrkB pathway. This study provides a likely candidate region to further investigate the function of lithium in bipolar individuals.

Keywords: Bipolar Disorder, Next Generation Sequencing, TrkB, Lithium Response

Disclosure: Nothing to Disclose.

M124. Distinct Network Structure of Depression Symptoms Among Responders and Non-Responders to Antidepressants and Placebo

\section{Venkat Bhat*, Xiamin Leng, Jennifer Larsen, Jane Foster, Sidney Kennedy}

University of Toronto, Toronto, Canada

Background: Major Depressive Disorder (MDD) is characterized by the presence of a minimum number and duration of symptoms. Specific symptoms may correspond to specific underlying dysfunctional neural circuits and to treatment outcomes in MDD such as treatment-response/relapse, chronicity and functioning. Studying the longitudinal structure of MDD symptomatology in relation to various network features such as centrality and connectivity could help identify key symptom hubs and their interrelations. This project examined data from several antidepressant trials to explore the network structure of treatment response to inform personalization of treatment approaches in MDD.

Methods: Data from three 6- week randomized placebocontrolled trials with duloxetine were included $(n=724$ at baseline) and MADRS scores were examined at the 4 available time points for subjects who completed the trial: baseline (week 0), week 2, 4 and 6 . The available data were categorized into 4 groups that included 264 duloxetine responders, 87 non-responders, 163 placebo responders, 210 placebo non-responders. Response was defined as $\geq 50 \%$ reduction in MADRS score by week 6 . The MADRS data at the four available time points were each converted into a weights matrix that codes the connectivity structure between nodes in a network in matrix form. The qgraph package for $\mathrm{R}$ is sensitive to network visualization of relationships in psychometric data and provides an interface to visualize data through network modelling techniques. Edges in the graph were examined for statistical significance (adjusted for multiple comparison in every analysis) during each of the 
4 time points using specific qgraph package modules. The emergent statistically significant network characteristics (e.g. nodes, degree, etc) were compared among subjects based on response to duloxetine/placebo and the trajectory of response to duloxetine (K-means based clustering of trajectory of response to duloxetine into fast, slow and non-responders). Results: Network characteristics specific to each of the four observed groups were noted at the four available time points. A consistent pattern of increasing connectivity with time was noted among the duloxetine responders and replicated among placebo responders. Further, a consistent pattern of decreasing connectivity was noted among duloxetine non-responders and replicated among placebo non-responders. Notably, duloxetine responders had a broad increase in connectivity across individual symptoms while the placebo responders had symptom improvement in a limited repertoire of symptoms, mainly apparent and observed sadness. Another notable network feature was the overall greater strength of connectivity among responders with greater magnitude of standard centrality features as compared to non-responders. In a manner similar to the response to medication/placebo analysis, network connectivity features that distinguished fast responders from non-responders were identified. Across the four time points longitudinally, the mean degree of connectivity in the MADRS scales increased from 0.6 to 9 among fast responders to duloxetine and decreased from 0.8 to 0.2 among non-responders to duloxetine.

Conclusions: So far as we are aware, this study represents the first characterization of the network architecture of MADRS in antidepressant response with consistent features seen with duloxetine and replicated with placebo among responders and non-responders. Features such as increasing connectivity among responders were notable longitudinal results in the duloxetine arm and were replicated among responders in the placebo arm. Further, many connectivity features are unique to the trajectory of response to duloxetine. The results point towards symptom specificity and their interconnectivity in antidepressant response. The network architecture is being currently examined for various other network parameters, and in the context of other variables such as gender, symptom severity and functioning. These network features could serve as potential signatures of antidepressant response and as starting points for treatment personalization by further investigation of molecular and neural circuit mechanisms underlying the connectivity patterns.

Keywords: Network-Analysis, Connectivity, Major Depressive Disorder, Treatment-Response, Antidepressants Disclosure: Nothing to Disclose.

M125. Higher Systemic Inflammation is Associated With Lower Insula Activity in Depression With Appetite Loss, but Higher Insula Activity in Depression With Increased Appetite

\section{W. Kyle Simmons*, Kaiping Burrows, Kent Teague, Wayne Drevets}

Laureate Institute for Brain Research, Tulsa, Oklahoma, United States

Background: Although clinicians have long noted the relationship between major depressive disorder (MDD) and changes in appetite, there exists little neuroscience evidence to explain why some individuals eat less when they become depressed and others eat more. Using fMRI, our lab has shown that MDD participants classified solely on their depression-related appetite changes exhibit differential activity within interoceptive insular cortex in response to food cues. This finding was particularly interesting in light of recent work showing that a severe atypical subtype of depression accompanied by increased appetite is associated with heightened systemic inflammation (Lamers et al., 2016, Transl. Psychiatry) and that inflammation in healthy, non-depressed participants modulates the activity of interoceptive regions of the insula (e.g., Harrison, et al., 2009, Biological Psychiatry). We therefore examined whether adults with depression-related increases and decreases in appetite exhibit differences in circulating markers of inflammation relative to non-depressed subjects, and whether these depression subtypes are associated with altered relationships between systemic inflammation and brain activity.

Methods: Eighty-nine participants meeting DSM 5 criteria for MDD underwent $\mathrm{fMRI}$ while viewing pictures of food and non-food objects. The participants were drawn from three groups: 22 unmedicated subjects with increased appetite during the current depressive episode (MDD-increase), 28 unmedicated subjects with decreased appetite during the current depressive episode (MDD-decrease), and 39 healthy control (HC) subjects. All three groups were matched for age. The two MDD groups were matched for body mass index, anhedonia, depression and anxiety severity. Blood plasma was collected immediately prior to fMRI imaging, and assayed to assess group differences in interleukin-1 receptor antagonist (IL-1RA), interleukin-6 (IL-6), and c-reactive protein (CRP). As a goal of the study was to identify whether group differences in circulating immune markers relate to group differences in the brain's response to food, we first identified regions exhibiting differences among the groups in responses to food pictures during fMRI. We used random-effects multivariate modeling in a whole-brain voxel-wise analysis to identify brain regions exhibiting a difference in activity between any two of the groups, with corrections for multiple comparisons $(p<.05$ corrected) applied separately for each statistical map contrasting the groups. This analysis identified eight brain regions exhibiting group mean activity differences. Next, we conducted robust regression using an $\mathrm{M}$ estimator to identify regions where the two depressed subgroups exhibited differences in the slope of the relationship between activity in these regions to food pictures and the inflammatory markers. Results: Plasma IL-1RA and CRP levels were greater in the MDD-increase participants than either the MDD-decrease participants (IL-1RA $p<0.004$; CRP $p<0.01$ ) or $\mathrm{HC}$ participants (IL-1RA $p<0.03$; CRP $p<0.04$ ). The MDDdecrease and HC participants did not differ on either IL-1RA $(p=0.84)$ or CRP $(p=0.35)$. Plasma IL-6 levels were greater in the MDD-increase participants than that the $\mathrm{HC}$ participants $(p<0.05)$. Although the two depressed groups did not differ in overall levels of IL-6 $(\mathrm{p}=.89)$, differences were observed among these groups in the slope of the relationship between circulating plasma IL-6 levels and hemodynamic activity in the posterior and dorsal midinsula to food pictures (dorsal mid-insula $\mathrm{p}=.041$; posterior insula $\mathrm{p}=.009$ ). In both insula regions, the MDD appetitedecrease participants who exhibited the highest IL-6 levels exhibited the weakest responses to foods cues $(r=-0.48)$, 
while the MDD appetite-increase participants who exhibited the highest IL-6 levels exhibited the highest responses to foods cues $(r=0.26)$.

Conclusions: Individuals with depression-related increases and decreases in appetite exhibit differential inflammatory activity, and systemic inflammation differentially predicts the activity of interoceptive neurocircuitry in these two depression phenotypes. The present findings lend support to the possibility that appetite changes in depression may be a marker for differential, inflammation-related pathophysiology among MDD phenotypes.

Keywords: Systemic Inflammation, Depression Subtypes, Insula, Eating, Functional MRI (fMRI)

Disclosure: Nothing to Disclose.

\section{M126. In Vivo Fiber Photometry Reveals Signature of Future Stress Susceptibility in Nucleus Accumbens}

Jessie Muir, Eric Nestler, Erin Calipari, Rosemary Bagot*

McGill University, Montreal, Canada

Background: Alterations in nucleus accumbens (NAc) activity have been linked to the pathophysiology of depression. Mice that exhibit depressive-like symptoms after chronic social defeat stress (CSDS) show distinct changes in NAc activity. However, the pre-existing individual differences that make certain mice resilient and others susceptible to stress are yet to be described. We hypothesized that individual differences in NAc activity present before exposure to stress may associate with future stress susceptibility.

Methods: Using in vivo fiber photometry calcium imaging in, we recorded activity in NAc D1- and D2- medium spiny neurons (MSN) in awake behaving mice before exposing mice to ten days of social defeat stress to identify resilient and susceptible individuals.

Results: We report that, prior to stress, mice that later become resilient have higher D1 - MSN activity than mice that later become susceptible, an effect observed both in baseline neuronal activity and during social interaction. We suggest that reduced D1-MSN activity before defeat may be a predisposing factor for stress susceptibility. We also observed differences in D2- MSN activity temporally correlated with behavior, pointing to an additional role for D2-MSN in susceptibility to stress.

Conclusions: These findings suggest a possible underlying mechanism of stress-induced susceptibility and offer the potential to predict at-risk individuals prior to encountering stress.

Keywords: Brain Based Markers for Depression, Chronic Social Defeat, Nucleus Accumbens, in vivo Calcium Imaging, Risk and Resilience

Disclosure: Nothing to Disclose.

M127. The Neurocircuitry of Approach-Avoidance Decisions in Depression: I. Neuroeconomic Modeling of Reward and Utility in Decision-Making

Min Su Kang*, Maria Ironside, Ken-ichi Amemori, Jeffrey Curry, Malavika Mehta, Callie McGrath, Ann Graybiel, Diego Pizzagalli

McLean Hospital, Belmont, Massachusetts, United States
Background: Functional and structural abnormalities within fronto-cingulate pathways - which have been found to play a key role in emotion regulation and decision-making - have been strongly implicated in the pathophysiology of major depressive disorder (MDD) and mechanisms of treatment response (Pizzagalli, 2011). One of the widely known maladaptive cognitive processes in MDD is a strong bias towards avoidance (Leventhal, 2008). Although several animal studies have suggested the role of fronto-cingulate in avoidance behaviors, little is known about parallel neural substrates in humans, especially in clinical samples. Using functional magnetic resonance imaging (fMRI) and an approach-avoidance (Ap-Av) decision-making task adapted from a prior macaque study (Amemori \& Graybiel, 2012), this study aimed to model the neurocircuitry of pessimistic evaluation in MDD.

Methods: During high temporal resolution fMRI acquisition, 41 females (20 unmedicated individuals with MDD) completed three runs of an Ap-Av task using reward points and aversive images-sound pairs ( 35 trials in each run). fMRI data were preprocessed using SPM12 using standard analyses. Hemodynamic responses were modeled using a canonical hemodynamic response function that was convolved with the onset times of task regressors in order to compute a general linear model (GLM) at the single-subject level. The GLM included six task-related regressors: three conditions related to approach decisions (decision, feedback, reward), and three conditions related to avoid decisions (decision, feedback, no reward). Contrasts of interest compared activations to Ap versus Av decisions. Linear utility models were calculated to fit individual subjective ratings of task stimuli after the fMRI paradigm.

Results: There were no group differences on the number of Av decisions (HC: 35\%, MDD: 36\%), reaction times, or slope of punishment ratings (all $p>.1$ ). Across the whole group $(N=41)$, whole brain corrected statistics $(p=.001, \mathrm{k}=20)$ revealed differences in activations for Ap versus Av decisions. Compared to Av decisions, Ap decisions were associated with greater activation in right inferior frontal gyrus, right superior orbital gyrus, left thalamus, right rectal gyrus and right parahippocampal gyrus/fusiform gyrus. Compared to Ap decisions, Av decisions were associated with greater activations in right dorsal/medial anterior cingulate cortex/midcingulate cortex, right supramarginal gyrus, right middle temporal gyrus, left posterior-medial frontal and the right insula. In order to identify brain regions that tracked utility values, an additional whole brain analysis was conducted, which showed a large network that was correlated with utility values across all decisions (Ap and Av): rostral and dorsal anterior cingulate cortex, leading into bilateral frontal sites, bilateral caudate and putamen, and the left insula. In addition, there were large clusters in the right hippocampus and thalamus and two clusters in the right insula.

Conclusions: These results suggest that fronto-cingulate and limbic system have distinct activation patterns during approach and avoidance behaviors in humans presented with simultaneous conflicting cues. Interestingly, the prior macaque study from which our task is adapted attributed the negative biasing to a subzone of pregenual ACC, which, as our analyses revealed, tracked utility of rewards in humans. These findings may contribute to our understanding of depression and comorbid anxiety disorders, and serve as a 
potential target for therapeutic interventions. Although many psychopharmacological interventions for anxiety and depression are informed by studies on non-human animal models, few studies aim to establish the parallel neural mechanisms across human and non-human primates, which is critical for therapeutics development. As such, future research on developing cross-species models of motivation and decisionmaking would be a crucial next step towards identifying neurobiologically-informed targets of treatment.

Keywords: Depression, Translational Neuroscience, Brain Imaging, fMRI, Human Brain Imaging, Approach/Avoidance Disclosure: Nothing to Disclose.

\section{M128. AMPA Receptor Subunit Expression and Receptor Binding in Patients With Major Depressive Disorder: A Systematic Review of Postmortem Studies}

Teruki Koizumi*, Hideaki Tani, Shinichiro Luke Nakajima, Nobuhiro Nagai, Takefumi Suzuki, Masaru Mimura, Hiroyuki Uchida

Keio University School of Medicine, Shinjuku-ku, Tokyo, Japan

Background: The dysfunction of glutamatergic synapses has been reported to be implicated in the pathophysiology of major depressive disorder (MDD). Among them, $\alpha$-amino-3hydroxy-5-methyl-4-isoxazolepropionic acid (AMPA) receptors are one of the centerpiece to regulate glutamatergic neural activity. While previous post-mortem studies of MDD have found altered trafficking of AMPA receptors, no systematic review on this topic has been conducted. In this systematic review, we assembled published data from postmortem studies that measured the AMPA receptor expression in MDD to summarize the findings and elucidate current situation in the field.

Methods: We conducted a systematic literature search for postmortem studies examining the expression of AMPA receptor subunits or receptor bindings in patients with MDD compared to healthy individuals in March 2017, using PubMed with the following search terms: ( $\alpha$-amino-3hydroxy-5-methyl-4-isoxazolepropionic acid) or AMPA or AMPAR) AND "depressi*" and filtered them by "Humans". Cross-reference and hand searches were also performed. We extracted the information regarding subject characteristics such as age, sex, postmortem interval, and cause of death, and study characteristics including quantification technique of receptor subunit expression or receptor binding, target regions, and target molecules.

Results: From 284 initial records, 9 relevant articles were identified. They consisted of 12 studies that examined AMPA receptor subunit (or related molecule) transcript expression, and/or AMPA receptor binding levels. The techniques used to quantify the molecules in these studies were quantitative polymerase chain reaction (qPCR) and in situ hybridization (ISH) (9 studies) and autoradiography (3 studies). The most frequently investigated region was dorsolateral prefrontal cortex (DLPFC) (4 studies) and following by anterior cingulate cortex (ACC) (2 studies), thalamus (2 studies), hippocampus (2 studies), and striatum (2 studies). Among the 4 DLPFC studies, 1, 1, and 2 studies showed an increase, a decrease, and no difference compared to healthy controls in the expression of AMPA, respectively. In the ACC, both of the 2 studies indicated an increase in the expression or binding of AMPA. With regard to the thalamus, 1 study each showed a decrease and no difference in expression of AMPA, respectively. Of the 2 studies examining hippocampus, a decrease in the expression was found in one study whereas there was no difference in the receptor binding in the other. In the striatum, no difference was observed in both studies. The subjects investigated were heterogeneous in terms of age, sex. Moreover, analysis techniques differed among studies. Other clinical information such as duration of illness and severity of disease was not available in most of the studies (9 studies).

Conclusions: Findings were inconsistent among studies, which may be due to the heterogeneity of this population as well as analysis techniques employed. Moreover, interpretation of the findings is difficult because of a limited number of available studies and the small sizes. In addition, postmortem studies are subject to physiological changes after death. Clinical information on the subjects studied was lacking or insufficient in general. These limitations of the previous studies clearly emphasize the need of examination of AMPA receptors in living human brains and thorough clinical characterization of the sample.

Keywords: Postmortem, AMPA, MDD, Depression

Disclosure: Nothing to Disclose.

\section{M129. Scope of Inflammation in Patients With Treat- ment Resistant Depression}

Yu Sun, Dai Wang, Jaskaran Singh, Giacomo Salvadore, James Palmer, Carla Canuso, David Hough, Benjamin Hsu, Qingqin Li, Gayle Wittenberg, Mark Curran, Wayne Drevets, Guang Chen*

Janssen Research \& Development, LLC of Johnson \& Johnson, San Diego, California, United States

Background: Accumulating data in the literature suggest that chronic inflammation contributes to both etiology and treatment response in depression. Several studies also suggest anti-inflammatory or anti-pro-inflammatory cytokine treatments may alleviate depressive symptoms. Using C-reactive protein (CRP) as the inflammation marker, the current study explores the extent of peripheral inflammation present in treatment resistant depression (TRD) patients mostly on antidepressants, and its relationship with depression features or subtypes, symptoms and other cytokines/inflammatory markers. To further understand the role of chronic inflammation in etiology of depression, this study also documents the prevalence of depressive symptoms in a very-high inflammation cohort comprised of rheumatoid arthritis (RA) subjects. Methods: The TRD data sets were obtained from Janssen ketamine and esketamine phase 2 trials (Singh et al., 2015 and 2016). Both the Montgomery-Åsberg Depression Rating Scale (MADRS) and Inventory of Depressive Symptomatology-Clinician Rated, 30-Item (IDS-30) scale were used in the studies. Eighty-eight subjects with C-reactive protein (CRP) data were included in the analysis, most of them were receiving oral antidepressant agents at the time of biosampling. Melancholic and atypical depression were classified based on DSM-5 and IDS-C30 items. Anxious depression was defined based on selected items from IDS-C30. Plasma 
levels of interleukin-6 (IL-6), interleukin-1 beta (IL-1 $\beta$ ), tumor necrosis factor alpha (TNF $\alpha$ ), serum amyloid A (SAA) and CRP were measured by ELISA. The RA data were from Janssen sirukumab Phase 2 and 3 trials (Smolen JS., et al. 2014; Aletaha D., et al. 2017, Takeuchi T., et al. 2016). The exclusion criteria included $\mathrm{CR} p<10$ (phase 2) or $<8$ (phase 3 ). In RA patients, depressive symptoms were measured by the 36-item short form health survey questionnaire (SF-36). RA patients $(n=1,593)$ were included in the analysis. Comparison of immune factor levels, age, BMI, and depression severity between patients with different clinical features were performed using the Wilcoxon rank sum test. Differences based on gender, race, and depression features were examined using Fisher's exact test.

Results: Forty-two percent of TRD patients had levels of CRP $\geq 3 \mathrm{mg} / \mathrm{L}$. Three percent of TRD patients had levels of CRP $\geq 10 \mathrm{mg} / \mathrm{L}$. Levels of SAA and IL-6, but not IL-1beta, TNF alpha, or BMI were significantly higher in TRD patients with $\mathrm{CRP} \geq 3 \mathrm{mg} / \mathrm{L}$. $\mathrm{CRP} \geq$ vs $<3 \mathrm{mg} / \mathrm{L}$ did not significantly differentiate depression severity, anxious, melancholic, or atypical features of depression. CRP levels were about 90- or 80 -fold lower in our TRD cohorts (mean \pm SD: $3.1 \pm 3.6 \mathrm{mg} /$ L) than in the RA cohorts (mean \pm SD: $28.1 \pm 29.1$ (phase 2) or $25.3 \pm 22.0$ (phase 3 ) $\mathrm{mg} / \mathrm{dL}$ ). Twenty-two percent of RA patients exhibited prevalent depressive symptoms.

Conclusions: Current data show that the pro-inflammatory or inflammation state in periphery as indicated by elevation of CRP presents in a sizable portion, but not the majority of TRD patients. Pro-inflammation or inflammation did not significantly influence the prevalence of depression features or subtypes, severities, and items scores of MADRS and IDS-30 in these TRD patients. Although inflammation may be a contributor, it does not appear to be sufficient as a causal factor of depressive symptoms, based on our study of very high inflammation RA cohorts showing that higher prevalence of core depression symptoms in RA patients than expected in general population and that majority of RA patients do not have these symptoms. It remains conceivable, however, that a subgroup of depressed patients exists who are more susceptible to the development of depressive symptoms under chronic inflammatory signaling.

Keywords: Depression, Cytokine, Inflammation

Disclosure: Part 1: JNJ, Employee, Part 2: JNJ, Employee, Part 5: JNJ, Employee.

M130. Sestrin 2 Modulator NV-5138 Shows KetamineLike Rapid Antidepressant Effects via Direct Activation of mTORC1 Signaling

Ronald Duman*, Taro Kato, Rong-Jian Liu, Catharine Duman, Rosemarie Terwilliger, George Vlasuk, Seung Hahm, Eddine Sajah

Yale University School of Medicine, New Haven, Connecticut, United States

Background: The NMDA receptor antagonist ketamine produces rapid antidepressant actions, even in patients considered treatment resistant, addressing a major limitation of currently available medications. In preclinical rodent models, the antidepressant behavioral actions of ketamine are associated with increased number and function of synapses in the medial prefrontal cortex (PFC) and these effects are dependent on mTORC1 signaling. The mTORC1 pathway is a cellular regulator of protein synthesis and is modulated by neuronal activity, endocrine and metabolic signals, including amino acids, notably leucine, which activates mTORC1 signaling via binding to sestrins 1 and 2 . Here, we examined the influence of NV-5138, a novel small molecule modulator of sestrin 2 that penetrates the blood brain barrier, on antidepressant behavioral responses and induction of spine synapses in the prefrontal cortex (PFC).

Methods: Behavioral analysis included analysis of NV-5138 (oral administration) in the forced swim test (FST), novelty suppressed feeding test (NSFT), female urine sniffing test (FUST), and sucrose preference test (SPT); chronic unpredictable stress (CUS) causes a decrease in SPT and is a measure of anhedonia. Signaling via the mTORC pathway was examined by western blot analysis of phosphorylated forms of mTOR, S6K, and 4EBP1 in the PFC. The influence of NV-5138 on synapse number and function was determined by analysis of layer $\mathrm{V}$ pyramidal neurons and western blot of synaptic proteins in the PFC.

Results: The results demonstrate that a single dose of NV-5138 (160 mg/kg) produced rapid antidepressant effects in the rat FST, NSFT, and FUST without effecting locomotor activity or home cage feeding. A single dose of NV-5138 also reversed anhedonia, measured in the SPT, caused by CUS exposure. The effects of NV-5138 were long-lasting in the FST, observed for up to 7 days following a single dose when there was no detectable brain or plasma exposure. As expected, NV-5138 activated the mTORC1 pathway in the PFC $1 \mathrm{hr}$ after administration, similar to ketamine, and the antidepressant actions of NV-5138 were blocked by infusion of the selective mTORC1 inhibitor rapamycin into medial PFC. We also tested synaptic responses of layer $\mathrm{V}$ pyramidal neurons in the medial PFC and found that NV-5138 administration rapidly increased the number and function of spine synapses in the apical dendrites. NV-5138 also increased levels of synaptic proteins in PFC, including GluA1 and synapsin1.

Conclusions: Taken together, the results demonstrate that NV-5138 produces rapid synaptic and antidepressant behavioral responses via sestrin 2 mediated activation of the mTORC1 signaling pathway, supporting the possibility that sestrin2 modulation is a novel target for development of rapid acting antidepressants.

Keywords: Stress, Synapse, Ketamine, Antidepressant, mTORC1, Depression

Disclosure: Part 1: Taisho, Consultant, Johnson \& Johnson, Consultant, Part 2: Psychogenics, Stock / Equity, Part 4: Allergan, Grant, Navitor, Grant, Lilly, Grant, Johnson \& Johnson, Grant, Relmada, Grant.

\section{M131. BDNF Release and Signaling are Required for the Antidepressant Actions of GLYX-13}

Taro Kato*, Manoela Fogaca, Satoshi Deyama, Xiao-Yuan Li, Kenichi Fukumoto, Ronald Duman

Sumitomo Dainippon Pharma Co., Ltd., Konohana-ku, Osaka, Japan

Background: Conventional antidepressant medications, which act on monoaminergic systems, have significant 
$\mathrm{S} 196$

limitations for the treatment of depression, including a time lag of weeks to months and low rates of therapeutic efficacy. GLYX-13 is a novel glutamatergic compound that acts as a modulator of the NMDA receptor with glycine-like partial agonist properties, and like the NMDA receptor antagonist ketamine produces rapid antidepressant actions in depressed patients and in preclinical rodent models. Importantly, GLYX-13 does not produce the dissociative and psychotomimetic side effects observed with ketamine. However, the mechanisms underlying the antidepressant actions of GLYX-13 have not been characterized.

Methods: Here, we use a combination of neutralizing antibody, mutant mouse, and pharmacological approaches to test the role of BDNF-TrkB signaling in the actions of GLYX-13.

Results: The results demonstrate that the antidepressant effects of GLYX-13 in three different models, the forced swim test, novelty suppressed feeding, and female urine sniffing test, are blocked by intra-mPFC infusion of an antiBDNF neutralizing antibody or in mice with a knock-in of the BDNF Val66Met allele, which blocks the processing and activity dependent release of BDNF. The results also demonstrate that pharmacological inhibitors of BDNFTrkB signaling or of L-type voltage dependent $\mathrm{Ca} 2+$ channels (VDCCs) block the antidepressant behavioral actions of GLYX-13. Finally, we examined the role of Rho GTPase proteins, which are required for BDNF mediated stabilization of spines. The results demonstrate that infusion of a selective inhibitor of Rac1 but not RhoA into the mPFC blocks the antidepressant effects of GLYX-13.

Conclusions: Together, these findings indicate that GLYX-13 causes activity dependent stimulation of VDCCs, leading to increased release of BDNF, subsequent TrkB-Rac1 signaling, and increased spine synapse number and stability that is required for the rapid and sustained antidepressant effects of GLYX-13.

Keywords: Rapastinel, BDNF, Fast-Acting Antidepressant, Rho Family GTPase

Disclosure: Part 5: Sumitomo Dainippon Pharma, Employee.

M132. Role of BDNF/mTORC1 Signaling in the Long-Lasting Antidepressant Effects of (2R,6R)-Hydroxynorketamine

\section{Kenichi Fukumoto*, Taro Kato, Xio-Yuan Li, Ronald Duman}

Yale University School of Medicine, New Haven, Connecticut, United States

Background: Recent studies report that (2 R,6 R)-Hydroxynorketamine ((2 R,6 R)-HNK), a metabolite of ketamine, produces rapid and long-lasting antidepressant effects in several different rodent models. Moreover, (2 R,6 R)-HNK does not appear to produce the side effects of ketamine, based on rodent models. However, the molecular signaling mechanisms underlying the actions of (2 R,6 R)-HNK remain unclear. While (2 R,6 R)-HNK increases AMPA receptor signaling similar to ketamine, initial studies have failed to demonstrate activity of $(2 \mathrm{R}, 6 \mathrm{R})$-HNK at the NMDA receptor, the initial cellular target underlying the actions of ketamine, or at other neurotransmitter receptor sites. To address this issue, we have initiated studies to determine if the mechanisms underlying the actions of (2 R,6 R)-HNK involve BDNF-TrkB-mTORC1 signaling, which is required for the antidepressant effects of ketamine. Methods: The antidepressant actions of (2 R,6 R)-HNK were tested in C57BL/6 J mice in the forced swim test (FST), novelty suppressed feeding test (NSFT), female urine sniffing test (FUST), and locomotor activity test. For mechanistic studies, we used a number of approaches. First, we tested the effects of an L-type voltage-dependent calcium channel (Ltype VDCC) antagonist, verapamil (i.p.), based on studies demonstrating that the effects of ketamine require activity dependent VDCC function and subsequent release of BDNF. We also used several approaches to test the role of BDNF and TrkB, including infusions of an anti-BDNF neutralizing antibody or a selective $\operatorname{TrkB}$ receptor antagonist, ANA-12 into the medial prefrontal cortex (mPFC). We also tested the effects of $(2 \mathrm{R}, 6 \mathrm{R})-\mathrm{HNK}$ in mice with a knockin of the BDNF Val66Met allele, which blocks the processing and activity dependent release of BDNF. Levels of the phosphorylated and activated form of mTOR were also determined by western blot at different time points after (2 R,6 R)-HNK administration.

Results: The results demonstrate that a single dose of (2 R,6 R)-HNK (i.p.) exerts dose-dependent, long-lasting antidepressant effects in the FST, NSFT, and FUST without influencing locomotor activity. These behavioral studies were conducted $24 \mathrm{hr}$ after (2 R,6 R)-HNK administration to avoid any acute effects of drug dosing. Microinjections of (2 R,6 R)$\mathrm{HNK}$ into $\mathrm{mPFC}$ were also sufficient to produce long-lasting antidepressant effects in mouse FST and NSF, demonstrating that mPFC is a key target region of $(2 \mathrm{R}, 6 \mathrm{R})-\mathrm{HNK}$, which is required for the inhibitor microinfusion studies described below. To determine if (2 R,6 R)-HNK acts via activity dependent release of BDNF and TrkB-mTORC1 signaling, we used several different approaches. First, we found that pretreatment with the L-type VDCC blocker verapamil (i.p.) blocked the antidepressant actions of (2 R,6 R)-HNK in the FST and NSFT, indicating a requirement for activity dependent BDNF release. This was confirmed by studies demonstrating that infusion of an anti-BDNF neutralizing antibody or a TrkB receptor antagonist, ANA-12 into the mPFC also block the antidepressant effects of (2 R,6 R)-HNK in the FST and NSFT; administration of these agents alone had no effect on FST, NSFT, or locomotor activity. The role of BDNF function and release was further tested in mutant mice with a knockin of Val66Met allele, a functional human polymorphism that blocks the processing and activity dependent release of BDNF. The results demonstrate that the antidepressant effects of (2 R,6 R)-HNK in the FST and NSFT are completely blocked in the BDNF Met knockin mice, providing further evidence that $\mathrm{BDNF}$ release is required for the actions of $(2 \mathrm{R}, 6 \mathrm{R})-\mathrm{HNK}$. Next, we examined the influence of ( $2 \mathrm{R}, 6 \mathrm{R})$-HNK mTORC1 signaling in the mPFC. The results demonstrate that $(2 \mathrm{R}, 6 \mathrm{R})$ $\mathrm{HNK}$ produces a rapid and transient increase in levels of phosphorylated $\mathrm{mTOR}$ at $30 \mathrm{~min}$, but not $60 \mathrm{~min}$ after administration. We also tested the role of mTORC1 signaling in the behavioral actions of (2 R,6 R)-HNK and found that infusion of a selective mTORC1 inhibitor, rapamycin into the $\mathrm{mPFC}$ completely blocked the antidepressant behavioral effects of (2 R,6 R)-HNK in the FST and NSFT. 
Conclusions: Our results indicate that (2 R,6 R)-HNK causes activity dependent release of $\mathrm{BDNF}$ in the $\mathrm{mPFC}$ via activation of L-type VDCCs, leading to activation of TrkBmTORC1 signaling, and that this pathway plays an essential role in the antidepressant behavioral effects of (2 R,6 R)-HNK. A similar signaling pathway is required for the antidepressant behavioral actions of ketamine. However, further studies are required to identify the initial cellular target for (2R,6R)$\mathrm{HNK}$, including experiments to test the role of NMDA receptors on glutamate pyramidal neurons vs. tonic firing GABA interneurons in the actions of (2 R,6 R)-HNK.

Keywords: Ketamine, Hydroxynorketamine, Depression, BDNF, mTOR

Disclosure: Part 5: Taisho Pharmaceutical Co. Ltd., Grant.

M133. Transdiagnostic Prediction of Memory and Cognitive Abilities From Functional Connectivity Data: A Multidimensional Connectome-Based Predictive Modeling Study

Dustin Scheinost*, Siyuan Gao, Abigail Greene, R. Todd Constable

Yale University School of Medicine, New Haven, Connecticut, United States

Background: Recent advances in human neuroimaging methods-particularly, functional connectivity (FC)-are revealing robust individual differences in patterns of neural activity that predict behavioral measures and clinical symptoms. FC is usually calculated using data acquired during rest; however, without tasks to perturb neural circuitry, these data may fail to represent the full range of individual differences in FC. In this work, we demonstrate that-much like a cardiac stress test identifies symptoms not observable at rest-FC matrices, or connectomes, derived from task-based fMRI can significantly predict memory and cognitive abilities in a sample of healthy individuals, as well as in samples of individuals diagnosed with schizophrenia, bipolar disorder, and attention-deficit/hyperactivity disorder (ADHD). Additionally, we demonstrate that these predictive models are transdiagnostic; that is, they generalize across three different mental health disorders. Finally, we show that a multidimensional approach-where information from seven different tasks, including resting-state, is optimally combined into a single predictive model-results in the best prediction of memory and cognitive abilities across individuals.

Methods: These analyses use fMRI data from the UCLA Consortium for Neuropsychiatric Phenomics LA5c Study. Each participant performed one resting-state and six task functional magnetic resonance imaging (fMRI) runs in the scanner. The tasks were the Balloon Analog Risk Task (BART), Stop Signal Task, Task Switching Task (TST), Spatial Working Memory Capacity Task (SCAP), Paired Associates Memory Encoding Task (PAMenc) and Paired Associates Memory Retrieval Task (PAMret). Only participants who completed all task and resting-state runs were included in analysis. The final sample consisted of 169 participants, composed of 73 healthy controls, 34 participants with bipolar disorder, 31 participants with schizophrenia, and 31 participants with ADHD. Memory abilities were measured using the Symbol Span, Visual Reproduction (I and II), and Digit Span scales from the Wechsler Memory Scale (WMS). Cognitive abilities were measured using the Letter Number Sequencing, Vocabulary, and Matrix Reasoning scales from the Wechsler Adult Intelligence Scale (WAIS).

The fMRI data were processed with standard methods and parcellated into 268 nodes. The mean timecourses of each node pair were correlated, generating seven, $268 \times 268$ FC matrices per participant. FC matrices were each submitted to the connectome-based predictive modeling (CPM) pipeline to generate cross-validated predictive models of memory and cognitive abilities. Briefly, CPM is a validated algorithm for generating predictive brain models of behavior using leaveone-out cross validation. Data were divided into training and testing datasets. FC matrices and behavioral data from a training dataset were correlated using regression analyses to identify predictive edges, or elements in the FC matrices. Following edge selection, single subject summary statistics were created by summing edge weights and entered into predictive linear models. These models were then applied to the novel subjects in the testing dataset to predict behavior. Model performance was quantified as the correlation between predicted and true values. Finally, to leverage independent information in task FC, we developed a multidimensional CPM approach that uses canonical correlation analysis to optimally combine data from all task FC matrices and create a single predictive model of behavior. Significance for all models was assessed use permutation testing with 5,000 permutations. Multiple comparisons were corrected for using False Discovery Rate correction.

Results: For all scales, CPM significantly predicted memory and cognitive abilities in novel subjects $(p<0.01$, corrected). In all but one case (predictive models of the WMS scales generated from the TST), models generated from task FC outperformed models generated from resting-state FC (task range: $r=0.11-0.42$, rest range: $r=0.1-0.32$ ). Models generated from the PAMenc performed the best across all measures (range $r=0.36-0.42$ ) except for the Visual Reproduction II scale, on which the PAMret performed the best $(\mathrm{r}=0.41)$. All models were complex and included multiple connections within and between canonical large-scale networks (e.g., frontoparietal, default mode, salience).

Models generated from the multidimensional CPM (range $\mathrm{r}=0.38-0.47$ ) performed numerically better than any models generated from a single task, explaining (on average) an additional 3\% of variance. Multidimensional models were significantly $(p<0.05$, corrected $)$ better than unidimensional models for all measures except the Visual Reproduction II and Symbol Span scales.

In an exploratory analysis, we performed a brute-force search of all combinations of tasks to examine which combinations generated the best predictive models. For the Vocabulary and Matrix Reasoning scales, all tasks were needed to generate the best performing multidimensional models. For the remaining scales (all of which are measures of memory), the BART, PAMenc, and PAMret were needed to generate the best performing multidimensional model.

Conclusions: These results suggest that multidimensional models that combine information from several task FC matrices can predict cognitive abilities in a transdiagnostic manner. Further, they suggest that a wide array of tasks is needed to optimize prediction for more general cognitive abilities (Vocabulary and Matrix reasoning), while scales 
more specific to a single construct (e.g. memory) can be predicted best by tasks related to that construct.

Keywords: Transdiagnostic, Dimensional, Predictive Models, fMRI Functional Connectivity

Disclosure: Nothing to Disclose.

\section{M134. Oxytocin Reduces a Chemosensory-Induced Fear Response Bias in Face Perception}

Ayline Maier, Dirk Scheele, Franny Spengler, Tugba Menba, Franziska Mohr, Birgit Stoffel-Wagner, Sahib Khalsa, Onur Güntürkün, Wolfgang Maier, Rene Hurlemann*

University of Bonn, Bonn, Germany

Background: There is abundant evidence that social signals of impending threat released by one individual induce fear neurocircuitry and stress axis responses in other individuals. This social transmission of fear is not restricted to visual or auditory stimuli, but extends to the olfactory domain, a phylogenetically more ancient sense. This is also of eminent relevance in patients with anxiety disorders who exhibit heightened sensitivity to social stressors conveyed via the olfactory pathway. An emerging theme is the question of how neural and endocrine systems including the hypothalamic peptide hormone oxytocin (OXT) influence and potentially interfere with the communication of social stress between conspecifics via olfactory cues such as body odors. Methods: In the present double-blind within-subjects functional MRI (fMRI) study, 58 healthy participants were administered OXT (40 IU) or placebo and exposed to a crossmodal task, in which the perception of stress-related body odors induced a face recognition bias towards fear. The presented body odors had been collected from an independent sample of 30 male volunteers undergoing an acute social stressor, with physical exercise as a control.

Results: Under placebo, stress odors facilitated the detection of fear signals in faces, evident in a reduction of reaction times and more accurate recognition of less salient stimuli. This crossmodal recognition bias towards fear was abolished as a result of OXT delivery. On the neural level, this effect of OXT was paralleled by diminished responses to stress odors in amygdala, anterior cingulate and hippocampus. Furthermore, OXT reinstated task-related functional connectivity between the anterior cingulate and the fusiform face area that was disrupted by stress odors under placebo.

Conclusions: Our findings shed light on a new modulatory role for OXT signaling, via interference with the communication of social stress signals between conspecifics. Specifically, we observed a crossmodal anti-stress effect of OXT that appears to result from a downregulation of stress odor-induced amygdala responses and concomitant strengthening of top-down control descending from the anterior cingulate to the fusiform face area. Future work is needed to describe the pathophysiology of this process in anxiety disorders, including whether interventions to normalize a pathological hypersensitivity to stress odors and resultant distortions of visual perception confers clinical benefit.

Keywords: Functional MRI (fMRI), Oxytocin, Amygdala, Fear, fMRI, Social Stress, Olfaction

Disclosure: Nothing to Disclose.

\section{M135. Menstrual Cycle Phase and Presynaptic Dopamine Function in Women}

Shau-Ming Wei*, Daniel P. Eisenberg, Philip Kohn, Catherine Hegarty, Angela Ianni, Michael Gregory, Peter Schmidt, Karen Berman

National Institute of Mental Health, Bethesda, Maryland, United States

Background: Dopamine-related neuropsychiatric disorders such as Parkinson's disease, drug addiction and schizophrenia are characterized by marked sex differences in the onset, duration, severity, and course of these diseases. It has been hypothesized that ovarian hormones contribute to these observed sex differences by interacting with the dopamine (DA) system. In naturally cycling women, estradiol and progesterone levels fluctuate along the menstrual cycle as a product of complex neuroendocrine interactions. In the early follicular phase, both estradiol and progesterone levels are low, whereas the late follicular phase is characterized by a peak in estradiol levels, and the luteal phase by high levels of both progesterone and estradiol. Ample preclinical evidence from animal studies has shown that central dopamine systems are sensitive to circulating ovarian steroid hormones. In particular, estradiol has been shown to increase the synthesis, release, reuptake and turnover of dopamine; affect downstream targets of its receptor; and modify basal firing rates of dopaminergic neurons. The literature addressing effects of progesterone on DA is less conclusive. Human neuroimaging studies have found that in the striatum and prefrontal cortex (PFC), estradiol facilitates cognitive and behavioral processes dependent on the DA system in healthy women, including reward processing and working memory. For example, our group has previously identified more robust striatal functional responses to rewarded stimuli - a dopamine-dependent phenotype - in women scanned during the follicular relative to luteal phase of the menstrual cycle. However, whether dopaminergic tone, measured directly with neurochemical imaging such as positron emission tomography (PET), shows similar menstrual cycle variation is unknown.

Methods: Carefully screened healthy, regularly menstruating women between the ages of 18 and 50 with no psychiatric history underwent [18 F]-FDOPA PET imaging to directly measure presynaptic dopamine synthesis capacity. Scans were obtained on a GE Advance scanner after at least a 6-hour fast and 4-hour abstinence from caffeine and nicotine. A transmission scan was used for attenuation correction, and a series of emission images were dynamically collected for 90 minutes beginning immediately after tracer injection. For each woman, striatal regions of interest ([ROIs]; dorsal caudate, dorsal putamen, and ventral striatum) and a cerebellar reference region were individually delineated on an anatomical MRI volume collected separately and coregistered to PET data. The specific uptake constant Ki was determined by the Gjedde-Patlak method using the cerebellar reference region as the input function. After analysis of the native space striatal ROI data, the PET frames were spatially normalized to standard space and similarly modeled, and exploratory whole-brain, voxel-wise analyses were carried out to examine menstrual-cycle-modulated DA 
function in cortical regions. Standard student's t-tests compared data for women studied during follicular (days 4-10 of an idealized 28- day menstrual cycle) to data for those studied during the luteal (days 18-24) phase. Additionally, to further investigate the role of estradiol in presynaptic DA function, we compared [18 F]-FDOPA uptake in women studied during the early follicular phase (days 1-4, low estradiol) to women studied during the late follicular phase (days 8-12, high estradiol). A threshold of $p=0.005$, uncorrected, was used for the voxel-wise analyses.

Results: Eleven women in the follicular phase (age $\pm \mathrm{SD}=$ $37.3 \pm 11.4$ ) and 12 women in the luteal phase (age $\pm \mathrm{SD}=$ $35.6 \pm 8.2, \mathrm{t}(22)=0.71, p=0.24)$ were studied. For the striatal ROI analyses, a trend toward increased [18 F]FDOPA uptake during the follicular phase compared to the luteal phase was observed in the right dorsal caudate nucleus $(p=0.06)$. Additionally, for women in the late follicular phase $(N=7$, age $\pm \mathrm{SD}=30.4 \pm 9.1)$ compared to those in the early follicular phase $(N=7$, age $\pm S D=40.3 \pm 12.6 ; \quad t$ $(13)=0.11, p=0.46)$, there was a trend toward increased FDOPA uptake in the left caudate nucleus $(p=0.08)$. The exploratory whole-brain voxel-wise analysis showed significantly increased [18 F]-FDOPA uptake in the follicular phase in the medial PFC (MNI $\mathrm{x}, \mathrm{y}, \mathrm{z}=2,50,39$; peak $p=8 \mathrm{x} 10-6$, uncorrected), and a similar $\mathrm{mPFC}$ area ( $\mathrm{x}, \mathrm{y}, \mathrm{z}=3,45,39$; peak $p=0.003$, uncorrected) was found to have greater uptake in the late follicular phase compared to the early follicular phase. Re-analyses with an age covariate did not substantially affect the results.

Conclusions: In this preliminary study, we observed trends for greater [18 F]-FDOPA uptake in women assessed during epochs of the menstrual cycle associated with relatively higher estradiol levels (follicular phase vs. luteal phase and late follicular phase vs. early follicular phase). These results are in line with previous preclinical and human studies showing that estradiol plays an important facilitative role in DA function. Moreover, the findings were localized to the dorsal caudate and $\mathrm{mPFC}$ - regions known to underlie cognitive and behavioral processes dependent on dopamine innervation. If confirmed in larger, longitudinal samples, these data may provide a reference for future studies in neuropsychiatric disorders in which both sexual dimorphic features and dopamine systems may play an interactive role in aspects of pathophysiology and treatment.

Keywords: Dopamine, PET Imaging, Sex Steroids, Sex Differences

Disclosure: Nothing to Disclose.

M136. Correlations Between Impulsivity and Gene Expression in Postmortem Brains: Preliminary Findings From a Psychological Autopsy Study in the UTHealth Brain Collection

Thomas Meyer*, Gabriel Fries, Laura Stertz, Elena Dyukova, Jair Soares, Glenn Sandberg, Consuelo Walss-Bass

University of Texas Health Science Center at Houston, Houston, Texas, United States

Background: Exploring the role of genetic factors in mental disorders and personality has a long tradition. However, the heterogeneity in presentations even within one diagnostic category such as bipolar disorder has complicated the identification of susceptibility genes. One way to address the issue that there is a) heterogeneity, b) symptom overlap, and c) high comorbidity rates, is to explore core characteristics or underlying vulnerability factors in line with the NIMH 'Research Domain Criteria (RDoC)' framework. 'Impulsivity' and its facets are discussed as being relevant across psychiatric disorders. It is often defined as a deficit in the ability to delay gratification and/or to inhibit responses without taking into account potential negative consequences. While it can be assessed as behavior and self-ratings, overall the evidence suggests that self-rated impulsivity has a higher stability and predictive validity. Furthermore, because it can be easily assessed even from an outside perspective based on observed behaviors, impulsivity is the ideal trait to investigate when the person of interest is not available for any reason to be interviewed in person or to complete questionnaires. Postmortem studies of psychiatric disorders often combine medical records with interviews with the next of kin, the latter sometimes called 'psychological autopsies'. The UTHealth Brain Collection performs psychological autopsies assessing mental health problems but also adopting a RDoC framework to assess personality traits including impulsivity. The current study presents data regarding reliability of impulsivity ratings and explores the association between impulsivity and gene expression in the prefrontal cortex of cases some of which have died from suicide.

Methods: Postmortem subjects and their next of kin are continuously being recruited from the Harris County Institute of Forensic Sciences (IFS), Houston, TX, regardless of the presence of a psychiatric disorder. Exclusion criteria are: a) obvious head trauma, b) identity of the subject is unknown, c) victims of fires, d) decomposed subjects; e) presence of a neurodegenerative disorder. Medical records are requested from healthcare providers. A psychiatric and psychological assessment is conducted using a structured interview schedule with the next of kin by phone ( $>5$ weeks after the death). It specifically probes for personality traits in addition to mental health and trauma. A consensus conference is also conducted discussing each case.

Gene expression: Based on prior genome-wide expression analysis in prefrontal cortex (BA9) from suicide cases and non-suicide controls from a combined cohort we identified MCTS1, CAMKK2, SNRPD3, NELL1, and FRMPD4 as the top-ranked differentially expressed genes between groups: Because associations between suicidality and impulsivity are discussed, these genes were chosen tor the preliminary current analyses.

Results: Based on joint interviews or recordings of 23 cases, Inter-rater reliability for impulsivity facets range from ICC $=.94-.98$.

We explored potential links between gene expression and impulsivity ratings by next of kin in 9 subjects. UPPSPerseverance, lack of self-regulation, and UPPS Negative Urgency did not significantly correlate with expression of any of the genes [( $\left.\begin{array}{llll}-.51 & -.57\end{array}\right),\left(\begin{array}{lll}-.41 & -.44\end{array}\right) ;\left(\begin{array}{lll}-.53 & -.35\end{array}\right)$ respectively]. Fun seeking (BAS) showed a significant negative correlation with MCTS1 $[\mathrm{r}=-.71, \mathrm{p}=.03]$ and non-significant negative ones with all other genes [-.60 -.22]. Asking directly for impulsive behaviors showed a trend for a negative association with CAMKK2 $[\mathrm{r}=-.63, \mathrm{p}=.07]$. 
Conclusions: As far as we know this is this the first study adopting an $\mathrm{RDoC}$ approach to assess potential associations between personality factors and gene expression in postmortem brain tissue. Most importantly, our results show that these ratings can be reliably assessed. Secondly, keeping in mind the very small sample at this stage, and the preliminary character of these analyses, we show that it is possible to explore whether personality traits are associated with gene expression in deceased individuals. In addition to utilizing much larger sample sizes and exploring other personality features such as behavioral inhibition or extraversion in relation to genetic markers in the brain, future research should also examine whether next of kin with different relationships to the deceased individual provide consistent information or whether the source needs to be factored in when analyzing such data.

Keywords: Impulsivity, Postmortem Brain Tissue, Gene Expression, Psychological Autopsy

Disclosure: Nothing to Disclose.

\section{M137. Modulation of Anxiety and Cognitive Behaviors by IL-33 in Mice}

Eisuke Dohi, Eric Choi, Minae Niwa, Shin-ichi Kano*

Johns Hopkins University, Baltimore, Maryland, United States

Background: Interleukin (IL)-33 is a member of the IL-1 family of cytokines. IL-33 is expressed in nuclei and secreted as alarmin upon cellular damage, to deliver a danger signal to the surrounding cells. Previous studies show that IL-33 is expressed in the brain and is involved in neuroinflammatory and neurodegenerative processes in both humans and rodents. Nevertheless, it is unclear whether IL-33 is required for physiological brain function and behavior. In this study, we have explored the role of IL-33 in mouse behaviors.

Methods: Mice deficient for Il33 gene (Il33-/- mice) were used to analyze their behavioral phenotypes in the open field test (OFT), elevated plus maze (EPM), and three-chamber social interaction test (SI). Expression of c-Fos as well as IL-33 proteins in various brain regions was assessed by immunofluorescent staining. Systemic administration of IL-33 was performed to address whether peripheral IL-33 can rescue the behavioral phenotypes of Il33-/- mice.

Results: In the adult mouse brain, IL-33 protein was mainly expressed in the nuclei of oligodendrocyte-lineage cells and astrocytes across various regions. Il33-/- mice exhibited reduced anxiety-related behaviors in the EPM and the OFT. Immunoreactivity of c-Fos, an indicator of neuronal activity, was altered in several brain areas related to anxiety-like behaviors including the medial prefrontal cortex, amygdala, and piriform cortex, suggesting that IL-33 deficiency may affect neuronal activity. Systemic injection of IL-33 did not rescue the anxiety-related behavioral phenotypes in Il33-/mice. Il33-/- mice also showed impaired social novelty recognition in the social interaction test.

Conclusions: Our data suggest that IL-33 plays a critical role in anxiety-related behaviors and cognitive functions. Although cell-type specific role of IL-33 and its contribution to brain development require further investigation, this study adds to increasing evidence that immune molecules can be critical regulators of brain function and behavior.

Keywords: Interleukin-33, Anxiety, Social Interaction Disclosure: Nothing to Disclose.

M138. Prenatal Stress Affects Commensal Microbes, Social Behavior, Serotonin, Oxytocin, and Neuroinflammation in Adult Male Offspring

Tamar Gur*, Aditi Vadodkar, Therese Rajaskera, Jacob Allen, Michael Bailey

Ohio State University College of Medicine, Columbus, Ohio, United States

Background: Recent studies demonstrate that exposure to stress changes the composition of the intestinal microbiota, which is associated with development of stress-induced behavioral changes. In utero and early neonatal exposure to maternal stress has been linked with psychiatric disorders, and the underlying mechanisms are currently being elucidated. Males have been demonstrated to be at increased risk of neurodevelopmental disorders, including autism spectrum disorders (ASD). As maternal stress is a risk factor for ASD, we used a prenatal stressor in pregnant mice to examine novel relationships between prenatal stress exposure, changes in the microbiome, and social behaviors.

Methods: Pregnant C57/BL6 females were randomly assigned to either the stressed experimental group or nonstressed control group ( $n=14$ /group). Mice were restrained between embryonic day (E) 10-E16, for a period of two hours using a well-validated restraint stress paradigm, or they were left undisturbed throughout pregnancy as a control. Placental tissue and amniotic fluid were collected from pregnant females and fetuses at E17.5 in one cohort of mice. Fetus gender was determined by microscopic inspection of existing reproductive structures through microdissection of the fetus. Microbial diversity was assessed using the Illumina MiSeq platform, for targeted $16 \mathrm{~S}$ ribosomal RNA gene sequencing. RT-PCR was used to examine gene expression. In a second cohort of mice, behavior was assessed with the elevated plus maze (EPM), the tail suspension test (TST), the novel object recognition test, and a social behavior paradigm when the offspring reached adulthood.

Results: Males exposed to prenatal stress show a significant reduction in social behavior in adulthood, with concomitant significant alterations in microbiome when compared to non-stressed controls. Prenatal stress lead to dysregulation of corticosterone response to a social stimulus in adulthood. Prenatal stress was associated with altered serotonin (5HT) metabolism in adulthood, as well as significant neuroinflammation and decreased oxytocin receptor.

Conclusions: Our findings suggest that gestation is a critical window in contributing to the development of adult psychopathology, and that the microbiome may be an important link between early life environment and later life behavioral changes as well as the emergence of sex differences. Targeting the microbiome during pregnancy may prove to be beneficial to offspring exposed to maternal stress in utero.

Keywords: Gut Microbiome, Social Behavior, Prenatal Stress Disclosure: Part 1: Abbott Nutrition, Honoraria. 
M139. Functional Connectivity Density has Neurovascular Origin

\section{Dardo Tomasi*, Nora Volkow}

National Institute on Alcohol Abuse and Alcoholism, Bethesda, Maryland, United States

Background: Brain functional connectivity density (FCD) studies in humans have shown FCD decreases in brain regions weakly activated/deactivated by continuous task performance and a negative association between the fMRI signal and the task-related FCD decreases, suggesting disconnection of task-irrelevant networks during task performance (Tomasi et al., 2014). This study, however, was carried in a relatively small sample $(N=45)$ using a specific visual attention task that limits the generalizability of the findings. We hypothesized the reproducibility and generalizability of the association between task-related changes in functional metrics, such as local FCD and the amplitude of low-frequency fluctuations (ALFF), and fMRI activation during standard fMRI task.

Methods: Thus, we investigated the association between task-related FCD changes and the concomitant fMRI signals during task performance on seven different functional domains: working memory (WM), relational processing, reward (gambling), motor function, social cognition, language, and emotion. We studied "rest" and "task" fMRI datasets from 426 healthy adults $(29 \pm 4$ years of age; 244 females) collected by the Human Connectome Project (HCP). We used FCD mapping (Tomasi and Volkow, 2010) for computing "resting-state" and "task-state" functional connectivity metrics, and general lineal modeling (GLM) for standard fMRI activation analyses. We studied the reproducibility of the findings, by splitting the sample between a discovery cohort (first 213 subjects) and a validation cohort (second 213 subjects) and using spatial correlation analyses across cohorts. Voxelwise correlation analyses across subjects were used to assess the linear association between FCD, ALFF and the BOLD signal in the brain. Stringent statistical criteria, based on the random field theory and a familywise error correction that complies with the current standards (Eklund et al., 2016), were used to assess the statistical significance of the findings.

Results: Task performance was associated with pronounced ALFF (0.01-0.10 Hz frequency bandwidth) increases in occipital and parietal cortices, and with decreases in the prefrontal cortex in the discovery cohort $(\mathrm{PFWE}<0.001)$. These task-related changes were less prominent for the language and gambling tasks than for the WM, relational, social, motor, and emotion tasks, and were not associated with the fMRI signals elicited by the tasks. The reproducibility of the task-related ALFF changes in the validation cohort was striking $(R>0.8)$ but not as good as for the fMRI activation patterns $(\mathrm{R}>0.9)$. Overall, the main fMRI activation clusters elicited by the tasks also showed taskrelated increases in FCD $(\mathrm{PFWE}<0.001)$, which were linearly associated across subjects with the blood-oxygenlevel dependent (BOLD) responses during fMRI $($ PFWE $<0.001)$. However, regions with weak fMRI activation and deactivated brain areas demonstrated task-related decreases in FCD $($ PFWE < 0.001), which were not associated with the BOLD signals across subjects. These task-related FCD increases and decreases were more pronounced for cognitive (WM, relational, social, language) and motor tasks than for the gambling and emotion tasks. Assuming that regions in which BOLD signals predominantly reflect neurovascular responses to the tasks would increase FCD, we identified regions in which BOLD signals likely reflect pure vascular phenomena (i.e. blood stealing). The former was remarkable in visual, auditory and motor areas for all tasks except gambling, which causes weak activation in reward regions. The latter was evident in the frontal lobe, especially during emotion processing which involves the fusiform face area, the amygdala and temporal cortex but not the frontal lobe.

Conclusions: The robust associations between FCD and ALFF with the BOLD signal demonstrate the neurovascular origin of the functional connectivity density. Thus, functional connectivity metrics can potentiate traditional fMRI studies and offer a complete picture of brain function by increasing the neurovascular specificity of fMRI.

Keywords: fMRI Functional Connectivity, fMRI, Neurovascular

Disclosure: Nothing to Disclose.

\section{M140. Naltrexone Implant Improves HIV Treatment Outcomes Compared to Oral Naltrexone in Opiate Addicted Patients}

George Woody ${ }^{\star}$, E. Krupitsky, E. Zvartau, E. Blokhina, E. Verbitskaya, D. Lioznov, T. Yaroslavtseva, V. Palatkin, M. Vetrova, N. Bushara, A. Burakov, D. Masalov, O. Mamontova, S. Poole, R. Gross

University of Pennsylvania, Philadelphia, Pennsylvania, United States

Background: Untreated opioid addiction is associated with poor ART adherence and outcome. Methadone or buprenorphine maintenance help but they are not always available and some patients do not want them. Extended release naltrexone may be a meaningful alternative.

Methods: 200 detoxified HIV+ opiate addicted patients starting ART in St. Petersburg were randomized 1:1 to 12 months of NI+ON placebo, or ON+NI placebo. All were offered every other week drug and adherence counseling.

Results: - 28\% females; Av. Age 32; no baseline differences between groups.

- More RNA suppression in NI than ON [66\% vs 50\%; OR $(95 \%$ CI $)=1.94(1.10-3.43)$

- Addiction treatment more often completed in NI than ON ( $32 \%$ vs $17 \%, p<0.05$ )

- ART retention better in NI than ON ( $46 \%$ vs $32 \%$; $p<0.05)$ - MEMS cap openings higher in those who continued naltrexone, regardless of group assignment: 73,7\% (95\%CI: $67.2-80.2 \%)$ vs. $64.9 \%$ (95\%CI: $59.7-70.1 \%$; F1, $198=4.37$; $\mathrm{P}=0.038)$.

- CD4 increase greater in those who continued naltrexone regardless of group assignment: $\mathrm{CD} 4$ change at the end of treatment vs baseline for completers vs. drop outs $9 \mathrm{M} \pm \mathrm{SD}$ ) 206.2 cells $/ \mathrm{mm} 3 \pm 201.8$ vs. 67.3 cells $/ \mathrm{mm} 3 \pm 159.4$, $p<0.001)$ 
- No group differences in AEs (32\% for ON vs. 30\% for NI, $p>0.5$ )

Conclusions: Extended release naltrexone is a meaningful alternative for HIV+ opioid addicted patients on ART who do not want agonist therapy or where it is unavailable.

Keywords: HIV, Addiction, Opiates

Disclosure: Nothing to Disclose.

M141. Relationship Between Marijuana Use and Hippocampal Response During Nonverbal Memory in College Students

Alecia Dager*, Madelynn Tice, Gregory Book, Sarah Raskin, Howard Tennen, Carol Austad, Rebecca Wood, Carolyn Fallahi, Godfrey Pearlson

\section{Yale University, Hartford, Connecticut, United States}

Background: Marijuana is one of the most widely used substances among college students, with peak use occurring between ages 18-22. Previous research suggests memory dysfunction occurs in adolescent and young adult marijuana users, but the neural correlates are unclear. Moreover, the hippocampus, a primary memory region, is particularly susceptible to marijuana effects. We examined hippocampal functional magnetic resonance imaging (fMRI) response to a figural encoding and recognition task among freshman college students with varying degrees of marijuana involvement.

Methods: Participants were 67 first-year college students ages 18-20 (BARCS Study) who performed a visual encoding and recognition task (Beason-Held et al., 2005) during fMRI. Substance use was ascertained for 3 months prior to scanning and groups were defined as follows: moderate/ heavy ( $>8$ times/month; $n=18$ ), light users with similar past 3-month alcohol use (1-2 times/month; $n=13$ ) or nonusers $(n=36)$. fMRI response was modeled during encoding based on whether targets were subsequently recognized (i.e., correct encoding), and during recognition based on target identification (i.e., hits). fMRI response in left and right hippocampal regions of interest was examined in relation to degree of marijuana use, controlling for past 3 months drinks/month use and sex. We predicted that heavier marijuana use would be related to reduced hippocampal activation during encoding and recognition.

Results: There were no relationships between marijuana use and fMRI response during encoding. However, fMRI response during correct recognition showed a marijuana group $\mathrm{x}$ sex interaction in both left $(\mathrm{F}(2,60)=5.37, \mathrm{p}=.007)$ and right $(\mathrm{F}(2,60)=5.65, \mathrm{p}=.034)$ hippocampus. More specifically, marijuana use was associated with lower hippocampal response in males, but not in females. Follow-up nonparametric correlations revealed that greater marijuana involvement was negatively related to left hippocampal response (Spearman's rho $=-0.26, \mathrm{p}=.037$ ). Alcohol use was not associated with hippocampal response during encoding or recognition.

Conclusions: This study provides preliminary evidence of sex differences in marijuana-related hippocampal dysfunction during recognition memory in college students. These results parallel earlier evidence of altered hippocampal response during verbal learning in adolescent marijuana users. Moreover, these findings may contribute to our previously identified decrements in academic performance in college marijuana users (Meda PLoS-1 2017), and could have substantial implications.

Funded by NIAAA (AA016599 Pearlson) and NIDA (DA038207, Dager)

Keywords: Cannabis Use, Functional MRI (fMRI), Memory Encoding and Retrieval

Disclosure: Nothing to Disclose.

M142. Corticotropin-Releasing Factor (CRF) Neurons in the Prefrontal Cortex (PFC) Impair Working Memory and PFC Neuronal Coding

\section{Sofiya Hupalo*, David Devilbiss, Craig Berridge \\ University of Wisconsin, Madison, Madison, Wisconsin, United States}

Background: The prefrontal cortex (PFC) and downstream striatal targets play a critical role in higher cognitive processes that guide goal-oriented behavior. Frontostriataldependent cognitive dysfunction is implicated in multiple psychopathologies, including attention deficit hyperactivity disorder (ADHD). Corticotropin-releasing factor (CRF)synthesizing neurons and CRF receptors are prominent in the PFC. We recently demonstrated that CRF receptor activation in the dorsomedial PFC (dmPFC) impairs frontostriatal-dependent working memory in rats. Conversely, intra-PFC and systemic administration of CRF antagonists improves working memory, similar to that seen with all FDA-approved treatments for ADHD. To examine the cognitive actions of PFC CRF neurons, current studies examined the effects of chemogenetic manipulations of PFC CRF neurons on working memory performance and neural coding within the dorsomedial frontostriatal circuit.

Methods: A dual adeno-associated virus approach was used to express excitatory or inhibitory designer receptors (DREADDs) in CRF neurons of the PFC. Rats received intra-dmPFC infusions of 2 viruses: one driving CRF promoter-specific expression of Cre-recombinase (CRFCre), and another driving Cre-conditional expression of hM3Dq or hM4Di transgenes (hSyn-DIO-hM3Dq/hM4DimCherry). At least three weeks later, animals were treated with vehicle or varying doses of clozapine-N-oxide (CNO; $0.3,1,3 \mathrm{mg} / \mathrm{kg}$ ) prior to performing in a delayed-alternation test of spatial working memory. To investigate the neurophysiological actions underlying PFC CRF neuronal activation, five CRF-hM3Dq animals and three CRF-mCherry animals (control virus lacking hM3Dq) were implanted with chronic recording electrodes in the dmPFC (layer V) and the dorsomedial striatum (dmSTR). Electrophysiological recordings were performed during working memory testing before and after vehicle or $\mathrm{CNO}$ treatment.

Results: Chemogenetic activation of PFC CRF neurons robustly impaired working memory performance, while suppression of these neurons improved working memory. Intra-PFC infusion of a CRF antagonist blocked the cognition-impairing actions of $\mathrm{CNO}$ treatment, indicating the cognitive actions of PFC CRF neurons are dependent on activation of local CRF receptors in the PFC. Working memory impairment induced by activation of PFC CRF 
neurons was associated with a robust suppression of PFC pyramidal neurons strongly activated/tuned to key task events (delay, reward, error). In contrast, PFC pyramidal neurons that were suppressed during task intervals became strongly activated with this manipulation. This suggests that activation of dmPFC CRF neurons elicits a robust degradation in task-related coding by PFC neurons. In contrast to that seen in the PFC, chemogenetic activation of PFC CRF neurons had no effect on activity of medium spiny neurons in the downstream dmSTR, regardless task-tuning. CNO treatment elicited no changes in working memory performance or spiking activity in animals receiving control virus. Conclusions: Collectively, these observations demonstrate CRF neurons in the PFC exert potent modulatory actions on higher cognitive function that involves degradation of taskrelated neuronal signaling in the PFC but not the dmSTR. The procognitive effects of $\mathrm{CRF}$ antagonists and $\mathrm{CRF}$ neuronal inhibition are similar to that seen with all FDAapproved drugs for ADHD, suggesting the PFC CRF system may represent a new target in the treatment of $\mathrm{ADHD}$ or other disorders associated with frontostriatal-dependent cognitive dysfunction.

Keywords: ADHD, Prefrontal Cortex, CorticotropinReleasing Factor (CRF), Striatum, Electrophysiology

Disclosure: Nothing to Disclose.

M143. What Leads to a Second Drink: Anxiety and Aggression as Mediators of Initial Alcohol Intake, a Nonhuman Primate Model

\section{J. Dee Higley*, John Capitanio, Elizabeth K. Wood, Laura Del Rosso}

\section{Brigham Young University, Provo, Utah, United States}

Background: Anxiety is widely held to be a contributing factor to some forms of alcohol dependency. As the origins of alcohol dependency typically occur before the legal drinking age, investigating factors contributing to alcohol use among teenagers is an important step in determining effective preventative measures and treatment. Some studies report that anxious adults self-medicate with alcohol. Few studies, however, have investigated whether anxiety is related to the induction of teen drinking. As anxiety is a relatively stable temperamental trait over time, it is hypothesized that anxiety in adolescence will be associated with higher initial alcohol intake.

Methods: In the present study, 16 adolescent male rhesus macaques (Macaca mulatta) were extensively tested for temperamental anxiety both as infants and again as adolescents (3-4-year-olds) using a standardized battery of tests including the Human Intruder Paradigm, a widely-used, standardized method for inducing and analyzing anxiety in the presence of a threat. One month after the adolescent Human Intruder testing, subjects were allowed to freely consume an $8.4 \%$ alcohol/aspartame sweetened solution (v/ v) for two hours each day, five days a week over a period of nine weeks.

Results: Analyses showed that interindividual differences in anxiety were stable from infancy into adolescence. When analyzing alcohol intake, we found a significant main effect for anxiety across a variety of individual measures (teeth grinding, behavioral withdrawal, vigilance, etc.). When variables were grouped using a Factor Analysis, results showed that subjects characterized as highly-anxious consuming more alcohol (about twice as much, $\mathrm{M}=0.34 \pm 0.04 \mathrm{~g} / \mathrm{kg}$ ) when compared to subjects characterized as having low anxiety $(\mathrm{M}=0.17 \pm 0.05 \mathrm{~g} / \mathrm{kg})$. Interestingly, measures of adolescent aggression during the Intruder Paradigm also showed a strong positive relationship with alcohol intake a finding that we have seen in other studies as well.

Conclusions: Our results suggest that temperamental anxiety is associated with the indication of alcohol intake in adolescent male rhesus monkeys and that aggressiveness is also a risk factor that is related to greater alcohol intake. To the extent our findings generalize to humans, they suggest that teens with high anxiety, and who act aggressively, are more likely to drink to excess when first consuming alcohol, perhaps indicating a risk for alcohol abuse and potentially future alcohol dependency. Measuring anxiety and aggressiveness in teens (at least in males) may contribute to our ability to accurately predict an individual's initial tendency to consume alcohol in a risky manner, leaving them susceptible the development of AUDs.

Keywords: Alcoholism, Nonhuman Primate, Adolescent Anxiety, Developmental Psychopathology

Disclosure: Nothing to Disclose.

\section{M144. OPRM1 Asp40 Interacts With COMT Val158Met in Predicting Naltrexone Response in an Alcohol Use Disorder Randomized Clinical Trial}

Raymond Anton*, Sarah Book, Konstantin Voronin, Patricia Latham, Patrick Randall

Medical University of South Carolina, Charleston, South Carolina, United States

Background: There is growing interest in "personalized" treatment of Alcohol Use Disorder (AUD) with the opiate antagonist, naltrexone. Whether the putative mu opioid receptor polymorphism OPRM1 asp40 status influences naltrexone response is controversial, with retrospective studies being positive (Oslin 2003, Anton 2008) and one prospective study reportedly negative (Oslin 2015) and another somewhat in between (Schacht 2017). We had previously reported in those not seeking treatment for AUD (Anton 2012), that naltrexone effects on drinking were influenced by an interaction of the OPRM1 asp40 allele and VNTR variation in the dopamine transporter gene (DAT1SLC6A3) and replicated this recently in treatment-seekers (ACNP 2016). We wanted to further explore whether other brain dopamine system genes might interact with the OPRM1 SNP to influence naltrexone response. We therefore examined whether another putative functional dopamine gene variation, catechol-o-methyl transferase (COMT) SNP val158met, might interact with OPRM1 asp40asn to influence naltrexone response.

Methods: 146 Caucasian alcohol dependent individuals (DSM IV) with a mean $80 \%$ heavy drinking days (HDD) were randomized to naltrexone $(50 \mathrm{mg}$ ) or placebo, based on their OPRM1 allele status (asp40 carriers vs. asn40 homozygotes - Taqman PCR) on a one to one basis in a blinded 
fashion. In an exploratory fashion, we also evaluated COMT allele status (met158 carriers vs, val158 homozygotes Taqman PCR) in these participants and evaluated interactions with OPRM1 genotype on naltrexone response.

Smoking (40\%), antidepressant use (33\%), and sex $(30 \%$ female) were equally distributed across groups. Nine sessions of Medical Management (wks. 1,2,3,4,6,8,10,12,16) were provided and drinking assessed by TLFB during the 16week treatment. Percent heavy drinking days (\%HDD) during the study were analyzed in a mixed model of medication (ntx vs. plac.) x OPRM1 allele (at least one asp40 allele vs. asn/asn homozygotes) x COMT (at least one met158 allele vs. val158 homozygotes).

Results: Of 146 evaluable individuals, 73 were OPRM1 asp40 carriers and 73 asn 40 homozygotes. COMT met158 carriers and val158 homozygotes were not significantly different across OPRM1 and medication groups $(p=.92)$ with group sizes ranging from 10-27. Study completers (76\%) and complete drinking data (88\%) did not differ across groups, $(p=.61)$ and $(p=.95)$ respectively.

There was a three-way interaction between medication, OPRM1 SNP and COMT SNP on heavy drinking day during the study $(p=0.065)$, such that OPRM1 Asp40 carriers, who also were COMT val158 homozygotes, responded better to naltrexone than placebo $(p=0.058)$ while those OPRM1 Asn40 homozygotes that were also COMT met158 carriers responded better to naltrexone than placebo $(p=0.03)$, while in other genotypes naltrexone and placebo response were similar. This pharmacogenetic prediction was not modified by sex, age, antidepressant use, or smoking status.

Conclusions: While great emphasis has been placed on the OPRM1 asp40 predicting naltrexone response, results have been inconsistent. One reason is that OPRM1 asp40 might be interacting with other genetic variants (epistasis). One important possibility is the functional COMT val158met variant. Many reports have indicated COMT met158 carriers have lower COMT function leading to increased synaptic dopamine availability. This could underpin more alcohol reward and cue sensitivity in met158 carriers leading to higher relapse risk. We had previously reported, in this same study population, that dopamine transporter gene (DAT1) VNTR variants also interact with the OPRM1 asp40asn SNP in a similar fashion. In our data, the COMT val158met interaction with OPRM1 predicting naltrexone response appears to be largely independent of the DAT1 interaction with OPRM1. In both cases, however, it was the OPRM1 asn40 homozygotes that responded better when they were also carriers of either DAT1 or COMT genotypes that favored more dopamine production. The OPRM1 asp40 carriers seem to respond better to naltrexone when they also carry genotypes favoring lower/normal dopamine production. Overall, these data suggest that genetically based dopamine system variation/tone may influence how opiate systems might respond and interact with medication. This could have both important scientific meaning and clinical implication in regards to pharmacogenetic treatment response and personalized medicine initiatives.

Supported by NIAAA R01AA017633 and K05AA017435

Keywords: Alcoholism, Pharmacotherapy, Pharmacogenetics Disclosure: Part 1: Alcohol Clinical Trails Initiative Alkermes, Indivior, Arbor, Otsuka, Lundbeck, Ethypharm,
Amygdala, Eli Lilly, Pfizer, Inc., Honoraria, CT Pharma, Consultant.

M145. Overexpression of the Endocannabinoid Anandamide Degrading Enzyme in the Basolateral Amygdala Paradoxically Decreases Anxiety and Fear Memory

Maria Morena*, Kira Leitl, Asim Rashid, Sheena Josselyn, Matthew Hill

University of Calgary, Calgary, Canada

Background: Inhibition of anandamide (AEA) hydrolysis by the enzyme fatty acid amide hydrolase (FAAH) within the basolateral complex of the amygdala (BLA) has been shown to reduce anxiety, neuroendocrine responses to stress and promote fear extinction. To determine if impairments in AEA signaling within the BLA would produce the opposite effects, and induce a stress-like state characterized by heightened anxiety and sustained fear, we examined the effects of overexpression of FAAH locally within the BLA on behavioural indices of anxiety and fear memory dynamics.

Methods: Male adult Sprague Dawley rats were bilaterally infused in the BLA with an Herpes simplex virus type 1 vector, which infects principal neurons, containing FAAH and green fluorescent protein (HSV-FAAH-GFP) or a control vector containing only GFP (HSV-GFP). Rats were, then, tested for biochemical measurements, anxiety or fear memory behaviour. All experimental procedures were in compliance with protocols approved by the University of Calgary Animal Care Committee and guidelines from the Canadian Council on Animal Care.

Results: Seventy-two hours following HSV administration, a time point in which protein transfection is maximal, we found increased FAAH-mediated AEA hydrolysis together with decreased AEA levels within the BLA, confirming that the virus did successfully increase FAAH expression. At this same time point, a separate cohort of rats was tested for anxiety behaviour in an elevated plus maze, a light/dark box and an open field task. Quite surprisingly, we found that the overexpression of FAAH induced consistent anxiolytic effects in all the three behavioural tasks we performed, relative to HSV-GFP rats. An additional cohort of animals was tested for fear memory in an auditory fear conditioning paradigm. Animals were bilaterally cannulated in the BLA, a week later were fear conditioned and $24 \mathrm{~h}$ after conditioning, the animals were injected with HSV-FAAH-GFP or its control vector. Seventy-two hours following HSV administration rats were re-exposed to the tone alone for 4 consecutive days to examine fear extinction dynamics. Unexpectedly, rats infused with the HSV-FAAH-GFP vector exhibited a dramatic reduction in fear expression during the extinction training and first extinction retrieval sessions when exposed to the tone, as compared to their HSV-GFP control rats. Moreover, both HSV-GFP and HSV-FAAHGFP animals showed reinstatement of fear memory, when given unsignaled foot shocks after the extinction had occurred in both groups and presented $24 \mathrm{~h}$ later the tone alone, thus demonstrating that the FAAH overexpression did not interfere with the ability to correctly acquire the conditioned-unconditioned stimulus association and to 
express freezing behaviour. Furthermore, the effects of FAAH overexpression on fear memory were blocked by intra-BLA injections of the FAAH inhibitor URB597 and by the GABAA antagonist bicuculline, suggesting that these effects might involve BLA GABAergic transmission.

Conclusions: These findings suggest that the exact modes of action of AEA within the amygdala in the regulation of emotional states and memory are still far from being clear, thus, opening the avenue to investigate new potential mechanisms by which these processes may occur.

Keywords: Endocannabinoids, Basolateral Amygdala, Fear Memory, Anxiety

Disclosure: Nothing to Disclose.

\section{M146. Prefrontal Top-Down Cortical Projection Controls Attentional Behavior}

Hiroyuki Koike*, Kevin Norman, Sarah Lopez, Elisa Nabel, Meghan Flanigan, Yury Garkun,

Zhe Dong, Michael Demars, Mark Baxter, Scott Russo, Hirofumi Morishita

Taisho Pharmaceutical Company, Ltd., Saitama, Japan

Background: Attention is a goal-directed process that facilitates discriminatory detection of relevant sensory stimuli from the environment. Attention deficit symptoms are commonly observed in psychiatric disorders, yet finite understanding of the neural circuits mediating attentional behavior has limited pathophysiologic insight. Previous studies across species have demonstrated that the frontal cortex-especially the anterior cingulate cortex (ACC) plays a key role in implementing attention through "topdown" control of sensory processing. However, the precise neural circuit mechanisms effecting attention remain largely unknown. Here we aim to identify the specific frontal cortex projecting neurons that mediate "top-down" control of visual attentional behavior in mice by integrating circuit-based techniques that monitor and manipulate neural activity in mice performing freely moving attentional behavior with a translational automated touchscreen system.

Methods: We use Bussey-Saksida TouchScreen-based 5choice serial reaction time task (5CSRTT), which requires mice to sustain and divide attention among 5 brief light stimuli presented in random order on touchscreen, to assess attention performance, processing speed, and response control in C57BL/6 mice. To identify the functional localization of the task-specific ACC projections implicated in top-down control, an activity- and tamoxifen-dependent neuron labeling method is used to fluorescently label projections from ACC activated during the 5CSRTT. To examine the causal contribution of neural projection from the ACC, we apply intersectional viral approach to express an inhibitory designer receptors exclusively activated by designer drug (DREADD) in projection specific manner. To identify when ACC projection neurons are activated during attentional behavior, we employ an intersectional top-down projection specific viral expression of genetically encoded calcium fluorescent indicator (GCaMP6) and fiber photometry imaging of neuronal activity in ACC while mice perform the 5CSRTT. Finally, to improve attentional behavior, we optogenetically modulate ACC projection neuron activity during 5CSRTT at time-points when the projection is physiologically activated.

Results: Our unbiased global mapping of neural activity specifically when mice are engaged in 5CSRTT reveals recruitment of a subpopulation of ACC neurons with the long-range cortico-cortical projection to the visual cortex. Selective chemogenetic inhibition of this long-range frontal top-down cortical projection impairs attention performance, without disrupting additional detectable readouts of decision-making capacity, motivational state, motor activity, impulsivity, and compulsivity. Consistently, our preliminary projection-specific fiber photometry imaging in free moving mice performing 5CSRTT shows enhanced activation of topdown projection neurons in correct trials over incorrect or omitted trials between trial initiation and visual stimulus onset when animals need to sustain attention. Optogenetic modulation of top-down projection within this behavioral context is underway in a genetic mouse model of $15 \mathrm{q} 13.3$ microdeletion syndrome to mitigate attention impairment.

Conclusions: Our data demonstrate that a long-range prefrontal-sensory cortical projection is a key enactor topdown control of attentional behavior. Our finding may provide circuit-based insight into the pathophysiology and intervention strategy for impaired visual attention in neurodevelopmental and psychiatric disorders, particularly attention deficit hyperactivity disorder, schizophrenia, and autism spectrum disorder when dysregulated functional connectivity is observed between the prefrontal cortex and visual cortex during visual attention tasks.

Keywords: sustained attention, anterior cingulate cortex, touchscreen, 5CSRTT, top-down control

Disclosure: Part 5: Taisho Pharmaceutical Co., Ltd., Employee.

M147. Metabotropic Glutamate Receptor Subtype 5 in Males With Autism Spectrum Disorder: Preliminary Findings of a [11C]ABP688 Positron Emission Tomography Study

Yoan Mihov*, Valerie Treyer, Funda Akkus, Gerrit Steinberg, Jennifer Niemeyer, Anass Johayem, Simon Ametamey, Alfred Buck, Gregor Hasler

University of Bern, Bern, Switzerland

Background: Preclinical investigations suggesting a therapeutic potential for metabotropic glutamate receptor subtype 5 (mGluR5) antagonists in fragile X syndrome raised interest in mGluR5 in neurodevelopment disorders in general, including autism spectrum disorder (ASD) (Davenport et al, 2016; Gogliotti and Conn, 2016; Scharf et al, 2015). To investigate the role of mGluR5 in ASD in vivo we carried out a positron emission tomography (PET) study with the mGluR5-selective radiotracer [11C]ABP688 in subjects with ASD and healthy controls.

Methods: Seventeen male subjects with ASD and 22 healthy male age-matched controls participated in this study. As our previous work showed that smoking profoundly and enduringly alters mGluR5 (Akkus et al, 2013), we matched both samples for smoking. As a result, the ASD sample included 11 non-smokers, 2 ex-smokers and four current smokers, and the control group 15 non-smokers, 2 exsmokers and five current smokers. 
We assessed psychopathology in both study groups with the Structured Clinical Interview for DSM-IV Axis I (SCID-I), Beck Anxiety Inventory (BAI), and Beck Depression Inventory (BDI). In addition, we evaluated subjects with ASD using the Autism Diagnostic Observation Schedule (ADOS), Yale-Brown Obsessive Compulsive Scale (Y-BOCS), Obsessive Compulsive Inventory-Revised (OCI-R), Autism Quotient (AQ), and Empathy Quotient questionnaires (EQ).

We acquired PET data with a bolus/infusion protocol, as previously reported (Burger et al, 2010). In brief, [11C]ABP688 was administered in a $50-\mathrm{mL}$ volume with an infusion pump. Our previous work shows that $40 \mathrm{~min}$ after the start of radioligand infusion equilibrium between [11C]ABP688 in tissue and blood is achieved. To calculate the relative distribution volume (DVR), we normalized the average of PET images acquired at $45-60 \mathrm{~min}$ after scan onset to the radioactivity concentration in the cerebellum. We used PMOD (PNEUROTool) and R for data analysis. We compared mGluR5 DVR in both groups in 33 brain regions using two-tailed Welch's t-tests, without correction for multiple comparisons.

Results: Age did not differ significantly between subjects with ASD and controls when comparing the entire samples or their subgroups (all p-values $>0.05$, two-tailed). Overall, subjects with ASD scored higher in BAI and BDI $(p<0.05$, two-tailed).

We found no significant differences in mGluR5 DVR in any of the brain regions when comparing the entire samples comprising smokers, ex-smokers, and non-smokers (all p-values $>0.05$, two-tailed). As expected, in both samples, smokers displayed globally decreased mGluR5 DVR $(p<0.05$, onetailed). Due to the profound effect of smoking, we repeated our analyses in non-smokers only. This analysis revealed increased mGluR5 DVR in ASD in two regions: the straight gyrus and the posterior superior temporal gyrus ( $p<0.05$, two-tailed). In addition, there was a trend for increased mGluR5 DVR in ASD in the postcentral gyrus $(p<0.1$, two-tailed). We did not find significantly decreased mGluR5 DVR in ASD in any brain region ( $p>0.05$, two-tailed).

Exploratory correlation analyses involving BAI, BDI, AQ, EQ, and mGluR5 DVR in the straight gyrus, posterior superior temporal gyrus, and postcentral gyrus in nonsmokers with ASD yielded only one significant relationship: higher, i.e., more aberrant, mGluR5 DVR in the postcentral gyrus corresponded to lower, i.e., more aberrant, EQ score (Spearman's rho $=-0.64, p<0.05$, two-tailed, uncorrected for multiple comparisons).

Conclusions: To our knowledge, this is the first in vivo investigation of mGluR5 in persons with ASD. Our findings suggest increased mGluR5 DVR in the straight gyrus, the posterior superior temporal gyrus, and the postcentral gyrus in male non-smokers with ASD. Due to the small sample size and the exploratory nature of the statistical analyses, these results remain preliminary. Nevertheless, our findings provide a preliminary support for the involvement of mGluR5 in ASD, encourage the study of mGluR5 in larger samples, and suggest a therapeutic potential for agents targeting the mGluR5 in autism (Mehta et al, 2011; Silverman et al, 2010).

Keywords: mGluR5 Receptors, Autism Spectrum Disorder, PET Imaging

Disclosure: Nothing to Disclose.
M148. Comprehensive Examination of the GABAergic System in Adults With Autism by Simultaneous [18F] Flumazenil-Positron Emission Tomography and Magnetic Resonance Spectroscopy

Lawrence Fung*, Ryan Flores, Kevin Liu, Meng Gu, Daniel Spielman, Frederick Chin, Antonio Hardan

Stanford University School of Medicine, Stanford, California, United States

Background: Dysfunction of the GABA neurotransmission system is one of the most accepted pathophysiologic mechanisms for autism spectrum disorder (ASD). Early evidence on the involvement of the GABAergic system in the pathophysiology of ASD relied on immunohistologic examination of postmortem brain samples of individuals with ASD. More recently, new imaging technologies allow for in vivo determination of GABA levels by magnetic resonance spectroscopy (MRS) and determination of GABAA receptor densities by [18 F]flumazenil-positron emission tomography ([18 F]FMZ-PET). However, studies published to date have only measured either GABA levels or GABAA receptor densities only. While these studies are very valuable, each of these measurements describes half of the full story. Due to the dynamic nature of neurotransmission systems in general, studying both GABAA receptor densities and GABA levels at the same time in individuals with ASD is highly desirable. In the present investigation, we report the first such attempt to simultaneously measure GABA levels and GABAA receptor densities in the brains of individuals with ASD using a stateof-the-art hybrid PET-MR neuroimaging system. The goal of this study is to examine the independent and interactive effects of GABAA receptor densities and GABA levels in the thalamus and DLPFC, on socio-communicative abilities.

Methods: Participants. Individuals with ASD and neurotypical controls aged 18-55 years. Image acquisition. Subjects were scanned on GE SIGNA PET/MR (Waukesha, WI). PET data were acquired in list mode (0-60 min p.i), dynamically reconstructed into 27 time-frames $(12 \times 15 \mathrm{~s}, 3 \times 1 \mathrm{~min}$, $3 \times 3 \mathrm{~min}, 9 \times 5 \mathrm{~min})$ and corrected for photon attenuation using both scanner-specific 8-channel headcoil correction and a MR-measured head atlas-based attenuation correction maps. During PET data acquisition, a series of MR structural sequences were acquired, including (a) T1-weighted MPRAGE sequence, (b) T2-weighted fast-spin-echo sequence. For the MRS measurement of GABA, IM-SPECIAL was performed on the left dorsolateral prefrontal cortex (DLPFC) and bilateral thalami with voxel sizes of approximately $15 \mathrm{cc}$ and TE/TR $=80 / 2000 \mathrm{~ms}, 15 \mathrm{~min}$ acquisition time. Neuropsychological assessments. Stanford Binet, 5th edition (SB5), Social Responsiveness Scale (SRS-2), Autism Quotient (AQ), Ritvo Autism Asperger Diagnostic Scale-Revised (RAADSR), and Berkeley Expressivity Questionnaire (BEQ). Image and data analysis. The dynamic PET and structural MR data were normalized to MNI (Montreal Neurological Institute) space (PMOD 3.8, Switzerland). Time-activity curves were extracted using pre-defined volumes-of-interest (VOIs) based on the Hammers atlas. A reference tissue model (Ichise model; MRTM0) was used to calculate binding potentials (BPND) with pons as the reference region. BPND is a surrogate for GABAA receptor densities. The IM- 
SPECIAL edited spectrum was obtained by subtracting the editing OFF spectrum from the editing ON spectrum. GABA levels were estimated from the integrated $3 \mathrm{ppm}$ peak area in the edited spectrum divided by the creatine (Cre) peak area and the water peak area.

Results: Eighteen adults with ASD and fourteen healthy volunteers were scanned. Highest uptake was observed in the neocortical regions and limbic system, intermediate in the cerebellum, thalami and basal ganglia, and low uptake in the brainstem. No group differences were found in BPND's and GABA levels of all VOIs. However, group differences were found in the product of the BPND and GABA/Cre of the left DLPFC. Furthermore, the BPND and GABA/water of the left DLPFC were found to have interactive effects on full scale IQ ( $\mathrm{p}<.001 ; \mathrm{R}=.919)$ in the ASD group; GABA/water of the thalami was found to correlate with the social cognition subscale of the SRS-2. In contrast, different correlations were revealed in the control group: the BPND and GABA/creatine of the left DLPFC were found to have interactive effects on the AQ ( $\mathrm{p}=.040$; $\mathrm{R}=.731)$, as well as the total score of the SRS-2 ( $\mathrm{p}=.023 ; \mathrm{R}=.777)$.

Conclusions: Preliminary results from the current study supported that the GABAergic system in adults with ASD was different from that of neurotypical individuals. While GABA levels of the thalami were found to correlate with social cognition in individuals with ASD, left DLPFC GABA levels interacted with left DLPFC GABAA receptor densities on overall socio-communicative function in neurotypical individuals. Further studies will be conducted to confirm these findings.

Keywords: GABA, Autism Spectrum Disorder, Positron Emission Tomography, Proton Magnetic Resonance Spectroscopy

Disclosure: Nothing to Disclose.

\section{M149. Effects of Valbenazine on Prolactin in Adults With Tardive Dyskinesia: Pooled Results From 3 Randomized, Double-Blind, Placebo-Controlled Trials}

Dimitri Grigoriadis*, Roger McIntyre, Scott Siegert, Dao Thai-Cuarto, Joshua Burke

Neurocrine Biosciences, Inc., San Diego, California, United States

Background: Prolactin secretion is regulated by dopamine neurons in the tuberoinfundibular pathway, which connects the hypothalamus to the pituitary gland. Exposure to dopamine receptor blocking agents (DRBAs), including antipsychotic medications, can result in hyperprolactinemia. At clinically relevant levels that depend on sex, age, pregnancy, and menopausal status, elevated prolactin can lead to reproductive, sexual, or metabolic adverse events. Patients who take DRBAs are also at risk for developing tardive dyskinesia (TD), a persistent and often debilitating drug-induced movement disorder. Valbenazine (INGREZ$\mathrm{ZA}$ ) is a novel and highly selective vesicular monoamine transporter 2 (VMAT2) inhibitor that is FDA-approved for the treatment of TD in adults. In valbenazine clinical trials, the majority of participants were taking at least 1 antipsychotic medication to manage their mental disorder (i.e., schizophrenia, schizoaffective disorder, mood disorder).
Data from randomized, double-blind, placebo-controlled trials were analyzed to assess the effects of once-daily valbenazine on serum prolactin levels.

Methods: For the current analyses, valbenazine doses $(40 \mathrm{mg}$, $80 \mathrm{mg}$ ) and placebo were pooled from three Phase $2 / 3$ studies (KINECT [NCT01688037], KINECT 2 [NCT01733121], and KINECT 3 [NCT02274558]). Descriptive analyses were conducted for the following outcomes: treatment-emergent adverse events (TEAEs) potentially related to hyperprolactinemia (e.g., blood prolactin increased, galactorrhea); median changes from baseline to Week 6 in serum prolactin concentrations; and shifts in prolactin concentration levels from baseline to any time during double-blind treatment. Shift levels (normal, low, high) were based on reference ranges for women $(3.8-23.2 \mathrm{ng} / \mathrm{mL})$ and men $(3.0-14.7 \mathrm{ng} /$ $\mathrm{mL}$ ). In addition, a logistic regression analysis and logistic model fit were applied to KINECT 3 data to determine the relationship between elevated prolactin concentration $(\geq 2$ fold increase from baseline) and exposure to valbenazine. For these analyses, exposure was based on predicted maximum plasma concentration (Cmax) and area under the curve (AUC) for the primary active metabolite of valbenazine, $[+]$ - $\alpha$-dihydrotetrabenazine $([+]-\alpha-H T B Z)$.

Results: The pooled safety population included 400 participants (40 mg, $n=110$; $80 \mathrm{mg}, n=112$; placebo, $n=178$ ), 336 (84.0\%) of whom were taking at least 1 antipsychotic medication. No participant had a TEAE potentially associated with hyperprolactinemia. At baseline, median prolactin concentrations (minimum, maximum; ng/mL) were: $40 \mathrm{mg}, 7.5$ (0.5, 108.6); $80 \mathrm{mg}, 8.2$ (1.4, 82.6); placebo, 8.2 $(0.6,100.6)$. Median changes (minimum, maximum; $\mathrm{ng} / \mathrm{mL}$ ) from baseline to Week 6 in serum prolactin concentrations were: $40 \mathrm{mg}, 3.0$ (-35.8, 130.1); $80 \mathrm{mg}, 4.7$ (-41.3, 108.4); placebo, $0.3(-37.0,45.1)$. In participants with available data (40 mg, $n=101 ; 80 \mathrm{mg}, n=108$; placebo, $n=169$ ), prolactin levels at baseline were as follows (normal, low, high): $40 \mathrm{mg}$ (63.4\%, 15.8\%, 20.8\%); $80 \mathrm{mg}(64.8 \%, 14.8 \%, 20.4 \%)$; placebo $(65.7 \%, 6.5 \%, 27.8 \%)$. The percentages of participants who had a shift from normal or low prolactin levels at baseline to high prolactin levels at any time during the 6-week doubleblind treatment period were: $40 \mathrm{mg}, 16.8 \%$ (17/101); $80 \mathrm{mg}$, $25.0 \%$ (27/108); placebo, $10.7 \%$ (18/169). In KINECT 3, the percentages of participants with $\geq 2$-fold increase in prolactin levels were as follows: $40 \mathrm{mg}, 31.4 \%$ (22/70); $80 \mathrm{mg}, 38.3 \%$ (28/73); placebo, $20 \%$ (15/75). The logistic regression analysis indicated that the odds of a $\geq 2$-fold increase in prolactin levels increased by $23 \%$ with each $10 \mu \mathrm{g} /$ $L$ increase in $[+]-\alpha-H T B Z$ Cmax and by $50 \%$ with each $1000 \mu \mathrm{g} \bullet \mathrm{hr} / \mathrm{L}$ increase in [+]- $\alpha-\mathrm{HTBZ}$ AUC. The logistic model fit indicated a linear relationship between the percentage of participants with $\geq 2$-fold increase in prolactin and increasing $[+]-\alpha-H T B Z$ exposure (predicted Cmax and AUC).

Conclusions: Once-daily treatment with valbenazine during placebo-controlled trials resulted in a dose-related increase in prolactin levels in adults with TD, many of whom were also receiving stable doses of their antipsychotic(s) to manage their primary psychiatric disorder.

Keywords: Valbenazine, Tardive Dyskinesia, Prolactin, Clinical Trials

Disclosure: Part 2: Neurocrine Biosciences, Inc., Employee, Part 5: Neurocrine Biosciences, Inc., Employee. 
M150. Structural and Blood Perfusion Abnormalities in Individuals With Autism Spectrum Disorder

Ariana Zargarian*, Ravi Bansal, Siddhant Sawardekar, Bradley Peterson

Keck School of Medicine of USC, Los Angeles, California, United States

Background: Autism Spectrum Disorder (ASD) is characterized by impaired verbal and nonverbal communication, social relationships, and behavioral flexibility. Understanding the underlying neural pathologies of this disorder can significantly contribute towards improving clinical care, diagnosis, and treatment of individuals with ASD. We acquired T1-weighted anatomical magnetic resonance imaging (MRI) and arterial spin labeling (ASL) MRI to understand how brain morphology, cortical thickness, and blood perfusion in individuals with ASD differ from those in healthy controls.

Previous studies of blood flow used positron emission topography (PET) and single-photon emission computed topography (SPECT) to understand how perfusion is abnormal in ASD. However, these techniques expose individuals to radioactivity and thus are difficult to acquire in children and in larger sample sizes. ASL MRI measures cerebral blood flow (CBF) using endogenous, magnetically labeled water molecules. Because this technique does not require the use of radioactive tracers, it is more acceptable to both individuals with autism and healthy controls, allowing us to increase and diversify our study population.

Methods: We acquired anatomical and ASL MRI on a 3 Tesla GE scanner using an 8-channel head coil in 104 (84 males, age 5 to 60 years) individuals with ASD and 130 (97 males) typically developing (TD) controls. Individuals with ASD were groupmatched with TD individuals on age, sex, IQ, handedness, and socioeconomic status. The anatomical MRI scans were preprocessed to correct for intensity nonuniformities, rotated into a standard orientation, scalp stripped to remove non-brain tissue, segmented to define gray and white matter, and subcortical gray matter removed for defining the cortex. Cortex was removed from the brain and morphological operators were applied to define cortical thickness at each point across the entire surface of the brain. All brain images were then coregistered using a similarity transformation (three rotations, three translations, and a global scale) into the coordinate space of a template brain. Each normalized individual brain was nonlinearly warped to the template brain for identifying corresponding points on the surface of the individual and template brain. These point correspondences permitted us to study and compare brain morphology, local volumes, and cortical thickness measures in individuals with ASD relative to TD controls. Individual brains were scaled to the template brain, and therefore, all morphological brain measures were scaled appropriately by the whole brain volume. We are currently statistically analyzing the ASL MRI data to assess differences in blood perfusion between individuals with ASD and TD controls. In the anatomical images, we performed statistical analyses at each point on the surface of the brain using multiple linear regression while covarying for age and sex. We used False Discovery Rate (FDR) to control for false positives from multiple statistical tests across the cerebral surface. We color-encoded and plotted the p-values that survived the FDR procedure on the surface of the template brain. Brain regions where individuals with ASD had a thinner cortex or reduced local volumes relative to TD controls are shown in cool colors (blue and purple), and those regions with thicker cortex or increased local volumes are shown in warm colors (red and yellow).

Results: Our study showed that individuals with ASD bilaterally had thinner cortex in the prefrontal cortex and thicker cortex in the orbitofrontal cortex, superior parietal cortex, occipital cortex, mesial prefrontal cortex, and cuneus region of the brain.

Conclusions: Our anatomical MRI findings suggest individuals with ASD have widespread abnormalities in brain morphology and cortical thickness, especially in brain regions that support cognition and emotional processing. We expect the ASL data to show greater blood perfusion in brain regions of greater cortical thickness in individuals with ASD.

Keywords: Autism Spectrum Disorders, Cerebral Blood Flow, Arterial Spin Labeling, Magnetic Resonance Imaging Disclosure: Nothing to Disclose.

\section{M151. Modeling Reward Decision Conflict: An fMRI Study}

Thilo Deckersbach*, Emily Hahn, Julia Felicione, Kristen Ellard, Todd Herrington, Shaun Patel, Angelique Paulk, Alik Widge, Emad Eskandar, Darin Dougherty

Massachusetts General Hospital, Charlestown, Massachusetts, United States

Background: Reward and punishment influence human behavior. The anticipation of reward motivates approach, whereas the expectation of punishment often leads to avoidance. Approach-avoidance behaviors are supported by a diverse collection of neural circuits, including those supporting reward valuation, e.g. orbitofrontal cortex (OFC), cingulate cortex, and the striatum; and those supporting threat monitoring, e.g. insular cortex, amygdala, and the hippocampus. In daily life, the chance of obtaining reward often comes at the risk of punishment, potentially creating a conflict with respect to decision-making. While the functional neuroanatomy of decision-making conflict has been demonstrated using task-based functional magnetic resonance imaging (fMRI), these methods have been criticized for not showing consistent results across studies. However, this may be an effect of modeling decision-making behavior. Here, we conducted two different fMRI paradigms that both involve decisions about reward. The same behavioral model was applied to both tasks. Functional activations were then compared across tasks.

Methods: Study participants were 34 healthy adults (13 female) who completed the Aversion-Reward-Conflict (ARC) paradigm and the WAR paradigm. In the ARC task, participants choose a risky or safe option. The risky option offers higher payment and chance of electric shock, while the safe option is always low payment and no shock. The WAR paradigm is a simplified version of the classic card game, 'War'. For this task, the subject and computer are dealt one card, and the participant must make a bet on which player's card hand is higher. Choosing the lower bet implies that the computer has a higher card, and choosing the higher bet implies the computer has a lower card. For the behavioral analysis, a state-space behavioral model was used to model 
decision conflict in both tasks. For each trial the degree of conflict about choosing reward was modeled. For example, decision-making conflict is highest when subjects exhibit a 50/50 chance of choosing the reward option. We modeled each trial as the distance from this "decision-making boundary", aligning them across a subjective decision-space. To integrate the behavioral modeling results into the fMRI models, the WAR and ARC AMRI distance to decision-making boundary values were inputted as a regressor (parametric modulator) into the first level General Linear Model. At the group level, a disjunction analysis was performed to identify unique neural substrates for each task (i.e., regions active in ARC but not $\mathrm{WAR}$, and the converse). The disjunction map was comprised of all voxels that survived multiple comparisons correction in the average activation map for each task exclusively. A conjunction analysis was performed to identify overlapping neural correlates between tasks. The conjunction map was comprised of all voxels that survived multiple comparisons correction in the average activation map for both tasks.

Results: For the fMRI conjunction/disjunction analysis, both ARC and WAR tasks revealed overlapping networks, including regions implicated in reward processing (striatum (caudate and nucleus accumbens), subthalamic nucleus) as well as cognitive control (dorsal anterior cingulate/dorsomedial prefrontal cortex, dorsolateral prefrontal cortex). Insula was also recruited for both tasks, further corroborating recent findings that suggest it is implicated in risk processing. The disjunction analysis revealed hippocampus as exclusive to ARC. On the contrary, no nodes were unique to WAR.

Conclusions: The appropriate modeling of conflict can provide measurable insight into what behavioral and neural processes give rise to maladaptive decision-making involving reward. Importantly, both ARC and WAR tasks effectively create decision-making conflicts. Regions activated by the tasks show great degree of overlap. Taken together, these results strengthen $\mathrm{fMRI}$ as a tool for functional mapping.

Keywords: Decision-Making, Approach/Avoidance, Impulsivity, Bayesian Modeling, fMRI

Disclosure: Nothing to Disclose.

\section{M152. Comparative Analysis of the Effects of Cannabidiol and $\triangle 9-T H C$ in Pilocarpine-Induced Epilepsy}

\section{Veronica Noches*, Carlos Rivera, Maria Andres \\ Pontificia Universidad Catolica de Chile, Santiago, Chile}

Background: Epilepsy is a neurological disorder characterized by recurrent spontaneous seizures. The treatment with cannabinoids for the resistant type of epilepsy is a promising therapy. It has been shown that the main psychoactive component of marijuana, delta-9-tetrahydrocannabinol $(\Delta 9$ THC), has anticonvulsant properties. In addition, the nonpsychotropic component of marijuana cannabidiol also has properties to prevent seizures. Our main objective is to relate the molecular and neurochemical effects of $\Delta 9$-THC and cannabidiol with their potential anticonvulsants properties. Methods: In our laboratory, we have implemented the epilepsy model in mice using $300 \mathrm{mg} / \mathrm{kg}$ of pilocarpine, a muscarinic cholinergic agonist that causes seizures in the temporal lobe of the brain. After 24 hours post pilocarpine injection, we did protein extraction from hippocampus and immunofluorescence on tissue to characterize epigenetic enzymes that have been involved in epilepsy. We evaluated the release of glutamate and GABA in hippocampus post-pilocarpine injection using in vivo microdialysis (AP: $-1.8, \mathrm{~L}:-1.5, \mathrm{~V}: 1.7$ relative to bregma, according to the atlas of Paxinos and Franklin). As well, the mice with pilocarpine were injected with cannabidiol $(100 \mathrm{mg} /$ $\mathrm{kg}$ ) after 20 minutes post pilocarpine injection.

Results: We observed by in vivo microdialysis an increase of both glutamate and GABA extracellular levels after pilocarpine injection. Preliminary data show that cannabidiol potentiates the epileptic seizure.

Molecularly, we are characterizing epigenetic enzymes that have been involved in epilepsy. In this regard, the lysinespecific demethylase 1 (LSD1/KDM1A) is an ubiquitously expressed histone H3Lys4 demethylase that acts as a transcriptional co-repressor in complex with its molecular partners CoREST and HDAC1/2 (LCH complex). LSD1 has a neuro-specific splicing variant by the inclusion of the exon E8a. NeuroLSD1-specific null mice are hypoexcitable and display decreased susceptibility to pilocarpine-induced seizure. We are studying the expression and subcellular location of all components of the LCH complex in the mice epilepsy model. Protein levels of LSD1 do not change after 24 hours of seizure. However, we observed a significant increase of CoREST2 and a decrease of CoREST1 specifically in the hippocampus of mice subjected to pilocarpine-induced seizure.

Conclusions: In summary, the data suggest that epigenetic modifications occur in the hippocampus during pilocarpineinduced seizure, which could be potentiated by cannabidiol. Keywords: Epilepsy, Cannabidiol, Epigenetic

Disclosure: Nothing to Disclose.

M153. Distinct Effects of Early-Life Experience and Trait Aggression on Cardiovascular Reactivity and Recovery

Ilan Kerman*, Phyllis Pugh, J. Michael Wyss, Sarah Clinton

Virginia Tech University, Roanoke, Virginia, United States

Background: Early-life experience (ELE) can significantly affect life-long health and disease, including cardiovascular function. Specific dimensions of emotionality also modify risk of disease, and aggressive traits along with social inhibition have been established as independent vulnerability factors for the progression of cardiovascular disease. Yet, the biological mechanisms mediating these associations remain poorly understood. We have previously demonstrated independent effects of ELE and trait aggression (TA) on resting heart rate (HR) and mean arterial pressure (MAP). Specifically, we found that pups that experienced early-life maternal separation (MS) exhibited lower resting HR accompanied by increases in HR variability irrespective of their propensity for aggression. In contrast animals with high TA (irrespective of their ELE) showed increased systolic, diastolic and mean arterial pressures, but no changes in resting heart rate. In this study, we sought to extend these findings by examining potential impact of TA and ELE on shaping stress-evoked cardiovascular responses.

Methods: The present study utilized the inherently stresssusceptible and socially inhibited Wistar-Kyoto rats that 
were bred in our laboratory. Pups were exposed to maternal separation (MS), consisting of daily 3 - $h$ separations of the entire litter from postnatal day (P)1 to P14, or to neonatal handling $(\mathrm{NH})$ consisting of 15 min daily separations from $\mathrm{P} 1$ to P14. The rats were weaned at P21, and as adults were instrumented for chronic radiotelemetry recordings of blood pressure and heart rate (HR). Adult aggressive behavior was assessed using the resident-intruder test, which demonstrated that TA was independent of MS exposure. Stressevoked HR and blood pressure responses were then recorded using radiotelemetry in response to different stressors, including: strobe light, exposure to a novel environment, exposure to an intruder rat, and restraint stress.

Results: Maximal HR and MAP responses to each stressor were quantified as indices of reactivity. One-phase exponential decay plots were then fitted to the data to quantify MAP and HR decay constants as a measure of recovery from stress. Strobe light was the weakest stressor, evoking the lowest increase in maximal HR of $22.56 \pm 3.57 \%$ as compared to $79.82 \pm 4.26 \%$ for intruder stress, $67.24 \pm 2.70 \%$ for restraint, and $58.74 \pm 2.00 \%$ novel environment. In addition, HR returned to baseline following strobe light and novel environment exposure, but not following intruder stress (at 10\% above baseline) or restraint (20-30\% above baseline). MAP responses showed the same pattern as HR.

Among these stressors, only responses to strobe light were significantly impacted by ELE, with MS-exposed rats (irrespective of their aggressive behavior) manifesting greater maximal HR and MAP responses as compared to their $\mathrm{NH}$ counterparts. In contrast, responses to a novel environment and to the intruder stress were significantly influenced by TA, but not ELE. TA animals exhibited increased maximum HR response and a decreased HR decay constant in a novel environment, along with increased $\mathrm{HR}$ and MAP decay constants with intruder stress. Finally, cardiovascular responses to restraint stress showed a significant interaction of ELE and TA in shaping the decay constants for HR and MAP.

Conclusions: Together these data indicate that cardiovascular stress reactivity is shaped by both ELE and TA. These effects depend on: 1) stress severity, 2) ability to choose a coping strategy in experiencing the stressor, and 3) presence of a social component. These data may help us understand the impact of stress exposure and subsequent risk for developing cardiovascular pathology as cardiovascular reactivity and recovery have been implicated in the emergence of hypertension and coronary artery disease.

Keywords: Wistar Kyoto Rat, Social Interactions, Aggression, Heart Rate, Blood Pressure

Disclosure: Nothing to Disclose.

M154. An Open Label PET Imaging Study to Evaluate the mGlu5 Receptor Occupancy Following ADX48621 (Dipraglurant) Administration

Dean Wong*, Hiroto Kuwabara, Sonia- Maria Poli, Lorena Gapasin, Anil Mathur, Elisa Carenza, James Brasic, Noble George, Josh Roberts, Kelly Kitzmiller, Thierry Duvauchelle

Johns Hopkins Medical Institutions, Baltimore, Maryland, United States
Background: ADX48621 is a selective, orally available, small molecule drug candidate which acts as a negative allosteric modulator (NAM) of the metabotropic glutamate receptor 5 (mGlu5 receptor). ADX48621 is selective for the mGlu5 receptor and does not have any significant activity or binding affinity to other mGlu receptors or CNS receptors, including serotonin, GABA and dopamine receptors.

Preclinical profiling of the safety of ADX485621 has been performed in vitro and in vivo to support up to three months of dosing in humans. ADX48621 is effective in the MPTP macaque model of Parkinson's disease (PD) levodopa induced dyskinesia (LID) and in addition, it has been shown to be efficacious in a number of preclinical pharmacology models of dystonia, anxiety, compulsion, depression, and parkinsonian motor symptom control.

Dipraglurant has been studied in three Phase 1 clinical studies and a Phase 2a LID Proof of Concept (POC) trial in PD patients, where it showed clinically meaningful improvements in LID scores at doses that were generally safe and well tolerated. To date, the clinical studies have enrolled 220 subjects (including $76 \mathrm{PD}$ patients). Among the total enrolled, 124 of the healthy subjects and 52 of the PD patients received at least one dose of dipraglurant.

A series of PET imaging studies with [18 F]FPEB were previously performed in non-human primates to determine mGlu5 receptor occupancy following intravenous injection of ADX48621. The collected data demonstrated that the receptors occupancy by $\mathrm{ADX} 48621$ was dose-dependent.

This study was intended:

1) to demonstrate brain penetration and target engagement of brain mGlu5 receptors after single or two oral doses of ADX48621in healthy subjects, using [18 F]-FPEB.

2) to assess the relationship between ADX48621 plasma concentration and its brain mGlu5 occupancy.

Methods: This was an open label, non-randomized, PET study, investigating the mGlu5 receptor occupancy after single or two oral doses of ADX48621 (dipraglurant) in healthy male subjects using [18 F]-FPEB using an adaptive dosing design where the dose administered could be changed after observation of the occupancy after each subject studied. Each subject was scanned using the HRRT (high resolution research tomograph) $2 \mathrm{~mm}$ axial resolution with [18 F]FPEB synthesized at the JHU radiochemistry/PET center. In Part 1, subjects underwent two [18 F]-FPEB PET imaging sessions: a baseline scan. then a second scan after treatment with one dose of ADX48621. The second scan was taken at Tmax for ADX48621 (1- hour post dose). In part 2, the dose that provided sufficient receptor occupancy as determined in Part 1, was studied in Part 2. Subjects received 2 doses of ADX48621 as a single dose on 2 separate days, and underwent three [18 F]-FPEB PET imaging sessions: a baseline scan, post dose scan 1 and post dose scan 2 .

The plasma reference graphical analysis (PRGA) was used to obtain distribution volume (VT) values of regions. It was examined that whether VT values of the cerebellum white matter (CW) remain stable across doses and $\mathrm{PK}$ values to evaluate whether CW could serve for reference region in occupancy scans. In parallel, distribution volume of nondisplaceable compartment (VND) was estimated for individual baseline-post-dose scan pairs using the inhibition plot (Gjedde and Wong et al.). 
Blood samples for PK were collected up to 3.00 hours post dose in Part 1 and up to 4.00 hours post dose in Part 2.

Results: A total of 12 subjects were studied. It was noted that VT values of CW decreased with doses and PK values ( $p<0.007$; simple regression), while VND values remained unchanged across doses $(1.5 \pm 0.8 \mathrm{~mL} / \mathrm{mL})$. Therefore, occupancy values were calculated as follows: Occupancy $(\%)=\Delta \mathrm{VT} /($ baseline VT $-\mathrm{VND}) \cdot 100$. Observed PK values ranged from 354 to $13699 \mathrm{ng} / \mathrm{mL}$, while occupancy values ranged from just above $10 \%$ to just above $80 \%$ across regions. The occupancy-PK plots fitted very well with the first-order Hill equation (occupancy $=\mathrm{PK} \cdot \mathrm{Omax} /(\mathrm{PK}+\mathrm{EC} 50)$; Omax $=$ $100 \%)$ across regions, including data of subjects who had second post-dose scans ( $n=5 ; \Delta \mathrm{PK}$ range: $655-5991 \mathrm{ng} / \mathrm{mL}$ ). Observed EC50 values averaged at $2910 \pm 152 \mathrm{ng} / \mathrm{mL}$ across regions, and EC70 values averaged at $6791 \pm 354 \mathrm{ng} / \mathrm{mL}$.

Conclusions: This study showed that occupancy of ADX48621 of mGluR5s obeys the first-order Hill equation as measured with [18 F]-FPEB. Five subjects had two postdose scans after separate single oral dose of ADX48621. Data points of these subjects existed along the model predicted curves in individual regions whether $\mathrm{PK}$ values were close to each other $(n=2)$ or very different $(n=3)$ between the two scans. These findings on double post-dose scans ensured the assertion, compared to the single post-dose scan design.

This study also yielded important findings regarding [18 F]FPEB. It was demonstrated that binding in CW was displaceable in a dose-dependent manner, and about $50 \%$ of VT in CW (slightly above $3 \mathrm{~mL} / \mathrm{mL}$ ) was displaceable, based on observed VND values (about $1.5 \mathrm{~mL} / \mathrm{mL}$ ). Therefore, drug occupancy studies could be misleading without employing arterial plasma data. In this study, EC50 values of tissue reference methods (no blood data) were about 2.5 times higher than EC50 values given by PRGA.

Keywords: PET, mGlu5, Target Engagement, Receptor Occupancy

Disclosure: Part 4: Roche Neuroscience, Grant, Dart Neuroscience, Grant, Acorda Therapeutics, Grant, Addex, Grant.

\section{M155. A Longitudinal, Prospective Study of DTI-Based White Matter Disturbances in Young Adults Born Prematurely}

Jonathan Sadik, Ravi Bansal, Stein Magnus Aukland, René Westerhausen, Kerstin Jessica Plessen, Morten Duus Odberg, Irene Bircow Elgen, Lars Ersland, Geir Egil Eide, Karen Rosendahl, Siddhant Sawardekar, Bradley Peterson*

Children's Hospital Los Angeles and University of Southern California, La Crescenta, California, United States

Background: Preterm low birth weight infants tend to display neurocognitive deficits later in life. Past work has shown that deficits in numerous brain areas, in both white and grey matter, contribute to this disparity. We aim to corroborate the brain regions affected by preterm birth, and explore the nature of these deficits, using diffusion tensor imaging (DTI) of preterm and healthy cohorts assigned at birth.

Methods: One hundred thirteen low birth weight $(<2000 \mathrm{~g})$ and one hundred controls, originally recruited at birth, had
DTI images taken at 19 years of age. Neurocognitive measures were also recorded at this time. The DTI images were processed using computer programs and numerous measures were calculated, including the average diffusion coefficient (ADC) and fractional anisotropy (FA). Statistical programs were used to compare differences in these values between the two groups.

Results: Subjects from the preterm cohort had significantly lower FA in the splenium of the corpus callosum and the inferior frontal gyrus. FA was higher in the hippocampus, lingual gyrus, and posterior corona radiata. ADC was widely increased in the preterm cohort, including in the thalamus, hippocampus, putamen, lingual gyrus, and right and left posterior corona radiata. ADC was lower in the cingulate gyrus.

All results were more pronounced in the very low birth weight group $(<1500 \mathrm{~g})$ compared to the upper low birth weight group ( $>1500 \mathrm{~g}$ and $<2000 \mathrm{~g}$ ).

Conclusions: Using a robust at-birth cohort selection, we were able to use DTI to confirm that various areas of the brain are affected by preterm birth. Our work also offers insight into the nature of these deficits. These findings are a step towards identifying preterm infants at-risk for later neurocognitive abnormality; early intervention programs may help prevent the progression of decline in these patients. Further work is needed to confirm the precise pathophysiology of these deficits, and the effectiveness of prevention programs.

Keywords: Preterm, Diffusion Tensor Imaging (DTI), White Matter, Low Birth Weight, Prematurity

Disclosure: Nothing to Disclose.

M156. Sleep Alters Subcortical Grey and White Matter Tissue Probabilities and Reduces Anisotropic Apparent Diffusion Coefficient Measures in Arousal Networks

Sukru Demiral*, Dardo Tomasi, Corinde Wiers, Ehsan Shokri Kojori, Joelle Sarlls, Carlo Pierpaoli, Gene-Jack Wang, Helene Benveniste, Nora Volkow

National Institute on Alcohol Abuse and Alcoholism, Bethesda, Maryland, United States

Background: Sleep deprivation can temporarily influence brain microstructure, leading to changes in white matter between rested and sleep deprived states (Elvsashagen et.al., 2015). Preclinical studies have shown that cerebrospinal fluid (CSF) transport into the brain-wide glymphatic pathway comprising peri-vascular compartment and brain parenchyma including grey mater (GM) leading to clearance of waste products from the interstitial fluid (ISF) space in particular during deep wave sleep (Xie et.al., 2014), however it is largely unknown whether or not glymphatic system dynamics during sleep observed in rodent brain are mirrored in the live human brain. Thus, in our study, we combined voxelbased morphometry analysis with Apparent Diffusion Coefficient (ADC) measures (Le Bihan, 2013) in order to understand whether structural changes related to sleep could trigger water diffusion changes in the human brain tissue. We examined WM (White Matter) and GM volume changes as well as ADC in healthy volunteers during sleep and the awake state via magnetic resonance imaging (MRI). 
Methods: Thirty healthy subjects ( 14 females, 16 males; ages 23-59) visited the clinic on two nights; one night they were allowed to sleep, and the other night they were kept awake under supervision of a nurse. In the following morning we ran MRIs. Sessions were done in randomized order. During the night, electroencephalography (EEG) recording was also used to assess the quality of sleep/awake. Structural and diffusion MRI scans were performed in a 3 T Siemens Prisma scanner with a 32-channel RF coil in the following mornings at 9AM. Participants stayed awake in the scanner after the rested night, but were asked to feel free to fall sleep after the sleep-deprived night. An MRI-compatible video camera was used to monitor sleep/arousal during MRI. Physiological signals (respiration and cardiac) were recorded using a BIOPAC system. High resolution T1 (3d MPRAGE, TR= $2400 \mathrm{~ms}$, $\mathrm{TE}=2.14 \mathrm{~ms}$, $\mathrm{TI}=1000 \mathrm{~ms}$, Flip Angle $=8 \mathrm{deg}$, $0.7 \mathrm{~mm}$ isotropic) images were entered into CAT12 software for voxel-based morphometry analysis for each session separately, which was then used in longitudinal analysis pipeline for calculating microstructural state changes. ADC calculation was conducted for slow (b-values of 300 and 1000 $(\mathrm{x} 10-3 \mathrm{~mm} / \mathrm{s})$ ), fast (b-values of 0,50 and $300(\mathrm{x} 10-3 \mathrm{~mm} /$ s) and full-model (using all b-values) diffusion components in three directions $\mathrm{x}, \mathrm{y}$, and $\mathrm{z}$. Additional average ADC values (trace) was also calculated.

Results: Paired t-tests were performed to compare brain volumetric measures during sleep versus rested wakefulness. Sleep relative to rested wakefulness showed a marginal increase in the CSF volume in overall brain $(>2 \mathrm{ml}, \mathrm{p}=.08$, 2-tailed). In addition, tissue probabilities in thalamus for GM decreased and for WM increased during sleep. Significant effects in fast ADC in thalamus (mainly in z-direction), slow ADC in hippocampus (mainly y-direction) and full-model in Nucleus Accumbens (x-direction) and Ventral Striatum (z-direction) were only observed for awake state.

Conclusions: Our preliminary results indicate that sleep results in microstructural changes, increasing CSF in the brain, and decreasing GM mainly in the arousal centers (i.e., thalamus). Strikingly, ADC in the arousal centers (i.e., thalamus, Nucleus Accumbens, Ventral Striatum, Cerebellum) was more isotropic during sleep, which might indicate increased hindered diffusion patterns under such microstructural environment. Our preliminary results indicate that glymphatic clearance hypothesis needs to be evaluated along with microstructural changes influenced by natural awakesleep state transitions.

Keywords: Sleep, Apparent Diffusion Coefficient, Glymhatic Clearance, Voxel-based Morphometry

Disclosure: Nothing to Disclose.

M157. [18F]FDG Uptake is Transiently Reduced in Mouse Brain Following Repetitive Blast-Induced Mild Traumatic Brain Injury

Garth Terry*, Kole Meeker, Marcella Cline, James Meabon, Robert Miyaoka, Murray Raskind, David Cook, Elaine Peskind

University of Washington, Seattle, Washington, United States

Background: There is growing concern that repetitive mild traumatic brain injury (mTBI) from either blast (e.g., battlefield-related explosions) or impact (e.g., contact sports such as American football or boxing) may increase the risk of developing neurodegenerative conditions that include $\mathrm{Alz}$ heimer's disease-related disorders and chronic traumatic encephalopathy (CTE). Neurodegenerative diseases often demonstrate reduced of [18 F]FDG brain uptake, representing reduced metabolic activity associated with disease progression. We have previously demonstrated that [18 F] FDG uptake in the cerebellum of Veterans is reduced in correlation to the number of blast mTBIs exposures.

Our mouse model of repetitive blast mTBI has demonstrated an anatomical pattern of injury in the cerebellum that are consistent with FDG-PET neuroimaging findings Veterans with blast-related mTBI. This vulnerability to neuron loss in these mice has also been associated with increased expression of phospho-tau in cerebellum, a marker associated with chronic neurodegenerative diseases. In this study we chose to image mice with [18 F]FDG at several time points following repetitive blast $\mathrm{mTBI}$ to assess if metabolic changes reflect the neurodegenerative pattern we have observed by histopathology.

Methods: All experiments and procedures were approved by the VA IACUC. Using a helium-driven pneumatic shock tube (which simulates battlefield-relevant shock waves), 3-4 month-old male C57BL/6 J mice anesthetized with isoflurane were exposed to eight (two consecutive exposures per day) primary blast overpressures (BOP; 19 psi peak intensity) or identical sham condition without blast exposure. At 4, 6, and 12 days post blast $(n=6)$ or sham $(n=10)$ exposure mice were imaged to assess the initial time course of acute injury. Additional mice (sham $n=3$, blast $n=2$ ) were imaged 40 days after blast representing more chronic injury. Mice undergoing imaging were fasted before being given $283+$ /30 microCi [18 F]FDG by intraperitoneal injection while under anesthesia. Mice were imaged on a Siemens Inveon PET/CT 50 to 70 minutes after [18 F]FDG injection. PET data was reconstructed into five minute frames to enable verification of [18 F]FDG steady state in brain and allow correction of any movement artifact. Tomographic images from 60 - 70 minutes after injection were analyzed with PMOD (PMOD Technologies Zurich, Switzerland). Radioactivity was decay-corrected to time of injection and expressed as percent standardized uptake value (\%SUV), which normalizes for injected activity and body weight, and provides comparison between mice. Statistical analysis was performed using two-tailed t-test comparing blast and sham exposed mice at individual time points; ANOVA was performed comparing those groups imaged repeatedly over time.

Results: Mice exposed to blast demonstrated 20\% less brain uptake of [18 F]FDG compared to sham controls four days after repetitive blast $\mathrm{mTBI}(p<0.05)$. There was no significant difference between groups at 6,12 , or 40 days after blast exposure. Blast mTBI mice imaged at multiple time points were significantly different between days 6 and 12; neither blast nor sham mice were significantly different across any other repeated measure over time.

Conclusions: These findings demonstrate [18 F]FDG uptake in mouse brain following blast is lower at least 4 days after blast exposure, suggesting hypometabolism resulting from acute injury. Resolution of the group differences at later time points might suggest recovery of brain neuronal metabolism 
over time. However, in light of our prior histopathology evidence demonstrating neurodegeneration 30 days after repetitive blast $\mathrm{mTBI}$, the lack of difference between blast and sham mice at later time points alternatively suggests that a compensatory hypermetabolic process (e.g., inflammation) might mask hypometabolism due to neuronal loss. Therefore, the poor specificity of [18 F]FDG might lead to limited sensitivity in detecting neuroinjury due to blast mTBI in mice.

Keywords: Mild Traumatic Brain Injury, Fluorodeoxyglucose, Blast

Disclosure: Nothing to Disclose.

\section{M158. Reduced Intracortical Facilitation as a Biomarker for Co-Morbid Attention Deficit Hyperactivity Disorder in Youth With Autism Spectrum Disorder}

Ernest Pedapati*, Craig Erickson, Lindsey Mooney, Steve Wu, Lauren Ethridge, John Sweeney, Donald Gilbert

Cincinnati Children's Hospital, Cincinnati, Ohio, United States

Background: The neurological correlates distinguishing youth with Autism (ASD) and youth with ASD and cooccurring Attention Deficit/Hyperactivity Disorder (ASD $+\mathrm{ADHD})$ is poorly understood, despite evidence that ASD +ADHD have higher rates of hospitalization, psychopharmacology, and behavioral concerns than ASD alone. In addition, youth with dual diagnoses have therapeutic implications including distinct treatment and neural correlates. Currently, there is a lack of an objective methodology for the diagnosis and management of ASD+ADHD, especially regarding pharmacotherapy response. Abnormalities in transcranial magnetic stimulation (TMS) evoked measure, short-interval cortical inhibition (SICI) and intracortical facilitation (ICF) has been previously associated with ADHD diagnosis and severity. Here we present baseline data which compares neurophysiological data and behavioral correlates between ASD and ASD+ADHD. In addition, we anticipate presenting novel, unblinded results of a single dose methylphenidate trial in subjects with ASD+ADHD pre/post differences in TMS, resting state dense array electroencephalography (EEG), and high-speed eye tracking.

Methods: Paired pulse transcranial magnetic stimulation (ppTMS) data and relevant behavioral measures were performed based on standard methods from an age and gender matched sample of ASD and ASD+ADHD. Short cortical inhibition (SICI) and ICF (intracortical facilitation), TMS measures of the efficiency of interneurons in the primary motor cortex (M1), were collected. The amplitude of TMS motor evoked potentials (MEP) by surface electromyography of the dominant hand was used as the primary outcome. These data are baseline measures from an ongoing randomized controlled, single dose methylphenidate study in youth with ADHD+ASD.

Results: Twenty-four ASD+ADHD subjects (mean age $=$ 14.5 ) and 15 ASD only subjects (mean age $=15.1$ ) were included in this analysis. As expected, Conners-3 Parent Rating Scale-Inattention (CPRS-IA), Hyperactivity (CPRS$\mathrm{H})$, and Executive Functioning(CPRS-EF) scores were significantly higher for the $\mathrm{ASD}+\mathrm{ADHD}$ group relative to the ASD group. ASD subjects demonstrated significantly enhanced intracortical facilitation (ICF) $(\mathrm{M}=1.26, \mathrm{SD}=$ $0.28, n=15)$ compared to ASD+ADHD subjects $(\mathrm{M}=1.01$, $\mathrm{SD}=0.22, n=24 ; \mathrm{t}=2.6, \mathrm{df}=37, p=0.01)$. ICF was significantly inversely correlated with CPRS-EF $(r=-0.350$; $p=0.029)$, CPRS-IA $(r=-0.394 ; p=0.013)$ but not CPRS-H $(r=-0.083 ; p=0.61)$. ICF also correlated with the ADHD-IV Rating Scale (ADHDRS) Inattention scale $(r=-0.432$; $p=0.007$ ) but not ADHDRS Hyperactivity scale.

Conclusions: This data suggests that ICF, a rapidly obtained ppTMS measure which is associated with glutamergic activity, is disturbed in ASD+ADHD. ICF is also associated with clinical variables representing the severity of inattention and executive function in ADHD. Further results are expected from the larger RCT which will clarify the impact of methylphenidate on these TMS measures, but also resting state electroencephalography and high-speed eye tracking. Keywords: Autism, Transcranial Magnetic Stimulation, ADHD, Quantitative Electroencephalography (qEEG)

Disclosure: Part 4: AACAP Junior Investigator Award, Grant.

M159. A Proof-of-Concept Open-Label Trial of COMT Inhibition to Ameliorate Cognitive and Behavioral Sequelae of Traumatic Brain Injury

Robert Schloesser*, Emily Berich, Alexander Maclay, Margo Lauterbach, Andrew Jaffe, Daniel Weinberger

Sheppard Pratt-Lieber Research Institute, Baltimore, Maryland, United States

Background: There are no FDA approved treatments for cognitive and behavioral problems experienced by patients after a traumatic brain injury (TBI). The catecholamine dopamine plays an important role in many aspects of cognition, including learning, working memory and inhibitory control. These cortical functions are often impaired in patients with TBI; some dopaminergic drugs have been shown to improve cognition in patients with a history of TBI. The goal of this project is to investigate whether improving cortical dopaminergic tone by inhibition of the major catabolic pathway of dopamine in the cortex can alleviate symptoms of cognitive impairment and behavioral dysfunction in patients with TBI. Specifically, we are examining the effects of Tolcapone, a brain-penetrant catechol-Omethyltransferase (COMT) inhibitor, on cognition and behavior in patients with a remote history of TBI. Inhibition of COMT by Tolcapone has been shown to improve prefrontal cortical functions in healthy controls and some studies show improvement of quality of life and neuropsychiatric problems in patients with Parkinson's disorder and other psychiatric conditions, however, whether Tolcapone can lead to clinically relevant improvements in patients with TBI is unknown.

Methods: This is an open-label clinical trial to examine the effects of COMT inhibition on cognitive and patient reported outcomes in patients with a history of TBI (mild-severe). Subjects take Tolcapone (200 mg TID) for 14 days. Cognitive and patient reported outcome measures were obtained prior to the start of and on the last day of Tolcapone treatment. 
Outcome measure: NIH Toolbox Cognitive Battery (including measures for Processing Speed, Attention, Working Memory, Executive Function, Episodic Memory and Language) as well as patient reported outcomes (22 domains of the TBI-Qol assessment, Frontal Systems Behavior Scale) and proxy reported outcomes (Frontal Systems Behavior Scale). Cohort: Participants were recruited from the Neuropsychiatry Clinic at the Sheppard Pratt Health System, a tertiary referral clinic specializing in care of patients with neuropsychiatric conditions, including patients with acquired brain injuries and neurodevelopmental disorders. 20 Subjects (7 female, 13 male) have completed this study. Mean age of the subjects is 46.80 years of age (SD 13.72).

Results: Open-label Tolcapone treatment significantly improved cognitive function in patients with a history of TBI. Group level analysis shows improvement in all 5 cognitive domains tested by the NIH Toolbox. The degree of improvement was largest in tests for episodic memory (Picture Sequence, $p=0.008^{*}$, effect size (delta T-scores): +7.0 ) and executive function (Dimensional Card Sorting Test, $p=0.017^{\star}$, effect size (delta T-scores): +5.6 ). However, there is substantial variability of improvement between subjects and cluster analysis separated 3 groups of subjects with distinctive "response patterns". As might be expected, severity of TBI is a major factor determining cluster distribution.

Conclusions: Data collected in this ongoing, open-label, proof-of-concept study suggest that Tolcapone could have beneficial effects on cognitive function and behavioral problems commonly experienced by subjects with a history of brain injury.

Interestingly, response to Tolcapone varies by subject. Three groups with distinctive "response characteristics" can be differentiated using cluster analysis of the improvement in cognitive performance while taking Tolcapone. Data from this study was used to inform the design of an ongoing randomized, placebo-controlled trial using the same intervention and outcomes measures to confirm efficacy of Tolcapone in this study population.

Keywords: Traumatic Brain Injury, Tolcapone, Cognition, Frontal Cortical, Neuropsychiatry

Disclosure: Nothing to Disclose.

\section{M160. The Delta Opioid Receptor as a Novel Therapeutic Target for Headache Disorders}

Amynah Pradhan*, Alycia Tipton, Laura Moye, Madeline Novack

Psychiatric Institute, University of Illinois at Chicago, Chicago, Illinois, United States

Background: Migraine is an extraordinarily common brain disorder for which therapeutic options are limited. We have previously shown that delta opioid receptor (DOR) agonists are effective in models of migraine-associated pain, aura, and negative affect. The aim of this study was to further characterize the effectiveness of DOR agonists in other models of headache associated pain, and to determine the specific role of peripheral DORs in migraine.

Methods: Medication overuse headache was induced by treating animals with sumatriptan $(0.6 \mathrm{mg} / \mathrm{kg})$ for 11 days.
For opioid induced hyperalgesia mice were injected with escalating doses of morphine over 4 days. We also tested conditional knockout mice in which DORs were deleted in Nav1.8-expressing ganglia, within a chronic migraine model in which mice were treated every other day for nine days with nitroglycerin $(10 \mathrm{mg} / \mathrm{kg})$. Immunohistochemical characterization of DOR relative to the pro-migraine neuropeptide CGRP was performed in DOR-eGFP knockin mice.

Results: Chronic treatment with sumatriptan or morphine produced significant mechanical hypersensitivity which was inhibited by the DOR agonist SNC80. Within the chronic migraine model, SNC80 inhibited migraine-associated pain in floxed controls, an effect that was partially blunted in DORNav1.8 cKOs. Furthermore, we observed that in DOReGFP mice there was a small amount of co-expression between DOR-eGFP and CGRP in trigeminal ganglia of naïve/pain-free animals.

Conclusions: Overall, our results show that DOR activation can inhibit other types of pain that contribute to migraine, and that peripheral/trigeminal ganglia are an important site of action for the anti-migraine effects of DOR agonists. Future studies will focus on how chronic headache disorders affect DOR expression and function.

Keywords: Hyperalgesia, Pain, Migraine

Disclosure: Nothing to Disclose.

\section{M161. Hemodynamic Response Function Characteristics in Pre- and Post-Treatment Obsessive-Compulsive Disorder}

Rangaprakash Deshpande*, Reza Tadayon-Nejad, Gopikrishna Deshpande, Joseph O'Neill, Jamie Feusner

University of California, Los Angeles, California, United States

Background: Functional MRI (fMRI) is used extensively for studying neural correlates of brain functioning, though it is an indirect measure of neural activity measuring changes in blood oxygenation. The non-neural component of fMRI, called the hemodynamic response function (HRF), is known to vary across brain regions and persons. While it may initially seem that the non-neural HRF is not useful, recent advances show it to be modulated by the underlying neurochemical metabolism. Although vasculature, neural activity and other factors also influence the HRF, it is now accepted that the HRF carries meaningful indirect information about local brain functioning, which may not be available through conventional fMRI-derived metrics like activation and connectivity.

Regional abnormalities in brain metabolism and activation have been observed in obsessive-compulsive disorder (OCD), which have been found to respond to cognitivebehavioral therapy (CBT) treatment. The aim of this study was to identify changes in HRF before and after CBT in adults with OCD. Our study also included a matched healthy control (HC) group to enable us to identify those HRF characteristics that were abnormal in OCD pre-treatment, and to subsequently compare post-treatment relative to the non-clinical group to identify if any abnormalities normalize. Additionally, we hypothesized that the HRF characteristics would be associated with OCD symptom severity. 
Methods: We obtained resting-state fMRI data from 69 adults (44 with DSM-IV OCD and 25 matched healthy controls) lasting $7 \mathrm{~min}$ in a $3 \mathrm{~T}$ Siemens Trio scanner using a $\mathrm{T} 2^{*}$-weighted EPI sequence, with TR/TE $=2000 / 25 \mathrm{~ms}$, flip angle $=78^{\circ}$, matrix $=64 \times 64$, voxel size $=3 \times 3 \times 3 \mathrm{~mm} 3$ and 32 interleaved slices. The primary clinical measure was the YaleBrown Obsessive Compulsive Scale (YBOCS, patients only). Data was obtained on two different days for each participant: (i) OCD participants, pre- and post- 4 weeks intensive CBT treatment, and (ii) HCs, sessions 1 and 2 separated by 4 weeks, to form an appropriate control for the pre- and post- treatment OCD scans.

Standard pre-processing was performed in SPM8 (realignment, normalization to MNI space, smoothing [8mm], regressing out white matter and CSF signals). Artifact detection tool (ART) was used for head motion calculation. Those with motion exceeding $2 \mathrm{~mm}$ or $2^{\circ}$ were excluded (nobody was excluded). There was no significant difference in motion between the groups.

Each voxel timeseries was then subjected to hemodynamic deconvolution. We employed the deconvolution technique proposed by $\mathrm{Wu}$ et al., which considers resting-state fMRI as a spontaneous pseudo-event-related signal and uses a variant of Weiner deconvolution. The HRF at each voxel in each participant was characterized by three parameters - response height (RH), time-to-peak (TTP), and full-width at half-wax (FWHM). Whole-brain two-sample t-tests were performed separately on the three parameters to obtain voxel-specific differences in HRF parameters $(p<0.05$, cluster-level and FDR thresholded, controlled for age, education and headmotion). The two primary comparisons were: (i) HC session-1 vs. OCD pre-treatment, and (ii) OCD pretreatment vs. post-treatment. The resulting statistical maps were also overlapped to determine brain regions conforming to both conditions. Associations between HRF parameters in significant voxels and the YBOCS score were obtained.

Results: In all the regions with HRF differences, we found shorter RH, faster TTP and narrower FWHM in OCD pretreatment compared to $\mathrm{HC}$, and this trend was reversed posttreatment. In corroboration, prior research has found glutamate, which is among the major neurochemicals exhibiting abnormalities in OCD, being associated with smaller TTP and FWHM. With the HC session-1 vs. OCD pre-treatment comparison, differences were found with $\mathrm{RH}$ in thalamus (pulvinar), caudate head, superior parietal and primary visual areas; with TTP in caudate tail; and with FWHM in superior parietal and primary visual areas. With the OCD pre-treatment vs. post-treatment comparison, differences were found with RH in precuneus; with TTP in caudate tail; and with FWHM in anterior insula. Overlapping the two maps, we found (with TTP) part of the caudate tail to be abnormal in OCD pre-treatment and changed towards the HC pattern post-treatment (no significant difference in these voxels between OCD post-treatment and HC session-2). There were no significant differences between HC session-1 and session-2.

These findings partially corroborate prior findings from conventional fMRI studies in OCD, and share similarities with prior MRS findings. The RH is comparable to BOLD signal strength, and hence might share characteristics with fMRI activation. As a secondary analysis, we computed the voxel-level fractional amplitude of low frequency fluctuations
(fALFF), a regional measure that quantifies the fraction of fMRI signal strength in the relevant low-frequency band. We found that fALFF differences were similar to the differences observed with RH, which lends credence to our findings. TTP and FWHM are, however, measures associated with BOLD latency, known to be strongly influenced by metabolites, but which cannot be directly quantified using fMRI activation or connectivity. Hence, they might provide unique insights into OCD pathophysiology. For example, TTP differences were found in caudate tail, a region seldom identified in conventional fMRI studies (although previously metabolic abnormalities have been identified) as opposed to the caudate head; however, it had significant association with YBOCS $\left(\mathrm{R}^{\wedge} 2=0.2294, p=.001\right)$ underscoring its relevance to symptom severity.

Conclusions: We uncovered HRF aberrations in adults with OCD, and observed changes towards normalization after CBT treatment. We found significant association between TTP of the caudate tail and OCD symptom severity. HRF may provide novel insights into OCD pathology, not available through conventional fMRI techniques.

Keywords: Obsessive-Compulsive Disorder (OCD), Cognitive-Behavioral Therapy, Resting State Functional Connectivity, Hemodynamic Response Function, Caudate Disclosure: Nothing to Disclose.

\section{M162. Seeking the Genetic Etiology of Domestic Behavior in House Cats and Tame Foxes as a Window Into Personality Disorders}

Carlos Driscoll ${ }^{*}$, Stephen Lindell, Qiaoping Yuan, Zhifeng Zhou, Bob Thompson, Juan Lopez, Markus Heilig, David Goldman, Wolfgang Sommer, J. Dee Higley, Stephen Suomi, Christina Barr

National Institute on Alcohol Abuse and Alcoholism, Rockville, Maryland, United States

Background: Adaptations to unpredictable or stressful environmental conditions can occur at both the species and the individual levels, and identification of genes that are critical to stress adaptation may be achieved by searching for presence of functionally similar variants that occur across species. Along these lines, searching for genes at which there is both shared genetic variation and epigenetic regulation may be particularly powerful for identifying genes that play critical roles in stress adaptation, development and survival. The rhesus macaque has been critically important in modeling how environment impacts behavior and in revealing gene by stress interactions, as many of the stress neurobiological pathways differ between catarrhine primates and other species. We have found that many of the findings derived from macaques translate to the human condition, but more recently, we have also been examining how genetic variation drives domestication among canids and felids, as it could reveal genes at which genetic polymorphism might influence behavior and susceptibility to human personality/ psychiatric disorders and the addictions. Recent studies demonstrate that among canids, genetic variation at a Williams' Syndrome-linked gene (GTF21) is predictive of hypersociability in the domestic dog, relative to the wolf ancestors. We wanted to examine whether early rearing 
condition (which is known to influence sociality), altered gene expression for GTF21 in archived macaque brain samples.

Methods: Macaques had either been reared in social groups composed of 8-14 females (about half of whom had sameaged infants) and two adult males (Mother-reared, MR) or nursery/peer-reared (PR) animals, or separated from their mothers at birth and hand-reared by human caregivers in a neonatal nursery for the first 37 days of life. Hippocampus had been identified in the right hemisphere slabs using gross anatomical landmarks and excised using a fine-toothed saw. RNA was extracted, and expression levels were determined using expression microarray and RNA-SEQ.

Results: We found that PR monkeys, raised by human caregivers, exhibited higher levels of hippocampal expression of GTF21.

Conclusions: This may assist in explaining how PR monkeys are more facile in their interactions with human investigators, despite being highly stress-reactive and impulsive relative to their MR counterparts. This could also suggest that both selection and environmental factors act at this gene to drive social behavior and could validate a role in driving animals' abilities to co-exist and socialize with nonconspecifics (aka, human caregivers). Further, these finding may suggest that expression differences, epigenetic effects or genetic polymorphism at this locus could contribute to human temperament and vulnerability to psychopathology.

Keywords: Stress, Macaque, Dog, GCF21, Sociality

Disclosure: Nothing to Disclose.

\section{M163. Intrinsic Activity and Intra-Individual Variability Differences Among B-SNIP Psychosis Cases Assessed by Single Trial EEG Time-Frequency Analyses}

Brett Clementz* Jun Wang, Godfrey Pearlson, Matcheri Keshavan, John Sweeney, Jordan Hamm, Lauren Ethridge, Elena Ivleva, Carol Tamminga

\section{University of Georgia, Athens, Georgia, United States}

Background: Abnormal intrinsic activity and large intraindividual variability have been reported among subgroups of psychosis cases and might reveal a genetically mediated core deficit in cortical functioning among subgroups of psychosis cases. If high intrinsic neural activity was a core feature of psychosis accentuated responses across a range of paradigms should be repeatedly observed. Although there are multiple reports of psychosis subgroups with augmented amplitude brain responses during multiple ERP-type paradigms, this is not the typical outcome. The overwhelming majority of psychosis studies use clinical definitions (like those in the DSM) to define psychosis subgroups (e.g., schizophrenia, schizoaffective disorder, bipolar disorder with psychosis). Schizophrenia and bipolar disorder with psychosis differ in predictable directions on many measures, but heterogeneity within and overlap between these theoretical constructs on a range of brain imaging, neurocognitive, and perceptual variables inspire doubts about the conformity of current clinical psychosis definitions with neurobiological reality. Clementz et al. (2016) proposed that a distinct subgroup of psychosis cases have high levels of intrinsic activity and excessive reactivity to sensory stimuli (so-called
Biotype-2s) while a different subgroup have diminished intrinsic activity and low levels of sensory reactivity (socalled Biotype-1 s). In addition, Keshavan (2011) developed a psychosis continuum Schizo-Bipolar Scale (SBS) based on types and proportions of psychotic and affective symptoms. This scale demonstrated advantages over DSM diagnoses for distinguishing psychosis cases. This study aimed to compare the intrinsic activity and intra-individual variability differences among psychosis subgroups defined by either clinical (Schizo-Bipolar Scale (SBS), a psychosis continuum scale) or neurobiological (Biotypes) features.

Methods: Auditory EEG data were collected while healthy persons $(N=224)$, psychosis probands $(N=573)$ and their first-order relatives $(N=557)$ from the BipolarSchizophrenia Network for Intermediate Phenotypes (BSNIP1) were administered oddball (OB) and paired stimuli (PS) tasks. These tasks have been described in multiple previous B-SNIP1 publications. Intrinsic activity was assessed by measuring the single trial power spectrum for pre-stimulus activity; intra-individual variability was assessed by measuring the strength and coefficient of variation (CV) of single trial power spectrum and Inter-trial Coherence (ITC). Probands were placed into Biotypes Groups (1,2, or 3) based on their Cognition, saccadic eye movement, and EEG data (after Clementz et al., 2016). Probands were also given SBS designations based on clinical information. The SBS is a 10 points scale, with scores at 0 being prototypical bipolar psychosis-like and those at 9 being prototypical schizophrenia-like. Probands scoring 0-2 were SBS-1, those scoring 3-6 were SBS-2, and those scoring 7-9 were SBS-3. Relatives were yoked to their respective proband. Results: For proband analyses, Biotype subgroups (derived from neurobiology) showed considerably better group separations and cleaner differentiations than did SBS subgroups (derived from clinical information). As predicted, Biotype-1 probands had lower levels of neural activity in the absence of stimulation, had restricted neural activity ranges in response to stimulation, and showed lower similarity in neuronal responses across trials. Also as predicted, Biotype-2 probands had higher pre-stimulus activity, and significantly more variable neural responses to stimulation. Biotype- 3 probands did not differ from healthy persons on these neural activity measures. For analysis of the relative data, biological relatives of the Biotype groups show similar, less extreme, but still statistically significant patterns of deviations as the probands groups: Biotype-1 relatives had attenuated responses, Biotype-2 relatives had accentuated responses, and Biotype- 3 relatives did not differ from healthy persons. SBS defined proband and relative groups showed nonspecific deficits on single trial power, intra-individual variability, and pre-stimulus activity compared to healthy controls.

Conclusions: It is important to highlight that these EEG measures were not used in Biotypes construction. These findings provide further support for necessity to develop biomarker based psychosis classification, accounting for neurobiological heterogeneity among psychosis groups. The present data are also consistent with pervious work indicating that level of intrinsic brain activity may be a genetically mediated marker of liability for a subgroup of persons with psychosis. They also indicate that there are multiple neurobiological pathways to psychosis, and that 
current means for classifying psychosis cases clinically do not efficiently capture these differences.

Keywords: Psychosis, EEG biomarkers, Relatives

Disclosure: Nothing to Disclose.

\section{M164. Prenatal Gene Networks Altered by Maternal Immune Activation}

Myka Estes, Deborah A. van der List, Iva Zdilar, Linda Su-Feher, A. Kimberley McAllister*, Alex S. Nord

University of California, Davis, Davis, California, United States

Background: Schizophrenia (SZ) and autism spectrum disorders (ASD) are complex diseases likely caused by a combination of genetic and environmental factors during early development. Recent work points to a central role for immune-related genes and immune responses to environmental stimuli in SZ and ASD, and specifically for maternal infection during early gestation as a risk factor for both disorders. The development of mouse and non-human primate models of maternal infection with strong face and construct validity for SZ and ASD has strengthened the link between maternal immune activation (MIA) and many of the abnormal behaviors and neuropathologic findings characteristic of these disorders. Results from these animal models and recent human studies have converged on the hypothesis that MIA causes a chronic immune-dysregulated state in the offspring that alters brain development and behavior. However, little is known about how MIA initiates the disease process and how alterations in the brains of offspring might change over time. Animal models of MIA provide the opportunity to identify the molecular signaling pathways that initiate the disease process. The aim of our study was to use RNAseq to identify gene networks that are altered in MIA offspring at multiple prenatal ages.

Methods: Pregnant mice were injected with saline or $30 \mathrm{mg} /$ $\mathrm{kg}$ poly(I:C) on E12.5 and postnatal offspring were examined at 3 prenatal ages ( 6 hours after polyI:C injection, E14.5, and E17.5) and at birth. Tissue from cerebral cortex was processed for RNAseq and epigenetic analysis and gene networks were identified that are altered by MIA over time. After RNA isolation and quality assessment, poly-A stranded cDNA libraries were prepared from polyA-selected mRNA and sequenced using a $50 \mathrm{bp}$ single end strategy on an Illumina instrument at the UC Davis DNA core. A total of 62 individual samples passed our QC metrics and were used to map changes in gene expression across time points after MIA. We examined global patterns via multi-dimensional scaling and principle components analysis (PCA). Next, gene-wise differential expression was assayed for each time point using generalized linear models in edgeR and functional enrichment (Gene Ontology (GO) Biological Process) and expression patterns was performed with topGO and co-expression analysis.

Results: In general, we found that MIA leads to long-lasting changes in gene expression in the brains of offspring that change with age. Comparison of global expression across all samples revealed that gestational age (e.g. DPC) was one of the strongest contributors to variation in expression. Surprisingly, MIA samples collected at the same time as matched saline control samples clustered independently, suggesting that MIA impacts stage-specific expression patterns in fetal cortex. These findings show that transcriptional age is perturbed in cerebral cortex following MIA due to alterations of developmental processes. Genes were differentially expressed at each time point, with some upregulated while others were down-regulated. MIA caused strong induction of gene expression in fetal mouse cortex across specific pathways in the hours and days after exposure. Six hours after MIA, induction is observed for stress response and immune signaling. In the days after MIA, a secondary induced response is observed, characterized by increased expression of genes associated with angiogenesis, the extracellular matrix, and cell migration and activation and decreased expression of genes involved in neurogenesis. Many of the genes induced downstream of the acute response are sensitive to, or regulate, cytokine signaling and the stress response and often have dual roles in immune and neuronal proliferation and migration. Next, there is a transcription signature across mid-to-late gestation suggesting decreased proliferation and a larger proportion postmitotic cells expressing synaptic genes. Finally, a third wave of changes in gene expression was characterized by changes in cell identity markers later in fetal development.

Conclusions: MIA causes dynamic changes in gene expression across prenatal development. Our results suggest three principle novel findings regarding pathological mechanisms caused by MIA. First, MIA appears to cause acute induction of a stress response leading to up-regulation of metabolic, angiogenesis, and extracellular matrix proteins. Second, MIA leads to perturbation of neurogenesis via a possible shift from proliferation to differentiation. Finally, MIA leads to a tertiary impact on structure, anatomy, and cellular make-up of the brain. We are currently testing the hypothesis that these three effects are functionally linked and represent sequential mechanisms of neurodevelopmental pathology after MIA. Understanding these initiating processes will be critical for developing new evidence-based intervention strategies and identifying biomarkers for diagnosis and novel candidates for drug development towards improving treatment in SZ and ASD.

Keywords: Immune Markers, Cytokines, Synapses, Schizophrenia, Autism, Angiogenesis, Neurogenesis, RNAseq, Stress Response

Disclosure: Nothing to Disclose.

\section{M165. White Matter Alterations and the Conversion to Psychosis: A Combined Diffusion Tensor Imaging and Magnetic Resonance Spectroscopy Study}

Pablo León-Ortiz, Francisco Reyes-Madrigal, Peter Kochunov, Laura Rowland, Camilo de la Fuente-Sandoval ${ }^{*}$

Instituto Nacional de Neurologia y Neurocirugia, Mexico City, Mexico

Background: Schizophrenia is a common, severe and chronically disabling mental illness. The study of individuals at clinical high risk (CHR) for the disease provides an important opportunity for unraveling pathological mechanisms underlying schizophrenia and related disorders. 
Diffusion tensor imaging (DTI) studies in CHR subjects who transitioned to a psychotic disorder have shown abnormalities in the microstructure of white matter tracts. On the other hand, proton magnetic resonance spectroscopy $(1 \mathrm{H}-$ MRS) is an important tool for identifying biomarkers that aid the understanding of early psychosis, as development of this condition may be associated with metabolite concentration changes. The current study explored the correlations between the fractional anisotropy (FA) and 1H-MRS glutamate levels in the frontal white matter of $\mathrm{CHR}$ subjects. Methods: A total of 33 individuals meeting CHR criteria and 38 healthy controls underwent $3 \mathrm{~T}$ magnetic resonance imaging, which included both DTI and 1H-MRS. Region of interest analysis was used for group comparisons of FA and $1 \mathrm{H}-\mathrm{MRS}$ scans were obtained from a voxel located in the right frontal white matter. Subjects at $\mathrm{CHR}$ were clinically followed for 2 years.

Results: Seven CHR subjects transitioned to a psychotic disorder during the follow-up period. DTI analysis showed statistically significant FA reduction in four white matter tracts (right anterior thalamic radiation, left cingulate, right posterior thalamic radiation and left cingulum cortex) in CHR subjects who converted to psychosis compared to both non-converters and controls. In the group of converters, a significant negative correlation $(r=-0.869 p=0.02)$ was found between the glutamate levels obtained by H-MRS, and the FA in the left cingulum tract. Moreover, correlations were also found for the right anterior thalamic radiation $(\mathrm{r}=$ $-0.885 p=0.01)$, right posterior thalamic radiation $(\mathrm{r}=$ $0.935 p=>0.01)$ and left cingulum cortex $(r=-0.869$ $p=0.02)$.

Conclusions: We present further evidence that white matter microstructure is abnormal in CHR individuals who transitioned to psychosis. Moreover, our findings suggest a relationship between glutamate levels and the alterations in white matter microstructure.

Keywords: Clinical High Risk for Psychosis, Diffusion Tensor Imaging (DTI), Fractional Anisotropy, Glutamate, 1H-MRS

Disclosure: Part 1: Janssen (Johnson \& Johnson), Consultant.

\section{M166. Effects of the Potent Anti-Inflammatory Agent Fingolimod in Schizophrenia}

Michael Francis*, Emily Liffick, Tom Hummer, Jenifer Vohs, Nikki Mehdiyoun, Ziyi Yang,

Ying Zhang, Alan Breier

Indiana University School of Medicine, Indianapolis, Indiana, United States

Background: New medications with novel targets are needed for schizophrenia. Several lines of evidence indicate that inflammatory processes including aberrant lymphocytic activity may account for the pathophysiology of this illness. These data suggest that agents with anti-inflammatory actions including modulation of lymphocytes and their inflammatory substrates may prove to be efficacious for schizophrenia. Fingolimod is a powerful anti-inflammatory agent that is used in the treatment of relapsing multiple sclerosis. It is a sphingosine-1-phosphate (S1P) receptor modulator and decreases circulating lymphocytes through sequestration in lymph tissues. In addition, evidence suggest that it stimulates oligodendrocytes and may enhance white matter integrity. The purpose of this study is to assess the effects of fingolimod in schizophrenia.

Methods: Subjects with schizophrenia $(N=40)$ were recruited through the Indiana University Psychotic Disorders Programs and randomized 1:1 in a double-blind, eight-week clinical trial of fingolimod $0.5 \mathrm{mg} / \mathrm{day}$ and placebo. Circulating total lymphocytes were determined and effects were assessed on symptoms (PANSS), cognition (BACS), plasma cytokines, white matter integrity (MRI DTI) and cortical connectivity (resting fMRI).

Results: Results revealed robustly significant decrease in lymphocytes in subjects taking fingolimod versus placebo (treatment $\mathrm{x}$ time; $\mathrm{F}=61.2, p<0.001$ ). Fingolimod treated subjects had a mean maximal drop in lymphocytes from baseline of $79.2 \%$ with all fingolimod treated subjects experiencing decrements greater than $60 \%$. This powerful biological effect did not result in significant improvement (treatment $\mathrm{x}$ time) in PANSS total $(\mathrm{F}=0.66, p=0.52)$, any of the PANSS subscales, or BACS composite score $(\mathrm{F}=0.54$, $p=0.44$ ). Biomarker data (plasma cytokines, MRI DTI and resting fMRI) will be presented. Serious side effects were not observed and a full safety report will be provided.

Conclusions: Fingolimod produced a strong antiinflammatory response with substantial reductions in circulating lymphocytes in all treated subjects. However, this response was not accompanied by improvements in symptoms or cognition. These data suggest that fingolimod's target of S1P modulation and robust anti-inflammatory actions may not mediate clinical benefits in schizophrenia.

Keywords: Schizophrenia, Inflammation, Imaging

Disclosure: Nothing to Disclose.

M167. Schizophrenia Polygenic Risk Score Predicts Antipsychotic Efficacy in First Episode Psychosis

Jianping Zhang*, Delbert Robinson, Jin Yu, Juan

Gallego, Wolfgang Fleischhacker, Rene Kahn,

Benedicto Crespo-Facorro, Javier Vazquez-Bourgon, John Kane, Anil Malhotra, Todd Lencz

Hofstra Northwell School of Medicine, Glen Oaks, New York, United States

Background: The genetic basis of antipsychotic drug efficacy is likely polygenic in nature. Genetic risks of schizophrenia may also be related to antipsychotic drug response. The Psychiatric Genomics Consortium (PGC) genome-wide association study (GWAS) provided evidence of association with schizophrenia risk for many single nucleotide polymorphism (SNP) across the genome. We examined whether polygenic risk scores (PRS) based on the PGC GWAS are predictive of antipsychotic efficacy in four cohorts of patients with first episode psychosis (total $n=510$ ).

Methods: The discovery cohort was the Zucker Hillside Hospital First Episode schizophrenia trial (ZHH-FE) with 77 patients (mixed ethnicity) randomized to risperidone or olanzapine. Three replication cohorts were: 1) European First Episode Schizophrenia Trial (EUFEST) with 141 patients (all Caucasian) randomized to four antipsychotics; 
2) Spanish First Episode Psychosis study (SFEP) with 192 patients (all Caucasian) on various antipsychotics; and 3) the clinical trial as part of the Center for Intervention Development and Applied Research at $\mathrm{ZHH}$ (CIDAR) with 100 patients (mixed ethnicity) randomized to risperidone or aripiprazole. Genotyping was performed using the Illumina Omni-1Quad (EUFEST and ZHH-FE) or Illumina Infinium HumanOmniExpressExome platform (CIDAR and SFEP). SNP imputation was conducted with IMPUTE2 against the full 1000 Genomes v3 reference panel. PRS was computed based on the results of the PGC GWAS using PRSice software for the discovery cohort with thresholds at $\mathrm{PT}<5 \mathrm{E}-8,0.001,0.01,0.05,0.10,0.20,0.50$. Based on the findings from the discovery cohort, PRS was computed for the three replication cohorts using a threshold of $\mathrm{PT}<0.01$. Symptom measure was the total score of Brief Psychiatric Rating Scale (BPRS) for ZHH-FE, SFEP, and CIDAR, or Positive and Negative Symptoms Scale (PANSS) for EUFEST. Hierarchical linear regression was performed on the 3-month symptom score with the PRS as the predictor while controlling for age, sex, and baseline symptom score. Genomic principal component scores were also covaried to control for population stratification for $\mathrm{ZHH}-\mathrm{FE}$ and CIDAR.

Results: In the ZHH-FE cohort, higher PRS at the thresholds of $\mathrm{PT}<0.01,0.05,0.10,0.20$, and 0.50 significantly predicted higher symptom scores at 3-month follow-up, explaining 6$8 \%$ of the variance (all p's $<0.05$ ). PT $<0.01$ gave the strongest result in the discovery sample, and was used to replicate the findings in the other three cohorts. Higher PRS significantly predicted worse symptoms in EUFEST and SFEP cohorts, explaining $3.5 \%$ and $3.7 \%$ of variance, ( $p$ 's $<0.01$ ), but not in the CIDAR sample. Combining the four cohorts in a meta-analysis, PRS was significantly predictive of 3-month symptom scores (pooled partial $r=0.18, p=0.002)$. Higher PRS was associated with higher symptom scores at 3-month follow-up, suggestive of less improvement in treatment. The overall results remained significant when only European ancestry individuals were included in the analysis.

Conclusions: These findings suggest that polygenic risk scores for schizophrenia may also be related to antipsychotic drug response. Patients with higher polygenic risk scores tended to have less improvement with antipsychotic drug treatment. Further analysis is needed to elucidate a more refined genomic profile for antipsychotic drug response.

Keywords: Pharmacogenetic Response, Antipsychotic Response, Antipsychotic-Naïve First-Episode Schizophrenia Disclosure: Nothing to Disclose.

M168. Different Patterns and Correlates of Polygenic Risk in Schizophrenia Subgroups Defined by Cognitive Development Trajectories

Dwight Dickinson*, Sofia Zaidman, Michael Gregory, Daniel Weinberger, Karen Berman

National Institute of Mental Health, Bethesda, Maryland, United States

Background: Generalized cognitive impairment is a core characteristic of schizophrenia. Previous work suggests that different patterns of premorbid vs. current IQ may reflect distinct trajectories of cognitive development through adolescence, separating subgroups of individuals with schizophrenia. We tested, first, whether subgroups defined on the basis of premorbid vs. current IQ patterns would emerge in data-driven analyses. Polygenic scores (PS) summarize the influence of numerous common genetic variants on a given phenotype (e.g., schizophrenia, cognition) based on appropriate GWAS results. Various work points to overlap in cognitive and schizophrenia genetics in affected individuals and families. Our second goal in this work was to test whether the cognitive trajectory subgroups differed in relation to Schizophrenia PS and Cognitive PS. Given that schizophrenia and cognition are interwoven behaviorally, our last objective was to test the association of Schizophrenia PS with cognitive performance.

Methods: 550 people with schizophrenia, 432 of their unaffected siblings, and 1127 community controls completed cognitive assessments and provided blood for genetics analyses, as part of the NIMH Study of Schizophrenia Genetics. We performed cluster analyses in the schizophrenia cases to identify cognitive subgroups, using premorbid (WRAT) and current (WAIS) IQ as clustering indicators. To investigate the familiality of the subgroups and their associations, subgroup assignments derived for schizophrenia cases were also assigned to their unaffected siblings. Schizophrenia and cognitive PS for all individuals were calculated at different thresholds based on phenotypeassociated genetic variants identified in separate GWAS by the Psychiatric Genetics Consortium (schizophrenia) and the Childhood Intelligence Consortium (IQ). We compared resulting PS across the derived cognitive subgroups. Across Schizophrenia PS thresholds, we used ordered hierarchical regression to test the association of PS with general cognitive ability ("g") in the subgroups, controlling for age, sex, and population stratification.

Results: Cluster analyses suggested a three-subgroup scheme: one subgroup with high scores on both WRAT and WAIS (Cognitively Stable), one with low scores on both WRAT and WAIS (Pre-adolescent Impairment), and one with high scores on the WRAT and low WAIS (Adolescent Decline), as found previously. Cognitive performance was significantly impaired and Schizophrenia PS were significantly elevated in all schizophrenia groups relative to controls. The Cognitively Stable subgroup was less impaired cognitively (mean difference p's $<1.0 x 10 \mathrm{E}-10$ ) and had lower Schizophrenia PS that the other trajectory subgroups. The Adolescent Decline subgroup had the highest levels of Schizophrenia PS among the subgroups, and the difference between the Adolescent Decline and Cognitively Stable subgroups was significant (e.g., at Sz PS_0.05 mean difference $p=.014)$. At the same time, the Adolescent Decline and Cognitively Stable subgroups both showed favorable Cognitive PS relative to the Pre-adolescent Impairment subgroup (e.g., at Cog PS_0.2 mean difference $p=.001$ and $p=.004$, respectively, for Cognitively Stable and Adolescent Decline vs. Pre-adolescent Impairment), and even (non-significantly) relative to siblings and controls. The Cognitively Stable subgroup showed significant associations between Schizophrenia PS and " $\mathrm{g}$ " at six of 10 PS thresholds (e.g., at Sz PS_0.05 $p=.002 ; \mathrm{R} 2=0.047$ ). The Pre-adolescent Impairment group showed a similar pattern. However, the 
Adolescent Decline subgroup showed no association of schizophrenia PS with "g" at any threshold - a surprising decoupling of schizophrenia risk from cognitive performance.

Conclusions: Three subgroups emerged from cluster analyses, which likely reflected different trajectories of cognitive development through adolescence. Although the subgroups were derived on the basis of WAIS and WRAT IQ scores, they showed quite distinct patterns on broad schizophrenia and cognitive polygenic scores. Thus, there was a convergence between stratification based on common cognitive measures and stratification based on schizophrenia and cognitive genetics. For most schizophrenia cases, summary scores indexing common genetic risk for the condition were predictive of cognitive impairment. Strikingly, for one subgroup (Adolescent Decline), despite significant cognitive impairment and high polygenic risk for schizophrenia, that risk was entirely decoupled from cognitive performance. One hypothesis is that different patterns of IQ performance identify subgroups with different trajectories of cognitive development and distinct illness genetics and etiologies.

Keywords: Schizophrenia Subtypes, Polygenic Scores, Cognition, Pre-Morbid IQ, Adolescent Development

Disclosure: Nothing to Disclose.

\section{M169. Greater Hippocampal Connectivity Predicts Poor Cognitive Function in Schizophrenia}

Jason Tregellas*, Korey Wylie, Ann Olincy, Josette Harris, Robert Freedman, Kristina Legget

University of Colorado/Denver VA Medical Center, Aurora, Colorado, United States

Background: Cognitive deficits continue to be the least well treated symptom of schizophrenia, leading to substantial functional impairment and decreased quality of life. As such, improving our understanding of the neurobiology underlying this dysfunction remains an important goal. Towards this end, the current study sought to use whole-brain multivariate connectivity metrics to determine how alterations in connectivity between different brain regions and networks contributes to poor cognitive function in schizophrenia.

Methods: Thirty-three outpatients with schizophrenia underwent functional magnetic resonance imaging at $3 \mathrm{~T}$ in the resting state for ten minutes. Cognitive function was measured with the MATRICS Consensus Cognitive Battery (MCCB). Five subjects were excluded due to excessive movement during scanning. All intrinsic connectivity networks (ICNs, also often called resting state networks) were extracted by group independent components analysis. The distance covariance statistic was used to examine relationships between all voxels in the brain, all ICNs, and MCCB composite scores. Follow-up correlation analyses examined the directionality of significant connections, in relation to MCCB scores.

Results: Whole-brain distance covariance analysis identified a cluster of voxels in the left hippocampus, whose connectivity was associated with MCCB composite T-scores $(p<0.05$, corrected). This association was driven by inverse correlations to default mode network (DMN) subnetworks, as well as to basal ganglia, right executive, auditory, and lateral sensorimotor ICNs ( $p<0.05$, corrected).

Conclusions: Greater intrinsic hippocampal connectivity may be associated with cognitive dysfunction in schizophrenia. This connectivity alteration includes associations between the hippocampus and the DMN, but extends to additional ICNs as well. These results suggest that altered hippocampal connectivity may be a useful target for therapeutic development.

Keywords: Schizophrenia, Hippocampus, Cognition

Disclosure: Nothing to Disclose.

\section{M170. First-Episode Psychosis Patients Display Increased Plasma IL-18 That Correlates With Cognitive Dysfunctions}

Lilly Schwieler*, Funda Orhan, Helena FatourosBergman, Simon Cervenka, Lena Flyckt, Lars Farde, Carl M Sellgren, Göran Engberg, Sophie Erhardt

Karolinska Institutet, Stockholm, Sweden

Background: Growing evidence implicate inflammation in the pathophysiology of schizophrenia. Previous studies have shown elevated blood levels of interleukin (IL)-18 in patients with chronic schizophrenia. Furthermore, several studies propose that immune-related pathophysiological processes may be specifically involved in cognitive impairment in schizophrenia. In the present study, we investigate cerebrospinal fluid (CSF) and plasma concentrations of this cytokine in first-episode psychosis (FEP) patients and study correlations to specific symptoms, including cognitive symptoms.

Methods: Plasma IL-18 levels from FEP patients and healthy controls were carried out using an electrochemiluminescence method (MesoScale, Gaithersburg, MD, USA; human IL-18 kit). The sample volume was $50 \mu \mathrm{l}$ and all samples were analyzed in duplicates and the intra-assay coefficient of variation (\%CV) was below 25\%. The Measurement and Treatment Research to Improve Cognition in Schizophrenia (MATRICS) Consensus Cognitive Battery, was used to evaluate cognitive function. This battery captures key cognitive domains relevant to schizophrenia including speed of processing, attention/vigilance, working memory, verbal learning, visual learning, reasoning and problem solving and social cognition. One psychologist administered all the tests. A majority of patients were naïve to antipsychotic drugs, and the remaining patients had an exposure of less than 2 weeks. Results: We found elevated plasma IL-18 levels in FEP patients $(n=35)$ compared to healthy controls $(n=19)$. Moreover, elevated plasma IL-18 levels associated with impaired cognitive performance in terms of processing speed. No association between IL-18 levels and ongoing antipsychotic and/or anxiolytic medication was observed among patients. The electrochemiluminescence assay did not allow for detection of CSF IL-18.

Peripheral levels of IL-18 are increased in FEP patients and correlate to speed of processing, in similarity to previous findings in chronic, medicated samples.

Conclusions: Further studies are warranted to investigate if these peripheral changes reflect a centrally disturbed immune-related homeostasis in schizophrenia that is of causal importance for cognitive dysfunction. Evaluation of 
blood biomarkers in schizophrenia is needed to rationally improve a biological basis for diagnosis. The findings of the present study, suggesting that plasma IL-18 may form part of a biomarker profile of cognitive impairments in schizophrenia, holds promise for such an approach.

Keywords: Immune System, Biomarker, Cognitive Symptoms

Disclosure: Nothing to Disclose.

\section{M171. fMRI GWAS Positive KCNV1 Interacts With Childhood Urbanicity}

Joseph Callicott*, Michael Gregory, Jessica Reed, Daniel R. Weinberger, Karen Berman

Clinical and Translational Neuroscience Branch, Bethesda, Maryland, United States

Background: Gene-environment interactions likely drive aspects of schizophrenia risk (Nicodemus et al., Mol Psychiatry 2008) and should be of significant effect in phenotypes associated with schizophrenia (Modinos et al., Schizophr Res 2013). Urbanicity is one such environmental variable that has been linked to increased risk for schizophrenia (van Os, Kenis and Rutten, Nature 2010). Urban environments carry both risks and benefits, but negative aspects include, for example, crime and over-population (Newbury et al., Schizophr Bull 2016; Villanueva et al. Acad Pediatr 2016). In animals, gene-environment interactions alter stress response, neurotransmission, and cognition (Kannan, Sawa and Pletnikov, Neuro Biol 2013).

Environmental factors are likely not restricted to the brain and therefore may influence a range of human traits. One of the more striking demonstrations using brain imaging were the London taxi drivers showing increased hippocampal size after acquiring navigational skills (Maguire et al., Proc Natl Acad Sci 2000). Childhood and adult urbanicity were associated with changes in the limbic system of healthy adults using a social-stress fMRI task (Lederbogen et al., Nature 2011) Here we used the environmental variable of urbanicity during childhood to examine the relationship between risk and urbanicity in two ways. First, we paired urbanicity together with the most significant result from an fMRI GWAS we performed examining working memory functioning - KCNV1. KCNV1 is highly expressed in brain and was associated with schizophrenia via case-control GWAS (PGC2-SCZ, Schizophrenia Working Group of the PGC, 2014). From the same case-control results, we constructed risk profile scores (RPS) to correlate with urbanicity. We asked whether these environmental and genetic risks for schizophrenia would interact. We predicted that cognitive neural function measured by our fMRI task would be sensitive to urbanicity as suggested by prior MRI findings (Lederbogen et al., 2011) and therefore would interact with a GWAS positive SNP. We also predicted that increased RPS would associate with urbanicity.

Methods: The fMRI GWAS was based on working memory data collected at $3 \mathrm{~T}$ using two samples, 501 healthy adults (mean age $=31.08 ; 56 \%$ female) from our lab and 348 healthy children (mean age $=15.7 ; 50 \%$ female) from the Philadelphia Neurodevelopmental Cohort. The two groups used slightly different forms of the Nback working memory task - a visuospatial task using numbers and a similar task using fractal images. After quality checks (Anderson et al., 2010), genetic data were phased using SHAPEIT and imputed to a 1000 Genomes Phase 3 reference using Impute2. BOLD signal was extracted from both cohorts and then tested separately voxel-wise against $\sim 5$ million SNPs. Urbanicity data were obtained during a SCID examination and categorized as follows: rural population $<10,000$; town $<100,000$; and urban $>100,000$. We tested for the interaction in SPM8 using a multiple regression controlling for age, sex, and performance. We took a complementary approach to the assumption of gene-environment interaction using risk profile scores (RPS). For 294 healthy subjects, we calculated RPS at different $p$ threshold ranging from 1 e- 8 to 1 . RPS scores were calculated using $\mathrm{p}$-values and odds ratios from PGC2-SCZ. The relationship between urbanicity and RPS was calculated as a simple regression controlling for age and sex.

Results: We observed that this KCNV1 SNP interacted with childhood urbanicity in right BA9. Specifically, this analysis showed an association between KCNV1 (rs36111028) and working memory activation in the right BA9 dorsolateral prefrontal cortex ( $p=3.12 \mathrm{E}-11$, Bonferroni corrected) in our discovery sample and then in the replication sample. Further, we found an interaction between rs36111028 and urbanicity where the risk allele showed greater BA9 BOLD activation in rural subjects during high working memory load, whereas it showed less BOLD activation in urban subjects - suggesting opposing effects of this risk SNP depending on childhood urbanicity status. Finally, in healthy controls, childhood urbanicity was significantly associated with RPS, suggesting a RPS-urbanicity interaction that may imply an urbanicity interaction across a broader set of risk genes in schizophrenia. Conclusions: We show that the KCNV1 SNP identified from a whole brain GWAS examination of working memory functioning with the environmental risk factor childhood urbanicity to impact the brain activity of the dorsolateral prefrontal cortex. The RPS-urbanicity relationship furthers the connections between risk SNPs and urbanicity, a relationship that includes many sub-threshold genome-wide signals. The KCNV1 finding suggests that an urban upbringing alters KCNV1's effect in brain during development, perhaps increasing risk for schizophrenia.

Keywords: Childhood Urban-Rural Upbringing, Polygenetic Risk Score, Gene-environment Interaction, Functional MRI (fMRI), Dorsolateral Prefrontal Cortex

Disclosure: Nothing to Disclose.

M172. Amygdala-Hippocampal Differences Across the Psychosis Spectrum: Diagnosis and Biotype Comparisons From the Bipolar-Schizophrenia Network on Intermediate Phenotypes (B-SNIP) Study

Synthia Guimond*, Sinead Kelly, Luke Mike, Mallar Chakravarty, John Sweeney, Godfrey Pearlson, Brett Clementz, Carol Tamminga, Matcheri Keshavan

Harvard Medical School, Boston, Massachusetts, United States

Background: A large body of research has demonstrated common hippocampal and amygdala volume abnormalities 
in psychotic disorders across schizophrenia (SZ), schizoaffective (SZA) and psychotic bipolar disorder (PBD), with varying degrees of effect. Alterations in shape of these structures may be particularly sensitive to detect group differences. However, finding specific biomarkers that differentiate patients across the psychosis spectrum has been challenging because of possible overlap between these disorders. This highlights the need of using different approaches from clinical phenomenology alone to classify patients in the search for meaningful biomarkers. Recent work from our group has identified three promising biotypes (biotype 1,2, and 3) that sort individuals with psychosis, based on cognitive and electrophysiological data, into distinctive groups that appear biologically relevant (biotype 1 being the most impaired). With the current study, we seek to validate this proof of concept by using amygdalahippocampal volume and shape data as an external validator for differences across the psychosis spectrum using biotype versus clinical categorization.

Methods: Data collection was completed across six sites in the Bipolar-Schizophrenia Network on Intermediate Phenotypes (B-SNIP) consortium. Following data quality control, a total of 790 participants were included in the study, including 186 individuals with SZ, 116 with SZA, 173 with PBD, and 315 healthy controls (HC). Structural magnetic resonance images were acquired on 3- $\mathrm{T}$ scanners using high-resolution T1-weighted sequences standardized across sites. Amygdalahippocampal volumes and surface area metrics were extracted with the Multiple Automatically Generated Templates (MAGeT)-Brain algorithm. General linear models were applied to test for main effect of biotypes and diagnosis, while covarying for confounding factors. Post-hoc independent t-tests were then used to localize differences and were corrected for multiple comparison using false discovery rate correction.

Results: We observed significantly smaller bilateral hippocampal and amygdala volume for SZ and SZA patients compared to HC (Cohen's d range: 0.19-0.33; $p<.05$ corrected). No significant difference was observed for the volume of these structures between any diagnosis, nor between PBD and HC (Cohen's d range: 0.07-0.18; $p>.10$ corrected). Significant abnormal shape of the bilateral amygdala and hippocampus was also observed in all diagnosis group $(p<.05$ corrected), and subtle shape difference was observed in the right hippocampus between SZ and SZA ( $p<.05$ corrected).

Using biotype classification, significant differences and larger effect sizes were observed when biotype 1 was compared to HC (Cohen's d range: 0.48-0.58), and to other biotypes (Cohen's d range: 0.31-0.54) for volume and shape of the bilateral hippocampus and amygdala ( $\mathrm{p}<.05$ corrected). No significant volume differences were observed between biotype 2 and 3, nor when comparing these biotypes to HC. Subtle shape differences were found in the right hippocampus between biotype 3 and HC, and between biotype 2 and 3 ( $p<.05$ corrected).

Conclusions: While diagnosis comparison results are consistent with previous findings, showing significant differences in the amygdala and hippocampus of patients with SZ and SZA compared to controls, larger effects were observed when using biotype classification. Patients in biotype 1 not only have significant volume and shape differences in these subcortical structures compared to healthy controls but also compared to other biotypes. Our results suggest that grouping patients by biotype, given that imaging data were not used in construction of these categories, may have better biological validity than diagnosis classification. Hence, using biotypes that are neurobiologically distinctive to classify patients could potentially lead to the identification of more precise biomarkers, and guide the development of more targeted and effective treatments.

Keywords: Biotypes, Biomarkers, Psychosis, Trans-diagnostic, Amygdala-Hippocampal Differences

Disclosure: Nothing to Disclose.

\section{M173. Neural Correlates of Social Cue Orienting in Schizophrenia}

Junghee Lee*, Eric Reavis, Michael Green

University of California, Los Angeles, California, United States

Background: Human beings are tuned for social stimuli. This tendency for individuals to prioritize processing social over nonsocial stimuli is referred to as social preference. Various lines of evidence emerging from basic science support the crucial role of social preference in the development of social cognitive skills and social functioning. It is plausible that disrupted social preference may play a role in social cognitive impairment and social dysfunction in schizophrenia. Hence, this study focused on one aspect of social preference, social cue orienting, and examined its neural correlates in schizophrenia using a functional magnetic resonance imaging (fMRI).

Methods: Twenty-five outpatients with schizophrenia and 25 demographically matched community controls participated in this study. To assess sensitivity to orienting to social versus nonsocial cues, a gaze cuing task was administered in the $3 \mathrm{~T}$ scanner at UCLA. At each trial, participants were asked to indicate the location of a target (i.e., the letter $\mathrm{X}$ in one of two peripheral boxes) following an uninformative central cue (either social or nonsocial). There were two stimulus onset asynchronies between the cue and the target (i.e., SOA of 150 or $750 \mathrm{~ms}$ ). It is important to note that both social and nonsocial cues were uninformative, meaning that the direction of the cue was consistent with the target location on half of the trials and inconsistent on the other half.

Results: Based on previous studies, we focused on two regions of interest (ROIs), the superior temporal gyrus and the inferior temporal gyrus. In the superior temporal gyrus, we did not observe any significant effect. However, in the inferior frontal gyrus, we observed a significant cue type by SOA by group interaction. At SOA 150, two group showed comparable neural activation. At SOA750, controls showed greater neural activation for social cues than nonsocial cues, whereas patients showed the opposite pattern (i.e., greater activation for nonsocial cues than social cues).

Conclusions: Our study found aberrant neural activation for social cue orienting in schizophrenia patients at a long SOA, but not at a short SOA, in the inferior frontal gyrus. We did not observe any group difference in the superior temporal gyrus. The findings of this study indicate that neural 
processes related to social cue orienting in schizophrenia may become dysfunctional when social cue orienting is less reflexive.

Keywords: Social Cue Detection, Social Preference, fMRI, Schizophrenia

Disclosure: Nothing to Disclose.

\section{M174. Evidence of Familial Factors Influencing Neuro- cognitive Functioning in Adult- and Childhood-Onset Schizophrenia}

Tim Bigdeli*, Keith H. Nuechterlein, Catherine Sugar, Kenneth L. Subotnik, Thomas Kubarych, Michael Neale, Kenneth Kendler, Robert Asarnow

SUNY Downstate Medical Center, Brooklyn, New York, United States

Background: The aggregation of neurocognitive deficits among both the non-psychotic first-degree relatives of adultand childhood-onset schizophrenia patients suggests that there may be a common etiology for these deficits in childhood- and adult-onset illness. However, there is considerable heterogeneity in the presentation of neurobiological abnormalities, and whether there are differences in the extent of familial transmission of specific domains of cognitive function has not been systematically addressed.

Methods: We employed variance components analysis, as implemented in SOLAR-Eclipse, to evaluate the evidence of familial transmission for empirically derived composite scores representing attention, working memory, verbal learning, verbal retention, and memory for faces. We contrast estimates of "heritability" for adult- and childhood-onset schizophrenia families and matched community control pedigrees, and compare our findings to previous reports based on analogous neurocognitive assessments.

Results: We observed varying degrees of familial transmission; attention and working memory yielded comparable, significant estimates for adult-onset and community control pedigrees; verbal learning was significant for childhoodonset and community control pedigrees; and facial memory demonstrated significant familial transmission only for childhood-onset schizophrenia. Model-fitting analyses indicated significant differences in "heritability" between adultand childhood-onset schizophrenia for attention, working memory, and verbal learning.

Conclusions: By comprehensively assessing a wide range of neurocognitive domains in adult- and childhood-onset schizophrenia families, we provide additional support for specific neurocognitive domains as schizophrenia endophenotypes. Whereas comparable estimates of familial transmission for certain dimensions of cognitive functioning support a shared etiology of adult- and childhood-onset neurocognitive function, observed differences in familial transmission may be taken as preliminary evidence of partially divergent multifactorial architectures.

Keywords: Neurocognitive Functioning, Childhood-Onset Schizophrenia, Endophenotypes, Familiality, Unaffected Relatives

Disclosure: Nothing to Disclose.
M175. Association of Within Person Variance With Data Quality Issues in Schizophrenia Clinical Trials

\author{
David Daniel*, Xingmei Wang, Gary Sachs, Alan Kott
}

Bracket Global, LLC, McLean, Virginia, United States

Background: Data quality monitoring in clinical trials seeks to identify aberrant rating patterns associated with poor interview or measurement technique. We have previously identified markers in screening associated with subsequent rating errors, placebo response and diminished drug-placebo differences (Kott and Daniel, 2016; Kott, Lee, Forbes et al, 2017). Within Person Variance (WPV) is a measure of symptom instability on subject level (Jahng, Wood, and Trull, 2008). In the current analysis, we assessed whether excessive WPV was associated with other markers of poor data quality in schizophrenia clinical trials.

Methods: Data from 17 phase 2 and phase 3 double blind, placebo controlled, multicenter schizophrenia studies were analyzed. The dataset consisted of 97,016 visits collected from 11,487 subjects at 1,323 research sites. For each subject in the database we calculated the WPV of the total PANSS score and within each study identified subjects with their WPV above 95th percentile as subjects with high WPV. Using a series of univariate logistic regressions we tested the effect of high WPV on the presence of the following indicators data concerns: 1) Within PANSS logical errors; 2) All 30/30 PANSS items rated identically across visits; 3 ) Discrepancies between change in CGI-S from baseline and CGI-I score; 4) Discrepancies between PANSS total score and CGI-S score; 5) Discrepancies between change from baseline in PANSS and CGI-S; 6) Erratic PANSS ratings.

Results: Out of 8,997 subjects who had sufficient number of visits to be able to calculate the PANSS WPV, we identified a total of $443(4.92 \%)$ subjects with WPV above the respective study 95th percentile. The probability of recording additional data findings from the tested list of indicators was significantly increased in subjects with high within person variance

o For Within PANSS logical errors by $10.03 \%(p<0.001)$

o For Identical PANSS ratings by $4.06 \%(p=0.019)$

o For Discrepancies between change in CGI-S from baseline and CGI-I score by $24.01 \%(p<0.001)$

o For Discrepancies between PANSS total score and CGI-S score by $23.25 \%(p<0.001)$

o For Discrepancies between change from baseline in PANSS and CGI-S by $26.44 \%(p<0.001)$

o For Erratic PANSS ratings by $6.61 \%(p<0.001)$

Conclusions: We have previously reported an association between high within person variance (WPV) in schizophrenia clinical trials and placebo response and diminished signal. The current analysis provides further evidence of an association between excessive WPV and data anomalies associated with both measurement error and subject behavior. The current findings should be considered preliminary at this time. Future reports will attempt to replicate these findings, address subject and rater characteristics associated with WPV and explore its utility as a marker in other therapeutic areas.

Keywords: Clinical Trials, Data Quality, Schizophrenia, Within Person Variance

Disclosure: Part 1: Bracket Global, LLC, Employee, Part 2: Bracket Global, LLC, Employee, Stock / Equity. 
M176. MRS Studies of Brain Glutathione and NAA in a Longitudinal Non-Human Primate Model of Maternal Immune Activation

Richard Maddock*, David Amaral, Jeffrey Bennett, Huan Wang, Ana-Maria Iosif, Judy Van de Water, Tyler Lesh, Melissa Bauman, Cameron Carter

University of California, Davis, Sacramento, California, United States

Background: There is growing evidence that immunemediated effects on neurodevelopment have a key role in the pathogenesis of major psychiatric disorders, including schizophrenia and autism. Immune molecules, such as cytokines and the major histocompatibility complex (MHC) have essential roles in brain development and function. Genetic studies show relatively strong associations between MHC genes and the risk for schizophrenia. Maternal exposure to specific viral infections during pregnancy is linked to increased risks for schizophrenia and autism. To further study maternal immune activation (MIA) effects on brain development and function, we have developed a non-human primate MIA model using a modified form of the viral mimic polyIC (polyICLC) injected at the end of the first trimester. Glutathione (GSH), the major antioxidant in the brain, can be measured non-invasively with magnetic resonance spectroscopy (MRS). Brain GSH is reduced in some neuroinflammatory disorders, and an age-dependent reduction in GSH was recently reported in a rodent MIA model. Age-dependent changes in n-acetylaspartate (NAA) were also reported. MRS findings in MIA models could enable translational studies of neuroimmune processes in neuropsychiatric disorders. Here we report initial findings from MRS studies in our longitudinal non-human primate MIA model.

Methods: Twenty-eight pregnant rhesus monkeys (Macaca mulatta) were randomized to either the MIA group or the control group. The 28 male offspring are undergoing longitudinal brain imaging studies on a Siemens Skyra $3 \mathrm{~T}$ scanner. Prefrontal cortex glutathione (GSH) was measured in 22 onemonth and 28 six-month old animals using a MEGA-PRESS sequence optimized to edit the cysteine methylene resonance of $\mathrm{GSH}$ at $2.95 \mathrm{ppm}(\mathrm{TE}=131 \mathrm{~ms}$, on and off resonance edit pulses at 4.56 and $4.90 \mathrm{ppm}$, bandwidth $=30 \mathrm{~Hz}$ ). Additional metabolite data were acquired with a short TE (33 ms) PRESS sequence in 27 six-month-old animals. The edited GSH peak is quantified by peak integration in the difference spectrum. Metabolites from PRESS spectra (and off-resonance MEGA-PRESS (MP) spectra) are quantified by LCModel and normalized to creatine.

Results: No behavioral abnormalities were evident in MIA animals at one or six months of age. The edited GSH peak at $2.95 \mathrm{ppm}$ was clearly visible in all animals. We found no difference in prefrontal cortex GSH/Cr in MIA compared to control animals at either one month (mean \pm s.d.:.059 \pm .024 and.063 \pm .018 , respectively, $\mathrm{t}=0.71, N=22, \mathrm{p}=.48$ ) or $\operatorname{six}$ months of age (.054 \pm .016 and.053 \pm .013 , respectively, $t$ $=.018, N=28, \mathrm{p}=.86)$. There was no significant change in prefrontal GSH/Cr from one month to six months in either group or the combined sample. TE-33 PRESS spectra were acquired only at six months; data from two animals were excluded for poor quality. These data showed elevated NAA/ $\mathrm{Cr}$ in MIA compared to control animals (mean \pm s.d.: 1.12 \pm .06 and $1.05 \pm .07$ respectively, $t=2.63, \mathrm{df}=23 \mathrm{p}=.015)$.
NAA/Cr values from TE-131 MP off-resonance spectra were highly correlated with NAA/Cr values from the TE-33 PRESS spectra acquired in the same voxel (Pearson's $r=.76, N=25$, $\mathrm{p}<.0001)$. This supported using the MP off-resonance spectra to examine longitudinal changes in NAA from one to six months of age. A repeated measures linear model analysis showed preliminary evidence of differences in NAA emerging over time. Although the group $\mathrm{x}$ time interaction was not significant $(\mathrm{F}=1.4, \mathrm{df}=22, \mathrm{p}=.25), \mathrm{NAA} / \mathrm{Cr}$ values were equivalent in the two groups at one month $(t=0.40$, $\mathrm{df}=21, \mathrm{p}=.69$ ) but began to diverge at six months, with higher NAA in the MIA group $(t=2.21, \mathrm{df}=25, \mathrm{p}=.037)$. No other metabolites differed significantly between MIA and control animals at one or six months.

Conclusions: Using a non-human primate MIA model, we found no changes in GSH in the prefrontal cortex of MIA animals at either one or six months of age. This negative finding early in development agrees with that of Vernon et al. (2015), who reported no change in GSH in adolescent MIA rats, but reduced GSH in MIA adults. Ongoing study of our cohort will show if GSH levels eventually decrease with maturation. Low GSH in MIA offspring could be a risk factor for neuropsychiatric disorders via increased vulnerability to oxidative stress in the brain. Prefrontal NAA/Cr was significantly increased in MIA monkeys at 6 months of age. NAA measurements from MEGA-PRESS off-resonance spectra provided preliminary evidence that the increase in prefrontal NAA is age-dependent, absent at one month and emerging at six months in the MIA animals. This pattern will be further examined as the animals mature. Vernon et al. (2015) previously reported an age-dependent effect of MIA on brain NAA in rats, with NAA higher in juvenile rats but lower in adult rats. $\mathrm{Li}$ et al. (2015) recently reported significantly higher cortical NAA levels in MIA compared to control mice. Increasing cortical NAA content is a hallmark of early brain development in humans. An agedependent increase in NAA levels could reflect an MIAinduced disturbance in the timing of some aspects of brain development. If so, measures of brain NAA during development may have value for studies investigating pathogenic mechanisms of maternal immune activation. Overall, these results highlight the potential value of brain MRS measurements across development in the non-human primate MIA model. Ultimately, this model may facilitate new insights into neuroimmune processes in the developmental pathogenesis of neuropsychiatric disorders.

Keywords: Maternal Immune Activation, Non-Human Primate, Neurodevelopment, Glutathione, N-acetylaspartate Disclosure: Nothing to Disclose.

M177. Akt Dysfunction Leads to Structure-Function Changes in the Voltage-Gated $\mathrm{Na}+$ Channel Complex at Axonal Initial Segment That Mimic Endophenotypes Associated With Schizophrenia

Wei-Chun J. Hsu, Pooran Negi, Miroslav N. Nenov, Thomas F. James, Cihan B. Kayasandik, Agenor Limon, Brooke E. Hjelm, Marquis P. Vawter, Saurabh Prasad, Demetrio Labate, Fernanda Laezza*

University of Texas Medical Branch, Galveston, Texas, United States 
Background: Recent genome-wide association studies have identified numerous loci significantly associated with schizophrenia (SZ) and other psychiatric disorders. Among those genes some code for proteins such as Ankyrin-G (ANK3), neurofascin (NFASC), spectrin (SPTBN1/4), and intracellular fibroblast growth factors (iFGFs, FGF11-14) that are part of the signaling complex associated with voltagegated $\mathrm{Na}+(\mathrm{Nav})$ channels at the axonal initial segment (AIS), the subcellular compartment that integrates synaptic inputs into an electrical output. Other genes relevant to SZ code for key signaling components such as protein kinase B (Akt). Previous in vitro studies have shown that glycogen synthase kinase-3 (GSK3), the primary target negatively regulated by Akt, establishes neuronal polarity and regulates FGF14:Nav assembly at the AIS, bidirectionally regulating Nav currents through a Nav isoform-dependent mechanism. Based on this evidence, we postulate that inhibition of Akt might recapitulate cellular endophenotypes that correlate with SZ by modulating Nav complex dynamics at the AIS and dysregulating neuronal firing.

Methods: The protein constructs Cluc-ANK3, CD4-Nav1.2II-IIIloop-Nluc, $\beta I V$-spectrin-14-15-Nluc, and NFASC-186Nluc were created by subcloning cDNA expressing Cluc and Nluc fragments of the enzyme luciferase enabling reconstitution of protein complexes through the split-luciferase complementation assay (LCA), which was performed by transient transfection of HEK293 cells with complementary Cluc and Nluc vectors. Relative binding of each protein pair was evaluated in the presence or absence of Akt and GSK3 inhibitors. Large-scale confocal microscopy with quantitative immunostaining was performed on primary rat hippocampal neurons treated with Akt and/or GSK3 inhibitors. To assess distribution and subcellular localization, images were processed to extract axonal and dendritic regions of interest (ROI) and pixel intensity values were reported as arbitrary units (AU). Whole-cell patch-clamp recordings were performed in HEK293 cells stably expressing Nav1.6, as well as in primary hippocampal neurons and acute hippocampal slices and firing pattern examined. In addition, mRNAsequencing of the dorso-lateral prefrontal cortex (DLPC) from $10 \mathrm{SZ}$ and 10 healthy controls (HC) was analyzed to measure gene expression of AIS proteins.

Results: Analysis of protein interacting pairs ANK3:NFASC, FGF14:NFASC, ANK3:CD4NavII-III, FGF14:Spectrin, FGF14:CD4NavII-III, and ANK3:Spectrin with LCA revealed that inhibition of Akt with triciribine globally enhanced complementation among protein pairs (FGF14/Spectrin ratio $1.17 \pm 0.23$ in triciribine ( $n=7$ replicates) vs $0.40 \pm 0.05$ in DMSO (20); $<<0.001, A n k 3 /$ Spectrin ratio of $2.59 \pm 0.62$ in triciribine $(n=12)$ vs $1.04 \pm 0.24$ in DMSO $(n=16 ; p<0.05)$ thereby predicting increased overall stability of the $\mathrm{Nav}$ complex. Using large-scale confocal microscopy to characterize neurite arclength intensities of hippocampal neurons treated with triciribine or CHIR-99021 (GSK3 inhibitor), we observed Ankyrin G $(522 \pm 60$ AU axonal AnkG with $5 \mathrm{uM}$ CHIR ( $n=25$ axons) vs $230 \pm 28$ in DMSO $(n=23 ; p<0.001)$, Neurofascin ( $177 \pm 18$ AU axonal NFASC ( $n=19$ axons) with 5 uM CHIR vs $345 \pm 42$ in DMSO $(n=21 ; p<0.01)$, and Spectrin $(185 \pm 11$ AU dendritic Spectrin with $30 \mathrm{uM}$ triciribine ( $n=26$ dendrites) vs $240 \pm 12$ in DMSO $(n=32$; $p<0.01)$ to be strongly perturbed. Through whole-cell patchclamp recordings, we observed that Akt inhibition significantly potentiates Nav1.6 peak current density (at -10 $\mathrm{mV}$ was $-47.8 \pm 9.4 \mathrm{pA} / \mathrm{pF}$ in control, $n=11$ versus $-99.4 \pm 14.6 \mathrm{pA} / \mathrm{pF}$ in triciribine group, $n=11 ; p<0.01$ with Student t-test). Akt inhibition also increases repetitive firing of CA1 pyramidal neurons (15.1 \pm 3.3 spikes in control, $n=10$ neurons vs. $22.9 \pm 2.4$ in the triciribine group, $n=10$ ); $p<0.05$ with Mann-Whitney test), and decreased current threshold $(124 \pm 17.1 \mathrm{pA}$ in control, $n=10$ versus $75 \pm 13.4$ in triciribine, $n=10) ; p<0.05$ with Student t-test) in hippocampal neurons. Analysis from mRNA sequencing revealed that individuals with $\mathrm{SZ}$ had increased expression of $\beta I V$-spectrin (SPTBN4) (ANOVA; $p=0.031$ ) along with higher expression ratios vs. other $\beta$-spectrins (SPTBN4/ SPTBN1; $p=0.02$ and SPTBN4/SPTBN2; $p=0.049)$. Furthermore, increased SPTBN4 in SZ correlated with an $\sim 18 \%$ increase in the proportion of Nav1.1 (SCN1A) compared to Nav1.2 (SCN2A) (SCN1A/SCN2A, $p=0.002$ ). In contrast, the ratios of $\mathrm{ANK} 1, \mathrm{ANK} 3$, and $\mathrm{AKT} 1$ versus SCN1A and SPTBN4 were reduced in SZ compared to HC. These results provide a mechanistic model that suggests alterations in AIS protein scaffolds may remodel Nav complexes and affect excitability.

Conclusions: Taken together, these results demonstrate that Akt inhibition results in marked remodeling of the Nav channel complex architecture, distribution, and function through effects on diverse complex members that reside at the AIS. Overall, these findings suggest that the level of Akt activity in a neuron might be a susceptibility marker for the dysregulation in excitability seen in SZ and other a broad range of psychiatric endophenotypes.

Funding: R01MH095995 (FL); R01MH111107 (FL); NSF DMS 1720487 (DL), GEAR 113491 (DL); R21MH113177 (AL, MPW); Predoctoral Kempner Fellowship (JH); Blocker Scholar Fellowship in Biomedical Research (JH); George Sealy Research Award in Neurology (JH); James E. Beall II Memorial Award in Anatomy and Neurosciences (JH).

Keywords: Ankryin-G, Axons, Excitabilty

Disclosure: Nothing to Disclose.

\section{M178. The Combination of Zonulin, a Regulator of Tight Junction Permeability and Peripheral Inflammation is Related to Psychiatric Symptoms in Schizophrenia}

Deanna Kelly*, Gregory Barber, Craig Sturgeon, Nicola Cascella, William Eaton, Robert McMahon, Alessio Fasano

University of Maryland School of Medicine, Baltimore, Maryland, United States

Background: Increasing evidence in schizophrenia suggests that the pathophysiology of the disease itself may be related to neuroinflammation. It remains unclear how immune dysfunction may relate to the pathogenesis of schizophrenia. Some evidence suggests that schizophrenia may be characterized by increased leakiness of the epithelial tight junctions of the intestine. Increased gut permeability has been demonstrated, in part by elevated levels of IgG-related antibodies in schizophrenia compared to healthy controls. This loss of barrier function allows the passage of macromolecules including endotoxins to cross into the body from the gastrointestinal tract. Subsequently the resultant immune 
response may lead to the establishment of chronic inflammation as suggested from many other chronic inflammatory diseases. The tight junctions of the gut are similar to those found in the brain and gut-brain crosstalk may lead to increased leakiness of the tight junctions of the blood brain barrier (BBB) as well. Zonulin has been shown to regulate tight junction permeability of the gut and the BBB. It has been identified as pre-haptoglobin 2, derived from chromosome 16, and is elevated in people with autoimmune diseases. Pre-haptoglobin 2 has been identified in some studies as a candidate gene for schizophrenia. As a stimulator of the endothelial growth factor receptor through transactivation of protease activates receptor 2 , its regulation of tight junction permeability is responsible for the movement of fluids, macromolecules, and leukocytes along the paracellular pathway. Zonulin's role in schizophrenia has not been previously studied.

Methods: We collected blood samples and symptom data from 98 male and female patients with DSM-IV-TR chart diagnosis of schizophrenia between the ages of 18 to 75 years. All participants were seen for a one time visit for clinical assessments and a lab draw. Serum zonulin was measured at the laboratory of Dr. Alessio Fasano at Massachusetts General Hospital. Cytokines were analyzed by the Cytokine Core Laboratory at the University of Maryland using multiplex (Luminex ) technology (IL-1B and TNF-a). Cut off scores for zonulin positivity were determined as $2.33 \mathrm{mg} / \mathrm{dL}$, the lower limit of detection. The Brief Psychiatric Rating Scale (BPRS) was used to examine psychiatric symptoms, including five symptom domains of the BPRS (activation, psychosis, hostility, negative symptoms and anxiety/depression).

Results: Within the group, the mean zonulin level in the schizophrenia cohort was 2.38 (SD 1.23). Fifty-six subjects (57.1\%) had a below-threshold zonulin of $<2.33 \mathrm{mg} / \mathrm{dL}$ and $42(42.9 \%)$ had a zonulin measure of $>2.33 \mathrm{mg} / \mathrm{dL}$. We find that independently there is no relationship of zonulin or cytokines (IL-1B, TNF-a) to psychiatric symptoms as measured by the BPRS, however multivariate models show that zonulin and IL-1B in combination predict total BPRS scores $(p=0.024)$ and a trend for zonulin and TNF-a $(p=0.067)$ is also observed.

Conclusions: These findings suggest that loss of gut barrier alone or an inflammatory state alone are not sufficient to produce neuroinflammation. The inappropriate movement of antigens secondary to zonulin up regulation and a subsequent inflammatory process together may explain the severity of psychotic symptoms in schizophrenia and underlying pathophysiology of the illness.

Keywords: Schizophrenia, Zonulin, Blood-Brain-Barrier Disclosure: Nothing to Disclose.

\section{M179. Correlation of FDG and Fractional Anisotropy in Never-Medicated Patients With Schizophrenia}

Monte Buchsbaum*, Brian Merrill, Serge Mitelman, Bradley Christian, Mehdi Adineh, Douglas Lehrer

University of California, San Diego, San Diego, California, United States

Background: Higher FDG uptake in white matter found in patients with schizophrenia has been hypothesized to reflect inefficient regional interconnections and less coherent fiber tracts assessed by diminished fractional anisotropy (FA). However, images of the same unmedicated patients using both patients have not previously been available.

Methods: To test whether high FDG uptake was associated with FA deficits in schizophrenia, we acquired FDG-PET images, diffusion tensor MRI images, and MRI anatomical images on 25 un-medicated (primarily never-medicated) patients with schizophrenia and 19 age and sex-matched normal controls. FDG-PET was acquired with ECAT EXACT HR+ in three-dimensional mode following injection of FDG (mean $7.8 \mathrm{mCi}$ ) with emission scanning beginning 40 minutes after administration of the tracer. Patients performed the Posner flanker task during uptake of FDG. Structural MRI images were acquired using a GE Signa NVi system $\left(\mathrm{TR}=24 \mathrm{~ms}, \mathrm{TE}=5 \mathrm{~ms}\right.$, flip angle $=40^{\circ}$, slice thickness $=1.2 \mathrm{~mm}$, pixel matrix $=256 \times 256$, field of view $=23 \mathrm{~cm}$, total slices $=128$ ). The DTI acquisition sequence acquired diffusion weighted images from 25 different directions. The parameters are: $\mathrm{TR}=11000 \mathrm{~ms}, \mathrm{TE}=87 \mathrm{~ms}$, flip angle $=90$, image matrix $=256 \times 256, B=1000$, slice thickness $=5 \mathrm{~mm}$, slice ga $p=2.5 \mathrm{~mm}$. The total time required for this sequence is approximately 10 minutes. We registered FA and FDG images to their own MRI and then used the transformation matrix of raw MRI to MNI MRI to create the first set of coregistered images. We then formed the average FA and average FDG templates. Each subject was then registered to the average FA and FDG templates. The average template was registered to the MNI template and the transformation matrix used to standardize each FA and FDG image to the MNI template (12-parameter affine registration model using FLIRT).

Results: Central gray matter disconnection dominated the deficit pattern suggesting relatively short pathways were important in the pathology. In healthy volunteers, large and high FA tracts including the corpus callosum showed positive correlations between FA and FDG while longitudinal fasciculi tended to show negative correlations. This pattern was less marked in patients with schizophrenia. The largest cluster of significant correlation differences was in the anterior tip of the left temporal lobe encompassing both gray and white matter and revealing significant negative correlations in healthy and positive correlations in patients. The largest cluster was in the left anterior temporal lobe (at Talairach xyz: $-55,9,37$, healthy $r=-.755$ patient $r=+.415$, $z=-4.33)$. The second largest cluster was located in the cerebellum in the region of the dentate nucleus and dentate/ cortical white matter connections (Talairach xyz: 25,-74, -43, healthy $\mathrm{r}=-.657$, patient $\mathrm{r}=0.613$, fisher $\mathrm{z}=4.53, p<0.001$ ). Areas where healthy have higher FA than patients and less negative correlation are in the centrum semiovale and anterior corpus callosum. The correlation across subjects between FDG and FA is may be expected if a mixed set of gray and white roi are plotted since gray tends to have greater FDG uptake, but is not necessarily expected when voxel by voxel correlations are carried out as in this analysis since all images have similar gray/white composition at each voxel.

Conclusions: These results indicate that 1 ) healthy subjects have positive FDG vs FA correlations in large tracts consistent with neural traffic and lower or negative correlations in smaller tract areas and 2) patients with schizophrenia tend 
to have lower FA in the centrum semiovale, and this is associated with higher FDG. Taken together, these results are consistent with the inefficiency model of diminished FA associated with greater FDG uptake in some but not all pathways in schizophrenia.

Keywords: Antipsychotic-Naive Schizophrenia, White Matter Fractional Anistropy, Fluorodeoxyglucose

Disclosure: Nothing to Disclose.

\section{M180. Comparing the Retest Reliability of Four Analytic Approaches to fMRI in Healthy \& Psychosis Patient Samples}

Angus MacDonald*, Andrew Poppe, Deanna Barch, Cameron Carter, James Gold, John Ragland, Steven Silverstein

University of Minnesota, Minneapolis, Minnesota, United States

Background: Because of its centrality to questions of statistical power, reproducibility and validity, the reliability of a measure is crucial for guiding researchers' data analytic choices, and to plan appropriately powered studies. Until recent years, the measurement properties of MRI have been largely overlooked, and many analytic approaches continue to be developed without regard to their capacity to reliably assess individual differences. Using a retest dataset collected across five different sites, the present study assessed and compared the reliability of four fMRI data analytic approaches and ascertained how susceptible each method was to data collection site effects and subject group effects.

Methods: As part of the Cognitive Neuroscience Task Reliability and Clinical Applications for Serious mental illness (CNTRaCS) initiative, 45 healthy controls (HC) and 41 people with schizophrenia (SZ) were scanned twice three weeks apart, across five data collection sites using $3 \mathrm{~T}$ scanners from three different manufactures. Participants performed an executive control task, and the groups showed predicted behavioral effects. Reliability was measured using intraclass correlation coefficients, and was calculated for general linear model (GLM using FEAT), independent component analysis (ICA using MELODIC), and tonic functional network connectivity (tFNC, following the Jafri et al., 2008 approach to optimization) and dynamic functional network connectivity (dFNC, following Sakoğlu et al., 2010 using a 15" sliding window). Analysis of variance (ANOVA) with ICC as the dependent variable and analysis method as the independent variable addressed whether observed differences in ICC's were statistically meaningful.

Results: Across regions for both patients and controls, ICA and tFNC had generally acceptable reliability whereas dFNC and GLM had significantly lower reliability. Additionally, ICA and tFNC had more stable reliability across data collection sites than GLM and dFNC. In terms of regional variation, ICA-identified executive components were more reliably measured (average ICC $>.70$ ) than other ICA components (average $<.60$ ), excepting the default mode and visual components (average ICC $<.70$ ). Finally, for both patients and controls, components with the higher tFNC magnitude were measured more reliably.
Conclusions: ICA and tFNC based on ICA components provided better retest reliability (and therefore better statistical power) than GLM or dFNC. This is because ICA takes advantage of the measurements across many different voxels and extracts the central tendency of those voxels rather than relying on each voxel (measured with noise) to provide a reliable signal. In this sense, the neurometrics of fMRI parallel that of test design where more items improve the retest reliability of a measure. In the case of dFNC, data is again reduced (this time by using a narrower time window), which seems to reduce the availability of a reliable signal. Practically, these findings suggest ICA and tFNC provide more power, and likely reproducibility, than GLM or dFNC in biomarker or treatment outcome fMRI studies.

Keywords: Functional MRI (fMRI), Test Retest, Task-Based Functional Connectivity, Psychosis

Disclosure: Nothing to Disclose.

\section{M181. Comparative Effectiveness of Adjunctive Phar- macotherapies in Schizophrenia}

Thomas Stroup*, Tobias Gerhard, Zhiqiang Tan, Melanie Wall, Steve Crystal, Chacku Mathai, Mark Olfson

\section{Columbia University, New York, New York, United States}

Background: Antipsychotics are the first-line treatment for schizophrenia. Their effectiveness is supported by strong evidence, but antipsychotics alone are rarely adequate to address all of the symptoms and functional limitations of individuals with schizophrenia. Other classes of psychotropic medications are frequently prescribed in addition to antipsychotics, but there is little high-quality evidence about the effectiveness of these adjunctive psychotropic medications. Among individuals with schizophrenia treated in routine outpatient clinical practice in the U.S. and treated with a single antipsychotic, the authors compared the effectiveness of initiating adjunctive treatment with a second antipsychotic, a mood stabilizer, an antidepressant, or a benzodiazepine.

Methods: U.S. national Medicaid data from 2001-2010 were used to examine treatment outcomes in patients diagnosed with schizophrenia and no current evidence of additional psychiatric conditions. The study cohort consisted of individuals who were stably treated with a single antipsychotic and then initiated treatment with an additional psychotropic medication. Outcomes were compared among cohorts of individuals, matched by multinomial propensity scores, who initiated treatment with a second antipsychotic $(N=23,507)$, a mood stabilizer $(N=8,085)$, an antidepressant $(N=22,118)$, or a benzodiazepine $(N=10,005)$. Propensity scores, based on a large number of demographic, clinical, and service use variables, were used to control for measured confounders. Individuals were followed for 365 days on an intent-to-treat basis. The primary outcome was hospitalization for a mental disorder. Secondary outcomes included emergency department visits for a mental disorder and all-cause mortality.

Results: Compared to initiating treatment with a second antipsychotic, initiating treatment with an antidepressant was associated with lower hospitalization rates (HR 0.81, 
95\% CI 0.77-0.85), initiating treatment with a mood stabilizer (HR 1.00, 0.94-1.07) was not different, and initiating treatment with a benzodiazepine was associated with higher hospitalization rates (HR 1.07, 1.00-1.15). Psychiatric emergency department visit rates were lower for antidepressants (0.90, 0.86-0.94), similar for mood stabilizers (1.02, 0.96-1.09) and higher for benzodiazepines (1.07, 1.01-1.14) in comparison to a second antipsychotic. Mortality rates were higher for those who initiated a mood stabilizer $(1.47,1.14-1.91)$ compared to those who initiated a second antipsychotic, but no different for antidepressants (1.06, 0.85-1.32) or benzodiazepines (1.07, 0.84-1.37).

Conclusions: In individuals diagnosed with schizophrenia treated with a single antipsychotic who started a new psychotropic medication, initiating treatment with an antidepressant was associated with lower hospitalization rates than initiating a second antipsychotic, a mood stabilizer, or a benzodiazepine. Compared to starting an antidepressant or a second antipsychotic, initiating treatment with a mood stabilizer or a benzodiazepine appeared to have no advantages but some disadvantages. The association of mood stabilizers with higher mortality rates in this population suggests that they should be used cautiously but requires further investigation.

Keywords: Schizophrenia, Pharmacotherapy, Comparative Studies, Mortality

Disclosure: Nothing to Disclose.

M182. Transient Recapitulation of the Face Emotion Recognition Deficits of Schizophrenia in Healthy Individuals

Cheryl Corcoran*, Jack Grinband, Jaimie Gowatsky, Casimir Klim, Matthew Hoptman, Daniel Javitt

Icahn School of Medicine at Mount Sinai, New York, New York, United States

Background: Profound deficits in face emotion recognition (FER) exist in schizophrenia (Sz), including during prodromal stages, causing social disability, though these can be partly remediated with computer-based training. Neurostimulation might augment remediation if critical nodes were identified. We aimed to 1) briefly recapitulate the FER deficits of $\mathrm{Sz}$ in healthy volunteers (HV) using rTMS to right posterior superior temporal sulcus (rpSTS); 2) identify connectivity of rpSTS regressed by FER in both $\mathrm{HV}$ and $\mathrm{Sz}$ patients; and 3) apply TMS to rpSTS with fMRI as readout. Methods: 1) Nine healthy volunteers had rTMS $(10 \mathrm{~Hz} ; 500$ msec; $110 \%$ RMT) to rpSTS or right occipital face area (rOFA) (counterbalanced; 10/10 system overlay with standard MRI) concurrent (1/3 trials) with stimuli (http://faces. mpdl.mpg.de/) for emotion or gender identification (button press). $14 \mathrm{Sz}$ patients completed these tasks without TMS.

2) Whole-brain resting-connectivity analyses, seeded by rpSTS, was applied in $27 \mathrm{Sz}$ and $35 \mathrm{HV}$ who also completed the UPenn FER task.

3) BOLD fMRI was obtained in $4 \mathrm{HV}$ pre- and post-TMS to rpSTS (1 Hz; 20 minutes).

Results: 1) In healthy volunteers, rTMS to rpSTS only (but not rOFA) significantly slowed reaction time for FER only (and not gender identification) (overall $\mathrm{F}$ test for $\log \mathrm{RT}$ $(p=.001))$. Post-hoc comparison showed significant decrease in log RT for face emotion recognition for rpSTS, as compared with rOFA stimulation $(p=.005)$, and as compared with non-stimulation trials $(p=.004)$. rpSTS stimulation recapitulated the slowed reaction time and lower accuracy for FER in $\mathrm{Sz}$ patients.

2) In both healthy volunteers and Sz patients, rpSTS had significant resting functional connectivity with V1 $(p=.0001)$, positively modulated by accuracy on the UPenn FER task.

3) Using the rpSTS ROI for connectivity analysis, network enhancement after rTMS showed increased connectivity to extrastriate regions, the right face fusiform area and cuneus. Conclusions: The right posterior superior temporal sulcus is a critical node in the face emotion recognition circuit, with connectivity to primary visual cortex that is modulated by FER accuracy. Its transient disruption in healthy individuals recapitulates FER deficits, making it a candidate target for neurostimulation-enhanced remediation of FER deficits in neuropsychiatric disorders. This is supported by the preliminary finding of network enhancement by lowfrequency TMS of face processing and visual areas.

Keywords: Face Emotion Processing, Transcranial Magnetic Stimulation, Cortical Circuit Function, Schizophrenia, Circuit Mapping, Resting State Functional Connectivity

Disclosure: Nothing to Disclose.

M183. Effect of NBI-98782, a Selective Vesicular Monoamine Transporter 2 (VMAT2) Inhibitor, on Neurotransmitter Efflux and Phencyclidine- and Amphetamine-Induced Locomotor Activity: Relevance to Tardive Dyskinesia, Psychosis, and Cognition

Mei Huang, Wenqi He, Andrea Kudwa, Dimitri Grigoriadis, Lakshmi Rajagopal, Herbert Meltzer*

Northwestern University, Chicago, Illinois, United States

Background: Tardive dyskinesia (TD) is an involuntary movement disorder, resulting primarily from dorsal striatal (dSTR) dysfunction, genetic vulnerability, and treatment with antipsychotic drugs (APDs). Valbenazine (Ingrezza), a selective VMAT2 inhibitor and the precursor of NBI-98782, was recently approved as the first medication indicated for the treatment of TD in adults. Its efficacy in TD may be due, in part, to inhibition of the release of vesicular neurotransmitters (NTs), including DA, norepinephrine (NE), 5-HT, acetylcholine (ACh), GABA and glutamate (Glu), which are also critically important for cognition and psychosis. TD has been shown to diminish the ability of atypical APDs to improve cognitive function. Inhibition of VMAT2 would be expected to diminish the release of some, or all, of these NTs from presynaptic neurons, which might influence some of the clinical effects of these drugs.

Methods: Our aim was to determine the effect of NBI-98782 and tetrabenazine on NT efflux from mPFC and dSTR using microdialysis in awake, freely moving mice, to characterize the interaction of NBI-98782 with atypical APDs, and to assess whether NBI-98782 can attenuate locomotor increases observed in the phencyclidine (PCP)- and amphetamineinduced mouse models of psychosis. 
Results: NBI-98782 and tetrabenazine $(10 \mathrm{mg} / \mathrm{kg}, \quad \mathrm{PO})$ decreased the efflux of DA, 5-HT, and NE, while increasing the efflux of DA metabolites DOPAC and HVA. and the 5-HT metabolite, 5-HIAA, without changing ACh, Glu or GABA efflux in medial prefrontal cortex (mPFC), dSTR, hippocampus, and nucleus accumbens, regions of interest for cognitive-, psychotic-, and rewarded behaviors. Sub-chronic NBI-98782 (7 days, $10 \mathrm{mg} / \mathrm{kg}$ per day, PO) decreased basal $\mathrm{DA}$ and 5-HT efflux in both mPFC and dSTR. Acute NBI-98782 $(10 \mathrm{mg} / \mathrm{kg}, \mathrm{PO})$ had similar effects on NT efflux in scNBI-98782 -treated mice. NBI-98782 $(10 \mathrm{mg} / \mathrm{kg}, \mathrm{PO})$ suppressed the atypical APDs, clozapine $(10 \mathrm{mg} / \mathrm{kg}$, ip) and olanzapine $(3 \mathrm{mg} / \mathrm{kg}$, ip)-induced DA efflux in both $\mathrm{mPFC}$ and dSTR, and ACh efflux in mPFC. NBI-98782 suppressed haloperidol $(0.5 \mathrm{mg} / \mathrm{kg}$, ip)-induced DA efflux in dSTR, but had minimal effect on GABA efflux, despite the fact that VMAT2 is involved in GABA transport.

Conclusions: These results suggest decreased release of dSTR DA may contribute to the beneficial effects of NBI-98782 on TD. NBI-98782 (10 mg/kg, PO) attenuated PCP $(10 \mathrm{mg} / \mathrm{kg}$, ip)-induced DA, 5-HT, NE and Glu efflux, and AMP $(2.5 \mathrm{mg} / \mathrm{kg}$, ip)-induced DA and NE efflux, in both $\mathrm{mPFC}$ and ASTR, and PCP- and AMP-induced hyperlocomotion. NBI-98782 may have actions similar to those of atypical APDs. Further study is needed to determine its effects on cognition and psychosis in patients.

Keywords: Schizophrenia, Tardive Dyskinesia, Valbenazine, Dopamine, Clozapine

Disclosure: Part 1: Neurocrine Biosciences, Grant, Part 2: Neurocrine Biosciences, Grant, Part 3: Neurocrine Biosciences, Grant, Part 4: Neurocrine Biosciences, Grant.

M184. Childhood Urbanicity Influences Prefrontal Response to Interpersonal Closeness, Stress and Genetic Risk for Psychosis

Hao Yang Tan*, Hao Yan, Jian Zhu, Xiao Zhang, Guang Yang, Xin Zhao, Shefali Shah, Zheng Dong, Debjani Saha, Xiaoxi Zhang, Qiang Chen, Sisi Jiang, Timothy Muse, Jinmin Liao, Yuyanan Zhang, Weihua Yue, Daniel Weinberger, Dai Zhang

Lieber Institute for Brain Development, Baltimore, Maryland, United States

Background: Working memory (WM) and prefrontal function is sensitive to social stress, and occurs in several neuropsychiatric disorders, including schizophrenia. Genetic and environmental contributions to risk for psychosis have been implicated. Less, however, is understood about how childhood environmental contexts may influence these brain functions. Indeed, urban social stressors and childhoods in urban environments may be associated with increased neuropsychiatric risk. Here, we further examined mechanisms by which WM prefrontal functions associated with genetic risk for psychosis in unaffected siblings of patients, may also be influenced by interpersonal stressors in relation to differing urban and rural childhoods.

Methods: We examined unaffected siblings of schizophrenia patients $(N=36)$ and healthy controls $(N=35)$ to define dorsolateral prefrontal cortical (DLPFC) functions influenced by genetic risk for psychosis in an event-related WM functional MRI paradigm in a $3 \mathrm{~T}$ scanner. To define the influence of childhood urbanicity on these brain functions, we examined a unique sample of healthy adult individuals currently living in Beijing but with divergent urban or rural childhoods before age $12(N=200,204)$. These subjects engaged a similar WM paradigm in a $3 \mathrm{~T}$ MRI scanner, but with additional events that induced interpersonal stress during WM. We then examined the effects of interpersonal stress on DLPFC function linked to psychosis risk, and the influence of interpersonal closeness in the diverging childhood environments on these brain functions.

Results: Reduced DLPFC function during manipulating information in WM was associated with increased genetic risk for psychosis in siblings. All results are significant at $p<0.05$ family-wise corrected within regions-of-interest (ROI) in the DLFPC. Within the DLPFC ROI, interpersonal stress also reduced DLPFC function. Interpersonal closeness in urban vs rural childhoods had divergent effects on DLPFC function. Higher interpersonal closeness in rural childhoods was associated with relatively less reduced DLPFC function during stress. Higher interpersonal closeness in urban childhoods, however, was associated with more stressrelated reduction in DLPFC function.

Conclusions: We suggest that childhoods in urban environments may influence risk for psychosis in part through brain mechanisms related to interpersonal stress. Interpersonal closeness may be less adaptive in urban than in rural life, potentially negating some of the protective effects of interpersonal relationships, at least on DLPFC function under interpersonal stress.

Keywords: Working Memory fMRI, Childhood Urban-Rural Upbringing, Social Defeat Stress

Disclosure: Nothing to Disclose.

\section{M185. GluN1 Hypomorph Disrupts Synaptic Protein- Protein Interactions}

Adam Funk ${ }^{*}$, Guillaume Labilloy, Catharine Mielnik, Rebekka Meeks, Ken Greis, Jarek Meller, Amy Ramsey, Robert McCullumsmith

University of Cincinnati, Cincinnati, Ohio, United States

Background: Inhibition or dysregulation of ionotropic glutamate receptors is a major hypothesis to describe the pathophysiology of schizophrenia. Additionally, there is substantial evidence that synaptic proteins are particularly involved in the genesis of schizophrenia. Therefore, we chose the GluN1 hypomorph mouse model to probe synaptic protein-protein interactions in an effort to better understand the intracellular milieu under normal and abnormal synaptic function.

Methods: Three groups, Wildtype $(N=6)$, GluN1 Knockdown $(N=6)$, and GluN1 Knockdown Rescue $(N=6)$, were included in the study. The frontal pole of each mouse was dissected, homogenized and prepped for anti-PSD-95 affinity purification. We targeted PSD-95 by conjugating antiPSD-95 antibody to magnetic dynabeads to isolate synaptic protein-protein interaction complexes. The PSD-95 interactome was then processed for mass spectrometry analyses on an ABSciex 5600+ mass spectrometer. 
Results: We explored the extent of protein-protein interactions affiliated with PSD-95 in the rodent frontal pole. First, we verified the specificity of this affinity purification by Western blot analysis. An isotype specific, negative control isolation showed zero PSD-95 signal. Mass Spectrometry analysis indicated roughly 650 co-isolated proteins, including key postsynaptic components. Unsupervised cluster analysis based on the correlation of peptide relative abundance revealed the Rescue group intermixed with the Wildtype samples, whereas the Knockdown group showed a distinctly independent cluster. Further analysis with non-negative matrix factorization (NMF) confirmed a unique Knockdown vs Wiltype/Rescue signature. We integrated the unsupervised and NMF signatures for both groups into a consensus signature and analyzed them by pathway analyses and through the iLINCS chemical and gene perturbation repository. The Knockdown signature includes Erbb4, Syngap1, Hspa8, Nptn, Syn2, and Glul. The Wildtype/Rescue signature includes DLG1, DLG2, CaMKIIa, Cntn1, Map1a, and Nefh. Conclusions: Our data establish a clear synaptic proteinprotein interaction signature for GluN1 Knockdown mice which implicates structural, cytoskelatal, and postsynaptic density integrity. Additionally, the knockdown signature predicts abnormal synaptic transmission, which has previously been reported in these mice. Further analyses suggest dysregulation of nicotinic acetylcholine receptor systems, the innate immune system, and endocytosis. Together, these data provide evidence that mice with decreased NR1 expression have substantial synaptic pathology leading to physiological and behavioral abnormalities. The data also provide testable hypotheses, whereby we can measure synaptic protein-protein interactions before and after pharmacological interventions.

Keywords: Glutamatergic Synapses, Schizophrenia, Synaptic Aberrations, Mouse Models, NMDA Receptor, PSD-95

Disclosure: Nothing to Disclose.

\section{M186. Effects of tDCS on Cognitive Control and Cortical Network Oscillations in Schizophrenia}

Katherine Scangos*, Megan Boudewyn, Brooke

Roberts, John Ragland, Charan Ranganath, Cameron Carter

University of California, San Francisco, Sacramento, California, United States

Background: Deficits in goal maintenance are consistently observed in patients with schizophrenia, are correlated with decreased activity in the frontal cortex, and may be improved by frontal transcortical direct current stimulation (tDCS). Oscillatory activity in brain networks is important for cognitive processes and deficits in gamma and theta activity are reported in schizophrenia. In this study, induced oscillatory activity in the electroencophologram (EEG) was examined during left dorsolateral prefrontal cortical (DLPFC) anodal tDCS to address the possibility that tDCS may restore task specific modulation of oscillatory activity important for cognitive control.

Methods: 17 subjects (18-31 y) diagnosed with schizophrenia or schizoaffective disorder within the previous 5 years received 2 sessions (sham, stim) of tDCS separated by 2-7 days. Exclusion criteria included substance use within 6 months, a history of traumatic brain injury, neurological disorder or medication changes within 1 month. The study design was a double blinded, sham controlled cross over design. Subjects received $20 \mathrm{~min}$ of sham or tDCS stimulation (StarStim32) at $2 \mathrm{~mA}$ while they performed a working memory task. The anode was located at F3 (left dorsolateral prefrontal cortex) and the cathode at FP2 (the right supraorbital region). Following stimulation 32 channel EEG was performed with the same system while patients performed the Dot Pattern Expectancy Task, a task of goal maintenance.

Results: Behaviorally, there was a trend toward more AY errors with tDCS, indicating improved use of the A cue to build context. No change in overall error rate was observed. Next, the effect of stimulation on task specific electrophysiological changes was examined through EEG by comparing activity on cognitively demanding $\mathrm{B}$ cue trials to less demanding A cue trials. We observed significantly increased theta power over the left central region, significantly increased beta power over the left frontal region, and a trend toward increased left frontal gamma power.

Conclusions: Left DLPFC tDCS led to partial improvement in goal maintenance and modulated task specific oscillatory activity related to cognitive control. These encouraging preliminary results suggest a causal role for left DLPFC in goal maintenance deficits in schizophrenia. Furthermore, they suggest that tDCS has therapeutic potential for restoring disruptions in synchronous oscillations that may underlie cognitive dysfunction.

Keywords: Cortical Circuit Function, Schizophrenia, Neural Oscillations, Goal Maintenance, Cognitive Control

Disclosure: Nothing to Disclose.

M187. Polygenic Interactions With Stress in Conferring Risk for Suicide

Vincenzo De Luca*, Etienne Sibille, Jerry Warsh, Samia Tasmim

University of Toronto, Toronto, Canada

Background: Approximately 5\% of patients with schizophrenia commit suicide, and $20 \%$ to $40 \%$ of them have at least one suicide attempt lifetime. Previous research has identified stress as potential risk factor for suicide attempt in schizophrenia. Familial, adoption, and twin studies suggest that suicidal behaviour is both heritable and under genetic influence. Suicide and suicide attempt seemingly cluster within families. The PGC found 108 loci associated with schizophrenia that have also been associated with suicide attempt and suicide ideation by other investigators.

Methods: The study design is cross-sectional and retrospective considering lifetime suicide attempt as the main dependent variable. We will also test the polygenic scores interaction with childhood trauma to prove our hypothesis that these risk loci are 'genetic hot spots' sensitive to environmental insults that can affect suicidal behavior. There were 129 non attempters and 47 multiple attempters. The attempts were recorded using the Columbia and the BSS. 
Results: When comparing the weighted scores in non attempters and multiple attempters we found a trend for higher risk with higher polygenic scores $(p=0.07)$.

Conclusions: The genetic risk for schizophrenia in combination with a history of childhood trauma may allow us to predict those who are at risk for suicide. The early identification of persons at risk will facilitate suicide prevention through counseling and early intervention. Finally, identifying genetic and gene-environment mechanisms that modulate suicide attempt may enhance our understanding of the pathways resulting in suicide, facilitating the development of novel treatments.

Keywords: Suicide, Schizophrenia, Genetics

Disclosure: Nothing to Disclose.

M188. Genome-Wide Association and Heritability Analyses of Clinical Dimensions and Age of Onset of Schizophrenia in the PGC

Tim Bigdeli, Roseann Peterson, Anna Docherty, Stephan Ripke, Silviu-Alin Bacanu, Richard Amdur, Pablo Gejman, Douglas Levinson, Brien Riley, David St. Clair, James Walters, Roel Ophoff, Andrew McQuillin, Hugh Gurling, Dan Rujescu, Patrick Sullivan, George Kirov, Michele Pato, Carlos Pato, Ole Andreassen, Michael Owen, Michael O'Donovan, Aiden Corvin, Anil Malhotra, Brian Mowry, Tonu Esko, Thomas Werge, Kenneth Kendler, Schizophrenia Working Group Psychiatric Genomics Consortium, Ayman Fanous*

SUNY Downstate Medical Center, Brooklyn, New York, United States

Background: Schizophrenia (SZ) is a clinically heterogeneous disorder, with individual patients varying with respect to age-of-onset (AOO), course of illness, response to treatment, prognosis, outcome, and the particular pattern of symptoms presented.

Methods: We sought to determine whether common genetic variants influence variation in clinical heterogeneity in symptom dimensions and $\mathrm{AOO}$, and to confirm a previous association between polygenic risk of SZ and the severity of negative symptoms. The Psychiatric Genomics Consortium (PGC) study of SZ comprised 8,432 cases of European ancestry with available clinical phenotype data, and 11,600 cases with available information on age-of-onset of illness. Lifetime symptoms were assessed by clinical interview and/ or review of medical records using the OPCRIT, PANSS, LDPS, SCAN, SCID, and CASH. Factor analyses of each constituent PGC study identified positive, negative, manic, and depressive symptom dimensions. We examined the relationship between each dimension and aggregate polygenic risk scores indexing SZ risk. We additionally performed a genome-wide association study (GWAS) of each quantitative trait using linear regression, adjusting for significant effects of sex and ancestry.

Results: The negative symptom factor was most strongly associated with polygenic risk for SZ, confirming a previous, suggestive finding by our group in a smaller sample, though explaining only a small fraction of the variance $(\mathrm{R} 2=0.004386 ; p=6.39 \times 10-9)$. In subsequent GWAS, we observed the strongest evidence of association for the positive and negative symptom factors, with SNPs in RFX8 on $2 \mathrm{q} 11.2(p=6.27 \times 10-8)$ and upstream of WDR72/ UNC13C on $15 q 21.3(p=7.59 \times 10-8)$, respectively. Using genome-wide summary statistics, we demonstrate the significant SNP-based heritability of age-of-onset (SNPh2 $=0.132$, 95\% CI:[0.083,0.181]; $p=1.02 \times 10-7)$.

Conclusions: To our knowledge, this is the first report that AAO of SZ is significantly heritable. We also report evidence of association of novel modifier loci for schizophrenia, though no single locus attained established genome-wide significance criteria. As this may have been due to insufficient statistical power, follow-up in additional samples is warranted. Importantly, we replicated our previous observation that polygenic risk of SZ explains at least some of the variance in negative symptoms, a core illness dimension.

Keywords: GWAS, Schizophrenia Subtypes, Negative Symptoms, Age of Onset

Disclosure: Nothing to Disclose.

\section{M189. Inflammatory Biomarkers in Early Psychosis}

Skylar Kelsven*, Camilo de la Fuente-Sandoval, Cris Achim, Francisco Reyes-Madrigal, Heline Mirzakhanian, Isabel Ribeiro Caldas Domingues, Kristin Cadenhead

San Diego State University/University of California, San Diego Joint Doctoral Program in Clinical Psychology, San Diego, California, United States

Background: There is evidence that perinatal inflammation may stand at the top of a cascade of neural events that disrupt cell structure and function, leading to later-life sensitivity to immune challenges. Specifically, it has been suggested that infection during the perinatal period may serve as a vulnerability factor for later-life alterations in cytokine production and changes in cognitive and affective behaviors throughout the remainder of the lifespan. Longterm changes in glial cell function are suggested to be triggered by altered brain cytokine responses following perinatal infection. In later life, the release of proinflammatory cytokines by prolonged microglial hyperactivity may lead to neuronal apoptosis which is seen in neurodegenerative disorders such as Parkinson's Disease and Alzheimer's Disease, and may be the cause for progressive loss of brain tissue observed in patients with schizophrenia. Neuroinflammatory processes were assessed in first episode psychosis patients, individuals at clinical high risk for psychosis, and normal comparison subjects. A panel of peripheral markers of neuroinflammation linked to cognitive impairments in HIV positive adolescents was investigated. The goal of this study was to collect pilot inflammatory biomarker data in preparation for a larger translational study that will investigate neuropathological mechanisms and intervention in early psychosis.

Methods: Subjects were included if they were in their first episode psychosis (FEP) (Psychosis NOS, Schizophreniform, Schizophrenia, or Schizoaffective disorders). Clinical high risk (CHR) for psychosis subjects met CHR criteria per the Structured Interview of Prodromal Syndromes (SIPS). 
Normal comparison subjects did not meet criteria for any diagnosis. A panel of peripheral biomarkers linked to neuroinflammation and neurocognitive abnormalities was obtained.

Results: The sample included 81 individuals (77.3\% male) ages 12-35 $($ Mean $=21.9 \pm 5.4), 58$ FEP (mean age $=$ $23.16 \pm 5.1$ ), $14 \mathrm{CHR}$ (mean age $=17.43 \pm 4.3$ ), and 9 normal comparison subjects (mean age $=21.56 \pm 5.3$ ). Groups significantly differed on mean plasma concentration of BDNF $(\mathrm{F} 2,78=29.04, p<0.001)$, Eotaxin $(\mathrm{F} 2,78=4.94$, $p=0.01), \quad$ IP-10 $\quad(\mathrm{F} 2,78=7.5, \quad p=0.001), \quad$ MCP-1 $(\mathrm{F} 2,78=60.98, p<0.001), \mathrm{MCD}(\mathrm{F} 2,78=10.24, p<0.001)$, MIP1b $(\mathrm{F} 2,78=18.81, p<0.001)$, and TARC $(\mathrm{F} 2,78=21.22$, $p<0.001)$. Bonferroni corrections were used for multiple comparisons. A variety of markers significantly differed between groups. These group differences were primarily accounted for by the CHR and antipsychotic naive FEP groups compared to normal comparison subjects. In addition, greater levels of inflammatory biomarkers were associated with more symptoms in early psychosis subjects. Conclusions: Overall, CHR subjects and antipsychotic naive FEP patients displayed higher concentrations of peripheral biomarkers linked to neuroinflammation compared to normal comparison subjects. Greater evidence of inflammation was associated with more symptoms and lack of antipsychotic medication exposure. These findings provide evidence that a period of hyper-plasticity or increased neuroinflammation is present during the at-risk phase of psychosis, which then presumably progresses to a stage of hypo-plasticity and reduced neuroinflammation after the onset of psychosis. If inflammation stands at the top of the complex cascade of events that may lead to the development of devastating neurodegenerative diseases such as schizophrenia, then treatment interventions must be prophylactic. The development and implementation of low-risk treatment interventions are necessary to help ameliorate, or possibly reverse, the damaging effects of neural inflammation which escalate cognitive deterioration in neurodegenerative disease. Keywords: Inflammation, Neuroinflammation, Early Psychosis, First Episode Psychosis, Neuroplasticity

Disclosure: Nothing to Disclose.

M190. Winter-Like Photoperiods Induce PsychiatryRelevant Behaviors in Mice: Evidence for Resiliency in Mice With Reduced Dopamine Transporter Expression

Zackary Cope*, Molly Kwiatkowski, Chuck van de Chappelle, Maria Lavadia, Davide Dulcis, Jared Young

University of California, San Diego, La Jolla, California, United States

Background: Winter gestation (Castrogiovanni et al., 1998) and reductions in functional dopamine transporter (DAT; Anand et al., 2011) have both been linked to elevated incidences of psychiatric conditions (Cope et al., 2016). Todate, investigations regarding the impact of winter gestation have focused on reduced vitamin D exposure or malnutrition. Recently however, we demonstrated that winter-like photoperiod lengths can elevate stress-related neurotransmission in rodents, whereas summer-like photoperiod potentiates catecholaminergic transmission (Dulcis et al,
2013). In addition, adult DAT hypomorphic mice with only one functional DAT allele (DAT-HT) exhibit exaggerated depression- and mania-relevant behaviors following such housing respectively in adulthood (Cope et al., 2017). The potential winter-like photoperiod gestation effects on psychiatry-relevant behaviors have yet to be examined, nor has their potential interactions with reduced DAT functioning. Here, we hypothesized that winter-like photoperiods would induce psychiatry-relevant behavior in normal mice but that this effect would be exaggerated in DAT HT mice. Methods: Thirty-four female DAT-WT dams were housed in custom photoperiod chambers for one week under normal photoperiod conditions (12 hours light:12 hours dark) to allow for initial acclimation. On day 8, half of the photoperiod chambers shifted to a short active condition (19 hours light: 5 hours dark). Mating triads (2 female: 1 male) were also formed at this time by pairing dams with DAT-HT sires to produce roughly equal numbers of DATHT and WT offspring. After a two-week pairing, pregnant dams were single housed. The resulting 237 pups were reared under these photoperiod conditions until P28, at which time they were weaned by sex and genotype into tetrads and moved into a standard vivarium room under normal photoperiod. Of this cohort, 75 male and female mice were trained to perform nose-poke responses in a five-choice operant chamber (Young et al. 2013). When all mice could reliably perform this behavior, they were tested on a progressive ratio breakpoint (PRB), probabilistic reward learning (PLT), and probabilistic reversal learning (PRL) tasks.

Results: In PRB, a significant genotype ${ }^{\star}$ perinatal photoperiod interaction was observed $(\mathrm{F}(1,66)=4.4, p<0.05)$ as breakpoint was decreased selectively in WT mice reared in SA photoperiod (WT-SA). In the PLT, significant gene ${ }^{*}$ photoperiod interactions were observed with WT-SA mice selectively exhibiting slowed mean target $(\mathrm{F}(1,66)=7.2$, $p<0.01)$ and nontarget $(\mathrm{F}(1,66)=4.0, p<0.05)$ response latencies. WT-SA mice also exhibited strong trends nearing interactions indicating reduced responsiveness such as decreased rewards achieved $(\mathrm{F}(1,66)=3.9, p=0.052)$, premature responses $(\mathrm{F}(1,66)=3.9, p=0.051)$, target responses $(\mathrm{F}(1,66)=3.0, \quad p=0.088)$, and timeout perseverations ( $\mathrm{F}$ $(1,66)=3.3, p=0.073)$. In the PRL, WT-SA mice produced a strong trend nearing a genotype $\mathrm{e}^{\star}$ photoperiod interaction to decrease switches completed $(\mathrm{F}(1,66)=3.712, p=0.058)$.

Conclusions: Winter-like photoperiod births in mice induce psychiatry-relevant behaviors such as reduced effortful motivation and impaired learning. Such impairments are observed clinically in depression and schizophrenia. Interestingly however, these results were only observed in the WT mice, as DAT-HT mice did not exhibit such SA photoperiod born effects. These results suggest that reduced DAT functioning during gestation may convey resiliency to the effects of perinatal SA photoperiod. Ongoing work will characterize epigenetic, synaptic, and/or system-level adaptations underlying developmental differences between WT and DAT-HT in SA photoperiod as well as defining critical periods throughout gestation and rearing that are driving these effects.

Keywords: Bipolar Disorder, Schizophrenia, Gene x Environment Interaction, Development

Disclosure: Nothing to Disclose. 
M191. MEG Investigation of Auditory Cortical DeltaGamma Cross-Frequency Coupling in Early Vs. Chronic Phase Schizophrenia

Nicholas Murphy, Nithya Ramakrishnan, Christopher Walker, Nicola Polizzotto, Danica Womack, Megan

Rafferty, Cristin Rodriguez, Raymond Cho*

University of Texas Health Science Center at Houston, Houston, Texas, United States

Background: Cognitive impairments in schizophrenia are associated with disturbances in cortical gamma oscillatory rhythms. There has been increasing attention to how these gamma rhythms are regulated in the form of cross frequency coupling (CFC), specifically, how they may modulate with the phase of low frequency (delta/theta) rhythm phase. The aim of this MEG study was to investigate phase-amplitude CFC, localized to the auditory cortex, during $40 \mathrm{~Hz}$ steady state stimulation and to track its potential disturbance across the early and chronic illness phases of schizophrenia.

Methods: $N=13$ early, and $N=15$ chronic schizophrenia patients were compared to $N=17$ healthy controls during the presentation of a $40 \mathrm{~Hz}$ auditory click train stimuli (1000 $\mathrm{ms}$ ), recorded using 305 channel magnetoencephalography (MEG). Data were processed using the MNE toolbox and custom scripts in the python environment. Source localization was performed using the dynamic statistical parametric maps (dSPM) method to generate dynamic imaging of coherent source (DICS) estimates. Hybrid anatomical/ functional regions of interest (ROI) were identified by masking the DICS maps with the anatomical co-ordinates for the combination of the superior and transverse temporal cortices, bilaterally. To assess CFC, we computed the mutual information between the delta phase $(2 \mathrm{~Hz})$ and the gamma amplitude $(40 \mathrm{~Hz})$ during the steady state entrainment response, and was normalized through subtraction of the pre-stimulus baseline CFC.

Results: Steady state gamma power showed an overall reduction in schizophrenia patients (healthy controls > early; healthy controls $>$ chronic), as well as an interaction between hemisphere and group (healthy controls right $>$ left hemisphere). The left hemisphere showed no differences between groups. All groups demonstrated coupling between gamma amplitude and delta phase, but there were no differences observed between groups or hemispheres.

Conclusions: Our findings demonstrate that despite impaired auditory cortical gamma oscillations in schizophrenia, the coupling of gamma rhythms to delta oscillatory phase appears intact. This implies that despite local disruption of the circuitry required to entrain activity to high frequency stimuli there is a relative preservation of the low-frequency modulation of these sensory cortical circuits across the illness phases of schizophrenia. Relevance of our findings to the cell type and anatomic specificity of disturbances in the pathophysiology of schizophrenia will be discussed.

Keywords: Schizophrenia, Oscillations, MEG

Disclosure: Nothing to Disclose.
M192. Effect of Tolcapone on Cognitive Control in Chronic Psychotic Disorder Patients

Savita Bhakta*, Gregory Light, Jo Talledo, Daniel Franz, Jared Young, Neal Swerdlow

University of California San Diego, San Diego, California, United States

Background: Functional impairment in chronic psychotic disorders such as schizophrenia is linked to deficits in cognitive processes, including those that facilitate goal-directed behavior and response selection, described in the Research Domain Criteria (RDoC) construct of "cognitive control (CC)." Putative pro-cognitive agents are ineffective in reducing these deficits, likely due in part to the clinical heterogeneity of schizophrenia, and the fact that neural mechanisms of cognitive control deficits remain poorly understood and hence are not directly targeted by these agents. We tested the CC enhancing effect of tolcapone, a reversible catechol O-methyl transferase (COMT) enzyme inhibitor, by using the Delis Kaplan Executive Function System (DKEFS) and an electroencephalogram (EEG)-based CC task - a reverse-translated 5-choice continuous performance test (5C-CPT) - to identify the neural targets underlying tolcapone's effect on CC in chronic psychotic disorder patients

Methods: Carefully screened, medically stable, second generation antipsychotic-treated schizophrenia or schizoaffective disorder, depressed type subjects between 18-45 yo, completed two test days separated by one week. On each test day, subjects received either tolcapone $200 \mathrm{mg}$ or placebo (PBO) p.o. in a double blind, randomized, counterbalanced, within-subject crossover design. Effects of tolcapone on DKEFS verbal fluency and color-word interference test, and 5C-CPT performance and ERP measures were analyzed using repeated measures ANOVA. Biomarkers predicting tolcapone's neurocognitive sensitivity were assessed, including demographic variables and baseline cognitive performance.

Results: 8 subjects have completed testing to date. Participants were in their mid-thirties ( $36 \pm 6$ yrs), high school educated $(12 \pm 1.5 \mathrm{yrs})$, men $(62.5 \%)$ with an intelligence quotient (IQ) of $94.5 \pm 9.1$, GAF score of $59.37 \pm 6.23$ and duration of illness $(20.25 \pm 9.82 \mathrm{yrs})$. Overall, tolcapone was well tolerated. Transient elevation in the liver enzyme, aspartate transaminase (AST; effect size $(d)=0.4$ ) was observed on follow-up liver function tests, however, these increased levels remained within normal limits and did not require clinical intervention. All participants showed mild elevations in blood pressure (mean increase systolic $\mathrm{B} p=2$ pts; $\mathrm{d}=0.3$ and mean increase diastolic $\mathrm{B} p=3$ pts; $\mathrm{d}=0.6$ ) with tolcapone in a time-dependent manner. Collectively, these findings indicate that $200 \mathrm{mg}$ of tolcapone was biologically active and well tolerated. Tolcapone reduced number of errors $(\mathrm{d}=0.5)$ compared to placebo during the color-word inhibition task (subjects were required to name the color of the ink that the letters were printed in and not read the word). We previously showed tolcapone enhanced verbal fluency performance in $N=27$ healthy subjects $(p=0.03)$. Compared to healthy subjects, SZ patients showed impaired verbal fluency performance $(\mathrm{d}=0.6)$, however, tolcapone failed to enhance verbal fluency performance in 
patients. Subject testing and analysis of neurophysiological (EEG) and behavioral (5C-CPT) data are ongoing and results will be presented in full.

Conclusions: Our preliminary findings suggest that tolcapone improved inhibition by reducing errors on an interference test in chronic psychotic disorder subjects. We previously reported enhanced response inhibition and associated EEG changes after administration of $200 \mathrm{mg}$ tolcapone in healthy subjects. Testing is ongoing, and a complete analysis of tolcapone effects on 5C-CPT performance and neurophysiological measures will be reported.

Keywords: Cognitive Control, Schizophrenia, Tolcapone Disclosure: Nothing to Disclose.

M193. PGC2 Schizophrenia Risk Genes Representation Within Gene Co-Expression Networks From Postmortem DLPFC of Affected and Unaffected Individuals

Eugenia Radulescu*, Richard E. Straub, Andrew E. Jaffe, Joo Heon Shin, Qiang Chen, Thomas M. Hyde, Joel E. Kleinman, Daniel R. Weinberger

Lieber Institute for Brain Development, Baltimore, Maryland, United States

Background: Schizophrenia polygenic risk is plausibly manifested by complex transcriptional dysregulation in the brain, involving networks of co-expressed and functionally related genes.

Methods: 1. Samples: DLPFC gene expression (RNA-Seq) was quantified (RPKM) in postmortem human brains from the LIBD Postmortem Human Brain Repositoryl (186 controls- CTRL, 126 schizophrenia- SCZ, Caucasians and African Americans, age: 16-80, RIN $\geq 7$ ). 2. Expression measures/ quality control: only genes with median RPKM $\geq$ $0.1 \quad(N=23023$ genes $)$ were used. Expression data was normalized by $\log 2$ transformation and adjusted for RNA quality measures (RIN, RIN2, PMI, total gene assignment, proportion of mitochondrial RNA and quality surrogate variables derived as described in Jaffe et al2). 3. Data analysis: WGCNA3 was applied to the adjusted expression data to construct co-expression networks, separately for CTRL and SCZ. Modules of co-expressed genes were detected employing the dynamic tree cutting method and summarized as "module eigengenes" for further analyses. 4. Modules of LIBD co-expression network (CTRL; SCZ) were tested for enrichment in Gene Ontology (GO) biological processes (BP) with clusterProfiler4. 5. The modules were also tested for overrepresentation in PGC2 genes within the 108 loci associated with the schizophrenia risk in the latest GWAS5 by using the WGCNA function userListEnrichment.

Results: 14 modules of co-expression were identified in the CTRL group and 15 in the SCZ group. PGC2 genes were over-represented in two CTRL modules $(p=0.000187$, $p=0.021192$ ). The CTRLs modules enriched for PGC2 genes were characterized by GO-BP including homophilic cell adhesion via plasma membrane adhesion molecules, cell-cell adhesion via plasma-membrane adhesion molecules, glutamate receptor signaling pathway via either peptidyl-lysine modification, chromatin modification, or histone modification as the second module. One SCZ module was enriched for PGC2 genes $(p=0.000568)$ and was identified by GO-BP as homophilic cell adhesion via plasma membrane adhesion molecules and modulation of synaptic transmission. Importantly, 57 PGC2 genes overlapped with the SCZ module (e.g., AKT3, CACNA1C, members of protocadherins family), whereas 7 and 14 PGC2 genes were overrepresented in the two CTRL modules, respectively. Of note, 41/57 overrepresented PGC2 genes in the SCZ module were not present in the CTRL modules enriched for PGC2. These specific 41 genes were part of GO biological processes such as chemical synaptic transmission (postsynaptic), regulation of postsynaptic membrane potential, and homophilic cell adhesion via plasma membrane adhesion molecules (among these 41 genes: CACNA1C, NLGN4X).

Conclusions: Our results illustrate the complexity of SCZ risk distribution within the DLPFC co-expression networks in postmortem brains of affected and unaffected individuals. Importantly, different PGC2 genes are members of co-expression modules in CTRL and SCZ, and they largely aggregate in separate modules. These observations may be explained by differential transcriptional regulation due to pleiotropy, epistasis, epigenetic dysregulation, or possibly hidden RNA quality artifacts. Future studies are necessary to elucidate the participation of SCZ risk genes in brain co-expression networks.

Bibliography:

1. BrainSeq: A Human Brain Genomics Consortium: BrainSeq: Neurogenomics to Drive Novel Target Discovery for Neuropsychiatric Disorders. Neuron. 2015 Dec 16;88 (6):1078-83.

2. Jaffe et al (2017): qSVA framework for RNA quality correction in differential expression analysis. Proc Natl Acad Sci U S A, 114(27):7130-7135.

3. Horvath S (2011): Weighted Network Analysis. Applications in Genomics and Systems Biology. Springer Book. ISBN: 978-1-4419-8818-8.

4. Guangchuang $\mathrm{Yu}$ et al (2012): clusterProfiler: an $\mathrm{R}$ Package for Comparing Biological Themes Among Gene Clusters. OMICS

5. Schizophrenia Working Group of the Psychiatric Genomics Consortium. Biological insights from 108 schizophreniaassociated genetic loci. Nature. 2014 Jul 24;511(7510):421-7. doi: 10.1038/nature13595.

Keywords: RNA-Seq, Gene Co-Expression, Schizophrenia, Genetic Risk

Disclosure: Nothing to Disclose.

M194. The Neurodevelopmental Disorder-Linked Gene, MEF2C, Regulates PCDH17 to Control Cortical Synapse Density

Adam Harrington*, Aram Raissi, Carly Hale, Ahlem Assali, Jennifer Cho, Kayla Blankenship, Alexandra Bowen, Nahom Gebreselassie, Jennifer Darnell, Kimberly Huber, Christopher Cowan

Medical University of South Carolina, Charleston, South Carolina, United States

Background: Numerous genetic variants associated with MEF2C are linked to risk for neurodevelopmental disorders (NDDs), including autism, intellectual disability (ID) and schizophrenia (SCZ), and MEF2C is highly expressed in developing and mature cortical neurons. We recently 
reported (Harrington et al, 2016, eLife) that MEF2C functions predominantly as a transcriptional repressor to regulate dendritic excitatory and inhibitory (E/I) synapse density on developing cortical pyramidal neurons, but not interneurons. We also showed that genetically depleting MEF2C in developing cortical pyramidal neurons leads to behaviors relevant to neurodevelopmental disorders, including repetitive behaviors and deficits in sociability, communication, cognition, and reward. Using an unbiased RNA sequencing approach, we identified $\sim 1000$ dysregulated genes in cortical tissue from adult MEF2C mutant mice, with many of the products of these gene target reported to function at synapses. In addition, we observed a significant enrichment of RNA transcripts reported to associate with the RNA binding protein, Fragile X Mental Retardation Protein (FMRP). In our current study, we sought to identify critical target genes that mediate neuronal activity- and MEF2dependent regulation of cortical E/I synapse development.

Methods: We used a variety of molecular and biochemical techniques (e.g. HITS-CLIP, synaptosomal fractionation, and immunoprecipitation) to identify and characterize the role of novel MEF2C/FMRP target genes. In addition, we employed cultured primary cortical neurons to study the subcellular localization and cell-autonomous functional roles of target genes, and we used multiple in vitro and in vivo approaches to examine E/I synapse regulation, dendritic complexity and NDD-related behaviors by common MEF2C/FMRP targets.

Results: Using the HITS-CLIP technique, we identified the d2 protocadherin, Pcdh17, as a MEF2C target gene whose RNA transcripts associate with FMRP. We find that PCDH17 levels are upregulated by MEF2C-VP16 (active mutant) and upregulated in the Mef $2 \mathrm{c}$ conditional knockout cortex. We find that PCDH17 is: (1) upregulated by neuronal activity, (2) expressed in the cortex throughout early and late brain development and into adulthood, (3) partially enriched in synaptosomal fractions in cortical tissues, and (4) detected in punctate immunostaining patterns in cortical neuron dendrites and near synapses. We find that overexpression of PCDH17 is sufficient to reduce cortical dendritic spine density, but not to increase GABAergic synapse density.

Conclusions: Our findings suggest that MEF2C plays a critical role as an activity-dependent regulator of E/I synapse balance, and it functions in part by regulating the expression of the activity-dependent gene target, Pcdh17. PCDH17 upregulation, which occurs in the Mef $2 \mathrm{c}$ cortical conditional knockout mice, appears sufficient to reduce dendritic spine density, but not to increase GABAergic synapse density onto dendrites of cortical pyramidal neurons. PCDH17 appears to be developmentally regulated during key periods of synapse remodeling in cortex, and it is localized, at least in part, at or near synapses and dendritic spines - positioning this neuronal cell adhesion molecule as a critical activitydependent regulator of glutamatergic synapses. Interestingly, Pcdh17 mRNA levels are reported to be dysregulated in human postmortem prefrontal cortical tissues from individuals with schizophrenia or major mood disorders. Our findings suggest that MEF2C-FMRP-PCDH17 likely function in the same pathway to regulate the development and remodeling of cortical synaptic connectivity, and position PCDH17 as a potential novel therapeutic target for treatment of multiple NDDs and neuropsychiatric disorders.
Keywords: Neurodevelopmental Disorders, Glutamatergic Synapses, Cortical Neurons, MEF2C, FMRP

Disclosure: Nothing to Disclose.

\section{M195. Relationship Between Indices of Cognitive Dysfunction and Dorsolateral Prefrontal Cortex Pyramidal Cell Activity in Schizophrenia}

Jill Glausier*, Mary Kelly, Samantha Salem, Dominique Arion, John Enwright, Kehui Chen, David Lewis

University of Pittsburgh, Pittsburgh, Pennsylvania, United States

Background: Cognitive impairment, a core clinical feature of schizophrenia, predicts important functional outcomes in affected individuals. Unfortunately, therapeutic options for cognitive deficits in schizophrenia are limited, with no available pharmacological treatments. The development of mechanistically-novel therapeutics requires an understanding of the neural substrates underlying cognitive dysfunction in schizophrenia. Achieving this goal requires investigation of the diseased brain using complementary approaches, including studies of postmortem human brain tissue to provide the required molecular, cellular, and circuitry levels of resolution. However, interpreting the contribution of biological findings from postmortem brain studies to cognitive impairments has, to date, relied on inferences drawn from studies of living subjects which show that subjects with schizophrenia have impaired cognition relative to healthy subjects, first-degree relatives, and subjects with mood disorders.

This limitation can be addressed by using multiple sources of information to quantify proxy measures of cognitive aptitude, such as educational and occupational attainment, that can be reliably obtained for deceased individuals and for their parents. Educational and occupational attainment are consistently correlated with global IQ, and have been used to accurately estimate IQ in living subjects. Within subjects with schizophrenia, cognitive ability is one of the best predictors of educational and occupational attainment, more so than psychotic symptoms. Moreover, much like cognitive ability, individuals with schizophrenia have lower educational and occupational attainment relative to healthy subjects, firstdegree relatives, and subjects with mood disorders.

Dysfunction of the circuitry in the dorsolateral prefrontal cortex (DLPFC) is thought to underlie cognitive impairments in schizophrenia. Within the DLPFC, firing of layer 3 pyramidal cells is essential for proper cortical activation during cognitive tasks. Neuronal firing is robustly indexed by expression levels of the rate-limiting electron transport chain enzyme, cytochrome c oxidase (COX).

To improve the ability of postmortem human brain studies to inform our understanding of the neural substrates of cognitive dysfunction in schizophrenia, we sought to answer the following three questions. First, are the expected differences in educational and occupational indices of cognitive ability present in postmortem subjects with schizophrenia relative to unaffected control and mood disorder subjects? Second, do postmortem schizophrenia subjects show the expected deviation from parental educational and occupational attainment? Third, does COX expression in DLPFC layer 3 pyramidal cells predict the deviation from parental indices of cognitive ability? 
Methods: Brain specimens $(N=522)$ were obtained during autopsies conducted at the Allegheny County Medical Examiner's Office (Pittsburgh, PA) after consent was obtained from the next-of-kin. Consensus DSM-IV diagnoses were made for each subject, and the Hollingshead categorical index was used to assess educational and occupational attainment of the decedent and their parents. Expression of COX subunits was quantified in a subset of 24 age- and sex-matched pairs of unaffected control and schizophrenia spectrum subjects. Frozen tissue sections containing DLPFC area 9 were stained for Nissl substance to identify pyramidal cells, and layer 3 pyramidal somata were dissected using a Leica laser microdissection system. Transcriptome profiling was performed by microarray using Affymetrix GeneChips specific to the human genome. Z-scores for each COX subunit were calculated per subject, and averaged for a composite COX Z-score per subject.

Results: The expected differences in educational and occupational indices of cognitive ability were present in postmortem subjects with schizophrenia spectrum disorders $(n=81)$ relative to postmortem unaffected control $(n=165)$ and mood disorder $(n=276)$ subjects. Consistent with findings in the clinical literature, postmortem unaffected control subjects had the highest attainment, schizophrenia spectrum subjects had the lowest attainment, and mood disorder subjects were intermediate. Postmortem schizophrenia spectrum disorder subjects also showed the expected deviation from parental educational and occupational attainment. Indeed, postmortem unaffected control and mood disorder subjects showed the expected generational improvement relative to their parents in educational and occupational attainment, but schizophrenia spectrum subjects showed no improvement or attained significantly less than their parents. Finally, composite COX Z Scores in DLPFC layer 3 pyramidal cells predicted the deviation in proband educational and occupational attainment from parental attainment.

Conclusions: The expected differences in educational and occupational attainment in schizophrenia spectrum subjects relative to multiple subject populations is measurable and present in postmortem human brain donors. The deviation in these attainment scores were predicted by a priori defined brain measures of neural components that mediate cognitive ability. These findings demonstrate, in the same subjects, that alterations in the activity of PFC layer 3 pyramidal cells could contribute to the neural substrate of cognitive dysfunction in schizophrenia.

Keywords: Postmortem Brain Tissue, Cognitive Impairments, Schizophrenia

Disclosure: Nothing to Disclose.

M196. Synaptic Protein Alterations in Schizophrenia are Robust, Highly Organized, and Suggest a Link Between Altered Glutamate and GABA Receptor Trafficking in Dynamic Spine Loss

Matthew MacDonald*, Megan Garver, Jason Newman, Ying Ding, Kenneth Fish, David Lewis, Nathan Yates, Robert Sweet

University of Pittsburgh Medical Center, Pittsburgh, Pennsylvania, United States
Background: Reduced dendritic spine density has been reproducibly observed in multiple brain areas in schizophrenia (Sz), such as the auditory cortex, and is believed to underlie deficits in cortical processing. We recently reported, in a cohort of $20 \mathrm{Sz}$ and 20 matched controls, that only small, presumably dynamic, spines are lost in the auditory cortex of Sz $(p=1 \mathrm{E}-3)$ while large, likely stable, spines are preserved. Here, in an expanded cohort of 50 pairs we utilized orthogonal microscopy and mass spectrometry (MS) approaches identity changes in synaptic protein levels and phosphorylation (Phos) that may underlie small spine loss Sz.

Methods: Left and right hemisphere auditory cortex grey matter from 50 pairs of $\mathrm{Sz}$ and matched control subjects was obtained from the Pitt Brain Tissue Donation Program. The left hemisphere was utilized in microscopy studies to assess deep layer 3 spine density and volume. The right hemisphere was utilized for 1 . Targeted MS to quantify the expression of 400 proteins in grey matter homogenates; 2. Biochemical fractionation - targeted MS to quantify 350 proteins in synaptosomes; 3. Differential MS to quantify over 2000 Phos events on 900 proteins in grey matter homogenates from a subset (16 pairs) of this cohort. In all experiments, the effects of postmortem interval and antipsychotics were assessed in animal models. MS peak integration was performed in Skyline. Statistical and network analyses are ongoing using ANCOVA and WGCNA algorithms. False discovery rates were calculated by Benjamini-Hochberg.

Results: Disease effects on synaptosome protein composition were robust, highly organized, and included multiple synaptic pathways. Levels of 165 proteins were altered in $\mathrm{Sz}$ synaptosomes $(\mathrm{q}<0.05)$, whereas differences in homogenate protein levels were modest $(61$ proteins, $\mathrm{q}<0.05)$. Protein Phos was altered at 166 sites on 102 proteins in $\mathrm{Sz}$ $(\mathrm{q}<0.1)$. Homogenate protein differences focused on mitochondrial function $(q=8 \mathrm{E}-3)$, while synaptosome alterations included proteins from multiple pathways implicated in Sz: glutamate and GABA receptor subunits, calcium channel subunits, mitochondrial proteins, and proteasome/ubiquitin components. Synaptosome levels of proteins from the same family or with similar function were highly co-regulated in both control and Sz. For example, unsupervised hierarchal clustering of synaptosome data identified one tightly co-regulated group of proteins enriched for the term Mitochondria $(q=5 \mathrm{E}-13)$ and another distinct group enriched for Postsynaptic Density $(q=3 E-2)$. Proteins with altered Phos in $\mathrm{Sz}$ were enriched for the terms Postsynaptic Density ( $p=1 \mathrm{E}-7)$ and Actin Binding $(p=1 \mathrm{E}-$ -3). More sophisticated WGCNA based approaches to fully characterize synaptosome and Phos protein co-regulation networks, and their relationship to small spine loss, are underway.

Of the 165 proteins with altered synaptosome levels, only 37 also differed in homogenates and 13 had altered phos. This suggests that protein trafficking to/from the synapse could be altered in $\mathrm{Sz}$ and that Phos may play a role. One representative finding that demonstrates the power of combining these analyses is discussed below.

We observed decreased synaptosome levels of the AMPA receptor subunit GRIA2 $(\downarrow 16 \%, p=1.6 \mathrm{E}-3)$ and the GABAA receptor subunit GBRB1 $(\downarrow 16 \%, p=7 \mathrm{E}-4)$, while their homogenate expression levels were unaltered. These decreases are unlikely to reflect spine/synapse loss as changes to 
PSD95 $(p=0.04)$ SYN1 $(p=0.18)$ did not reach significant under Benjamini-Hochberg. Additionally, GRIA2 and GBRB1 were found in a different cluster than PSD95 and SYN1. GRIA2 and GBRB1 synaptosome levels were exceptionally well correlated with each other in both $\mathrm{Sz}$ and control $(r=0.86, p<1 \mathrm{E}-4)$. GRIA2 and GBRB1 synaptosome levels were also correlated with small spine density in $\mathrm{Sz}$ but not control (GRIA2: $r=0.6, p=5 \mathrm{E}-3$, GBRB1: $r=0.5$, $p=0.04)$. Endocytosis of both GRIA2 and GBRB1 is mediated by the AP2 family of adaptor proteins, two of which had increased synaptosome levels in Sz: AP2B1 ( $\uparrow 12 \%$ 7.2E-3) and AP2M1 ( $\uparrow 14 \% 7.8 \mathrm{E}-4)$. Protein trafficking by AP2 is regulated by Phos and AP2M1 Phos was also altered in $\mathrm{Sz}$ (T156, $\downarrow 2.4$-fold, $p=4.0 \mathrm{E}-3$ ). This analysis utilized spine density and volume data from the original 20 pairs. Analysis of the expanded cohort is near completion. Antipsychotics and postmortem interval had no effect on any of these findings in animal models.

Conclusions: Our findings strongly suggest that cortical synaptic protein trafficking and Phos is altered in Sz. GRIA2 and GBRB1 were highly correlated with each other, decreased at the synapse, and correlated with small spine loss in Sz. AP2 alterations suggest that increased endocytosis could drive decreases in synaptic GRIA2 and GBRB1. A population of dual glutamate and GABA innervated dendritic spines has recently been characterized. These spines are highly stable and found in close proximity to small dynamic spines whose stabilization they are believed to mediate via local calcium gradients. As GRIA2 containing AMPA receptors are $\mathrm{Ca} 2+$ impermeable and GABA receptor activation at spines suppresses local $\mathrm{Ca} 2+$ one interpretation of our findings is that decreased GRIA2 and GBRB1 at these dual innervated spines impairs the stabilization of nearby small dynamic spines. This hypothesis, and others generated by this data set, will be confirmed in future microscopy studies in patient tissue and functional studies in animal and culture models.

Keywords: Postmortem Brain Tissue, Schizophrenia, Proteomics, Phosphorylation, Protein Trafficking

Disclosure: Nothing to Disclose.

M197. Impaired Eye Tracking and TPJ-pSTS Connectivity Predicts Impaired Comprehension of Social Situations During Naturalistic Scene Processing in Schizophrenia

Gaurav Patel*, Sophie Arkin, Laura Bartel, Juan Sanchez-Pena, Antigona Martinez, Daniel Javitt

Columbia University, New York, New York, United States

Background: Deficits in social functioning are a major cause of psychosocial disability in schizophrenia patients (SzP). The brain networks involved in the visual scanning and comprehension of social situations-visual, face-emotion recognition (FER), attention, and theory of mind networksall have nodes in the right temporoparietal junction/posterior superior temporal sulcus (TPJ-pSTS), suggesting that this region serves as a hub for quick integration and propagation of information necessary for these abilities. Deficits in one or more of these networks, then, may also propagate through this hub, especially in complex and dynamic situations that require quick integration between these systems.
We examined the relationship between a naturalistic test of social cognition with connectivity and activation by naturalistic stimuli of these areas. Subjects performed The Awareness of Social Inference Test (TASIT), which uses video-based stimuli to test understanding of the mental state of actors in social situations involving lies or sarcasm, while eye-movements were recorded. We correlated eye-movement patterns with performance on answering questions about the social scene. We also correlated these measures to the connectivity of the involved areas, defined by separate localizer tasks. Lastly, we examined the synchronization of activity in these areas during the viewing of dynamic naturalistic stimuli (cinematic movies).

We predicted that SzP would be specifically impaired in comprehension of social situations involving sarcasm, which depends on use of FER more than lie situations. We expected that this performance would correlate with eye-tracking measures, and that the degree of impairment would correlate with social functioning. We also expected that these deficits would correlate with connectivity of right hemisphere TPJpSTS areas, and that the synchronization of activity in these areas during naturalistic movies would be accordingly diminished.

Methods: 39 SzP and 28 HC performed TASIT with eyetracking. The percentage of time spent further than 2 std. dev. from the mean of the HC eye-position for each subject across all video frames (HCs' eye-position compared to all other $\mathrm{HC}$ ) was correlated to performance on answering questions about each TASIT social situation. We then separated patients into high and low performing groups with $\mathrm{k}$-means clustering, and examined the neuropsychological and social functioning differences of these two groups. The subjects also underwent task, resting-state and moviewatching functional magnetic resonance imaging (fMRI) using Human Connectome Project compatible multiband sequences and processing pipelines. We used task fMRI data to localize the visual, FER, attention, and theory of mind areas, and then measured inter-areal connectivity resting state functional connectivity. Inter-areal connectivity was also used to calculate inter-network connectivity, pathlength, and correlations with TASIT performance. Similar procedures were used to evaluate the inter-areal synchronization of BOLD activity during movie-watching.

Results: SzP demonstrated significantly worse comprehension of TASIT social situations that involved sarcasm versus lies (repeated measures ANOVA, $\mathrm{F}(1,64)=9.425, p<.01$ ), and this performance correlated across groups with eyetracking performance. K-means clustering separated SzP into two groups; the lower performing group exhibited both slower processing speed and lower interpersonal functioning measured by both SLOF and SFS. In accordance, functional connectivity of the TPJ-pSTS areas involved in TASIT was also significantly decreased in strength, leading to increased path length between visual and theory of mind areas in SzP vs. HC. Contrary to expectations, however, within-SzP performance on TASIT did not correlate with the degree of TPJ-pSTS connectivity; rather better performance correlated most closely with increased connectivity between prefrontal attention/task control and theory of mind areas. Comparing connectivity of the better and worse performing clusters of SzP confirmed this, showing that the main difference between the two clusters was that lower performing SzP 
lacked connectivity between lateral prefrontal cortex attention/working memory areas and lateral theory of mind areas. Movie-watching fMRI data revealed that theory of mind TPJ-pSTS areas remained significantly less synchronized with other TPJ-pSTS areas compared to HC, suggesting a lack of synchronization of TPJ-pSTS areas in the processing of complex dynamic naturalistic social stimuli. Following the functional connectivity results, $\mathrm{SzP}$ also demonstrated increased synchronization between attention/task-control areas and theory of mind areas.

Conclusions: In the present study, we have connected impaired visual scanning of naturalistic scenes with both resting state functional connectivity and inter-areal synchronization deficits in TPJ-pSTS evoked by viewing of naturalistic social situations. Moreover, we have found evidence of compensatory mechanisms that involve use of theory of mind and attention/task-control networks to overcome these deficits. Our findings not only demonstrate the utility of naturalistic stimuli in the study of social functioning in psychiatric disorders, but also help localize both impairments and compensatory mechanisms that may be targeted with cognitive rehabilitation and/or neuromodulatory techniques.

Keywords: Social Cognition, Attention, Functional MRI (fMRI), Resting State Functional Connectivity, NetworkAnalysis

Disclosure: Part 1: Pfizer, Inc., Employee, Spouse, Part 2: Pfizer, Inc., Stock / Equity, Spouse, Part 3: Pfizer, Inc., Employee, Spouse.

M198. Diffusion Measures of Extracellular Free Water in a Non-Human Primate Model of Maternal Immune Activation - Exploring Neuroimmune Mechanisms of Psychiatric Disorders

Tyler Lesh*, Costin Tanase, Jeffrey Bennett, Ana-Maria Iosif, Judy Van de Water, Richard Maddock, David Amaral, Melissa Bauman, Cameron Carter

University of California, Davis, Sacramento, California, United States

Background: Evidence has been accumulating for an immune-based component of psychiatric disorder etiology, particularly schizophrenia. One of the first indications of such a link comes from early epidemiological studies, which found an increased incidence of schizophrenia in offspring of mothers who had an infection (e.g., influenza) during pregnancy. Recent work has identified genetic links to the major histocompatibility complex, pro-inflammatory cytokine elevations in cerebrospinal fluid and plasma, as well as mixed evidence of microglial activation. While these findings provide strong evidence of an immune component, human studies are inherently limited by the heterogeneity of the sample and inability to make strong inferences about causality. Consequently, we have developed a non-human primate (NHP) model of maternal immune activation (MIA) using a modified form of the viral mimic polyIC (polyICLC) to test the hypothesis that maternal immune response contributes to changes in the developing brain and behavior of NHP offspring. In a previous cohort of MIA-exposed offspring, our group observed increased pre-synaptic dopamine levels in the striatum using 6-[18 F]fluoro-L-mtyrosine (FMT) positron emission tomography, in addition to pubertal-onset behavioral abnormalities, which may model part of the neurodevelopmental pathway towards psychosis. The current study builds on this model and examines the effect of maternal immune activation on a promising biomarker of neuroinflammation in vivo-extracellular free water-a diffusion magnetic resonance imaging measure obtained with a multi-shell acquisition (i.e., Pasternak, Shenton, and Westin, 2012). We sought to test the hypothesis that offspring of pregnant monkeys who received polyICLC injections at the end of the first trimester would show increased extracellular free water compared to control offspring.

Methods: Fourteen pregnant rhesus monkeys (Macaca mulatta) received polyICLC and 10 pregnant monkeys received saline injections at the end of the first trimester. An additional four offspring were added to reach target enrollment of 14 control animals. The offspring from both groups (all of whom were male) underwent a diffusion MRI scan on a 3 Tesla Siemens Skyra scanner in which multiple b-value shells were acquired to improve estimation of extracellular free water. Diffusion data was collected when the offspring were one month, 6 months, and 12 months of age, although only 6-month preliminary findings are currently presented. Diffusion images were aligned to individual subject MPRAGE scans, and existing segmented MPRAGE masks were used to define whole-brain gray- and white-matter free water estimates. Individual subject structural scans were then nonlinearly aligned to generate a common group average template and the group average template was subsequently nonlinearly aligned to a neurodevelopmental rhesus atlas. Finally, regional parcellations from the rhesus atlas were brought back into individual subject space via inverse transformations in order to generate subject-specific regions of interest. For this preliminary analysis, the frontal cortex was selected as an a priori region of interest in addition to the more global whole-brain gray and white matter masks. Group differences were assessed using repeated measures ANOVA and independent samples t-tests implemented in SAS.

Results: Six-month-old MIA-exposed rhesus offspring showed a trend for increased whole-brain white matter extracellular free water $(p=.09)$ with no significant difference in whole-brain gray matter free water $(p=.27)$ compared to control offspring. However, analysis of the frontal region of interest revealed significantly increased gray matter free water in the left hemisphere $(p=.013)$ with a trend towards increased gray matter free water in the right hemisphere $(p=.081)$. There were no significant differences between MIA-exposed and control offspring in basic motor and reflex development or growth trajectories.

Conclusions: These data suggest that despite the lack of behavioral abnormalities at this early age, extracellular free water values are increased in MIA-exposed offspring, particularly in frontal gray matter. More global whole-brain free water group differences, however, did not reach statistical significance, which may indicate some regional specificity to these changes early in development. The addition of the 12-month imaging timepoint, due to be completed in two months, will provide important context and give a better indication of the trajectory of free water 
changes both at global and local levels. Nonetheless, the NHP MIA model complements the human schizophrenia literature in which extracellular free water increases have been repeatedly identified. Further support for the NHP MIA model is provided by findings from our previous cohort of MIA-treated NHP offspring, which showed significantly higher striatal [18 F]FMT binding potential, along with abnormal behaviors. Ultimately, these data provide validation of the clinical relevance of the NHP MIA model and improve our understanding of neuroimmune mechanisms in the development of psychiatric disorders, particularly schizophrenia.

Keywords: Nonhuman Primate Model, Diffusion Weighted Imaging, Neuroinflammation, Schizophrenia, Maternal Immune Activation

Disclosure: Nothing to Disclose.

M199. How Population-Based Pharmacokinetic Modeling (PopPK) is Used in the Development and Approval of Long-Acting Injectable Medications Using the 2Month Dosing Regimen of Aripiprazole Lauroxil as an Example

Sheldon Preskorn*, Peter Weiden, Marjie Hard, Angela Wehr, Lisa von Moltke

University of Kansas School of Medicine, Wichita, Kansas, United States

Background: US FDA guidance has encouraged the use of population pharmacokinetic (PK) modeling and simulation methods that are now common quantitative analyses to routinely inform the clinical development of new drugs. Population PK (PopPK) modeling combines PK data from multiple clinical PK studies to yield more accurate estimates of drug concentration than would be possible from a single PK study. Notably, PopPK models have been developed for all recently approved long-acting injectable (LAI) antipsychotics. Given the prolonged exposure resulting from LAI antipsychotics, model-based approaches are particularly useful to evaluate clinical scenarios that extend beyond the experimental state.

Here, we present the PopPK model used in the development work that led to the recent approval of the LAI antipsychotic aripiprazole lauroxil (AL) $1064 \mathrm{mg}$ injection at a 2-month dosing interval for the treatment of schizophrenia. AL is a prodrug of aripiprazole that results in extended systemic release of aripiprazole after intramuscular (IM) administration. Prior to this approval, the highest approved dose of $\mathrm{AL}$ was $882 \mathrm{mg}$ and the longest dose interval was every 6 weeks (q6wk). The PK profile of AL is characterized by a steady rise in plasma concentrations, a broad peak and prolonged exposure, with a half-life of 53.9-57.2 days. Based on these facts, it was hypothesized that extending the highest AL dose from $882 \mathrm{mg}$ to $1064 \mathrm{mg}$ would result in steady-state aripiprazole concentrations appropriate for its use at 2month dose intervals.

Methods: To test this hypothesis, a phase 1 study was conducted to determine whether higher doses of $\mathrm{AL}$ at a longer interval could produce similar exposure to other lower $\mathrm{AL}$ doses given at more frequent intervals. Next, a modelbased approach was used to combine PK data from this phase 1 study of AL $1064 \mathrm{mg}$ (NCT02320032) with four prior studies $(N=576)$ to develop a 2-month PopPK (2MPopPK) model to inform the regulatory review of AL $1064 \mathrm{mg}$ every 8 weeks (q8wk), and to illustrate the process of using PopPK modeling to inform LAI development programs.

A PopPK analysis is conducted according to a model analysis plan, which predefines the purpose of the model, the data to be used in the construction of the model, the handling of the data, the modeling approach, the means by which the model will be evaluated, and the intended simulations. Model evaluation, a critical step in the process, may include assessments that evaluate how well the model describes the observed data. Predictive simulations are conducted once the model has been established and evaluated to answer questions such as what to do when a dose is missed.

Results: Based on the 2MPopPK model, the dosing regimen of AL $1064 \mathrm{mg}$ q8wk resulted in steady-state aripiprazole concentrations that were within the concentration range associated with other efficacious and well-tolerated doses of AL. The model showed that median steady-state concentrations of aripiprazole for the $1064 \mathrm{mg}$ q8wk regimen were comparable with the $882 \mathrm{mg}$ q6wk and $662 \mathrm{mg}$ every 4 week (q4wk) regimens. Model-based simulations provided guidance regarding administration of AL $1064 \mathrm{mg}$ in different clinical contexts, which confirmed that initiation of $\mathrm{AL}$ $1064 \mathrm{mg}$ q8wk in conjunction with 21 consecutive days of oral aripiprazole is sufficient to achieve therapeutic aripiprazole concentrations. Next, the model was extended to different clinical scenarios of what to do when a dose is missed. The model demonstrated that no oral supplementation is required if the time since the last injection is $\leq 10$ weeks. In the event an $\mathrm{AL}$ dose needs to be administered early, as is sometimes needed for practical reasons (i.e. travel, life event), the increased aripiprazole exposure from administration of $1064 \mathrm{mg}$ up to 6 weeks early did not exceed Cmax of $882 \mathrm{mg}$ q4wk.

Conclusions: PopPK modeling is a common quantitative analysis used in the development of LAI antipsychotics. It is an important step in the clinical development of LAI formulations due to the number of clinical questions that may practically limit the ability to study all clinically relevant scenarios associated with missed dosing. The phase 1 study and the subsequent PopPK modeling showed that a 2-month dosing interval for AL $1064 \mathrm{mg}$ resulted in aripiprazole concentrations within the AL therapeutic window established in the phase 3 study and supported the approval of this dose regimen.

Keywords: Population Pharmacokinetics, Long-Acting Injectable Antipsychotics, Aripiprazole

Disclosure: Part 1: Alkermes, Consultant.

M200. Antipsychotic Use in Drug-Naïve Emerging Adults: Metabolic Indices and Abdominal MR Imaging

Araba Chintoh*, Sri Mahavir Agarwal, Nicole MacKenzie, Gary Remington, Margaret Hahn

Centre for Addiction and Mental Health, Toronto, Canada

Background: Atypical antipsychotics (AAPs) are known to cause metabolic dysregulation, and a growing literature 
suggests that children and adolescents are especially vulnerable to these effects. The anthropometric measures routinely used to monitor these metabolic side effects may, in fact, underestimate risk in the pediatric population as they are not a good index of pericardial, hepatic and visceral adipose tissue, key risk factors for the development of cardiovascular disease (CVD). With increased antipsychotic prescriptions for authorized indications, as well as growing off-label use in the pediatric population, it is imperative we understand the extent of these side effects given their association with CVD and type 2 diabetes mellitus (T2DM) - two of North America's major public health concerns.

We focus on a population of adolescents who are particularly susceptible to the metabolic effects of antipsychotics adolescents being treated for their first-episode of psychosis. This is a critical area of study given emerging data suggesting that accrual of cardiovascular risk occurs early in illness, contributing to the 20-year reduction in life expectancy seen in the schizophrenia population. The contributions of medications to this phenomenon in the earliest stages of illness may have important implications for prescribing practices (i.e. off-label use), risk/benefit assessment, and for prompting early behavioral and pharmacological interventions to mitigate these substantial treatment effects.

Ours is a naturalistic study whose strengths include 1) a within subject design, comparing pre- and post-treatment MRI scans; 2) transitional age youth who are at high risk for developing metabolic side effects with AAPs; 3) gold standard MRI measurements of pericardial and hepatic adipose tissue, which, to our knowledge, have not previously been investigated in adolescents treated with AAPs.

The purpose of this project is to obtain thoracic and abdominal magnetic resonance images (MRI) to measure the metabolic side-effects and quantify the accumulation of pericardial, hepatic and visceral fat induced by first-time use of antipsychotics in adolescents.

Methods: Patients are recruited from the emergency department and youth-focused programs at CAMH. Patients complete the following baseline assessments that are repeated again after 3 months.

- Patient demographics (age, gender, ethnicity, indication for antipsychotic treatment)

- Medical history and physical examination, including anthropometric measurements (weight, height, and waist circumference)

- Thoracic and abdominal MRI

- Baseline bloods (fasting): complete blood count (CBC), liver enzymes, glucose, insulin, lipid profile, $\mathrm{HbA1C}$, serum bicarbonate/electrolytes, thyroid status, leptin; Glucagon-like polypeptide-1 (GLP-1), glucagon inhibitory peptide (GIP) and ghrelin will be measured during the OGTT

- Oral glucose tolerance test (OGTT)

- 3- day dietary history, the International Physical Activity Questionnaire (IPAQ), Food Cravings Questionnaire(FCQ)

- Urine sample for drug screen

MRI: Sixty to 80 axial $5 \mathrm{~mm}$ contiguous slices will be acquired, covering from the top of liver to L5 vertebrae (estimated resolution $1.5-2.5 \mathrm{~mm}$; TR $9-11 \mathrm{~ms}$; TE1 $=0.57 \mathrm{~ms}$, TE2 $=1.7 \mathrm{~ms}$ and TE3 $=2.9 \mathrm{~ms}$ allowing, at 3 Telsa, approximation of the following phase relations between water and fat: (90deg, 270deg and 450deg). Volumetric data are acquired in 5-6 breath holds (10-15 seconds each). Measured adipose tissue volumes in $\mathrm{ml}$ (subcutaneous and visceral components), pericardial and liver fat fraction averages are compared across time.

Results: Compared to baseline measurements, at 3 months follow-up, data indicate significant increases in weight, waist circumference and body mass index as well as low-density lipoprotein levels. Our data does not support a significant change in glucose indices after 12 weeks. MRI quantification of fat changes in the right posterior lobe of the liver positively correlate with the change in weight in patients over time.

Conclusions: Our preliminary results suggest AAP treatment in youth - especially with those agents considered lowliability for metabolic side effects - has pronounced early, negative metabolic side-effects. Our findings have important implications for prescribing practices (i.e. off-label use), analyzing the risks and benefits of medicating youth, as well as for prompting early behavioral and pharmacological interventions to mitigate these treatment effects.

Keywords: Antipsychotic Treatment, Metabolic Side Effects, MRI, Youth

Disclosure: Nothing to Disclose.

\section{M201. Stress-Induced Prefrontocortical Dopamine Re- sponse is Altered in Subjects at Clinical High Risk for Psychosis Using Cannabis}

Christin Schifani*, Huai-Hsuan Tseng, Miran Kenk, Abanti Tagore, Alan A. Wilson, Sylvain Houle, Pablo M. Rusjan, Romina Mizrahi

Centre for Addiction and Mental Health, Toronto, Canada

Background: Dopamine (DA) hyperactivity in the striatum is a neurobiological hallmark of schizophrenia and is associated with psychotic symptoms. Stress and cannabis are both risk factors for the development and relapse to psychosis. Recently, we reported that subjects at elevated risk for psychosis exhibit a higher striatal DA response to acute stress compared to healthy volunteers (HV) and that this DA response is absent in those with chronic cannabis use. However, it is not known if this abnormal DA response to stress extends to the prefrontal cortex, a brain region involved in regulating cognition and stress response. Therefore, we investigated the effect of acute psychosocial stress on (dorsolateral and medial) prefrontal cortex DA in subjects with clinical high risk for psychosis (CHR) with and without concurrent cannabis use using [11C]FLB 457 positron emission tomography (PET).

Methods: We recruited CHR without cannabis use $(n=14)$, CHR chronic cannabis users (CHR-CU, $n=8$ ) and HV $(n=12)$ matching in demographics. All subjects underwent two PET scans while performing: 1) a Sensory Motor Control Task (SMCT) (non-stress) and 2) the Montreal Imaging Stress Task (MIST), a validated challenge to induce psychological stress. DA release in response to stress was calculated as \% difference in [11C]FLB 457 binding potential (BPnd) between the SMCT and MIST scans. Subjective perception of stress was assessed before and after each PET session by state anxiety questionnaires. Group effect in \% difference in DA release per region of interest was evaluated by one-way analyses of variance (ANOVAs). Fischer's LSD 
test was used post hoc for comparisons between study groups. Changes in the subjective perception of stress per study group were evaluated by two-way repeated measures ANOVA.

Results: The MIST task lead to significantly elevated ratings of subjective stress in all study groups (main effect of task: $\mathrm{F}$ $(1,31)=196.69, p<0.0001$; main effect of study group: $F$ $(2,31)=10.43, p<0.0001$, task $\times$ study group: $\mathrm{F}(2,31)=0.88$, $p=0.43)$. Analysis of stress-induced DA release revealed a trend of study group in the medial prefrontal cortex (F $(2,31)=2.96, p=0.067)$. While $\mathrm{CHR}$ and $\mathrm{HV}$ did not differ $(p=0.74), \mathrm{CHR}-\mathrm{CU}$ showed lower DA release in response to stress (CHR-CU vs CHR: $p=0.026$; $\mathrm{CHR}-\mathrm{CU}$ vs $\mathrm{HV}$ : $p=0.057)$. No study group differences were observed in the dorsolateral prefrontal cortex $(\mathrm{F}(2,31)=0.92, p=0.41)$.

Conclusions: This study provides first evidence of an altered response to stress in prefrontal cortex DA signaling in $\mathrm{CHR}$ cannabis users. Given the global trend to legalize cannabis, this study is important as it highlights the effects of chronic cannabis use on cortical DA function in high-risk youth.

Keywords: Clinical High Risk for Psychosis, Cannabis, Positron Emission Tomography, Dopamine, Cortex

Disclosure: Nothing to Disclose.

\section{M202. Circular RNAs in Schizophrenia}

Torsten Klengel*, Claudia Klengel, Guia Guffanti, Anne Boyer-Boiteau, Fadi Hamati, Gabriele Chelini, Harry Pantazopoulos, Fabio Macciardi, Kerry Ressler, Sabina Berretta

McLean Hospital, Harvard Medical School, Belmont, Massachusetts, United States

Background: Non-protein coding transcripts dominate the transcriptional output of the mammalian genome producing a wide range of non-coding RNAs. Among them, circular RNAs (circRNAs) recently gained attention due to the unique properties of the circular structure and their enrichment in neuronal tissue. However, their biological function in the healthy and diseased brain remains largely unexplored.

Methods: We profile circRNAs in post-mortem Dorsal Lateral Prefrontal Cortex (DLPFC) in a discovery and independent replication sample of individuals with schizophrenia (SZ) and controls (CTRL) and investigate the biological function of candidate circRNAs by in-vitro molecular studies.

Results: Using post-mortem brain RNA-seq data from individuals with SZ $(n=10)$ and CTRL $(N=10)$, we detect over 25,000 circRNAs expressed in the human DLPFC with 18 circRNAs upregulated and 5 circRNAs down regulated in individuals with SZ compared to CTRL $(p<0.006)$. In an independent cohort, we replicate the increased expression of circLARP1B in the DLPFC of SZ $(N=14)$ compared to CTRL $(N=10)(p=0.0048)$. Concurrently, we can show a downregulation in the expression of linear isoforms from the LARP1B locus $(p=0.039)$ suggesting a functional interaction between circular and linear transcripts. In vitro experiments including the overexpressing and inhibiting circLARP1B indeed provide evidence for a regulatory role of this circRNA on the transcription of the linear counterparts.
Conclusions: The non-coding transcriptome may have an important but largely unexplored regulatory function potentially contributing to the development of psychiatric disorders. We show a dysregulation of circRNAs in SZ and provide data suggesting that circRNAs may contribute to transcriptional alterations in SZ.

Keywords: RNA, Non-Coding, Schizophrenia

Disclosure: Nothing to Disclose.

\section{M203. White Matter Pathology in Schizophrenia in Three Major White Matter Fasciculi}

\section{Rosalinda Roberts*, Kirsten Schoonover, Charlene Farmer, Andrew Cash}

University of Alabama, Birmingham, Birmingham, Alabama, United States

Background: White matter in the brain is comprised of axonal projections between brain regions and forms the basis for connectivity in the brain. People with schizophrenia exhibit abnormal connectivity termed "dysconnectivity" in several white matter tracts, including the cingulum bundle, corpus callosum and arcuate fasiculus.

Methods: This study used Western blot analysis to compare protein levels of myelin basic protein (MBP), neurofilament heavy (NFH), autophagosome marker LC3, and microtubule marker $\alpha$-tubulin in postmortem cingulum bundle, corpus callosum, and arcuate fasiculus in schizophrenia subjects (SZ; $n=16)$ and matched controls (NCs; $n=15)$. Additionally, SZ cases were subdivided by treatment status: off-(OFF; $n=8$ ) or on-medication ( $\mathrm{ON} ; n=8$ ). Off drug cases were not medicated within the last six months of life. Brain tissue came from the Maryland and Alabama Brain Collections.

Results: The combined SZ group exhibited less NFH protein versus NCs $(p=0.02)$ in the corpus callosum. In the cingulum bundle, OFF subjects had increased $\alpha$-tubulin protein versus $\mathrm{ON}(p=0.02)$ and NCs $(p=0.04)$. There were significant correlations between $\alpha$-tubulin and all other proteins, but only in the cingulum bundle. A strong negative relationship was detected in NCs between $\alpha$-tubulin \& MBP $(\mathrm{r}=-0.905)$ and $\alpha$-tubulin \& LC3 $(\mathrm{r}=-0.680)$ that was absent in SZs $(r=0.474 \& r=0.094$, respectively); coefficients of comparison were significant $(p<0.001 \& p=0.028$, respectively). Race analyses revealed that African American SZ (AASZ) had less $\alpha$-tubulin than AANCs $(p=0.02)$ and Caucasian SZ (CSZ; $p=0.003$ ) in the arcuate fasciculus. AASZ had less NFH than AANCs in the corpus callosum and cingulum bundle ( $p=0.03 \& p=0.01$, respectively). AANCs expressed more MBP in the corpus callosum and more NFH in the cingulum bundle than CNC $(p=0.047 \& p=0.04$, respectively). CSZ had more LC3 than CNC $(p=0.048)$ in the cingulum bundle.

Conclusions: The results show tract and protein specific abnormalities in relationship to diagnosis, treatment and race. These data provide evidence of cytoskeletal abnormalities in all three tracts. These data suggest dysregulation of the relationship between $\alpha$-tubulin and other observed markers of $\mathrm{CB}$ white matter integrity in schizophrenia.

Keywords: Myelin, Cytoskeleton, Autophagosome, Neurofilament, Microtubule

Disclosure: Nothing to Disclose. 
M204. Elevated Nuclear Factor-KB Transcriptional Activity Across the Visuospatial Working Memory Circuit in Schizophrenia

Paven Lidstone*, Erin Sipple, Alicia Locker, David Volk

University of Pittsburgh Medical Center, Western Psychiatric Institute \& Clinic, Pittsburgh, Pennsylvania, United States

Background: Cognitive impairments, including deficits in visuospatial working memory, are debilitating features of schizophrenia. Immune activation, indicated by higher transcript levels for cytokines and interferon-induced transmembrane proteins (IFITMs), has been reported in the prefrontal cortex (area 9) in schizophrenia. The transcription factor nuclear factor- $\mathrm{\kappa B}(\mathrm{NF}-\mathrm{\kappa B})$ regulates the expression of these IFITM variants as well as the same cytokines that are overexpressed in schizophrenia. We recently found higher mRNA levels for multiple NF- $\kappa B$ family members (NF- $\kappa B 1$, NF- $\kappa B 2$, RelA, cRel) and initiation receptors (IL-1 R, TNFR) in the canonical NF- $\mathrm{kB}$ activation pathway in PFC area 9 of schizophrenia subjects relative to unaffected comparison subjects. Consequently, we examined whether levels of these NF- $\kappa B-$ related markers are higher throughout cortical regions in the visuospatial working memory circuit, including dorsolateral prefrontal (area 46), posterior parietal (area 7), and visual (area 17) cortices in 40 schizophrenia subjects relative to 40 unaffected comparison subjects.

Methods: Quantitative PCR was employed to measure mRNA levels for NF- $\mathrm{kB}$-related markers in cortical areas 46,7 , and 17 from postmortem tissue of 40 schizophrenia subjects individually matched to 40 unaffected comparison subjects.

Results: Overall, schizophrenia subjects had significantly higher mRNA levels for all measured NF-кB-related markers relative to unaffected comparison subjects. Post-hoc tests revealed that in schizophrenia subjects, NF- $\mathrm{\kappa B}$ family

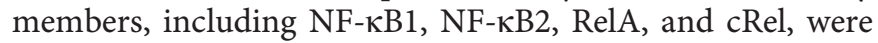
significantly (or nearly significantly) elevated in each cortical region relative to unaffected comparison subjects. Statistically significant regional differences were also seen for many measured NF- $\mathrm{KB}$ family members in both schizophrenia subjects and unaffected comparison subjects. NF- $\mathrm{KB}$ family member mRNA levels were consistently higher in primary visual cortex relative to posterior parietal and prefrontal cortex. Finally, diagnosis by region interactions were significant (or nearly significant) for NF- $\mathrm{\kappa B}$ family members

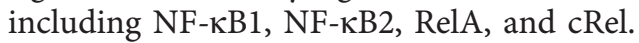

Conclusions: Defining the pathophysiology underlying cortical dysregulation and subsequent cognitive impairments in schizophrenia is critical to the discovery of therapeutic treatments of this disorder. Our findings indicate that levels of NF- $\mathrm{kB}$-related markers are elevated across the entire visuospatial working memory circuit in schizophrenia subjects and these increases are most prominent in visual cortex. These data suggest that elevations in nuclear factor$\kappa \mathrm{B}$ transcriptional activity within the visuospatial circuit may play a role in the disruption of cortical function associated with schizophrenia.

Keywords: Immune Markers, Cytokines, Synapses, Schizophrenia, Autism, NFkB, IFITM, Visuospatial Working
Memory, Cortical Circuit Function, Schizophrenia, Postmortem Human Brain

Disclosure: Nothing to Disclose.

M205. Neuromelanin-Sensitive MRI: A Novel, NonInvasive Proxy Measure of Dopamine Function in Neuropsychiatric Illness

Guillermo Horga*, Clifford Cassidy, Anissa Abi-Dargham

Columbia University, New York, New York, United States

Background: Neuromelanin-sensitive magnetic resonance imaging (NM-MRI) purports to detect the content of neuromelanin (NM), a by-product of dopamine metabolism that slowly accumulates in neurons of the substantia nigra (SN) over the lifespan. NM accumulation can be pharmacologically induced in rodents by increasing dopamine synthesis in midbrain neurons, suggesting that the excess pre-synaptic dopamine in nigro-striatal pathways in psychosis may be accompanied by an increase in NM accumulation in the SN. NM-MRI has proven to be a sensitive neuroimaging marker for degeneration of dopamine neurons in Parkinson's disease but its utility as a marker of dopamine function in non-neurodegenerative conditions is unclear, making its application to psychotic and other psychiatric illnesses dubious. Here, we validated NM-MRI as a marker of neuromelanin content and a proxy for dopamine function using in vivo and ex vivo data. We further tested whether this non-invasive MRI technique could be a proxy for the wellestablished dopamine dysfunction of the nigro-striatal pathway associated with psychosis in schizophrenia. Finally, we tested in whether NM-MRI may further capture this dopamine-related phenotype in relation to psychosis in a population at clinical high risk for schizophrenia.

Methods: We obtained high-resolution scans of the midbrain $(0.39 \mathrm{~mm}$ in-plane resolution) using a $2 \mathrm{D}$ gradient-echo NM-MRI sequence with magnetization transfer on a $3 \mathrm{~T}$ GE MR750 scanner. We validated SN NM-MRI ex vivo, against spectrophotometric measurements of regional NM concentration in post-mortem tissue $(n=7$ specimens of the midbrain from individuals without histopathology compatible with Parkinson's disease). We also validated this technique in vivo, against a Positron Emission Tomography (PET)-based measure of dopamine release capacity (based on amphetamine-induced displacement of the radiotracer [11C] raclopride) obtained in individuals without a neurodegenerative condition $(n=18)$. We further collected in vivo data in 24 individuals with Parkinson's disease and 11 agematched healthy controls in order to validate a novel, unbiased, voxelwise measure of signal to noise (relative to a midbrain white-matter reference region). Finally, to test the utility of NM-MRI as a proxy for psychosis-related dopamine dysfunction, we collected data in 25 unmedicated individuals diagnosis of with schizophrenia, 23 unmedicated individuals at clinical high risk for psychosis, and 46 healthy controls. For voxelwise analyses, we used a permutationbased method for correction for multiple comparisons and report corrected results for correlation and regression analyses at $p<0.05$. 
Results: Regional NM-MRI signal intensity in post-mortem midbrain specimens highly correlated with regional neuromelanin concentration $(r=0.73, p=0.005)$. Consistent with prior reports, we observed a profound reduction in the NMMRI signal in patients with Parkinson's disease compared to age-matched controls $(\mathrm{T}(35)=5.2, p=0.00002)$, a reduction that was regionally specific and thus more apparent using unbiased voxelwise analyses within the SN. Voxelwise analysis within the SN also revealed a cluster in which NM-MRI signal-to-noise positively correlated with striatal dopamine release capacity $(r=0.48, p=0.044)$. Similar voxelwise analyses in the psychiatric populations identified overlapping clusters where higher NM-MRI signal-to-noise in the SN correlated with more severe psychotic symptoms both in patients with schizophrenia $(r=0.53, p=0.006)$ and in individuals at clinical high risk $(r=0.7, p=0.00008$; conjunction test $p<0.05$ ).

Conclusions: Our results indicate that NM-MRI is indeed a sensitive marker of NM content in midbrain tissue, even in the absence of neurodegenerative pathology of the midbrain, and thus they highlight its potential utility as a marker of (non-neurodegenerative) psychiatric illness. Our unbiased voxelwise method, in combination with the high spatial resolution afforded by NM-MRI ( a 5-to-10-fold increase compared with standard PET protocols), revealed regional differences within the SN that may correspond to specific neuroanatomical pathways with differential involvement across neurobiological processes and neuropsychiatric conditions. Finally, our results showing that NM-MRI can capture a psychosis- and dopamine-related phenotype in schizophrenia and clinical-high-risk individuals suggest that this non-invasive MRI method may be a promising imaging tool in psychosis. Future work should evaluate the utility of NM-MRI as a predictive biomarker to stage risk of conversion to illness in at-risk populations and to predict response to anti-dopaminergic treatments for psychosis.

Keywords: MRI, Dopamine, Psychosis

Disclosure: Nothing to Disclose.

\section{M206. The Missing Piece in the Puzzle: Cognitive Decline in Schizophrenia and Bipolar Patients After the First Episode}

Abraham Reichenberg*, Jolanta Zanelli, Sven Sandin, Josephine Mollon, Paola Dazzan, izabela Pilecka, Paul Fearon, Tiago Reis Marques, Gillian Doody, Kevin Morgan, Anthony David, Craig Morgan, Peter Jones, Robin Murray

Icahn School of Medicine at Mount Sinai, New York, New York, United States

Background: Schizophrenia is associated with a severe cognitive impairment. While it is widely believed that cognitive deficits in schizophrenia remain stable after illness onset, few studies have comprehensively examined longerterm cognitive change from soon after the first episode through adulthood. We examined whether schizophrenia patients experience cognitive decline following the first episode, whether decline is generalized or confined to individual neuropsychological functions, and whether decline is specific to schizophrenia.
Methods: Participants were from a population-based, casecontrol study of patients with first-episode psychosis followed prospectively up to 10 years post first admission. A neuropsychological battery was administered to patients with a diagnosis of schizophrenia $(N=91)$ and bipolar disorder or mania $(N=37)$ at index presentation and at follow-up, as well as at two time points to healthy comparison subjects $(N=103)$.

Results: The schizophrenia group exhibited declines in IQ and individual neuropsychological functions, particularly those tapping verbal knowledge, executive function, language and visual memory. The ages when progression of deficits occurred differed between functions. There was little evidence for decline in verbal memory and processing speed. These functions showed large deficits at the first episode, which remained static thereafter. Cognitive decline in IQ, verbal knowledge and language was not specific to schizophrenia and was also apparent in the bipolar/mania group. Psychiatrically healthy individuals with low IQ, on the other hand, showed no evidence of decline, suggesting that a progressive course of cognitive impairment is specific to psychosis.

Conclusions: Schizophrenia and bipolar/mania patients experience cognitive decline after onset of psychosis. Cognitive remediation efforts should target individual functions during specific time periods.

Keywords: Schizophrenia, Cognition, Neuropsychology, Bipolar Disorder, Cognitive Decline

Disclosure: Nothing to Disclose.

M207. Identification and Characterization of ML321: A Novel and Highly Selective D2 Dopamine Receptor Antagonist That Demonstrates Efficacy in Animal Models That Predict Antipsychotic Activity

David Sibley*, R. Benjamin Free, Jingbo Xiao, Marc Ferrer, Noel Southall, Jonathan Javitch, Robert Mach, Jeremiah Bertz, Lei Shi, James Woods, Juan Marugan, William C. Wetsel

National Institute of Neurological Disorders \& Stroke, Bethesda, Maryland, United States

Background: Schizophrenia is a devastating illness that affects approximately $1.1 \%$ of the adult human population. It is characterized by a combination of positive symptoms (hallucinations, etc.), negative symptoms (flat affect) and cognitive impairment. Current treatment consists of antipsychotic drugs that are primarily effective in treating the positive symptoms of the disorder. These consist of typical (first generation) and atypical (second generation) antipsychotics, the latter of which are defined by fewer extrapyramidal side-effects (EPS). Despite much research, the mechanism of "atypicality" observed with second generation antipsychotics is not known. There is consensus, however, that antagonism of the D2 dopamine receptor (D2R) is primarily responsible for the efficacy of these drugs in treating the positive symptoms of schizophrenia. Unfortunately, all antipsychotics, both typical and atypical exhibit numerous other side effects (sedation, weight gain, diabetes, etc.), primarily due to their interaction with various receptors, transporters and other signaling proteins. A 
potent and highly selective D2R antagonist, which has not been previously available in the pharmacological armamentarium, might thus be particularly effective for treating schizophrenia while exhibiting fewer side effects than the antipsychotics in current use.

Methods: High throughput screening was performed using the NIH small molecule repository ( $\sim 400,000$ compounds) and a D2R functional assay. Active hit compounds were confirmed and counter-screened using a D1R functional assay. Active D2R/inactive D1R compounds were furthered screened using a D3R functional assay. A small pool of compounds were identified that were $>10$-fold D2/D3 selective. The most promising antagonist candidate was further optimized using medicinal chemistry resulting in ML321. The global pharmacology of ML321 was further evaluated using the DiscoverX gpcrMAX panel (168 known GPCRs) and the NIH Psychoactive Drug Screening Program ( $\sim 8$ GPCRs, transporters and ion channels). Further experiments were performed as described. All animal experimentation was approved by an Institutional Animal Care and Use Committee and conducted in accordance with the National Institutes of Health Guide for the Care and Use of Laboratory Animals.

Results: Using high throughput screening techniques, we identified, optimized, and characterized a lead D2R antagonist with high selectivity - ML321. In a functional profiling screen of 168 different GPCRs (using $10 \mathrm{uM}$ of ML321 in both agonist and antagonist modes), ML321 showed little activity beyond potent inhibition of the D2R, and to a lesser extent the D3R, demonstrating exceptional GPCR selectivity. Interestingly, we found that the binding of ML321 to the $\mathrm{D} 2 \mathrm{R}$ is entirely dependent on $\mathrm{Na}+$. The extreme D2R selectivity of ML321 may be related to the fact that, unlike other monoaminergic ligands, ML321 lacks a protonatable (i.e., positively charged) amine group and adopts a unique binding pose within the orthosteric binding site of the D2R. PET imaging studies in non-human primates demonstrated that ML321 can penetrate the CNS and occupy the D2R in a dose-dependent manner. Preliminary in vitro ADME studies showed that ML321 is metabolically stable and does not block hERG channels, whereas pharmacokinetic (PK) studies in mice demonstrated elevated plasma and brain levels of ML321 for up to $8 \mathrm{hrs}$. Behavioral paradigms in rats demonstrated that that ML321 can selectivity antagonize a D2R-mediated response (hypothermia) while not affecting a D3R-mediated response (yawning) using the same dose of drug, thus demonstrating good in vivo selectivity. We also investigated the effects of ML321 in animal models that are predictive of antipsychotic efficacy in humans. We found that ML321 can attenuate both PCP and amphetamineinduced locomotor activity and pre-pulse inhibition (PPI) in a dose-dependent manner. Surprisingly, using doses that were maximally effective in both the locomotor and PPI studies, ML321 promoted little-no catalepsy compared with the non-selective antipsychotic haloperidol. This observation suggests that ML321, or a derivative thereof, may prove to be an "atypical" D2R antagonist and produce fewer extrapyramidal side effects (EPS) than observed with currently FDAapproved D2R antagonists.

Conclusions: We have identified a novel, highly selective D2R antagonist, ML321, that will be useful as a probe for dissecting D2R function in animal models and may also serve as a drug lead for developing novel antipsychotic agents. Among 168 different GPCRs, and additional transporters and ion channels, ML321 demonstrated remarkable selectivity for the D2R and this selectivity was borne out using in vivo behavioral paradigms. ML321 demonstrated the ability to penetrate the CNS, engage the D2R, and exhibit efficacy in animal models that are predictive of antipsychotic efficacy. Due the extreme receptor selectivity of ML321, the potential number of off-target side effects are predicted to be nil. While ML321 might still possess liability for EPS (thought to be an on-target effect), our results showing that ML321 produces little to no catalepsy suggests that this may not be the case. Further experiments investigating these behavioral effects of ML321 are in progress.

Keywords: Antipsychotics, Dopamine, D2 Receptor, Drug Discovery, Drug Development

Disclosure: Nothing to Disclose.

M208. Effect of Brexpiprazole on Patient Functioning in Patients With Schizophrenia: Results From a LongTerm, Randomized, Double-Blind, Placebo-Controlled, Maintenance Study

Francois Therrien*, Catherine Weiss, Na Jin, Ross A. Baker, Erin MacKenzie, Stine R. Meehan

Otsuka Canada Pharmaceutical, Inc., St-Laurent, Canada

Background: For a long-term therapy to be considered successful in schizophrenia, it must positively influence a variety of domains beyond positive and negative symptoms. Symptomatic treatment alone is not sufficient for successful occupational performance and interpersonal relations. Remission and, ultimately, recovery are advocated as achievable long-term treatment goals; however, in order to obtain recovery, treatment goals must be expanded to include domains such as cognitive performance, quality of life, and patient functioning. The latter may be defined as a patient's ability to perform his/her role in society as a member of a family and/or career fulfillment, and has been viewed as a prerequisite to recovery.

There are relatively simple instruments available to measure patient functioning. The Personal and Social Performance (PSP) scale is a single-score, clinician-rated scale designed to measure the severity of dysfunction in four sub-domains of personal and social performance (1: socially useful activities, 2: personal and social relationships, 3: self-care, and 4: disturbing and aggressive behavior). The validity and reliability of the PSP has been described in patients with schizophrenia over the course of treatment. The Global Assessment of Functioning (GAF) scale is a single-item, clinician-rated scale that assesses overall psychological symptoms, social, and occupational functioning, and previous research has reported good reliability and validity. The present analyses investigated the effect of brexpiprazole (a serotonin-dopamine activity modulator that is a partial agonist at 5-HT1A and dopamine D2 receptors, and an antagonist at 5-HT2A and noradrenaline alpha1B/2C receptors, all at similar potency) on patient functioning during maintenance treatment in patients with schizophrenia. 
Methods: Patient functioning data were collected during a 52-week, randomized, double-blind, placebo-controlled maintenance study to assess the efficacy, safety, and tolerability of long-term therapy with brexpiprazole for the treatment of schizophrenia (Equator [NCT01668797]). Patients enrolled in the maintenance phase had previously been stabilized on brexpiprazole for 12 consecutive weeks (single-blind), and eligible patients were randomized to continue receiving brexpiprazole $1-4 \mathrm{mg}$ /day or to switch to placebo. Efficacy was demonstrated at a prespecified interim analysis and thus the study was terminated early. The following analyses were performed to comprehensively estimate the effect of brexpiprazole versus placebo on patient functioning, as measured by the PSP and GAF scales during the maintenance phase:

- Least squares (LS) mean change from baseline to Week 52 in PSP and GAF scores (Last Observation Carried Forward [LOCF] analysis, Analysis of Covariance [ANCOVA])

- LS mean change from baseline to Week 52 in PSP subdomain scores (LOCF, ANCOVA)

- Descriptive proportion of patients achieving PSP score thresholds at baseline of $\leq 30,31-50,51-70$, and $\geq 71$ and descriptive proportion of patients having improved or deteriorated at least one PSP threshold category at Week 52 (LOCF)

- Proportion of patients achieving an increase in PSP score of at least 10 points (defined as functional response) between baseline and Week 52 (LOCF, Fisher's exact test)

Results: A total of 194 patients were included in the present analysis (brexpiprazole $1-4 \mathrm{mg}, n=94$; placebo, $n=100$ ). The improvements in PSP Total score were greater with brexpiprazole than with placebo (LS mean difference at Week 52: +4.8 ; 95\% CI: $1.3,8.2 ; p=0.0071)$. LS mean GAF score remained stable during maintenance treatment with brexpiprazole ( +0.6 point) while a deterioration (LS mean of -6.0 points) was observed in the placebo group $\left(\mathrm{p}^{<} 0.001\right)$. Brexpiprazole produced significant benefits versus placebo on personal and social relationships and disturbing and aggressive behaviors, and numerical benefits on socially useful activities including work and study, and self-care. A higher proportion of patients receiving brexpiprazole improved at least one PSP score threshold category at endpoint compared to patients receiving placebo (60\% versus $50 \%$, respectively). Also, $35 \%$ and $5 \%$ of brexpiprazole patients compared to $44 \%$ and $6 \%$ of placebo patients remained in the same PSP category or deteriorated by at least one category, respectively. The proportion of patients meeting criteria for functional response was significantly higher in the brexpiprazole group (64\% versus $47 \%, \mathrm{p}^{<} 0.05$ ). Conclusions: It is necessary to not only consider the reduction in symptoms but also the long-term successful psychosocial reintegration of patients when defining positive outcomes in the treatment of schizophrenia. The present comprehensive analyses demonstrate that brexpiprazole treatment is associated with clinically relevant long-term improvement in the level of patient functioning relative to placebo.

Keywords: Brexpiprazole, Patient Functioning, Personal and Social Performance (PSP) and Global Assessment of Functioning (GAF), Schizophrenia, Long-Term Treatment
Disclosure: Part 5: Otsuka Canada Pharmaceutical Inc., Employee.

\section{M209. Somatostatin Interneurons Facilitate Hippocampal- Prefrontal Synchrony and Prefrontal Spatial Encoding During Working Memory}

Atheir Abbas*, Marina Sundiang, Eric Myhre, Britt Henoch, Mitchell Morton, Scott Bolkan, Alexander Harris, Christoph Kellendonk, Joshua Gordon

Columbia University/New York State Psychiatric Institute, New York, New York, United States

Background: Working memory, a critical cognitive function, is impaired in schizophrenia and other psychiatric disorders including bipolar disorder, unipolar major depression, autism, and attention deficit hyperactivity disorder. Current treatments, however, do not effectively treat cognitive impairments despite their contribution to poor functioning in psychiatric illness. Thus, elucidating the neural basis for cognitive symptoms in psychiatric disorders may inform new treatment strategies which address cognitive deficits and reduce the impact of disease on functioning.

Methods: To address these gaps, we used the light-activated proton pump Arch3.0 to selectively silence prefrontal PV or SOM interneurons in the medial prefrontal cortex of mice performing the delayed non-match to sample T-maze test of spatial working memory. We simultaneously recorded neural activity in the medial prefrontal cortex (mPFC) and other brain areas known to be involved in working memory, including the dorsal and ventral hippocampus (dHPC and vHPC), and mediodorsal thalamus (MD). We used measures of synchrony such as pairwise phase consistency (PPC) to characterize the functional connectivity between these various structures.

Results: We found that silencing SOM interneurons during the sample or delay epochs of the task significantly impaired working memory performance when the delay length was 10 or 60 seconds. SOM silencing during the sample epoch of the working memory task was associated with a decrease in coherence between mPFC and HPC theta oscillations and a decrease in phase locking, as measured by pairwise phase consistency, between mPFC neurons and vHPC and dHPC theta oscillations. SOM silencing during the sample epoch was also associated with impaired spatial encoding within the mPFC. Silencing PV interneurons had no effect on synchrony, mPFC spatial encoding, or working memory performance.

Conclusions: The evidence we present is consistent with SOM interneurons supporting spatial encoding during working memory and facilitating hippocampal-prefrontal synchrony. These findings suggest that interneuron dysfunction may contribute to cognitive deficits in schizophrenia by disrupting long range synchrony between the hippocampus and prefrontal cortex.

Keywords: Interneurons, Medial Prefrontal Cortex, Working Memory, Somatostatin, Parvalbumin

Disclosure: Nothing to Disclose. 
M210. The Effect of Brexpiprazole in Patients With Schizophrenia Experiencing or Not Experiencing Activating or Sedating Adverse Events: A Post-Hoc Analysis of Short-Term Studies

Catherine Weiss ${ }^{\star}$, Peter Zhang, Ross A. Baker,

Stine R. Meehan

Otsuka Pharmaceutical Development \&

Commercialization, Inc., Princeton, New Jersey, United States

Background: Side effects of treatments for schizophrenia can impose a significant overall burden on patients. Patients with schizophrenia report activating and sedating side effects as being among the most 'bothersome' side effects that they experience.

Brexpiprazole is a serotonin-dopamine activity modulator that is a partial agonist at 5-HT1A and dopamine D2 receptors, and an antagonist at 5-HT2A and noradrenaline alpha $1 \mathrm{~B} / 2 \mathrm{C}$ receptors, all at similar potency. Brexpiprazole is approved in the United States, Canada and Australia for the treatment of schizophrenia, and in the United States for use as adjunct to antidepressants in the treatment of major depressive disorder.

Here, we assess if activating (akathisia, restlessness, agitation, insomnia/anxiety) or sedating (sedation/somnolence/fatigue) adverse events affect functioning and the efficacy of brexpiprazole in patients with an acute relapse of schizophrenia.

Methods: In two similarly designed short-term studies (Vector [NCT01396421] and Beacon [NCT01393613]) patients with acute schizophrenia were randomly assigned to fixed once-daily doses of brexpiprazole $2 \mathrm{mg}, 4 \mathrm{mg}$, or placebo. (An additional treatment group was included in each study [0.25 mg and $1.0 \mathrm{mg}$ ] to evaluate the lower dose range; these doses were not included in the analysis.) In this post-hoc analysis, the primary endpoint was change from baseline in PANSS Total score in brexpiprazole-treated patients with and without activating or sedating adverse events. The effect on functioning was assessed by looking at the change from baseline in PSP Total score. LOCF analyses were conducted with an ANCOVA model, with country as main effect and baseline value as covariate, to estimate the mean change at Week 6 for each group. Correlation coefficients between change from baseline in PANSS Total score or PSP Total score, and with/without activating or sedating adverse events were calculated.

Results: In these studies, of 718 patients receiving brexpiprazole $2 \mathrm{mg}$ or $4 \mathrm{mg}, 196$ patients experienced activating $(n=166)$ or sedating adverse events $(n=40)$. LS mean change (SE) from baseline at Week 6 in PANSS Total score was -14.5 (2.8) for patients experiencing activating adverse events $(n=166)$ and $-19.7(1.5)$ for those without activating adverse events $(n=552)$. LS mean change (SE) from baseline at Week 6 in PANSS Total score was -19.5 (4.4) for patients experiencing sedating adverse events $(n=40)$ and -18.6 (1.0) for those without sedating adverse events $(n=678)$. The correlation coefficient between improvement in PANSS Total score and those experiencing activating adverse events was -0.0656 $(p=0.0790)$, and -0.0297 $(p=0.4270)$ for patients experiencing sedating adverse events.
LS mean change (SE) from baseline in PSP Total score was $7.3(2.0)$ in patients with activating adverse events $(n=163)$ and 10.0 (1.1) in patients without activating adverse events $(n=522)$. LS mean change (SE) from baseline in PSP Total score was 9.5 (3.1) in patients with sedating adverse events $(n=39)$ and $9.4(1.0)$ in patients without sedating adverse events $(n=639)$. The correlation coefficient between improvement in PSP Total score and those experiencing activating adverse events was $-0.0896(p=0.0190)$, and $-0.0168(p=0.6609)$ for patients with sedating adverse events.

Conclusions: In the brexpiprazole short-term studies, there does not appear to be a correlation between the presence of activating or sedating adverse events and the efficacy of brexpiprazole in patients with acute schizophrenia, although there was a trend toward a correlation between activating adverse events and reduced efficacy in terms of PANSS Total score. In functioning, there was a clearer trend toward activating adverse events and less functional improvement, and no correlation between sedating side effects and functioning. Results must be viewed with caution, particularly due to low rates of sedating adverse events in patients with schizophrenia participating in brexpiprazole short-term clinical studies. Taken together, the results do remind clinicians that side effect profiles and active management of early side effects should be taken into account when using antipsychotics for the treatment of schizophrenia.

Keywords: Brexpiprazole, Schizophrenia, Affect of Adverse Events on Efficacy, Affect of Adverse Events on Functioning, Short-Term Clinical Studies

Disclosure: Part 5: Otsuka Pharmaceutical Development \& Commercialization, Inc., Employee.

M211. Effect of Brexpiprazole on Patient Functioning in Patients With Acute Exacerbation of Schizophrenia: Results From Three Short-Term, Randomized, DoubleBlind, Placebo-Controlled Studies

Catherine Weiss, Francois Therrien, Na Jin, Erin MacKenzie, Stine R. Meehan, Ross A. Baker*

Otsuka Pharmaceutical Development \& Commercialization, Inc., Princeton, New Jersey, United States

Background: For a therapy to be considered successful in schizophrenia, it must positively influence a variety of domains beyond positive and negative symptoms. Symptomatic treatment alone is not sufficient for successful longterm occupational performance and interpersonal relations. One such domain is patient functioning, which may be defined as a patient's ability to perform his/her role in society as a member of a family and/or career fulfillment. Functional benefits are unlikely to be maximized during short-term treatment with antipsychotics, and progress achieved after 4 to 6 weeks of treatment is important for clinicians, patients, and caregivers, to ascertain when deciding on a future course of treatment.

There are relatively simple instruments available to measure patient functioning. The Personal and Social Performance (PSP) scale is a single-score, clinician-rated scale designed to measure the severity of dysfunction in four sub-domains of 
personal and social performance (1: socially useful activities, 2: personal and social relationships, 3: self-care, and 4: disturbing and aggressive behavior). Its validity and reliability has been described in patients with schizophrenia during the acute phase of the illness. The present analyses investigated the effect of brexpiprazole, a serotonin-dopamine activity modulator that is a partial agonist at 5-HT1A and dopamine D2 receptors, and an antagonist at 5-HT2A and noradrenaline alpha $1 \mathrm{~B} / 2 \mathrm{C}$ receptors, all at similar potency, on patient functioning during short-term treatment in patients with acute exacerbation of schizophrenia.

Methods: Patient functioning data were pooled from three 6week, double-blind, placebo-controlled trials conducted in hospitalized patients with acute exacerbation of schizophrenia (Vector [NCT01396421, Beacon [NCT01393613], Lighthouse [NCT01810380]). These studies demonstrated the efficacy, safety, and tolerability of brexpiprazole for the short-term treatment of schizophrenia at doses between 2 and $4 \mathrm{mg} /$ day. The following analyses were performed on the same dataset to comprehensively estimate the effect of brexpiprazole versus placebo on patient functioning as measured by the PSP scale:

- Least squares (LS) mean change from baseline to Week 6 in PSP score (Mixed Model Repeated Measures [MMRM] analysis with fixed factors of study, treatment, visit, treatment visit interaction, baseline value, and baseline visit interaction as covariates)

- LS mean change from baseline to Week 6 in PSP subdomain scores (MMRM)

- Descriptive proportion of patients achieving PSP score thresholds at baseline of $\leq 30,31-50,51-70$, and $\geq 71$ and descriptive proportion of patients having improved or deteriorated at least one PSP threshold category at Week 6 (Last Observation Carried Forward [LOCF] analysis)

- Proportion of patients achieving an increase in PSP score of at least 10 points (functional response) between baseline and Week 3 and Week 6 (LOCF)

- Proportions were compared using a Cochran-MantelHaenszel row mean scores differ test stratified by study.

Results: A total of 1,321 patients were included in the present analysis (brexpiprazole 2-4 mg, $n=831$; placebo, $n=490$ ). Brexpiprazole produced a significant benefit versus placebo on mean PSP scores with LS mean increases of 12.2 for brexpiprazole and 9.0 points for placebo at Week 6

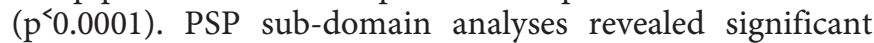
improvements across all four domains in patients rando-

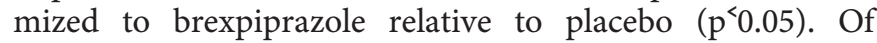
patients receiving brexpiprazole, $44 \%$ improved at least one PSP score threshold category at endpoint compared to $35 \%$ of patients receiving placebo.

The proportions of patients meeting criteria for functional response were significantly higher in the brexpiprazole group at both Week 3 (31\% versus $\left.26 \%, \mathrm{p}^{<} 0.05\right)$ and Week $6(48 \%$ versus $\left.37 \%, \mathrm{p}^{<} 0.0001\right)$.

Conclusions: It is necessary to not only consider the reduction in symptoms but also the improvement of patient functioning when defining positive outcomes in the shortterm treatment of schizophrenia. The present comprehensive analyses demonstrate that brexpiprazole treatment is associated with a clinically relevant short-term improvement in symptoms of schizophrenia and the level of patient functioning relative to placebo.
Keywords: Brexpiprazole, Short-Term Studies, Functioning, Personal and Social Performance (PSP)

Disclosure: Part 5: Otsuka Pharmaceutical Development \& Commercialization, Inc., Employee.

M212. A Phase II, Randomized, Double-Blind Study to Evaluate the Efficacy, Safety and Tolerability of ALKS 3831 in Subjects With Schizophrenia and Alcohol Use Disorder

Mary Brunette, Christoph Correll, Bernard Silverman, Adam Simmons, Ying Jiang, Lauren DiPetrillo, David McConnell, Alan Green*

Geisel School of Medicine at Dartmouth, Lebanon, New Hampshire, United States

Background: Over a third of people with schizophrenia have a co-occurring alcohol use disorder (AUD), which worsens the overall outcome of the disease. At present, there is no approved treatment specifically for this tough-to-treat patient population and there is a dearth of clinical trial data evaluating pharmacologic interventions for schizophrenia with AUD. In this study, ALKS 3831 was composed of a flexible dose of the antipsychotic olanzapine (OLZ) and a fixed dose $(10 \mathrm{mg})$ of the $\mu$-opioid receptor antagonist samidorphan (SAM). The aim of this phase 2, randomized, double-blind study was to assess the efficacy, safety and tolerability of ALKS 3831 (OLZ+SAM) compared with OLZ and placebo $(\mathrm{OLZ}+\mathrm{PBO})$.

Methods: Adult patients (18-65 years old) diagnosed with schizophrenia (DSM-IV-TR) and AUD (DSM-5), with a recent exacerbation of schizophrenia were included. Exclusion criteria were intolerance to OLZ, positive test for opioids, and first episode of schizophrenia. The study consisted of a screening phase (30 days), an open-label phase ( 6 weeks' treatment: 4 weeks of OLZ followed by 2 weeks of ALKS 3831), and a double-blind treatment period (9-15 months). In the double-blind phase, 234 patients were randomized (1:1) to receive either daily flexibly dosed OLZ (determined by investigator) or ALKS 3831. The primary outcome was time to exacerbation of disease symptoms as assessed by the event of exacerbation of disease symptoms (EEDS), based on the occurrence of eight pre-specified events related to worsening of disease symptoms of schizophrenia and/or AUD, and assessed by a blinded Independent Adjudication Committee. Other outcomes included change from baseline in alcohol use (WHO drinking level). Time to first EEDS event rate was estimated by Kaplan-Meier method. The log-rank test was used for treatment comparison and the Cox proportional-hazards model was used to estimate the hazard ratio, adjusting for relevant covariates. For the secondary endpoints, time to recurrent EEDS was analyzed by an Andersen-Gill mean/rate intensity model and improvement in WHO drinking level was analyzed by a logistic regression model. Descriptive summary statistics were used for all safety measures.

Results: Of 229 randomized and dosed patients, 58 (49.6\%) receiving OLZ+PBO completed the study compared with 53 (47.3\%) receiving ALKS 3831. Baseline characteristics were similar between treatment groups - mean age was 45.7 years and $78.6 \%$ of patients were male. The mean $( \pm$ standard 
deviation) dose of OLZ prescribed after randomization was $14.0 \pm 6.6 \mathrm{mg}$ in the ALKS 3831 group and $15.0 \pm 6.8 \mathrm{mg}$ in the OLZ+PBO group. During the 9-15 months of the double-blind study phase, there was no significant group difference in time to first EEDS (hazard ratio [HR] 0.91; 95\% confidence interval [95\% CI]: $0.53,1.56, p=0.746$ ), or time to recurrent EEDS (HR 0.77; 95\% CI: 0.43, 1.37, $p=0.372$ ). Alcohol use decreased in both groups during the study, without group difference. Assessing differences between groups on the individual EEDS components revealed that patients treated with ALKS 3831 had numerically lower rates versus OLZ+PBO of hospitalization for psychopathology (3.6\% $[n=4]$ vs. $8.5 \%[n=10], p=0.137)$, emergency-room visits $(1.8 \%[n=2]$ vs. $5.1 \%[n=6], p=0.156)$, aggression/ suicide $(8.9 \%[n=10]$ vs. $11.1 \%[n=13], p=0.557)$ and rescue medication $(3.6 \%[n=4]$ vs. $9.4 \%[n=11], p=0.098)$. A similar proportion of patients improved by at least $1 \mathrm{WHO}$ drinking level at Week $24(40.5 \%[n=45]$ in the ALKS 3831 group and $37.9 \%[n=44]$ in the OLZ+PBO group), without group difference. Treatment-emergent adverse events (AEs) were reported in $59.0 \% \quad(n=69)$ of OLZ+PBO-treated patients vs. $57.1 \%(n=64)$ of ALKS 3831 -treated patients. Serious AEs were experienced by $10.3 \%(n=12)$ of patients in the OLZ+PBO group and 6.3\% $(n=7)$ in the ALKS 3831 group; additionally, AEs leading to treatment discontinuation were $11.1 \%(n=13)$ in the OLZ+PBO group compared with $8.9 \%(n=10)$ in the ALKS 3831 group. The most common AE in the double-blind phase was weight increased for both groups $(12.0 \%[n=14]$ in OLZ+PBO and $14.3 \%$ $[n=16]$ in ALKS 3831-treated patients).

Conclusions: Both OLZ+PBO and ALKS 3831 were generally well tolerated in this patient population. ALKS 3831 (OLZ, flexible dose+SAM, $10 \mathrm{mg}$ ) treatment in patients with schizophrenia and co-occurring AUD was not associated with a statistically significant difference in time to first EEDS compared with OLZ+PBO treatment. ALKS 3831-treated patients had numerically lower rates of hospitalizations and other pre-defined EEDS events. Both groups decreased alcohol use during the study, without group difference.

Keywords: Schizophrenia, Dopamine, Antipsychotics, Alcohol Use Disorder, Clinical Trials

Disclosure: Part 4: Alkermes, Grant, Self, Novartis, Grant.

M213. The Benefit of Minocycline on Negative Symptoms of Schizophrenia: Extent and Mechanisms (The BeneMIn Study)

Bill Deakin*, John Suckling, Paola Dazzan, Eileen Joyce, Nusrat Husain, Rachel Upthegrove, Stephen Lawrie, Imran Chaudhry, Graham Dunn, Peter Jones, Lisiecka Danuta, Shon Lewis, Thomas Barnes, Steve Williams, Emma Knox, Richard Drake

University of Manchester, Manchester, United Kingdom

Background: Progressive loss of cortical grey matter has been reported in early schizophrenia. In a previous trial we tested the hypothesis that the putative neuro-protective or anti-inflammatory effects of minocycline might prevent grey matter loss and thus improve clinical outcome over one year when added to treatment as usual (Chaudhry et al 2012, PMID: 22526685). We reported a beneficial effect on negative symptoms. The BeneMIn study aimed to replicate the benefit and to determine whether there was associated preservation of grey matter, reduction in circulating inflammatory cytokines and enhancement of cognition. The objective of the trial was to determine the efficacy of minocycline on negative and other symptoms of schizophrenia and the mechanistic role of neuro-protective, antiinflammatory and cognitive enhancing actions.

Methods: Two hundred and seven patients with a current research diagnosis of schizophrenia within 5 years of onset were randomised to minocycline $(300 \mathrm{mg} /$ day) or matching placebo as an adjunct to their continuing treatment provided by their community mental health care team. The primary efficacy outcome variable was the negative symptom subscale from the Positive and Negative Syndrome Scales at 2,6,9 and 12 months. Secondary outcome measures included positive symptom scores and measures of depression, cognition, and social and occupational functioning. The primary mechanistic (biomarker) variables were i) medial prefrontal grey matter volume (GMV); ii) circulating cytokine IL6 concentration; and iii) dlpfc fMRI activations during performance of the N-back task. Secondary mechanistic outcome variables assessed other regional brain MRI regions and responses, and, cytokines. Movement disorder, side effects and treatment adherence were monitored throughout the study.

Results: Compared with placebo, the addition of minocycline had no effect on the severity of negative symptoms (treatment effect difference -0.186 [95\% CI -1.225 to 0.854]) across the 2,6, 9 and 12 months follow-up visits. None of the mechanistic biomarkers was influenced by minocycline: eg left GMV -91.2 [95\%CI -303.8 to 121.4] and IL6 0.072 [95\% CI -0.118 to 0.262 ]. There were no statistically significant treatment effects on any of the secondary outcomes and no group differences at baseline. Most measures were stable over the course of the study. There was no evidence of deterioration in negative symptoms or medial prefrontal GMV overall or in the placebo-treated participants over the 12 months of the study. There was no deterioration in outcome in the 3 months following cessation of trial medication. 25 of the 29 serious adverse events were due to hospital admission for worsening psychiatric state affecting 10 minocycline treated participants and 7 placebo treated.

Conclusions: Limitations: Although the revised recruitment target of 207 was attained on time, the overall drop-out rate was large with only $43 \%$ (89) of 207 randomised patients completing 12 months of the study instead of the target of 170. Since the follow-up imaging and cognitive biomarker data were only measured at 12 months, the planned power of the study to detect treatment effects on the mechanistic variables was considerably reduced. In contrast, the clinical efficacy assessments were carried out at each visit and $83 \%$ of those who started treatment remained on it for 6 months. Patients were not selected for the presence of negative symptoms and their initial overall psychopathology was at most moderate and therefore less likely to show treatment effects.

Conclusions: The results of the study do not support the use of adjunctive minocycline for the treatment of negative or other symptoms of schizophrenia in recent psychosis. There is no evidence for a neuro-protective, anti-inflammatory or cognitive enhancing action of minocycline in this indication. It is unlikely that persistent neurodegeneration or 
inflammation are targets for novel therapies since neither process was detectable in the present study in terms of an overall change in their measures. We cannot exclude the possibility that minocycline might benefit an inflammatory process in the prodrome, in treatment resistant patients or in those with the deficit syndrome.

Keywords: Immune Markers, Cytokines, Synapses, Schizophrenia, Autism, Neurodegeneration, Inflammatory Markers, Minocycline

Disclosure: Nothing to Disclose.

M214. Heritability of Mismatch Negativity in Patients With Schizophrenia and Healthy Controls: Results From a Danish Register Based Twin Study

Bob Oranje*, Rikke Hilker, Maria Jensen, Christian Legind, Simon Anhøj, Brian Broberg, Birgitte Fagerlund, Birte Glenthoj

Center for Neuropsychiatric Research, Copenhagen University Hospital, Mental Health Center Glostrup, Glostrup, Denmark

Background: Reduced mismatch negativity ( $M M N)$ has been suggested as a candidate endophenotypic marker for schizophrenia. Although there is some evidence indicating that MMN is reduced in patients with schizophrenia, reports on its heritability are sparse. In the current study, we investigated heritability of MMN both in healthy twins as well as in twin pairs either concordant or discordant for schizophrenia.

Methods: Twenty-four dizygotic (DZ) and 22 monozygotic (MZ) twin pairs either concordant $(n=3)$ or discordant for schizophrenia spectrum disorders were tested in a typical auditory MMN paradigm containing three types of deviants based on either frequency, duration or a combination of both. Their results were compared with those of $18 \mathrm{DZ}$ and $24 \mathrm{MZ}$ healthy twin pairs matched on age, gender and zygosity.

Results: The results showed no differences in MMN amplitude between the proband from the affected twin pairs and the healthy control twins, regardless of zygosity or type of deviant. Nor did we find any significant differences in MMN amplitudes between the probands and their healthy co-twin. Furthermore, correlation analyses for MMN amplitude between the two co-twins of the monozygotic and dizygotic twin pairs showed similar values (Spearman's rho) for duration MMN yet moderately lower values for DZ compared to MZ twins for frequency MMN and frequency/ duration MMN (patients and controls combined).

Conclusions: In conclusion, we found no reduced $\mathrm{MMN}$ amplitude in our patients with schizophrenia using a standard MMN paradigm. Furthermore, our results indicate low levels of heritability for frequency MMN, medium heritability levels for frequency/duration MMN, but virtually no heritability of duration MMN. Taken together, our data do not support the theory that any of the currently assessed three types of MMN are endophenotypic markers for schizophrenia.

Keywords: Mismatch Negativity, Endophenotype, Schizophrenia

Disclosure: Nothing to Disclose.
M215. Increased Soluble ICAM-1 is Related to Verbal Memory, Reasoning and Processing Speed Deficits in Schizophrenia

Thomas Weickert*, Helen Cai, Maryanne O'Donnell, Ryan Balzan, Ruth Wells, Dennis Liu, Cherrie Galletly, Cynthia Shannon Weickert

University of New South Wales, Randwick, Australia

Background: Schizophrenia is a disabling and often unremitting mental illness with an unknown cause that is characterized by heterogeneity in psychotic symptom presentation, cognitive deficits and treatment response. There is accumulating evidence for the role of inflammation in the etiology of schizophrenia. Inflammatory markers have been identified in the brains and peripheral blood of chronically ill patients with schizophrenia and in first episode patients and these markers have been associated with structural and functional brain abnormalities and cognitive deficits. Intercellular adhesion molecule 1 (ICAM-1) is a transmembrane protein expressed on endothelial cells which binds to leukocyte receptors that promotes transmigration of white blood cells into tissue. ICAM-1 is elevated in schizophrenia relative to control brains; however, the extent to which peripheral levels of soluble ICAM-1 (sICAM-1) is increased in relation to cognitive impairment in schizophrenia is unknown.

Methods: sICAM-1 was measured with a Luminex immunoassay from the plasma of 78 chronically ill patients with schizophrenia (all receiving antipsychotic medication) and 73 healthy controls. All participants received the following cognitive assessments: Wechsler Adult Intelligence Scale 3rd edition to assess current IQ, Controlled Oral Word Association Test verbal fluency and Wechsler Memory ScaleRevised to assess verbal memory. Pearson's or Spearman's correlations were performed between cognitive measures and sICAM1 levels as appropriate in schizophrenia patients and healthy controls.

Results: sICAM1 was elevated by $29.2 \%$ in patients with schizophrenia compared to healthy controls, $\mathrm{t}(140)=-3.988$, $p<0.01$. In patients with schizophrenia, sICAM1 was inversely correlated with immediate verbal memory $(\mathrm{r}=$ $0.30, p=0.01)$, delayed verbal memory $(\mathrm{rho}=-0.29, p=0.01)$, verbal abstract reasoning $(\mathrm{r}=-0.23, p=0.05)$, and processing speed (rho $=-0.28, p=0.02$ ). In healthy controls, sICAM1 levels were inversely correlated with verbal fluency $(r=-0.27$, $p=0.03$ ) and processing speed (rho $=-0.26, p=0.03$ ).

Conclusions: sICAM-1, a cleavage product of ICAM which enables white blood cell migration into tissue (including brain) is significantly elevated in peripheral blood of patients with schizophrenia. sICAM-1 is associated with poor verbal memory, reasoning and processing speed in people with schizophrenia and accounts for variation in cognition of healthy controls. This suggests that increased inflammatory processes, measured in blood, may reflect brain related cognitive deficits that are the hallmark of schizophrenia. Anti-inflammatory treatments may reverse cognitive impairment in schizophrenia.

Keywords: Schizophrenia, Immune Markers, Cytokines, Synapses, Schizophrenia, Autism, Verbal Memory, Visual Information Processing 
Disclosure: Part 1: Lundbeck, Consultant, Spouse, Astellas, Grant, Spouse, Part 4: Astellas, Grant, Spouse.

M216. Lower Levels of Prostaglandin-Endoperoxide Synthase 1 in the Dorsolateral Prefrontal Cortex From Subjects With Schizophrenia and After Treatment With Antipsychotic Drugs in Conjunction With Aspirin

Brian Dean*, Andrew Gibbons, Andrea Gogos, Madhara Udawela, Elizabeth Thomas, Elizabeth Scarr

Florey Institute for Neuroscience and Mental Health, Parkville, Australia

Background: It has been reported that using aspirin (acetylsalicylic acid), which targets prostaglandinendoperoxide synthase 1 (PTGS1: COX1), as an adjunct to antipsychotic drug treatment improves therapeutic outcomes when treating schizophrenia (Aïd and Bosetti, 2011). Significantly, our microarray data (Narayan et al, 2008) showed levels of PTGS1 mRNA to be higher in the dorsolateral prefrontal cortex (DLPFC) from subjects with schizophrenia of long duration of illness compared to that in age / sex matched controls. Hence, we hypothesised that aspirin could add to the effects of antipsychotic drugs by lowering PTGS1 expression in the cortex of subjects with the disorder to give a better therapeutic outcome and that changes in levels of cortical PTSG1 levels would be associated with an ongoing immune mediated process.

Methods: To test our hypotheses we measured levels of PTGS1 protein using Western blotting in the DLPFC, anterior cingulate and frontal pole from subjects with schizophrenia and age / sex matched controls. We also measured levels of PTGS1 protein in i) the DLPFC from subjects with mood disorders, ii) rat cortex after treatment with antipsychotic drugs, iii) mouse cortex after treatment with polyinosinic-polycytidylic acid sodium salt (Poly I:C; a well-established model of immune activation) and iv) human derived CCF-STTG1 cells, a human derived astrocytic cell line, after treatment with antipsychotic drugs, aspirin or a combination of these drugs.

Results: Compared to controls, PTGS1 levels were $41 \%$ lower in the DLPFC $(p<0.01)$, but not the anterior cingulate or frontal pole, from subjects with schizophrenia; unlike for mRNA (Narayan et al, 2008), the lower level of PTGS1 was independent of duration of illness. Levels of PTGS1 were not changed in the DLPFC in mood disorders or in the cortex of rats treated with antipsychotic drugs. There was a strong trend $(p=0.05)$ to lower cortical Ptgs1 10 months after mice were treated postnatally with Poly I:C. In CCF-STTG1 cells, aspirin caused a dose dependent decrease in PTGS1 which was decreased further with the addition of risperidone but treatment with risperidone alone did not affect levels of PTGS1.

Conclusions: Our data suggests that low levels of PTGS1 in the DLPFC could be involved in the pathophysiology of schizophrenia and that levels of mRNA are increased (Narayan et al, 2008) to compensate for low levels of protein. However, our data from CFF-STTG1 cells suggests that treatment with aspirin lowers levels of PTGS1 and this effect is more pronounced in the presence of risperidone. This raises the possibility that the improved therapeutic outcome from treating schizophrenia with antipsychotic drugs augmented with aspirin may be because such treatment lowers cortical PTGS1. Our combined data could therefore suggest that the lower levels of PTGS1 in the DLPFC in schizophrenia already reflects an attempt to maintain cortical homeostasis by counteracting other changes caused by the pathophysiology of the disorder and that treating with antipsychotic drugs and aspirin augments this process. The lower levels of cortical Ptgs1 10 months after Poly I:C likely reflects a long-lasting outcome from the impact of postnatal immune activation. Hence, lower levels of PTGS1 in the DLPFC from subjects with schizophrenia may be associated with the immune / inflammatory component of the aetiology of the disorder ( $\mathrm{Na}$ et al, 2014).

Aïd S, Bosetti F (2011). Targeting cyclooxygenases-1 and -2 in neuroinflammation: therapeutic implications. Biochimie 93(1): 46-51.

$\mathrm{Na}$ KS, Jung HY, Kim YK (2014). The role of proinflammatory cytokines in the neuroinflammation and neurogenesis of schizophrenia. Prog Neuropsychopharmacol Biol Psychiatry 48: 277-286.

Narayan S, Tang B, Head SR, Gilmartin TJ, Sutcliffe JG, Dean B, et al (2008). Molecular profiles of schizophrenia in the CNS at different stages of illness. Brain Res 1239: 235248.

Keywords: Schizophrenia, Aspirin, Dorsolateral Prefrontal Cortex, Prostaglandin-Endoperoxide Synthase 1, Postmortem Human Brain

Disclosure: Nothing to Disclose.

\section{M217. Antipsychotic-Induced Hypothalamic Inflamma- tion as a Potential Mediator of Metabolic Side Effects}

Chantel Kowalchuk, Denise Belsham, Gary Remington, Margaret Hahn*

Centre for Addiction and Mental Health, Toronto, Canada

Background: Antipsychotics (AP)s are the cornerstone treatment for schizophrenia but cause serious metabolic dysregulation. The hypothalamus is the primary brain region responsible for energy regulation, and inflammation in this region has been implicated in impaired energy homeostasis resulting in insulin resistance and weight gain. Thus, hypothalamic inflammation could be involved in the metabolic disturbances seen with AP use. Data in relation to AP-associated changes in inflammatory markers in schizophrenia has been inconsistent, owing in part due to confounds of illness- related factors (e.g. diet, smoking), and secondary effects of weight gain.

Methods: To examine direct, molecular effects of APs in the hypothalamus, an immortalized rat hypothalamic cell line, rHypoE-19, was treated with 2.5 and $100 \mathrm{uM}$ of olanzapine and clozapine. Quantitative real-time PCR was performed to determine changes in the mRNA expression of interleukin (IL)-6, IL-10, TNF-alpha and brain derived neurotrophic factor (BDNF). Western blots were used to detect changes in the activation of AMPK, components of the insulin signaling pathway (AKT, GSK3B), and components of the MAPK pathway (ERK1/2, JNK, p38), the latter which are linked to inflammation. 
Results: There was a significant increase in IL-6 and trend increase in TNF-alpha mRNA expression with $100 \mathrm{uM}$ olanzapine treatment versus the control, along with a significant increase in ERK1/2 and JNK protein expression. Clozapine (100 uM) also significantly increased ERK1/2 protein expression, along with increases in AMPK phosphorylation. Clozapine (100uM) inhibited insulin-mediated phosphorylation of AKT. Both antipsychotics increased BDNF expression.

Conclusions: Olanzapine may induce hypothalamic inflammation, which would be predicted to result in metabolic dysregulation. While clozapine did not activate pathways directly linked to inflammation, it increased AMPK phosphorylation (activation) an orexigenic energy sensor, and inhibited the insulin signalling pathway; both effects would be expected to disrupt energy and glucose homeostasis. Clozapine and olanzapine also increased BDNF suggesting a potential therapeutic effect as decreases in BDNF have been linked to the underlying etiology of schizophrenia. Taken together, our findings suggest a differential effect across the two most metabolically active antipsychotics (i.e. clozapine and olanzapine) on neuroinflammation and energy sensing in the hypothalamus, warranting further exploration.

Keywords: Antipsychotic, Metabolic Side-Effects, Neuroinflammation, BDNF, Schizophrenia

Disclosure: Nothing to Disclose.

\section{M218. Cognitive Training Effects on Emotion Regula- tion, Impulsivity, and Aggression in Schizophrenia}

\section{Anthony Ahmed*, Matthew Hoptman, Jean-Pierre Lindenmayer}

\section{Weill Cornell Medical College, White Plains, New York,} United States

Background: Cognitive deficits contribute to functional impairments in people with schizophrenia including aversive social behaviors such as aggression. Overlap in the neural circuitry implicated in cognitive deficits, emotion regulation abnormalities, impulsivity, and reactive aggression suggest that emotion regulation and impulse control are part of a causal chain that links cognitive deficits to aggression. Recent studies suggest that cognitive training interventions may decrease aggression risk in people with schizophrenia. The current study attempted to elucidate a mechanism of cognitive training effects on aggression by examining training effects on emotion regulation, impulse control, and reactive aggression. In particularly, changes in behavioral expressions of emotion regulation and impulsivity as a result of cognitive training were the focus of the study.

Methods: Participants were 24 people with schizophrenia and schizoaffective disorder recruited from NewYork Presbyterian Hospital (NYPH) and Manhattan Psychiatric Center (MPC). Participants were randomized to one of two cognitive training groups-a Combined Cognitive Remediation and Social Cognition Training (CRT+SCT) group or Cognitive Remediation alone (CRT). Participants assigned to the CRT+SCT group completed 24 hours of CRT and 12 hours of SCT. Participants in the CRT only group completed 24 hours of CRT and 12 hours of mundane computer activities.
Participants completed the MATRICS Consensus Cognitive Battery (MCCB), the Reading the Mind in the Eyes Task (Eyes Task), and the Penn Emotion Recognition Task (ER-40). We indexed emotion regulation capacity using the Positive and Negative Affect Scale (PANAS) and by obtaining heart rate, respiration, and electrodermal activity while participants viewed pictures selected from the International Affective Picture System (IAPS). IAPS pictures were neutral or emotionally-evocative. Emotionally-evocative pictures were preceded by either a description of the picture or a reappraisal statement. Participants completed the Go No-go task and the Emotional Stop Signal task as measures of impulsivity. Aggression was assessed using two behavioral tasks-the Taylor Aggression Paradigm and the Point Subtraction Aggression Paradigm.

Results: Preliminary pre-post analysis of the major study outcome variables showed that both cognitive training groups demonstrate improvements from baseline to endpoint assessments.

Aggression: On the primary study outcome variableaggression-there was a reduction in aggressive incidents as measured by the Overt Aggression Scale (OAS) (Cohen's $\mathrm{d}=-0.98$ ) and the provoked trials of the Point Subtraction Aggression Paradigm (PSAP) (Cohen's $d=-0.39$ ). There were minimal differences between the CRT alone and the $\mathrm{CRT}+\mathrm{SCT}$ groups in reductions in aggression.

Cognition: Although both cognitive training groups showed improvements in cognition from baseline (Cohen's $d=0.78$ ), we are starting to see some separation with the CRT+SCT demonstrating greater improvements on the composite cognition score of the MATRICS Consensus Cognitive Battery (MCCB) (Cohen's d = 0.22).

Both groups also improved in their ability to recognize facial emotions in others as measured by the Emotion Recognition40 (ER-40) (Cohen's $d=1.26)$, and in their theory-of-mind or mentalizing capacity (Cohen's $\mathrm{d}=1.27$ ) as measured by the Reading the Mind in the Eyes Task (Eyes Task). The CRT +SCT group demonstrated greater improvements in the ability to recognize facial emotions in others (Cohen's $\mathrm{d}=0.21$ ), and in their mentalizing capacity (Cohen's $\mathrm{d}=0.39)$.

Emotion Regulation: Although both groups demonstrated reductions in PANAS negative affectivity scores from baseline (Cohen's $\mathrm{d}=-0.48$ ), reductions were greater in the CRT+SCT group (Cohen's $\mathrm{d}=-0.24$ ).

We computed Heart Rate Variability scores differenced for emotionally-evocative pictures relative to neutral pictures. Both groups demonstrated pre-to-post reductions in their Low Frequency/High Frequency heart rate variability ratio (Cohen's $\mathrm{d}=-0.83$ ). Both groups also showed pre-to-post reductions in skin conductance (Cohen's $d=-0.48$ ). Group differences in HRV and skin conductance were very minimal.

Impulsivity: Both groups demonstrated large pre-to-post reductions in misses on the No-Go trials of the Go No-Go Task (Cohen's $d=-1.74$ ). Reductions were greater in the CRT+SCT than the CRT only group (Cohen's $d=0.49$ ) suggesting that the CRT+SCT group show greater improvements in impulse control following cognitive training.

Conclusions: Although results are very preliminary, cognitive training appears to contribute to improvements in emotion regulation and impulse control capacity. Changes 
apparent in all behavioral measures were associated with reductions in aggression incidents. Cognitive deficits may be viable targets for enhancing emotion regulation and impulse control capacity and decreasing the risk of aggressive behavior in people with schizophrenia.

Keywords: Cognitive Remediation, Aggression, Social Cognition, Emotion Regulation, Impulsivity

Disclosure: Nothing to Disclose.

M219. Chronic THC Administration and Post-THC Abstinence Produce Prolonged Sleep Disturbances in Mice in an Antagonist-Free Abstinence Paradigm

David Lovinger*, Karina Abrahao, Matthew Pava

National Institute on Alcohol Abuse and Alcoholism, Rockville, Maryland, United States

Background: Use of cannabinoid drugs is widespread throughout the world, and the DSM-V describes criteria for cannabis withdrawal syndrome. However, the diagnosis of cannabis withdrawal is contentious because reliable, objective measures of withdrawal have been difficult to observe with this drug. This is due, in part, to the long biological half-life of phytocannabinoids like delta-9tetrahydrocannabinol (THC), the major psychoactive ingredient in cannabis derivatives. Indeed, it is not clear how many of these withdrawal symptoms can be modeled in experimental animals where withdrawal is usually precipitated by administration of an antagonist for the cannabinoid type 1 (CB1) receptor in rodent studies. This does not mimic the human situation involving simple cessation of drug taking. Sleep disturbances (e.g. insomnia, vivid dreams, etc.) during acute abstinence following chronic cannabis use are a central feature of the criteria for cannabis withdrawal syndrome. We sought to determine if cannabis withdrawalinduced changes in sleep can be modeled in an antagonistfree rodent paradigm.

Methods: We hypothesized that a well-characterized method of reliably inducing cannabinoid tolerance in rodents (Bass \& Martin, 2000) would result in impaired sleep. We tested this hypothesis using electrocorticogram and electromyogram recordings from chronically implanted mice combined with our fully automated sleep analysis system to score sleep before, during and after chronic treatment with either THC or vehicle (1:1:18 mixture of DMSO, Cremaphor, and $0.9 \%$ saline). Subjects were maintained on a 12:12 light:dark cycle throughout the experiment. Baseline data ( $48 \mathrm{hr}$ ) were obtained prior to systemic administration of vehicle to all mice just before the onset of the dark photoperiod (ZT 11:30). Subjects were then divided into vehicle- and chronic THC-treatment groups. In total, 8 injections were administered over 6 days during this treatment regimen.

Results: The first THC injection augmented total time spent in non-rapid eye movement (NREM) sleep, but this effect was significantly attenuated following the last injection in the chronic treatment regimen. The reduction in the acute hypnogenic effect of THC is consistent with other physiological indices of tolerance observed with this treatment regimen. Measurements obtained over 6 days following cessation of THC treatment revealed that time spent in NREM sleep was reduced largely because of a decrease in NREM bout duration. Rapid eye movement (REM) sleep was also reduced on the first day of acute THC administration and was enhanced 6 days after the end of treatment. The REM augmentation during the abstinence phase was due to an increase in the number of REM bouts, and this effect persisted throughout the 6 days of abstinence. None of these changes were observed with vehicle treatment. Paradoxically, the power of delta oscillations $(0-4 \mathrm{~Hz})$ was no different between THC and vehicle during the first day of abstinence, but THC-treated mice displayed markedly less delta power by the last day of abstinence. Thus, impairment of processes contributing to slow oscillations in the cortex appears to gradually manifest over recovery from chronic THC exposure. To assess the effects of extended abstinence from chronic THC treatment, $48 \mathrm{hr}$ recordings were obtained from these mice after 77 days of abstinence. Preliminary findings suggest that NREM sleep time may be augmented following extended abstinence from chronic THC, and the reduction in delta power during NREM appears to persist at this time point. Thus, the mechanisms contributing to altered cortical delta oscillations persist throughout prolonged abstinence after chronic THC treatment.

Conclusions: These findings indicate that a widely-used chronic THC regimen can produce overt withdrawal symptoms without antagonist precipitation, and in addition, extended abstinence from chronic THC may reveal allostatic processes that emerge during the recovery from acute withdrawal. Further studies will examine the neurobiological mechanisms underlying these chronic THC actions.

Keywords: Cannabis Use, Tolerance, Drug Use

Disclosure: Part 1: Elsevier, Ltd., Honoraria.

\section{M220. Nonclinical Evaluation of Abuse Liability for a Novel Dual Orexin Receptor Antagonist Lemborexant - Comparison to Suvorexant}

Shoji Asakura*, Motohiro Shiotani, David Gauvin, Atsushi Fujiwara, Takashi Ueno, Nancy Bower, Carsten Beuckmann, Margaret Moline

Eisai Co., Ltd., Tsukuba, Japan

Background: Most sleep medications including suvorexant (SUV), approved in Japan, the US and Australia, are controlled substances regulated under existing federal laws. Sleep-promoting drugs with less abuse liability are desirable. Lemborexant (LEM), a novel dual orexin receptor antagonist (DORA), is currently being developed for the treatment of insomnia and irregular sleep-wake rhythm disorders. Nonclinical studies were conducted to evaluate the abuse liability potential of LEM according to the recommendations of ICH M3(R2), and included SUV as an active comparator, a drug in the same pharmacological class (DORA) for which the results of the nonclinical studies to evaluate abuse liability have been published. In the US, SUV is a schedule IV substance, and has been reported to demonstrate partial generalization with zolpidem (ZOL, GABA agonist) in rats and also similar reinforcing properties 
as ZOL during clinical trials for insomnia disorder (79 FR 52143, 08-28-2014).

Methods: Three nonclinical studies, a physical dependence study in rats, self-administration study in monkeys, and drug discrimination study in rats, were conducted to evaluate the potential for the abuse liability of LEM. All LEM doses were selected as to achieve sufficiently higher plasma concentrations than would be needed to promote sleep in humans, but not to show significant toxicity in animals. In the physical dependence study, LEM (200 and $600 \mathrm{mg} / \mathrm{kg} /$ day) was administered orally by gavage to SD rats twice a day for 28 days followed by a 7-day withdrawal period; diazepam was the positive control in the study. In the selfadministration study, catheterized rhesus monkeys were allowed to self-administer LEM for 2 hours/day with a descending dose-manner from 0.3 to $0.003 \mathrm{mg} / \mathrm{kg} /$ infusion for 4 to 7 days/dose. Pentobarbital was used as a positive control, and the response to the positive control was confirmed in each animal before self-administration of LEM. In the drug discrimination study, a standard twochoice lever press response under a FR10 schedule of food deliveries was used to train female SD rats to differentiate between oral gavage dose of Zolpidem $(3 \mathrm{mg} / \mathrm{kg} /$ day $)$ and vehicle. Once trained, cross generalization tests were conducted with LEM $(10,30,100$ and $1000 \mathrm{mg} / \mathrm{kg})$ and SUV (30, 100, 300 and $1000 \mathrm{mg} / \mathrm{kg})$. ZOL-like response was presented as mean \% of ZOL-appropriate responding in 6 rats in each dose.

All experimental protocols in animal studies were approved by the Institutional Animal Care and Use Committee and were conducted by the applicable requirements of the institution.

Results: Expected results were obtained for positive control treatments in all studies conducted, and therefore these studies were considered to be valid. In the physical dependence study ( 28 days of doses up to $600 \mathrm{mg} / \mathrm{kg} / \mathrm{day}$ ), no physical dependence in rats was noted during the 7 - day withdrawal period. In the self-administration study, there were no gross behavioral changes or increase in the number of self-administrations of LEM, compared to that of the vehicle. In the drug discrimination study, LEM did not produce a dose-dependent generalization (increase of ZOLlike responses) at doses from 10 to $1000 \mathrm{mg} / \mathrm{kg} /$ day; however, SUV produced a dose-dependent partial generalization, i.e. $49 \%$ ZOL-like response at the highest dose, in this study. Results obtained for SUV in the study were comparable to results of a drug discrimination study with SUV reported by Born et al. (2017), in which SUV produced ZOL-like responses up to a maximum of approximately $40 \%$.

Conclusions: Neither physical dependency nor reinforcing effects in the self-administration study were identified in animals treated with LEM. A lack of cross-generalization to ZOL was observed in rats treated with LEM, which contrasts with a partial generalization reproduced in rats treated with SUV in the current study. No abuse potential of LEM was identified in the standard battery of nonclinical abuse liability studies described in the ICH guidelines.

Keywords: Abuse Liability, Preclinical, Orexin Receptor Antagonist, Irregular Sleep/Wake Rhythm Disorder

Disclosure: Nothing to Disclose.
M221. Efficacy of the Novel Dual Orexin Receptor Antagonist Lemborexant in a Preclinical Animal Model for Irregular Sleep Wake Rhythm Disorder

Carsten Beuckmann*, Yoshihide Osada, Margaret Moline, Patricia Murphy, Andrew Satlin

Eisai Co., Ltd., Tsukuba, Japan

Background: Irregular Sleep Wake Rhythm Disorder (ISWRD) is a common feature in patients with Alzheimer's disease (AD). While patients with ISWRD and insomnia have disrupted nighttime sleep, in contrast to insomnia, ISWRD is a circadian rhythm sleep disorder, where patients also have disrupted daytime wakefulness, with several mostly involuntary naps, and where the sleep/wake patterns are irregular from day to day. There are no approved treatments for ISWRD. Improving nighttime sleep and daytime wakefulness may also improve daytime function in $\mathrm{AD}$ patients.

There are currently no nonclinical pharmacological models described for ISWRD in any corresponding population (AD or other) or its treatment. In this study, we aimed to identify a mouse model for ISWRD relevant for AD and which is amenable to pharmacological intervention with an orexin antagonist.

Methods: Animal experiments were approved by the Animal Care and Use Committee of Eisai Co., Ltd. Male mice, senescence-accelerated mouse prone 8 strain (SAMP8) and senescence-accelerated mouse resistant 1 strain (SAMR1) as control (Japan SLC, Inc., Hamamatsu, Japan) were maintained under a 12-hour light-dark cycle, with food and water available ad libitum. SAMP8 and SAMR1 mice were implanted with epidural brain electroencephalogram (EEG) and nuchal muscle electromyogram (EMG) electrodes at 18-19 weeks of age. Three weeks later, mice were dosed orally with vehicle, 3 or $30 \mathrm{mg} / \mathrm{kg}$ lemborexant (LEM), a novel dual orexin receptor antagonist, at onset of light (Zeitgeber time $0.0-0.5$ hours) in a randomized, 3-way cross-over design on three consecutive days, with each treatment period being 1 day with continuous EEG/EMG recording. Vigilance states (wakefulness, non-REM sleep, REM sleep) were automatically determined based on EEG/EMG signals for individual 10- seconds epochs (SleepSign software v3.3.9.1609; Kissei Comtec, Japan), and were verified and, if necessary, corrected by a trained observer for 8 animals per strain. Vigilance states were expressed as $\%$ of time (11.5 hrs for light period, $12.0 \mathrm{hrs}$ for dark period). Circadian phase of wakefulness distribution was calculated using RStudio software (v1.0.136, RStudio, Inc.). Statistical analysis of wakefulness, non-REM sleep, REM sleep and latency, and sleep latency was performed with a linear mixed model which specified animal as random effect, and day, strain, treatment and the interaction between strain and treatment as fixed using SAS software (v9.3, SAS Institute Inc., Cary, NC, USA).

Results: Vehicle-treated SAMP8 mice $(n=8)$ showed significantly more absolute amounts of wakefulness, less non-REM sleep, and less REM sleep time than vehicletreated SAMR1 mice $(n=8)$ during the light (resting) period. When administered at the beginning of light period, in a dose-dependent manner, LEM significantly both reduced absolute amounts of wake and increased non-REM sleep in both strains of mice. The amounts of wake and non-REM sleep in LEM-treated SAMP8 mice normalized to the level of 
vehicle-treated SAMR1 mice. Similarly, a significant and dose-dependent increase in the amount of REM sleep in both strains in the light period was observed. Furthermore, LEM also significantly and dose-dependently reduced the latency to sleep onset and REM sleep in both strains of mice.

During the dark period, previous administration of LEM at $30 \mathrm{mg} / \mathrm{kg}$ to SAMR1 mice during the light period led to a significant decrease of wakefulness and increase of non-REM sleep. In contrast, dosing with LEM led to a significant and dose-dependent increase in wakefulness, and decreases in non-REM sleep and REM sleep in SAMP8 mice.

Analysis of circadian phase of wakefulness indicated that it is advanced in vehicle-treated SAMP8 mice relative to vehicletreated SAMR1 mice. Treatment with LEM resulted in a dose-dependent and significant delay (right-shift) of the wake phase in SAMP8 mice, resulting in a circadian phase similar to that in vehicle-treated SAMR1 mice. Interindividual variability in the phase of wakefulness in SAMP8 mice was higher than in SAMR1 mice. LEM reduced this variability, resulting in a more consistent, stable phase among SAMP8 mice following treatment.

Conclusions: SAMP8 mice have previously been described by others to have cognitive, behavioral, and pathophysiological deficits closely resembling AD.

In this study, this strain showed increased wakefulness time and reduced sleep time during the resting (light) period compared to SAMR1 control mice, comparable to nighttime ISWRD, since mice are nocturnal animals. Upon treatment with LEM at the onset of the resting period (like dosing before bedtime for ISWRD patients), SAMP8 mice had shorter sleep and REM sleep onsets as well as increased non-REM and REM sleep times during the resting period to levels seen in control mice. However, LEM also increased wakefulness during the active (dark) period, the equivalent of daytime in patients. LEM also normalized circadian phase and reduced intersubject variability. These preclinical effects support a scientific rationale for the clinical development of LEM in ISWRD. A Phase 2 trial is currently in progress.

In conclusion, we believe that SAMP8 mice are potentially the first animal model of ISWRD, and antagonism of the orexin pathway has the potential to be an effective treatment for ISWRD.

Keywords: Orexin Receptor Antagonist, Irregular Sleep/ Wake Rhythm Disorder, Circadian Rhythm, Mouse Model Disclosure: Part 1: Eisai Co., Ltd., Employee, Part 2: Eisai Co., Ltd., Employee, Part 3: Eisai Co., Ltd., Employee, Part 5: Eisai Co., Ltd., Employee.

M222. A Randomized, Placebo-Controlled, Phase 3 Study of Solriamfetol (JZP-110) in Patients With Narcolepsy: Effects on Wakefulness, Excessive Sleepiness, and Health-Related Quality-of-Life

Michael Thorpy ${ }^{\star}$, Colin Shapiro, Geert Mayer, Bruce Corser, Dan Chen, Joan Li, Lawrence Carter, Hao Wang, Jed Black, Helene Emsellem, Giuseppe Plazzi, Yves Dauvilliers

Albert Einstein College of Medicine, Bronx, New York, United States

Background: Solriamfetol (formerly known as JZP-110) is a selective dopamine and norepinephrine reuptake inhibitor with wake-promoting effects. Two phase 2 clinical studies in patients with narcolepsy type 1 and $2(\mathrm{NT} 1 / 2)$ showed that solriamfetol significantly improved wakefulness and reduced excessive sleepiness (ES). This phase 3 study provides further understanding of the safety and efficacy of solriamfetol in patients with $\mathrm{NT} 1 / 2$ and how this potentially translates into benefits on patient-reported function and health-related quality-of-life (HRQoL).

Methods: In this double-blind, randomized, placebo-controlled, parallel-group study, patients were randomized $(1: 1: 1: 1)$ to receive solriamfetol $75 \mathrm{mg}, 150 \mathrm{mg}, 300 \mathrm{mg}$, or placebo for 12 weeks. Key eligibility criteria were diagnosis of NT1/2 according to International Classification of Sleep Disorders- 3 criteria; mean sleep latency $<25$ minutes on the Maintenance of Wakefulness Test (MWT); Epworth Sleepiness Scale (ESS) score $\geq 10$; usual nightly sleep time $\geq 6$ hours. Key exclusion criteria were use of medication that could affect ES or cataplexy; nighttime or variable shift work; conditions other than narcolepsy that cause ES. Coprimary endpoints were change from baseline to week 12 in MWT mean sleep latency and ESS score. The percentage of patients reporting improvement on the Patient Global Impression of Change (PGI-C) was the key secondary endpoint, and HRQoL was assessed using the 36-item Short Form version 2 (SF-36v2). A mixed model with repeated measures was used for continuous variables, and chi-square test for the categorical variable of PGI-C. Safety evaluation included incidence of treatment-emergent adverse events (TEAEs), laboratory tests, and vital signs.

Results: A total of 239 patients were randomized, and 236 received $\geq 1$ dose of study drug (safety population), of whom $65.3 \%$ were female and $80.1 \%$ were white. Mean \pm standard deviation baseline values were $17.2 \pm 3.2$ for ESS, $7.5 \pm 5.7$ minutes for MWT mean sleep latency, and $46.3 \pm 8.6$ and $45.4 \pm 9.4$ on the SF-36v2 Physical Component Summary (PCS) and Mental Component Summary (MCS) scores, respectively. Efficacy analyses were based on a modified intent-to-treat population $(n=231)$. Solriamfetol $300 \mathrm{mg}$ and $150 \mathrm{mg}$ significantly increased MWT mean sleep latency at week 12 relative to placebo. Least squares (LS) mean (standard error [SE]) change from baseline was 2.1 (1.3) minutes for placebo; 12.3 (1.4) for $300 \mathrm{mg}$ and 9.8 (1.3) for $150 \mathrm{mg}$ (both $p<.0001$ ); and 4.7 (1.3) for $75 \mathrm{mg}$ (nonsignificant). ESS scores significantly decreased at week 12 with solriamfetol relative to placebo; LS mean (SE) changes from baseline were $-1.6(0.7)$ for placebo, -6.4 (0.7) for $300 \mathrm{mg}(p<.0001),-5.4(0.7)$ for $150 \mathrm{mg}(p<.0001)$, and -3.8 $(0.7)$ for $75 \mathrm{mg}(p<.05)$. At week 12, more patients reported improvement on the PGI-C with solriamfetol relative to placebo $(39.7 \%) ; 84.7 \%$ for $300 \mathrm{mg}(p<.0001), 78.2 \%$ for $150 \mathrm{mg}(p<.0001)$, and $67.8 \%$ for $75 \mathrm{mg}$ (nominal $p<.05$ ). Improvements with solriamfetol were observed as early as week 1 on the MWT and ESS (300 and $150 \mathrm{mg}$ ), and PGI-C (all doses). At week 12, the mean changes from baseline on the SF-36v2 were significantly greater than placebo $(p<.05)$ for the specific domains of Vitality at all doses of solriamfetol, General Health for the $75 \mathrm{mg}$ dose, and Role Physical and PCS for 150 and $300 \mathrm{mg}$ doses; there were no significant differences on the MCS or other domains. The most common TEAEs ( $\geq 5 \%$ across all solriamfetol doses) were generally dose dependent and included headache (21.5\%), nausea (10.7\%), decreased appetite (10.7\%), 
nasopharyngitis (9.0\%), dry mouth (7.3\%), and anxiety (5.1\%). One patient in the solriamfetol $150 \mathrm{mg}$ group had 2 serious TEAEs (non-cardiac chest pain and anxiety) that the investigator considered to be not related to study drug. Discontinuations due to TEAEs in solriamfetol $150 \mathrm{mg}$ $(n=3)$ and $300 \mathrm{mg}$ groups $(n=5)$ were more frequent than with placebo $(n=1)$.

Conclusions: In this phase 3 study in patients with narcolepsy, the increased wakefulness and reduced ES observed with solriamfetol were dose dependent; the $300 \mathrm{mg}$ dose resulted in the greatest benefits, which were observed as early as week 1. Changes in wakefulness and sleepiness were generally paralleled by patient-reported improvements in specific domains related to HRQoL such as Vitality. The safety profile was consistent with prior clinical studies of solriamfetol in narcolepsy and obstructive sleep apnea.

Support: Jazz Pharmaceuticals.

Keywords: Narcolepsy, Excessive Sleepiness, Solriamfetol, JZP-110

Disclosure: Part 1: Jazz Pharmaceuticals, Consultant, Merck \& Co, Inc., Consultant, Part 4: Jazz Pharmaceuticals, Grant, Merck \& Co, Inc., Grant.

M223. Transdiagnostic Effects of Ventromedial Prefrontal Cortex TMS on Drug Cue Reactivity: 3 Studies, 3 Drugs of Abuse

Tonisha Kearney-Ramos*, Logan Dowdle, Warren Bickel, Colleen Hanlon

Medical University of South Carolina, Charleston, South Carolina, United States

Background: Through advances in preclinical research over the last decade, we now have an unprecedented understanding of the neural correlates of drug-taking behavior. In both rodent models and clinical neuroimaging studies, it is apparent that drug cue-triggered behavior is regulated by medial frontal-striatal-limbic circuits. While preclinical studies can use invasive stimulation techniques to inhibit drug cue-evoked behavior, clinical neuroscientists are pursuing non-invasive theta burst stimulation (TBS) as a novel therapeutic tool to inhibit drug cue-associated behavior. Preliminary data from our laboratory has demonstrated that continuous TBS (cTBS) targeted at the ventromedial prefrontal cortex (VMPFC) can attenuate activity in these circuits in cocaine users. To extend upon our preliminary studies, and to evaluate the transdiagnostic relevance of VMPFC TMS on drug cue reactivity, we performed 3 independent shamcontrolled studies. We tested the hypothesis that VMPFC cTBS could selectively attenuate drug cue-evoked functional connectivity in these frontal-striatal-limbic circuits.

Methods: A total of 74 individuals were enrolled in 1 of 3 studies (Study 1: cocaine users, $n=25$; Study 2: alcohol users, $n=24$, Study 3: nicotine smokers, $n=25$ ). Functional neuroimaging data were acquired from all participants while they performed a drug cue reactivity task tailored to their drug of choice. Following the cue-reactivity paradigm, participants received 3600 pulses of real or sham VMPFC cTBS (110\% resting motor threshold) while they were primed to envision their last drug use session (using standardized imaginal techniques from exposure therapy). The cue-reactivity paradigm was repeated after the cTBS session. In Studies $1 \& 2$, participants received a session of real and sham cTBS (1-2 weeks apart). In Study 3, participants received 5 daily sessions of either real or sham cTBS. Generalized psychophysiological interaction (GPPI) analysis of fMRI data was used to characterize changes in drug cue-related functional connectivity following real and sham cTBS.

Results: Across all 3 studies, real but not sham VMPFC cTBS led to a specific decrease in cue-evoked functional connectivity between the VMPFC and putamen (a dorsal striatal structure typically implicated in habitual aspects of drug taking). In the cocaine users (Study 1), cTBS led to a decrease in cue-evoked functional connectivity between the VMPFC, left putamen, left caudate, and left insula. In the alcohol users (Study 2), cTBS led to a decrease in cue-evoked functional connectivity between the VMPFC, left putamen, right caudate, and ACC. After 5 days of cTBS, the smokers that received real cTBS also showed lower cue-evoked functional connectivity between the VMPFC and putamen than those that received sham cTBS, though the effect size did not reach significance.

Conclusions: These are the first studies to demonstrate the transdiagnostic efficacy of VMPFC TMS to modulate both striatal and salience craving circuits, thus, corroborating prior evidence from our lab indicating VMPFC as a viable TMS treatment target. These findings facilitate the next critical steps in developing inhibitory VMPFC TMS as an innovative, non-invasive neurobiologically-targeted intervention in substance use disorders.

Keywords: Transdiagnostic, Substance Abuse, Transcranial Magnetic Stimulation, Cue Reactivity, Functional Connectivity Disclosure: Nothing to Disclose.

\section{M224. BDNF is a Critical Regulator of Memory System Competition Following Withdrawal From Cocaine}

Eric Harvey, Nick Angelillis, Pamela Kennedy*

University of California, Los Angeles, California, United States

Background: Learned behaviors are regulated by both goaldirected and habit brain systems, and these systems can act cooperatively or competitively to mediate action selection and behavioral choice (McDonald \& White, 2013). Disruption in the balance between these two systems is a persistent and possibly pervasive symptom of the addicted phenotype (Everitt \& Robbins, 2016; Balleine \& O'Doherty, 2010). Whether maladaptive behaviors characteristic of drug addiction are supported by enhancements in habit learning systems (including the dorsolateral striatum, DLS), impairments in goal-directed systems (including the hippocampus, HC) or a combination of both remains poorly understood. In humans, deficits in tasks requiring the use of hippocampaldependent memory processing predict poor treatment outcomes in cocaine users and preference towards the use of striatal-dependent learning strategies has been associated with addictive drug use (Aharonovich et al., 2006; Bohbot et al., 2013). More recent data suggests that oral cocaine selfadministration selectively impairs hippocampal-dependent 
extinction learning and that dorsal striatal-dependent memory is enhanced by repeated cannabinoid exposure (Gabriele et al., 2015; Goodman and Packard, 2015). The neural mechanisms supporting the dissociable effects of drugs of abuse on memory systems in the brain remain poorly understood. Here we use behavioral, molecular and pharmacological approaches to identify neural adaptations that may support drug-mediated shifts from cognitive, goaldirected to habit learning systems.

Methods: To determine the extent to which withdrawal from repeated cocaine exposure biases competition between memory systems, adult male Long-Evans rats were given daily injections of cocaine $(20 \mathrm{mg} / \mathrm{kg} \cdot 14$ days). Three weeks later rats were trained and tested in the dual-solution plus maze task (Packard \& McGaugh, 1996). This task has been used previously to dissociate HC and DLS memory system function, and it has been shown that early in training, rats will use an HC-dependent (spatial) strategy to solve the task but that with overtraining there is a transition to DLSdependent (response-based) solutions. Rats were trained in daily sessions to approach a consistently baited goal arm from the same start arm. Probe trials in which rats were started from the opposite start arm were administered to test whether rats adopted an HC-dependent spatial strategy or a DLS-dependent response strategy to solve the task. A second cohort of animals received weekly injections of the kappa opioid receptor antagonist, JDTic $(20 \mathrm{mg} / \mathrm{kg}$, i.p.), throughout withdrawal and maze training and testing. The DLS and $\mathrm{HC}$ were collected 1 hour after behavioral testing to assess differential regulation of gene transcription associated with learning strategy.

Results: Saline and cocaine treated animals learned the task at the same rate. However, saline treated rats adopted a spatial, HC-dependent learning strategy whereas rats withdrawn from cocaine utilized a response/habit learning strategy dependent on the DLS. This memory system bias was associated with bidirectional regulation of brain derived neurotrophic factor (BDNF) in the HC and DLS, upregulated in DLS and downregulated in HC. JDTic administration throughout cocaine withdrawal normalized levels of BDNF in $\mathrm{HC}$ and restored HC-dependent learning.

Conclusions: Our results suggest that withdrawal from cocaine is associated with the pervasive use of inflexible habit memory systems in DLS, in lieu of a more flexible, rapidly updated memory system that involves HC. Further, we find that downregulation of $\mathrm{BDNF}$ in the $\mathrm{HC}$ may be a critical regulator of the transition from $\mathrm{HC}$-dependent to DLS-dependent behavioral learning following cocaine withdrawal and that this may be mediated through dynorphin signaling. The efficacy of many pharmacotherapeutic agents in treating cocaine addiction is enhanced by regular cognitive-behavioral therapy (CBT). Investigations in clinical populations have reported that long-term success of CBT treatment is positively correlated with cognitive and memory faculties that depend upon the hippocampus (HC). Our data reveal a complex and differential relationship between dynorphin and BDNF signaling in the HC and DLS and associated memory system function following withdrawal from cocaine. Further studies are underway to investigate these mechanisms.

Keywords: JDTic, BDNF, Hippocampus

Disclosure: Nothing to Disclose.
M225. Buprenorphine Integrated Care Delivery Project: Correlates of Mental Health Screening and Primary Care Outcomes

Tanya Alim*, Finie Richardson, Beverlyn Settles-Reaves, Suneeta Kumari, Elizabeth Akinfiresoye, Edwin Chapman, Walter Bland, Mark Johnson

Howard University, White Plains, Maryland, United States

Background: The Buprenorphine Integrated Care Delivery Project is a collaboration between Howard University and a community-based medical practice that seeks to deliver accessible, high-quality care to patients receiving buprenorphine (BUP) for chronic opiate addiction by linking them to comprehensive healthcare services via care coordination.

When mental illness and substance use co-occur, these illnesses interact leading to even greater disability, with lower rates of medication adherence, increased risk for psychiatric and substance use relapses, higher psychological distress, and worse treatment retention.

Care managers conducted assessments and developed individualized treatment plans to provide patients a medical home and access to appropriate clinical and non-clinical services. This care coordination seeks to address additional hurdles such as homelessness, unemployment, legal problems, mental health illnesses, and co-occurring diseases (e.g. HIV, hepatitis C, diabetes mellitus) that may hamper BUP treatment success.

Objectives of the study are to:1.-To describe the relationship between mental health screening and primary care outcomes and 2. To describe correlation between mental health screening, retention and barriers to treatment.

Methods: Population: Participants receiving buprenorphine therapy were recruited at the Howard University Department of Community and Family Medicine and Department of Psychiatry as well as a community-based medical practice. Inclusion criteria: Participants were age $\geq 18$ year old, met DSM-IV criteria for opioid dependence or undiagnosed, had medical insurance in DC, had the ability to provide informed consent, and ability to communicate in English.

Exclusion criteria: Patients that were pregnant or unwilling to use contraception (including OCPs, patch, Depo-Provera), breastfeeding or unwilling to stop breastfeeding, liver dysfunction (acute hepatitis, liver failure or hepatic dysfunction), suicidal ideation, and hypersensitivity to buprenorphine.

Behavioral Health Surveys: Mental health surveys were administered to patients using the M3 Behavioral Screening Tool (M3). M3 is a 27-question validated mental health assessment tool that is completed in three to five minutes and designed to screen for the symptoms of four common mental health conditions: Depression, Anxiety, Bipolar, and Posttraumatic Stress (PTSD) Disorders. M3 assessment was conducted during intake process to establish patient mental status at time of enrollment. HUUHI utilized M3 assessments to facilitate longitudinal measurement, monitoring for communication among care team members, which is an essential part of the Patient Centered Medical Home (PCMH).

HUUHI team used the M3 results, the accompanying risk assessment and patient discussion as the basis for 
formulating a comprehensive treatment plan. M3 is not designed to diagnose illness on its own however, a referral to a specialist may be recommended. The care management team completed the assessment at enrollment and will track, measure changes over time, and monitor patient's progress via the care plan.

Results: Of the patients screened, $56 \%$ of patients had total M3 scores of 33 or greater (Range $=33-94$ ). The mean total M3 score was 49 . The majority of participants, received subscores for Depression, Anxiety, PTSD and Bipolar Disorder. Of these Sub-scores, 63\% had elevated Depression scores, $82 \%$ had elevated Anxiety scores, $60 \%$ had indications of PTSD and $60 \%$ had scores indicative of Bipolar Disorder. More than half, had two or more combinations of the subscores and approximately half had sub-score combinations that were significantly high. These results indicate that many of the patients from this population had significant indications of mental disorders with combination pathology. The median retention rate of our patient population is 121 weeks or 847 days compared to the 53 day national standard recently reported by the RAND Corporation. The median retention rate is the best available quality benchmark for buprenorphine medicated assisted treatment, which addresses patient satisfaction and adequate dosing.

Other factors that lead to gaps in treatment include social determinants such as lack of housing or stable housing (which includes a large portion of this population), lack of employment or income, catastrophic events which might include neighborhood violence, death of loved ones, severe illness due to co-morbid conditions and incarceration.

Conclusions: The presence of mental illness (Dual-Diagnoses) present a significant challenge and barrier to successful substance use disorder treatment. Using M3, physicians can more effectively treat mood and anxiety disorders that impede compliance with medical treatments and adversely affect recovery from illness and physical health in general. Care coordination with mental health providers, primary care providers and other social services organizations and facilities helps manage patient treatments better, and improve patient outcomes. The Howard University Urban Health Initiative Program is a collaborative effort to better serve this population by improving patient access to care, adherence to prescribed medications and treatment regimen compliance, while maximizing the efficacy of the medications prescribed, as the cost in human suffering of these clients, as well as expense of current treatments are significant.

Keywords: Buprenorphine-Naloxone, Integrative Health, Care Coordination

Disclosure: Nothing to Disclose.

\section{M226. Human Neuronal-Like Cells Transdifferentiated From Blood Circulating Monocytes Express Cannabinoid Receptor 1 (CB1)}

\section{Dylan Van Kampen, Adam Lescallette, Alfredo Bellon*}

Penn State Milton S. Hershey Medical Center, Hershey, Pennsylvania, United States

Background: The cannabinoid receptor 1 (CB1) has been involved not only in the addictive effects of cannabis but also in the pathophysiology of schizophrenia. But the exact role of $\mathrm{CB} 1$ on these illnesses remains elusive, at least in part, due to our inability to access neurons that, on one hand, express this receptor and on the other, come directly from patients. Methods: We have developed a protocol to transdifferentiate blood circulating monocytes into neuronal-like cells by combining different growth factors, antioxidants and conditioned media. This model is a practical, inexpensive and noninvasive approach to obtain neuronal-like cells directly from patients. Unlike other models such as Induced Pluripotent Stem cells (IPSCs), the cell's genome is not altered with viral insertion (reprograming) which can become a confounder in illnesses with a strong but still misunderstood genetic component.

Results: We have transdifferentiated monocytes into neuronal-like cells from over 50 individuals and established that transdifferentiated neuronal-like cells resemble human neurons early in development, express several neuronal markers and present spontaneous action potentials as well as postsynaptic inhibitory and excitatory currents. During differentiation, these cells undergo similar structural stages to those present in neurons while developing from rounded neuroblasts. We have also determined that when these neuronal-like cells are exposed to either dopamine or colchicine, they respond similarly to neurons by retracting their neuronal arborizations. Moreover, transdifferentiation of monocytes deliver reproducible results in samples from the same individuals. In addition, we recently established via flow cytometry and RT-PCR that Monocyte-DerivedNeuronal-like cells (MDNCs) express CB1. Our results also indicate that CB1 agonists modify the structure of MDNCs in a similar way than what has been described in neurons.

Conclusions: Monocytes can be consistently transdifferentiated into neuronal-like cells that resemble human neurons during early brain development. Moreover, MDNCs express numerous neuronal proteins including CB1. Activation of CB1 in MDNCs elicits structural changes comparable to those observed in neurons. Therefore, this model opens the opportunity to study CB1 in vitro directly in neuronal-like cells from patients with schizophrenia and cannabis addition. Keywords: Cannabis Use Disorder, Psychosis, Schizophrenia; Technology

Disclosure: Nothing to Disclose.

\section{M227. Fronto-Striatal Deficits Associated With Self- Regulatory Control in Cannabis-Using Youth}

Marilyn Cyr, Gregory Tau, Martine Fontaine, Frances Levin, Rachel Marsh*

Columbia University Medical Center, New York, New York, United States

Background: Disturbances in self-regulatory control are involved in the initiation and maintenance of substance use disorders (SUDs), including cannabis use disorder (CUD) [Bechara, 2005; Chambers et al., 2003]. Most adults with SUDs began having problems with drugs and alcohol during adolescence [Chambers et al., 2003; Johnston et al., 2009], a period during which the neural circuits underlying selfregulatory capacities continue to mature [Tau \& Peterson, 2010]. Since, cannabis is the most commonly used illegal 
substance among adolescents, investigating the functioning of these circuits in adolescents with CUD may shed light on the emergence and maintenance of SUDs during this critical developmental period. The Simon Spatial Incompatibility Task requires the engagement of self-regulatory control to resolve cognitive conflict [Horga et al., 2011]. Thus, we used the Simon task with fMRI to investigate the functioning of the fronto-striatal and fronto-parietal circuits underlying self-regulatory control in cannabis-using youth.

Methods: Functional magnetic resonance imaging was acquired from 28 adolescents with cannabis use disorder (CUD) and 28 age-matched healthy adolescents during their performance on a Simon task. Briefly, participants were presented with a leftward or rightward pointing arrow on each trial that was either congruent or incongruent with its position (left or right) on the screen. They were instructed to respond as quickly and accurately as possible to the direction of the arrow by pressing a button on a response box using the index finger for left and the middle finger for right. For each participant, preprocessed time series data were modeled using a GLM and an Incongruent versus Congruent (Conflict) contrast was entered in a group level analysis.

Results: Adolescents from both group responded slower to Incongruent than to Congruent trials. There was no Group-byCondition interaction, indicating that both groups responded similarly to conflict. Imaging analyses revealed significant group differences in conflict-related (Incongruent versus Congruent) activation in fronto-striatal and fronto-parietal regions typically engaged during this task. Specifically, relative to their healthy counterparts, adolescents with CUD showed reduced activation in right inferior frontal gyrus (IFG), dorsolateral prefrontal cortex (dlPFC), as well as in bilateral ventral striatum, anterior cingulate (ACC) and orbitofrontal (OFC) cortices, and inferior and superior parietal gyri.

Conclusions: Our findings of reduced fronto-striatal and fronto-parietal activation in response to cognitive conflict in adolescents with CUD are consistent with previous neuroimaging findings showing altered function [Nestor et al., 2011], structure [Sorg et al., 2012], and connectivity [Worhunsky et al., 2013] across regions and circuits that support self-regulatory control. They are also consistent with reductions dopamine function in the striatum and decreased metabolism in prefrontal regions implicated in impulsivity and controlled behaviors, which likely contribute to impulsive drug taking in addiction [Volkow et al., 2011]. Future studies should examine whether dysfunction in these circuits can be used as a reliable marker of substance use and addiction, as well as targets for circuit-based interventions and prevention strategies in children.

Keywords: Functional MRI (fMRI), Cognitive Control, Substance Abuse Disorders, Adolescence, Self-Regulation Disclosure: Nothing to Disclose.

M228. The Effects of Methamphetamine Self-Administration on Social Preferences in Male and Female Prairie Voles

Adam Perry*, Bruce Cushing

University of Texas at El Paso, El Paso, Texas, United States

Background: While methamphetamine abuse has numerous physiological consequences, it is also associated with deficits in social cognition that often lead to interpersonal conflict and the erosion of important social connections- which affects both the person struggling with addiction and their loved ones. Therefore, it is essential to have a socially relevant animal model system to study the impact of methamphetamine use on social behavior. Here we use the prairie vole (Microtus ochrogaster) as they display many human-like characteristics (e.g. forming long-lasting pair bonds) that make it an ideal model system for investigating the effects of methamphetamine use on social relationships. Methods: Adult males and females were trained to selfadminister a palatable food reward for one week, and then methamphetamine was added to the pellets. Voles were given 6-hour self-administration sessions with dosage being increased every week (dose series: $0.0025,0.005$, or $0.01 \mathrm{mg}$ per pellet). This was repeated for 3 cycles, totaling 9 weeks of methamphetamine self-administration. Subjects remained housed with their same-sex sibling for the first two cycles and were then paired with a novel member of the opposite sex for the final cycle. Female voles do not display a spontaneous ovarian cycle and require exposure to a novel male in order to undergo estrus induction. Partner preferences were tested by giving animals a choice between their familiar mate or a novel member of the opposite sex and was conducted three times during the course of the experiment following 8, 22 and 23 days of cohabitation. The first two preference tests were conducted under withdrawal conditions, whereas the final test was conducted immediately after self-administration while voles were under the influence of methamphetamine. Thus, this study examined: 1) patterns of methamphetamine self-administration in naïve and pairbonded individuals, and 2) how methamphetamine use affected partner preferences in males and females.

Results: Preliminary findings suggest that methamphetamine intake is fairly equivalent in naïve males and females, but females appear to have higher breaking points suggesting greater motivation to self-administer methamphetamine compared to males. The pattern of oral methamphetamine intake suggests a dose-dependent effect, but this is primarily due to over-consumption during the first hour, which may occur due to the slower onset of drug effects following ingestion (as opposed to the more rapid effects following intravenous route of administration). Voles subsequently adjusted the number of rewards they earned in a dosedependent fashion to deliver a relatively stable amount of methamphetamine for the rest of the daily session. Partner preference results are currently being scored and will be presented along with other data, including the effects of methamphetamine self-administration on adrenal weights.

Conclusions: The prairie vole appears to be a potentially useful model, as we have demonstrated that operant oral methamphetamine self-administration is feasible. Furthermore, we have established dose response curves in males and females and shown that voles can regulate their drug intakepresumably in response to the interoceptive effects of methamphetamine.

Keywords: Methamphetamine, Self-Administration, Prairie Voles, Sex Differences, Social Behaviors

Disclosure: Nothing to Disclose. 
M229. Environmental Enrichment and Length of Abstinence Alter Motivation for Cocaine Revealing Novel RNA Expression Changes in the Nucleus Accumbens Correlated With Cocaine Seeking

Janet Neisewander*, Gregory Powell, Madeleine St. Peter, Annika Vannan, Trisha Chaudhury, Thomas Benson, Daniela Alcazar, Ryan Bastle, Nora PerroneBizzozero

\section{Arizona State University, Tempe, Arizona, United States}

Background: Following acquisition of cocaine self-administration, animals express increased cocaine-seeking behavior when denied drug access for extended periods of time (i.e., incubation effect). However, this behavior is reduced when animals are housed in environmental enrichment (EE) rather than in isolation during the abstinence period. Recent research into the effects of $\mathrm{EE}$ during adolescence prior to drug self-administration has shown significant alterations in the nucleus accumbens (NAc) transcriptome. Here we examined if EE utilized as a post-self-administration treatment incurs similar transcriptomic effects and whether such effects correspond to changes in cocaine seeking in male rats given a brief (1 day) or prolonged (21 days) abstinence period after cocaine self-administration. We hypothesized that cocaine-seeking behavior would positively correlate with expression of genes commonly linked to motivation for cocaine and negatively correlate with neuroprotective genes.

Methods: Male Sprague-Dawley rats were trained to selfadminister cocaine $(0.75 \mathrm{mg} / \mathrm{kg} /$ infusion i.v., paired with a light+tone cue) across a minimum of 21 sessions. A separate group of saline rats that received infusions timed to a yoked cocaine animal served as a control. Following self-administration, rats were placed into abstinence for either 1 or 21 days, in either standard isolation housing or an enriched environment. EE chambers contained 3-6 rats, a running wheel, tubes, toys, and communal food and water. Upon completion of abstinence, animals were given a 1 - $\mathrm{h}$ test session during which cocaine-paired cues were presented response-contingently but no cocaine was available. Rats were immediately sacrificed after the session. Brain tissue was extracted, flash frozen, and tissue punches of the NA core and shell were collected and processed for RNA analysis using RNA-seq and RT-qPCR for validation.

Results: Following an abstinence period of 21 days, animals in isolation housing demonstrated increased cocaine-seeking behavior as measured by lever presses during the 1-hr cuereactivity test compared to animals in abstinence for 1 day. This effect was blocked by EE, which caused a significant reduction in active lever pressing after 21 days of abstinence compared to animals in standard housing conditions. RNAseq analysis revealed multiple genes differentially altered by cocaine self-administration, housing environment, and/or abstinence length. Ingenuity Pathway Analysis (IPA) designated the top network of genes affected by housing following 21 days of enrichment as Cell-Mediated Immune Response, Cellular Development, and Cellular Function and Maintenance. Other top networks include Psychological Disorders, Cell-To-Cell Signaling and Interaction, Cell Death and Survival, Cellular Movement, Nervous System
Development and Function, and Immunological Disease. Furthermore, gene expression results indicate over 400 genes significantly correlated with cocaine-seeking behavior. Specific genes with differential expression observed from RNAseq results include BDNF, complement component 3 (C3), heat shock $70 \mathrm{kDa}$ protein $1 \mathrm{~B}$ (Hspalb), multiple mitochondrial genes, and Satbl. The Satb1 gene is known to be regulated by epigenetic factors previously linked to cocaine exposure.

Conclusions: EE provides robust protection against increased cocaine-seeking behavior due to abstinence, comparable to the known protective effects of enrichment when given prior to drug use. Additionally, RNA-seq of nucleus accumbens tissue revealed multiple genes differentially affected by abstinence from cocaine self-administration or housing environment, indicative of potential pathways for treatment to reduce cocaine-seeking behavior. In particular, IPA analysis focusing on genes within the Cell-Mediated Immune Response, Cellular Development, and Cellular Function and Maintenance network provides a unique dataset for investigation of potential treatments linked to EE. Keywords: Cocaine Seeking, Environmental Enrichment, RNA-seq, Abstinence

Disclosure: Nothing to Disclose.

M230. Decreased Pro-Inflammatory Cytokine Expression Supports That Microglia Have an M2-Like Phenotype After Four-Day Binge Alcohol Exposure in Adolescent Rats

Kimberly Nixon*, Hui Peng

University of Kentucky, Lexington, Kentucky, United States

Background: Activation of the innate immune system may play a role in the development of alcohol use disorders (AUDs), a condition which often originates with adolescent alcohol use and abuse. Microglia, the macrophage of the central nervous system, play a key role in the innate immune system. Although several groups report "activated" microglia upon the insult of excessive or chronic alcohol exposure, microglia have different phenotypes that range on a continuum from pro-inflammatory/cytotoxic (M1-like) to anti-inflammatory/reparative (M2-like). While binge-like alcohol exposure results in beneficial or M2-like microglia in adult rats, it is not known what happens in adolescent rats which have a greater susceptibility to alcohol-induced pathological effects, including neuroimmune activation. Therefore, studies were designed to determine microglia phenotype in a 4- day binge model of alcohol dependence in adolescent rats.

Methods: Postnatal Day 35 male rats were gavaged with 25\% $(w / v)$ ethanol or isocaloric control diet every 8 hours for 4 days following a modified Majchrowicz protocol. Rats received $11.9 \pm 0.9 \mathrm{~g} / \mathrm{kg} /$ day ethanol, which yielded peak blood ethanol concentrations of $388.5 \pm 123.5 \mathrm{mg} / \mathrm{dl}$, measured on the third day of exposure. Withdrawal severity was scored 10-26 hours after the binge which peaked at $3.5 \pm 0.3$ indicated that most animals had some form of tremor and averaged $1.9 \pm 1$ on a scale of $0-4(3.8=$ convulsions, most severe). Rats were then sacrificed 2 days later by anesthetic 
overdose followed by transcardial perfusion of heparinized saline. Brains were rapidly extracted, the hippocampus dissected on ice and homogenized. Microglia were isolated from hippocampal homogenates by Percoll density gradient centrifugation. Some cells were labeled with microglia surface antigens and analyzed by flow cytometry while an aliquot was pelleted and lysed in TRIzol for later PCR detection of cytokine and growth factor gene expression.

Results: Consistent with our recently published studies in adult rats, isolated cells from adolescent rats were a highly enriched population of microglia/macrophages ( $>95 \%$ pure) according to immunoreactivity for microglia/macrophage surface protein, CD11b. Polarization states of microglia, identified as CD11b+CD45low, were determined by expression of M1 surface markers, major histocompatibility complex (MHC) II, CD32, and CD86, and M2 surface marker, CD206 (mannose receptor). As shown previously, microglia isolated from hippocampi of ethanol-treated adolescent rats show the most dramatic increases in M1 and M2 markers at T2 where expression of M1 markers, MHC-II, CD86, CD32, and M2 marker, CD206, were all increased significantly. Assessment of gene expression for cytokines and growth factors in these isolates showed decreases in pro-inflammatory gene expression: $62 \%$ decrease in TNF $(p<0.05), 50 \%$ decrease in CCL2 $(p<0.05)$, $65 \%$ decreases in IL1ß $(p<0.05)$ and IL6 $(p<0.05)$ and a $77 \%$ decrease in TGFß $(p<0.05)$. Simultaneously, a two-fold increase in BDNF expression $(p<0.05)$ was observed, though surprisingly, a $75 \%$ decrease in IGF1 expression was detected $(p<0.05)$.

Conclusions: These decreases in pro-inflammatory cytokines and increased BDNF gene expression complement the 2-fold increase in CD206 expression in microglia isolated from the hippocampus at T2. Altogether, surface marker expression coupled with gene expression supports that microglia may be more M2-like, i.e. anti-inflammatory or reparative, at 2 days after ethanol exposure in a binge model of an AUD. Determining microglia phenotype is critical for understanding the role of innate immune system activation in the development of AUDs.

Keywords: Microglial Activation, Alcohol Use Disorder, Adolescence, Neuroimmune, Ethanol

Disclosure: Nothing to Disclose.

\section{M231. Risk Taking and Valence: Dissociating Mesial Prefrontal Opioidergic and Dorsal Striatal Serotonergic Mechanisms}

Valerie Voon*, Juho Joutsa, Joonas Majuri, Eveliina Arponen, Sarita Forsback, Valterri Kaasinen

University of Cambridge, Cambridge, United Kingdom

Background: The evaluation of risk is intrinsic to our daily decisions and can underlie psychiatric pathology. Risk attitude can differ dependent on whether the outcome is rewarding or aversive. We have previously shown that goaldirected and habit control was associated with opposing ventral striatal mu-opioid receptor and serotonin transporter binding potential as a function of valence. Here we examine the neurochemical correlates of risk taking as a function of valence using three PET ligands to evaluate presynaptic dopamine function, mu-opioid receptor and serotonin transporter binding potential.

Methods: Thirty-nine subjects (17 healthy controls, 15 pathological gamblers, 7 binge eating disorders) were scanned using 3 PET ligands ([18 F]fluoroDOPA, [11C] carfentanil and [11C]MADAM) and tested on an anticipatory risk-taking task for reward and loss outcomes. Two multiple regression analyses were used to assess reward and loss separately for the relationship between risk taking probability weighting in four regions implicated in risk taking (striatum, ventromedial prefrontal cortex/medial orbitofrontal (vmPFC/mOFC) and insula) and controlling for the opposite valence (Bonferroni corrected $p<0.025$ ). Pearson correlations were also assessed between probability weighting and the same regions (Bonferroni corrected $p<0.004)$.

Results: No group differences were observed in risk taking propensity between groups. Across all subjects, the best fitting model for risk taking to reward was positively correlated with vmPFC and dorsal cingulate [11C]carfentanil $(\mathrm{F}=5.83, p=0.022 ; \mathrm{F}=21.32, p<0.0001)$. Greater risk taking to losses $(\mathrm{F}=7.61, p=0.002)$ was positively correlated with striatal [18 F]fluroDOPA and [11C]MADAM. The striatal findings were significant for both caudate and putamen but not ventral striatum. The Pearson correlation findings confirmed the multiple regression analyses in all except the [18 F]fluoroDOPA findings.

Conclusions: We show distinct neurochemical substrates underlying risk taking with the mesial prefrontal cortex muopioid receptor binding potential associated with rewards and dorsal striatal serotonin transporter binding potential associated with losses. These findings have implications for novel therapeutic substrates.

Keywords: Mu-Opioid Receptors, Serotonin Transporter, Risky Decision-Making

Disclosure: Nothing to Disclose.

M232. Achieving Analgesia in Buprenorphine Maintained Persons: A Comparison of Intravenous Buprenorphine and Hydromorphone

Andrew Huhn*, Eric Strain, George Bigelow, D. Andrew Tompkins

Johns Hopkins University School of Medicine, Baltimore, Maryland, United States

Background: Medication-assisted therapy (MAT) is a highly effective and safe treatment for opioid use disorder (OUD). The most widely used MAT in the United States is the partial mu-opioid agonist, buprenorphine. A common clinical concern regarding MAT is how to achieve analgesia for acute, moderate to severe pain in buprenorphine patients when needed in emergency or peri-operative settings. The high affinity of buprenorphine for the mu opioid receptor attenuates the efficacy of other opioid analgesics. To address this concern, we performed a double-blind, placebocontrolled human laboratory study comparing the analgesic effects of intravenous (IV) hydromorphone and IV buprenorphine on quantitative sensory testing (QST) in buprenorphine-maintained patients. QST is a set of experimentally induced pain methodologies (cold, heat, and 
pressure) that provides quantification of analgesic effects and has been used to evaluate opioid analgesia. We hypothesized that hydromorphone, a full mu opioid agonist, would provide superior analgesia compared to buprenorphine, and that both hydromorphone and buprenorphine would provide superior analgesia compared to placebo.

Methods: Medically stable and pain-free participants maintained on $12-16 \mathrm{mg}$ of once daily sublingual buprenorphine $(n=13)$ underwent three residential, double-blind controlled sessions examining the effect of IV placebo and of increasing doses of IV hydromorphone and IV buprenorphine on QST measures. Participants provided negative urine screens for all illicit substances (but positive for buprenorphine) at the beginning of each session. Each session began 17 hours after maintenance dose, with baseline measures scheduled to occur at 9:00 AM and first IV drug administration at 10:00 AM. Sessions were at least seven days apart, and employed a cumulative dose design in the active conditions: $4 \mathrm{mg}, 4 \mathrm{mg}$, $8 \mathrm{mg}, 16 \mathrm{mg}$ for both the hydromorphone and buprenorphine conditions (for cumulative doses $4 \mathrm{mg}, 8 \mathrm{mg}, 16 \mathrm{mg}$, $32 \mathrm{mg}$ ) with each injection administered 90 minutes apart. For safety reasons, rescue doses of naloxone were available (but never needed) in the event of significant respiratory depression; also, a physician was present throughout each session. QST occurred prior to the first IV challenge dose (baseline), as well as during six subsequent time points; four time points occurred 15 minutes after each IV test dose administration (to allow for the IV drug to reach peak effect), and the additional two time points occurred 90 and 180 minutes after the last injection to examine waning analgesic effects. QST outcomes included the cold pressor test (CPT), thermal pain test, and pressure pain test. The primary QST outcome was the CPT wherein the participant's hand was submerged in a circulating water bath held at $4 \mathrm{oC}$. $\mathrm{CPT}$ tolerance was defined as the duration the hand remained in the water bath prior to removal due to pain (up to 300 seconds). To control for individual differences on baseline CPT, change from baseline values were calculated for CPT threshold and tolerance. Repeated measures ANOVAs were used at each time point to examine cumulative dose effects on QST outcomes. Tukey's honestly significant difference (HSD) tests were used for planned post-hoc analyses looking at differences between conditions at each time point.

Results: CPT tolerance at each time point (measured in seconds) was subtracted from baseline measurements to examine drug-related differences in analgesic response. A cumulative dose of $16 \mathrm{mg}$ of hydromorphone elicited greater CPT tolerance in seconds $\mathrm{M}(\mathrm{SD}) 16.0(24.2)$, compared to $16 \mathrm{mg}$ of buprenorphine $1.2(14.6)$, or placebo $-1.8(6.7)(\mathrm{F}(2$, $12)=4.19, p=.028)$. A cumulative dose of $32 \mathrm{mg}$ of hydromorphone also elicited greater CPT tolerance in seconds $\mathrm{M}(\mathrm{SD}) 49.2(76.4)$, compared to $32 \mathrm{mg}$ of buprenorphine $6.5(15.9)$, or placebo $-1.8(5.3)(\mathrm{F}(2,11)=4.34$, $p=.026)$. Ninety minutes following the cumulative dose of $32 \mathrm{mg}$, hydromorphone still elicited greater CPT tolerance in seconds $\mathrm{M}(\mathrm{SD}) 39.8(71.3)$, compared to buprenorphine 2.1 (15.5), or placebo $-2.6(5.5)(\mathrm{F}(2,12)=3.69, p=.04)$. There were no significant differences in thermal or pressure pain. Importantly, there were no incidences of significant respiratory depression that led to participant withdrawal or need for use of naloxone.
Conclusions: These data represent the first controlled trial of acute analgesia in buprenorphine maintained individuals. Importantly, CPT tolerance was an effective measure of analgesic response in this population. In the current study, a cumulative dose of $16 \mathrm{mg}$ of hydromorphone was necessary to provide analgesia, and was given without significant respiratory depression. Thus, much higher doses of hydromorphone will likely be required in clinical settings to overcome buprenorphine tolerance for acute pain management.

Keywords: Opioid Agonist Treatment, Analgesia, Pain, Opioid Disclosure: Nothing to Disclose.

M233. Characterizing the Reinforcing Properties of Ethanol Within the Central Nucleus of the Amygdala: Sex Differences, Effect of Adolescent Alcohol Consumption, and Modulation by Neuropeptides

Christopher Knight, Sheketha Hauser, Robert A. Waeiss, Lauren Pratt, William McBride, Richard Bell, Zachary Rodd*

Indiana University School of Medicine, Indianapolis, Indiana, United States

Background: Alcohol has both stimulator and anxiolytic actions. Ethanol $(\mathrm{EtOH})$ is directly self-infused to the posterior ventral tegmental area (pVTA), nucleus accumbens shell (AcbSh) and the Central Nucleus of the Amygdala (CeA). Research examining the effects of comparable experimenter microinjections of $\mathrm{EtOH}$ into these brain structures has indicated that $\mathrm{EtOH}$ is anxiolytic in only the $\mathrm{CeA}$ (determined by social interaction, startle response, and open field behaviors). One of the main projections of the $\mathrm{CeA}$ is the bed nucleus of the stria terminalis (BNST). The CeA-BNST pathway is sexually dimorphic, and is thought to be a potential biological basis for the gender differences in the rate of anxiety and PTSD disorders in humans. In addition, there is a sex difference in rats for the number of $\mathrm{CRF}+$ neurons in the $\mathrm{CeA}$ and the effect of adolescent alcohol consumption on the expression of CRF neurons in the CeA. The current experiments were designed to determine the effect of sex on the reinforcing properties of $\mathrm{EtOH}$ in the $\mathrm{CeA}$, the effect of adolescent alcohol consumption on the reinforcing properties of $\mathrm{EtOH}$ in the $\mathrm{CeA}$ in both males and females, and to determine the role of neuropeptides mediating EtOH reward in the CeA.

Methods: Male and female Wistar rats were exposed to alcohol (intermittent exposure model; AIE) or water during adolescence (post-natal day \{PND\} 28-48, 4 g/kg gavage; $25 \%$ v/v EtOH, every other day). On PND 90, rats were implanted with a guide cannula aimed at the CeA. One week after surgery, rats were placed in two-lever (active and inactive) operant chambers and were able to directly self-administer 0 , $75,100,150,200$, or $250 \mathrm{mg} \% \mathrm{EtOH}$ directly into the CeA. In vitro experiments suggest that the effects of $\mathrm{EtOH}$ on GABAergic and glutamatergic transmission within the CeA are modulated by activity at the corticotropin releasing factor 1 receptor (CRF1) and by the neuropeptide Nociceptin. Experiments were designed to investigate the possibility that co-infusion of either the CRF1 antagonist NBI 35965 or Nociceptin would be able to block the rewarding effects of 
EtOH within the CeA. For this intra-cranial self-administration (ICSA) experiment, adult male Wistar rats were implanted unilaterally with guide cannulas aimed at the CeA. One week after surgery, rats were able to directly self-administer $200 \mathrm{mg} \% \mathrm{EtOH}$, a dose which is readily selfadministered into the CeA. All rats were allowed to selfadminister $\mathrm{EtOH}$ for the first 4 sessions (acquisition). During sessions 5 and 6 , rats were randomly assigned to groups receiving co-infusion of $200 \mathrm{mg} \% \mathrm{EtOH}$ and either $5 \mathrm{nM}$, $200 \mathrm{nM}, 25 \mu \mathrm{M}$, or $50 \mu \mathrm{M}$ of the CRF1 antagonist NBI 35965, or 100 or $500 \mathrm{nM}$ Nociceptin.

Results: The data indicated that naïve female Wistar rats will readily self-administer 75 or $100 \mathrm{mg} \% \mathrm{EtOH}$ directly into the $\mathrm{CeA}$, while male rats required concentrations of 200 or $250 \mathrm{mg} \%$ to support self-administration behaviors. Exposure to the AIE protocol results in a leftward and upward shift of the $\mathrm{EtOH}$ dose-response curve in both male and female Wistar rats. The pharmacological data indicated that in naïve Wistar rats, CRF antagonists did not alter EtOH selfadministration into the $\mathrm{CeA}$, but co-administration of Nocicpetin enhanced EtOH self-administration.

Conclusions: The current data are the first data set that indicates a major sex difference for the reinforcing properties of $\mathrm{EtOH}$ within a brain region. In addition, adolescent $\mathrm{EtOH}$ exposure enhances the rewarding properties of $\mathrm{EtOH}$ in the CeA during adulthood. Together, the data indicate that $\mathrm{EtOH}$ reward in the $\mathrm{CeA}$ is complex in nature and is readily influenced by sex, past alcohol history, and regulated by neuropeptides.

Keywords: Alcohol Self-Administration, Sex Differences, Adolescent Alcohol

Disclosure: Nothing to Disclose.

M234. Differential Modulation of Striatal Dopamine Release by Nicotinic Receptors in Adolescent and Adult Rats

Mark Ferris*, Ashley Fennell, Lacey Sexton

Wake Forest School of Medicine, Winston-Salem, orth Carolina, United States

Background: Variability in the rate in which animals will acquire self-administration of psychostimulants, including nicotine, is a preclinical model for vulnerability to abuse drugs in humans. The variability in animals' acquisition rate can be predicted by individual differences in animals' locomotor response to a novel environment. Therefore, we investigated whether the propensity to explore a novel environment might also predict individual differences in how nicotinic acetylcholine receptors (nAChR) located on dopamine terminals in the core of the nucleus accumbens modulate rapid dopamine signals. We chose $\mathrm{nAChRs}$ given their well-documented ability to modulate rapid dopamine signals that are critical for acquisition behavior. Finally, we investigated whether $\mathrm{nAChR}$ modulation of dopamine release is similar in adolescent vs. adult rats.

Methods: We assessed locomotor activity in an inescapable novel environment followed by measurements of dopamine release using voltammetry in brain slices from adolescent (PD 28-35) and adult (PD 75-80) rats. Following assessment of dopamine release under single pulse, paired-pulse, and multiple pulse conditions across a range of frequencies, mecamylamine (non-selective $\mathrm{nAChR}$ antagonist), $\mathrm{DH} \beta \mathrm{E}$ (Beta2-selective nAChR antagonist), $\alpha$-conotoxin PIA (alpha6-selective $\mathrm{nAChR}$ antagonist), and nicotine was bath applied to different slices from the same animal and dopamine release was reassessed.

Results: We found no relationship between the animals' locomotor response to a novel environment and the magnitude of electrically-stimulated dopamine release in the nucleus accumbens of either cohort. In fact, no relationship between these parameters was found when dopamine was elicited under single-pulse conditions and under multiple-pulse conditions across a range of frequencies $(5 \mathrm{~Hz}-100 \mathrm{~Hz})$. Following assessment of dopamine release, mecamylamine, $\mathrm{DH} \beta \mathrm{E}, \alpha$-conotoxin PIA, and nicotine was bath applied to different slices from the same animal. Only adult animals with low response to a novel environment (LR) showed inhibition in dopamine release to $\mathrm{nAChR}$ blockade or desensitization under single pulse and low frequency, multiple-pulse stimulations that reflect tonic firing of dopamine neurons. All adolescent animals regardless of locomotor activity and adult animals with a high response to a novel environment (HR) showed greater facilitation of dopamine release to $\mathrm{nAChR}$ blockade or desensitization under multiple-pulse, high-frequency conditions that reflect phasic firing of dopamine neurons.

Conclusions: LR adult animals are more sensitive to the dopamine inhibitory effects of nAChR blockade under tonic firing-like conditions while adolescent and adult HR animals are more sensitive to the dopamine facilitative effect of nAChR blockade under phasic firing-like conditions. The increased sensitivity to $\mathrm{nAChR}$-induced facilitation of dopamine signals in $\mathrm{HR}$ adult animals and adolescent animals may be mechanistically linked to faster and more robust acquisition of drugs of abuse and vulnerability to nicotine addiction in these populations.

Keywords: Nicotinic Acetylcholine Receptors, Dopamine, Ventral Striatum, Nicotine

Disclosure: Nothing to Disclose.

\section{M235. Examining Phenotypes in the Expression of Withdrawal From Opioids}

Kelly Dunn*, Elise Weerts, Andrew Huhn, Jennifer Schroeder, D. Andrew Tompkins, George Bigelow, Eric Strain

Johns Hopkins University School of Medicine, Baltimore, Maryland, United States

Background: Opioid use disorder (OUD) is a leading cause of morbidity and mortality related to opioids and is the leading cause of accidental death among adults in the United States. Individuals with opioid physical dependence experience a withdrawal syndrome when tapering off opioids. Treatments that manage withdrawal symptoms have highly variable success, due in part to individual differences in withdrawal symptom expression. Outcomes could be improved by tailoring pharmacotherapies and concomitant medications to the individual needs of a patient, but the mechanisms underlying the individual differences in withdrawal are not well-understood. This secondary analysis 
evaluated whether behavioral phenotypes of opioid withdrawal symptomology could be identified. This is a necessary first step towards determining the mechanisms underlying differences in opioid withdrawal symptom expression, which may ultimately be used to inform individualized treatment approaches.

Methods: Participants $(N=106)$ with OUD who were physically-dependent on opioids were admitted to a residential clinical research unit as part of a randomized controlled treatment trial (NCT00301210). All participants were maintained on morphine (30 mg, SC, QD) for 4-7 days before undergoing a precipitated withdrawal session that was conducted by administering the opioid antagonist naloxone $(0.4 \mathrm{mg}$ IM). Withdrawal ratings were collected every 15 minutes using the Subjective Opiate Withdrawal Scale (SOWS), a 16-item self-report rating of withdrawal severity. Participants were then randomly assigned to one of three double-blind, double-dummy groups to receive decreasing doses of clonidine, tramadol-ER, or buprenorphine over a 7- day period. Self-report, observer-rated, and physiological indices of withdrawal were collected daily and participants were monitored for an additional 7-days in a post-taper placebo-dosing period. Phenotypes were evaluated by deriving area-under-the-curve (AUC) values from the naloxone challenge session SOWS ratings for each participant and assessing whether participants clustered together using $\mathrm{K}$-means clustering. The associations of cluster assignment with demographics as well as subsequent outcomes from the primary trial were explored.

Results: Participants were a mean 40.6 years old, $15.1 \%$ female, and 54.7\% African American. A 3-cluster solution was originally identified, however examination revealed the 3 rd cluster was composed of persons with BMI $>30$, suggesting results may have been driven by differential drug absorption. When K-means clustering was restricted to individuals with BMI 18-30 $(N=89)$, a 2-cluster solution was identified that represented Low (cluster $1 ; N=52$ ) and High (cluster 2, N=37) Withdrawal phenotypes. BMI was not significantly associated with withdrawal phenotypes. Sex was significantly associated with cluster membership, with women representing $2 \%$ and $27 \%$ of the Low and High Withdrawal phenotypes, respectively $(p<.001)$. Evaluation of primary study outcomes revealed the Low Withdrawal phenotype was associated with more mild withdrawal during the primary trial, whereas the High Withdrawal phenotype was associated with higher levels of withdrawal across several different indices (self-report, observer, and physiological) and independent of experimental group assignment. Phenotype was also associated with differential likelihood of completing the taper, with $75 \%$, $76 \%$, and $100 \%$ of Low Withdrawal Phenotype participants in the clonidine, tramadol-ER, and buprenorphine groups completing the taper, in contrast to $39 \%, 60 \%$, and $80 \%$ of the High Withdrawal phenotype participants completing the taper, respectively.

Conclusions: These results have several implications. First, data suggest meaningfully different phenotypes in the expression of opioid withdrawal, characterized here as Low or High levels of withdrawal. Second, the phenotypes identified within the precipitated withdrawal session were associated with the outcomes of a clinical taper that was conducted under double-blind, double-dummy conditions, suggesting they are of clinical significance. Third, data suggest the results obtained from a precipitated withdrawal session correspond to clinical outcomes, which supports the use of precipitated withdrawal sessions to evaluate medications for opioid use disorder. Finally, few demographic variables were significantly associated with cluster membership, indicating that prospective research designed to examine the mechanisms underlying these results is necessary. Further exploration of these withdrawal phenotypes can be used to advance a personalized medicine approach to opioid withdrawal treatment approaches.

Keywords: Opioid, Opioid Withdrawal, Phenotypes Disclosure: Nothing to Disclose.

\section{M236. Persistent Cocaine Seeking is Controlled by Dendritic Reorganization via Stable Induction of Microtubule Proteins in Medium Spiny Neurons}

\section{Erin Calipari*, Arthur Godino, Marine Salery, Emily} Peck, Michael Cahill, Diane Damez-Werno, Amy Gancarz, Hannah Cates, Joseph Landry, Deena Walker, Drew Kiraly, Yasmin Hurd, David Dietz, Eric Nestler

Icahn School of Medicine at Mount Sinai, New York, New York, United States

Background: Drug addiction is a chronic relapsing disorder characterized by maladaptive learning about drugs and predictive cues that leads to compulsive drug seeking. Reward learning is a complex process controlled by a computational network of neural connections interacting with transcriptional and molecular mechanisms within each cell to precisely guide behavior. This process, which is dysregulated in addiction, has been attributed, in part, to the formation of new synaptic connections on medium spiny neurons in the nucleus accumbens (NAc). Currently, the molecular mechanism by which this process occurs, and how it is dysregulated in addiction, is not well understood; however, one important factor is to understand how longterm stabilization of these spines, and thus, cocaineassociated memories, is maintained and acts to drive drives drug seeking. Here we define the microtubule associated protein, end binding protein 3 (EB3), as a critical regulator of this process and directly link its ability to stabilize spines to persistent cocaine seeking.

Methods: Following a 1 day or 30- day withdrawal period from cocaine self-administration, western blot hybridization on the synaptic fraction was used to determine levels of the dendritic spine/microtubule associated proteins (MAPs): EB3, $\beta$-tubulin, Src kinase, and the NR2B subunit of the NMDA receptor. Using confocal imagining and dendritic spine analysis, alterations in spine density and morphology in the NAc following cocaine exposure were determined. Further, the role of microtubule polymerization in the rewarding/reinforcing effects of cocaine was confirmed with behavioral analysis, whereby the microtubule inhibitor podophyllotoxin was infused directly into the NAc before daily conditioned place preference (CPP) sessions. Viralmediated gene transfer in self-administering animals was used to over-express targets (EB3 and Srcin1-a negative regulator of Src kinase) via HSV vectors to determine their 
effects on the reinforcing efficacy of cocaine and directly link changes in these proteins to motivated behavior.

Results: Following short (1 day) withdrawal from cocaine self-administration increases in thin spines were observed, however, following long withdrawal periods (30 days) stubby spines were increased, suggesting that initial increases in immature spines served as a scaffold for the formation of mature, long-lasting synaptic connections that encode cocaine-associated information. Accordingly, following a 1- day withdrawal period Src phosphorylation at the active site (Y416) was increased and overexpression of Srcin1 blocked this effect. The initial changes in Src were also critical in the subsequent changes that occurred during withdrawal, where overexpressing Srcin1 reduced stubby spine formation 30 days later. These changes were positively correlated with drug seeking and motivation. The formation of mature synapses following longer withdrawal periods was linked to increased synaptic levels of beta-tubulin and EB3 which were positively correlated with both synaptic strength and cocaine consumption. Finally, the long-term increases in EB3 were shown to be via transcriptional processes, suggesting a role for epigenetic modifications to the EB3 gene in the long-term stabilization of synaptic function associated with cocaine self-administration.

Conclusions: We show that cocaine self-administration leads to the formation of immature dendritic spines which serve as a scaffold for the subsequent stabilization of cocaineassociated memories. During withdrawal microtubule entry into spines enhances EB3-dependent signaling cascades leading to long-term stabilization of spines and timedependent enhancement of cue-reward associations which drive addiction-related behaviors. The interplay between rapid, temporally specific neuronal activation, which is controlled via synaptic connections, and longer-term changes in transcription, which stabilizes these connections, is of critical importance in the expression of appropriate, or in the case of drug addiction, inappropriate behaviors. Understanding the complex interplay between these two factors will allow us to formulate novel epigenetic therapies that are likely to normalize maladaptive reward behaviors, with a goal of modulating addictive, but not natural reward behaviors.

Keywords: Dendritic Spines, Cocaine Self-Administration, Nucleus Accumbens, Brain Transcription, Addiction Circuitry

Disclosure: Nothing to Disclose.

\section{M237. The Gut Microbiome Modulates Motivational Properties of Self-Administered Cocaine}

Emily Peck, Arthur Godino, Nicholas Mervosh, Erin Calipari, Drew Kiraly*

Icahn School of Medicine at Mount Sinai, New York, New York, United States

Background: The resident bacteria of the gastrointestinal tract, collectively dubbed the gut microbiome, have been shown to have profound effects on both brain and behavior. The microbiome and its metabolites affect neuronal architecture, microglial responsiveness, function of the blood brain barrier, and many other aspects of brain function. To date, the majority of research into the effects of the microbiome in neuropsychiatric pathology has focused on affective and neurodegenerative disorders. However, our group recently demonstrated that altering the gut microbiome has a marked effect on behavioral and neurobiological responses to cocaine. Animals with depleted gut flora exhibited increased locomotor sensitization and a lower threshold for conditioned place preference for cocaine. Additionally, these mice had altered regulation of the genes encoding brain-derived neurotrophic factor, the D1 dopamine receptor, and other important regulators of behavioral response to cocaine. These data suggest the possibility that targeted manipulations of the endogenous gut flora, may be able to affect the course of addiction behavior in patients with cocaine use disorder - a recalcitrant condition with significant morbidity for which there are no currently available pharmacotherapies.

Methods: To knock down the gut microbiome, SpragueDawley rats were given a cocktail of non-absorbable broadspectrum antibiotics (Neomycin $2 \mathrm{mg} / \mathrm{ml}$, Bacitracin $0.5 \mathrm{mg}$ / $\mathrm{ml}$, Vancomycin $0.4 \mathrm{mg} / \mathrm{ml}$ ) via their drinking water for two weeks prior to any experimental manipulation. Rats were then implanted with indwelling jugular venous catheters, and trained to self-administer cocaine $(0.8 \mathrm{mg} / \mathrm{kg} /$ infusion $)$ on an FR1 schedule until they were stably responding. We then assessed drug seeking and intake utilizing a behavioral economics threshold task in which the "price" (lever presses/ mg cocaine) is slowly increased throughout the session. After completing this task, the animals were returned to FR1 responding for several days before going through five days of extinction training in which lever presses were not associated with any drug reward. Once responding was extinguished, animals were returned to their home cage for ten days to allow for incubation of cocaine craving. Following this incubation, animals were returned to test boxes and underwent either cue or cocaine-induced reinstatement of drug seeking. Following this final behavioral analysis, brains were dissected and processed for analysis of both protein and gene expression changes.

Results: While control and antibiotic-treated animals showed no differences in FR1 acquisition of cocaine administration, animals with depleted gut flora showed significantly enhanced responding for cocaine particularly at low levels of reinforcement. When the rats were subsequently put through an extinction task, the antibiotic-treated animals showed a more rapid extinction of drug seeking behaviors most pronounced in the first two days of the task. After a period of incubation in the home cage, reinstatement of cocaine-seeking behavior was assessed. Interestingly, the antibiotic-treated animals showed a significant increase in cocaine but not cue-induced reinstatement of cocaine seeking. Ongoing molecular analyses have demonstrated alterations in activation of key dopamine-responsive signaling pathways in the nucleus accumbens of antibiotic-treated animals following final cocaine-induced reinstatement.

Conclusions: These experiments demonstrate that manipulations of the gut microbiome significantly affect drug intake and seeking in a translationally-relevant animal model of drug addiction. Interestingly, depletion of gut flora affected all phases of the behavioral response. Additionally, molecular analyses suggest that changes in key molecular 
signaling pathways are altered in a manner that correlates with these behaviors. While these findings are still preliminary and will require considerably more follow-up and mechanistic insight, they do suggest that targeting the gut microbiome may represent a tractable therapeutic strategy for altering the course of substance use disorders in human patients.

Keywords: Gut Microbiome, Cocaine Self-Administration, Inflammation

Disclosure: Nothing to Disclose.

M238. Genes, Proteins, and Mechanisms Underlying PPAR $\gamma$-Mediated Attenuation of Cocaine Cue Reactivity

Larry Denner, Andrea Dimet, Irma Cisneros, Robert Fox, Sonja Stutz, Joy Schmitz, Scott Lane, Kathryn Cunningham, Kelly Dineley*

University of Texas Medical Branch, Galveston, Texas, United States

Background: Cocaine is a potent psychostimulant that can induce behavioral and cognitive changes through altered brain structure and function. Cocaine use disorder is a chronic relapsing condition characterized by compulsive drug seeking and drug taking, even after prolonged abstinence periods. Environmental cues repeatedly paired with drug effects can acquire conditioned reinforcing, rewarding and motivational properties through learning processes; themselves altered through repeated drug exposure. As a result, vulnerability to drug-associated cues ("cue reactivity") can promote craving and relapse in abstinent cocaine-dependent humans and drug-seeking behaviors in rodents.

Structurally, cocaine use disorder manifests as altered white matter (WM) tracts and gray matter (GM) structures utilized for higher-order neural circuit processing that supports cue reactivity: decision making, attention, and impulsivity. Rat cocaine self-administration (SA) and forced abstinence (FA) results in enduring parallel alterations, supporting the utility of rats as a preclinical model to study the brain alterations underlying cocaine cue reactivity. Signaling networks centered on ERK (extracellular-signal regulated kinase) are major mediators of these changes.

Our research has inter-related ERK and the peroxisome proliferator-activated receptor- $\gamma(\operatorname{PPAR} \gamma)$ in memory processes. We have exploited this relationship to discover that the FDA-approved PPAR $\gamma$ agonist pioglitazone (PIO, Actos) attenuates cocaine-seeking behavior (cue reactivity) in rats with PIO treatment during FA from cocaine SA. Importantly, the PPAR $\gamma$ antagonist GW9662 reversed this effect. This discovery prompted us to initiate a two-arm doubleblind randomized controlled proof-of-concept pilot trial of PIO (or placebo) to examine its effectiveness to suppress cue reactivity and improve WM integrity in cocaine-dependent subjects. We recently published that PIO reduced cocaine cue reactivity and improved WM integrity in cocainedependent subjects. Thus, PIO shows promise as a relapse prevention treatment for cocaine use disorder, consistent with our strong preclinical data.

We hypothesize that PPAR $\gamma$ agonism attenuates cocaine cue reactivity through the induction of a gene and protein repertoire that counteracts cocaine-mediated damage to WM and GM structures to rejuvenate circuit function and structural integrity.

Methods: All experiments were carried out in accordance with the National Institutes of Health Guide for the Care and Use of Laboratory Animals (National Research Council) and with the approval of the Institutional Animal Care and Use Committee at UTMB. Rats with right jugular vein-implanted intravenous catheters were trained to self-administer cocaine $(0.75 \mathrm{mg} / \mathrm{kg})$ via active lever press in 3-hour sessions; upon active lever press, rats received both the cocaine stimulus and discrete cues. Following 14 days of cocaine SA, rats underwent FA and received PIO treatment $(\sim 3.83 \mathrm{mg} / \mathrm{kg} /$ day). On FA day 30, animals underwent a 1-hour cue reactivity test where responding to the previously active lever was measured with the delivery of discrete cues, but no cocaine provided. Immediately following the cue reactivity test, rats were decapitated, brains subdissected into prefrontal cortex (PFC), hippocampus (HIP), amygdala (AMG), nucleus accumbens (NAC), and ventral tegmental area (VTA). RNA or nuclear extracts were prepared. RNA was sent for sequencing; nuclear extracts were stored frozen at -80 o C until analyzed.

A set of "no cue reactivity test" rats were harvested as a control for the behavioral experience of the cue reactivity session. Thus, our data describe effects of re-exposure to cocaine-related cues in the presence and absence of PIO.

RNA-sequencing and Ingenuity Pathway Analysis identified gene interaction networks. Quantitative immunoblot using Protein Simple Wes ${ }^{\mathrm{rx}}$ technology and immunofluorescence analyzed the expression of proteins. In vitro experiments investigated cocaine-related mechanisms and the effect of PIO using DNA fragmentation and antiviral/inflammatory markers as readouts.

Results: Ingenuity Pathway Analysis identified immune function, cell structural and functional integrity, and survival as top-ranking gene networks regulated by PPAR $\gamma$ agonism during cocaine FA and re-exposure to cocaine-related cues. Genes identified within these networks contain putative PPAR response elements (PPREs) and ERK-dependent cyclic AMP response elements (CREs), further supporting the inter-relationship of PPAR $\gamma$ and ERK in circuit function. Immunoblot and immunofluorescence results supported genomic results and our overall hypothesis that the expression of key proteins important to WM and GM integrity are modulated by PPAR $\gamma$ agonism during cocaine FA (e.g., GFAP, aquaporin 4) and/or by chronic cocaine exposure, FA, and re-exposure to cocaine-related cues (e.g., ERK2, pERK2, PPAR $\gamma$ ). Protein evaluation also showed regional specificity (e.g., PFC vs. HIP). Finally, in vitro experiments found that PPAR $\gamma$ agonism attenuates inflammatory signaling and DNA damage induced by cocaine, supporting our ex vivo results.

Conclusions: PPAR $\gamma$ agonism during FA from cocaine SA led to the induction of gene and protein repertoires that appear to rejuvenate circuit function underlying cue reactivity to attenuate cocaine-seeking behavior.

Keywords: Relapse Prevention, Cue Reactivity, Cocaine Seeking

Disclosure: Nothing to Disclose. 
M239. Healthy Human Male Dopamine D3 Receptor Uptake of [18F]Fluortriopride (FTP) Before and After Lorazepam Challenge

Robert Doot*, Jacob Dubroff, Kyle Labban, Jenny Cai, Hsiaojou (Sharon) Lee, Erin Schubert, Chia-Ju Hsieh, Catherine Hou, Regan Sheffer, Kuiying Xu, Shihong Li, Alex Schmitz, Finbarr O'Sullivan, Robert Mach

University of Pennsylvania, Philadelphia, Pennsylvania, United States

Background: Dopamine D3 receptors are localized in rewardrelevant brain regions, and D3 receptor dysfunction has been implicated in many diseases including drug addiction, Parkinson's disease, and schizophrenia. The distribution of D3 receptors, indicated by autoradiography of human brain tissue $\{$ Sun et al. 2012. PloS one; 7:e49483\}, was reflected in Positron Emission Tomography (PET) images of [18 F] fluortriopride (FTP) uptake in anesthetized non-human primates after administration of a benzodiazepine agonist, lorazepam \{Mach et al. 2011. Synapse; 65:724-732\}. Thirty minutes before injection of FTP, one $\mathrm{mg} / \mathrm{kg}$ of lorazepam was administered to block intrinsic dopamine release in these male rhesus monkeys \{Mach 2011\}. This lorazepam challenge led to increases in the nondisplaceable binding potential (BPnd) of FTP to D3 receptors in the caudate, putamen, and thalamus regions compared to baseline FTP scans \{Mach 2011\}. The purpose of this study was to assess if there is a quantifiable increase in measured FTP radiotracer uptake in dopamine D3 receptor rich brain regions of healthy human male brains following lorazepam treatment.

Methods: Three healthy white male volunteers $(28,37$, and 52 years old) participated in a double-blind, placebo controlled serial PET/CT study. Each subject underwent two dynamic [18 F]FTP PET/CT brain scans on a Philips Ingenuity PET/CT scanner on two separate days. On one scan day, the subject received an IV injection of normal saline (placebo) approximately 30 minutes prior to the FTP brain PET/CT scan, and on the other scan day the subject received an IV injection of $4 \mathrm{mg}$ of lorazepam (mean: $0.044 \mathrm{mg} / \mathrm{kg} \pm 0.006 \mathrm{mg} / \mathrm{kg}, \quad 0.040-$ $0.051 \mathrm{mg} / \mathrm{kg}) 30$ minutes prior to the planned FTP injection. Then, each underwent 2 hours of dynamic PET brain imaging following the intravenous administration of FTP (mean dose: $252.3 \mathrm{MBq} \pm 8.7 \mathrm{MBq}, 237.8$ - 258.6 MBq). Each participant also underwent MRI T1 imaging with isotropic voxels for coregistration with the PET data. Specialized statistical recovery software \{O'Sullivan et al. 2010. IEEE Trans Med Imaging; 29:610-624\} was used to extract arterial input functions from FTP images to avoid the need for invasive arterial blood sampling. Kinetic image analyses (Pmod Technologies LLC v3.7 software) of FTP uptake using image-extracted arterial blood input functions via a 2-compartment model yielded total distribution volumes $(\mathrm{Vt})$ and $\mathrm{BPnd}$ for the caudate nucleus, nucleus accumbens, pallidum, putamen, and nigra, and thalamus. The difference between Vt and BPnd values from the with and without lorazepam FTP PET/CT scans for each brain region was examined using a 2-tailed, paired Student $\mathrm{t}$ test. $\mathrm{P}$ values less than 0.05 were considered significant.

Results: The 4 mg lorazepam dose to 3 human male subjects resulted in an average lorazepam concentration $0.044 \mathrm{mg} /$ $\mathrm{kg} \pm 0.006 \mathrm{mg} / \mathrm{kg} \quad(0.040-0.051 \mathrm{mg} / \mathrm{kg})$ that was 23 times lower than the $1 \mathrm{mg} / \mathrm{kg}$ dose provided to monkeys in the preclinical trials. Average Vt measures of FTP uptake without and with lorazepam challenge with units of $\mathrm{mL} /$ $\mathrm{ccm}$ were $1.31 \pm 0.75 \& 1.49 \pm 0.69$ in caudate nucleus, $1.83 \pm 1.14 \& 4.72 \pm 3.56$ in nucleus accumbens, $1.96 \pm 1.28 \&$ $2.08 \pm 0.85$ in pallidum, $1.90 \pm 1.12 \& 1.81 \pm 0.73$ in putamen, and $2.43 \pm 1.66 \& 2.06 \pm 0.85$ in thalamus. Average BPnd unitless measures of FTP uptake without and with lorazepam challenge were $1.55 \pm 1.84 \& 2.34 \pm 1.18$ in caudate nucleus, $1.98 \pm 1.68 \& 4.34 \pm 0.80$ in nucleus accumbens, $2.76 \pm 1.05 \&$ $3.32 \pm 2.24$ in pallidum, $2.03 \pm 1.11 \& 1.94 \pm 0.71$ in putamen, and $2.83 \pm 0.76 \& 2.14 \pm 1.21$ in thalamus. Vt and BPnd measures of FTP uptake in the caudate nucleus, nucleus accumbens, pallidum, putamen, and thalamus regions of healthy human male brains were not significantly increased following lorazepam treatment $(P \geq 0.21)$.

Conclusions: Differences in both Vt and BPnd measures of FTP uptake were not significantly different $(P \geq 0.21)$ between PET/CT scans of subjects with and without lorazepam challenge. The lack of significant differences in FTP measures following lorazepam administration may be due in part to low subject sample size $(n=3)$ and an average lorazepam dose that was 23 times lower than in the previous non-human primate study. Future studies with a larger cohort are needed to understand the impact of lorazepam challenge on FTP uptake in regions rich in dopamine D3 receptors.

Keywords: Dopamine D3 Receptors, PET Imaging, Human Brain Imaging, Human Neuroimaging, Benzodiazepine

Disclosure: Nothing to Disclose.

\section{M240. Mapping an Insular Cortex Dynorphinergic Microcircuit}

\section{Melanie Pina*, Thomas Kash}

University of North Carolina School of Medicine, Chapel Hill, North Carolina, United States

Background: The insular cortex (IC) generates interoceptive states by integrating internal and external cues to drive motivated behavior. This has broad implications for IC involvement in drug dependence, with previous work indicating it plays a role in both drug reward and the affective aspects of withdrawal (Li et al., 2013; Wills et al., 2016). We have recently identified a microcircuit within the agranular IC that signals through the Goi/o-coupled Kappa Opioid Receptor (KOR) and its endogenous ligand Dynorphin (Dyn). This is particularly interesting, as the Dyn/KOR system also appears to regulate affective states in humans and is involved in stress and addiction-related processes (Shippenberg, 2009). In the present series of experiments, we mapped Dyn expression and assessed intra- and inter-laminar connectivity in the IC of mice. We also tested the functional impact of KOR signaling within this IC Dyn microcircuit and assessed its laminar- and cell-type specific expression. Finally, we traced long-range outputs from the IC to determine its subcortical targets.

Methods: To label and map Dyn cells in the IC, Dyn-IRESCre mice (Krashes et al., 2014) were crossed with mice expressing the EGFP::L10a fusion gene to create a Dyn reporter strain (Dyn-GFP). The impact of KOR signaling in the IC was assessed using whole-cell patch clamp electrophysiology. Excitability and synaptic transmission were 
assessed during baseline and after bath application of the KOR agonist Dynorphin-A. To visualize interneurons in the IC, a vGAT reporter mouse strain (vGAT-GFP) was used and was generated by crossing vGAT-IRES-Cre mice to mice expressing EGFP::L10a. For long-range circuit mapping, a combination of retrograde (Fluoro-Gold, cholera toxin B subunit) and anterograde (AAV9-EF1a-DIO-synaptophysinmCherry, AAV8-CamKIIa-eGFP) tracers were used. These tracers were injected stereotaxically into the substantia nigra (retrograde) and IC (anterograde) of Dyn-Cre and Dyn-GFP mice. In addition, channelrhodopsin-2 assisted circuit mapping (via AAV5-Ef1a-DIO-hChR2(H134R)-mCherry) was also used to assess intra- and inter-laminar connectivity within the IC and confirm functional connectivity between the IC and SN.

Results: In our Dyn reporter strain of mouse, we found laminar-specific distribution of Dyn in the IC, where it is predominately confined to layer $2 / 3$ and densely expressed. Conversely, KOR expression, which is virtually absent from layer $2 / 3$, is localized to deep layer 5 of the IC. Within layer 5 , we found that KOR is expressed on GABAergic interneurons, as bath application of Dyn reduced the excitability of vGAT-expressing cells. We next traced outputs from layer 5 to determine their subcortical targets. We discovered that a subpopulation of principle cells in layer 5 of the IC project to the substantia nigra (SN). Optical stimulation of IC terminals released glutamate onto $\mathrm{SN}$ neurons, indicating that this IC$\mathrm{SN}$ projection is functional. Finally, in recordings from this SN-projecting subpopulation of IC layer 5 cells, we found that bath application of Dyn increased excitatory synaptic transmission and decreased inhibitory synaptic transmission. Conclusions: We have identified a microcircuit within the IC that signals through Dyn/KOR. Within this microcircuit, Dyn is robustly expressed in layer 2/3, while KOR is localized to deep layer 5 and expressed on GABAergic interneurons. We found that Dyn-induced activation of KOR on layer 5 GABA cells reduced their excitability. This, in turn, led to an increase in the excitatory to inhibitory balance (E/I ratio) in layer 5 principle cells that project to the SN. Thus, our findings suggest that Dyn may modulate the activity in an IC microcircuit by directly inhibiting layer 5 interneurons, thereby disinhibiting principle neurons, including those that send a functional projection to subcortical sites. Our future work will determine the impact of opioid withdrawal on this IC Dyn microcircuit and it synaptic connectivity with the SN. Results of these experiments may reveal a discrete neural circuit contributing to the pathophysiology of opioid withdrawal.

Keywords: Dynorphin, Kappa Opioid Receptor, Insula, Electrophysiology, Circuit Mapping

Disclosure: Nothing to Disclose.

\section{M241. Distinct Midbrain Dopamine Circuits Regulate Individual Alcohol Drinking Behaviors}

Barbara Juarez*, Carole Morel, Stacy Ku, Yutong Liu, Hongxing Zhang, Hilledna Gregoire, Sarah

Montgomery, Marshall Crumiller, Jessica Walsh, Yingbo Zu, Song Zhang, Dipesh Chaudhury, Allyson Friedman, Ming-Hu Han

University of Washington, Seattle, Washington, United States
Background: Alcohol-use disorders are the leading substance-use disorder worldwide, yet there is incredible individual variability in alcohol consumption. While some individuals maintain controlled alcohol drinking behaviors, others succumb to pathological alcohol consumption. The need to understand the neural circuits that underlie individual alcohol drinking behaviors is crucial for the field's development of effective pharmacotherapeutics. Dysfunction in the ventral tegmental area (VTA) dopaminergic circuit is known to be involved in mediating the initial stages of alcohol addiction. There is incredible heterogeneity in VTA dopamine (DA) neurons, with projection-specific populations have diverse intrinsic ion channel compositions that regulate neuronal firing activity. These distinct DA populations project to neural substrates that regulate reward processing and executive control. Studies have shown that ethanol $(\mathrm{EtOH})$ can activate VTA DA neurons directly to cause DA release downstream. Yet, few studies have investigated how these heterogeneous dopamine neurons may regulate individual alcohol drinking behaviors.

Methods: In order to determine how VTA DA neurons regulate individual alcohol drinking behavior we designed a two-bottle choice, continuous access alcohol-drinking paradigm. C57BL/6 J male mice or TH-BAC-Cre male mice were exposed to escalating $\mathrm{EtOH}$ concentrations to parse out individual alcohol drinking behaviors. High alcohol drinking (HAD) mice were found to have $\mathrm{EtOH}$ preferences of $60 \%$ or higher and EtOH intakes greater than $10 \mathrm{~g} / \mathrm{kg} / 24 \mathrm{~h}$. Low alcohol drinking (LAD) mice displayed EtOH preferences of $40 \%$ or less and EtOH intakes less than $10 \mathrm{~g} / \mathrm{kg} / 24 \mathrm{~h}$. We then performed anaesthetized in vivo single unit electrophysiology of VTA DA neurons of EtOH naïve, LAD and HAD mice. To parse out the intrinsic neurophysiological dopaminergic heterogeneity of the VTA between alcohol drinking groups, we performed circuit-dissecting in vitro slice physiology of either the NAc (VTA-NAc) or the mPFC (VTA-mPFC) pathways.

Next, in order to determine if these firing alterations were causal to alcohol drinking behaviors, we turned to optogenetics for cell-type specific and temporally precise manipulations. We injected Cre-inducible, blue-light activated excitatory channelrhodopsin (ChR2) into the VTA of THBAC-Cre mice to express ChR2 into DA neurons. Firingpattern specific optogenetic manipulations were performed for 15 minutes then alcohol-drinking behaviors were monitored in $\mathrm{HAD}$ or EtOH naïve mice. Finally, we expressed ChR2 in either VTA-NAc or VTA-mPFC neurons and determined how projection-specific alterations regulated alcohol drinking behaviors.

Results: A majority of C57BL/6 J male mice developed high alcohol drinking behaviors, yet a subset of mice developed low alcohol drinking behaviors. Interestingly, we discovered that LAD mice had significantly higher VTA dopaminergic in vivo firing and burst activity than $\mathrm{EtOH}$ naïve mice. Surprisingly, HAD mice had firing and burst activity similar to that of EtOH naïve mice, seemingly unchanged after the alcohol-drinking paradigm. Our circuit-dissecting electrophysiological investigation revealed projection specific differences between the VTA-NAc and the VTA-mPFC pathways. LAD mice had increased firing activity of VTANAc neurons, yet VTA-NAc activity of HAD mice resembled $\mathrm{EtOH}$ naïve mice, similar to what we observed in vivo. 
However, we discovered no differences in VTA-mPFC neuronal firing between the two alcohol drinking groups and $\mathrm{EtOH}$ naive mice. Furthermore, VTA-NAc neurons of HAD mice showed reduced excitatory $\mathrm{Ih}$, an ion channel adaptation that could underlie the seemingly unchanged firing rate. Remarkably, VTA-NAc neurons of LAD mice have enhanced excitatory responses to EtOH, yet VTA-NAc neurons of HAD mice lose this responsivity.

Causally, we found that phasically activating, but not tonically activating, VTA DA neurons in HAD mice reduced $\mathrm{EtOH}$ preference for 48 hours. Furthermore, this reduction in $\mathrm{EtOH}$ preference was specific to mice that had HAD experience, as we did not see lower EtOH preferences in $\mathrm{EtOH}$ naïve mice following phasic stimulation. Finally, this reduction in $\mathrm{EtOH}$ preference in $\mathrm{HAD}$ mice was specific to phasic induction of VTA-NAc, but not VTA-mPFC, neurons.

Conclusions: These investigations identified firing patternspecific, experience-specific and circuit-specific adaptations of VTA dopamine neurons that regulate individual alcohol drinking behaviors.

Keywords: Dopamine, Alcohol and Substance Use Disorders, Neurocircuits, Neurophysiology, Optogenetics

Disclosure: Nothing to Disclose.

\section{M242. Amphetamine-Paired Stimuli Induce Conditioned Brain Activity in Healthy Adults}

Kathryne Van Hedger, Sarah Keedy, Markus Heilig, Harriet de $\mathrm{Wit}^{*}$

\section{University of Chicago, Chicago, Illinois, United States}

Background: Previously neutral stimuli paired with drugs of abuse are known to acquire conditioned incentive properties through Pavlovian conditioning, and these conditioned responses may facilitate future drug-seeking behavior. In this study we investigated the acquisition of conditioned responses after visual and auditory stimuli were paired with single oral doses of methamphetamine in healthy volunteers. The conditioned response was assessed by presenting the conditioned stimuli in the fMRI scanner, in a drug-free state. Methods: Healthy volunteers $(N=51)$ aged $18-35$ participated in four conditioning sessions followed by a scanning test session. During the conditioning sessions, they received oral methamphetamine $(20 \mathrm{mg})$ paired with one compound stimulus (visual+auditory), and placebo paired with another stimulus, on two occasions each. Drugs were administered under double blind conditions. During the scanning session when no drug was administered, subjects completed a choice task in which the stimuli were presented, and neural responses to the stimuli were assessed using a Philips Achieva $3.0 \mathrm{~T}$ scanner. We hypothesized that the stimuli paired with methamphetamine would elicit a greater brain response than the stimuli paired with placebo, especially in brain areas associated with reward and positive affect.

Results: The stimuli paired with drug elicited greater neural activity than the stimuli paired with placebo, providing evidence of conditioning. Unexpectedly, however, the locations of the effect of greater drug-paired stimuli activation were bilateral visual and auditory cortices.
Conclusions: The results suggest that conditioning developed in relation to the arousing effects of the stimulant enhancing early perceptual system responsivity, rather than affective system effects. These findings raise important questions about the nature of conditioned stimuli and how they affect subsequent behavior.

Keywords: Pavlovian Conditioning, Methamphetamine, $\mathrm{Hu}$ man Neuroimaging

Disclosure: Nothing to Disclose.

\section{M243. Subgroups of Smokers Derived From Broad Based Characterization of Cognitive and Affective Function and Personal History}

\section{Betty Jo Salmeron*, Xiaoyu Ding, Thomas Ross, Elliot Stein}

National Institute on Drug Abuse, Baltimore, Maryland, United States

Background: To date, successful subdivision of nicotine addiction has been limited to those based primarily on severity of dependence and metabolism of nicotine. In this study, we hypothesized that subgroups may exist in nicotine addicts, which might be revealed using measures assessing demographic variables, personality, psychiatric comorbidities and trauma history, as well as dependence severity, and further understood with neuroimaging data.

Methods: 102 cigarette smokers were enrolled under several protocols approved by NIDA-IRP IRB. Features included gender, education, socioeconomic status (SES), IQ, smoking history (containing cigarette per day (CPD), smoking years, age of first smoking, and lifetime usage), frequency of alcohol usage, ADHD Self-Report Scale (ASRS), Beck Anxiety Inventory (BAI), Beck Depression Inventory (BDI-II), Fagerström Test for Nicotine Dependence (FTND), K10, Toronto Alexithymia Scale (TAS-20), Childhood Trauma Questionnaire (CTQ), and Temperament and Character Inventory (TCI). Hierarchical clustering was used to reveal subgroups.

65 subjects also had resting-state fMRI data acquired on a $3 \mathrm{~T}$ Siemens Allegra MRI scanner. Data preprocessing was done in AFNI using standard methods including regressing out the first 3 principal components of WM and CSF. Group differences in the following resting-state features were examined: amplitude of low frequency fluctuations (ALFF), regional homogeneity (ReHo), voxel-mirrored homotopic connectivity (VMHC), and 10 large-scale brain networks (sensorimotor, auditory, default-mode, salience, cerebellum, left and right executive-control, and 3 visual networks).

Results: The dendrogram resulting from hierarchical clustering revealed 2 subgroups or 3 subgroups, while still retaining reasonable numbers in each subgroup. The twosubgroup result included one group with higher smoking severity, persistence and minimization/denial and with greater education/IQ and more ADHD, depressive, alexithymic symptoms and greater childhood emotional abuse/ neglect. The three-subgroup result split the high severity group into one with higher minimization/denial and lower education $(11.9 \pm 1.6$ years), psychiatric symptoms and childhood abuse and one with unique elevations in selfdirectedness, persistence and cooperativeness as well as 
elevations in education/IQ, ADHD/depressive symptoms, childhood trauma and difficulty identifying feelings shared with the original lower severity group that also had elevations in difficulty describing feelings. Education in these subgroups was $13.4 \pm 2.3$ vs. $14 \pm 2.3$ years, respectively. For the 2-subgroup division, two-sample T-tests (age and gender controlled, voxel-wise $p<0.005$, corrected $p<0.05$ ) on the ALFF, ReHo, VMHC, and spatial maps of the brain networks showed multiple discriminative regions. Interactions between the occipital gyrus and the default mode network, as well as that between the lingual gyrus and the right executive control network were higher in the high severity group in contrast to the low severity group, as was ReHo in the precuneus. For the three-subgroup division, group differences were seen in ALFF in the middle cingulate cortex (MCC) and in the cuneus interaction with the lateral visual network, both of which were also seen in the two-subgroup division and driven by differences between both high severity groups and the lower severity group. ALFF in MCC correlated negatively with Difficulty Describing Feelings (TAS-20) while cuneus interaction with lateral visual network correlated negatively with severity of nicotine dependence (FTND).

Conclusions: Subgroups of smokers were identified that included smokers with subclinical mood, anxiety and attention/hyperactivity symptoms and childhood trauma who nevertheless achieved education levels beyond a high school diploma and those with higher severity, high on minimization/denial and averaging less than a high school education. Higher achievement despite various forms of adversity may indicate smokers for whom nicotine is useful in dealing with mild mood, anxiety and attention problems. High minimization/denial combined with low educational achievement and high severity of nicotine dependence may identify smokers for whom intensive motivational enhancement will be needed in order to engage them in smoking cessation. Imaging results highlight a role for attention and emotion regulation in distinguishing smokers of different subtypes as well as primary visual areas. Subtyping smokers based on a broad range of cognitive, affective and personal history characteristics may identify smokers who might benefit from different treatment approaches (nicotine replacement and further evaluation of mild affective and attentional symptoms versus intensive motivation enhancing interventions).

Keywords: Nicotine Dependence, Subtypes, Clustering, Resting-State fMRI

Disclosure: Nothing to Disclose.

\section{M244. Combining GWAS and Transcriptome Data to Define Denes and Pathways in Alcoholism}

Dayne Mayfield*, Manav Kapoor, Sean Farris, Jen Wang, Sarah Bertelsen, Howard Edenberg, Adron Harris, Yunlong Liu, Alison Goate

University of Texas at Austin, Austin, Texas, United States

Background: Alcohol dependence (AD) is a complex disorder with high heritability (50-60\%). The excessive use of alcohol results in changes in global gene expression across different tissues including brain.
Methods: RNA sequencing was used to compare the transcriptomic organization of the Dorso-Lateral Pre-Frontal Cortex (DLFPC) in 143 post-mortem brain samples from 66 uncomplicated alcoholics and 77 matched controls. Differential gene expression (DGE) was performed (DESeq2) after correcting for batch effects such as age, gender and RNA integrity number (RIN).

Results: We identified 481 genes that were differentially expressed between alcoholics and controls (10\% FDR). Ingenuity pathway analysis (IPA) of differentially expressed genes showed gene enrichment for GADD45 signaling, interferon signaling and inflammatory response. We also performed Weighted Gene Co-expression Network Analysis (WGCNA), which clustered the genes into 52 modules (modules with correlations of greater than $75 \%$ were merged). Module-trait correlation analysis identified a module containing 731 genes that was positively correlated with alcoholism classification (DSM4, DSM5) and alcohol consumption. This module was also significantly correlated with age and the number of years of drinking. Age-corrected module-trait correlation analysis improved the association signal for DSM4, DSM5 and case-control classification, which showed that this module was significantly correlated with alcoholism independent of age. SNPs overlapping with this module of genes were highly enriched for association signals with age at onset of alcohol dependence in the COGA European American (EA) dataset. In addition, the COGA extended $\mathrm{AD}$ families were enriched for common factors increasing risk for $\mathrm{AD}$. Genome-wide Cox proportional hazards regression model was used to test for the association between age at onset of AD and SNPs. This important gene module was found to be highly enriched for genes related to immune function.

Conclusions: By leveraging power from transcriptome and GWAS data, we identified novel genes and networks associated with alcoholism.

Keywords: Transcriptome, GWAS, Alcoholism

Disclosure: Nothing to Disclose.

\section{M245. Abstinence Induced Deficits in Target-But Not Flanker-Processing in Smokers}

John Fedota*, Michael McKenna, Allison Matous, Betty Jo Salmeron, Thomas Ross, Elliot Stein

National Institute on Drug Abuse, Baltimore, Maryland, United States

Background: Prominent theories of addiction posit that deficits in prefrontal cortical function and impaired cognitive control contribute to substance abuse. The putative mechanism of these contributions include an inability to inhibit prepotent behaviors related to drug seeking as well as the biased processing of interoceptive signals associated with drug craving. However, to date empirical evidence of specific cognitive control dysfunction in sated and abstinent smokers has been inconsistent. This is in stark contrast to the clear and consistent negative effects of nicotine abstinence on affect and mood.

Recent evidence from our lab illustrates attentional control deficits in smokers that are related to onset of attentional modulation as opposed to the discrete ability to recruit 
control at all. Thus, it does not appear that cognitive control is broadly dysfunctional, but instead may be selectively impaired as a function of both chronic and acute nicotine exposure. These results suggest that the influence of nicotine on cognitive control may be subtler than previously described. To more fully characterize abstinence-induced deficits in attentional control, here we employ a previously validated dual stage computation model of selective attention. In combination with subjective measures of affective disruption, the current study seeks to identify specific cognitive constructs impacted by acute nicotine abstinence in smokers.

Methods: In a within subjects design, 30 otherwise healthy smokers completed all experimental procedures on two occasions-during sated smoking and again following approximately 48 hours of full, biochemically assessed nicotine abstinence. To assess affective and cognitive disruptions associated with acute nicotine abstinence, participants completed subjective measures of withdrawal symptoms and a cognitive task battery including a previously described parametric flanker task. Individual participant flanker task behavioral results in each condition were separately fit to a dual-stage two-phase (DSTP) computational model to derive seven model parameters serving as indices of cognitive constructs relevant to task performance. These parameters were then tested for STATE (abstinencesated) effects.

Results: Participant abstinence was verified physiologically (expired CO) and subjectively. Across physiological and subjective measures, a significant STATE effect was observed with reduced $\mathrm{CO}$ and enhanced subjective withdrawal. In contrast, flanker task performance (reaction time and accuracy) did not show STATE effects. Nonetheless, a subset of the calculated DSTP model parameters were modulated as a function of STATE. Specifically, during the early stage of task stimulus processing when attention is diffusely distributed, abstinence reduced the rate of evidence accumulation for targets, but not flankers. In addition, a parameter for non-decision time (i.e. stimulus encoding, response execution) was reduced in abstinence. Beyond these modulations, no changes in response bias or criterion were observed as a function of STATE.

Conclusions: Behaviorally, the consequence of acute abstinence appears to be a misinformed control system as opposed to a hypofunctional control system. Nicotine abstinence decreases early capture of attention by salient targets-but importantly not flankers. That is, the attentional capture of targets (as indexed by target drift rate in the DSTP model) is reduced in the initial stage of stimulus processing prior to the application of attentional selectivity. This STATE dependent modulation in target drift rate occurs independently from flanker drift rate, which remains unchanged. The separation of target and distractor processing in attentional selectivity agrees with prior evidence showing attentional control works by amplification of task relevant information, as opposed to inhibition of distracting information.

The observed deficit in evidence accumulation may be driven by a reduced attentional capacity for task performance due to the increase in negative affect more consistently associated with acute abstinence. That is, endogenous dysphoria during withdrawal may absorb attentional capacity that would otherwise be applied to task performance. Taken together, the current results suggest that nicotine abstinence in smokers selectively influences basic attentional functions as opposed to top down control. This shift in emphasis may explain, in part, why less sensitive characterizations of performance have failed to illustrate the abstinence-induced deficits predicted. In addition, the insight into the attentional processes disrupted in abstinence may guide future cessation treatment development.

Acknowledgement: Supported by the Intramural Research Program of the NIH/NIDA and FDA grant NDA13001-00100000 to EAS.

Keywords: Cognitive Control, Abstinence, Computational Modeling

Disclosure: Nothing to Disclose.

\section{M246. A New Murine Model for Aggressive Motivation: The Role of CRF in Persistent Escalation by Alcohol}

\section{Herbert Covington, III, Emily Newman, Steven Tran,} Joseph DeBold, Klaus Miczek*

\section{Tufts University, Medford, Massachusetts, United States}

Background: Activation of the HPA stress axis by CRF and CRF action in the ventral tegmental area (VTA) are important for the initiation of motivated behaviors. The introduction of a novel method permits the study of the neurocircuitries mediating aggressive motivation as distinct from aggressive acts.

Methods: The motivation to initiate aggressive acts, as well as the execution of aggressive outbursts, were experimentally examined in inbred $\mathrm{C} 57 \mathrm{BL} / 6 \mathrm{~J}$ mice. Mice were initially conditioned to respond on a fixed interval ten-minute (FI10) schedule with aggression serving as a reward. The progressively accelerating pattern of responding established by the FI schedule allows for direct, stable and quantifiable measurements of aggressive motivation prior to the measures of aggressive performance. Subsequent to the completion of each FI schedule, an intruder was presented, and aggressive acts in one bout of fighting were examined. The role of corticotrophin releasing factor receptor 1 (CRF-R1) was examined on both, motivation and fighting performance.

Results: Tolerance and sensitization became evident with repeated oral administrations of alcohol $(1.8 \mathrm{~g} / \mathrm{kg}$, gavage) in the motivation to engage in aggressive acts. Interestingly, low doses of the selective CRF-R1 antagonist CP 376395 blocked the expression of alcohol-induced sensitization of aggressive motivation, but without influencing fighting performance. Finally, measurements of crf, crfr1 and crfr2 mRNA across the VTA, DRN and LH reveal that the emergence of alcoholinduced sensitization of aggressive motivation occurs with a persistent augmentation of CRF tone in distinct extrahypothalamic CRF circuits. These circuits can be selectively activated in CRH-IRES-Cre mice during individual components of escalated motivation and measures of fighting performance using optogenetics.

Conclusions: These experiments begin to identify discrete neural targets for intervention to modify intense aggressive motivation vs. those controlling reactive "hot" aggressive acts. 
Keywords: Aggression, Ventral Tegmental Area (VTA), Corticotropin-Releasing Factor (CRF), Alcohol, Sensitization Disclosure: Nothing to Disclose.

\section{M247. Augmented LH Orexin/Hypocretin Cell Function Underlies the Expression of an Addicted State in Rats}

Morgan James*, Colin Stopper, Benjamin Zimmer, Nikki Koll, Hannah Bowrey, Shayna O'Connor, Gary Aston-Jones

Rutgers University, Piscataway, New Jersey, United States

Background: The hypothalamic orexin (hypocretin) system has been highlighted as a promising target for pharmacotherapies designed to treat addiction. However, a role for orexin in the expression of a multifaceted addiction phenotype has not been shown. Here, we used a novel intermittent access (IntA) cocaine self-administration paradigm (Zimmer et al., 2012) in rats to recapitulate the pattern of drug use reported by human addicts. Following IntA, we examined the expression of multiple addiction-like behaviors, and the extent to which the orexin system is important for their expression.

Methods: Rats were given IntA to cocaine in daily sessions for two weeks, before being tested for economic demand for cocaine (Bentzley et al., 2012) and a range of other addictionlike behaviors. In a subgroup of animals, we quantified the numbers of orexin-expressing cells and the reactivity of these cells to a drug-associated context both immediately and 150d after IntA training. To test the functional role of orexin in the expression of addiction-like behaviors following IntA, we either treated animals with the selective orexin-1 receptor antagonist SB-334867 (SB; $10 \mathrm{mg}, 30 \mathrm{mg} / \mathrm{kg}$, i.p.), or used an antisense morpholino to selectively reduce orexin expression in either the medial (perifornical/dorsomedial) or lateral orexin cell fields.

Results: IntA produced a persistent increase in motivation for cocaine (decreased alpha) without affecting animals' hedonic set point for cocaine (Q0) on the behavioral economics task. This change in motivation persisted for at least 50d following Int A training. IntA was also associated with higher expression of various behavioral analogs of key addiction criteria, including compulsive (punished) responding for cocaine, reinstatement of extinguished drug-seeking, and expression of depression- and anxiety-like behavior following protracted withdrawal from cocaine. Remarkably, IntA was associated with an increase in both the number and activity of orexin cells in LH that persisted for at least $150 \mathrm{~d}$ following IntA. Normalization of orexin cell numbers via morpholino knockdown reversed the IntA-induced addiction multi-phenotype. Further, the IntA phenotype could also be reversed by a dose of SB that is 3-fold lower than that generally found to affect motivated behavior for cocaine.

Conclusions: IntA to cocaine self-administration induced a persistently addicted state characterized by the expression of multiple addiction-like behaviors that reflect key diagnostic criteria for addiction. This addicted state was associated with a persistent increase in the number and activation of $\mathrm{LH}$ orexin neurons, and normalization of orexin numbers reversed the addicted state. Moreover, rats that exhibited an addicted state following IntA were more susceptible to the anti-drug-seeking effects of SB, highlighting the potential clinical utility of orexin-based therapies for the treatment of addiction. These findings are particularly timely given the recent FDA approval of the dual orexin receptor antagonist suvorexant (BelsomraTM) for the treatment of insomnia.

Keywords: Cocaine Addiction, Orexin Receptor Antagonist, Orexin, Animal Model, Behavioral Pharmacology

Disclosure: Nothing to Disclose.

\section{M248. Development of a Virtual Reality Conditioned Place Preference Paradigm for in Vivo Hippocampal Imaging During Morphine-Conditioned Contextual Learning}

Sidney Williams*, Moises Arriaga, Edward Han, Jose Moron-Concepcion

Washington University in St. Louis, Saint Louis, Missouri, United States

Background: Long term associations between a specific environment and drugs of abuse, such as opioids, can trigger craving and relapse in people with a previous drug use history. We have previously reported that these maladaptive contextual associations may be partly formed by structural and functional changes in the dorsal hippocampus. Our lab has shown that morphine conditioned place preference (mCPP) decreases the number of dendritic spines on hippocampal CA1 neurons, yet the timing and dynamics of these events and their potential relationship to the association between morphine reward and context are unknown. To observe neural networks in real time (as mCPP and reinstatement take place), we have designed a virtual reality conditioned place preference (VR-CPP) paradigm paired with two-photon imaging. This strategy will allow us to observe the spatio-temporal neuronal dynamics of druginduced contextual memories.

Methods: We designed a three chamber VR environment composed of two conditioning chambers with distinct visual cues connected by a third neutral chamber. The visual VR environment is displayed on an array of five computer monitors that tile around the animal. Mice are head-fixed and allowed to freely run on a Styrofoam ball suspended by air pressure. Movement of the ball is tracked by a computer mouse and converted to forward and yaw velocities by custom written software (LabView) that feeds into the VR engine (Virmen, Matlab), which updates the projected visual scene. Before animals begin VR training, they are injected with a genetically encoded Ca2+ indicator (AAV1.Syn.NESjRGECO1a.WPRE.SV40) in the CA1 region of the dorsal hippocampus. After two weeks, we implant an imaging cannula directly above CA1 region of the hippocampus for two-photon calcium imaging during training. To motivate animals to explore in the VR environment, animals are water restricted, then undergo two training trials per day during which they receive a $\mathrm{H} 2 \mathrm{O}$ reward for spending $15 \mathrm{~s}$ in the $\mathrm{H} 2 \mathrm{O}$-paired chamber of the VR-CPP, whereas no rewards were given in the opposite chamber. Within 8-12d the animals reach the learning criteria ( $>70 \%$ of time spent in the $\mathrm{H} 2 \mathrm{O}$ - paired chamber for 4 consecutive trials). Mice were then submitted to a biased $\mathrm{mCPP}$ protocol in the VR environment. Briefly, a baseline preference test measured 
time spent in each compartment of VR-CPP, followed by 8 days of contextual saline/morphine $(20 \mathrm{mg} / \mathrm{kg})$ conditioning. A post-conditioning preference test confirms the development of a preference for the morphine conditioned chamber.

Results: Following $\mathrm{H} 2 \mathrm{O}-$ paired contextual conditioning, the mice undergo a CPP test $24 \mathrm{hrs}$ after the last conditioning trial where they are placed back in the VR environment in the absence of $\mathrm{H} 2 \mathrm{O}$. Mice demonstrate a significant preference (\% time) for the $\mathrm{H} 2 \mathrm{O}$-paired chamber compared to the opposite chamber $(n=17$; unpaired T-test, $p<0.0001)$. The $\mathrm{H} 2 \mathrm{O}$-paired contextual conditioning is repeated with the $\mathrm{H} 2 \mathrm{O}$ now paired with the opposite contextual chamber. Mice quickly demonstrate operant conditioning to the new $\mathrm{H} 2 \mathrm{O}-$ paired chamber and a switch in preference for that newly conditioned chamber in the VR environment $(n=17$, $p<0.0001)$. Extinction of conditioned place preference for $\mathrm{H} 2 \mathrm{O}$ is achieved before beginning the mor-induced contextual conditioning. After 8 days of mor-paired contextual conditioning, mice demonstrate a significant shift in place preference (\% time pre vs. post) for the VR mor-paired chamber following conditioning $(n=17$; unpaired T-test, $p=0.013)$. Throughout the entire operant $\mathrm{H} 2 \mathrm{O}$-conditiong, mor-paired contextual conditioning, and CPP testing we are able to track and measure single cell $\mathrm{Ca} 2+$ dynamics and CA1 network activity. Our preliminary data suggests that CA1 pyramidal neurons reveals a region-specific firing pattern based on the animal's preference for the morconditioned chamber, meaning that the neurons are more active when the animal is in the mor-conditioned chamber. Conclusions: Now that we have successfully established a novel virtual reality paradigm for $\mathrm{mCPP}$ and operant conditioning behavioral testing, we will be able to study not only structural plasticity with high spatio-temporal resolution, but also changes in network neuronal activity using genetically encoded calcium sensors, allowing us to build a comprehensive picture of neuronal changes during $\mathrm{CPP}$, from the scale of spines to networks. This cutting-edge technique, combining virtual reality with two-photon imaging, will allow for a more detailed investigation of neuronal and circuit level mechanisms underlying a broad range of behavioral outputs.

Keywords: Virtual reality, CPP, Hippocampus, Novel Tool, Two-Photon

Disclosure: Nothing to Disclose.

\section{M249. A Rodent Model of Social Function Deficits in Opiate Use Disorder}

\section{Seven Tomek, Gabriela Steggmann, M. Foster Olive*}

\section{Arizona State University, Tempe, Arizona, United States}

Background: The current opioid epidemic is a significant public health problem, and the high rates of relapse underscore the need for improved treatment approaches, particularly those that expand beyond traditional opioid substitution therapies. Diagnostic criteria for opiate use disorder set forth by the American Psychiatric Association include impaired social functioning as a result of excessive drug use. In addition, social support networks are critical for successful attempts at recovery. The purpose of the present study was to develop a rodent model of deficits in prosocial behavior in the context of opiate addiction. Prosocial behavior was assessed using a "rescuing" paradigm, whereby rats were allowed to free their cage-mate from a modified plastic restrainer. Prosocial behavior was assessed before and after acquisition of intravenous heroin self-administration, or as a control, self-administration of sucrose.

Methods: Male Sprague-Dawley rats (250-300 g) were pairhoused upon arrival. Following daily handling, one rat in each pair was randomly selected to be the "trapped" rat, and the other was designated as the "rescuer" rat. In daily sessions, rescuer rats were given the opportunity to release their trapped cagemate from a modified plastic restrainer, and the occurrence of and latency to free the confined rat was recorded. After two weeks of baseline rescuing behavior was established, rats were randomly assigned to selfadminister either heroin $(0.06 \mathrm{mg} / \mathrm{kg} /$ infusion, i.v. $)$ or sucrose pellets $(45 \mathrm{mg})$. Operant self-administration was conducted daily for 10-14 days. Next, rats were retested for rescuing behavior once daily for 3 consecutive days, during which they were provided with the opportunity to free the trapped cagemate or self-administer their respective reinforcer. All experimental procedures were approved by the ASU Institutional Animal Care and Use Committee and were conducted in accordance with the National Institutes of Health Guide for the Care and Use of Laboratory Animals. Results: Prior to acquisition of heroin or sucrose selfadministration, both groups of rats displayed similar proportions of rescuing behavior. Following the selfadministration phase, the proportion of rats in the sucrose group that rescued their cagemate instead of selfadministering sucrose was $76 \%$, whereas the proportion of rats in the heroin group that rescued their cagemate was $0 \%$ which was characterized by continued heroin selfadministration. In addition, rats that lost catheter patency during the self-administration, and therefore had a history limited heroin intake, continued to display rescuing behavior.

Conclusions: Unlike rats with a history of sucrose selfadministration, those with a history of heroin selfadministration show deficits in prosocial behavior, consistent with diagnostic criteria for opiate use disorder. These deficits appear to be related to the amount of prior heroin intake. Utilizing this novel paradigm, future investigations into the neural substrates of opiate-induced deficits in prosocial behavior may reveal novel approaches to restoring social functioning and promoting recovery in opiate addiction.

Keywords: Heroin, Self-Administration, Social Deficits, Rat Models, Opiate

Disclosure: Nothing to Disclose.

\section{M250. Encoding of Conditioned Motivation by Midbrain Dopamine Neurons}

Benjamin Saunders*, Jocelyn Richard, Patricia Janak

Johns Hopkins University, Baltimore, Maryland, United States

Background: Conditioned motivational states evoked by rewards and associated stimuli (conditioned stimuli, CSs) are 
essential for adaptive reward seeking, but they also contribute to a variety of psychiatric disorders, such as addiction. Despite this, we lack a circuit-level understanding of how the brain creates motivation under normal and pathological conditions. Dopamine neurons originating from the ventral tegmental area (VTA) and substantia nigra (SN) have received considerable attention for their role in rewardrelated processes, but it remains unclear what information is signaled by CS-evoked neural activity within these systems, and how that maps on to conditioned behaviors.

Methods: To explore this issue, we used fiber photometry to measure activity of dopamine neurons in TH-cre rats expressing GCaMP6, during Pavlovian conditioning of a sensory cue with optogenetic activation of dopamine neurons.

Results: We found that through Pavlovian conditioning, sensory cues paired with optogenetic activation of VTA dopamine neurons become CSs that evoke dopamine neuron activity in the VTA, and elicit conditioned behavioral responses. Furthermore, the magnitude of the cue-evoked dopamine signal predicted the vigor of cue-evoked conditioned behavior.

Conclusions: These results suggest that rather than simply signaling the learned predictive value of a Pavlovian CS, VTA dopamine neurons encode its incentive motivational value to actually drive specific conditioned actions. We are currently investigating if cue-evoked dopamine neuron signals vary as a function of the sensory modality of the predicted outcome (i.e., optogenetic brain stimulation versus delivery of an external, consumable reward, such as sucrose or cocaine) and across dopamine neuron subpopulations (e.g., VTA versus SN). The goal of these studies is to better understand how phasic dopamine neuron activity contributes to cueevoked motivational states that drive reward-specific behaviors.

Keywords: Dopamine, Incentive Motivation, Photometry Disclosure: Nothing to Disclose.

\section{M251. Gene Expression Changes Associated With Anhedonia in Cocaine Use Disorder}

Sarwar Khan, Gabriel Fries, Elena Dyukova, Consuelo Walss-Bass, Scott Lane, Joy Schmitz, Margaret Wardle*

University of Texas Health Science Center at Houston, Houston, Texas, United States

Background: Cocaine use disorder (CUD) affects almost 1 million people in the United States. Current CUD treatments are variably effective, so it is important to understand factors associated with poor treatment outcomes. One such factor is anhedonia, i.e. difficulties experiencing pleasure in non-drug rewards. Anhedonia is associated with worse treatment outcomes in cocaine, nicotine and opiate use disorders. There are no CUD treatments that explicitly target this symptom, and indeed, anhedonia appears resistant to medications for depression. To generate hypotheses about the potential biological underpinnings of anhedonia in CUD and indicate future directions for treatment research, we conducted a preliminary study of differences in gene expression in individuals with CUD who reported high vs. low anhedonic symptoms. One hypothesis, based on a prior analysis of a smaller sample, was that inflammatory pathways would be altered, as inflammation can produce anhedonia-like "sickness behavior".

Methods: Individuals with CUD $(N=48)$ were recruited from the greater Houston area. Exclusion criteria were: potentially inflammatory medical conditions (except high blood pressure or cholesterol), psychotic disorders, pregnancy, menopause or oral contraceptive use. Anhedonia was measured with the Snaith-Hamilton Pleasure Scale (SHAPS), and a median split was used to produce high and low anhedonia groups (each $n=24$ ). Groups were matched on demographics, substance use, other psychiatric diagnoses, and blood pressure/cholesterol. RNA was extracted from peripheral blood mononuclear cells in heparinanticoagulated blood samples. Following assessment of quality and integrity, biotinylated RNA samples were loaded onto a HumanHT-12 v4 Expression BeadChip (Illumina) and scanned with the Illumina iScan reader. Differential gene expression was assessed using Genome Studio. We conducted a stringent analysis with a false discovery rate (FDR) correction of $1 \%$ and alpha of 0.05 , and an exploratory analysis of genes with uncorrected $\mathrm{p}$ values $<0.05$. Any genes identified were subjected to Ingenuity Pathway Analysis (IPA) to identify major altered pathways and networks.

Results: No significantly differentially expressed genes (DEGs) were present with FDR correction, but 167 DEGs were indicated by uncorrected $\mathrm{p}$-values $<0.05$. IPA found several networks with significant enrichment based on these DEGs, with the top differentially expressed network being one involved in cell death, cellular organization and cellular compromise. The top cellular and molecular functions identified were enriched for metabolic functions, including lipid and nucleic acid metabolism. In contrast to prior findings with a smaller sample, inflammatory pathways were not directly implicated.

Conclusions: This preliminary study suggests that metabolism may be altered in individuals with high anhedonia and CUD. Surprisingly, results did not indicate direct involvement of inflammation. In the event this result is confirmed in larger studies, adjunctive therapies to address metabolic functioning should be investigated in anhedonic individuals with CUD. This is consistent with early positive results for metabolism-altering drugs such as pioglitazone in CUD.

Keywords: Cocaine Addiction, Substance Use Disorder, Anhedonia, Gene Expression

Disclosure: Nothing to Disclose.

\section{M252. Functional MRI Changes During Marijuana- Intoxicated Driving}

Godfrey Pearlson*, Lindsey Repoli, Diana King, Stephanie Novotny, Erwin Boer, Vince Calhoun, Nicholas Ward, Michael Stevens

Yale Institute of Living, Hartford, Connecticut, United States

Background: The issue of driving while intoxicated by cannabis $(\mathrm{CNB})$ has become prominent as more states legalize $\mathrm{CNB}$ for both medical and recreational use. Although numerous studies provide evidence that recent CNB use can 
impair performance on tests of cognitive abilities thought to be important for optimal motor vehicle operation, there is little understanding of exactly how CNB affects the brain to give rise to such impairments. A corresponding challenge is translating laboratory findings to actual driving behaviors to more clearly determine if CNB use increases risky driving. Our ongoing 5-year NIDA-funded study (R01DA038807) is examining $\mathrm{CNB}$-induced driving-related neurocognitive impairment with an immersive, realistic simulation to assess multiple driving behaviors during functional neuroimaging. Here, we report preliminary results from that validate our experimental approach and provide the first evidence that CNB use alters driving-related brain activation in a dosedependent way.

Methods: fMRI data have been collected so far from $n=6$ regular CNB users (near-daily use of 1 or more "joints", at least 4 times per week for the prior 3 months) with no DSM$\mathrm{V}$ psychiatric diagnoses. On three separate days, participants used a paced inhalation method with vaporizer to smoke marijuana, randomly receiving $0.5 \mathrm{gm}$ of either moderatedose (13.4\% THC), low-dose (5.9\% THC), or placebo. On each visit, participants underwent fMRI where they engaged in $>30 \mathrm{~min}$ of simulated driving using Realtime Technologies, Inc. (RTI) software in paradigms customized for fMRI to reduce the need for large head movements and assess common driving skill domains. These included strategic planning (Gap Acceptance - deciding when to overtake a car in the face of oncoming traffic), tactical decisions about vehicle maneuvers (Car Following - responding to the acceleration or deceleration of a lead car), and operational vehicle control (Lane Keeping - maintaining car position despite minor unpredictable wind gusts). Multiple instances of these driving demands were naturalistically embedded into the drive with sufficient frequency that BOLD signal could be estimated for each separate event. Each event onset was extracted, then modeled in SPM12 to create overall activation maps for each condition and to contrast study doses. As data collection is ongoing, preliminary results were examined using $p<.05$ uncorrected significance levels.

Results: Each driving task was associated with a distinct statistically significant BOLD change profile. Vehicle position corrections during Lane Keeping engaged the dorsal and ventral attention networks and disengaged the default mode network. When participants noticed lead vehicle speed changes during the Car Following task, activation in supplemental motor area, right frontoparietal cortex, and posterior middle temporal gyri increased. In contrast, activation decreased in bilateral postcentral gyri and posterior insular cortex. Overtaking decisions during the Gap Acceptance task more greatly engaged supplemental motor area regions, right dorsolateral (BA 46) and ventrolateral prefrontal cortex, while visual cortex regions disengaged. These and other brain regions were affected by CNB use. Common to all driving tasks, bilateral putamen was less engaged when participants had recently ( $1 \mathrm{~h})$ used CNB. All other CNB effects differed by task. For Lane Keeping, supplemental motor area and secondary visual cortex activity were reduced after both low and moderate CNB doses. Both doses were also associated with greater right dorsolateral, ventrolateral, and ventromedial prefrontal cortex activity, but this effect was more extensive and stronger during the moderate CNB dose condition. For Car Following, putamen deficits were dose specific, with higher doses linked to lower activation. Other dose-specific effects included bilateral precentral gyri \& left frontoparietal cortex deficits. After both doses, there was greater activity in visual association, motor, premotor, and supplementary motor cortices. During Gap Acceptance, putamen deficits were only detected after a moderate CNB dose. However, both doses showed extensive right hemisphere fronto-parietal deficits that direct comparison revealed were most impaired in the low dose condition. Both doses resulted in lower anterior cingulate cortex activation. The moderate dose was also associated with greater activity in left dorsolateral and ventrolateral prefrontal cortices.

Conclusions: These results are preliminary due to the currently small sample and our use of liberal statistical thresholds. But they confirm the validity of the experimental approach - it is possible to directly assess brain activation related to specific driving behaviors. These preliminary results also showcase widespread effects of recent CNB use on brain function - some of which are observed regardless of CNB dose, others that are either deleterious or possibly compensatory in a dose-dependent manner. Although we focus here only on fMRI data collected $1 \mathrm{~h}$ after CNB dosing, the protocol includes 2 other, later fMRIs, as well as repeated neurocognitive testing and blood/oral fluid assays. Ultimately, when the planned $n=80$ final sample of both regular and occasional CNB users is complete, it should be possible both to describe dose-dependency of any driving related neural impairment and predict how long it takes these deficits to resolve over the course of the day.

Keywords: Marijuana, Functional MRI (fMRI), Acute Effects Disclosure: Part 1: Astellas Pharm, Consultant.

\section{M253. Measuring Plaque Inflammation in Asympto- matic Cocaine Addicted Individuals With 18F-FDG Using Simultaneous PET/MR Imaging}

\section{Keren Bachi*, Venkathesh Mani, Rita Goldstein,} Zahi Fayad, Nelly Alia-Klein

Icahn School of Medicine at Mount Sinai, New York, New York, United States

Background: Cocaine use is involved in $40 \%$ of emergency department visits, where positive toxicology for illicit drugs have been associated with stroke, coronary artery disease and myocardial infarction, resulting in severe impairments or sudden mortality, even in the absence of prior vascular disease symptoms. Indeed, cocaine, a powerful vasoconstrictor and a sodium channel blocker, decreases basal antiinflammatory markers (Interleukin 10) and increases proinflammatory cytokines (Tumor necrosis factor alpha), contributing to progressive vascular inflammation (atherosclerosis). The carotid arteries supply blood to brain regions that are implicated in the higher-order cognitive impairments documented in individuals with cocaine use disorder (iCUD). Hence, structural and/or functional damage in the carotid arteries may impact cognitive and behavioral functioning even before substantial arterial narrowing results in clinical symptoms. We hypothesized that iCUD have significant vascular inflammation, which is modulated by history of drug use. 
Methods: Using Positron Emission Tomography/Magnetic Resonance (PET/MR), we imaged the internal carotid arteries to assess atherosclerosis in 10 healthy iCUD aged 43 to 58 with cocaine lifetime use of $22.6 \pm 7.3$ years and without a history of neurological or cerebrovascular disease (CVD). Analyses for additional 4 iCUD and 10 matched healthy controls are ongoing. We compared results to findings in a sample at risk for CVD, aged 64.6 \pm 7.8. Amount of inflammation, measured with PET with $18 \mathrm{~F}$ fluorodeoxyglucose (18 F-FDG), was calculated by the maximum arterial wall (target) to background (blood) ratio (TBR). To measure enlargement of wall area and thickness of the vessel, we used MR with 3-Dimensional dark-blood sequence.

Results: Results show that $78 \%$ of iCUD had inflamed plaque in arteries [TBRmax. (mean, SE) right $(1.89, .12)$ left $(1.7, .11)$; notably, $\mathrm{TBR} \geq 1.6$ is indicative of inflamed plaque]. Furthermore, in one sample t-tests using the comparison group's mean values, iCUD had thicker wall (mm; 1.63,.03 versus $1.27, .04, \mathrm{t}(8)=8.84, p<.001)$ and larger wall area $(\mathrm{mm} 2 ; 38.45,1.48$ versus $32.28,1.43, \mathrm{t}(8)=3.34, p=.01)$ indicating the presence of more plaque in the carotid than the much older comparison sample at risk for CVD. These PET/MR findings correlated significantly with cocaine use indices (lifetime use, craving, and addiction severity) and with nicotine and alcohol lifetime use where the more severe the drug use, the greater the carotid abnormalities $(.53 \leq \mathrm{r}$ $\leq .85, p<.01)$.

Conclusions: Replicating and extending previous results in an independent sample of healthy controls, these preliminary results show markers of carotid disease in CVDasymptomatic iCUD, which may exacerbate cognitive and behavioral impairments, of paramount clinical significance for combating silent disease progression.

Keywords: Cocaine Addiction, MR-PET, Cardiovascular, Neuro-Inflammation

Disclosure: Nothing to Disclose.

M254. The D3-Selective Dopamine Receptor Tracer [18F]Fluortriopride (FTP) Shows Differential Uptake in Limbic and Striatal Regions of Recently-Abstinent Cocaine Patients: Preliminary Studies

Anna Rose Childress*, Robert Doot, Jacob Dubroff, Christopher Ward, Kyle Labban, Jenny Cai, Hsiaojou (Sharon) Lee, Erin Schubert, Chia-Ju Hsieh, Catherine Hou, Kuiying Xu, Shihong Li, Alex Schmitz, Stefanie Darnley, Charles O'Brien, Kyle Kampman, Robert Mach

University of Pennsylvania Perelman School of Medicine, Philadelphia, Pennsylvania, United States

Background: After more than 30 years of the national cocaine epidemic, there are still no FDA-approved medications for this painful disorder. Finding precise medication targets for addiction depends on highly-specific tracers to label the neuroreceptors implicated in reward and motivational dysfunctions. Both D2 and D3 dopamine receptors may be dysregulated in addiction: preclinical data show that chronic stimulant exposure is associated with downregulation of the dopamine D2 receptors, but up-regulation of the D3 receptor (especially in reward-relevant regions). However, for more than two decades, dopamine receptor studies in humans could not parse the potentially distinct roles of these two receptor types. The most commonly-used PET radiotracer for dopamine receptor imaging, [11C] raclopride, is not specific for D3 vs. D2 receptors and development of more specific tracers (and candidate medications) has been hindered by the high overlap (up to $80 \%)$ in amino acids comprising the D2 vs. D3 receptors. The new availability of a highly-D3-selective (164-fold preference for D3 vs. D2 receptors) radiotracer, [18 F] fluortriopride (FTP), enabled the first scans in human cocaine patients, with the prediction of higher tracer uptake in regions known to contain D3 receptors.

Methods: Participants for these initial [18 F]FTP studies in a cocaine population were five treatment-seeking males who met standard inclusion/exclusion criterion for MR imaging, with additional criteria related to PET imaging (e.g., restrictions on recent use of medications known to impact dopamine transmission). The cocaine participants were representative of our local cocaine population, i.e., they were middle-aged (age range 47-53) African American males with heavy use of cocaine in the past month (range 12-27 days of the past 30), and chronic histories of cocaine (e.g.,14-34 years of self-reported regular use, at least $3 \mathrm{x}$ weekly). PET scanning was scheduled shortly after admission to a closed inpatient treatment unit (e.g., 1-3 days). [18 F]FTP studies in $(n=5)$ younger healthy male controls (see adjacent posters by DuBroff et al. 2017 and Doot et al., 2017 at this ACNP conference, for details) offered an informal comparison.

On scan day (Philips Ingenuity TF PET/CT scanner), lines were placed for bilateral upper extremity IV access and left upper extremity arterial line access. Following a CT attenuation scan, patients received an intravenous bolus administration of [18 F] FTP (dose range of $5.98 \mathrm{mCi}$ $7.15 \mathrm{mCi}$ ), and underwent 2 hours of dynamic PET brain imaging. Dynamic arterial sampling was performed for subsequent metabolite analysis. On a separate occasion, each participant also received a high-resolution $(1 \mathrm{~mm})$ structural MRI (MPRAGE; Siemens $3 \mathrm{~T}$ PRISMA) for later coregistration with the functional [18 F]FTP PET data. Kinetic analyses of time-activities curves extracted from coregistered $\mathrm{PET}$ and $\mathrm{MR}$ images estimated regional brain distribution volumes of total ligand uptake in tissue relative to total concentration of parent ligand in plasma (VT) using measurements from patient blood samples (PMOD Technologies, Ltd, version 3.7).

Results: Encouragingly, the regional pattern of [18 F]FTP uptake in these initial cocaine patients showed higher mean values in several brain regions known to contain D3 receptors (e.g., caudate, putamen, pallidum, n. accumbens, thalamus, amygdala and substantia nigra). Tracer uptake in the other regions was generally lower. There was considerable individual variation in [18 F]FTP uptake within the cocaine cohort. Average [18 F]FTP uptake in cocaine patients for the caudate, putamen, and pallidum, $\mathrm{n}$. accumbens, and amygdala regions were higher than in the healthy young male controls; this comparison was significant for the putamen (2-sample t-test, equal variance, 2-tailed, $p<0.033)$.

Conclusions: This study of [18 F]FTP in cocaine patients is the first use of this tracer in a clinical population. The 
preliminary results indicate increased uptake of [18 F]FTP in cocaine subjects for brain regions having a high density of D3 receptors. Upcoming studies to inform these initial findings include a blocking study with a D3-preferring compound and recruitment of age-matched controls. This future research will help determine whether the increase [18 F]FTP uptake is indeed related to a change in D3 receptor function in chronic cocaine users. As pre-clinical research demonstrates a clear role for dopamine D3 receptors in cue-triggered motivation for several rewarding drugs of abuse, confirming up-regulation of D3 receptors in cocaine patients would offer a clear brain target for antirelapse agents. PET tracers with increased specificity, such as [18 F]FTP, can play a critical role not only in parsing the primary brain dysfunctions in addiction, but also in screening and developing candidate medications to address underlying receptor dysfunctions.

Keywords: PET Imaging, Dopamine D3 Receptors, Cocaine Addiction, [18- F] fluortriopride

Disclosure: Nothing to Disclose.

\section{M255. A Phase 2, Multicenter, Randomized, Double-Blind, Placebo-Controlled Study of the Safety and Efficacy of Samidorphan in Adults With Alcohol Dependence}

Stephanie O'Malley*, Mark Todtenkopf, Elliot Ehrich, Bernard L. Silverman

Yale University School of Medicine, New Haven, Connecticut, United States

Background: Samidorphan (SAM) is an opioid modulator that functions as an antagonist at $\mu$-opioid receptors. Nonclinical studies in rats demonstrated that oral SAM administration results in a significant reduction in alcohol drinking behavior. This Phase 2 study evaluated the safety and efficacy of samidorphan in adults with alcohol dependence.

Methods: This was a multi-center, randomized, doubleblind, placebo-controlled study. Adults with a diagnosis of alcohol dependence and heavy drinking on at least 24 of 60 days prior to screening, and at least 4 , but not more than 10 consecutive days of abstinence were randomized to SAM $(1,2.5$ or $10 \mathrm{mg})$ or matching placebo. The primary endpoint was the percent of subjects abstinent from heavy drinking (PSNHDD) during Weeks 5 through 12 as self-reported using Timeline Follow-Back. Acknowledging these measures provide only indirect evidence of treatment benefit and cannot quantify the impact of treatment on how a patient actually feels or functions. tTe Food and Drug Administration considers PSNHDD to be acceptable within the clinical trial setting (Center for Drug Evaluation and Research, 2015) The European Medicines Agency also recommends the use of the various secondary endpoints including, for example, craving/urge to drink, or evaluation of the proportion of patients with a significant categorical shift in World Health Organization (WHO) risk levels of drinking (EMA, 2010). As such, additional post hoc analyses examined a shift down in World Health Organization (WHO) risk level of alcohol use (very high risk, high risk, moderate risk and low risk based on grams of alcohol consumed) by treatment group as a categorical response measure. Finally, the effects of SAM were examined for patient global assessment of response, craving, emotional fluctuations, and sleep habits.

Results: 406 subjects were included in the full analysis set. Participants were predominantly male (71\%) and white (64\%), with a mean age of 43 years (range 18-77). On the primary outcome, there was an overall decrease in the PSNHDD in SAM-treated subjects that was not statistically significant when compared to placebo. Post-hoc analyses revealed significantly ( $p=0.0047)$ fewer heavy drinking days occurred with SAM $10 \mathrm{mg}$ vs. placebo. For the cumulative event rate for daily heavy drinking, the relative reduction was $41 \%$ with SAM $10 \mathrm{mg}$ vs. placebo $(p=0.0006)$. The proportion of subjects who shifted down by at least 1 WHO risk category ranged from $61.4 \%-68.4 \%$ for the SAM groups compared to $50.6 \%$ for the placebo group.

Patient global assessment of response to therapy was significantly higher $(p<0.05)$ vs. placebo at Weeks 4,8 , and 12 with SAM 2.5 and $10 \mathrm{mg}$. Reductions in alcohol craving scores were significantly greater with SAM 2.5 and $10 \mathrm{mg}$ ( $p=0.0068$ and 0.0032 , respectively) vs. placebo at Week 4 and with $10 \mathrm{mg}$ vs. placebo $(p=0.0014)$ at Week 6 . At Week 8 , time to fall asleep $(p=0.0026)$ and overall quality of sleep $(p=0.0127)$ were significantly improved with SAM $10 \mathrm{mg}$ vs. placebo. In the SAM $10 \mathrm{mg}$ group, significant reductions in HAM-A total score were recorded at Weeks 4, 8 , and 12 compared with placebo $(p<0.01, p<0.01$, and $p<0.05$, respectively). HAM-D total score was significantly reduced at Weeks 8 and 12 following treatment with SAM $10 \mathrm{mg}$ compared with placebo $(p<0.01$, and $p<0.05$, respectively).

Nausea was the most frequently reported treatment emergent adverse event. There were no deaths or serious AEs considered related to study drug nor other clinically relevant changes.

Conclusions: Although the primary outcome measure, PSNHDD, was not met, treatment with SAM over 12 weeks was associated with significant improvement in clinically relevant measures of alcohol consumption (cumulative heavy drinking days, change in WHO drinking-risk level) compared with placebo. There was also improvement in patient global assessment of response to therapy, alcohol craving, anxiety, depression, and overall sleep quality following SAM treatment, particularly at the highest dose. Thus, results for PSNHDD were at variance with improvements noted on other measures of alcohol consumption and several measures important to patients. These findings highlight the importance of understanding outcomes most relevant to patients and appropriately prioritizing patient-centered outcomes in assessing interventions for AUD.

Keywords: Alcohol Dependence, Opioid Antagonist Treatment, Clinical Trial

Disclosure: Part 1: Alkermes, Advisory Board, Amygdala, Advisory Board, Cerecor, Advisory Board, Mitsubishi Tanabe, Advisory Board, Opiant, Advisory Board, American Society of Clinical Pharmacology Alcohol Clinical Trials Initiative, Board Member, Part 4: Pfizer, Inc., Grant. 
M256. Impairments in Resting State Activity are Associated With Cannabis use and Major Depressive Disorder

Nina Levar*, Milena Radoman, A. Eden Evins, Jodi Gilman

Massachusetts General Hospital, Boston, Massachusetts, United States

Background: Little is known about the effect of marijuana (MJ) use in people with major depressive disorder (MDD). MJ use among depressed patients is particularly concerning because both MJ use and MDD are independently associated with similar psychological, cognitive and neural impairments, such as anhedonia, amotivation, and dysphoria, raising the possibility that the combination of both may have a particularly detrimental impact on functioning. This study examined differences in neural activity among marijuana users and non-users with and without MDD in the three core networks implicated in psychopathology: the default-mode network (DMN), the salience network (SN), and the central executive network (CEN).

Methods: Eighty-one participants between the ages 18-25 years completed this neuroimaging study, consisting of four groups: Control participants with neither MDD nor MJ use (CON; $n=24)$, control participants with MJ use (MJ; $n=19)$, MDD participants without MJ use (MDD; $n=19$ ), and MDD participants with MJ use (MDD+MJ; $n=19)$. MRI scans were acquired at the Martinos Center for Biomedical Imaging. A high resolution structural T1 scan and a resting-state scan were acquired. Participants completed a timeline follow-back interview assessing cannabis and alcohol use over the past 90 days. Depressive and anxiety symptoms were assessed using the Mood and Anxiety Symptom Questionnaire and a clinical interview for diagnosis (SCID).

Resting state networks were extracted using independent component analysis (ICA) in Melodic in FSL. Components representing the DMN, SN, and the left and right CEN were identified based on standard network definitions. Permutation tests were used to assess differences in network activity between groups for the four networks $(p<0.05)$. Partial correlations between networks were calculated for $\mathrm{HC}, \mathrm{MJ}$, $\mathrm{MDD}$, and MDD+MJ groups separately. BOLD activity within resting state networks were correlated with demographic and clinical measures across groups.

Results: Five ICA components were selected for the anterior DMN (aDMN), posterior DMN (pDMN), SN, and the left and right CEN. MDD had increased resting state activity compared with all other groups in the aDMN (e.g. frontal pole, ACC, frontal medial cortex), the salience network (e.g. insula, caudate, putamen), and left and right CEN (e.g. precuneus, lateral occipital cortex, superior parietal lobe). MDD also showed greater activity compared to CON and MJ in the pDMN (e.g. posterior cingulate gyrus, angular gyrus). MDD-MJ had increased activation in the pDMN compared to $\mathrm{MJ}$ without MDD. There was a significant correlation for age of marijuana use onset and the aDMN and rCEN (aDMN: $r=0.36, \quad p=0.023 ; \quad r C E N: \quad r=0.42 ; p=0.008$ ). Anxiety symptoms correlated with activity in the pDMN $(\mathrm{r}=0.33, p=0.041)$.

Conclusions: Results show that cannabis use and symptoms of major depressive disorder are associated with differences in resting state activity in the DMN, SN, and CEN, with depression having the largest effect across networks. Cannabis use is associated with increased activity compared to non-using controls, while depressed cannabis users show reduced activation compared to MDD in all networks except the pDMN. Future work is needed to examine whether connectivity between networks is linked with differences functional outcomes including MDD symptoms and cognitive functioning. It is possible that cannabis use reduces aberrant resting state activity associated with MDD, but might disrupt the interplay between networks, thereby impairing cognitive processes such as episodic memory and attention.

Keywords: Resting State Brain Imaging, Marijuana, Major Depressive Disorder, Default Mode Network (DMN), Salience Network

Disclosure: Nothing to Disclose.

\section{M257. Peripheral TNF $\alpha$ Levels Correlate With Hippo- campal Volume in Alcoholism but not in HIV Infection}

Natalie Zahr*, Dongjin Kwon, Kilian Pohl, Edith

Sullivan, Adolf Pfefferbaum

Stanford University, Menlo Park, California, United States

Background: A rich literature suggests that elevated levels of inflammatory biomarkers are associated degenerative disease. High levels of pro-inflammatory cytokines, including tumor necrosis factor alpha $(\mathrm{TNF} \alpha)$, have been associated with poor cognitive function, smaller hippocampal volume, and Alzheimer's disease. Recent mechanisms proposed to contribute to neuropathology in Alcohol Use Disorder (AUD) include elevations in peripheral cytokines that sensitize the brain to the damaging effects of alcohol. In HIV infection, significant evidence supports the view that brain alterations are due to inflammatory processes. This study was conducted to determine whether relations between elevated TNF $\alpha$ levels and smaller hippocampal volumes are also evident in AUD and HIV.

Methods: Four groups of human participants were included: healthy controls $(n=39,18$ women, $52.3 \pm 9.8$ years $)$, individuals with $\operatorname{AUD}(n=72,23$ women, $51.3 \pm 9.1$ years), those infected with HIV ( $n=39,15$ women, $56.0 \pm 7.6$ years), and those comorbid for AUD and HIV ( $n=38,15$ women, $55.3 \pm 6.2$ years). The Human Immune Monitoring Center (http://iti.stanford.edu/himc/) performed immunological assays using 41-plex kits (i.e., 41 analytes, HCYTOMAG-60 K, $n=7$ ) purchased from EMD Millipore and used according to manufacturer's recommendations. SPoiled Gradient Recalled Echo (SPGR; TR $=7 \mathrm{~ms}, \mathrm{TE}=2.2 \mathrm{~ms}, \mathrm{TI}=300 \mathrm{~ms}$, thick $=$ $1.25 \mathrm{~mm}$, ski $p=0 \mathrm{~mm}, 124$ slices) and dual-echo fast spin echo $(\mathrm{FSE} ; \mathrm{TR}=8583 \mathrm{~ms}, \mathrm{TE} 1 / 2=13.5 / 108.3 \mathrm{~ms}$, thick $=2.5$ $\mathrm{mm}$, ski $p=0 \mathrm{~mm}, 62$ slices) sequences were collected on a GE 3.0 T Signa whole-body system with an 8-channel phasedarray head coil. After registration, skull stripping, and reformatting, regions of interest were quantified: regional gray matter volumes were parcellated using Freesurfer; white matter regions were parcellated using the SRI24 atlas.

Results: Of 41 analytes, only interferon gamma-induced protein 10 (IP10) and TNFo were higher in HIV and HIV +AUD groups than control or AUD groups. A 2-group 
analysis revealed that $\mathrm{TNF} \alpha$ was higher in AUD than controls, but this difference was due to higher TNF $\alpha$ in AUD patients sero-positive for Hepatitis $\mathrm{C}$ virus (HCV).

An Akaike information criterion (AIC) model, conducted independently for each patient group (AUD, HIV, AUD + HIV), determined which of 9 brain regions (i.e., thalamus, hippocampus, caudate, putamen, pallidum, pons, corpus callosum, cerebellar cortex, and cerebellar white matter) was relevant to TNF $\alpha$ levels in AUD and HIV.

In the AUD group, the initial AIC model indicated the hippocampus as the only relevant brain region $(\mathrm{F}(70)=3.3$, $p=.07$ ), contributing $4.5 \%$ to the variance in TNF $\alpha$ levels. Results were similar for the HIV+AUD group, with the AIC indicating only the hippocampus $(\mathrm{F}(36)=3.6, p=.07)$, which contributed $9.0 \%$ to the variance in TNF $\alpha$ levels. For the HIV group, the relevant brain regions identified by the AIC included the thalamus, caudate, pallidum, and cerebellar cortex. A multiple regression including all 4 of these regions was significant $(\mathrm{F}(39)=5.6, p=.0014)$. Together, these 4 regions explained $40 \%$ of the variance in TNF $\alpha$ levels. In the multiple regression, thalamic volume was significant $(p=.04)$ in explaining the variance in TNF $\alpha$ levels (caudate $p=.07$, pallidum $p=.07$, cerebellar cortex $p=.10$ ), and independently predicting $24 \%$ of the variance.

In separate AIC models conducted independently in each patient group, further variables were considered. In addition to hippocampal and thalamic volumes, each AIC model predicting $\mathrm{TNF} \alpha$ levels included a cognitive test (performance on Trails B), age, gender, race, education, BMI, blood pressure, smoking status, physical activity, depressive symptoms (BDI), and total gray matter volume. In the AUD group, the AIC model indicated that variables relevant to $\mathrm{TNF} \alpha$ levels included hippocampal volume, depressive symptoms, and performance on Trails B. A multiple regression including these 3 variables was significant $(p=.0014)$, explained $24 \%$ of the variance in TNF $\alpha$ levels, and was driven by performance on Trails B $(p=.003)$. In the $\mathrm{HE}$ group, this AIC model indicated the hippocampus $(p=.02)$ as the only relevant variable. In the HIV group, the AIC specified thalamic volume and performance on Trails B. Together, a in a multiple regression including these 2 variables explained $33 \%$ of the variance in TNF $\alpha$ levels, with thalamic volume driving significance $(p=.0005)$.

Conclusions: Our results suggest that in AUD, with or without HIV comorbidity, higher levels of $\mathrm{TNF} \alpha$ were associated with smaller hippocampal volumes. This relation, which persisted even when considering a number of additional, potentially relevant variables, comports with the Alzheimer's disease literature. Further, in agreement with the literature, this study also reveals that high $\mathrm{TNF} \alpha$ levels are associated with poor cognitive performance and depressive symptoms.

In HIV, this relation was not evident. Instead, TNF $\alpha$ levels correlated with several brain regions; of them, the thalamus was responsible for explaining a large percentage of the variance of the model. This relation, which persisted after considering a number of other variables, may emerge because of the sensitivity of the thalamus to HIV infection. Keywords: Magnetic Resonance Imaging, Cytokines, Hepatitis C, Regions of Interest, Brain Volumes

Disclosure: Nothing to Disclose.
M258. Noradrenergic Function After Chronic Stress and Ethanol Exposure

Carol den Hartog, Stephanie Fedorchak, Michael Grampetro, Owen Jordan, David Moorman, Elena Vazey*

University of Massachusetts Amherst, Amherst, Massachusetts, United States

Background: Stress is a risk factor implicated in the transition from initial alcohol drinking to dependence, as well as in relapse following abstinence. Chronic stress further increases ethanol consumption in rodents with a history of ethanol dependence. Identifying the neural circuits and behavioral functions disrupted by concomitant stress and ethanol exposure may help identify new treatments for alcohol use disorders and for those at risk of stress related relapse. Candidate circuits include those within the locus coeruleus (LC) norepinephrine (NE) system, a stress sensitive nucleus that is dysregulated in addiction. Candidate behaviors include cognitive control and anxiety, both of which can be influenced by LC-NE. After repeated stress and ethanol exposure we investigated NE function during anxiety and prefrontal dependent cognition using the alpha adrenergic antagonist prazosin. We also measured the activation of LC-NE neurons in these subjects to identify neuronal correlates of disrupted behavior.

Methods: Adult male and female C57BL/6 J mice were trained to drink ethanol $(15 \%, \mathrm{v} / \mathrm{v})$ on a $1 \mathrm{hr} /$ day 1-bottle choice schedule until a baseline was established. Mice were then exposed to weekly cycles of chronic intermittent ethanol (CIE) or air-control vapor exposure (Air), followed by test cycles of $1 \mathrm{hr}$ /day ethanol drinking. Mice were split into nonstress control (NS) or stress groups that received 10 minutes of forced swim stress (FSS) 4 hours before each drinking test. This schedule produced four experimental groups: control, Air/NS; ethanol-dependent no stress, CIE/NS; nondependent stress, Air/FSS; or ethanol-dependent stress, CIE/FSS. After stress and ethanol history was established, all animals were tested for prefrontal-dependent cognition using object/context recognition (OCR) and anxiety-like behavior with a marble burying test (MBT) with or without prazosin $(0.75 \mathrm{mg} / \mathrm{kg})$. At the end of behavioural testing we recorded single unit LC activity and sensory responsiveness before and after alcohol. We used voluntary ethanol consumption to determine individual alcohol dosing to investigate how preferred alcohol levels impacted LC activity. Results: Combined stress and ethanol history disrupted prefrontal dependent cognition and increased anxiety as measured by disrupted OCR performance and increased marble burying. Prazosin treatment impacted cognitive function in males but not females. Prazosin decreased anxiety in animals with CIE/FSS history in both sexes. During in-vivo recording ethanol and stress history altered baseline activity and sensory responsiveness of LC neurons, with a bias towards increased LC activity in alcohol and/or stress groups. Furthermore, treatment with preferred alcohol levels ameliorated some changes in LC activity.

Conclusions: Noradrenergic function is sensitive to stress and ethanol history, although impact varies between males and females. Addressing LC-NE function, either through 
manipulation of LC-NE neurons or pharmacological treatment at specific NE receptor targets, may be a future therapeutic strategy for alcohol use disorders, particularly in cases of interactions between chronic stress and alcohol use. Keywords: Locus Coeruleus, Electrophysiology, Sex Differences

Disclosure: Nothing to Disclose.

M259. Changes in Computational Markers of DecisionMaking are Proximally Tied to Heroin Use in Opioid Users Followed Longitudinally Through the First Months of Treatment

Anna Konova*, Silvia Lopez-Guzman, Adelya

Urmanche, Stephen Ross, Kenway Louie, John

Rotrosen, Paul Glimcher

New York University, New York, New York, United States

Background: Opioid overdose deaths have increased fourfold in the last decade and are now the leading cause of accidental death in the U.S. Relapse to opioid (heroin and painkiller) use during abstinence or opioid replacement treatment significantly increases the risk for overdose. Identifying predictors of relapse, and ultimately preventing relapse, in abstaining and/or treatment-seeking individuals is thus of critical importance. Here we tested the hypothesis that computational markers of decision-making are proximately tied to heroin use in individuals seeking treatment for their opioid addiction. We specifically hypothesized that the value of risky prospects is enhanced when these individuals are most vulnerable to return to heroin use, and that the neurobiological basis of this vulnerability would involve the brain's valuation system comprised of the striatum and ventromedial prefrontal cortex (VMPFC).

Methods: 79 chronic opioid users were serially studied over the first 7 months of initiating opioid replacement treatment $(1-15$ study sessions/subject; mean $=6, \mathrm{SD}=3.9)$. At each session, we characterized individual decision-making and documented any return to heroin use by self-report and random (weekly) urine toxicology tests. To derive computational markers of decision-making we used a validated task amenable to neuroeconomic modeling. In the task completed at each session, subjects repeatedly chose between certain gain of real money and a chance to play a lottery. Lotteries differed in possible monetary gain and in the probability of experiencing that gain. In some trials, the probability of winning the lottery was known (risky prospects), in others, the probability was only partially known (ambiguous prospects). This overall design allowed us to perform timelagged analyses predicting heroin use next session from decision-making behavior on the current session via mixedeffects logistic regression. A subset of the larger cohort ( $n=12$, study ongoing) also completed a multi-band fMRI session where they performed the same decision-making task and resting-state scans to isolate the neural mechanism of the decision process involved.

Results: Of 605 total sessions, 288 (47\%) were heroin positive. Only an increase in an ambiguity tolerance decision parameter was significantly predictive of prospective heroin use at the timescale examined [ $\mathrm{t}(577)=3.39, p<0.001]$, supporting our hypothesis that the value of these (more risky) prospects is enhanced when individuals are most vulnerable. The preliminary fMRI data revealed activity in the striatum and VMPFC encoded the value of the ambiguous prospects on a trial-by-trial basis, increasing as their subjective value increased. Activity in these regions in turn correlated with connectivity between them at rest, together suggesting that coordinated activity in the brain's valuation system might underlie both the observed decisionmaking behavior and heroin use vulnerability.

Conclusions: Treatment monitoring and intervention efforts aimed at decision processes for risky prospects (and thus the valuation system) may help reduce incidence of relapse in a population at high risk for overdose death.

Keywords: Computational Psychiatry, Opioid Dependence, Decision-Making, Relapse and Treatment Outcome, Brain Imaging, fMRI

Disclosure: Nothing to Disclose.

M260. Varenicline Reduces Nicotine Self-Administration, Naltrexone Inhibits Alcohol Consumption, but Both Fail to Alter Concurrent Ethanol and Nicotine SelfAdministration

Sheketha Hauser*, R. Aaron Waeiss, Christopher Knight, Lauren Pratt, Richard Bell, William McBride, Zachary Rodd

Indiana University School of Medicine, Indianapolis, Indiana, United States

Background: Simultaneous alcohol and nicotine usage occurs in the majority of alcoholics and nicotine dependent individuals. Varenicline (Var) is clinically used to assist in the cessation of nicotine use, while naltrexone $(\mathrm{Nal})$ is the standard treatment for alcoholism. Despite the growing evidence that ethanol (EtOH) and nicotine (Nic) co-use produces unique neuroadaptations and clinical outcomes, preclinical research has focused on examining the effects of potential pharmacotherpeutics on a single reinforcer. The current experiments examined the effects of $\mathrm{Var}$ and $\mathrm{Nal}$ on $\mathrm{EtOH}$, Nic, or $\mathrm{EtOH}+\mathrm{Nic}$ self-administration.

Methods: For both experiments, there were 4 drinking conditions (water only, Nic only, EtOH only, and $\mathrm{EtOH}$ $+\mathrm{Nic}$ ). The effects of Var and $\mathrm{Nal}$ on maintenance and relapse self-administration were determined for 4 consecutive days.

Results: Var reduced maintenance Nic self-administration and inhibited the increase in Nic self-administration following deprivation, but had no effect on EtOH or EtOH + Nic self-administration in either test conditioned. Conversely, $\mathrm{Nal}$ reduced $\mathrm{EtOH}$ maintenance and relapse drinking, but had no effect on $\mathrm{Nic}$ or $\mathrm{EtOH}+\mathrm{Nic}$ self-administration. Relapse drinking was increased and prolonged in rats concurrently consuming $\mathrm{EtOH}+\mathrm{Nic}$.

Conclusions: The results indicate that while the standard pharmacological treatment for Nic dependence and alcoholism was effective at reducing the consumption of the targeted reinforcer, neither was effective at reducing $\mathrm{EtOH}+\mathrm{Nic}$ couse/abuse. Overall, the findings suggest that the failure to develop successful treatment for alcoholism and Nic dependency maybe the result of a limitation in the models used to create such treatments. 
Keywords: Co-Abuse, Varenicline, Naltrexone, Alcohol, Nicotine

Disclosure: Nothing to Disclose.

M261. Neuronal Activation Within the Orbitofrontal Cortex: Subregional Analysis Following Cue-Induced Reinstatement

Amy Arguello*, Bal Aneesh, Butler Rachel, Verma Mansi

Michigan State University, East Lansing, Michigan, United States

Background: Cocaine-use disorders are characterized by repeated relapses to drug-seeking and taking behavior following acute or extended periods of abstinence. While several brain regions have been implicated in precipitating relapse to drug-associated cues, we focused on the lateral orbitofrontal cortex (IOFC) and basolateral amygdala (BLA) based on their involvement in both adaptive and maladaptive forms of associative learning. We previously found that optogenetic inhibition of monosynaptic lOFC $\bullet$ BLA projections impaired explicit conditioned stimuli (CS)-induced cocaine-seeking behavior; whereas, inhibition of monosynaptic BLA $\bullet$ IOFC projections did not impair this behavior. There is evidence that subregions of the OFC and BLA can play distinct roles in cue-induced reinstatement and rewardseeking behaviors. Therefore, as a first step to follow up on our unexpected results, we aimed to determine whether subregions within these monosynaptic circuits are differentially activated following exposure to previous drugassociated cues.

Methods: In order to examine subregional neural activation in the OFC in response to drug-associated cues, rats were trained to lever press for cocaine infusions paired with light +tone CS (2-hour sessions, at least 10 days) followed by extinction training (2-hour sessions, at least 7 days). To examine the pattern of neural activation in response to cocaine-paired cues, rats underwent a 1-hour reinstatement test with response-contingent CS presentation or NO CS presentation. Thirty minutes later rats were overdosed with a ketamine+xylazine cocktail and then transcardially perfused with $4 \%$ paraformaldehyde. Brains were extracted, cryoprotected in 30\% sucrose, then serially sectioned at $30 \mathrm{um}$. OFC sections were slide-mounted, stained with C-fos primary antibody and visualized using 3'3-diaminobenzidine.

Results: Rats acquired lever-pressing for cocaine infusions during self-administration training and exhibited decreased responses by the 7 th day of extinction training. Rats exhibited an increase in the number of lever responses following a 1-hour reinstatement test, with responsecontingent CS-presentation. We observed a qualitative increase of Cfos-immunoreactive (IR) cells within the OFC when compared to the control, no CS-exposure group. Future analysis will quantify the number of Cfos-IR cells within subregions of both the IOFC and BLA.

Conclusions: The current study aimed to map neural activation in the OFC and BLA to determine whether subregions within this circuit might differentially respond to cues that were previously associated with drug taking. Given that new methodologies are available to investigate the potential relevance of OFC subregions in humans, the present studies may provide essential information for the development of effective treatments for cocaine addiction. Keywords: Cue Reinstatement, Orbitofrontal Cortex (OFC), Neural Activity

Disclosure: Nothing to Disclose.

M262. Placebo (Under Conditions of Analgesic Expectation) Moderates Neuroimmune Interactions Between Brain Dopamine Release at D2 Receptors and Plasma IL-18 Concentration in Healthy Humans

Alan Prossin*, Robert Dantzer, Phillip Campbell, Alisa Koch, Jon-Kar Zubieta

\section{University of Texas Health Science Center at Houston, Houston, Texas, United States}

Background: Ongoing work in our lab shows that placebo administration, under conditions of analgesic expectation, reduces plasma concentration of IL-18, a stress reactive, potent IL-1 family cytokine with hyper-nociceptive properties. Preliminary data shows that extent of placebo-derived reduction in plasma IL-18 is linearly proportional to both endogenous opioid release and subsequent reduction in pain experienced during a standardized, experimental sustained pain challenge in healthy humans. While clinical implications of these findings are likely wide ranging, substantial inter-individual variance underlying placebo analgesia suggests that knowledge of underlying mechanisms is critical in developing applications seeking to enhance medical precision in a patient specific manner. Prevailing mechanistic theories are driven by knowledge acquired from conditioning experiments in animal models with very little evidence in humans. However, prior "non-placebo" evidence in both animal models and humans suggests dopamine D1 and D2 receptor activation within specific brain regions modulates central and peripheral immune processes via NLRP3 dependent and independent mechanisms. In this manner, activation of dopamine receptors is capable of regulating plasma concentration of IL-1 family cytokines (IL-1 $\beta$ and IL-18).

Methods: To enhance understanding of mechanisms underlying placebo modulation of systemic inflammatory state, we analyzed neuroimaging data and plasma IL-18 from 36 healthy humans who completed a 11C-raclopride (RCL) PET neuroimaging study. More specifically, we sought to determine whether placebo activation of brain dopamine D2 receptors (under expectation of analgesia) modulated plasma concentration of IL-18. Subjects included both males and females between 18 and 45 years of age. All subjects completed 2 RCL PET scans (1 with placebo pre-treatment under conditions of analgesic expectation and 1 without). Each RCL PET scan included a standardized sustained pain challenge as previously reported. In brief, the pain challenge included 2 conditions, a control condition involving masseteric injection of isotonic saline followed by a painful condition involving injection of hypertonic saline. Using a flexible factorial model within SPM12 we sought to confirm that placebo induced increases in dopamine release during pain anticipation (reduction in RCL binding as previously described) co-varied with placebo-induced reduction in 
plasma IL-18. Plasma IL-18 was quantified via standard ELISA using whole blood samples obtained following the control condition (pain anticipation) on each of 2 PET scan days, 1 without placebo pre-treatment and 1 with placebo pre-treatment under conditions of analgesic expectation.

Results: Placebo pre-treatment, under conditions of analgesic expectation significantly reduced plasma IL-18 (T36 = $3.8 ; p<0.001)$ with a mean reduction of $46 \%$. Placebo had a significant moderating effect on linear relationships between brain dopamine release and plasma Il-18. Reduction in plasma IL-18 was linearly proportional to extent of dopamine release (and activation of D2 receptors) following placebo administration. This placebo induced moderation of neuroimmune interactions occurred in a bilateral and symmetric pattern across brain regions critically involved in processing emotionally salient, and stressful events, in large part replicating data from animal models. Results are as follows:

Left Thalamus Proper: (XYZ: -14,-14,7; F1,140=20.1; $p<0.00002$ )

Left Post Thalamus: (XYZ: $-24,-28,1 ; \quad F 1,140=20.2$; $p<0.00002$ )

Left Anterior Cingulate: (XYZ: -2,41,4; F1,140=20.6; $p<0.00002$ )

Left Amygdala: (XYZ: -13,-4,-19; F1,140=20.1; $p<0.00002$ ) Right Anterior Cingulate: (XYZ: 5,36,18; F1,140=21.2; $p<0.00002$ )

Right Amygdala: (XYZ: 20,-2,-26; F1,140=20.0; $p<0.00002$ )

Brain Stem - Pons: (XYZ: 6,-12,-22; F1,140 =20.2; $p<0.00002$ )

Conclusions: In-vitro evidence in macrophages and in-vivo evidence in stroke models in small animals suggests dopamine agonists, via activation of either D1 or D2 receptors, negatively regulate systemic activation and production of IL-1 family cytokines via either NLRP3 dependent or independent mechanisms. The data presented support our original hypothesis that placebo (under analgesic expectation) induces release of dopamine, subsequently modulating peripheral immune function as evidenced by reduction in plasma IL-18 concentration. The evidence of expectation induced moderation of neuroimmune interactions we report has wide-ranging applications in stress exacerbate illnesses (i.e. pain states, prescription drug abuse, heart disease, malignant spread of tumors, etc.). However, replication of findings and further clarification of both underlying mechanistic pathways and variance factors are needed in order to translate these findings to clinical care. Based on existing evidence, we believe that dopamine release within brain regions with inherent direct or indirect projections to the anterior pituitary, are capable of modulating plasma IL-18 concentration. However, whether changes in Il-18 derive from dopamine modulation of production, dopamine inhibition of NLRP3 activation, or both and whether dopamine related effects occur directly via D2 activation, D1 activation, or both, remain unclear. Subsequent studies that incorporate an additional radio-tracer capable of quantifying D1 receptor activation will help to clarify these remaining mechanistic issues and in the long term, will facilitate clinical translation of these exciting findings.

Keywords: Placebo Response, Molecular Mechanisms, Neuroimmune Interaction, Immune Modulation, Psychoneuroimmunology

Disclosure: Nothing to Disclose.
M263. Meta-Analysis of Drug Cue Reactivity: Towards Neuromarkers of Craving and Relapse

\author{
Hedy Kober*, Ross MacLean, Tor Wager \\ Yale University, New Haven, Connecticut, United States
}

Background: The experience of craving, defined in DSM-5 as 'a strong desire for drugs', is a diagnostic criterion for addiction that predicts drug use, and relapse after treatment. Exposure to drug-related stimuli is known to elicit cueinduced craving, as well as physiological responses, called cue reactivity, which have been linked to drug use and relapse across addictions. Imaging studies have further linked neural cue-reactivity to drug use and relapse. Given the clinical importance of cue-reactivity and cue-induced craving across drugs, it is imperative to identify their underlying neural mechanisms. This will inform our understanding of addictive behavior, and ultimately, improve our ability to predict it and treat it.

Consistently, hundreds of neuroimaging studies have already investigated the neural correlates of drug cue-reactivity and cue-induced craving. These studies vary in drug type (e.g., cigarettes, alcohol, cocaine), cue type (e.g., pictures, movies, paraphernalia), cue length (seconds to minutes), design (event-related vs. block), participant treatment status (treatment-seeking vs. not), pre-scan abstinence (long vs. short), and other factors. Early meta-analyses have shown that cuereactivity and/or craving are generally associated with increased activity in brain regions including the ventral striatum, and medial prefrontal cortex (mPFC). However, there has been surprisingly little consensus in findings, and an incomplete understanding of which of the variables listed above are important drivers of differences in brain activity. Methods: We used state-of-the-art meta-analytic methods (multilevel kernel density analysis) to summarize the literature on neural cue reactivity and cue-induced craving, including 118 neuroimaging studies published to date, representing 3513 participants (3x larger than any prior meta-analysis). This work builds on our prior methodological developments as well as meta-analytic work in other domains, uses optimized inclusion criteria, employs appropriately-stringent correction methods, and accounts for multiple methodological differences in studies across substances (e.g., cigarettes, alcohol, cocaine, cannabis).

Results: Across all drugs, we found consistent cue-related neural activity in mPFC, ventral striatum, dorsal striatum, bilateral amygdala, insula, thalamus, and posterior cingulate cortex. Analyses of individual drug types indicated that striatal and $\mathrm{mPFC}$ regions were consistently activated; however, cigarette cues were more strongly associated with insula activity, for example. We found additional differences associated with different cue-types (e.g., pictures vs. video), pre-scan abstinence, and treatment-seeking status. Magnitude of self-reported cue-induced craving was correlated with neural activity in mPFC, ventral striatum, and left amygdala. Conclusions: This meta-analysis serves several functions. For example, it provides a consensus view and systematic understanding of the neural mechanisms of cue-reactivity and cue-induced craving across addictions, which can be compared with preclinical findings and guide future research. Importantly, it will also help us move towards the 
development of biomarkers for craving that may then be used to assess treatment efficacy and predict clinical outcomes. In work recently-funded by R01 DA043690, we are going beyond this meta-analysis, to address an urgent need in addiction research: development of predictive biomarkers, which are stable indicators of biological processes (as have been developed in other areas of medicine). To do this, we are combining the meta-analytic results described above with person-level imaging data from our lab (of cue-induced craving) using machine learning, to establish a multivariate pattern of neural activity that can predict craving self-report from neural activity - a predictive neuromarker for craving, and ultimately, of relapse. Given the associations between neural cue reactivity, craving, drug use, and relapse, a multivariate neuromarker could provide a powerful neural predictor of drug-related outcomes.

Keywords: Craving, Cue-Induced Craving, Cue Reactivity, Addiction Circuitry, Meta-Analysis

Disclosure: Nothing to Disclose.

M264. Posterior Cerebellar Volume, Iowa Gambling Task Performance and Substance Use Disorder Outcome in Young Adult Offspring From Families at Ultra-High Risk for Alcohol Dependence

Shirley Hill*, Jessica O'Brien, Brian Holmes, Jeannette Locke-Wellman

Western Psychiatric Institute and Clinic, Pittsburgh, Pennsylvania, United States

Background: Cerebellar regional differences have been seen in association with familial risk for alcohol dependence. Risk for developing substance use disorders may be related to cerebellar/striatal functioning and their association with decision-making capacity. We hypothesized that we would find: (1) volumetric difference in high-risk (HR) offspring vs. low-risk (LR) controls in the posterior cerebellum that might have implications for decision-making capacity; and that (2) these factors may confer differences in SUD survival time. Methods: MRI scans (3 T) from $120 \mathrm{HR}$ offspring $(25.7+4.5$ years) and 108 controls $(24.1+5.1$ years $)$ that included 116 males and 112 females were analyzed using manual tracing to region of interest (ROI) analyses. Participants were administered the Iowa Gambling Task (IGT).

Results: A Cox survival analysis showed a significant relationship between posterior cerebellar volume but not the anterior cerebellar region, scores on the IGT, familial risk and survival time. Scores on the final block of trials where greater improvement is generally seen in normal controls due to increased practice, was a significant predictor of SUD survival time (Wald $=6.89, \mathrm{df}=1, p=0.009$ ). Significant effects for total posterior cerebellar volume was also marginally significant (Wald $=3.69$, df $=1, p=0.055$ ), though anterior cerebellar volume was not a significant predictor of SUD survival outcome.

Conclusions: The inferior posterior cerebellar region has been linked to working memory deficits. IGT performance has been reported to predict SUD survival. The present analysis shows that the posterior cerebellum and IGT performance are each independent predictors of SUD outcome.
Keywords: Cerebellum, Iowa Gambling Task, Alcohol and Substance Use Disorders

Disclosure: Nothing to Disclose.

M265. Neuropsychopharmacology: Left- Censoring and Truncation as Sources of Bias in Effect Estimates

James Anthony*, Olga Vsevolozhskaya

Michigan State University, College of Human Medicine, East Lansing, Michigan, United States

Background: Within neuropsychopharmacology, a subset of research projects is conducted by epidemiologists and statisticians, and we can anticipate a growing number of these studies in the 21st century 'big data' and bioinformatics era. 'Neuropsychopharmacoepidemiologists' study hazards or benefits of medicine or other drug-taking of the compounds used in psychiatry and neurology, and in other medical specialties, often beginning with cross-sectional or case-control observational research designs. For several reasons, estimates from these first-stage studies cannot be described as 'definitive evidence.' Rather, whenever possible, the next step is to design and to complete prospective or longitudinal studies, or randomized trials, in order to acquire more definitive evidence. However, the set of information requirements for design of any second-step prospective, longitudinal, and RCT clinical research project includes prerequisites such as a set of plausible starting effect size estimates (e.g., for statistical power calculations). It follows that the just-mentioned initial observational study estimates sometimes become especially valuable as empirically derived and plausible effect size estimates in the design of RCT and other prospective studies. In this report, we describe results from simulation research in order to quantify the degree of bias and error that might distort estimates from initial crosssectional and case-control studies, using examples within domains of drug dependence research and neuropsychopharmacology.

Methods: Data for our simulations are from United States National Surveys on Drug Use and Health (NSDUH), 20112015 , with more than 250,000 participants, cross-classified in relation to their computer-assisted self-interview assessment of use of internationally regulated drugs such as cannabis, cocaine, and opioids. We focus on estimation and forecast of (1) rapid transition from first drug use to dependence outcomes, and (2) biases as might vary across drug subtypes, such as cannabis (for which the pre-assessment case fatality rate (CFR) is essentially $0 \%$ ) versus opioids such as heroin, fentanil, or carfentanil (with larger CFR).

Results: Our simulations, based on both cross-sectional survey and case-control designs, yield modeled estimates of two missing data mechanisms (left-censoring and lefttruncation). These simulations make it clear that, in general, for low CFR compounds (e.g., cannabis), the estimates show little effect size distortions. In contrast, when CFR is larger (e.g., opioids), left-censoring and left-truncation mechanisms create larger distortions of effect size estimates. The conclusions can be generalized for missing data mechanisms such as jail/prison incarceration and non-participation biases, and for research on race-ethnicity subgroups subject to differential rates of incarceration or CFR variations. 
Conclusions: We anticipate that our simulations will help ACNP members understand how research design issues such as left-censoring and left-truncation must be taken into account when epidemiological cross-sectional survey estimates are used to forecast neuropsychiatric drug hazards. In some instances, such as cannabis research, these issues might prove to be trivial. In relation to other instances, such as opioids research, these issues might be substantial.

Keywords: Experimental Design, Left-Truncation, Expectation Bias

Disclosure: Nothing to Disclose.

\section{M266. Postpartum Depression and Opiate Exposure Affect Maternal Brain Physiology According to Parenting Measures}

James Swain*, Shaun Ho

Stony Brook University Medical Center, Stony Brook, New York, United States

Background: Sensitive maternal parenting depends on evolutionarily conserved attachment behaviors and associated thoughts for parents to respond to infant cues and shape infant development. Upon hearing their baby cry, for example, mothers may address baby needs and safety as well as sooth and provide other care. Parenting behaviors and thoughts toward their infant may depend on reward/ motivation/attachment circuit responses to approach their distressed infant, accurate representation of their infant and decreased negative emotions, such as anger, toward their infant. However, the capacity of mothers to generate caring behaviors and thoughts toward their infant may be compromised by the accumulation of risk factors, including postpartum depression and opiate exposure. For this longitudinal magnetic resonance imaging study, we hypothesize that: 1 . Among mothers, brain response changes over the early postpartum weeks in response to own baby-cry in affiliative and emotion regulation circuits will be affected by depression and opiates. 2. Opiate exposure will affect Own Baby Cry responses according to measures from structured parenting interviews.

Methods: We studied 79 mothers in early PP, and 42 of which completed structural and functional magnetic resonance imaging (MRI) scans at two time-points: T1 (2-4 weeks PP) and T2 (3-6 months PP). The participants reported depressive symptoms using Beck Depression Inventory (BDI) and underwent structured interviews on parenting (Working Model of the Child Interview) and at T1 \& T2. According to the BDI at T1 and prescription opioid usage, we divided the participants into three groups, including a group of 11 mothers who were on prescription opioid medication (bupreoprhine dosed to prevent withdrawal) before gestation until the time of study, a group of 12 mothers who had elevated depressive symptoms without the influences of prescription opioid (Depressed Controls), and a group of 56 mothers who were not depressed nor on opioid (Healthy Controls). At T1 and T2, the participants underwent functional magnetic resonance imaging (fMRI) scans while performing a baby-cry task, wherein they listened to own and other's baby cry and respective control sounds in a block design ( $30 \mathrm{~s}$ per block, 5 blocks per condition). The
fMRI images were processed and analyzed in SPM 8 to examine the differential neural responses to Own vs. Other Baby-Cry $(\mathrm{OBC})$. The structured interviews were analyzed for word count and linguistics (LIWC2015).

Results: BDI scores at T1 and T2 were not different between opioid group (OG) and depressed controls (DC) groups and were higher in these two groups as compared to healthy control (HC) group. The BDI scores did not differ between $\mathrm{T} 1$ and $\mathrm{T} 2$ in all three groups. For the OG, T1-to-T2 increases in own baby cry ( $\mathrm{dOBC}$ ) response in bilateral posterior hippocampus, caudate, insula, and middle temporal cortex were associated with T1-to-T2 BDI increases (dBDI), while those in the left dorsolateral prefrontal cortex were inversely associated with $\mathrm{dBDI}$. In examining the group differences in dOBC, OG and DC groups, as compared to $\mathrm{HC}$ group, showed greater $\mathrm{dOBC}$ in bilateral caudate and left insula and the OG, as compared to DC, showed greater $\mathrm{dOBC}$ in the left precuneus and parietal lobe but lesser dCOI in the rostral anterior cingulate cortex (rACC)/medial orbitofrontal cortex (mOFC). Some of these group differences in $\mathrm{dOBC}$ were related to maternal parenting behaviors. We found that the $\mathrm{dOBC}$ in $\mathrm{rACC} / \mathrm{mOFC}$ were inversely associated with the increases in the frequency of angerrelated words in the maternal discourse about the child (dAnger), when the OG group showed T1-to-T2 increases and the DC showed decreases in dAnger. Moreover, the $\mathrm{dCOI}$ in the right caudate, which were greater in OG than the other two groups, were associated with reduction in maternal affiliation-oriented behaviors.

Conclusions: During the early postpartum, the adaptation of maternal brain and behaviors were influenced by depressive symptoms and opiate exposure. First, increases in Own Baby-Cry oriented neural responses in the caudate, insula and other regions were associated with increases in depressive symptoms, compared to healthy controls and depressed controls. Second, mothers influenced by opioids showed altered Own Baby-Cry responses in reward neurocircuitry, including caudate and rACC, which were associated with decreasing maternal child-oriented affiliation behaviors and increasing anger (anti-rewards), respectively. These findings suggest that neuroimaging may detect risks to maternal behavior and child development beyond those detected using basic depression screening.

Keywords: Functional MRI (fMRI), Maternal Depression, Opiates, Baby-Cry

Disclosure: Nothing to Disclose.

\section{M267. Evaluating Early Exercise Interventions for Cocaine Abuse: Sex Matters}

\section{Chloe Jordan*, Susan Andersen}

National Institute on Drug Abuse, Baltimore, Maryland, United States

Background: Adolescence is a vulnerable period in development when individuals are more likely to engage in risktaking behaviors, including experimenting with drugs of abuse. However, drug use before the age of 14 is associated with a four-fold greater risk of developing substance use disorder (SUD) in adulthood. Early intervention in individuals at risk for drug use is therefore critical to reducing the 
prevalence of SUD. Aerobic exercise can reduce drug use in adults, potentially through stimulating neurogenesis of inhibitory GABAergic interneurons. However, few studies have investigated the utility of exercise as a preventative intervention in young subjects, before vulnerability to drug abuse manifests. We hypothesized that engaging in aerobic exercise during adolescence would protect against future cocaine-seeking behaviors. We investigated neurogenesis of GABAergic calretinin-expressing interneurons in the nucleus accumbens as one mechanism by which early exercise exerts its long-term effects on cocaine use, in both males and females.

Methods: Beginning in early adolescence (post-natal days [P] 25-40) male and female Sprague-Dawley rats were allowed access to running wheels for 2-hr daily (exercise intervention) or remained in homecages (control). In Experiment 1, rats ( $n=8-12 /$ sex/group) were implanted with intravenous jugular catheters on P42 and were allowed to self-administer cocaine $(0.5 \mathrm{mg} / \mathrm{kg})$. Self-administration training began with 2-hr sessions under a fixed ratio 1 (FR1) schedule of reinforcement and gradually increased to 6-hr binge sessions under a variable interval (VI) 120 - sec schedule to promote high levels of cocaine seeking. In adulthood $(\sim$ P120) rats were sacrificed and examined for neurogenesis and GABAergic interneuron expression in the nucleus accumbens using immunohistochemical staining for doublecortin and calretinin, respectively, followed by stereological analysis. In Experiment 2, separate groups of rats ( $n=5-8 /$ sex/group) were sacrificed on $\mathrm{P} 42$ and examined for doublecortin expression in the nucleus accumbens to verify that neurogenesis is observed acutely after adolescent wheel running. Results: Compared to males, females ran significantly more during adolescence $(p<0.001)$ and made more cocaineseeking responses in adulthood ( $\mathrm{ps}<0.05)$. Contrary to our hypothesis, linear regression revealed that adolescent wheel running was associated with increased cocaine seeking under the VI 120 and binge schedules in adult females (Rsquared = 0.21 and 0.28 , respectively, ps $<0.05$ ). Adolescent wheel running had no long-term impact on cocaine selfadministration in males. In accordance with behavioral observations, wheel running was associated with neurogenesis (increased doublecortin expression in rats that ran more) in the nucleus accumbens core of adolescent females (Rsquared $=0.46, p<0.03$ ), but not males. Adolescent wheel running further interacted with early puberty onset to predict doublecortin and GABAergic calretinin interneuron co-expression in the accumbens core of adult females (Rsquared $=0.37, p<0.05$ ). No changes in the accumbens shell were observed as a result of wheel running. However, adult females exhibited increased co-expression of doublecortin and calretinin in the accumbens shell compared to males, irrespective of exercise condition $(p<0.05)$.

Conclusions: In contrast to prior reports in adults, adolescent aerobic exercise may increase future cocaineseeking behaviors in females, in part through augmented neurogenesis and GABAergic interneuron expression in the nucleus accumbens core. Future work must determine whether continuation of exercise into adulthood can mitigate increased cocaine abuse risk in females.

Keywords: Aerobic Exercise, Cocaine Self-Administration, Adolescence, Sex Differences

Disclosure: Nothing to Disclose.
M268. Adolescent Social Isolation-Induced Increased Ethanol Intake is Mediated by Increased Kappa Opioid Receptor Function in the Nucleus Accumbens and Basolateral Amygdala of Rats

\author{
Anushree Karkhanis*, Jeff Weiner, Sara Jones
}

Wake Forest School of Medicine, Winston-Salem, North Carolina, United States

Background: Chronic early life stress exposure, such as neglect during childhood, increases the risk of developing alcohol use disorders during adulthood. Similarly, rats reared in social isolation (aSI) during adolescence show increased ethanol intake in adulthood compared to group housed controls $(\mathrm{aGH})$. Acute stress elevates dynorphin levels, a kappa opioid receptor (KOR) ligand, which regulates dopamine. Activation of KORs inhibits dopamine release in the NAc and BLA. The NAc and BLA are interconnected and play integral roles in the neurobiology of stress, anxiety and reward-seeking behavior. Recent literature shows that the KOR system regulates drug seeking following chronic exposure to ethanol. We used a multi-disciplinary approach to understand the potential underlying mechanisms driving the isolation-induced increases in ethanol intake. Additionally, we examined the effect of increased KOR function driving elevated ethanol intake.

Methods: Dopamine in the NAc and BLA was measured using in vivo microdialysis in freely moving rats that were either housed in groups (4 rats/cage) or individually (1 rat/ cage) for six weeks (PD 28-74). After reaching adulthood, acute ethanol was administered after establishing stable dopamine baselines. A separate group of rats was treated with norBNI ( $10 \mathrm{mg} / \mathrm{kg}$; i.p.) $24 \mathrm{hrs}$ prior to ethanol challenge. To investigate the KOR function specifically on dopamine terminals, ex vivo voltammetry in conjunction with optogenetics was used to record the effects of U50,488 (10-1000 nM), a KOR agonist, on optically stimulated dopamine release in slices containing NAc and BLA from $\mathrm{aGH}$ and aSI rats. To test causality, Cre dependent KOR overexpressing virus was infused into the ventral tegmental area (VTA) of aGH TH:Cre positive rats and ethanol intake was measured.

Results: Baseline levels of dopamine were significantly lower in the NAc and BLA of aSI compared to aGH rats. KORs increased baseline dopamine levels in both NAc and BLA. The aSI rats showed increased dopamine responses to ethanol $(2 \mathrm{~g} / \mathrm{kg})$ in both NAc (200\% of baseline) and BLA ( $280 \%$ of baseline) and this increase was significantly greater in BLA than NAc. Ethanol augmented dopamine responses in the NAc of aSI rats pre-treated with norBNI, but attenuated responses in the BLA. The inhibitory effects of U50,488 on optically stimulated dopamine release were enhanced in the NAc and BLA of aSI rats suggesting that chronic stress increases the functional responsiveness of KORs. Overexpressing KORs in dopamine neurons projecting from the VTA to the NAc and BLA resulted in elevated ethanol intake.

Conclusions: Increased dopamine elevations after ethanol in both regions of SI rats are consistent with the stimulant literature; however, the mechanisms behind the distinct effects of ethanol-induced dopamine in the presence and 
absence of norBNI in NAc and BLA are unclear. It is possible that KOR differences may explain in part the effects of ethanol on behaviors related to specific brain regions, for example, augmentation of dopamine in the NAc results in increased reinforcement, whereas augmentation in dopamine in the BLA may contribute to decreased anxiety.

Keywords: Adolescent Stress, Dopamine, Kappa Opioid Receptor, Alcohol

Disclosure: Nothing to Disclose.

M269. GABA Hyposensitivity of DA VTA Neurons During Alcohol Withdrawal is Reversed by in Vivo Treatment With a Histone Deacetylase Inhibitor: Evidence of Epigenetic Regulation of DA VTA Neuronal Physiology by Chronic Ethanol

Mark Brodie ${ }^{\star}$, Chang You, Bertha Vandegrift, Huaibo Zhang, Subhash Pandey, Amy Lasek

University of Illinois at Chicago, Chicago, Illinois, United States

Background: Alteration of neuronal physiology by chronic use of drugs of abuse likely underlies the sequelae of addiction, including compulsive drug craving and seeking. Agents that could reverse of those physiological changes would ameliorate the symptoms of addiction. Neurons of the ventral tegmental area (VTA) are important for the rewarding and reinforcing properties of drugs of abuse, including ethanol. Chronic or repeated ethanol treatment induces specific alteration of the physiology of VTA neurons, including increased responsiveness to acutely administered ethanol, and decreased responsiveness to the inhibitory neurotransmitter GABA. We have previously shown that in vitro administration of the histone deacetylase (HDAC) inhibitor SAHA reverses this GABA hyposensitivity of VTA neurons, but the action of systemic SAHA on these same phenomena are unknown.

Methods: We used Sprague-Dawley rats treated for 15 days using the Lieber-DeCarli diet method. Alcohol diet was removed from the rats in the "withdrawal" group 24 hours before sacrifice. Rats were injected with SAHA (vorinostat, $50 \mathrm{mg} / \mathrm{kg}$ ) or DMSO vehicle two hours before sacrifice. We used brain slice electrophysiological techniques to assess the effect of GABA on DA VTA neurons.

Results: Inhibitory responses to GABA were reduced significantly in VTA neurons from rats in ethanol withdrawal and treated with DMSO vehicle compared to control diet rats treated with DMSO.In contrast, there was no difference in response to GABA of VTA neurons from rats in withdrawal or control rats after treatment with SAHA. There was no difference between the GABA responses in VTA neurons from control diet treated rats or either group of rats treated with SAHA. Concurrent with this reduction in GABA sensitivity was a significant increase in immunolabeling for HDAC2 an isoform of histone deacetylase, and a significant decrease in acetylated histone protein (H3K9/H3K14). The level of acetylated histone protein was normalized by SAHA treatment.

Conclusions: Taken together with our previously published results, our studies demonstrate that GABA sensitivity in the VTA is reduced by chronic ethanol treatment, and that this
GABA hyposensitivity is under epigenetic control that is modulated by histone acetylation. At least a portion of the physiological function in the VTA altered during ethanol withdrawal may be normalized by treatment with HDAC inhibitors. Supported by NIH grant AA022538.

Keywords: Ventral Tegmental Area (VTA), Alcohol Withdrawal, Epigenetics

Disclosure: Nothing to Disclose.

\section{M270. A Tale of Two Atypical DAT Inhibitors and Their Potential to Treat Cocaine Use Disorders}

\author{
Amy Newman*, Jianjing Cao, Guohua Bi, Gianluigi \\ Tanda, Zheng-Xiong Xi \\ DHHS/National Institute on Drug Abuse, Baltimore, \\ Maryland, United States
}

Background: In an effort to discover potential pharmacotherapeutics for the treatment of psychostimulant abuse, we have designed and synthesized novel analogues of modafinil and discovered several lead candidates (Cao et al., 2016). JJC8-088 and JJC8-091 both bind to the Y156F DAT mutant differently than cocaine, suggesting a preference for the inward facing conformation that has been associated with an atypical behavioral profile in rodents (Loland et al., 2008, 2012.).

Methods: Both JJC8-088 and JJC8-091 were tested in rat models of cocaine self-administration (FR2 and PR schedules) and optical intracranial self-stimulation (oICSS). In addition, both compounds were evaluated for their effects on Nucleus Accumbens shell (NAS) dopamine, by in vivo brain microdialysis, and their addictive potential was evaluated in rats previously trained to self-administer cocaine.

Results: Despite being close structural analogues, JJC8-088 and JJC8-091 demonstrated significantly different behavioral profiles. Whereas, both compounds decreased cocaine selfadministration and cocaine intake, JJC8-091 was more effective in the PR model in which the workload is progressively increased for cocaine reward. In contrast, JJC8-088 was more effective in the FR2 model in which cocaine is essentially freely available. To determine whether this decrease in cocaine self-administration was due to enhancement of cocaine's rewarding effects, and therefore, less cocaine is required to achieve satiation, or due to a reduction in cocaine reward, and therefore, animals lose interest in the drug, we used a new animal model of optical intracranial self-administration (oICSS) maintained by photoactivation of midbrain dopamine neurons in transgenic DAT-cre mice. We found that JJC8-088 produced a cocainelike, dose-dependent upward and leftward shift in the stimulation-rate response oICSS curve, suggesting a potentiation of optical brain-stimulation reward. JJC8-091 produced an opposite effect, shifting the stimulation-rate response curve rightward and downward. To further confirm these findings, we used in vivo brain microdialysis to measure NAS dopamine response to these compounds. Again, JJC8-088 (0.5-10 mg/kg i.p.) produced a dosedependent cocaine-like increase in extracellular dopamine in the NAS, with a maximum increase of about $400 \%$ after $30 \mathrm{~min}$ from injection of the highest dose. On the other hand, JJC8-091 (10-56 mg/kg i.p.) had a significantly lower effect 
on dopamine levels in this brain region, with a maximum increase of about $250 \%$ at $30 \mathrm{~min}$ after injection of the highest dose. Finally, we used a classical drug substitution test and found that JJC8-088 was able to maintain stable selfadministration behavior in rats previously trained to selfadminister cocaine, while JJC8-091 did not. Moreover, when JJC8-091 was substituted for cocaine, the rats extinguished self-administration behavior.

Conclusions: Although both modafinil analogs inhibited cocaine self-administration or cocaine intake, the underlying mechanisms appear to be different. JJC8-088 is a high affinity DAT inhibitor $(\mathrm{Ki}=2.6 \mathrm{nM})$ that displays a cocaine-like agonist or substitute therapy profile in several rodent models. In contrast, JJC8-091, a DAT inhibitor with moderate DAT affinity $(\mathrm{Ki}=289 \mathrm{nM})$ was not cocaine-like in these animal models of drug reward, but effectively blocked cocaine selfadministration, supporting an atypical DAT inhibitor behavioral profile. In addition, JJC8-091 showed no rewarding potential by itself, while JJC8-088 may have addictive liability, as assessed in both oICSS and the drug substitution test in the cocaine self-administration model. Collectively, these data support the potential utility of JJC8-091 as a treatment for cocaine abuse. Further investigation of these and other modafinil analogues toward understanding their different mechanisms of action at DAT and the role of off target sites, such as sigmal and dopamine D2-like receptors are underway. These and additional behavioral studies will be conducted to determine their potential for the treatment of cocaine use disorders.

Keywords: Cocaine, Medication Development, Dopamine Transporter

Disclosure: Part 1: EncepHEAL, Inc., Patent.

\section{M271. Ventral Pallidal Contributions to Alcohol Seeking Depend on the Underlying Associative Structure}

Jocelyn Richard*, Anne Armstrong, Deanna Acs, Patricia Janak

Johns Hopkins University, Baltimore, Maryland, United States

Background: A critical area of inquiry in the neurobiology of alcohol abuse is the neural mechanisms by which cues gain the ability to elicit craving and relapse. The ventral pallidum (VP) has been suggested to act as part of a common pathway for relapse to drug and alcohol seeking. We previously showed that the activity of VP neurons in response to a cue predicting reward availability encodes both the likelihood and latency of subsequent instrumental reward-seeking actions (Richard et al., 2016). Here, we assessed the role of VP activity in cue-elicited alcohol seeking as well as whether VP cue responses would encode and contribute similarly to the vigor of Pavlovian versus instrumental reward seeking behaviors, when these responses consist of superficially similar locomotor response patterns, and are driven by similar levels of reward expectancy.

Methods: During Pavlovian conditioning, male and female Long Evans rats were trained to associate one auditory cue (the CS+) with delivery of $10 \%$ liquid sucrose reward or $20 \%$ ethanol (not contingent on the animal's behavior) and alternative auditory cue (CS-) with no delivery of reward. In the instrumental task, one auditory cue (the discriminative stimulus; DS) signaled availability of the same sucrose or ethanol reward, if the animal made an entry into the reward port during the cue period; the alternative cue (NS) signaled no reward availability. Rats were trained in one of these tasks until they entered the reward port on $>70 \%$ of reward cue trials (CS+ or DS), and $<30 \%$ of control cue trials (CS- or $\mathrm{NS}$ ), and then were implanted with drivable microwire arrays aimed at VP. We also assessed the functional contribution of VP activity to Pavlovian alcohol seeking using optogenetic inhibition: following training, we photoinhibited VP (ArchT expression and $532 \mathrm{~nm}$ light) during $50 \%$ of cue presentations to assess the impact on cue-elicited behavior.

Results: We found that, as expected, many ( $40 \%)$ of VP neurons were excited by a cue predicting alcohol delivery (CS $+)$ and these excitations were significantly greater to CS+ presentations that are followed by an entry to the alcohol port. Additionally, VP inhibition just during the alcohol cue period reduced the likelihood of a port entry response during the cue. Surprisingly, VP responses to the CS+ did not predict the latency of reward seeking. When we assessed VP responses to Pavlovian cues for sucrose we also found that VP cue excitations were predictive of port entry likelihood, but not of port entry latency. We next assessed VP responses to a cue predicting reward availability contingent on entry to the port during the cue period, to determine whether the vigor of this superficially similar instrumental reward seeking response would be encoded by VP neurons. We found that, similarly to our previous report, cue elicited activity in $\sim 25 \%$ of VP neurons significantly predicts the latency of instrumental reward seeking, even when it consists of a much simpler, Pavlovian-like behavior: entry to a reward port.

Conclusions: Here we find that VP activity contributes to reward seeking in distinct ways depending not only on the rewarding outcome or locomotor response pattern, but on the underlying associative structure that drives this reward seeking. This makes VP circuitry a promising target for future attempts to limit maladaptive behaviors driven by diverse dysfunctions in reward-related processing.

Keywords: Ventral Pallidum, Incentive Motivation, Alcohol and Substance Use Disorders, Optogenetics, Electrophysiology

Disclosure: Nothing to Disclose.

\section{M272. Neural Correlates of Discounted Subjective Value Track Heroin Use in Treatment-Seeking Opioid Users}

Silvia Lopez-Guzman*, Anna Konova, Adelya

Urmanche, Stephen Ross, Kenway Louie, John

Rotrosen, Paul Glimcher

New York University, New York, New York, United States

Background: Increased impulsive decision-making has been widely observed in individuals with substance use disorders. Several studies have pointed to brain regions in the valuation network as necessary computational neural substrates for this behavior, and posited that dysfunction in this circuitry in drug users might be at the basis of these disorders. Although identifying these regions is important, more pressing and 
clinically relevant questions remain unanswered: how does this dysfunction relate to the severity of drug use? And, in the case of treatment-seeking individuals, how does it relate to treatment success/failure, i.e. concomitant use, relapse and dropout? Focusing on the current opioid epidemic, we addressed these questions by following a cohort of patients with Opioid Use Disorder (OUD) receiving treatment, and assessing their neural activity in a temporal discounting (TD) task at 2 time points over the course of their treatment. Critically, this longitudinal approach allowed us to follow their dynamics in symptomatology, drug use and impulsivity and test how our neural measures relate to recovery or treatment failure.

Methods: We conducted 2 fMRI sessions in OUD patients starting standard outpatient treatment $(\sim 4$ and $\sim 12$ weeks post treatment entry). Patients completed a TD task in the scanner in which they were asked to choose between immediate and delayed monetary rewards. Patients' symptoms, drug use (by self-report and toxicology) and impulsive decision-making were assessed during the fMRI sessions and in multiple separate sessions over the course of 7 months. fMRI and behavioral testing was also performed in a cohort of matched community controls.

Results: In preliminary results $(n=13)$, we find similar taskbased activation of valuation circuit areas, e.g. ventromedial prefrontal cortex and posterior cingulate cortex, across both OUD patients and controls. Interestingly, in OUD patients, dorsolateral prefrontal cortex (DLPFC) subjective value coding for delayed rewards decreased as a function of recent and imminent heroin use.

Conclusions: Our results point to a role for DLPFC recruitment as a contributor to treatment success and suggest it could be a potential candidate area for therapeutic intervention. Current efforts in this study focus on increasing our sample size and establishing whether neural activity elicited by our task is truly predictive of future drug use, relapse and dropout from treatment.

Keywords: Addiction, Delay Discounting, Impulsivity, Opioid, fMRI

Disclosure: Nothing to Disclose.

\section{M273. Nucleus Accumbens D1 Receptor-Expressing Neurons Control Autoshaping Behavior}

Tom Macpherson, Takatoshi Hikida*

Institute for Protein Research, Osaka University, Osaka, Japan

Background: The striatum of the basal ganglia is known to control learning about the properties of environments, as well as the appropriate behavioral response that will maximize reward or minimize harm in these environments. Indeed, dysfunction of the striatum is known to contribute to a number of decision-making-associated neuropathologies, including drug addiction and schizophrenia. However, it is still unclear how attribution of motivational value (incentive salience) towards primary rewards, or environmental stimuli predictive of rewards, may be controlled by the activity of different striatal subregions and neuron types.

Methods: We used a reversible neurotransmission blocking (RNB) technique (as described in Hikida et al, 2010) to separately inhibit neurotransmission in dopamine D1- and D2-receptor-expressing medium spiny neurons (D1-/D2MSNs) originating from three striatal subregions, the nucleus accumbens (NAc), the dorsomedial striatum (DMS), and the dorsolateral striatum (DLS), in order to explore their role in mediating Pavlovian approach behaviour in an autoshaping task in touchscreen operant chambers. Over the course of 6 daily sessions, a food reward was paired with the presentation of one conditioned stimulus (CS+), but not the presentation of another conditioned stimulus in a different location (CS-). During these conditioning sessions, we measured approaches to the CS+ or food magazine during $\mathrm{CS}+$ presentation as a measure of sign-tracking and goaltracking, respectively.

Results: Sign-tracking behavior was impaired in NAc D1MSN-blocked RNB mice over the course of 6 daily sessions compared to WT and NAc D2-MSN-blocked RNB mice. Blockade of neurotransmission in D1 or D2-MSNs in either the DMS or DLS did not alter the ability to acquire signtracking behavior. Goal-tracking behavior decreased over the course of 6 daily sessions in all animals, with the exception of NAc D1-MSN-blocked RNB mice.

Conclusions: We revealed a specific role of NAc D1expressing neurons in controlling Pavlovian approach behavior to an environmental cue associated with a natural food reward. Blockade of NAc D1-expressing neuron activity impaired sign-tracking responses to the CS, while leaving goal-tracking responses intact. These findings provide insight into the neural circuits underlying Pavlovian conditioning, and may be important in the identification of therapeutic targets for the treatment of disorders associated with a loss of impulse control, including drug addiction and schizophrenia.

Keywords: Basal Ganglia, Dopamine Receptors, Conditioning

Disclosure: Nothing to Disclose.

\section{M274. Effects of the Classic Hallucinogen Psilocybin and the Dissociative Hallucinogen Dextromethorphan on Cognition}

Frederick Barrett*, Theresa Carbonaro, Matthew Johnson, Roland Griffiths

Johns Hopkins University School of Medicine, Baltimore, Maryland, United States

Background: Classic hallucinogens (serotonin $2 \mathrm{~A}$ receptor agonists) and dissociative hallucinogens (NMDA receptor antagonists) have a wide range of neuropsychological effects. Drugs of both classes are being pursued as novel treatments for mood disorders. While classic and dissociative hallucinogens have different pharmacologic mechanisms of action, they may have similar subjective and neuropsychological effects. The objective of this double-blind, placebo controlled crossover study was to compare the neuropsychological effects of multiple doses of the classic hallucinogen psilocybin with the effects of a single high dose of the dissociative hallucinogen dextromethorphan.

Methods: Twenty hallucinogen users $(11 \mathrm{~F}, 9 \mathrm{M})$ completed five drug administration sessions lasting 6 to 8 hours each. During each session, volunteers self-administered capsules 
containing placebo, one of three doses $(10,20$, and $30 \mathrm{mg} /$ $70 \mathrm{~kg}$ ) of psilocybin, or a dose dextromethorphan (DXM; $400 \mathrm{mg} / 70 \mathrm{~kg}$ ). Dose order was counter-balanced across volunteers using a 5-order Williams design. Before capsule administration and at peak drug effect, volunteers were assessed for overall cognitive impairment using the MiniMental State Examination. Computerized neuropsychological tasks measuring psychomotor performance (the Motor Praxis test), working (the Letter N-Back test) and episodic memory (word encoding, recall, and recognition), executive function and associative learning (the Digit Symbol Substitution Task, or DSST), and visual perception (the Penn Line Orientation Task, or PLOT) were assessed at various points throughout the timecourse of drug effects. The DSST and episodic memory tasks were programmed within the Presentation software package. The Motor Praxis, Letter N-Back, and PLOT tasks were administered as part of the Penn Computerized Neurocognitive Battery (CNB). Multiple task forms were administered in order to minimize learning effects. Task order was counterbalanced across participants, and orthogonalized in relation to dose order. Outcome measures for each task were analyzed using a mixed-effects repeated measures ANCOVA using the lmer function of the lme4 library and the lmerTest library within the R statistical environment. Responses were nested within subject, testing a main effect of drug condition and using average mpraxis response times for the given assessment period as a covariate to control for general effects of psychomotor slowing on performance of each task. For the DSST and Letter N-Back analyses, responses were also nested within drug condition and a main effect of time point (for the DSST) or task condition (for the Letter N-Back) was included, as DSST was assessed at multiple timepoints within each session, and the Letter N-Back contained multiple task conditions.

Results: Overall cognitive impairment, as assessed with the Mini-Mental State Examination, was not observed with psilocybin or DXM. Orderly and dose-dependent effects of psilocybin were observed, and consisted of impairments of psychomotor performance, working memory, episodic memory, associative learning, and visual perception. Effects of a high dose of DXM were observed, and consisted of impairments of psychomotor performance, working memory, visual perception, and associative learning that were in the range of effects of a moderate to high dose $(20$ to $30 \mathrm{mg} /$ $70 \mathrm{~kg}$ ) of psilocybin. Psilocybin had greater effects than DXM on measures of working memory. DXM had greater effects than all doses of psilocybin on measures of word recognition sensitivity, response inhibition, and executive control.

Conclusions: Psilocybin exerted selective effects on working memory, whereas DXM selective effects on episodic memory, response inhibition, and executive control. Notably, this is the first time that dose-dependent effects of psilocybin were demonstrated for measures related to verbal working memory, episodic memory, and executive function. Future studies should determine whether any of these component processes of cognition are necessary, sufficient, or supportive of therapeutic outcomes associated with classic psychedelics or dissociative hallucinogens.

Keywords: Hallucinogens, Comparative Neuropsychopharmacology, Cognitive Functioning, Psilocybin, Dextromethorphan/Quinidine

Disclosure: Nothing to Disclose.
M275. Stress Promotes Drug Seeking Through Glucocorticoid-Dependent Endocannabinoid Mobilization in Prelimbic Cortex

John Mantsch*, Jayme McReynolds, Elizabeth

Doncheck, Yan Li, Oliver Vranjkovic, Evan Graf, Daisuke Ogasawara, Benjamin Cravatt, David Baker, Qing-song Liu, Cecilia Hillard

Marquette University, Milwaukee, Wisconsin, United States

Background: Clinical evidence suggests that, rather than directly driving cocaine use, stress may create a biological context within which other triggers for drug use become more potent. We hypothesize that stress-induced increases in corticosterone "set the stage" for relapse via a cannabinoid receptor 1 (CB1R) endocannabinoid-induced attenuation of inhibitory transmission in the prelimbic cortex (PL) and increased excitability of pyramidal neurons that project from the PL to the nucleus accumbens (NAc) core.

Methods: We have established a rat model for the stagesetting effects of stress. In this model, neither a stressor (electric footshock) nor corticosterone administration $(2.0 \mathrm{mg} / \mathrm{kg}$, ip) alone reinstates cocaine seeking following self-administration and extinction, but each treatment potentiates reinstatement in response to an otherwise subthreshold cocaine priming dose $(2.5 \mathrm{mg} / \mathrm{kg}$, ip). We determined the contributions of endocannabinoid signaling in the PL to the effects of stress-level corticosterone on PL neurotransmission and cocaine seeking using intra-PL micro-infusions. Endocannabinoid-dependent effects of corticosterone on inhibitory synaptic transmission in the rat PL were determined using whole-cell recordings in layer $\mathrm{V}$ pyramidal neurons. Effects on PL neurons that project to the NAc core were determined by recording from neurons labelled using intra-NAc RetroBead injections. The relationship between activation of the cortico-accumbens pathway and corticosterone-potentiated reinstatement was assessed by analyzing PL Fos expression in cholera toxin B (ctB) immunoreactive neurons following intra-NAc ctB injections. Results: Electric footshock stress potentiated cocaineinduced reinstatement, an effect that was recapitulated by corticosterone administration, at a dose that reproduced stress levels in blood, and by intra-PL corticosterone injections. Corticosterone application attenuated inhibitory synaptic transmission in the PL via CB1R- and 2arachidonoylglycerol (2-AG)-dependent inhibition of GABA release without altering postsynaptic responses. These CB1Rdependent effects of corticosterone were also observed specifically in RetroBead-labeled PL neurons that project to the NAc core. Both shock- and corticosterone-potentiated were blocked by intra-PL injections of the CB1R antagonist, AM251. Moreover, the ability of systemic stress-level corticosterone treatment to potentiate cocaine-primed reinstatement was recapitulated by intra-PL injections the CB1R agonist WIN 55-212,2, or the monoacylglycerol lipase inhibitor URB602 and attenuated by intra-PL injections of the diacylglycerol lipase inhibitor, DO34, indicating a role for increases in PL 2-AG. Preliminary results suggest that corticosterone-potentiated cocaine seeking is associated with a Fos response in PL neurons that project to the NAc core as 
indicated by increased Fos expression in ctB-positive neurons in the PL following intra-NAc ctB injections.

Conclusions: These findings suggest that stress-induced increases in corticosterone promote cocaine seeking by mobilizing 2-AG in the $\mathrm{PL}$, resulting in CB1R-mediated attenuation of inhibitory transmission in this brain region.

Keywords: Relapse, Endocannabinoids, Glucocorticoids, Stress, Prefrontal Cortex

Disclosure: Part 1: Promentis Pharmaceuticals, Consultant.

M276. The Persistence and Dose-Dependency of Acute Marijuana Effects on Brain Circuits Underlying Time Estimation

Michael Stevens*, Nic Ward, Erwin Boer, Vince Calhoun, Diana King, Lindsey Repoli, Godfrey Pearlson

Yale University School of Medicine, Hartford, Connecticut, United States

Background: As more states legalize cannabis (CNB) for both medical and recreational use, both scientists and public policy-makers have become interested in understanding how CNB alters cognition. It is important to learn what brain activation changes underlie well-described effects of CNB on cognitive functions whose impairment might pose safety risks during motor vehicle operation or in the workplace. A well-known effect of CNB intoxication is distorted time perception. Several neurocognitive studies have confirmed this subjective effect by finding CNB increases one's "internal clock," resulting in over-estimation of time intervals. This study used fMRI to examine CNB effects on brain activation during mental time interval discrimination. Study goals included describing dose-dependency of immediate CNB effects on brain function and determining with repeat fMRI scans at what point those effects dissipate after use. We hypothesized that CNB use would impair activation of cerebellum and basal ganglia regions known to underlie time discrimination, as well as alter the engagement of attention and executive neural circuits. Because the limited available literature suggests most CNB-induced cognitive decrements are not long-lived, we predicted any neural effects would largely resolve by 5 hours after CNB use.

Methods: As part of an ongoing 5-year NIDA-funded study (R01DA038807), fMRI data were collected from $n=7$ regular CNB users (near-daily use of 1 or more joints, at least 4 times per week for the prior 3 months). Structured clinical interview (SCID-V) confirmed the absence of all current DSM-V psychiatric diagnoses. On three separate occasions, participants used a paced inhalation method with vaporizer to smoke marijuana, receiving either moderate-dose $(13.4 \%$ THC), low-dose (5.9\% THC), or placebo in a randomized order across visits. On each visit, participants underwent fMRI three separate times after dosing $\left(1^{1 / 2} \mathrm{~h}, 3^{1 / 2} \mathrm{~h}\right.$ and $51 / 2 \mathrm{~h}$ post-drug use). In each session, they completed a time estimation fMRI task that required them to judge which of two successively presented square stimuli was on the screen for a longer duration. Because prior research has shown a preferential role for cerebellum and basal ganglia in processing sub- versus supra-second intervals, respectively, the task included both interval lengths. Whole brain fMRI maps representing activation to either sub-second or suprasecond conditions were examined in a series of different SPM12 statistical models. As data collection is ongoing, preliminary results were examined using $p<.05$ uncorrected significance levels.

Results: One hour after $\mathrm{CNB}$ use, time estimation-elicited brain activation profiles differed from placebo in comparable ways for both low and moderate doses, and for both supraand sub-second interval discrimination. Compared to placebo, CNB reduced the level of activation in bilateral putamen - regions rich in CB1 receptors and known to be engaged for time interval discrimination. However, this effect did not differ by dose. CNB also reduced activation in brain regions that comprised parts of the frontoparietal executive and ventral attention networks. By way of possible compensation, the dorsal anterior cingulate, supplementary motor and motor cortices were more greatly engaged after CNB use compared to placebo in all conditions and doses. Recovery from these CNB effects depended on dose. Each dose had a different profile of brain function changes throughout the day. For the moderate CNB dose condition, executive and ventral attention network activation increased over time, while cerebellar, caudate, and right ventrolateral prefrontal activation decreased. For the low CNB dose condition, brain regions in the dorsal attention network increased their activation levels over time, while cinguloopercular salience network regions decreased. In contrast to our prediction, by 5 hours after CNB administration brain activation had not yet returned to normal. Putamen activation was still lower than placebo for the moderate, but not low dose condition. Numerous brain regions that were part of both dorsal and ventral attention networks continued to show lower activity than placebo after all doses and in both fMRI task conditions.

Conclusions: Although these results are preliminary due to the small sample collected so far, the observed effects are noteworthy because they provide evidence that brain function-altering effects of CNB likely persist well after the typical, relatively brief period of intoxication. CNB use resulted in lower activation for striatal brain regions known to play an important role in mental timekeeping cognitive processes, as well as for a variety of distributed neural systems engaged for higher-order cognition. As the study sample accrues towards an eventual $n=80$ planned sample size of both regular and occasional CNB users, it will be possible to examine behavioral performance deficits on the time estimation task, link any performance decrements to brain function to isolate which neural changes might underlie the observable effects of $\mathrm{CNB}$ and detect possibly smaller effects of $\mathrm{CNB}$ dose on regional brain function changes. Ultimately upon study completion, the results should provide a useful context to evaluate national and state policy determinations about CNB use decriminalization, penalties for operating motor vehicles while under the influence, and might provide information that guides development of roadside CNB intoxication detection.

Keywords: Marijuana, Functional MRI (fMRI), DoseResponse Analyses, Time Estimation

Disclosure: Nothing to Disclose. 
M277. Endocannabinoid Receptor-Mediated Effects on Cocaine Memory Reconsolidation and Subsequent Drug Context-Induced Cocaine-Seeking Behavior

Jessica Higginbotham, Nicole Jones, Shi Tan, Rong Wang, Mark Presker, Rita Fuchs*

Washington State University, Pullman, Washington, United States

Background: Environmentally induced drug craving and relapse are major impediments to the successful treatment of cocaine addiction. This phenomenon depends on the availability of salient drug-context associative memories. Remarkably, associative memories are not permanently preserved in long-term memory stores after consolidation. Instead, they can become unstable upon retrieval and must undergo subsequent restabilization, or reconsolidation, to be maintained over time. Therefore, targeted disruption of cocaine-memory reconsolidation may weaken drug-context memories and reduce subsequent drug context-induced cocaine-seeking behavior.

Endocannabinoid receptor 1 (CB1R) stimulation facilitates presynaptic inhibition and regulates synaptic plasticity associated with memory processes, including memory reconsolidation. However, the role of CB1Rs in cocaine memory reconsolidation has not been examined in instrumental models of drug relapse. Hence, here we evaluated the effects of systemic or intra-basolateral amygdala CB1R antagonist administration following memory reactivation on long-term memory integrity following memory reconsolidation, as well as on serum corticosterone concentrations and on known cellular mediators of memory reconsolidation following memory retrieval.

Methods: Rats were trained to lever press for cocaine infusions in a distinct environmental context during 10 daily two-hour sessions, followed by extinction training in a different context during seven daily two-hour sessions. On post-cocaine day 8 , rats were exposed to the cocaine-paired context for 15 minutes to reactivate cocaine memories and trigger memory reconsolidation. Groups received vehicle or the CB1R antagonist, AM251, systemically (3 mg/kg, IP) or into the basolateral amygdala (BLA; $0.3 \mathrm{ug} / 0.5 \mathrm{ul} /$ side), a brain region involved in cocaine memory reconsolidation. These manipulations were administered immediately after the memory reactivation session or after a no reactivation control manipulation (i.e., 6 - $\mathrm{h}$ after the memory reactivation session or after home cage stay). In experiment 1 , the effects of these manipulations were assessed on reinstatement of extinguished cocaine-seeking behavior (nonreinforced lever presses) in the previously cocaine-paired context rats after two additional extinction sessions in the extinction context. In experiment 2, samples for serum corticosterone assays were collected from intra-BLA AM251- and vehicle-treated groups immediately before and at 30-minute intervals after the memory reactivation session. In experiment 3 , rats were euthanized 45 minutes after the memory reactivation session. Their brains were dissected for the analysis of zinc finger 268 (zif268) expression using Western blotting.

Results: Systemic AM251 treatment impaired, whereas intraBLA AM251 treatment enhanced, the ability of the cocainepaired context to reinstate extinguished cocaine-seeking behavior, relative to vehicle. The effects of AM251 were dependent on memory reactivation, indicating AM251induced alterations in cocaine memory reconsolidation. Systemic AM251 treatment inhibited a memory reactivation-associated increase in zif268 expression in the BLA. Furthermore, intra-BLA AM251 treatment potentiated a cocaine memory reactivation-associated increase in serum corticosterone levels, relative to vehicle.

Conclusions: The findings suggest that CB1R populations are functionally heterogeneous. CB1R in the BLA play an inhibitory role in cocaine-memory reconsolidation, possibly by stabilizing HPA axis function following memory retrieval. However, stimulation of other, as yet unidentified CB1R populations plays a facilitating role in cocaine memory reconsolidation, possibly by diminishing zif268 and downstream gene expression.

Keywords: Cocaine Seeking, Memory Reconsolidation, CB1 Receptor, Basolateral Amygdala

Disclosure: Nothing to Disclose.

M278. Effects of the CHRNA5 rs16969968 Genetic Polymorphism on Anterior Cingulate-Striatal Circuit Strength and Alcohol Use in Social Drinkers

Joshua Gowin*, Salvatore Torrisi, Alyssa Schneider, Melanie Schwandt, David Goldman, Elliot Stein, Monique Ernst, Reza Momenan, Vijay Ramchandani

National Institute on Alcohol Abuse and Alcoholism, Bethesda, Maryland, United States

Background: Alcohol use disorder prevalence is substantially higher in smokers than non-smokers, and neurobiological evidence implicates the nicotinic acetylcholine receptor activity near the ventral striatum in the reinforcing properties of alcohol consumption. While the CHRNA5 Asp398Asn genetic polymorphism has been associated with both increased risk of nicotine dependence and decreased resting state functional connectivity between the dorsal anterior cingulate cortex and the ventral striatum, no previous study has examined its effect on intrinsic functional connectivity in relation to alcohol use. Prior evaluation of alcohol selfadministration data in a previous study revealed a preliminary finding of lower alcohol consumption in a human lab setting in non-smoking minor A-allele carriers. Thus, we hypothesized that the Asp398Asn polymorphism would be associated with lower alcohol consumption and reduced functional connectivity between the dorsal anterior cingulate and the ventral striatum.

Methods: We examined a previously collected sample of non-smoking social drinkers who had undergone a functional MRI scan at rest. We restricted our sample to 66 individuals of European ancestry because the Asp398Asn frequency is small in other ancestries. Allele frequencies did not differ from Hardy-Weinberg equilibrium. We excluded individuals with excessive head motion during their scans. Motion scrubbing was performed at time points with $>0.2 \mathrm{~mm}$ motion between them, and criteria for subject exclusion was if scrubbing was performed on $>20 \%$ of a given subjects' data. Our final sample included 27 398Asn homozygotes and 28 Asp398Asn allele carriers. Analyses were performed with FreeSurfer and AFNI. Left and right 
dorsal anterior cingulate seeds were generated by FreeSurfer and warped to MNI space. Time courses were extracted from these seeds and correlated with voxel activity across the brain. Primary group analyses included a region-of-interest analysis of circuit strength to bilateral nucleus accumbens masks, also generated by FreeSurfer. An exploratory whole brain analysis was conducted via a linear mixed-effects model covarying for scanner type, as scanning was conducted on Siemens and GE scanners.

Results: Groups did not differ on gender, age, IQ, or socioeconomic status. Groups also did not differ on current drinking patterns or drinking and smoking history. Across groups, there was robust functional connectivity between seeds in the left and right dorsal anterior cingulate cortex and the medial prefrontal cortex, bilateral insula, and posterior cingulate cortex. Region of interest analysis revealed that Asp398Asn carriers did not differ from 398Asn homozygotes (all Cohen's $\mathrm{d}<0.35$, all $p>0.20$ ). Circuit strength was not correlated with drinking patterns for any of these regions. Whole-brain analysis revealed no significant clusters.

Conclusions: The results of this retrospective candidate gene study do not support a role of the CHRNA5 rs16969968 polymorphism on alcohol consumption in social drinkers. They also do not support the hypothesis that this polymorphism is associated with altered functional connectivity between seeds in the dorsal anterior cingulate and ventral striatum. Further work is ongoing to better understand the relationship between this polymorphism and altered cortico-striatal functional connectivity as well as with alcohol consumption and response phenotypes in social drinkers.

Keywords: Resting State Functional Connectivity, Human Genetics, Nicotinic Acetylcholine Receptors, Nucleus Accumbens, Anterior Cingulate Cortex

Disclosure: Nothing to Disclose.

\section{M279. Divergent Changes in Brain and Behavior Caused} by Different Patterns of Morphine Exposure in Mice

Kerry Trotter, Emilia Lefevre, Marc Pisansky, David Leipold, Federico Buffaldi, Marco Pravetoni, Patrick Rothwell ${ }^{*}$

University of Minnesota, Minneapolis, Minnesota, United States

Background: Many studies have characterized the behavioral changes that develop during chronic opiate exposure in rodents. While antinociceptive tolerance is commonly observed, other behavioral effects (like psychomotor activation and conditioned place preference) can be either enhanced or reduced by prior opiate exposure. This variability is often related to the pattern of chronic opiate administration, with intermittent exposure causing sensitization and continuous exposure causing tolerance. However, few studies have conducted well-controlled comparisons between different patterns of opiate exposure, and investigated how behavioral changes correlate with adaptations in the mesolimbic dopamine system.

Methods: In an initial series of experiments, we assessed morphine-induced antinociception and psychomotor activation across a range of doses, and compared the effects of intermittent exposure (daily injections) with continuous exposure (via subcutaneously implanted osmotic minipumps). We used two additional strategies to manipulate the continuity of morphine exposure. First, we implanted osmotic minipumps to continuously deliver morphine, and administered daily naloxone injections to interrupt opioid receptor stimulation. Second, we implanted miniaturized mechanical pumps (iPrecio SMP-300) that were programmed to alternate between 24 hours of continuous morphine delivery and 24 hours of inactivity.

Results: All patterns of chronic morphine exposure caused a similar degree of antinociceptive tolerance. However, intermittent exposure via daily injection caused long-lasting sensitization of psychomotor activation, whereas continuous exposure with osmotic minipumps caused transient psychomotor tolerance. These behavioral differences were apparent after controlling for factors such as total drug dose and peak analgesic effect. Interrupting the continuity of drug delivery, by either daily naloxone injection or programmed periods of pump inactivity, reversed the development of psychomotor tolerance and instead tended to cause sensitization. These behavioral differences were not related to either brain or serum morphine levels. Instead, preliminary data suggest changes in psychomotor activation are correlated with changes in the size of dopamine neurons in the ventral tegmental area, as well as altered inhibitory synaptic transmission in the nucleus accumbens.

Conclusions: These studies provide a framework for our lab's ongoing investigation of the distinct neurobiological consequences of different patterns of opiate exposure. Our results may inform the way opiates are used clinically to treat pain, and suggest new strategies to reduce opiate abuse.

Keywords: Morphine, Mouse Behavior, Sensitization, Dopamine, Nucleus Accumbens

Disclosure: Nothing to Disclose.

M280. Sex-Dependent Differences in Nicotine SelfAdministration on a Second-Order Schedule and Varenicline Treatment Efficacy

Christopher Larsen, Brady Thompson, Wendy Huynh, Isabella Catalano, Jordan Wordekemper, Scott Barrett, Rick Bevins, Jennifer Murray*

University of Nebraska-Lincoln, Lincoln, Nebraska, United States

Background: Varenicline is commonly prescribed as a smoking cessation aid (Chantix ${ }^{\circ}$ ) and is the most effective pharmacotherapy available. In humans, the treatment regimen involves a period of pre-quit exposure, in which the individual takes the varenicline in advance of their quit date and continues for a time into abstinence. However, most of the basic research investigating varenicline as a pharmacotherapy in rodent models uses it during extinction or as a way to prevent relapse. Those studies that do use repeated treatment during self-administration have only tested male rats. In these studies, repeated varenicline reduced nicotine self-administration in a simple schedule of reinforcement in male rats. Further, acute treatment with varenicline disrupted drug-induced reinstatement of nicotine selfadministration in males but not females. Notably, in human 
smokers, women, but not men, benefit from extended varenicline treatment in advance of smoking cessation. These effects suggest that short-term pre-quit treatment may be effective for males to reduce nicotine self-administration, however, females are likely to require prolonged exposure for any direct therapeutic effects of varenicline to develop.

Methods: Male and female Sprague-Dawley rats were implanted with indwelling jugular catheters under general anesthesia with analgesic relief. Following seven full days of recovery, rats were trained to self-administer nicotine $(0.03 \mathrm{mg} / \mathrm{kg} /$ infusion $)$ in daily 2 -hr sessions. The initial schedule of reinforcement was a FR1 (fixed ratio 1). On this schedule, a single depression of the active lever (active and inactive lever assignment counterbalanced across rats) resulted in a 1-sec infusion of nicotine coincident with the start of a $20-\mathrm{sec}$ timeout. During the timeout, both levers were retracted, the house light was extinguished, and the cue light above the active lever was illuminated. After eight training sessions on a FR1, the reinforcement schedule was gradually shifted to a FI5(FR2:S) (fixed interval 5; fixed ratio 2:stimulus) second-order schedule. In this schedule, nicotine seeking was maintained over 5-min delays by $1-\mathrm{sec}$ presentations of the light cues for every other active lever press. Use of this schedule allows for the assessment of active lever pressing to be separated from that of the number of drug infusions. Following stabilization, half of each sex began chronic varenicline testing $(1 \mathrm{mg} / \mathrm{kg})$. These rats received subcutaneous varenicline injections $30 \mathrm{~min}$ before each standard nicotine self-administration session; the remaining rats received saline injections. After a stable reduction in nicotine self-administration, nicotine was removed and the effects of varenicline treatment on extinction were assessed. All procedures were conducted in accordance with University of Nebraska-Lincoln Institutional Animal Care and Use Committee (IACUC \#1477). The animal welfare assurance number for UNL is A3459-01. University of Nebraska animal facilities are AAALAC accredited.

Results: Female rats initially showed higher levels of nicotine self-administration on the FR1 schedule than male rats. This level was stable across the FR1 training phase, whereas male rats increased their intake across sessions. As training progressed to the fixed interval schedules, however, males began to show higher levels of nicotine infusions compared to females. In contrast, females and males showed similar levels of active lever pressing until females stabilized at a lower level of behavior at the end of second-order schedule training. Varenicline treatment during this phase significantly reduced active lever pressing and nicotine selfadministration in males faster than in females.

Conclusions: This study included chronic varenicline treatment during nicotine self-administration sessions to emulate the pre-quit period of human tobacco users with an explicit emphasis on investigating sex differences. Females in the present study showed more stable levels of nicotine selfadministration than males. Females also required a longer treatment with varenicline during continued selfadministration sessions to reduce their nicotine intake compared with males. This suggests differential sensitivity to direct pharmacological manipulation of the nicotine action. Further, the results corroborate findings with human smokers that women benefit from extended varenicline treatment in advance of a quit attempt. Combined, the research implies that clinical practice may be improved by incorporating different treatment protocols for women versus men.

Keywords: Pharmacotherapy, Varenicline, Sex Differences, Nicotine, Intravenous Drug Self-Administration

Disclosure: Nothing to Disclose.

M281. N-Acetylcysteine Inhibits Cue-Induced Nicotine Seeking in a GLT-1-Dependent Manner and Suppresses Proinflammatory TNFo Expression in the Nucleus Accumbens Core

Cassandra Gipson-Reichardt*, Mark Namba, Gregory Powell, Julianna Goenaga, Armani Del Franco, Joseph McCallum

\section{Arizona State University, Tempe, Arizona, United States}

Background: Nicotine self-administration is associated with decreased expression of the glial glutamate transporter (GLT-1) and the cystine-glutamate exchange protein $\mathrm{xCT}$. Additionally, chronic use of nicotine leads to alterations in brain homeostasis, including increased oxidative stress and glutamatergic hyperactivity. Thus, addiction to nicotine may be characterized as a systemic pro-inflammatory condition. $\mathrm{N}$-acetylcysteine (NAC) is an antioxidant used in clinical studies in the treatment of various substance use disorders including tobacco. Importantly, NAC restores key glutamatergic proteins associated with increased drug relapse vulnerability. However, the specific molecular mechanisms driving its inhibitory effects on cue-induced nicotine relapse are unknown.

Methods: In the present study, rats were trained to selfadminister nicotine $(0.02 \mathrm{mg} / \mathrm{kg} /$ infusion $)$ and underwent extinction training, where they received chronic NAC and an antisense vivo-morpholino designed to selectively suppress GLT-1 expression in the nucleus accumbens core (NAcore). Following extinction, rats were tested for cue-induced nicotine reinstatement. NAcore GLT-1 and proinflammatory tumor necrosis factor alpha $(\mathrm{TNF} \alpha)$ protein expression was then assessed using Western blot.

Results: Chronic NAC treatment $(100 \mathrm{mg} / \mathrm{kg})$ significantly attenuated cue-induced nicotine seeking. As well, GLT-1 antisense vivo-morpholino significantly suppressed GLT-1 levels in the NAcore, which blocked the attenuating effect of NAC on reinstatement. Unlike what has been observed with cue-induced cocaine reinstatement, inhibition of GLT-1 expression and administration of NAC did not augment reinstatement over and above control conditions. Intriguingly, chronic NAC treatment significantly reduced proinflammatory $\mathrm{TNF} \alpha$ expression in the NAcore following reinstatement, suggesting a potential anti-inflammatory mechanism underlying NAC's therapeutic potential.

Conclusions: These results suggest that while GLT-1 may be a conserved neurobiological substrate underlying relapse vulnerability across drugs of abuse, there may be neuroimmunological mechanisms that modulate nicotine relapse and associated glutamatergic plasticity. As well, NAC decreased expression of a nicotine-induced neuroinflammatory marker illustrating neuroimmunomodulation as a key component to its efficacy in promoting nicotine use cessation. 
Keywords: Neuroinflammation, Nicotine Addiction, Glutamate Homeostasis, Glutamate Transport, Relapse

Disclosure: Nothing to Disclose.

\section{M282. Cycooxygenase-2 Inhibition for Cannabis Withdrawal and Relapse: A Human Laboratory Study}

Margaret Haney*, Sachin Patel, Ziva Cooper, Gillinder Bedi, Evan Herrmann, Stephanie Reed, Richard Foltin

Columbia University, New York, New York, United States

Background: Treatment outcome for Cannabis Use Disorder (CUD) is poor, with few patients achieving continued cannabis abstinence. One factor that contributes to high relapse rates is cannabis withdrawal (negative mood, disrupted sleep and food intake), which may be related to deficits in the physiological function of endogenous cannabinoid (eCB) systems. Increasing brain levels of the eCBs anandamide (AEA) and 2-arachidonoylglycerol (2-AG) by inhibiting their degradation with FAAH or MAGL inhibitors, respectively, reduces signs of precipitated cannabinoid withdrawal in mice. Preclinically, we have identified cyclooxygenase-2 (COX-2) as a third enzyme that inactivates brain eCBs, and have demonstrated that blocking COX-2 activity with non-steroidal anti-inflammatory drugs increases central eCB levels. There is also preclinical evidence that $\Delta 9$-tetrahydrocannabinol (THC) administration increases brain COX-2 expression via CB1 activation. These findings suggest that: (1) chronic THC exposure up-regulates COX-2 expression, which in turn inactivates eCBs, (2) this eCB deficiency contributes to continued cannabis use, and (3) blocking COX-2 activity may normalize eCB levels and reduce withdrawal and relapse. The primary objective of this placebo-controlled study was to test the efficacy of the FDAapproved COX-2 selective inhibitor, Celecoxib, in a human laboratory model of CUD that assesses medication effects on cannabis' positive subjective effects, withdrawal symptoms and a model of relapse. A secondary objective was to assess the effects of celecoxib on plasma levels of eCBs and related compounds under conditions of cannabis abstinence and non-abstinence.

Methods: This within-subject study comprised two 11- day phases, with one phase testing celecoxib (200 mg BID) and the other testing placebo ( $0 \mathrm{mg}$ BID) in counter-balanced order. An outpatient phase ( $>14$ days) with no medication administration separated the two inpatient phases to provide time for medication clearance. On the first two inpatient days, participants smoked active cannabis (5.6\% THC) 6 times/day under controlled conditions; this standardized recent cannabis exposure and allowed assessment of celecoxib's effects on measures of cannabis intoxication. For the next 4 days, only placebo cannabis $(0.0 \%$ THC) was available for self-administration (allowing for measures of cannabis withdrawal), followed by 3 days when active cannabis was available at a financial cost for selfadministration (a laboratory measure of relapse). Ratings of mood, drug-related effects, sleep, daily caloric intake and cognitive task performance were assessed repeatedly. Plasma was drawn upon awakening (8:30AM) under non-abstinent
(11 hours post-cannabis) and abstinent (4.5 days postcannabis) conditions during both celecoxib and placebo maintenance conditions.

Results: Male $(n=12)$ and female $(n=3)$ daily cannabis smokers $(5.3+5.5$ grams/day) completed the study. Cannabis Intoxication: On days of controlled cannabis administration, celecoxib significantly improved ratings of sleep (sleep satisfaction, number of hours slept), while also increasing ratings of cannabis craving relative to placebo. Cannabis Abstinence: Under placebo conditions, abstinence from active cannabis produced significant symptoms of withdrawal relative to active cannabis administration, including: (1) negative mood (increased ratings of 'miserable,' 'anxious,' 'irritable;' decreased ratings of 'friendly,' (2) sleep disruption (decreased sleep efficiency, increased latency to fall asleep, increased dreaming) and (3) weight loss. Celecoxib significantly reversed some abstinence-related effects. Relative to placebo, celecoxib improved sleep (number of hours slept) and prevented weight loss; there was a trend $(p<0.08)$ for celecoxib to decrease withdrawal-related anxiety while increasing ratings of fatigue. Celecoxib also increased cannabis craving. Cannabis relapse: Under placebo medication conditions, $40 \%$ of the participants 'relapsed,' i.e., paid to self-administer cannabis after 4 days of abstinence, purchasing an average of $9.9 \pm 2.7$ cannabis puffs/day. Celecoxib did not alter the amount of cannabis selfadministered relative to placebo. Plasma Measures: Celecoxib did not significantly alter plasma levels of AEA, 2-AG, arachidonic acid, oleoyl ethanolamide (OEA) or 2-oleoyl glycerol (2-OG). However, cannabis abstinence was associated with significant increases in AA and OEA and reductions in 2-OG relative to non-abstinent conditions. The physiological significance of these changes is unclear at this time, however that cannabis abstinence is associated with changes in eCB-related lipids regulated by FAAH and MAGL suggests the possibility that alterations in the enzymatic activities of these enzymes could occur during cannabis abstinence. Data analysis for other measures (caloric intake, cognitive task performance) are underway.

Conclusions: These preliminary findings suggest that although celecoxib was well tolerated and had some positive effects on sleep and mood during cannabis withdrawal, our hypothesis that this dose of medication would increase plasma eCBs, attenuate a range of withdrawal symptoms and ultimately reduce a laboratory measure of cannabis relapse was not supported. Further, cannabis craving was consistently enhanced by celecoxib, although there was no concomitant increase in self-administration. For this proof of concept study, we only tested one dose of medication, selected based on estimated maximal tolerability. There were signals that centrally-mediated eCBs may have been affected by celecoxib given the changes in mood, body weight, and sleep observed after celecoxib treatment. Given the low incidence of side effects at $200 \mathrm{mg}$ BID, higher FDAapproved doses could be considered in future studies.

Keywords: Marijuana, Endocannabinoids, Cannabis Use Disorder

Disclosure: Nothing to Disclose. 